\title{
Transformer Efficiency Assessment - Okinawa, Japan
}

Jason W. Bush

Robert J. Turk

Kurt S. Myers

Jake P. Gentle

Thomas L. Baldwin

May 2012

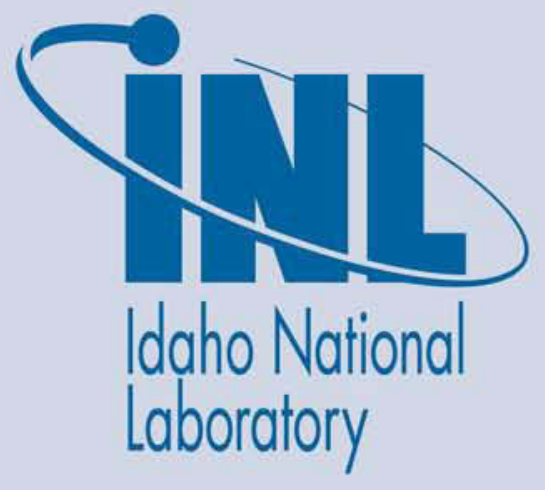

The INL is a U.S. Department of Energy National Laboratory operated by Battelle Energy Alliance 
INL/EXT-12-26145

Revision 1

\title{
Transformer Efficiency Assessment - Okinawa, Japan
}

\author{
Jason W. Bush \\ Robert J. Turk \\ Kurt S. Myers \\ Jake P. Gentle \\ Thomas L. Baldwin
}

May 2012

\author{
Idaho National Laboratory \\ Idaho Falls, Idaho 83415 \\ http://www.inl.gov
}

\author{
Prepared for the \\ U.S. Department of Energy \\ Assistant Secretary for Energy Efficiency \& Renewable Energy \\ Under DOE Idaho Operations Office \\ Contract DE-AC07-05ID14517
}




\section{DISCLAIMER}

This information was prepared as an account of work sponsored by an agency of the U.S. Government. Neither the U.S. Government nor any agency thereof, nor any of their employees, makes any warranty, expressed or implied, or assumes any legal liability or responsibility for the accuracy, completeness, or usefulness, of any information, apparatus, product, or process disclosed, or represents that its use would not infringe privately owned rights. References herein to any specific commercial product, process, or service by trade name, trade mark, manufacturer, or otherwise, does not necessarily constitute or imply its endorsement, recommendation, or favoring by the U.S. Government or any agency thereof. The views and opinions of authors expressed herein do not necessarily state or reflect those of the U.S. Government or any agency thereof. 


\section{Transformer Efficiency Assessment - Okinawa, Japan}

INL/EXT-12-26145

Revision 1

May 2012

Author

Title [optional]

Approved by:

Name

Title [optional]

Name

Title [optional]

Name

Title [optional]

Date
Approved by:

Date
Date

\section{Date}




\section{EXECUTIVE SUMMARY}

The United States Army Engineering \& Support Center in Huntsville, Alabama (USAESCH), and the U.S. Marine Corps Base (MCB) in Okinawa, Japan, retained the Idaho National Laboratory (INL) to conduct a Transformer Efficiency Assessment of various "key" distribution transformers located on Camps Foster, Hansen, and Kinser in Okinawa, Japan. This assessment supports MCB Okinawa in evaluating medium-voltage distribution transformers for potential efficiency upgrades. The original scope of work (SOW) included the MCB providing actual transformer nameplate data, manufacturer's factory test sheets, electrical system data $(\mathrm{kWh})$, demand data $(\mathrm{kWd})$, power factor data, and electricity cost data, but the MCB's actual data was not available. As such, INL has had to make some assumptions in place of actual data. Any similar nameplate data, photos of similar transformer nameplates, and basic electrical details from one-line drawings (provided by $\mathrm{MCB}$ ) are not to be considered replacements for actual load loss test data.

This report covers a generalized assessment of three sizes of modern U.S. market transformers (500 $\mathrm{kVA}, 750 \mathrm{kVA}$, and $1000 \mathrm{kVA}$ ) in three efficiency categories: Low-Level efficiency, Medium-Level efficiency, and High-Level efficiency. 36 Energy life-cycle cost (LCC) analyses for this project. The following assumptions were used for the LCC analyses:

- Operating period of 30 years

- On-line date of $1 / 1 / 13$

- Discount rate of $3 \%$

- Other rates and factors given by 2012 Federal Emergency Management Program rates

- $100 \%$ government funded

- $\mathrm{MCB}$ energy rates of $\$ 0.229$ per $\mathrm{kWh}$.

The LCC analyses brought to light several facts. Running transformers at light loading, such as the $20 \%$ load analyzed, makes any transformer efficiency upgrade hard to justify because the Simple Payback Periods (SPP) are greater than the 30-year life expectancy of the transformer. If at all possible, transformers should be loaded to at least $35 \%$. INL also found that low-level transformers would only be an option if initial price was the only factor for purchasing transformers and the energy losses experienced over the life of the transformer did not matter. This is because of INL's assumption that the transformers already in place in Okinawa are of a higher quality and efficiency than the low-level transformers evaluated. This analysis also showed that replacing a current transformer with a high- or medium-level transformer is justified as both levels of transformers have the ability to pay for themselves over their expected 30-year lifetime.

In the economic analysis, INL found that on some occasions replacing a transformer early with a high-level transformer is justified. INL recommends that load measurements are performed on the high and low sides of each transformer to better quantify actual load losses, demand data, and power factor data. INL also recommends that actual data, when available, be inserted by MCB Okinawa where assumptions have been made and that the LCC and economic analyses be updated, accordingly. INL believes if actual data is collected and inserted in place of the assumed data, other transformers will show to be ideal candidates for early replacement.

Transformer efficiency improvements are not the only place where energy savings can be recognized. Electrical energy savings is the primary goal, and potentially greater savings with a higher SIR could be achieved by considering building efficiency upgrades, such as insulation, lights, windows, doors, appliances, smart-grid meters, micro-grid control systems, and so forth. 
This page intentionally left blank. 


\section{CONTENTS}

Executive Summary iii

Acronyms vii

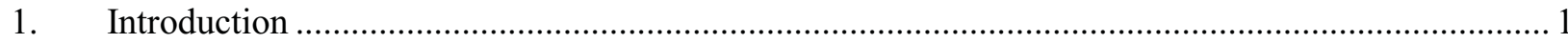

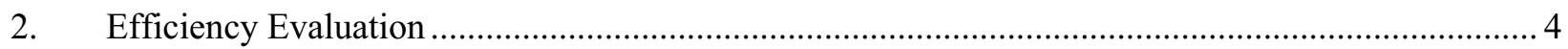

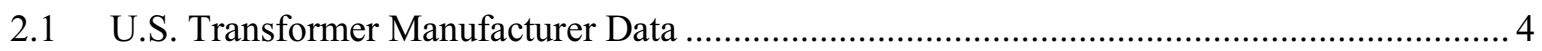

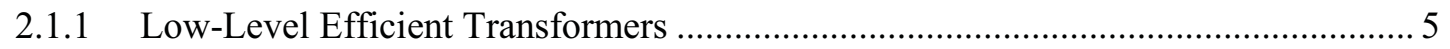

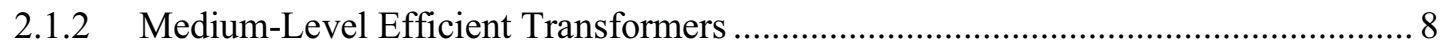

2.1.3 High-Level Efficient Transformers ................................................................. 12

2.2 Okinawa Transformer Assumptions ........................................................................... 15

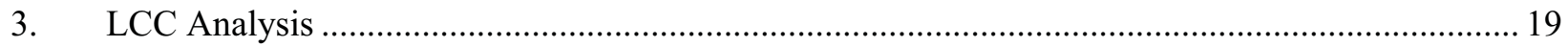

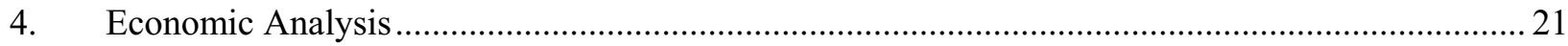

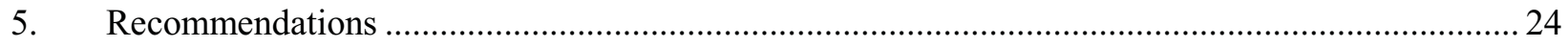

\section{FIGURES}

Figure 1. 30\% \& 70\% Efficiency Similarities .................................................................................... 3

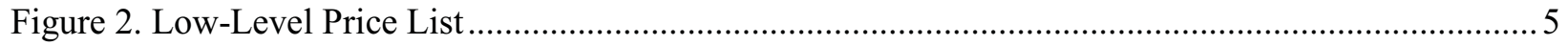

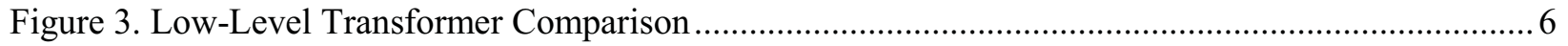

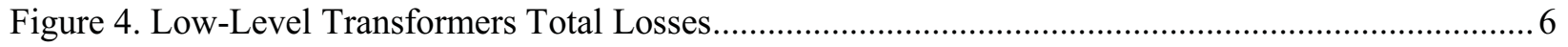

Figure 5 Efficiency Curve for Low-Level 500 kVA XFMR …............................................................ 7

Figure 6. Efficiency Curve for Low-Level 750 kVA XFMR ................................................................ 7

Figure 7. Efficiency Curve for Low-Level 1000 kVA XFMR .............................................................. 8

Figure 8. Medium-Level Price List .................................................................................................

Figure 9. Medium-Level Transformer Comparison............................................................................... 9

Figure 10. Medium-Level Transformer Total Losses .......................................................................... 10

Figure 11. Efficiency Curve for Medium-Level 500 kVA XFMR ........................................................ 10

Figure 12. Efficiency Curve for Medium-Level 750 kVA XFMR ....................................................... 11

Figure 13. Efficiency Curve for Medium-Level 1000 kVA XFMR ..................................................... 11

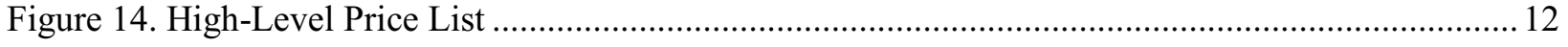

Figure 15. High-Level Transformer Comparison ............................................................................ 13

Figure 16. High-Level Transformers Total Losses .............................................................................. 13

Figure 17. Efficiency Curve for High-Level 500 kVA XFMR............................................................ 14

Figure 18. Efficiency Curve for High-Level 750 kVA XFMR............................................................. 14 
Figure 19. Efficiency Curve for High-Level 1000 kVA XFMR......................................................... 15

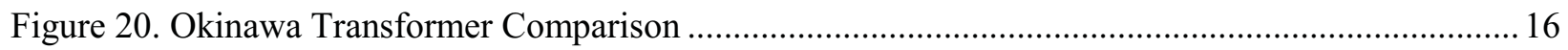

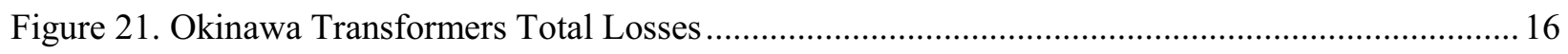

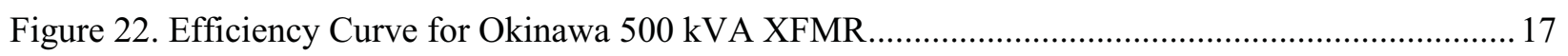

Figure 23. Efficiency Curve for Okinawa 750 kVA XFMR ................................................................. 17

Figure 24. Efficiency Curve for Okinawa 1000 kVA XFMR .............................................................. 18

\section{TABLES}

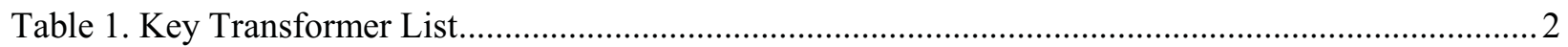

Table 2. Transformers Loaded (with Construction Costs) at 60\% LCC Results Summary.......................20

Table 3. Transformers Loaded (without Construction Costs) at 60\% LCC Results Summary.................. 20

Table 4. Transformer \$ Loss to EOL comparison at 60\% Loading ....................................................... 22

Table 5. Deltas between Okinawa Transformers and Medium/High Level Transformers ........................23 


\section{ACRONYMS}

$\begin{array}{ll}\text { AIRR } & \text { Adjusted Internal Rate of Return } \\ \text { DC } & \text { Design Cost } \\ \text { DOE } & \text { Department of Energy } \\ \text { ECIP } & \text { Energy Conservation Investment Program } \\ \text { EOL } & \text { End of Life } \\ \text { EULA } & \text { End User Lease Agreement } \\ \text { ESPC } & \text { Energy Savings Performance Contract } \\ \text { FEMP } & \text { Federal Emergency Management Program } \\ \text { INL } & \text { Idaho National Laboratory } \\ \text { kVA } & \text { Kilovolt-Ampere } \\ \text { kW } & \text { Kilowatt } \\ \text { kWd } & \text { Kilowatt-demand } \\ \text { kWh } & \text { Kilowatt-hour } \\ \text { LCC } & \text { Life cycle cost } \\ \text { MCB } & \text { Marine Corps Base } \\ \text { PF } & \text { Power Factor } \\ \text { SIOH } & \text { Supervision, Inspection, \& Overhead } \\ \text { SIR } & \text { Savings to Investment Ratio } \\ \text { SOW } & \text { Statement of Work } \\ \text { SPP } & \text { Simple Payback Period } \\ \text { TDOL } & \text { Total Discounted Operational Loss } \\ \text { TDOS } & \text { Total Discounted Operational Savings } \\ \text { UESC } & \text { Utility Energy Service Contract } \\ \text { VAR } & \text { Volt-amperes reactive power } \\ \text { XFMR } & \text { Transformer } \\ & \end{array}$


This page intentionally left blank. 


\section{Transformer Efficiency Assessment - Okinawa, Japan}

\section{Introduction}

The United States Army Engineering \& Support Center in Huntsville, Alabama (USAESCH), and the U.S. Marine Corps Base (MCB) in Okinawa, Japan, retained the Idaho National Laboratory (INL) to conduct a Transformer Efficiency Assessment of various "key" distribution transformers located on Camps Foster, Hansen, and Kinser in Okinawa, Japan. This assessment supports MCB Okinawa in evaluating medium voltage distribution transformers for potential efficiency upgrades. The original scope of work (SOW) included the MCB providing actual transformer nameplate data, manufacturer's factory test sheets, electrical system data $(\mathrm{kWh})$, demand data $(\mathrm{kWd})$, power factor data, and electricity cost data for use in the analysis, but that data was not available. As such, INL has had to make some assumptions in place of actual data. This report covers a generalized assessment of three sizes $(500 \mathrm{kVA}, 750 \mathrm{kVA}$, and $1000 \mathrm{kVA}$ ) of modern U.S. market transformers in three efficiency categories: Low-Level efficiency, Medium-Level efficiency, and High-Level efficiency.

An original list of 651 transformers (provided in Appendix A) has been reduced to a more manageable list of 50 transformers for the purpose of this report. This was done by identifying transformers that were 10 years old or older and by limiting transformers to the $500 \mathrm{kVA}-1000 \mathrm{kVA}$ size range. This list was given to the MCB who then identified transformers on that list that were already earmarked for replacement. The remaining transformers make up the final list of key transformers used in this evaluation, as shown in Table 1. 
Table 1. Key Transformer List

\begin{tabular}{|c|c|c|c|c|}
\hline Camp & Location & Capacity kVA & Mfr_Date & Serial_ID \\
\hline Foster & $5704 \mathrm{TS}$ & 500 & 1989 & 892221701 \\
\hline Foster & $5703 \mathrm{TS}$ & 500 & 1989 & 892221702 \\
\hline Foster & $478 \mathrm{TS}$ & 500 & 1997 & 9721148 \\
\hline Foster & $5696 \mathrm{TS}$ & 500 & 1991 & 3SD1250A1 \\
\hline Foster & $370 \mathrm{TS}$ & 500 & 1991 & 3SD1253A1 \\
\hline Foster & $481 \mathrm{TS}$ & 500 & 1996 & B50348AT1 \\
\hline Foster & $445 \mathrm{TS}$ & 500 & 1998 & 1N6330T1 \\
\hline Foster & $490 \mathrm{TS}$ & 500 & 1999 & DD9891001 \\
\hline Foster & $5670 \mathrm{TS}$ & 500 & 1989 & $89 \mathrm{~J} 495273$ \\
\hline Hansen & $2860 \mathrm{TS}$ & 500 & 1994 & 9421620 \\
\hline Hansen & $2519 \mathrm{TS}$ & 500 & 1999 & 9821621 \\
\hline Hansen & $2622 \mathrm{TS}$ & 500 & 1990 & 3SD1232A1 \\
\hline Hansen & $2665 \mathrm{TS}$ & 500 & 1990 & 3SD1122A1 \\
\hline Hansen & $2530 \mathrm{TS}$ & 500 & 1992 & 3SD1424A1 \\
\hline Hansen & $2340 \mathrm{TS}$ & 500 & 1990 & B90330A01 \\
\hline Hansen & $2441 \mathrm{TS}$ & 500 & 1993 & $\mathrm{~A} 24006 \mathrm{~T} 1$ \\
\hline Hansen & $2141 \mathrm{TS}$ & 500 & 1991 & P817332TYF \\
\hline Hansen & $2442 \mathrm{TS}$ & 500 & 1990 & 1P9691T1 \\
\hline Hansen & $2221 \mathrm{TS}$ & 500 & 1996 & $1 \mathrm{~T} 8311 \mathrm{~T} 1$ \\
\hline Hansen & $2610 \mathrm{TS}$ & 500 & 1999 & 1N6714T1 \\
\hline Hansen & $2165 \mathrm{TS}$ & 500 & 1994 & DH4649001 \\
\hline Hansen & $2518 \mathrm{TS}$ & 500 & 1996 & DN3220001 \\
\hline Hansen & $2245 \mathrm{TS}$ & 500 & 1999 & 99000692 \\
\hline Kinser & $1225 \mathrm{TS}$ & 500 & 1990 & B00288A01 \\
\hline Kinser & $616 \mathrm{TS}$ & 500 & 1993 & B30146A01 \\
\hline Kinser & $99 \mathrm{TS}$ & 500 & 1988 & DM4692001 \\
\hline Foster & $464 \mathrm{TS}$ & 750 & 1998 & 98J597224 \\
\hline Foster & $5907 \mathrm{TS}$ & 750 & 2001 & 1703349 \\
\hline Foster & $11 \mathrm{TS}$ & 750 & 2001 & 1P5419T1 \\
\hline Foster & $480 \mathrm{TS}$ & 750 & 1996 & DM2743001 \\
\hline Foster & $363 \mathrm{TS}$ & 750 & 2001 & DY1357001 \\
\hline Hansen & $2667 \mathrm{TS}$ & 750 & 1993 & 9223017 \\
\hline Hansen & $2537 \mathrm{TS}$ & 750 & 1992 & 3SD1429A1 \\
\hline Hansen & $2327 \mathrm{TS}$ & 750 & 1993 & 3SD1605A1 \\
\hline Hansen & $2725 \mathrm{TS}$ & 750 & 1992 & B20168A01 \\
\hline Hansen & $2635 \mathrm{TS}$ & 750 & 1999 & 23339821 \\
\hline Hansen & $2893 \mathrm{TS}$ & 750 & 1992 & 1R8626T1 \\
\hline Kinser & $1202 \mathrm{TS}$ & 750 & 1993 & 9222379 \\
\hline Kinser & $1226 \mathrm{TS}$ & 750 & 1991 & 3SD1272A1 \\
\hline Kinser & $1210 \mathrm{TS}$ & 750 & 1992 & $\mathrm{~B} 10310 \mathrm{~A} 01$ \\
\hline Kinser & 104-A TS & 750 & 1993 & A30954T1 \\
\hline Kinser & $100 \mathrm{TS}$ & 750 & 1989 & DP3113001 \\
\hline Foster & $1002 \mathrm{TS}$ & 1000 & 1993 & Q108279-TNI \\
\hline Foster & $1004 \mathrm{TV}$ & 1000 & 1989 & PAW4176-0101 \\
\hline Hansen & $2365 \mathrm{TS}$ & 1000 & 2001 & $\mathrm{~B} 10215 \mathrm{~A} 01$ \\
\hline Hansen & $2654 \mathrm{TS}$ & 1000 & 2000 & 23589 \\
\hline
\end{tabular}


The transformers on this list are from the three different Marine Corp camps (Foster, Hansen, and Kinser) and include 46 transformers (26-500 kVA, 16-750 kVA, 4-1000 kVA). Loading for these transformers is unknown and most likely will vary per transformer. The original Scope of Work (SOW) called for analyzing base loads at 30\% and 70\%; however, a closer look of transformer efficiencies noted that $30 \%$ and $70 \%$ are very similar on most transformers. It was determined that $20 \%$ and $60 \%$ would provide a better analysis that shows the differences in the smaller load and the larger load. Figure 1 shows an efficiency curve from Howard Transformers and an example of how similar $30 \%$ and $70 \%$ are in the curve.

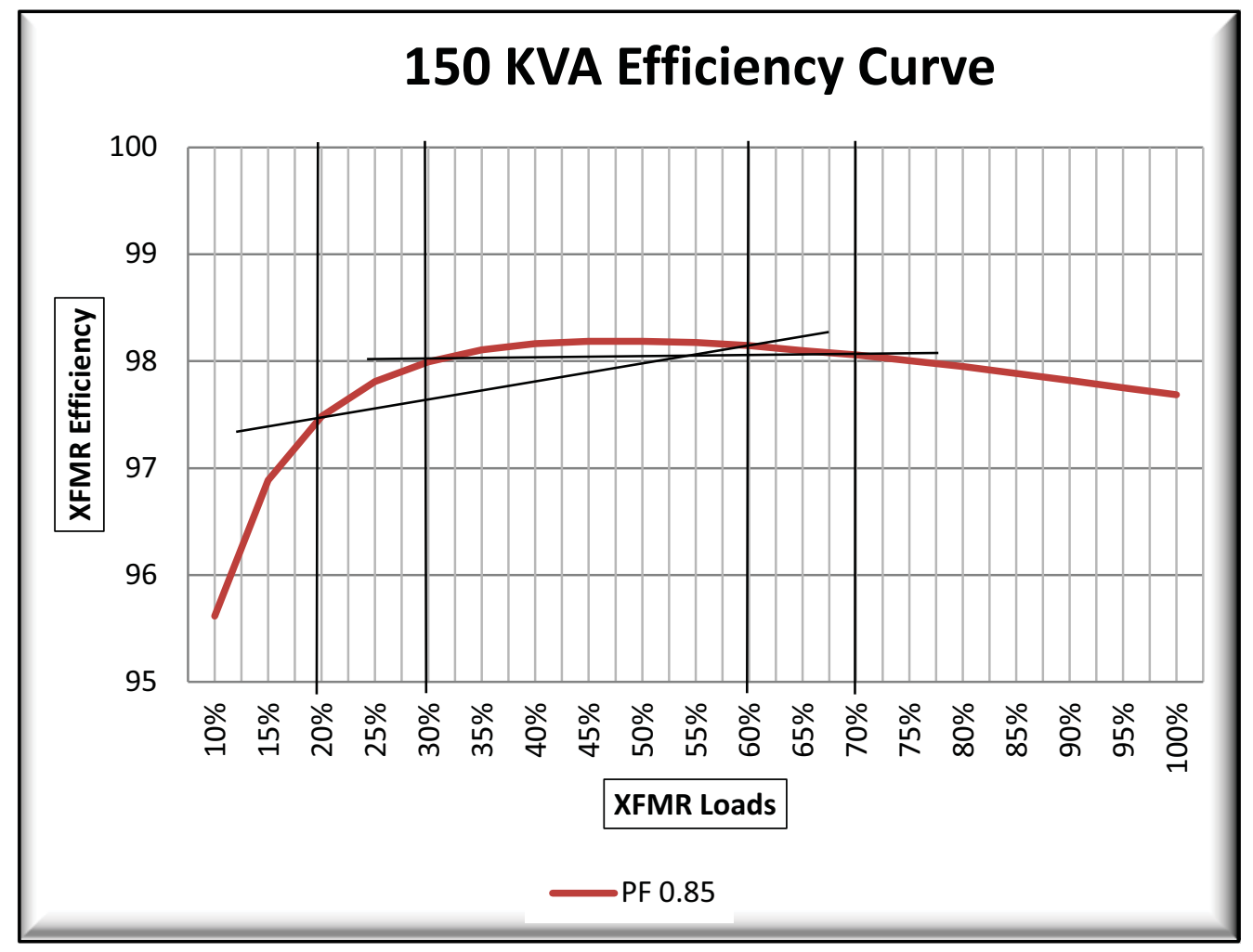

Figure $1.30 \%$ \& $70 \%$ Efficiency Similarities 


\section{Efficiency Evaluation}

\subsection{U.S. Transformer Manufacturer Data}

INL contacted several U.S. transformer manufacturers and used information provided by Howard Transformers as well as a price list consisting of General Electric (GE) transformer prices. INL utilized the Department of Energy's (DOE) list of transformer standard and premium efficiencies, along with a European standards list. The information obtained was then compiled into an analysis spreadsheet containing expected transformer efficiencies for low-level, medium-level, and high-level transformers in the $500 \mathrm{kVA}, 750 \mathrm{kVA}$ and $1000 \mathrm{kVA}$ sizes. Some of the analysis involved using the Linear Interpolation formula, as shown in Equation 1. Two other formulas were also used: the second formula for determining transformer efficiency, and the third formula for applying the Power Factor (PF) conversion. These formulas are shown in Equations 2 and 3, respectively. Based on INL's past experiences with military bases, a power factor of 0.85 has been assumed for the MCB in Okinawa, Japan, and used in the life-cycle cost (LCC) and economic analyses that are detailed in this report.

$$
\begin{gathered}
y_{2}=\frac{\left(x_{2}-x_{1}\right)\left(y_{3}-y_{1}\right)}{\left(x_{3}-x_{1}\right)}+y_{1} \\
\eta=\frac{\text { POut }}{\text { PIn }} \times 100 \%=\frac{(\text { PIn }- \text { PLoss })}{\text { PIn }} \times 100 \% \\
\eta=\frac{((k V A \times P F)-k W)}{(k V A \times P F)} \times 100 \%
\end{gathered}
$$




\subsubsection{Low-Level Efficient Transformers}

Low-level transformers are the lowest efficient transformers and come from the manufacturers with no extra features or options. These transformers have the smallest amount of material, the highest resistance, the smallest cores, and therefore have the highest amount of both load losses and no-load losses. However, these transformers are the cheapest priced on the market and should cost somewhere around $\$ 15,000$ - $\$ 20,000$ for a $500 \mathrm{kVA}, \$ 25,000-\$ 30,000$ for a $750 \mathrm{kVA}$, and $\$ 40,000-\$ 45,000$ for a $1000 \mathrm{kVA}$ version. These prices vary depending on metal market fluctuations, manufacturer, windings type, options, and so forth and could possibly be improved upon through a government contract bidding process. Figure $2-7$ shows the manufacturer listed prices for the Low-Level Efficient GE Transformers. This price list was used in the report analysis and LCC's for low-level transformers.

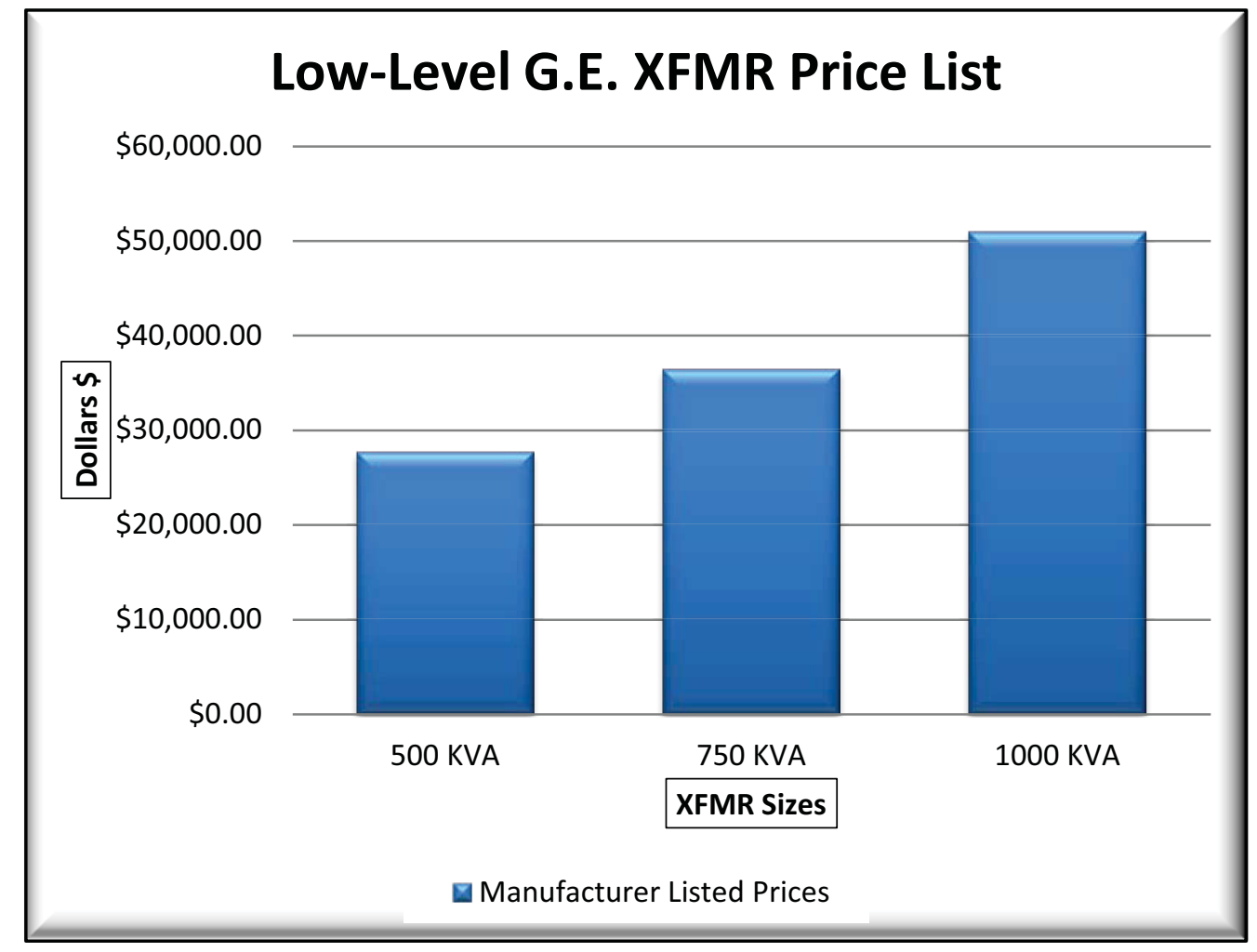

Figure 2. Low-Level Price List

Figures $3-7$ show efficiency comparisons (at the 0.85 power factor), total losses, and efficiency curves for $500 \mathrm{kVA}, 750 \mathrm{kVA}$, and $1000 \mathrm{kVA}$ sizes. 


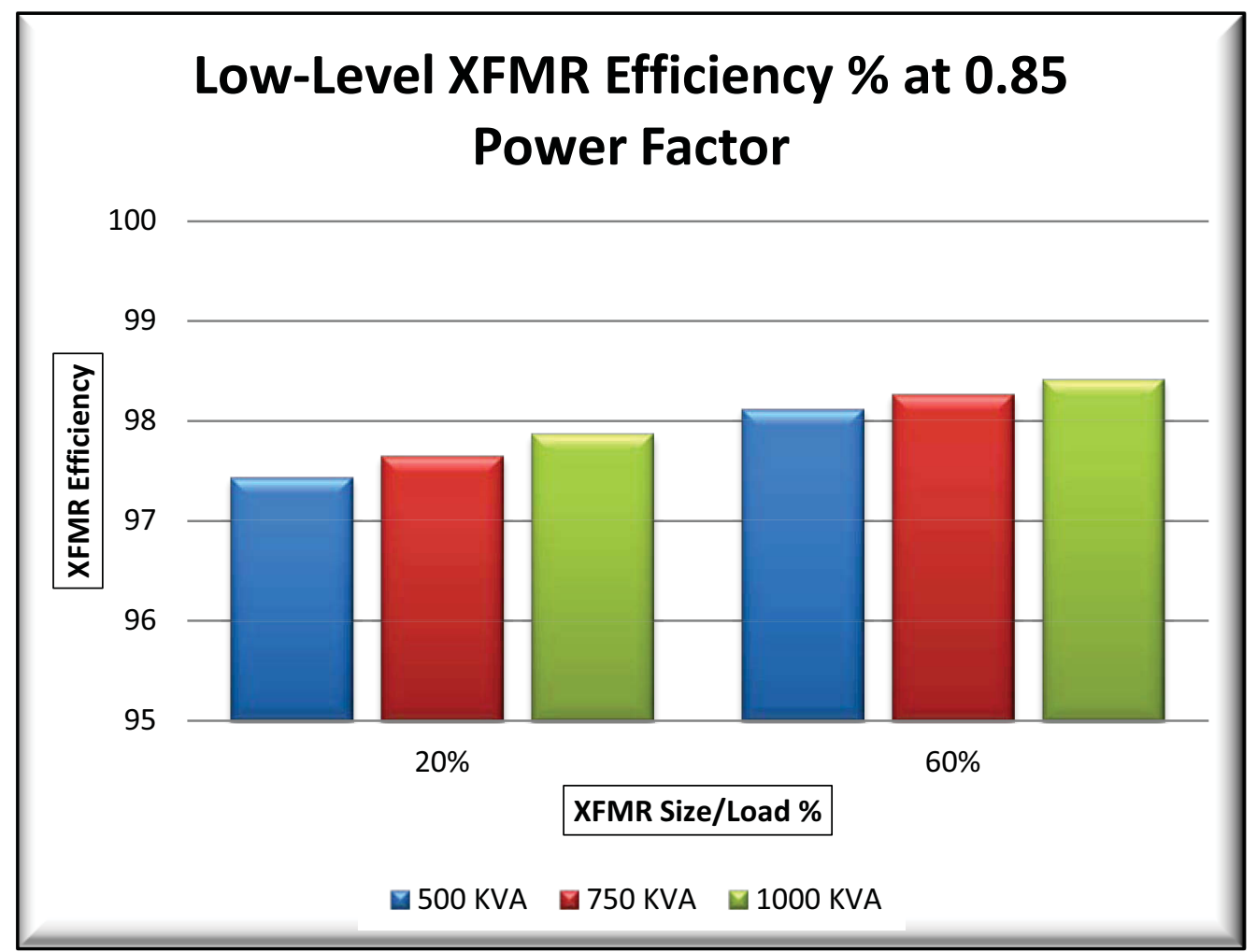

Figure 3. Low-Level Transformer Comparison

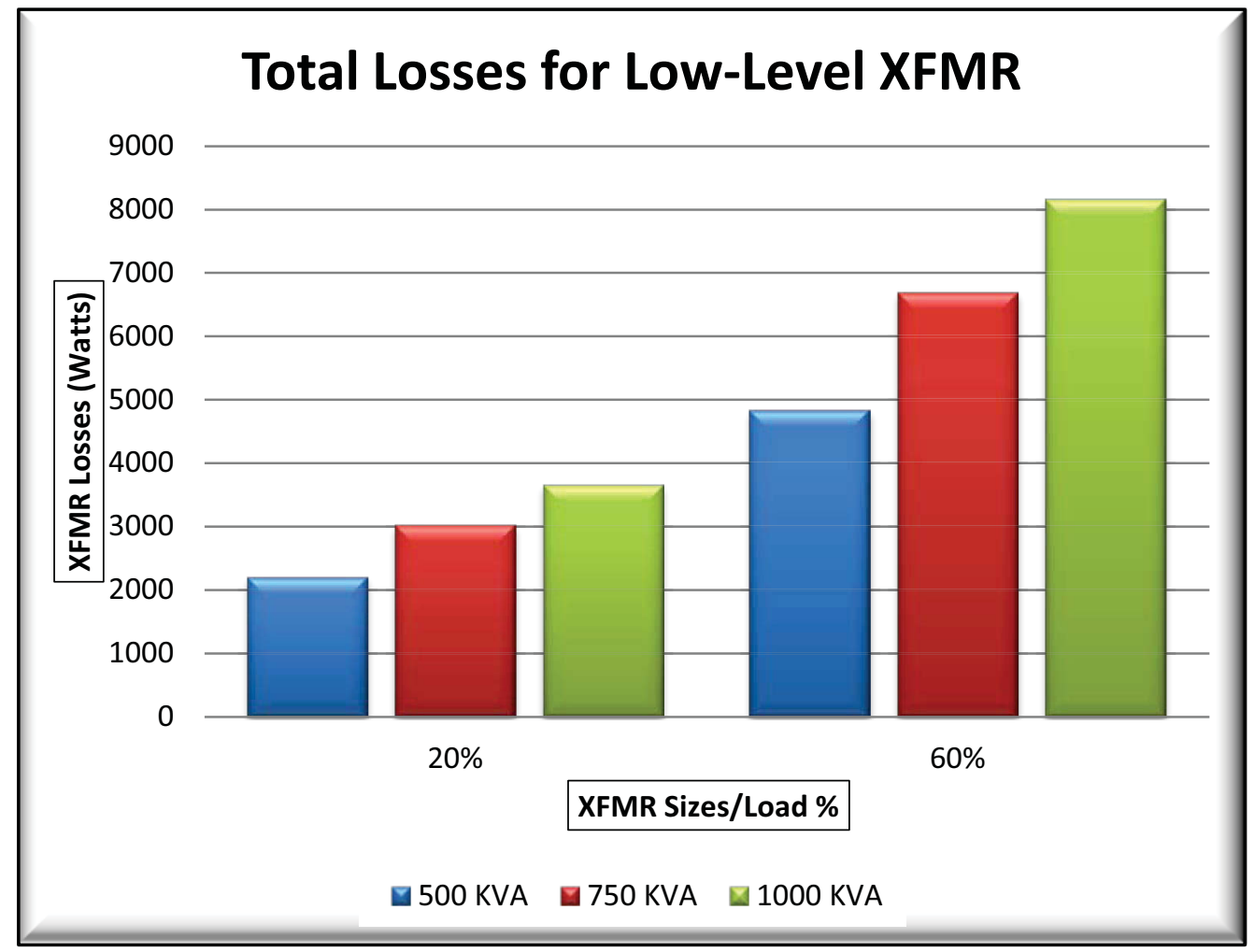

Figure 4. Low-Level Transformers Total Losses 


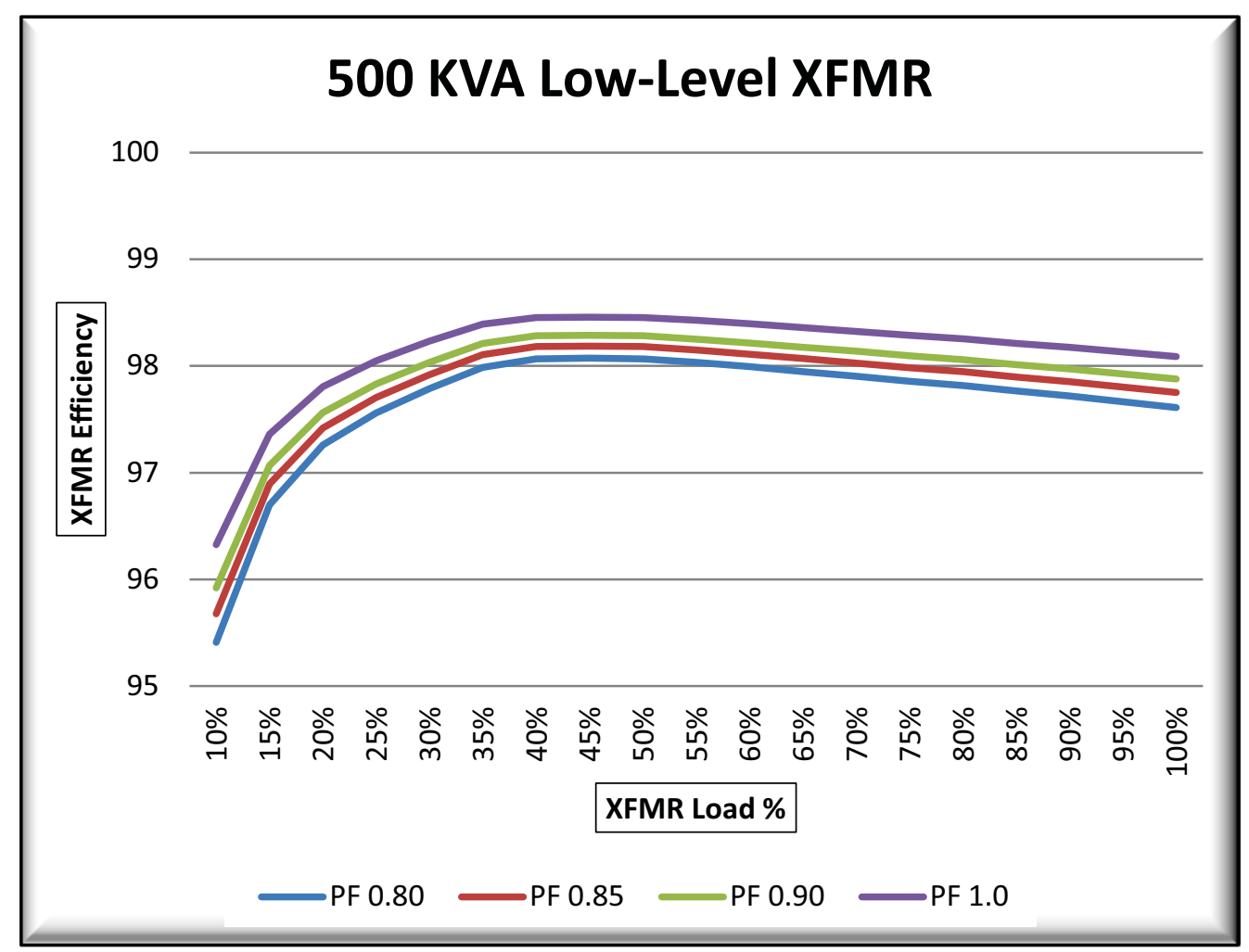

Figure 5 Efficiency Curve for Low-Level 500 kVA XFMR

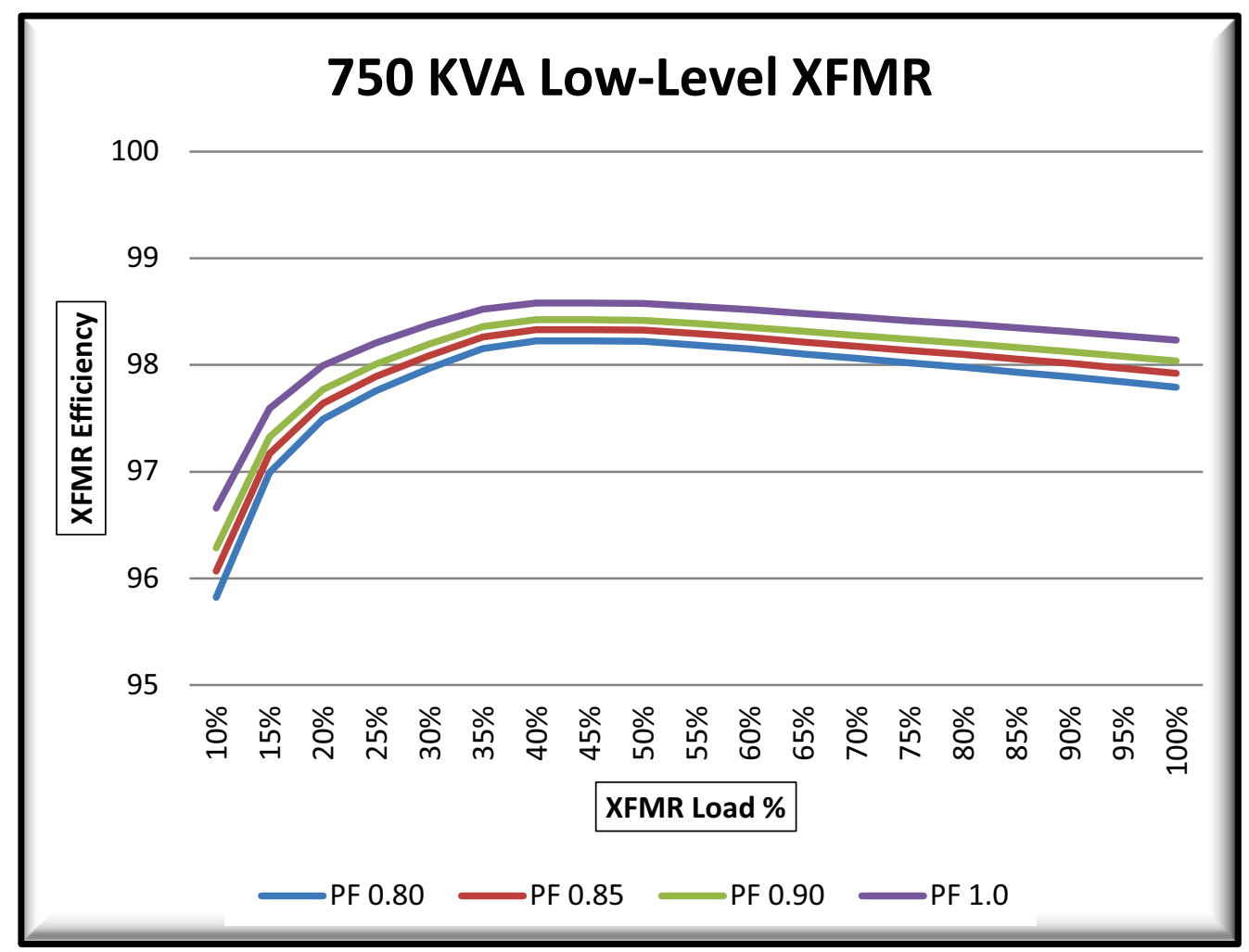

Figure 6. Efficiency Curve for Low-Level 750 kVA XFMR 


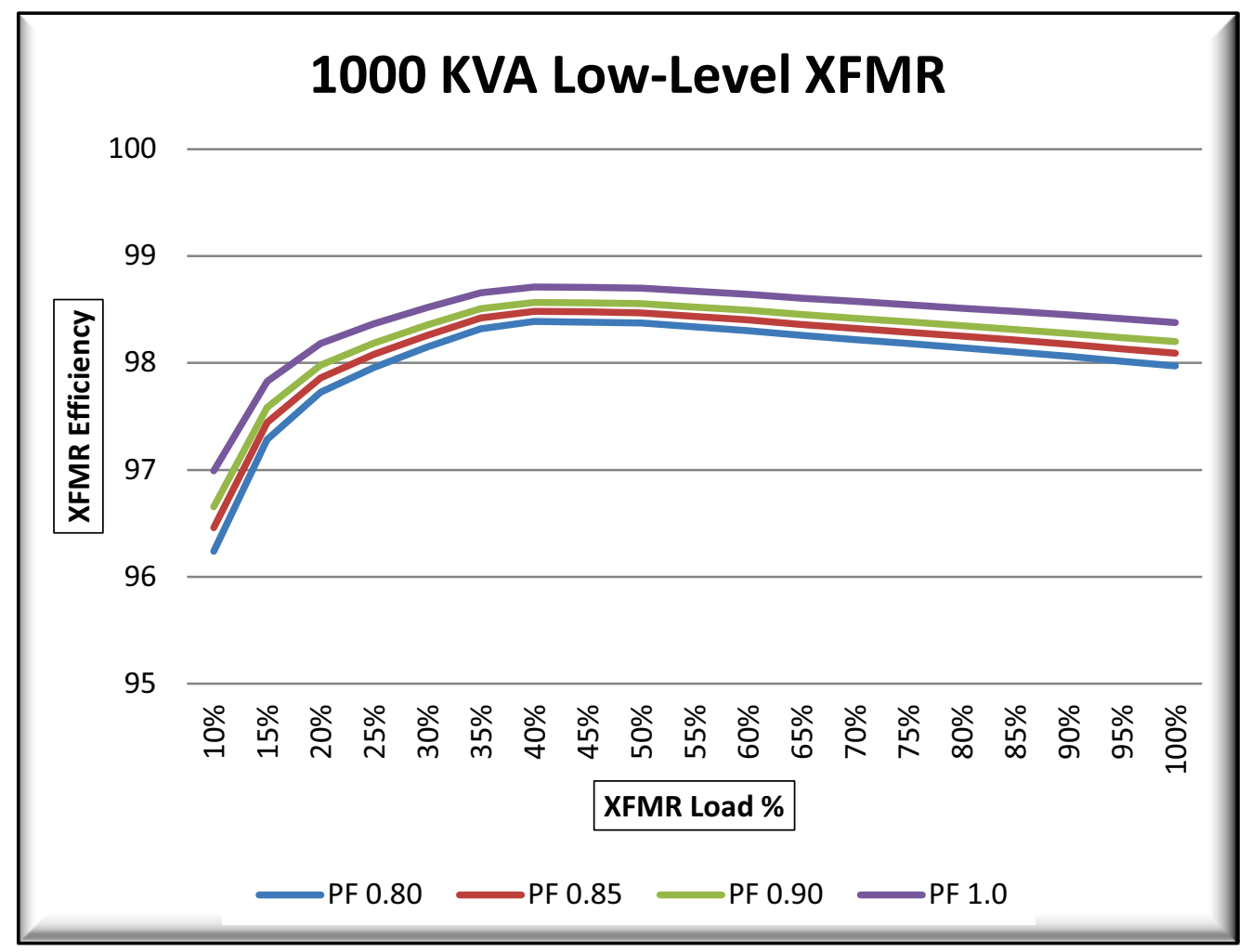

Figure 7. Efficiency Curve for Low-Level 1000 kVA XFMR

\subsubsection{Medium-Level Efficient Transformers}

The medium-level transformers have better efficiencies than the lowest efficient transformers discussed in Section 2.1.1, but they are not as efficient as the high-level transformers discussed in Section 2.1.3. These transformers tend to have improvements in winding material and, therefore, have slightly better load loss ratios than the low-level transformers. These transformers usually included extra features/options that also help improve transformer efficiency. However, these transformers are more expensive in price and should cost somewhere around $\$ 30,000-\$ 35,000$ for a $500 \mathrm{kVA}, \$ 40,000$ $\$ 45,000$ for a $750 \mathrm{kVA}$, and $\$ 60,000-\$ 65,000$ for a $1000 \mathrm{kVA}$ version. These prices also vary depending on metal market fluctuations, manufacturer, windings type, options, and so forth and could possibly be improved upon through a government contract bidding process. Figure 8 shows the manufacturer listed prices for the Medium-Level Efficient GE Transformers. This price list was used in the report analysis and LCC's for medium-level transformers. Figures $9-13$ show efficiency comparisons (at the 0.85 power factor), total losses, and efficiency curves for $500 \mathrm{kVA}, 750 \mathrm{kVA}$, and $1000 \mathrm{kVA}$ sizes. 


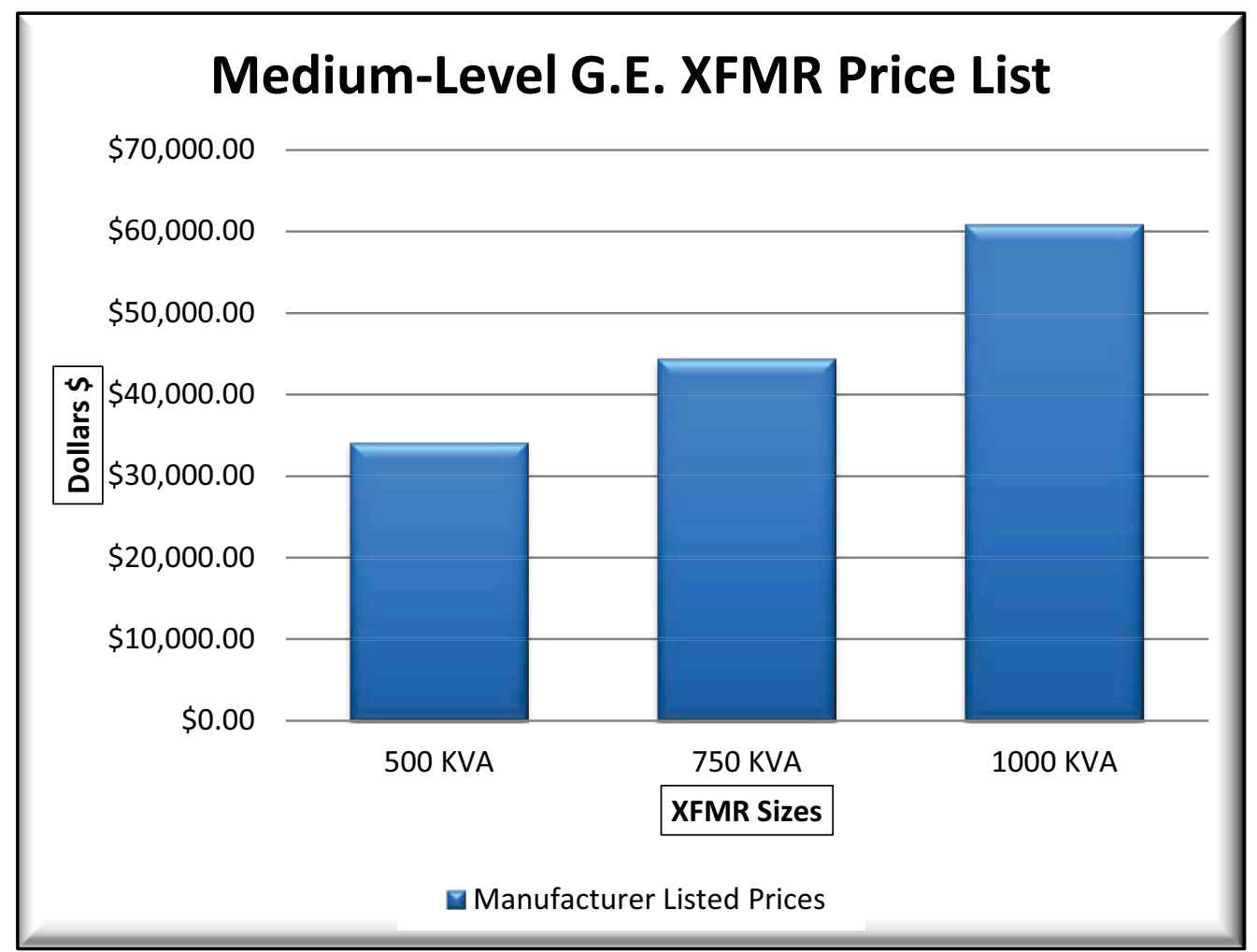

Figure 8. Medium-Level Price List

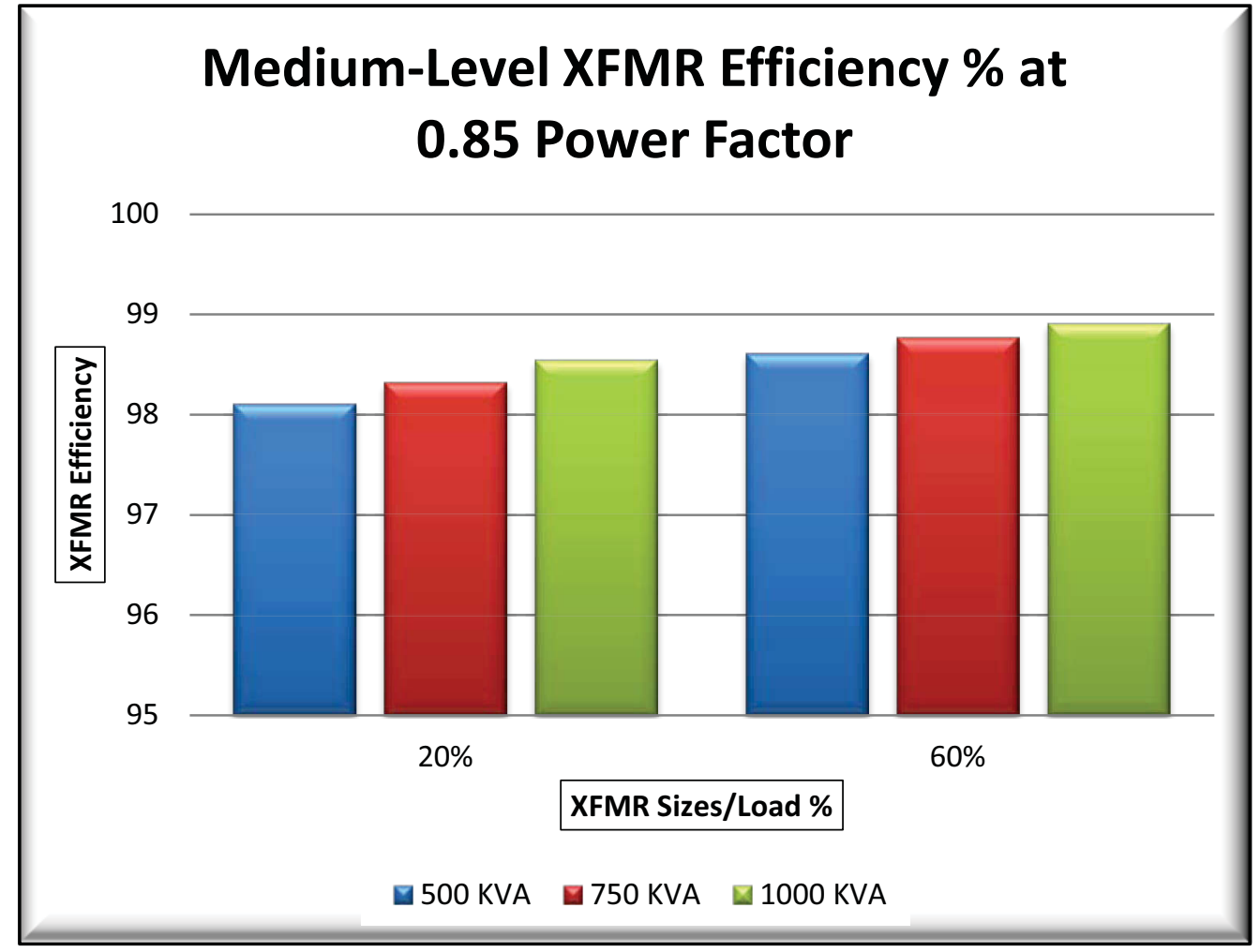

Figure 9. Medium-Level Transformer Comparison 


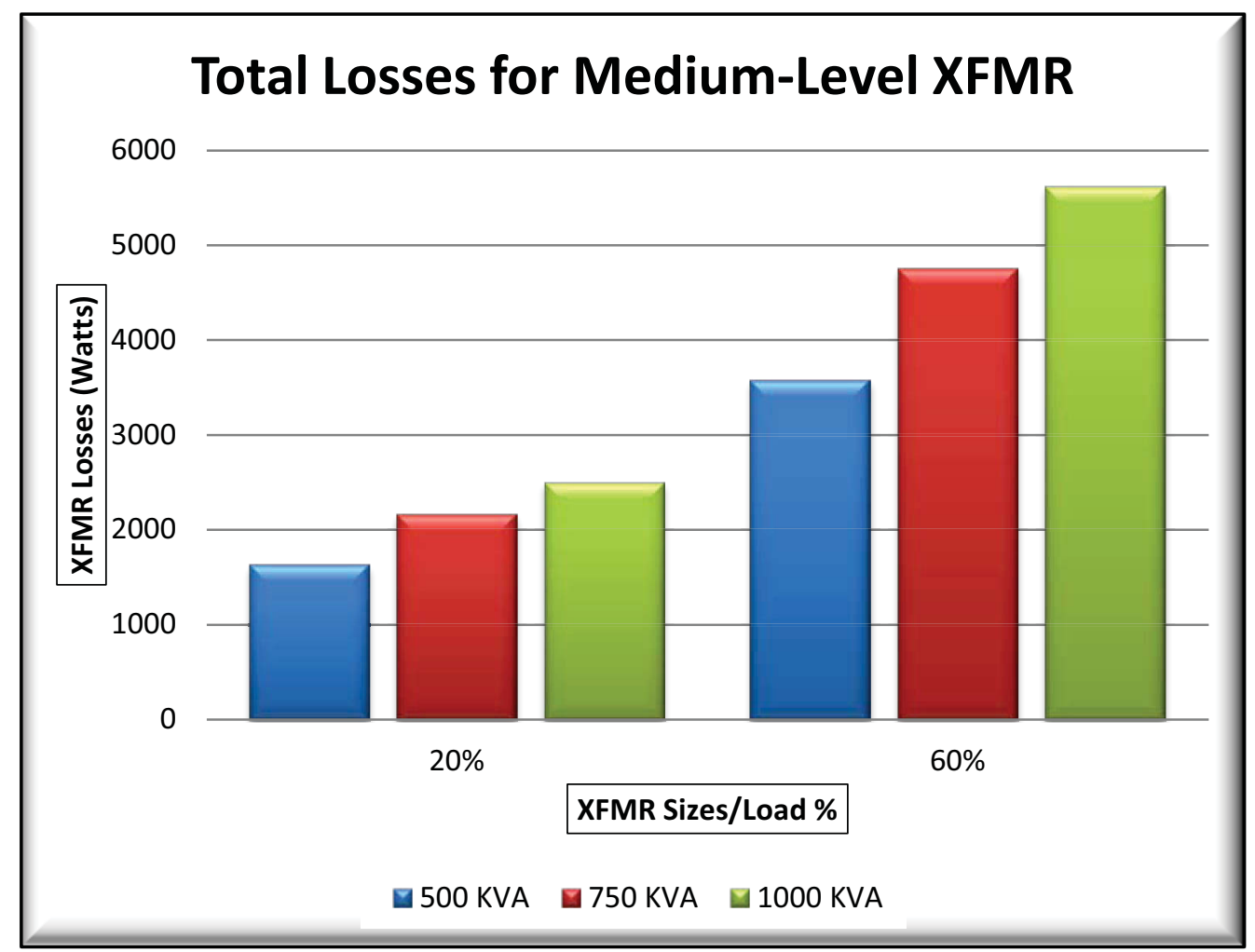

Figure 10. Medium-Level Transformer Total Losses

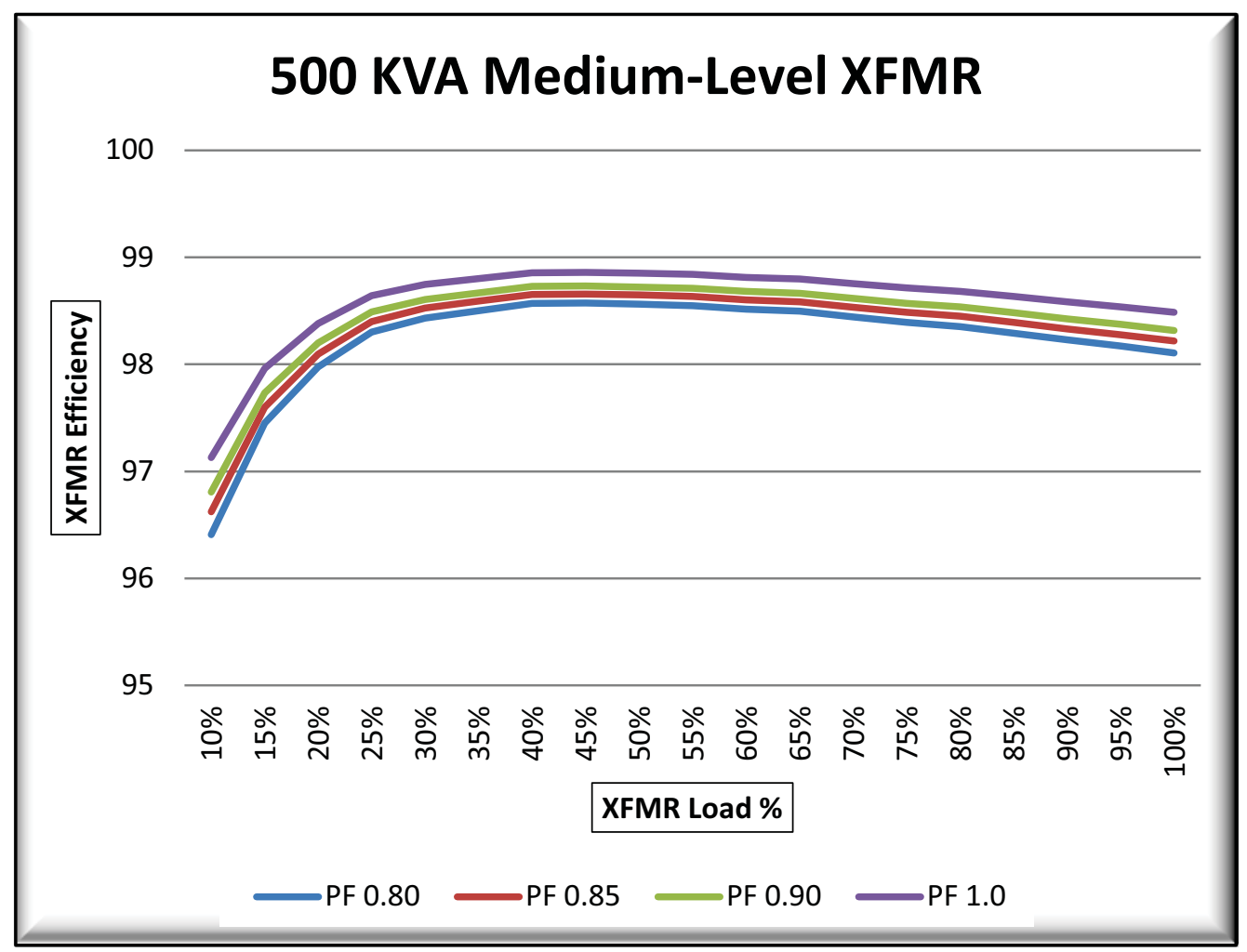

Figure 11. Efficiency Curve for Medium-Level 500 kVA XFMR 


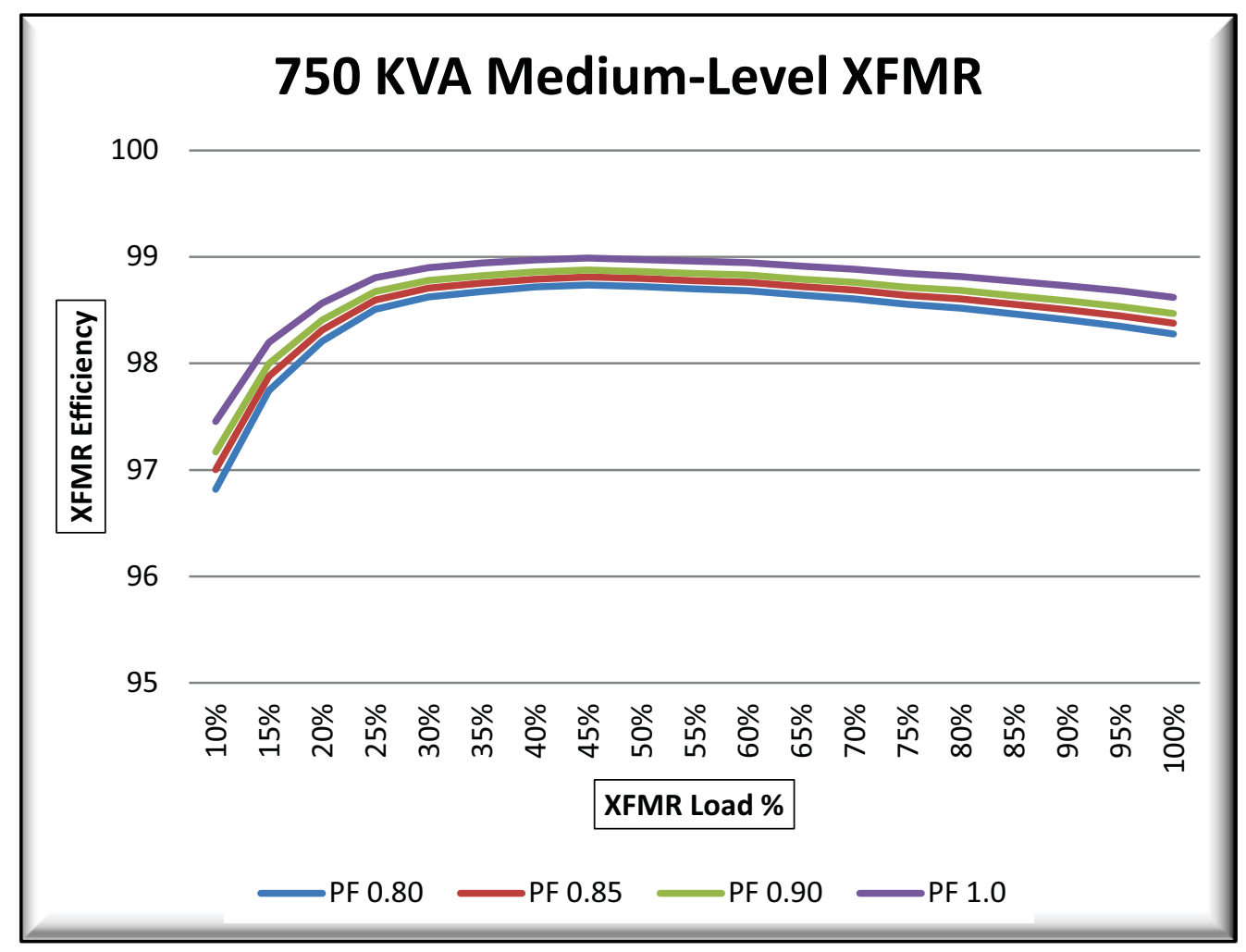

Figure 12. Efficiency Curve for Medium-Level 750 kVA XFMR

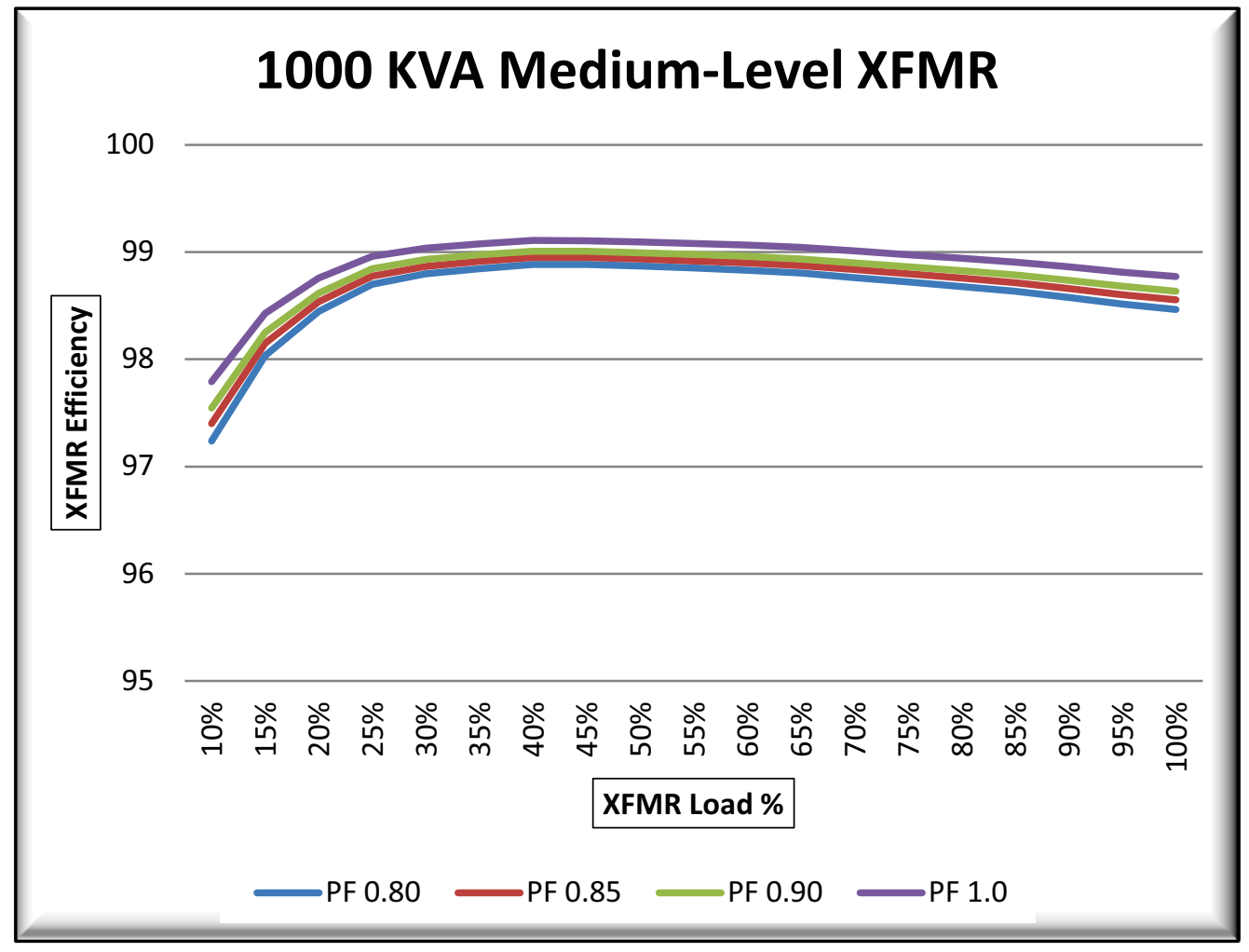

Figure 13. Efficiency Curve for Medium-Level 1000 kVA XFMR 


\subsubsection{High-Level Efficient Transformers}

The high-level transformers are the highest efficient transformers and have the best extra features or options for improving efficiency. These transformers have the highest quality winding material and, therefore, have the lowest amount of both load losses and no-load losses. However, these transformers are the most expensive and should cost somewhere around $\$ 55,000$ - $\$ 60,000$ for a $500 \mathrm{kVA}, \$ 65,000$ $\$ 70,000$ for a $750 \mathrm{kVA}$, and $\$ 80,000-\$ 85,000$ for a $1000 \mathrm{kVA}$ version. Again, these prices vary depending on metal market fluctuations, manufacturer, windings material, options, and so forth and could possibly be improved upon through a government contract bidding process. Figure 14 shows the manufacturer listed prices for the High-Level Efficient GE Transformers. This price list was used in the report analysis and LCC's for High-Level transformers.

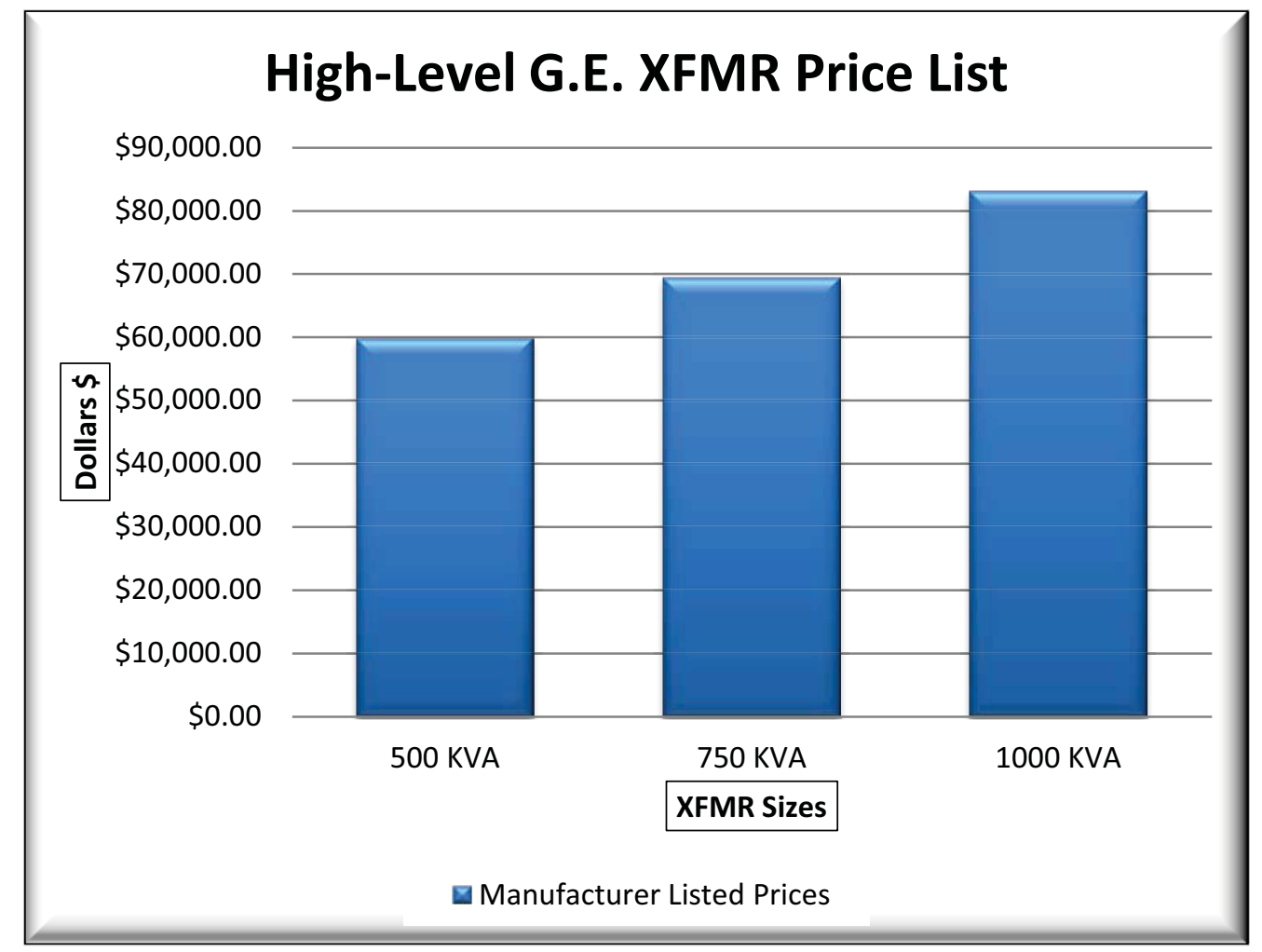

Figure 14. High-Level Price List

Figures $15-19$ show efficiency comparisons (at the 0.85 power factor), total losses, and efficiency curves for $500 \mathrm{kVA}, 750 \mathrm{kVA}$, and $1000 \mathrm{kVA}$ sizes. 


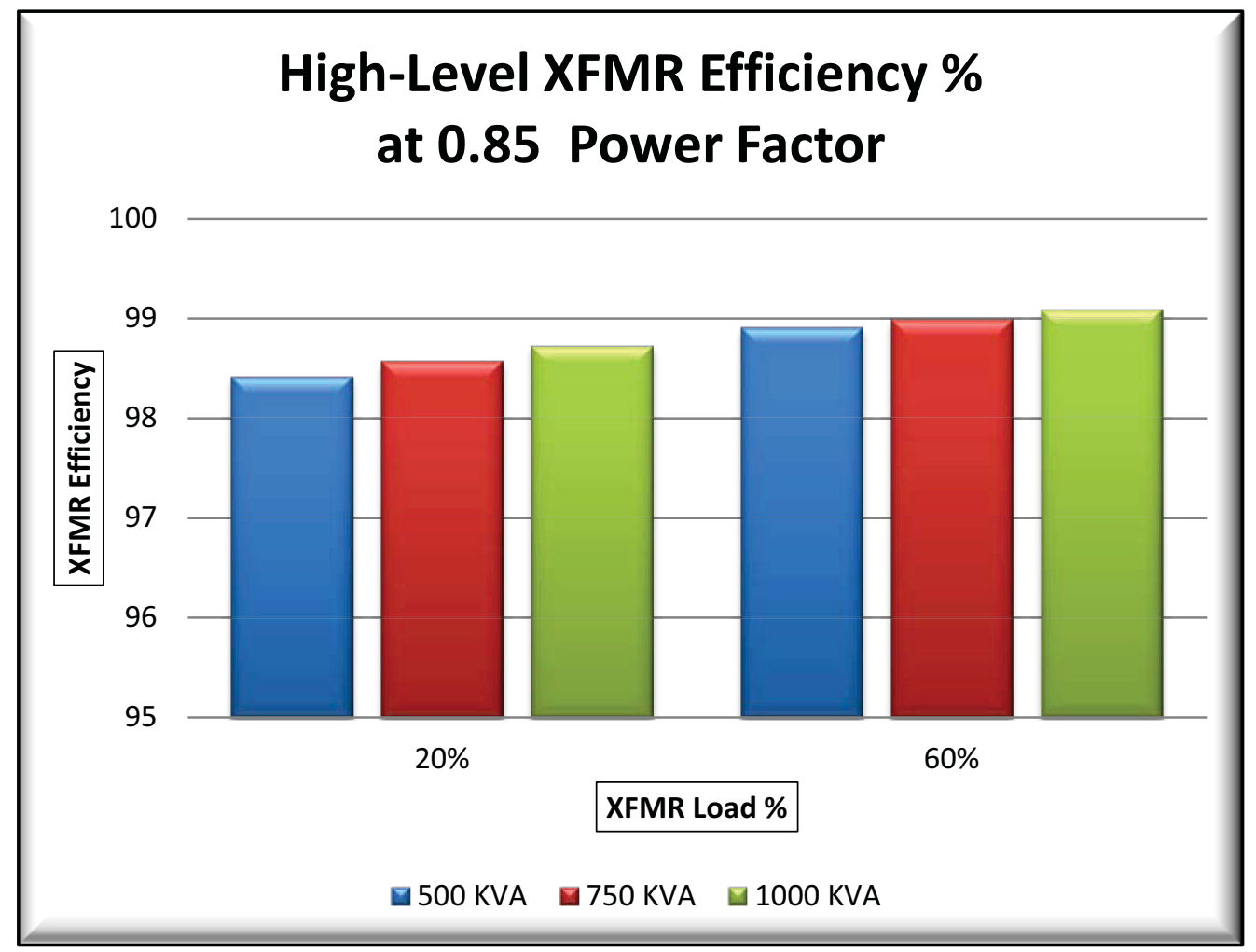

Figure 15. High-Level Transformer Comparison

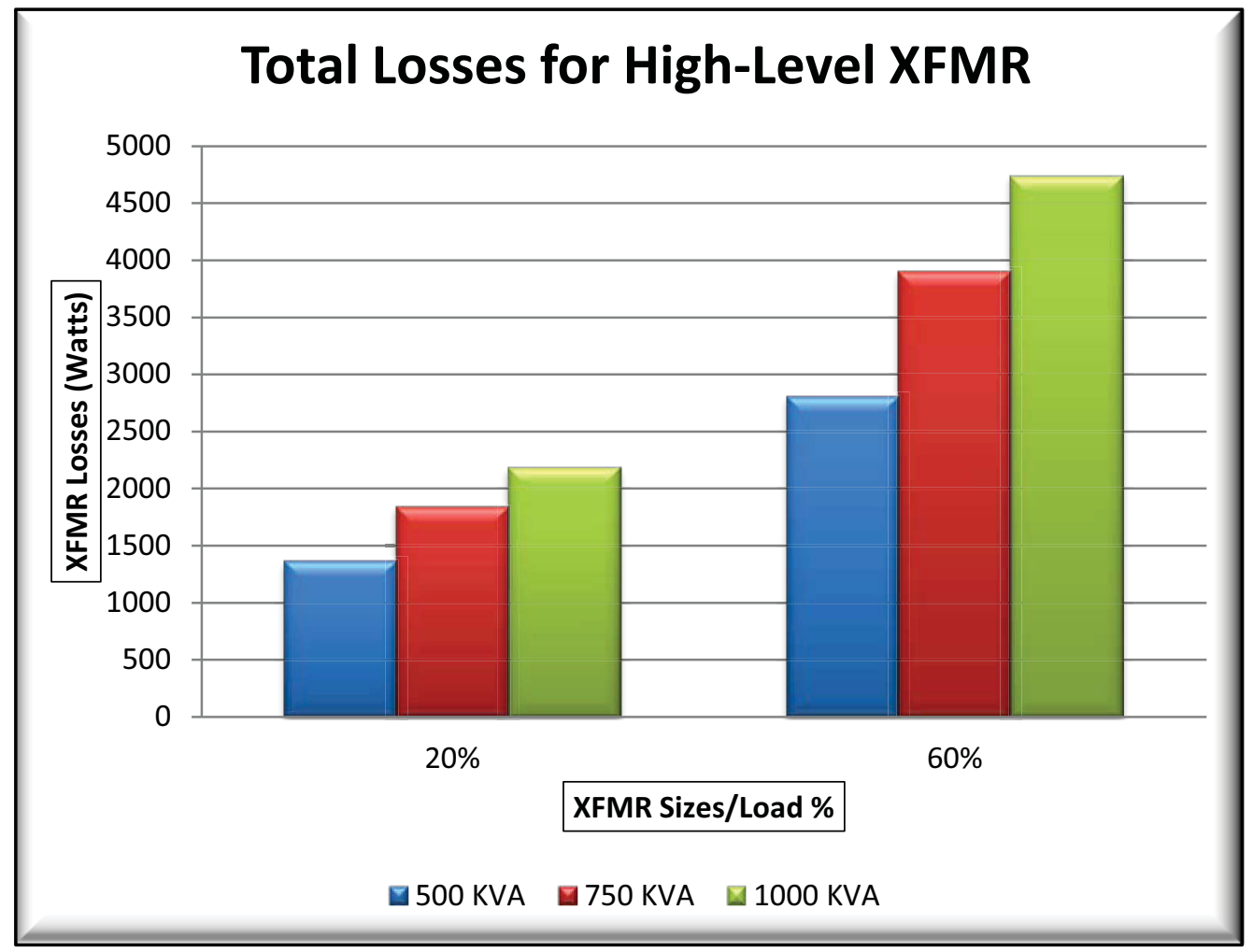

Figure 16. High-Level Transformers Total Losses 


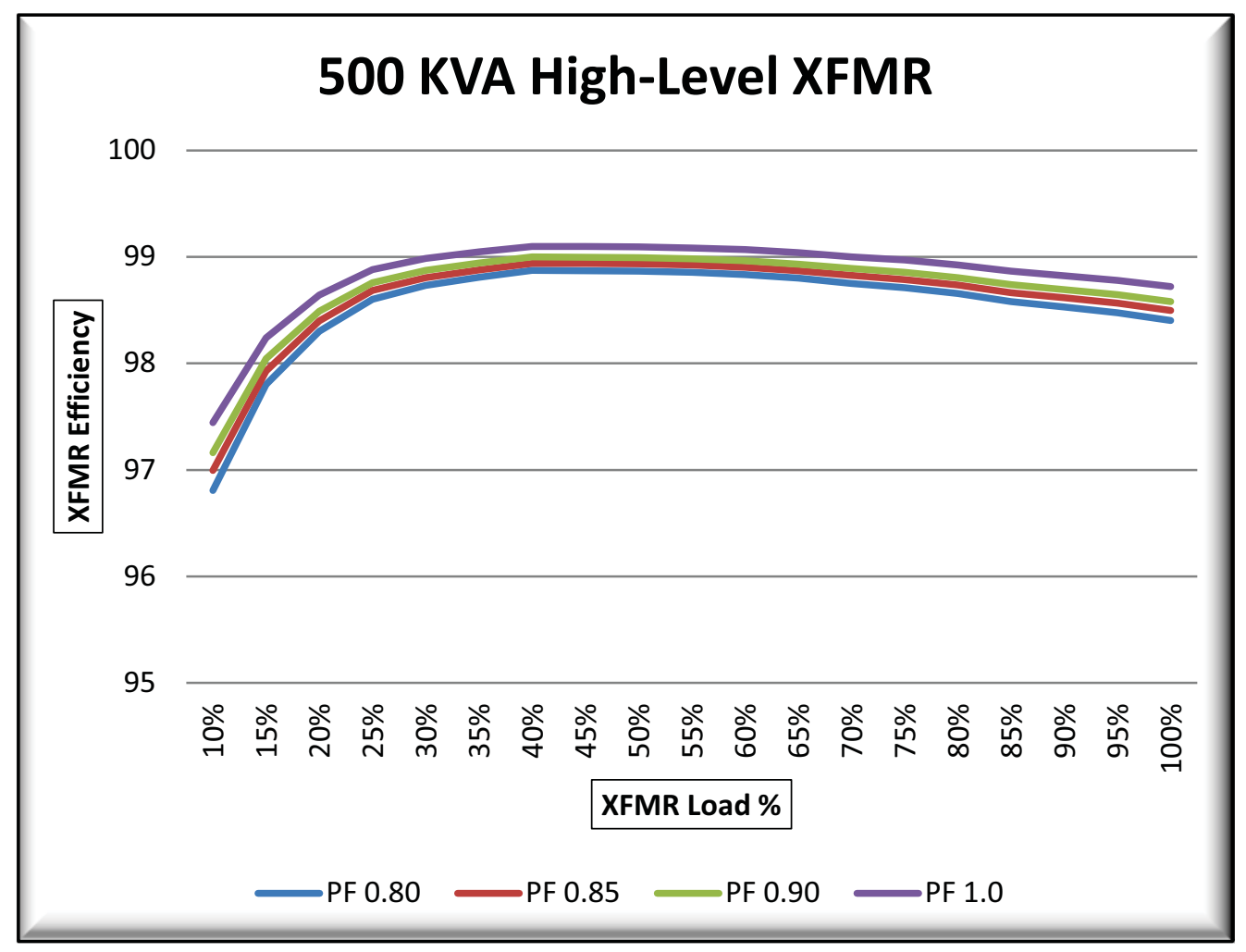

Figure 17. Efficiency Curve for High-Level 500 kVA XFMR

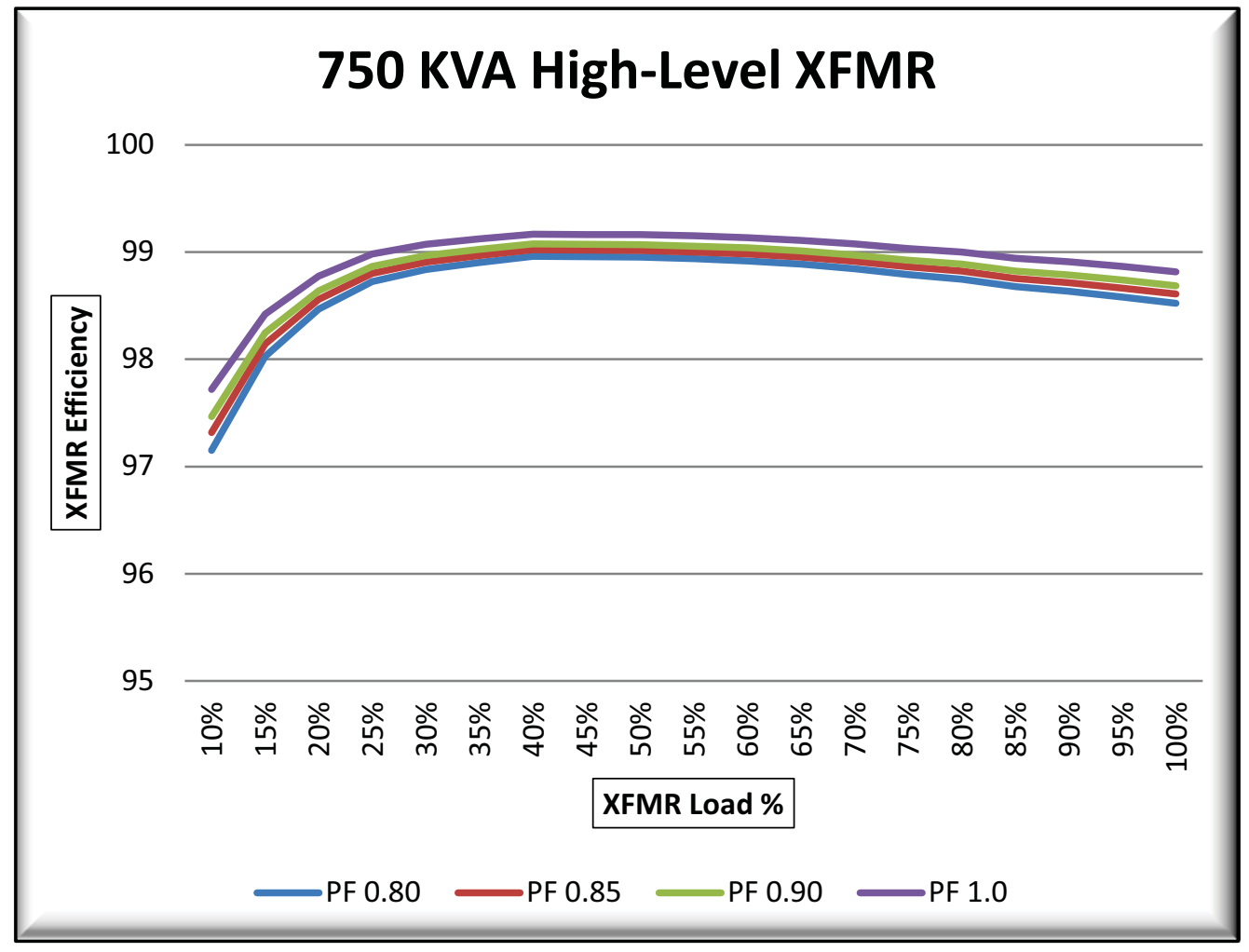

Figure 18. Efficiency Curve for High-Level 750 kVA XFMR 


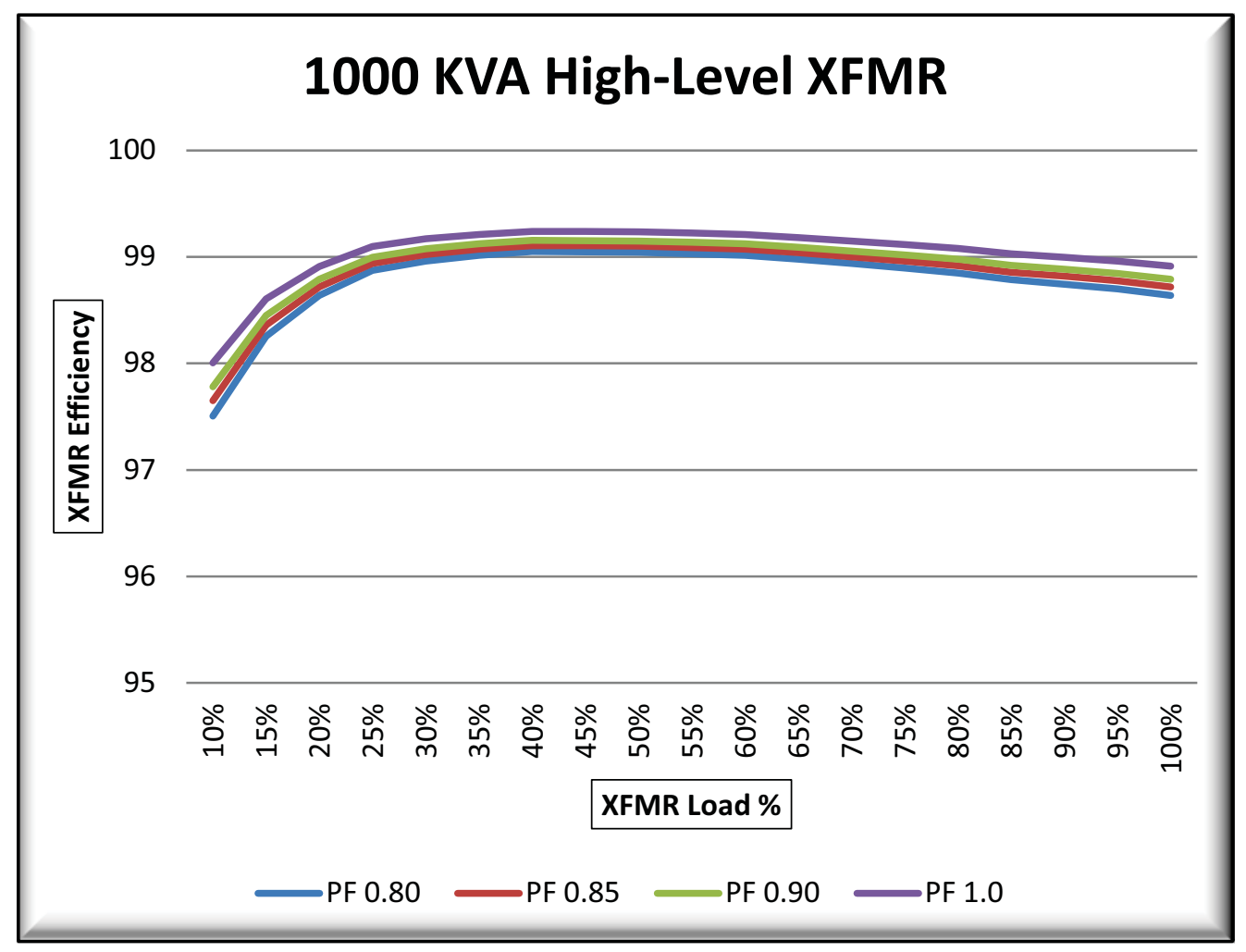

Figure 19. Efficiency Curve for High-Level 1000 kVA XFMR

\subsection{Okinawa Transformer Assumptions}

Based on INL's past experiences with military bases, it is INL's opinion that the transformers already in place at Okinawa are probably somewhere between the low-level and medium-level. The efficiency curves from the 1980's Howard Transformers were used to represent the MCB transformer data. Figures $20-24$ show the assumed efficiency comparisons (at the 0.85 power factor), total losses, and efficiency curves for $500 \mathrm{kVA}, 750 \mathrm{kVA}$, and $1000 \mathrm{kVA}$ Okinawa transformers. 


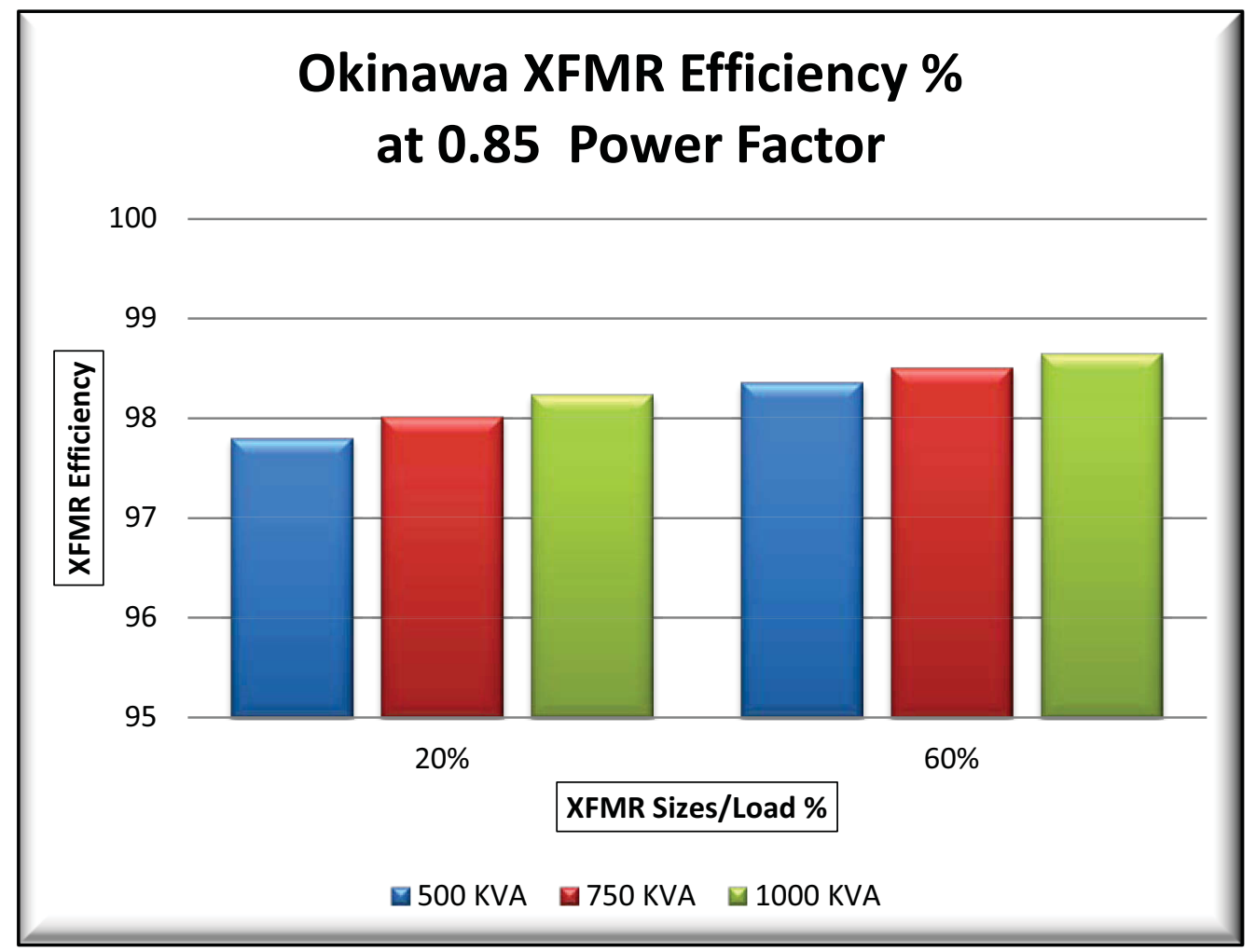

Figure 20. Okinawa Transformer Comparison

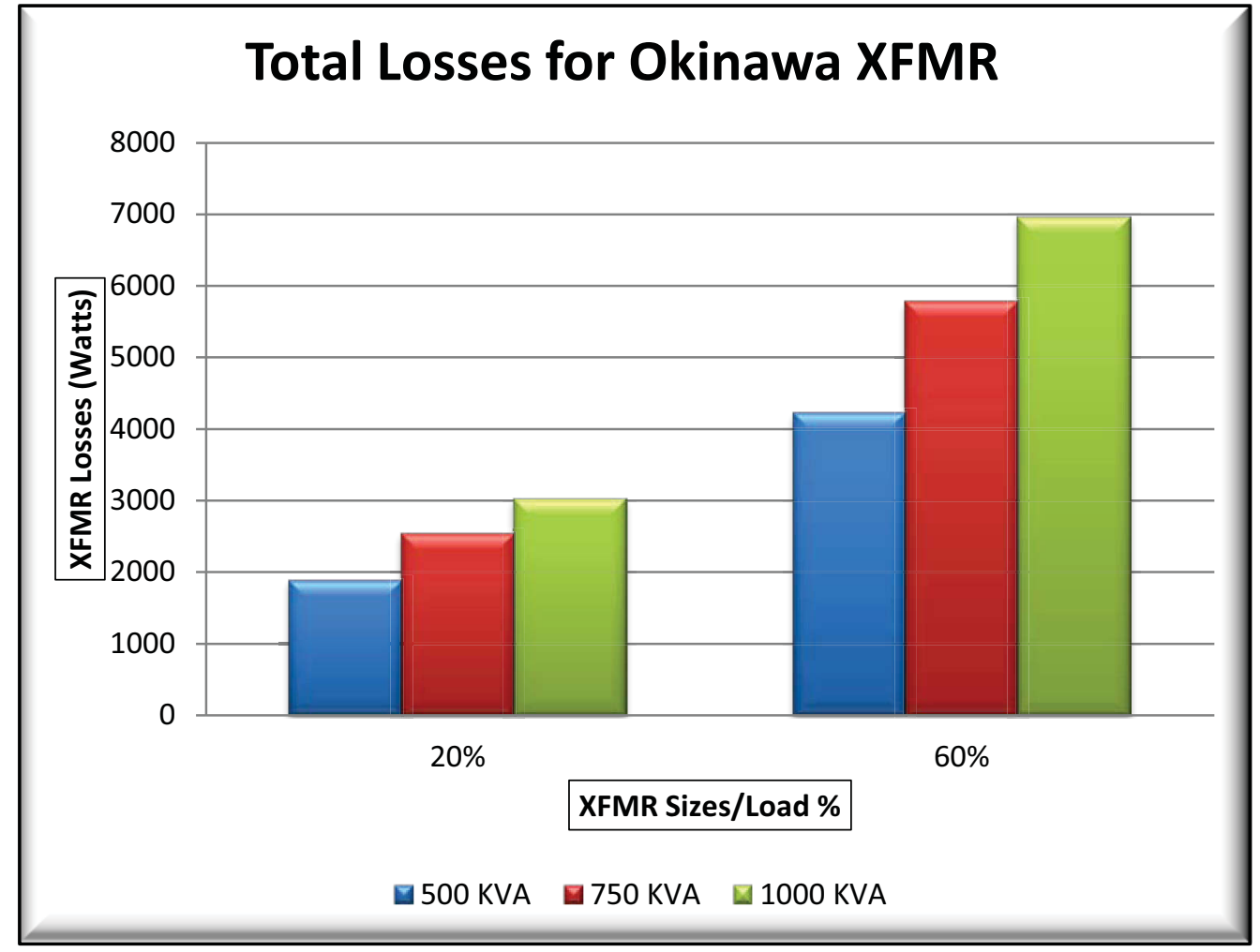

Figure 21. Okinawa Transformers Total Losses 


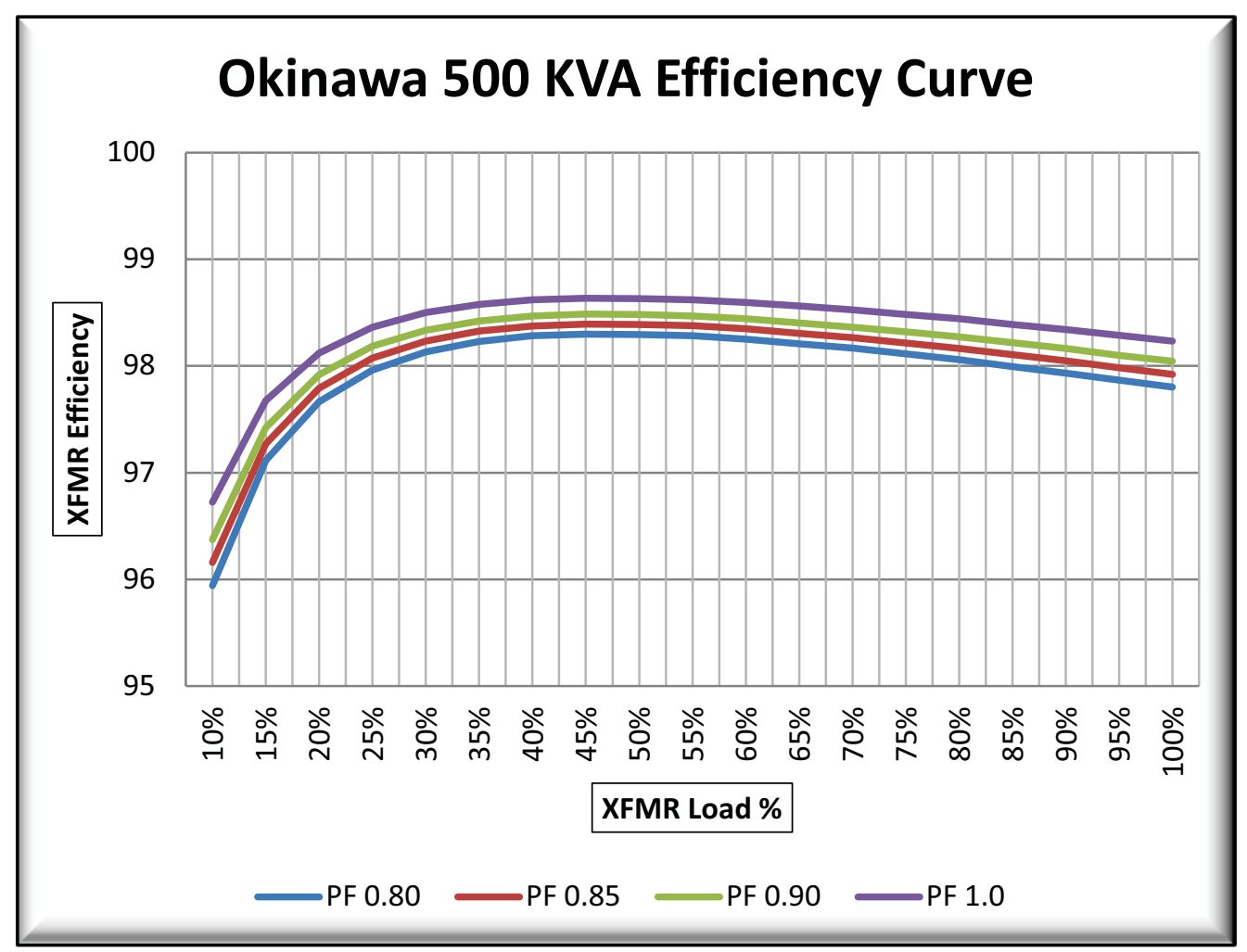

Figure 22. Efficiency Curve for Okinawa 500 kVA XFMR

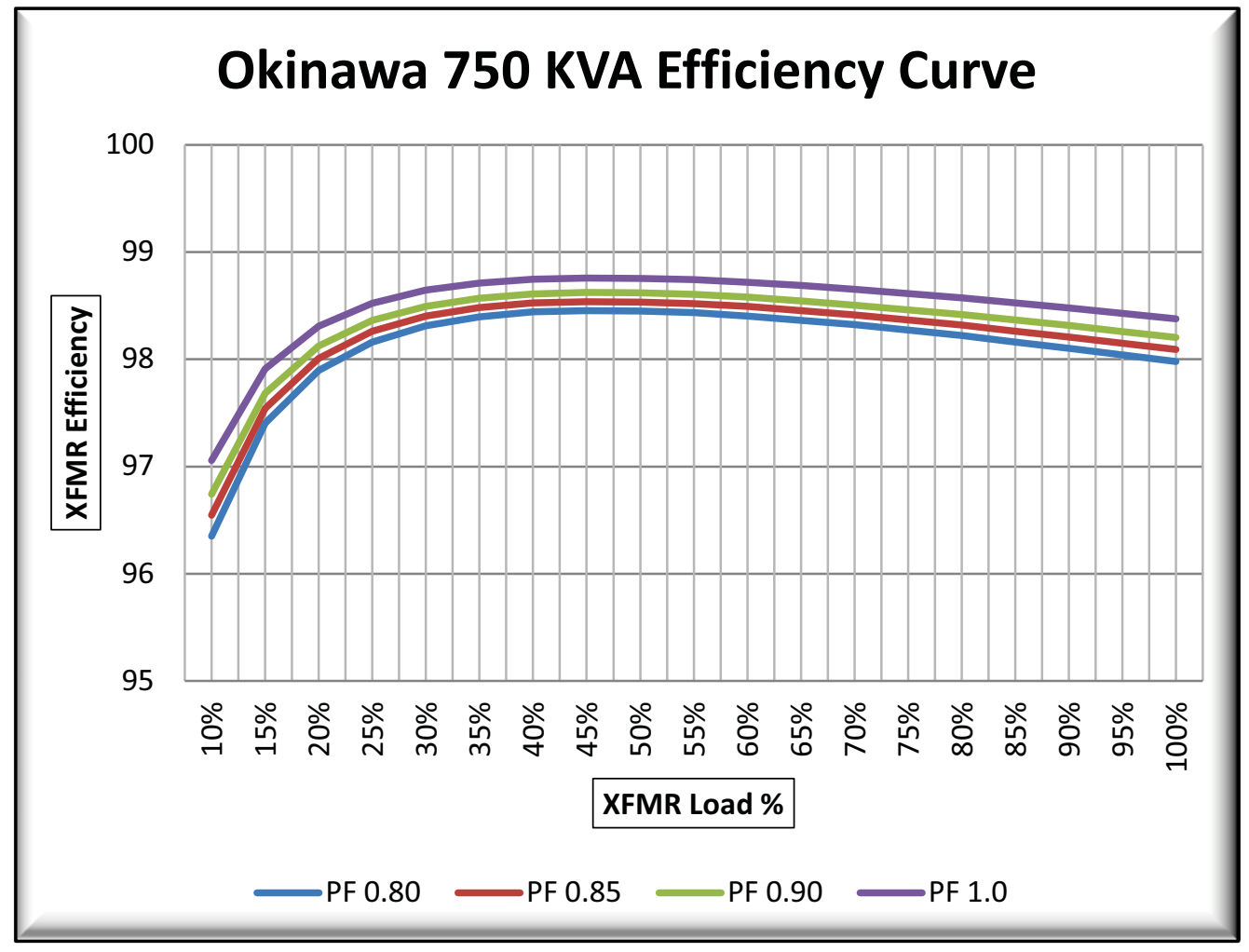

Figure 23. Efficiency Curve for Okinawa 750 kVA XFMR 


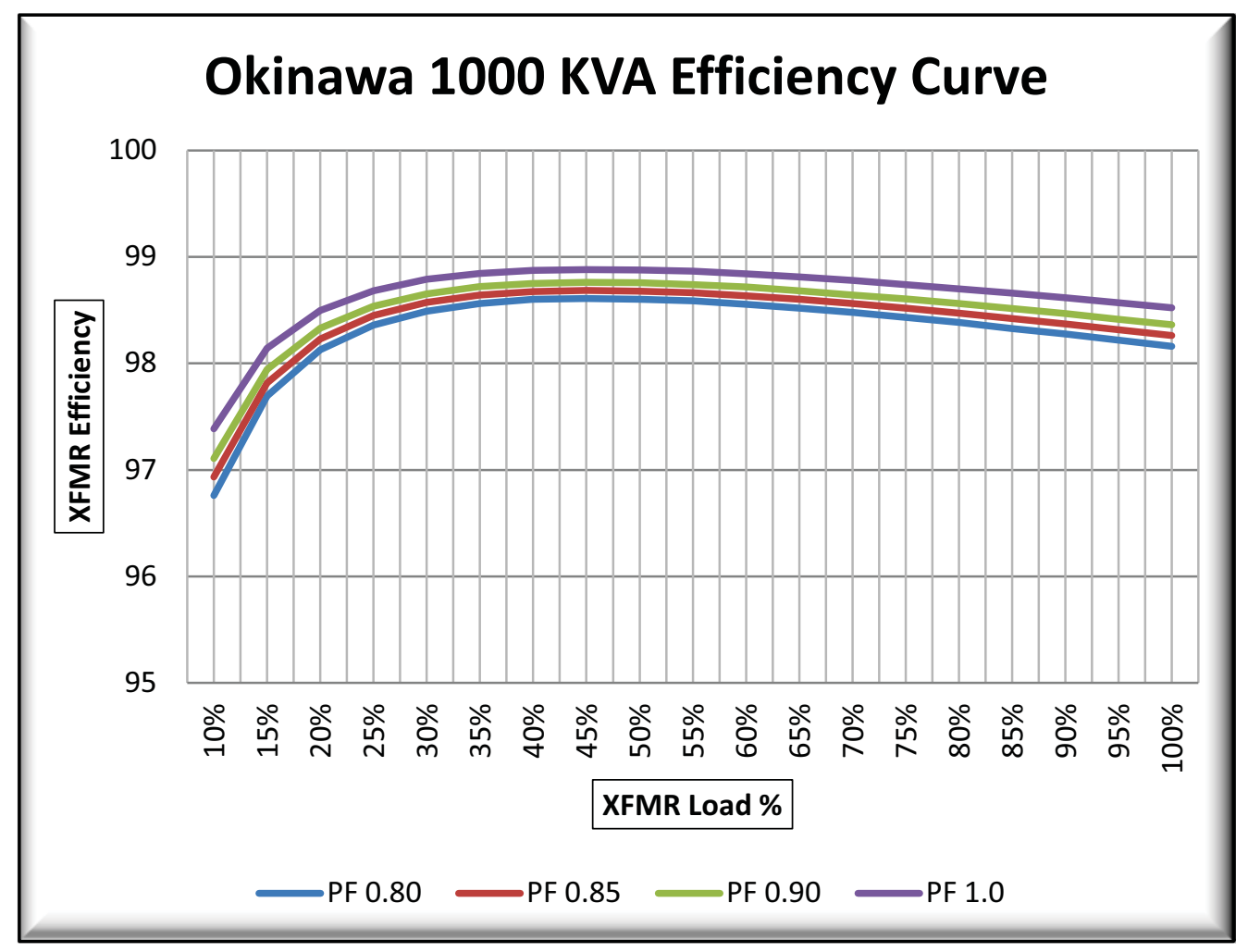

Figure 24. Efficiency Curve for Okinawa 1000 kVA XFMR 


\section{LCC Analysis}

This analysis centered on the assumption that a potential transformer replacement project will be using $100 \%$ government funding. Some other types of business models and financing options for project development on federal land could include the Energy Conservation Investment Program (ECIP), Utility Energy Service Contracts (UESC), Energy Savings Performance Contracts (ESPC), End-User Lease Agreements (EULA), private financing, and/or other options.

Two different sets of LCC analysis scenarios (one with construction costs and one without) were run for this assessment. The first set evaluated total costs using assumptions for construction costs that included the following:

- Operating period of 30 years

- On-line date of January 1, 2013

- Discount rate of $3 \%$

- Other rates and factors given by 2012 Federal Emergency Management Program (FEMP) rates

- $100 \%$ government funding

- Base energy rates of $\$ 0.229$ per $\mathrm{kWh}$

- Install and removal costs at approximately $\$ 25,000$; Supervision, Inspection, and Overhead $(\mathrm{SIOH})$ cost of $6 \%$; and design cost of $4 \%$.

Removal/install costs, transformer prices, and base energy rates could vary significantly depending on the situation, so any approximations used in this report should be refined for better matching to actual project details of future transformer replacements. INL felt these results could be improved upon by removing construction aspects and looking solely at transformer prices. As a result, the second set of LCC analysis was created and used for the primary analysis in this report.

The second LCC analysis scenario was similar to the first except that the LCC input was narrowed to evaluate only the transformer price (install/removal, $\mathrm{SIOH}$, and design cost were removed). INL believes that these costs will vary only slightly whether the replacement is done today or ten years from now. By running the analysis without these extra inputs, the results show a clearer picture of the differences in transformer levels (low, medium, and high).

The LCC analysis for transformers loaded at 20\% can be found in Appendix D and will not be discussed here as both sets of analysis show that transformers with $20 \%$ load have poor Simple Payback Period (SPP), Savings to Investment Ratio (SIR), and Adjusted Internal Rate of Return (AIRR). Transformers loaded at $60 \%$ show better economics, as seen in the two sets of LCC analysis shown in Tables 2 and 3. 
Table 2. Transformers Loaded (with Construction Costs) at 60\% LCC Results Summary

\begin{tabular}{|c|c|c|c|c|c|c|c|}
\hline & $\begin{array}{c}\text { New } \\
\text { XFMR } \\
\text { Price\$ }\end{array}$ & $\begin{array}{c}\text { Total } \\
\text { Cost } \\
\text { includes } \\
\text { install/removal, } \\
\text { SIOH, \& DC) }\end{array}$ & $\begin{array}{c}\text { Operating } \\
\text { Annual } \\
\text { Savings } \$\end{array}$ & $\begin{array}{c}\text { SPP } \\
\text { SYRS) }\end{array}$ & $\begin{array}{c}\text { Total } \\
\text { Discounted } \\
\text { Operational } \\
\text { Savings } \$\end{array}$ & SIR & AIRR \\
\hline 500 Low & $\$ 27,569.00$ & $\$ 57,826.00$ & $-\$ 1,209.00$ & -47.85 & $-\$ 23,716.00$ & -0.41 & $\mathrm{n} / \mathrm{a}$ \\
\hline $500 \mathrm{Med}$ & $\$ 36,354.00$ & $\$ 64,894.00$ & $\$ 1,307.00$ & 49.67 & $\$ 25,638.00$ & 0.4 & $-0.14 \%$ \\
\hline $500 \mathrm{High}$ & $\$ 59,626.00$ & $\$ 93,089.00$ & $\$ 2,843.00$ & 32.74 & $\$ 55,795.00$ & 0.6 & $1.26 \%$ \\
\hline $750 \mathrm{Low}$ & $\$ 36,354.00$ & $\$ 67,489.00$ & $-\$ 1,801.00$ & -37.47 & $-\$ 35,345.00$ & -0.52 & $\mathrm{n} / \mathrm{a}$ \\
\hline $750 \mathrm{Med}$ & $\$ 44,299.00$ & $\$ 76,229.00$ & $\$ 2,056.00$ & 37.07 & $\$ 40,354.00$ & 0.53 & $0.84 \%$ \\
\hline $750 \mathrm{High}$ & $\$ 69,350.00$ & $\$ 103,785.00$ & $\$ 3,758.00$ & 27.62 & $\$ 73,746.00$ & 0.71 & $1.83 \%$ \\
\hline $1000 \mathrm{Low}$ & $\$ 50,794.00$ & $\$ 83,373.00$ & $-\$ 2,386.00$ & -34.94 & $-\$ 46,822.00$ & -0.56 & $\mathrm{n} / \mathrm{a}$ \\
\hline $1000 \mathrm{Med}$ & $\$ 60,742.00$ & $\$ 94,316.00$ & $\$ 2,692.00$ & 35.04 & $\$ 52,824.00$ & 0.56 & $1.03 \%$ \\
\hline $1000 \mathrm{High}$ & $\$ 82,958.00$ & $\$ 118,754.00$ & $\$ 4,458.00$ & 26.64 & $\$ 87,471.00$ & 0.74 & $1.96 \%$ \\
\hline
\end{tabular}

Table 3. Transformers Loaded (without Construction Costs) at 60\% LCC Results Summary

\begin{tabular}{|c|c|c|c|c|c|c|}
\hline & $\begin{array}{c}\text { New } \\
\text { XFMR } \\
\text { Price\$ }\end{array}$ & $\begin{array}{c}\text { Operating } \\
\text { Annual } \\
\text { Savings } \$\end{array}$ & $\begin{array}{c}\text { SPP } \\
\text { (YRS) }\end{array}$ & $\begin{array}{c}\text { Total Discounted } \\
\text { Operational Savings \$ }\end{array}$ & SIR & AIRR \\
\hline $500 \mathrm{Low}$ & $\$ 27,569.00$ & $-\$ 1,209.00$ & -22.81 & $-\$ 23,716.00$ & -0.86 & $\mathrm{n} / \mathrm{a}$ \\
\hline $500 \mathrm{Med}$ & $\$ 36,354.00$ & $\$ 1,307.00$ & 27.83 & $\$ 25,638.00$ & 0.71 & $1.81 \%$ \\
\hline $500 \mathrm{High}$ & $\$ 59,626.00$ & $\$ 2,843.00$ & 20.97 & $\$ 55,795.00$ & 0.94 & $2.77 \%$ \\
\hline $750 \mathrm{Low}$ & $\$ 36,354.00$ & $-\$ 1,801.00$ & -20.18 & $-\$ 35,345.00$ & -0.97 & $\mathrm{n} / \mathrm{a}$ \\
\hline $750 \mathrm{Med}$ & $\$ 44,299.00$ & $\$ 2,056.00$ & 21.54 & $\$ 40,354.00$ & 0.91 & $2.68 \%$ \\
\hline $750 \mathrm{High}$ & $\$ 69,350.00$ & $\$ 3,758.00$ & 18.45 & $\$ 73,746.00$ & 1.06 & $3.21 \%$ \\
\hline $1000 \mathrm{Low}$ & $\$ 50,794.00$ & $-\$ 2,386.00$ & -21.29 & $-\$ 46,822.00$ & -0.92 & $\mathrm{n} / \mathrm{a}$ \\
\hline $1000 \mathrm{Med}$ & $\$ 60,742.00$ & $\$ 2,692.00$ & 22.56 & $\$ 52,824.00$ & 0.87 & $2.52 \%$ \\
\hline $1000 \mathrm{High}$ & $\$ 82,958.00$ & $\$ 4,458.00$ & 18.61 & $\$ 87,471.00$ & 1.05 & $3.18 \%$ \\
\hline
\end{tabular}

As noted above, the second LCC analysis was conducted by only taking into account transformer prices. For example, this analysis shows that replacing a current Okinawa $1000 \mathrm{kVA}$ transformer with a High-Level $1000 \mathrm{kVA}$ transformer will have an SPP of 18.61 years, SIR of 1.05, and an AIRR of 3.18\%. Using these results, replacing a current transformer with a High or Medium transformer would be justified as both levels of transformers have the ability to pay for themselves in their lifetimes. This is because their efficiency improvements (lower energy losses) are enough to cover their higher costs, when taking into account the assumptions INL made about current Okinawa transformers. 


\section{Economic Analysis}

INL performed further analysis by performing a straight-line depreciation comparison of the transformers from the "key" transformer list. The comparison was based on a replacement date of now (2012) until the transformers actual end of life (EOL) date. This analysis provides details of the differences in replacing a transformer now or waiting until the EOL of the transformer. In certain situations, however, this analysis may not apply because there are other justifications besides the cost difference for replacing transformers early. Those other issues would include maintenance issues, such as bad oil sample tests; any additional indications a unit is failing; a unit history of suspect reliability; and/or possibly liability concerns. Table 4 shows an analysis of the amount of energy losses (in U.S. dollars) for each transformer from the present date to the EOL for the transformers on the "key" transformer list. Table 5 represents the deltas between replacing a transformer early with a medium- or high-level transformer and not replacing an Okinawa transformer. From this analysis, INL finds that on some occasions replacing a transformer early is justified. For example, transformer 2365 TS at Camp Hansen has 19 years until EOL. By replacing this transformer now with a high-level transformer (instead of leaving the current transformer in operation for 19 more years), the difference recognized in energy loss dollars is $\$ 84,693.79$. In this example, the difference would cover the price of the new transformer (High-Level $1000 \mathrm{kVA}=\$ 82,958.00$ ). The transformers that appear to have similar benefits for early replacement would be:

- Camp Foster, 5907 TS, 750 kVA

- Camp Foster, 11 TS, 750 kVA

- Camp Foster, 363 TS, 750 kVA.

Several other transformers show near similar results for possible early replacement, and INL suspects that if actual data is used in place of assumed data, there will be other transformers that are ideal candidates for early replacement. 
Table 4. Transformer \$ Loss to EOL comparison at $60 \%$ Loading

\begin{tabular}{|c|c|c|c|c|c|c|}
\hline Camp & Location & kVA & $\begin{array}{c}\text { Years until } \\
\text { EOL }\end{array}$ & $\begin{array}{c}\text { Okinawa XFMR } \\
\text { \$ Loss } \\
\text { Present to EOL } \\
\end{array}$ & $\begin{array}{c}\text { New Medium-Level XFMR } \\
\text { \$ Loss } \\
\text { Present to EOL } \\
\end{array}$ & $\begin{array}{c}\text { New High-Level XFMR } \\
\text { \$ Loss } \\
\text { Present to EOL } \\
\end{array}$ \\
\hline Kinser & $99 \mathrm{TS}$ & 500 & 6 & $\$ 50,727.74$ & $\$ 42,888.69$ & $\$ 33,667.77$ \\
\hline Foster & $5704 \mathrm{TS}$ & 500 & 7 & $\$ 59,182.36$ & $\$ 50,036.80$ & $\$ 39,279.07$ \\
\hline Foster & $5703 \mathrm{TS}$ & 500 & 7 & $\$ 59,182.36$ & $\$ 50,036.80$ & $\$ 39,279.07$ \\
\hline Foster & $5670 \mathrm{TS}$ & 500 & 7 & $\$ 59,182.36$ & $\$ 50,036.80$ & $\$ 39,279.07$ \\
\hline Hansen & $2622 \mathrm{TS}$ & 500 & 8 & $\$ 67,636.98$ & $\$ 57,184.91$ & $\$ 44,890.36$ \\
\hline Hansen & $2665 \mathrm{TS}$ & 500 & 8 & $\$ 67,636.98$ & $\$ 57,184.91$ & $\$ 44,890.36$ \\
\hline Hansen & $2340 \mathrm{TS}$ & 500 & 8 & $\$ 67,636.98$ & $\$ 57,184.91$ & $\$ 44,890.36$ \\
\hline Hansen & 2442 TS & 500 & 8 & $\$ 67,636.98$ & $\$ 57,184.91$ & $\$ 44,890.36$ \\
\hline Kinser & $1225 \mathrm{TS}$ & 500 & 8 & $\$ 67,636.98$ & $\$ 57,184.91$ & $\$ 44,890.36$ \\
\hline Foster & $5696 \mathrm{TS}$ & 500 & 9 & $\$ 76,091.61$ & $\$ 64,333.03$ & $\$ 50,501.66$ \\
\hline Foster & $370 \mathrm{TS}$ & 500 & 9 & $\$ 76,091.61$ & $\$ 64,333.03$ & $\$ 50,501.66$ \\
\hline Hansen & $2141 \mathrm{TS}$ & 500 & 9 & $\$ 76,091.61$ & $\$ 64,333.03$ & $\$ 50,501.66$ \\
\hline Hansen & $2530 \mathrm{TS}$ & 500 & 10 & $\$ 84,546.23$ & $\$ 71,481.14$ & $\$ 56,112.95$ \\
\hline Hansen & $2441 \mathrm{TS}$ & 500 & 11 & $\$ 93,000.85$ & $\$ 78,629.26$ & $\$ 61,724.25$ \\
\hline Kinser & $616 \mathrm{TS}$ & 500 & 11 & $\$ 93,000.85$ & $\$ 78,629.26$ & $\$ 61,724.25$ \\
\hline Hansen & $2860 \mathrm{TS}$ & 500 & 12 & $\$ 101,455.48$ & $\$ 85,777.37$ & $\$ 67,335.54$ \\
\hline Hansen & 2165 TS & 500 & 12 & $\$ 101,455.48$ & $\$ 85,777.37$ & $\$ 67,335.54$ \\
\hline Foster & $481 \mathrm{TS}$ & 500 & 14 & $\$ 118,364.72$ & $\$ 100,073.60$ & $\$ 78,558.13$ \\
\hline Hansen & $2221 \mathrm{TS}$ & 500 & 14 & $\$ 118,364.72$ & $\$ 100,073.60$ & $\$ 78,558.13$ \\
\hline Hansen & $2518 \mathrm{TS}$ & 500 & 14 & $\$ 118,364.72$ & $\$ 100,073.60$ & $\$ 78,558.13$ \\
\hline Foster & $478 \mathrm{TS}$ & 500 & 15 & $\$ 126,819.35$ & $\$ 107,221.71$ & $\$ 84,169.43$ \\
\hline Foster & 445 TS & 500 & 16 & $\$ 135,273.97$ & $\$ 114,369.83$ & $\$ 89,780.72$ \\
\hline Foster & $490 \mathrm{TS}$ & 500 & 17 & $\$ 143,728.59$ & $\$ 121,517.94$ & $\$ 95,392.02$ \\
\hline Hansen & $2519 \mathrm{TS}$ & 500 & 17 & $\$ 143,728.59$ & $\$ 121,517.94$ & $\$ 95,392.02$ \\
\hline Hansen & $2610 \mathrm{TS}$ & 500 & 17 & $\$ 143,728.59$ & $\$ 121,517.94$ & $\$ 95,392.02$ \\
\hline Hansen & $2245 \mathrm{TS}$ & 500 & 17 & $\$ 143,728.59$ & $\$ 121,517.94$ & $\$ 95,392.02$ \\
\hline Kinser & $100 \mathrm{TS}$ & 750 & 7 & $\$ 81,015.20$ & $\$ 66,619.81$ & $\$ 54,708.37$ \\
\hline Kinser & $1226 \mathrm{TS}$ & 750 & 9 & $\$ 104,162.40$ & $\$ 85,654.04$ & $\$ 70,339.34$ \\
\hline Hansen & $2537 \mathrm{TS}$ & 750 & 10 & $\$ 115,736.00$ & $\$ 95,171.15$ & $\$ 78,154.82$ \\
\hline Hansen & $2725 \mathrm{TS}$ & 750 & 10 & $\$ 115,736.00$ & $\$ 95,171.15$ & $\$ 78,154.82$ \\
\hline Hansen & $2893 \mathrm{TS}$ & 750 & 10 & $\$ 115,736.00$ & $\$ 95,171.15$ & $\$ 78,154.82$ \\
\hline Kinser & $1210 \mathrm{TS}$ & 750 & 10 & $\$ 115,736.00$ & $\$ 95,171.15$ & $\$ 78,154.82$ \\
\hline Hansen & $2667 \mathrm{TS}$ & 750 & 11 & $\$ 127,309.60$ & $\$ 104,688.27$ & $\$ 85,970.30$ \\
\hline Hansen & $2327 \mathrm{TS}$ & 750 & 11 & $\$ 127,309.60$ & $\$ 104,688.27$ & $\$ 85,970.30$ \\
\hline Kinser & $1202 \mathrm{TS}$ & 750 & 11 & $\$ 127,309.60$ & $\$ 104,688.27$ & $\$ 85,970.30$ \\
\hline Kinser & 104-A TS & 750 & 11 & $\$ 127,309.60$ & $\$ 104,688.27$ & $\$ 85,970.30$ \\
\hline Foster & $480 \mathrm{TS}$ & 750 & 14 & $\$ 162,030.40$ & $\$ 133,239.61$ & $\$ 109,416.74$ \\
\hline Foster & $464 \mathrm{TS}$ & 750 & 16 & $\$ 185,177.60$ & $\$ 152,273.84$ & $\$ 125,047.71$ \\
\hline Hansen & $2635 \mathrm{TS}$ & 750 & 17 & $\$ 196,751.20$ & $\$ 161,790.96$ & $\$ 132,863.19$ \\
\hline Foster & $5907 \mathrm{TS}$ & 750 & 19 & $\$ 219,898.40$ & $\$ 180,825.19$ & $\$ 148,494.15$ \\
\hline Foster & $11 \mathrm{TS}$ & 750 & 19 & $\$ 219,898.40$ & $\$ 180,825.19$ & $\$ 148,494.15$ \\
\hline Foster & 363 TS & 750 & 19 & $\$ 219,898.40$ & $\$ 180,825.19$ & $\$ 148,494.15$ \\
\hline Foster & $1004 \mathrm{TV}$ & 1000 & 7 & $\$ 97,675.76$ & $\$ 78,832.24$ & $\$ 66,472.78$ \\
\hline Foster & $1002 \mathrm{TS}$ & 1000 & 11 & $\$ 153,490.48$ & $\$ 123,879.23$ & $\$ 104,457.23$ \\
\hline Hansen & 2654 TS & 1000 & 18 & $\$ 251,166.24$ & $\$ 202,711.47$ & $\$ 170,930.01$ \\
\hline Hansen & $2365 \mathrm{TS}$ & 1000 & 19 & $\$ 265,119.92$ & $\$ 213,973.21$ & $\$ 180,426.13$ \\
\hline
\end{tabular}


Table 5. Deltas between Okinawa Transformers and Medium/High Level Transformers

\begin{tabular}{|c|c|c|c|c|c|}
\hline Camp & Location & kVA & $\begin{array}{c}\text { Years until } \\
\text { EOL }\end{array}$ & $\begin{array}{c}\text { Okinawa XFMR } \\
\text { \$ Loss } \\
\text { minus } \\
\text { Medium-Level XFMR \$ Loss } \\
\end{array}$ & $\begin{array}{c}\text { Okinawa XFMR } \\
\text { \$ Loss } \\
\text { minus } \\
\text { High-Level XFMR \$ Loss }\end{array}$ \\
\hline Kinser & $99 \mathrm{TS}$ & 500 & 6 & $\$ 7,839.05$ & $\$ 17,059.97$ \\
\hline Foster & $5704 \mathrm{TS}$ & 500 & 7 & $\$ 9,145.56$ & $\$ 19,903.30$ \\
\hline Foster & 5703 TS & 500 & 7 & $\$ 9,145.56$ & $\$ 19,903.30$ \\
\hline Foster & $5670 \mathrm{TS}$ & 500 & 7 & $\$ 9,145.56$ & $\$ 19,903.30$ \\
\hline Hansen & $2622 \mathrm{TS}$ & 500 & 8 & $\$ 10,452.07$ & $\$ 22,746.62$ \\
\hline Hansen & $2665 \mathrm{TS}$ & 500 & 8 & $\$ 10,452.07$ & $\$ 22,746.62$ \\
\hline Hansen & 2340 TS & 500 & 8 & $\$ 10,452.07$ & $\$ 22,746.62$ \\
\hline Hansen & 2442 TS & 500 & 8 & $\$ 10,452.07$ & $\$ 22,746.62$ \\
\hline Kinser & $1225 \mathrm{TS}$ & 500 & 8 & $\$ 10,452.07$ & $\$ 22,746.62$ \\
\hline Foster & $5696 \mathrm{TS}$ & 500 & 9 & $\$ 11,758.58$ & $\$ 25,589.95$ \\
\hline Foster & $370 \mathrm{TS}$ & 500 & 9 & $\$ 11,758.58$ & $\$ 25,589.95$ \\
\hline Hansen & $2141 \mathrm{TS}$ & 500 & 9 & $\$ 11,758.58$ & $\$ 25,589.95$ \\
\hline Hansen & $2530 \mathrm{TS}$ & 500 & 10 & $\$ 13,065.09$ & $\$ 28,433.28$ \\
\hline Hansen & $2441 \mathrm{TS}$ & 500 & 11 & $\$ 14,371.60$ & $\$ 31,276.61$ \\
\hline Kinser & $616 \mathrm{TS}$ & 500 & 11 & $\$ 14,371.60$ & $\$ 31,276.61$ \\
\hline Hansen & $2860 \mathrm{TS}$ & 500 & 12 & $\$ 15,678.10$ & $\$ 34,119.93$ \\
\hline Hansen & $2165 \mathrm{TS}$ & 500 & 12 & $\$ 15,678.10$ & $\$ 34,119.93$ \\
\hline Foster & $481 \mathrm{TS}$ & 500 & 14 & $\$ 18,291.12$ & $\$ 39,806.59$ \\
\hline Hansen & $2221 \mathrm{TS}$ & 500 & 14 & $\$ 18,291.12$ & $\$ 39,806.59$ \\
\hline Hansen & $2518 \mathrm{TS}$ & 500 & 14 & $\$ 18,291.12$ & $\$ 39,806.59$ \\
\hline Foster & $478 \mathrm{TS}$ & 500 & 15 & $\$ 19,597.63$ & $\$ 42,649.92$ \\
\hline Foster & $445 \mathrm{TS}$ & 500 & 16 & $\$ 20,904.14$ & $\$ 45,493.25$ \\
\hline Foster & $490 \mathrm{TS}$ & 500 & 17 & $\$ 22,210.65$ & $\$ 48,336.57$ \\
\hline Hansen & $2519 \mathrm{TS}$ & 500 & 17 & $\$ 22,210.65$ & $\$ 48,336.57$ \\
\hline Hansen & $2610 \mathrm{TS}$ & 500 & 17 & $\$ 22,210.65$ & $\$ 48,336.57$ \\
\hline Hansen & 2245 TS & 500 & 17 & $\$ 22,210.65$ & $\$ 48,336.57$ \\
\hline Kinser & $100 \mathrm{TS}$ & 750 & 7 & $\$ 14,395.39$ & $\$ 26,306.83$ \\
\hline Kinser & $1226 \mathrm{TS}$ & 750 & 9 & $\$ 18,508.36$ & $\$ 33,823.06$ \\
\hline Hansen & 2537 TS & 750 & 10 & $\$ 20,564.85$ & $\$ 37,581.18$ \\
\hline Hansen & $2725 \mathrm{TS}$ & 750 & 10 & $\$ 20,564.85$ & $\$ 37,581.18$ \\
\hline Hansen & $2893 \mathrm{TS}$ & 750 & 10 & $\$ 20,564.85$ & $\$ 37,581.18$ \\
\hline Kinser & $1210 \mathrm{TS}$ & 750 & 10 & $\$ 20,564.85$ & $\$ 37,581.18$ \\
\hline Hansen & $2667 \mathrm{TS}$ & 750 & 11 & $\$ 22,621.33$ & $\$ 41,339.30$ \\
\hline Hansen & 2327 TS & 750 & 11 & $\$ 22,621.33$ & $\$ 41,339.30$ \\
\hline Kinser & 1202 TS & 750 & 11 & $\$ 22,621.33$ & $\$ 41,339.30$ \\
\hline Kinser & 104-A TS & 750 & 11 & $\$ 22,621.33$ & $\$ 41,339.30$ \\
\hline Foster & $480 \mathrm{TS}$ & 750 & 14 & $\$ 28,790.79$ & $\$ 52,613.66$ \\
\hline Foster & $464 \mathrm{TS}$ & 750 & 16 & $\$ 32,903.76$ & $\$ 60,129.89$ \\
\hline Hansen & $2635 \mathrm{TS}$ & 750 & 17 & $\$ 34,960.24$ & $\$ 63,888.01$ \\
\hline Foster & $5907 \mathrm{TS}$ & 750 & 19 & $\$ 39,073.21$ & $\$ 71,404.25$ \\
\hline Foster & $11 \mathrm{TS}$ & 750 & 19 & $\$ 39,073.21$ & $\$ 71,404.25$ \\
\hline Foster & $363 \mathrm{TS}$ & 750 & 19 & $\$ 39,073.21$ & $\$ 71,404.25$ \\
\hline Foster & $1004 \mathrm{TV}$ & 1000 & 7 & $\$ 18,843.52$ & $\$ 31,202.98$ \\
\hline Foster & $1002 \mathrm{TS}$ & 1000 & 11 & $\$ 29,611.25$ & $\$ 49,033.25$ \\
\hline Hansen & $2654 \mathrm{TS}$ & 1000 & 18 & $\$ 48,454.77$ & $\$ 80,236.23$ \\
\hline Hansen & $2365 \mathrm{TS}$ & 1000 & 19 & $\$ 51,146.71$ & $\$ 84,693.79$ \\
\hline
\end{tabular}




\section{Recommendations}

INL's analysis shows that transformers in the $750 \mathrm{kVA}$ to $1000 \mathrm{kVA}$ size range will see the greatest energy savings from efficiency improvements. Replacing a current transformer with a high- or mediumlevel transformer would be justified as both levels of transformers have the ability to pay for themselves in their lifetimes. INL also found that on some occasions replacing a transformer early is justified and that several other transformers show potential for possible early replacement.

INL suspects that if actual data is used in place of assumed data, there will be other transformers that are ideal candidates for early replacement. As such, INL recommends that load measurements be performed on the high and low sides of each transformer to better quantify actual load losses, demand data, and power factor data. INL also recommends that actual data, when available, be inserted by MCB Okinawa where assumptions have been made and that the LCC analysis and economic analysis be updated accordingly.

Other justifications exist for replacing transformers early besides the energy savings perspective. Those other justifications would include maintenance issues, such as bad oil sample tests; any additional indications unit is failing; a unit history of suspect reliability; and/or possibly liability concerns. Market conditions should also continue to be monitored to determine if economy fluctuations and changes could affect the timing of the project and procurements. INL believes that under the current market conditions, several manufacturers/models of transformers could respond to a contracting/procurement process to supply transformers to this proposed project.

Transformer efficiency improvements are not the only place where energy savings can be recognized. Electrical energy savings is the primary goal, and potentially greater savings with a higher SIR could be achieved by considering building efficiency upgrades, such as insulation, lights, windows, doors, appliances, smart-grid meters, micro-grid control systems, and so forth. 


\section{Appendix A}

\section{Military Base Transformer List}


APPENDIX A

Pad Mounted Transformer List

\begin{tabular}{|c|c|c|c|c|c|c|}
\hline CAMP & LOC & CAPACITY KVA & MANUFACT & MFR_DATE & Serial_ID & Type \\
\hline HANSEN & 307Q-8 POLE & $10 \mathrm{KVA}$ & $\mathrm{AlCHI}$ & 2007 & 07211926 & Pad Mount \\
\hline CTNY & 4354 TS & $100 \mathrm{KVA}$ & OSAKA & 1985 & 3ND0577A1 & Pad Mount \\
\hline CTNY & $4399 \mathrm{TS}$ & $100 \mathrm{KVA}$ & MITSUBISHI & 2005 & DA3657001 & Pad Mount \\
\hline CTNY-TG & 4137 TS & $100 \mathrm{KVA}$ & DAIHEN & 1988 & 3ND0823A1 & Pad Mount \\
\hline FOSTER & $5641 \mathrm{TS}$ & $100 \mathrm{KVA}$ & $\mathrm{AlCHI}$ & 1993 & 9222477 & Pad Mount \\
\hline FOSTER & 5970 TS & $100 \mathrm{KVA}$ & $\mathrm{AlCHI}$ & & 9221168 & Pad Mount \\
\hline FOSTER & 362 TS & $100 \mathrm{KVA}$ & $\mathrm{AlCHI}$ & 2000 & 0701085 & Pad Mount \\
\hline FOSTER & 5666 TS & $100 \mathrm{KVA}$ & $\mathrm{AlCHI}$ & 2002 & 02704192 & Pad Mount \\
\hline FOSTER & 497 TS & $100 \mathrm{KVA}$ & $\mathrm{AlCHI}$ & 1999 & 8960322 & Pad Mount \\
\hline FOSTER & 5833 TS & $100 \mathrm{KVA}$ & $\mathrm{AlCHI}$ & 2000 & 0700486 & Pad Mount \\
\hline FOSTER-1 & SHOP STOCK & $100 \mathrm{KVA}$ & FUJI & $93 \mathrm{JUN}$ & A30946T1 & Pad Mount \\
\hline FOSTER-1 & SHOP STOCK & $100 \mathrm{KVA}$ & DAIHEN & 1994 & 2TF436801 & Pad Mount \\
\hline HANSEN & $2346 \mathrm{TS}$ & $100 \mathrm{KVA}$ & $\mathrm{AlCHI}$ & 1992 & 9220879 & Pad Mount \\
\hline HANSEN & 2653 TS & $100 \mathrm{KVA}$ & MEIDEN & JUN 1992 & 1R8544T1 & Pad Mount \\
\hline HANSEN & 2609 TS & $100 \mathrm{KVA}$ & MITSUBISHI & 1993 & DF0790001 & Pad Mount \\
\hline HANSEN & 2175 TS & $100 \mathrm{KVA}$ & HITACHI & 2002 & 24614221 & Pad Mount \\
\hline HANSEN & 2174 TS & $100 \mathrm{KVA}$ & $\mathrm{AlCHI}$ & 2005 & 05207302 & Pad Mount \\
\hline HANSEN & 2464 TS & $100 \mathrm{KVA}$ & TOSHIBA & 2002 & 02011230 & Pad Mount \\
\hline HANSEN & 2715 TS & $100 \mathrm{KVA}$ & FUJI & DEC 1996 & $\mathrm{~B} 60382 \mathrm{~A} 01$ & Pad Mount \\
\hline HANSEN & 2474 TS & $100 \mathrm{KVA}$ & $\mathrm{AlCHI}$ & 2008 & 08213938 & Pad Mount \\
\hline HANSEN & 2439 TS & $100 \mathrm{KVA}$ & FUJI & $1990 \mathrm{JUN}$ & A01329T 1-1 & Pad Mount \\
\hline HANSEN & 2240 TS & $100 \mathrm{KVA}$ & FUJI & 1991 FEB & B00364A01 & Pad Mount \\
\hline KINSER & 104-C TS & $100 \mathrm{KVA}$ & DAIHEN & ОСТ 1986 & 3ND0657A1 & Pad Mount \\
\hline
\end{tabular}


Page 2 of 24

\begin{tabular}{|c|c|c|c|c|c|c|}
\hline KINSER & 424 TS & $100 \mathrm{KVA}$ & $\mathrm{AlCHI}$ & 1986 & 8622191 & Pad Mount \\
\hline KINSER & $520 \mathrm{TS}$ & $100 \mathrm{KVA}$ & DAIHEN & & 3SD146001 & Pad Mount \\
\hline KINSER & 1305-A TS & $100 \mathrm{KVA}$ & $\mathrm{AlCHI}$ & SEP 1992 & 9123095 & Pad Mount \\
\hline KINSER & $611 \mathrm{TS}$ & $100 \mathrm{KVA}$ & $\mathrm{AlCHI}$ & 1999 & 9860231 & Pad Mount \\
\hline KINSER & 5 -E TS & $100 \mathrm{KVA}$ & JIMELCO & $1991 \mathrm{MAR}$ & AA00201406 & Pad Mount \\
\hline MCAS & $681 \mathrm{TS}$ & $100 \mathrm{KVA}$ & DAIHEN & 1992 & 3SD1209A1 & Pad Mount \\
\hline MCAS & $687 \mathrm{TS}$ & $100 \mathrm{KVA}$ & DAIHEN & & 3SD1206A2 & Pad Mount \\
\hline MCAS & 675 TS & $100 \mathrm{KVA}$ & DAIHEN & & 3ND0819A1 & Pad Mount \\
\hline MCAS & $683 \mathrm{TS}$ & $100 \mathrm{KVA}$ & $\mathrm{AlCHI}$ & & 8820697 & Pad Mount \\
\hline FOSTER & 5972 TV & $1000 \mathrm{KVA}$ & $\mathrm{AlCHI}$ & & 9221416 & Pad Mount \\
\hline FOSTER & 1004 TV & $1000 \mathrm{KVA}$ & WESTING HOUSE & OCT 1989 & PAW4176-0101 & Pad Mount \\
\hline $\begin{array}{l}\text { FOSTER } \\
\end{array}$ & 1002 TS & $1000 \mathrm{KVA}$ & G.E. & JUN 1993 & Q108279-TNI & Pad Mount \\
\hline FOSTER & 122-F TS & $1000 \mathrm{KVA}$ & MITSUBISHI & & D52035001 & Pad Mount \\
\hline FOSTER & 148-B TS & $1000 \mathrm{KVA}$ & DAIHEN & 1988 & 2TE141401 & Pad Mount \\
\hline FOSTER & 5866 TS & $1000 \mathrm{KVA}$ & VANTRAN & & $90 \mathrm{~V} 6400$ & Pad Mount \\
\hline FOSTER & $5718 \mathrm{TS}$ & $1000 \mathrm{KVA}$ & COOPER & JUL 2008 & 0850011715 & Pad Mount \\
\hline FOSTER-1 & SHOP STOCK & $1000 \mathrm{KVA}$ & COOPER & 2005 JAN & 0526000153 & Pad Mount \\
\hline HANSEN & 2365 TS & $1000 \mathrm{KVA}$ & FUJI & 2001 JUN & B10215A01 & Pad Mount \\
\hline HANSEN & 2654 TS & $1000 \mathrm{KVA}$ & TOSHIBA & 2000 & 00023589 & Pad Mount \\
\hline HENOKO & 1024 TS & $1000 \mathrm{KVA}$ & DAIHEN & & 3SD145701 & Pad Mount \\
\hline KINSER & $831 \mathrm{TS}$ & $1000 \mathrm{KVA}$ & MITSUBISHI & & DB5361001 & Pad Mount \\
\hline KINSER & $107 \mathrm{TV}$ & $1000 \mathrm{KVA}$ & SIEMENS & & PYG-0619 & Pad Mount \\
\hline KINSER & $67-C$ TS & $1000 \mathrm{KVA}$ & RTE & & 886000358 & Pad Mount \\
\hline KINSER & $107 \mathrm{TS}$ & $1000 \mathrm{KVA}$ & SIEMENS & & PZE-0484 & Pad Mount \\
\hline KINSER & 301 TS & $1000 \mathrm{KVA}$ & COOPER & 2005 FEB & 0537003818 & Pad Mount \\
\hline MCAS & $633 \mathrm{TS}$ & $1000 \mathrm{KVA}$ & FUJI & & B50328A01 & Pad Mount \\
\hline MCAS & 49-A-2 TS & $1000 \mathrm{KVA}$ & WESTING HOUSE & APR 1989 & 89J385097 & Pad Mount \\
\hline MCAS & 505 TS & $1000 \mathrm{KVA}$ & COOPER & JUN 2007 & 0759001399 & Pad Mount \\
\hline
\end{tabular}


Page 3 of 24

\begin{tabular}{|c|c|c|c|c|c|c|}
\hline CTNY & 4352 TS & $112.5 \mathrm{KVA}$ & HOWARD & & 2252112195 & Pad Mount \\
\hline CTNY & 4206 TS & $112.5 \mathrm{KVA}$ & COOPER & 2005 FEB & 0537004313 & Pad Mount \\
\hline CTNY & $4436 \mathrm{TS}$ & $112.5 \mathrm{KVA}$ & VANTRAN & & $05 \mathrm{~V} 6858$ & Pad Mount \\
\hline CTNY-TG & 4108 TS & $112.5 \mathrm{KVA}$ & RTE & & 896003384 & Pad Mount \\
\hline FOSTER & 13-A TS & $112.5 \mathrm{KVA}$ & ABB & JAN 1991 & 91J817095 & Pad Mount \\
\hline FOSTER & $301-C$ TS & $112.5 \mathrm{KVA}$ & COOPER & NOV 1995 & 959006282 & Pad Mount \\
\hline FOSTER & 33-D TS & $112.5 \mathrm{KVA}$ & PAUWELS & NOV 2004 & 04J129802 & Pad Mount \\
\hline FOSTER & $371 R$ TS & $112.5 \mathrm{KVA}$ & COOPER & AUG 2008 & 0850013516 & Pad Mount \\
\hline FOSTER-1 & SHOP STOCK & $112.5 \mathrm{KVA}$ & ABB & DEC 2008 & 08J162068 & Pad Mount \\
\hline FOSTER-1 & SHOP STOCK & $112.5 \mathrm{KVA}$ & COOPER & AUG 2008 & 0850012805 & Pad Mount \\
\hline FOSTER-1 & SHOP STOCK & $112.5 \mathrm{KVA}$ & COOPER & APR 2009 & 0950003921 & Pad Mount \\
\hline FOSTER-1 & SHOP STOCK & $112.5 \mathrm{KVA}$ & COOPER & 2005 FEB & 0537003338 & Pad Mount \\
\hline FOSTER-1 & SHOP STOCK & $112.5 \mathrm{KVA}$ & PAUWELS & NOV 2004 & 04J129805 & Pad Mount \\
\hline FOSTER-1 & SHOP STOCK & $112.5 \mathrm{KVA}$ & COOPER & 2005 FEB & 0537003337 & Pad Mount \\
\hline FOSTER-1 & SHOP STOCK & $112.5 \mathrm{KVA}$ & COOPER & SEP 2007 & 0750017354 & Pad Mount \\
\hline FOSTER-1 & SHOP STOCK & $112.5 \mathrm{KVA}$ & $G$ & FEB 2010 & 20111020441 & Pad Mount \\
\hline FOSTER-1 & SHOP STOCK & $112.5 \mathrm{KVA}$ & COOPER & JAN 2010 & 1050000517 & Pad Mount \\
\hline FOSTER-1 & SHOP STOCK & $112.5 \mathrm{KVA}$ & COOPER & JUL 2009 & 0950006596 & Pad Mount \\
\hline FOSTER-1 & SHOP STOCK & $112.5 \mathrm{KVA}$ & COOPER & DEC 2009 & 0950011341 & Pad Mount \\
\hline HANSEN & 2406 TS & $112.5 \mathrm{KVA}$ & COOPER & 2006 SEP & 0650016683 & Pad Mount \\
\hline HANSEN & 2892 TS & $112.5 \mathrm{KVA}$ & COOPER & FEB 2005 & 0537004310 & Pad Mount \\
\hline HANSEN & $2435 \mathrm{TS}$ & $112.5 \mathrm{KVA}$ & COOPER & 2005 FEB & 0537004314 & Pad Mount \\
\hline HANSEN & 2139 TS & $112.5 \mathrm{KVA}$ & COOPER & MAY 2008 & 0850007604 & Pad Mount \\
\hline HANSEN & 2887 TS & $112.5 \mathrm{KVA}$ & COOPER & 2007 MAY & 0750009544 & Pad Mount \\
\hline HANSEN & $2171 \mathrm{TS}$ & $112.5 \mathrm{KVA}$ & COOPER & 2008 JAN & 0850000733 & Pad Mount \\
\hline HANSEN & $2523 \mathrm{TS}$ & $112.5 \mathrm{KVA}$ & COOPER & AUG 2007 & 0750015787 & Pad Mount \\
\hline HANSEN & $2538 \mathrm{TS}$ & $112.5 \mathrm{KVA}$ & ABB & DEC 2008 & 08J162067 & Pad Mount \\
\hline HENOKO & $1 \mathrm{TS}$ & $112.5 \mathrm{KVA}$ & ABB & & 98J584270 & Pad Mount \\
\hline
\end{tabular}


Page 4 of 24

\begin{tabular}{|c|c|c|c|c|c|c|}
\hline HENOKO & 5 TS & $112.5 \mathrm{KVA}$ & VANTRAN & & 03V6401-1 & Pad Mount \\
\hline HENOKO & 2 TS & $112.5 \mathrm{KVA}$ & VANTRAN & & 03V6401-2 & Pad Mount \\
\hline HENOKO & $6 \mathrm{TS}$ & $112.5 \mathrm{KVA}$ & COOPER & & 0437009015 & Pad Mount \\
\hline HENOKO & 9-A TS & $112.5 \mathrm{KVA}$ & PAUWELS & SEP 2003 & $03 G 122433$ & Pad Mount \\
\hline HENOKO & 4 TS & $112.5 \mathrm{KVA}$ & VANTRAN & & 03V6401-3 & Pad Mount \\
\hline KINSER & 30-A TS & $112.5 \mathrm{KVA}$ & COOPER & & 929000640 & Pad Mount \\
\hline MCAS & 33-A TS & $112.5 \mathrm{KVA}$ & COOPER & 2008 FEB & 0850003442 & Pad Mount \\
\hline MCAS & $38 \mathrm{TS}$ & $112.5 \mathrm{KVA}$ & WESTING HOUSE & & 88JB100180 & Pad Mount \\
\hline MCAS & 11-C TS & $112.5 \mathrm{KVA}$ & COOPER & & 959006334 & Pad Mount \\
\hline MCAS & $667 \mathrm{TS}$ & $112.5 \mathrm{KVA}$ & WESTING HOUSE & & $88 \mathrm{~J} 246046$ & Pad Mount \\
\hline MCAS & $416 \mathrm{TS}$ & $112.5 \mathrm{KVA}$ & COOPER & 2005 FEB & 0537004311 & Pad Mount \\
\hline MCAS & $690 \mathrm{TS}$ & $112.5 \mathrm{KVA}$ & PAUWELS & APR 2007 & 20060002086 & Pad Mount \\
\hline MCAS & 11-B1 TS & $112.5 \mathrm{KVA}$ & PAUWELS & & 20060002087 & Pad Mount \\
\hline MCAS & 2B-3 TS & $112.5 \mathrm{KVA}$ & COOPER & MAY 2008 & 0850007605 & Pad Mount \\
\hline MCAS & $13-4$ TS & $112.5 \mathrm{KVA}$ & WESTING HOUSE & & 80JG262280 & Pad Mount \\
\hline MCAS & $435 \mathrm{TS}$ & $112.5 \mathrm{KVA}$ & COOPER & DEC 2003 & 0326002279 & Pad Mount \\
\hline KINSER & 202 TS & $1200 \mathrm{KVA}$ & FUJI & 1993 DEC & B30228A01 & Pad Mount \\
\hline MCAS & $518 \mathrm{TV}$ & $15 \mathrm{KW}$ & COOPER & & 2209 & Pad Mount \\
\hline CTNY & 4233 TS & $150 \mathrm{KVA}$ & DAIHEN & 1985 & 3ND0760A1 & Pad Mount \\
\hline CTNY & 4444 TS & $150 \mathrm{KVA}$ & MEIDEN & & 1N8218T1 & Pad Mount \\
\hline CTNY & $4438 \mathrm{TS}$ & $150 \mathrm{KVA}$ & PAUWELS & OCT 2005 & 05G135695 & Pad Mount \\
\hline CTNY & 4344 TS & $150 \mathrm{KVA}$ & VANTRAN & & $05 \bigvee 6855$ & Pad Mount \\
\hline CTNY & 4412 TS & $150 \mathrm{KVA}$ & $\overline{\mathrm{AlCHI}}$ & 2004 & 04205391 & Pad Mount \\
\hline CTNY & $4411 \mathrm{TS}$ & $150 \mathrm{KVA}$ & CUTLER-HAMMER & OCT 2003 & 03J908112 & Pad Mount \\
\hline CTNY-TG & 4135 TS & $150 \mathrm{KVA}$ & COOPER & SEP 2003 & 0337014224 & Pad Mount \\
\hline FOSTER & 1005 TS & $150 \mathrm{KVA}$ & $\mathrm{AICHI}$ & 1997 & 9720755 & Pad Mount \\
\hline FOSTER & $371 S$ TS & $150 \mathrm{KVA}$ & COOPER & & 0326000060 & Pad Mount \\
\hline FOSTER & $5821 \mathrm{TS}$ & $150 \mathrm{KVA}$ & $\mathrm{AlCHI}$ & 1999 & 9960222 & Pad Mount \\
\hline
\end{tabular}


Page 5 of 24

\begin{tabular}{|c|c|c|c|c|c|c|}
\hline FOSTER & $14 \mathrm{TS}$ & $150 \mathrm{KVA}$ & ABB & JAN 1991 & 91J817147 & Pad Mount \\
\hline FOSTER & 7-E TS & $150 \mathrm{KVA}$ & $\overline{A B B}$ & NOV 2003 & 03J924313 & Pad Mount \\
\hline FOSTER & $5838 \mathrm{TS}$ & $150 \mathrm{KVA}$ & DAIHEN & 2000 & KSB0151B1 & Pad Mount \\
\hline FOSTER & $5968 \mathrm{TS}$ & $150 \mathrm{KVA}$ & MITSUBISHI & & AM6336001 & Pad Mount \\
\hline FOSTER & 5655 TS & $150 \mathrm{KVA}$ & FUJI & 1997 & B70345AT1 & Pad Mount \\
\hline FOSTER & 151B TH & $150 \mathrm{KVA}$ & $\mathrm{AlCHI}$ & & 03203947 & Pad Mount \\
\hline FOSTER & 355 TS & $150 \mathrm{KVA}$ & MITSUBISHI & 2005 & DA3627001 & Pad Mount \\
\hline FOSTER & $1006 \mathrm{TS}$ & $150 \mathrm{KVA}$ & HITACHI & 2004 & 23011421 & Pad Mount \\
\hline FOSTER & 215 TS & $150 \mathrm{KVA}$ & DAIHEN & & 3SD1083A1 & Pad Mount \\
\hline FOSTER & 5969 TS & $150 \mathrm{KVA}$ & DAIHEN & & 3SD1433A1 & Pad Mount \\
\hline FOSTER & 1620 TS & $150 \mathrm{KVA}$ & DAIHEN & 1992 & 3SD1434A1 & Pad Mount \\
\hline FOSTER & $455 \mathrm{TS}$ & $150 \mathrm{KVA}$ & DAIHEN & 1992 & 3SD1519A1 & Pad Mount \\
\hline $\begin{array}{l}\text { FOSTER } \\
\end{array}$ & $206 \mathrm{TS}$ & $150 \mathrm{KVA}$ & HITACHI & 2003 & 24615221 & Pad Mount \\
\hline FOSTER & $12 \mathrm{TS}$ & $150 \mathrm{KVA}$ & WESTING HOUSE & & 85JJ513092 & Pad Mount \\
\hline FOSTER & 264-A TS & $150 \mathrm{KVA}$ & WESTING HOUSE & & 87JC873224 & Pad Mount \\
\hline FOSTER & $5631 \mathrm{TS}$ & $150 \mathrm{KVA}$ & COOPER & JUL 2009 & 0950006597 & Pad Mount \\
\hline FOSTER & 5668 TS & $150 \mathrm{KVA}$ & $\mathrm{AlCHI}$ & & 06208829 & Pad Mount \\
\hline FOSTER-1 & SHOP STOCK & $150 \mathrm{KVA}$ & COOPER & 1995 MAR & 959001470 & Pad Mount \\
\hline FOSTER-1 & SHOP STOCK & $150 \mathrm{KVA}$ & MITSUBISHI & 1993 & D90879004 & Pad Mount \\
\hline FOSTER-1 & SHOP STOCK & $150 \mathrm{KVA}$ & PAUWELS & OCT 2005 & 05G135694 & Pad Mount \\
\hline FOSTER-1 & SHOP STOCK & $150 \mathrm{KVA}$ & COOPER & 2005 FEB & 0537004315 & Pad Mount \\
\hline FOSTER-1 & SHOP STOCK & $150 \mathrm{KVA}$ & COOPER & 2005 FEB & 0537003967 & Pad Mount \\
\hline FOSTER-1 & SHOP STOCK & $150 \mathrm{KVA}$ & COOPER & 2005 FEB & 0537003964 & Pad Mount \\
\hline FOSTER-1 & SHOP STOCK & $150 \mathrm{KVA}$ & PAUWELS & NOV 2004 & 04J129804 & Pad Mount \\
\hline FOSTER-1 & SHOP STOCK & $150 \mathrm{KVA}$ & COOPER & 2005 FEB & 0537003966 & Pad Mount \\
\hline FOSTER-1 & SHOP STOCK & $150 \mathrm{KVA}$ & COOPER & SEP 09 & 0950008496 & Pad Mount \\
\hline HANSEN & SHOP STOCK & $150 \mathrm{KVA}$ & PAUWELS & $2007 \mathrm{JUN}$ & 20110703497 & Pad Mount \\
\hline HANSEN & 2354 TS & $150 \mathrm{KVA}$ & COOPER & JUN 2007 & 0750011232 & Pad Mount \\
\hline
\end{tabular}


Page 6 of 24

\begin{tabular}{|c|c|c|c|c|c|c|}
\hline HANSEN & 2440 TS & $150 \mathrm{KVA}$ & HOWARD & & 1312150504 & Pad Mount \\
\hline HANSEN & $2137 \mathrm{TS}$ & $150 \mathrm{KVA}$ & VANTRAN & & 92V8997 & Pad Mount \\
\hline HANSEN & 2859 TS & $150 \mathrm{KVA}$ & MEIDEN & DEC 1995 & 1T8309T1 & Pad Mount \\
\hline HANSEN & 2418 TS & $150 \mathrm{KVA}$ & MITSUBISHI & 1994 & DH8709001 & Pad Mount \\
\hline HANSEN & 2148 TS & $150 \mathrm{KVA}$ & MOLONEY & JUN 1964 & 2055688 & Pad Mount \\
\hline HANSEN & 2485-A TS & $150 \mathrm{KVA}$ & BALTEAU & & PZB-0165 & Pad Mount \\
\hline HANSEN & 2143 TS & $150 \mathrm{KVA}$ & $\mathrm{AICHI}$ & 2002 & 02704781 & Pad Mount \\
\hline HANSEN & $2451 \mathrm{TS}$ & $150 \mathrm{KVA}$ & DAIHEN & 1989 & 3SD1027A1 & Pad Mount \\
\hline HANSEN & 2208 TS & $150 \mathrm{KVA}$ & VANTRAN & & $88 \mathrm{~V} 3331$ & Pad Mount \\
\hline HANSEN & 2376 TS & $150 \mathrm{KVA}$ & PAUWELS & JUN 2007 & 200110703497 & Pad Mount \\
\hline HANSEN & R4 TS & $150 \mathrm{KVA}$ & $\mathrm{AlCHI}$ & 2007 & 07211925 & Pad Mount \\
\hline HANSEN & 2484 TS & $150 \mathrm{KVA}$ & $\mathrm{AlCHI}$ & 2006 & 06210656 & Pad Mount \\
\hline HANSEN & 2140 TS & $150 \mathrm{KVA}$ & COOPER & SEP 2007 & 0750017355 & Pad Mount \\
\hline HANSEN & 2864 TS & $150 \mathrm{KVA}$ & WESTING HOUSE & & 87JK022149 & Pad Mount \\
\hline HANSEN & 2901-A TS & $150 \mathrm{KVA}$ & VANTRAN & & 04V6756 & Pad Mount \\
\hline HANSEN & 2885 TS & $150 \mathrm{KVA}$ & $\mathrm{AlCHI}$ & 2005 & 05207424 & Pad Mount \\
\hline KINSER & 54-D TS & $150 \mathrm{KVA}$ & DAIHEN & 1987 & 3ND0696A1 & Pad Mount \\
\hline KINSER & 73-A TS & $150 \mathrm{KVA}$ & DAIHEN & 1986 & 3ND0612A1 & Pad Mount \\
\hline KINSER & 90-B TS & $150 \mathrm{KVA}$ & DAIHEN & AUG 1986 & 3ND063501 & Pad Mount \\
\hline KINSER & 864 TS & $150 \mathrm{KVA}$ & DAIHEN & 1995 & 3SD1867B1 & Pad Mount \\
\hline KINSER & 64-A TS & $150 \mathrm{KVA}$ & HOWARD & & 4423985003 & Pad Mount \\
\hline KINSER & 27-A TS & $150 \mathrm{KVA}$ & SIERRA & & $9476-1$ & Pad Mount \\
\hline KINSER & 114 TS & $150 \mathrm{KVA}$ & $\mathrm{AlCHI}$ & & 9221897 & Pad Mount \\
\hline KINSER & 1040R TS & $150 \mathrm{KVA}$ & COOPER & & 0326000061 & Pad Mount \\
\hline KINSER & $79 \mathrm{TH}$ & $150 \mathrm{KVA}$ & $\begin{array}{c}\text { MGM } \\
\text { TRANSFORMER }\end{array}$ & & $\begin{array}{c}03-02-02- \\
10569 \mathrm{~A}\end{array}$ & Pad Mount \\
\hline KINSER & 13-C TH & $150 \mathrm{KVA}$ & H. K.PORTER & & W-228059 & Pad Mount \\
\hline KINSER & 1052 TS & $150 \mathrm{KVA}$ & PAUWELS & & 05E134380 & Pad Mount \\
\hline MCAS & $2 \mathrm{~A}-5 \mathrm{TS}$ & $150 \mathrm{KVA}$ & VANTRAN & & $88 \mathrm{~V} 4357$ & Pad Mount \\
\hline
\end{tabular}


Page 7 of 24

\begin{tabular}{|c|c|c|c|c|c|c|}
\hline MCAS & 77 TS & $150 \mathrm{KVA}$ & PAUWELS & & 06F142079 & Pad Mount \\
\hline MCAS & 38-J1 TS & $150 \mathrm{KVA}$ & COOPER & 2008 MAR & 0850004240 & Pad Mount \\
\hline MCAS & 2B-1 TS & $150 \mathrm{KVA}$ & PAUWELS & OCT 2005 & 05G135693 & Pad Mount \\
\hline MCAS & 682 TS & $150 \mathrm{KVA}$ & $\mathrm{AlCHI}$ & & 9222794 & Pad Mount \\
\hline MCAS & 8-6 TS & $150 \mathrm{KVA}$ & MITSUBISHI & & D5147901 & Pad Mount \\
\hline MCAS & $461 \mathrm{TS}$ & $150 \mathrm{KVA}$ & PAUWELS & JUL 2005 & $05 E 134378$ & Pad Mount \\
\hline MCAS & $220 \mathrm{TS}$ & $150 \mathrm{KVA}$ & COOPER & 2005 FEB & 0537003965 & Pad Mount \\
\hline MCAS & $438 \mathrm{TS}$ & $150 \mathrm{KVA}$ & COOPER & DEC 2008 & 0850020422 & Pad Mount \\
\hline MCAS & $444 \mathrm{TS}$ & $150 \mathrm{KVA}$ & COOPER & DEC 2008 & 0850020421 & Pad Mount \\
\hline MCAS & 11-A1 TS & $150 \mathrm{KVA}$ & $\mathrm{AlCHI}$ & 1986 & 8621626 & Pad Mount \\
\hline MCAS & 49-A-1 TS & $150 \mathrm{KVA}$ & RTE & & 866005009 & Pad Mount \\
\hline MCAS & 13-1B TS & $150 \mathrm{KVA}$ & PAUWELS & 2007 MAR & 20060001660 & Pad Mount \\
\hline MCAS & $38-\mathrm{N}$ TS & $150 \mathrm{KVA}$ & WESTING HOUSE & & 87JH983095 & Pad Mount \\
\hline MCAS & 505-B TS & $150 \mathrm{KVA}$ & BALTEAN & & PUK-0972 & Pad Mount \\
\hline FOSTER & 5675 TS & $1500 \mathrm{KVA}$ & ABB & & 96J031306 & Pad Mount \\
\hline FOSTER & 480A TS & $1500 \mathrm{KVA}$ & TAKAOKA & 2004 & 03011288 & Pad Mount \\
\hline HANSEN & 2419 TS & $1500 \mathrm{KVA}$ & HOWARD & & 3630173005 & Pad Mount \\
\hline KINSER & 618 TS & $1500 \mathrm{KVA}$ & TOSHIBA & 2002 & 01057451 & Pad Mount \\
\hline KINSER & $425 \mathrm{TS}$ & 1500/1725 KV & COOPER & & 9926000262 & Pad Mount \\
\hline CTNY & 4456 TS & $200 \mathrm{KVA}$ & FUJI & JAN 1990 & B-90404A01 & Pad Mount \\
\hline CTNY & 4453 TS & $200 \mathrm{KVA}$ & MITSUBISHI & & DM0852001 & Pad Mount \\
\hline CTNY-TG & $4119 \mathrm{TS}$ & $200 \mathrm{KVA}$ & $\mathrm{AlCHI}$ & 1985 & 8521470 & Pad Mount \\
\hline CTNY-TG & $4149 \mathrm{TS}$ & $200 \mathrm{KVA}$ & MITSUBISHI & 1988 & DM4693001 & Pad Mount \\
\hline FOSTER & 5665 TS & $200 \mathrm{KVA}$ & $\mathrm{AlCHI}$ & 2003 & 03203998 & Pad Mount \\
\hline FOSTER & $150 \mathrm{TS}$ & $200 \mathrm{KVA}$ & FUJI & & A30947T1 & Pad Mount \\
\hline FOSTER & 302-F3 TS & $200 \mathrm{KVA}$ & JIMELCO & MAR 1991 & AA00201411 & Pad Mount \\
\hline FOSTER & 5971 TS & $200 \mathrm{KVA}$ & FUJI & & $\mathrm{B} 10285 \mathrm{~A} 01$ & Pad Mount \\
\hline FOSTER & 5626 TS & $200 \mathrm{KVA}$ & $\mathrm{AlCHI}$ & 1993 & 9221396 & Pad Mount \\
\hline
\end{tabular}


Page 8 of 24

\begin{tabular}{|c|c|c|c|c|c|c|}
\hline FOSTER & 97-A TS & $200 \mathrm{KVA}$ & MITSUBISHI & 1978 & D51836001 & Pad Mount \\
\hline FOSTER & 130-B TS & $200 \mathrm{KVA}$ & MITSUBISHI & 1978 & D51838001 & Pad Mount \\
\hline FOSTER & 129-K TS & $200 \mathrm{KVA}$ & DAIHEN & 1995 & 3SD1834B1 & Pad Mount \\
\hline FOSTER & $5831 \mathrm{TS}$ & $200 \mathrm{KVA}$ & MEIDEN & DEC 1991 & 1Q9558T1 & Pad Mount \\
\hline FOSTER & 496 TS & $200 \mathrm{KVA}$ & MEIDEN & NOV 1992 & 1R8668T1 & Pad Mount \\
\hline FOSTER-1 & SHOP STOCK & $200 \mathrm{KVA}$ & MITSUBISHI & & D52039001 & Pad Mount \\
\hline HANSEN & $2533 \mathrm{TS}$ & $200 \mathrm{KVA}$ & $\mathrm{AlCHI}$ & 2005 & 05207386 & Pad Mount \\
\hline HANSEN & 2865 TS & $200 \mathrm{KVA}$ & DAIHEN & 1995 & 3SD1947B1 & Pad Mount \\
\hline HANSEN & $2821 \mathrm{TS}$ & $200 \mathrm{KVA}$ & MITSUBISHI & 2000 & DN9113001 & Pad Mount \\
\hline HANSEN & $2241 \mathrm{TS}$ & $200 \mathrm{KVA}$ & $\mathrm{AlCHI}$ & 1994 & 9420698 & Pad Mount \\
\hline HANSEN & $2138 \mathrm{TS}$ & $200 \mathrm{KVA}$ & HITACHI & 2003 & 024670321 & Pad Mount \\
\hline HANSEN & 2489-E TS & $200 \mathrm{KVA}$ & $\mathrm{AlCHI}$ & 2009 & 0921447 & Pad Mount \\
\hline HANSEN & 2183 TS & $200 \mathrm{KVA}$ & $\overline{\mathrm{AlCHI}}$ & 2004 & 04206635 & Pad Mount \\
\hline HANSEN & 2457 TS & $200 \mathrm{KVA}$ & TOSHIBA & 2002 & 02028889 & Pad Mount \\
\hline HANSEN & $2858 \mathrm{TS}$ & $200 \mathrm{KVA}$ & $\mathrm{AICHI}$ & 2006 & 06208938 & Pad Mount \\
\hline KINSER & $5 G \mathrm{TS}$ & $200 \mathrm{KVA}$ & DAIHEN & & 3SD0927A1 & Pad Mount \\
\hline KINSER & 52-A TH & $200 \mathrm{KVA}$ & $\mathrm{AlCHI}$ & & 8421663 & Pad Mount \\
\hline KINSER & 90-C TS & $200 \mathrm{KVA}$ & $\mathrm{AlCHI}$ & 1993 & 92231117 & Pad Mount \\
\hline KINSER & 104-B TS & $200 \mathrm{KVA}$ & $\mathrm{AlCHI}$ & 1978 & 8922063 & Pad Mount \\
\hline KINSER & $415 \mathrm{TS}$ & $200 \mathrm{KVA}$ & HITACHI & & 24754321 & Pad Mount \\
\hline MCAS & $261 \mathrm{TS}$ & $200 \mathrm{KVA}$ & $\mathrm{AICHI}$ & & 9222795 & Pad Mount \\
\hline MCAS & $685 \mathrm{TS}$ & $200 \mathrm{KVA}$ & DAIHEN & & 3SD1123A1 & Pad Mount \\
\hline MCAS & $646 \mathrm{TS}$ & $200 \mathrm{KVA}$ & MEIDEN & NOV 1993 & 1R9946T1 & Pad Mount \\
\hline CTNY & $4234 \mathrm{TS}$ & $225 \mathrm{KVA}$ & WESTING HOUSE & & 87JA840043 & Pad Mount \\
\hline CTNY & $4218 \mathrm{TS}$ & $225 \mathrm{KVA}$ & $\mathrm{ABB}$ & & 96J164212 & Pad Mount \\
\hline CTNY & $4300 \mathrm{TS}$ & $225 \mathrm{KVA}$ & $B \& B$ & & 0505920 & Pad Mount \\
\hline CTNY & $4320 \mathrm{TS}$ & $225 \mathrm{KVA}$ & PAUWELS & OCT 2005 & $05 G 135691$ & Pad Mount \\
\hline CTNY-TG & $4123 \mathrm{TS}$ & $225 \mathrm{KVA}$ & COOPER & FEB 2006 & 0650003885 & Pad Mount \\
\hline
\end{tabular}


Page 9 of 24

\begin{tabular}{|c|c|c|c|c|c|c|}
\hline FOSTER & $330 \mathrm{TS}$ & $225 \mathrm{KVA}$ & COOPER & & 969002966 & Pad Mount \\
\hline FOSTER & $320 \mathrm{TS}$ & $225 \mathrm{KVA}$ & COOPER & JAN2005 & 0537000671 & Pad Mount \\
\hline FOSTER & $5-B$ TS & $225 \mathrm{KVA}$ & COOPER & FEB2005 & 0537000672 & Pad Mount \\
\hline FOSTER & 183-A TS & $225 \mathrm{KVA}$ & WESTING HOUSE & & 79JH038017 & Pad Mount \\
\hline FOSTER & 33-C TS & $225 \mathrm{KVA}$ & COOPER & & 906008978 & Pad Mount \\
\hline FOSTER & 95-C TS & $225 \mathrm{KVA}$ & PAUWELS & & 20110704947 & Pad Mount \\
\hline FOSTER & 89-C TS & $225 \mathrm{KVA}$ & PAUWELS & NOV 2004 & 20110704948 & Pad Mount \\
\hline FOSTER & 5699 TS & $225 \mathrm{KVA}$ & COOPER & APR 2008 & 0850006947 & Pad Mount \\
\hline FOSTER & 264-C TS & $225 \mathrm{KVA}$ & PAUWELS & 2007 MAR & 20060001856 & Pad Mount \\
\hline FOSTER-1 & SHOP STOCK & $225 \mathrm{KVA}$ & COOPER & JUN 2008 & 0850010005 & Pad Mount \\
\hline FOSTER-1 & SHOP STOCK & $225 \mathrm{KVA}$ & COOPER & MAY 2008 & 0850007864 & Pad Mount \\
\hline FOSTER-1 & SHOP STOCK & $225 \mathrm{KVA}$ & PAUWELS & JUN 2007 & 20110703029 & Pad Mount \\
\hline FOSTER-1 & SHOP STOCK & $225 \mathrm{KVA}$ & COOPER & DEC2004 & 0437020478 & Pad Mount \\
\hline FOSTER-1 & SHOP STOCK & $225 \mathrm{KVA}$ & COOPER & DEC2004 & 0437020477 & Pad Mount \\
\hline FOSTER-1 & SHOP STOCK & $225 \mathrm{KVA}$ & COOPER & & 0537005787 & Pad Mount \\
\hline FOSTER-1 & SHOP STOCK & $225 \mathrm{KVA}$ & $B \& B$ & $\mathrm{~N} / \mathrm{A}$ & 1215981 & Pad Mount \\
\hline FOSTER-1 & SHOP STOCK & $225 \mathrm{KVA}$ & COOPER & DEC 2004 & 0437020880 & Pad Mount \\
\hline FOSTER-1 & SHOP STOCK & $225 \mathrm{KVA}$ & ABB & JUL 2002 & 02J602094 & Pad Mount \\
\hline HANSEN & 2206 TS & $225 \mathrm{KVA}$ & COOPER & AUG 2007 & 0750016080 & Pad Mount \\
\hline HANSEN & 2444 TS & $225 \mathrm{KVA}$ & COOPER & 2007 AUG & 0750015008 & Pad Mount \\
\hline HANSEN & 2617 TS & $225 \mathrm{KVA}$ & COOPER & FEB 2005 & 0537000623 & Pad Mount \\
\hline HANSEN & $2618 \mathrm{TS}$ & $225 \mathrm{KVA}$ & COOPER & FEB 2005 & 0537000624 & Pad Mount \\
\hline HANSEN & 2510 TS & $225 \mathrm{KVA}$ & COOPER & FEB 2005 & 0537000625 & Pad Mount \\
\hline HANSEN & $2460 \mathrm{TS}$ & $225 \mathrm{KVA}$ & COOPER & JUN 2005 & 0537000670 & Pad Mount \\
\hline HANSEN & 2449 TS & $225 \mathrm{KVA}$ & COOPER & DEC 2004 & 0537000622 & Pad Mount \\
\hline HANSEN & $2631 \mathrm{TS}$ & $225 \mathrm{KVA}$ & PAUWELS & JUL 2005 & 05E134081 & Pad Mount \\
\hline HANSEN & 2148-A TS & $225 \mathrm{KVA}$ & VANTRAN & & $88 V 3333$ & Pad Mount \\
\hline HANSEN & 2728 TS & $225 \mathrm{KVA}$ & VANTRAN & & $88 V 3332$ & Pad Mount \\
\hline
\end{tabular}


Page 10 of 24

\begin{tabular}{|c|c|c|c|c|c|c|}
\hline HANSEN & 2176 TS & $225 \mathrm{KVA}$ & PAUWELS & APR 2005 & $05 C 132897$ & Pad Mount \\
\hline HANSEN & 2407 TS & $225 \mathrm{KVA}$ & $\mathrm{ABB}$ & OCT 2003 & 03J898241 & Pad Mount \\
\hline HANSEN & 2149 TS & $225 \mathrm{KVA}$ & ABB & 2007 FEB & 07J716216 & Pad Mount \\
\hline HANSEN & $2620 \mathrm{TS}$ & $225 \mathrm{KVA}$ & COOPER & OCT 2007 & 0750020176 & Pad Mount \\
\hline HANSEN & 2857 TS & $225 \mathrm{KVA}$ & COOPER & MAY 2007 & 0750010141 & Pad Mount \\
\hline KINSER & 1202-A TS & $225 \mathrm{KVA}$ & $A B B$ & DEC 2008 & 08J162069 & Pad Mount \\
\hline KINSER & 40-D TS & $225 \mathrm{KVA}$ & COOPER & JUN 2009 & 0950005374 & Pad Mount \\
\hline KINSER & 37-A TS & $225 \mathrm{KVA}$ & VANTRAN & & $88 \mathrm{~V} 3352$ & Pad Mount \\
\hline KINSER & 42-A TS & $225 \mathrm{KVA}$ & VANTRAN & & $88 \mathrm{~V} 3351$ & Pad Mount \\
\hline KINSER & 1216 TS & $225 \mathrm{KVA}$ & PAUWELS & & 05E134080 & Pad Mount \\
\hline KINSER & 40-B TS & $225 \mathrm{KVA}$ & SIERRA & & $9104-1$ & Pad Mount \\
\hline MCAS & $15 \mathrm{TS}$ & $225 \mathrm{KVA}$ & CUTLER-HAMMER & OCT 2003 & 03J908084 & Pad Mount \\
\hline MCAS & 38-BA TS & $225 \mathrm{KVA}$ & ABB & MAY 1996 & 96J094049 & Pad Mount \\
\hline MCAS & 13-2 TS & $225 \mathrm{KVA}$ & ABB & & 96J067063 & Pad Mount \\
\hline MCAS & 71B TS & $225 \mathrm{KVA}$ & $A B B$ & NOV 2003 & 03J914087 & Pad Mount \\
\hline MCAS & 665-A TS & $225 \mathrm{KVA}$ & COOPER & DEC2004 & 0437020474 & Pad Mount \\
\hline MCAS & 17-B2 TS & $225 \mathrm{KVA}$ & COOPER & DEC2004 & 0437020475 & Pad Mount \\
\hline MCAS & 11-E TS & $225 \mathrm{KVA}$ & COOPER & DEC2004 & 0437020476 & Pad Mount \\
\hline MCAS & $2 \mathrm{~A}-1 \mathrm{TS}$ & $225 \mathrm{KVA}$ & $A B B$ & & 91J822137 & Pad Mount \\
\hline MCAS & 14 TS & $225 \mathrm{KVA}$ & ABB & $1991 \mathrm{FEB}$ & 91J824197 & Pad Mount \\
\hline MCAS & 17-B1 TS & $225 \mathrm{KVA}$ & COOPER & & 04J102349 & Pad Mount \\
\hline MCAS & 39-A TS & $225 \mathrm{KVA}$ & COOPER & & 959006283 & Pad Mount \\
\hline MCAS & 539 TS & $225 \mathrm{KVA}$ & COOPER & DEC 2007 & 0750023420 & Pad Mount \\
\hline MCAS & 13-1 TS & $225 \mathrm{KVA}$ & PAUWELS & 2007 MAR & 20060001855 & Pad Mount \\
\hline CTNY & 4446 TS & $250 \mathrm{KVA}$ & MITSUBISHI & 2005 & DA3652001 & Pad Mount \\
\hline CTNY & $4224 \mathrm{TS}$ & $250 \mathrm{KVA}$ & OSAKA & 1985 & 3ND0487A1 & Pad Mount \\
\hline CTNY-TG & 4118 TS & $250 \mathrm{KVA}$ & $\mathrm{AlCHI}$ & 1985 & 8521471 & Pad Mount \\
\hline HANSEN & $2123 \mathrm{TS}$ & $250 \mathrm{KVA}$ & DAIHEN & 1988 & 2TE140701 & Pad Mount \\
\hline
\end{tabular}


Page 11 of 24

\begin{tabular}{|c|c|c|c|c|c|c|}
\hline CTNY & $4217 \mathrm{TS}$ & $30 \mathrm{KVA}$ & MITSUBISHI & 1978 & D51789001 & Pad Mount \\
\hline FOSTER-1 & SHOP STOCK & $30 \mathrm{KVA}$ & DAIHEN & & 3SD1766B1 & Pad Mount \\
\hline FOSTER-1 & SHOP STOCK & $30 \mathrm{KVA}$ & ABB & NOV 2003 & 03J924055 & Pad Mount \\
\hline KINSER & 54-A TH & $30 \mathrm{KVA}$ & DAIHEN & & 2TF909101 & Pad Mount \\
\hline MCAS & 47A-5 POLE & $30 \mathrm{KVA}$ & $\mathrm{AlCHI}$ & & 8622697 & Pad Mount \\
\hline FOSTER & $129 \mathrm{RH}$ & $30 \mathrm{KW}$ & WESTING HOUSE & & 1PXB09073 & Pad Mount \\
\hline FOSTER & $129 \mathrm{RH}$ & $30 \mathrm{KW}$ & WESTING HOUSE & & 1PXB13074 & Pad Mount \\
\hline CTNY & 4419 TS & $300 \mathrm{KVA}$ & PAUWELS & & 20110703034 & Pad Mount \\
\hline CTNY & $4231 \mathrm{TS}$ & $300 \mathrm{KVA}$ & $\mathrm{AlCHI}$ & & 9222797 & Pad Mount \\
\hline CTNY & $4440 \mathrm{TS}$ & $300 \mathrm{KVA}$ & MEIDEN & JAN 2004 & 4A5588TP1 & Pad Mount \\
\hline CTNY & 4405 TS & $300 \mathrm{KVA}$ & TOSHIBA & 1997 & 97060796 & Pad Mount \\
\hline CTNY & $4451 \mathrm{TS}$ & $300 \mathrm{KVA}$ & FUJI & & A24004T1 & Pad Mount \\
\hline CTNY & 4409 TS & $300 \mathrm{KVA}$ & COOPER & OCT 2005 & 0559002005 & Pad Mount \\
\hline CTNY & $4316 \mathrm{TS}$ & $300 \mathrm{KVA}$ & COOPER & 2005 FEB & 0537001402 & Pad Mount \\
\hline CTNY & $4335 \mathrm{TS}$ & $300 \mathrm{KVA}$ & COOPER & 2005 JAN & 0537001397 & Pad Mount \\
\hline FOSTER & 5624 TS & $300 \mathrm{KVA}$ & $\mathrm{AlCHI}$ & 1997 AUG & 9720746 & Pad Mount \\
\hline FOSTER & $5618 \mathrm{TS}$ & $300 \mathrm{KVA}$ & $\mathrm{AlCHI}$ & 2003 & 03204858 & Pad Mount \\
\hline FOSTER & 91-A TS & $300 \mathrm{KVA}$ & COOPER & APR 1996 & 969002967 & Pad Mount \\
\hline FOSTER & $5713 \mathrm{TS}$ & $300 \mathrm{KVA}$ & $\mathrm{AlCHI}$ & 2004 & 04206081 & Pad Mount \\
\hline FOSTER & $361 \mathrm{TS}$ & $300 \mathrm{KVA}$ & $\mathrm{AlCHI}$ & & 9420508 & Pad Mount \\
\hline FOSTER & 5634 TS & $300 \mathrm{KVA}$ & MITSUBISHI & 1990 & DX3611001 & Pad Mount \\
\hline FOSTER & $5835 \mathrm{TS}$ & $300 \mathrm{KVA}$ & MITSUBISHI & 2003 & DJ7988001 & Pad Mount \\
\hline FOSTER & 5949 TS & $300 \mathrm{KVA}$ & FUJI & 2000 DEC & B00344A01 & Pad Mount \\
\hline FOSTER & 437 TS & $300 \mathrm{KVA}$ & HITACHI & APR 2004 & 23012421 & Pad Mount \\
\hline FOSTER & $14 \mathrm{TS}$ & $300 \mathrm{KVA}$ & DAIHEN & & 3SD2084B1 & Pad Mount \\
\hline FOSTER & $371 \mathrm{TS}$ & $300 \mathrm{KVA}$ & COOPER & 2005 JAN & 0537001399 & Pad Mount \\
\hline $\begin{array}{l}\text { FOSTER } \\
\end{array}$ & 5679 TS & $300 \mathrm{KVA}$ & $\mathrm{AICHI}$ & & 9023259 & Pad Mount \\
\hline FOSTER & $5900 \mathrm{TS}$ & $300 \mathrm{KVA}$ & WESTING HOUSE & & 83JB885015 & Pad Mount \\
\hline
\end{tabular}


Page 12 of 24

\begin{tabular}{|c|c|c|c|c|c|c|}
\hline FOSTER & $4 \mathrm{TS}$ & $300 \mathrm{KVA}$ & CUTLER-HAMMER & AUG 2004 & 04J102272 & Pad Mount \\
\hline FOSTER & 499 TS & $300 \mathrm{KVA}$ & $\overline{\mathrm{AlCHI}}$ & FEB 1989 & 8822210 & Pad Mount \\
\hline FOSTER & $5910 \mathrm{TS}$ & $300 \mathrm{KVA}$ & COOPER & & 0850016223 & Pad Mount \\
\hline FOSTER & $16 \mathrm{C} \mathrm{TS}$ & $300 \mathrm{KVA}$ & $A B B$ & SEP 2007 & 07J872209 & Pad Mount \\
\hline FOSTER & 1017 TS & $300 \mathrm{KVA}$ & ALSTOM & JUN 2003 & PIF-0427 & Pad Mount \\
\hline $\begin{array}{l}\text { FOSTER } \\
\end{array}$ & $90 \mathrm{TS}$ & $300 \mathrm{KVA}$ & COOPER & OCT 2007 & 0759002459 & Pad Mount \\
\hline FOSTER-1 & SHOP STOCK & $300 \mathrm{KVA}$ & VANTRAN & $\mathrm{N} / \mathrm{A}$ & $96-6655$ & Pad Mount \\
\hline FOSTER-1 & SHOP STOCK & $300 \mathrm{KVA}$ & PAUWELS & SEP 2008 & 20110811295 & Pad Mount \\
\hline FOSTER-1 & SHOP STOCK & $300 \mathrm{KVA}$ & $A B B$ & JUN 98 & 98J505238 & Pad Mount \\
\hline FOSTER-1 & SHOP STOCK & $300 \mathrm{KVA}$ & COOPER & OCT 06 & 0650019150 & Pad Mount \\
\hline FOSTER-1 & SHOP STOCK & $300 \mathrm{KVA}$ & COOPER & DEC2004 & 0437020529 & Pad Mount \\
\hline FOSTER-1 & SHOP STOCK & $300 \mathrm{KVA}$ & $\begin{array}{l}\text { COOPER } \\
\text {. }\end{array}$ & JAN 05 & 0437020528 & Pad Mount \\
\hline FOSTER-1 & SHOP STOCK & $300 \mathrm{KVA}$ & COOPER & MAY 2010 & 1050004104 & Pad Mount \\
\hline FOSTER-1 & SHOP STOCK & $300 \mathrm{KVA}$ & COOPER & MAY 2010 & 1050004102 & Pad Mount \\
\hline FOSTER-1 & SHOP STOCK & $300 \mathrm{KVA}$ & PAUWELS & NOV 2004 & 04J129803 & Pad Mount \\
\hline FOSTER-1 & SHOP STOCK & $300 \mathrm{KVA}$ & COOPER & AUG 2004 & 0437014260 & Pad Mount \\
\hline FOSTER-1 & SHOP STOCK & $300 \mathrm{KVA}$ & COOPER & NOV 2009 & 0950010824 & Pad Mount \\
\hline FOSTER-1 & SHOP STOCK & $300 \mathrm{KVA}$ & COOPER & MAY 2010 & 1050004105 & Pad Mount \\
\hline FOSTER-1 & SHOP STOCK & $300 \mathrm{KVA}$ & $\mathrm{AlCHI}$ & 2003 & 03203801 & Pad Mount \\
\hline FOSTER-1 & SHOP STOCK & $300 \mathrm{KVA}$ & COOPER & JUL 2009 & 0950006598 & Pad Mount \\
\hline HANSEN & 2215 TS & $300 \mathrm{KVA}$ & COOPER & $2007 \mathrm{JUL}$ & 0750014503 & Pad Mount \\
\hline HANSEN & 2534 TS & $300 \mathrm{KVA}$ & $\mathrm{AlCHI}$ & 2005 & 05207385 & Pad Mount \\
\hline HANSEN & 2867 TS & $300 \mathrm{KVA}$ & MITSUBISHI & & L24257121 & Pad Mount \\
\hline HANSEN & 2386 TS & $300 \mathrm{KVA}$ & HITACHI & 2000 & 23601921 & Pad Mount \\
\hline HANSEN & $2134 \mathrm{TS}$ & $300 \mathrm{KVA}$ & $\mathrm{AlCHI}$ & 1992 & 9221188 & Pad Mount \\
\hline HANSEN & $2130 \mathrm{TS}$ & $300 \mathrm{KVA}$ & COOPER & 2005 FEB & 0537001398 & Pad Mount \\
\hline HANSEN & 2505 TS & $300 \mathrm{KVA}$ & PAUWELS & APR 2005 & 05C132898 & Pad Mount \\
\hline HANSEN & 2142 TS & $300 \mathrm{KVA}$ & COOPER & JUY 2009 & 0950005375 & Pad Mount \\
\hline
\end{tabular}


Page 13 of 24

\begin{tabular}{|c|c|c|c|c|c|c|}
\hline HANSEN & $2716 \mathrm{TS}$ & $300 \mathrm{KVA}$ & DAIHEN & 2000 & KNB0165B1 & Pad Mount \\
\hline HANSEN & 2616 TS & $300 \mathrm{KVA}$ & MITSUBISHI & 1994 & DH8717001 & Pad Mount \\
\hline HANSEN & 2455 TS & $300 \mathrm{KVA}$ & $\mathrm{AlCHI}$ & 1999 & 9821620 & Pad Mount \\
\hline HANSEN & 2383 TS & $300 \mathrm{KVA}$ & COOPER & NOV 2006 & 0650021781 & Pad Mount \\
\hline HANSEN & $2420 \mathrm{TS}$ & $300 \mathrm{KVA}$ & $\mathrm{AlCHI}$ & 2006 & 06210657 & Pad Mount \\
\hline HANSEN & $2345 \mathrm{TS}$ & $300 \mathrm{KVA}$ & MEIDEN & 2004 JAN & 4A5611TP1 & Pad Mount \\
\hline HANSEN & 2397 TS & $300 \mathrm{KVA}$ & ABB & APR 2002 & $02 J 544290$ & Pad Mount \\
\hline HANSEN & 2375 TS & $300 \mathrm{KVA}$ & $\mathrm{AlCHI}$ & 2000 & 0700900 & Pad Mount \\
\hline HANSEN & 2323 TS & $300 \mathrm{KVA}$ & FUJI & $2001 \mathrm{MAY}$ & B00422A01 & Pad Mount \\
\hline HANSEN & $2828 \mathrm{TS}$ & $300 \mathrm{KVA}$ & $\overline{\mathrm{AlCHI}}$ & 2006 & 06208939 & Pad Mount \\
\hline HANSEN & 2814 TS & $300 \mathrm{KVA}$ & $\mathrm{AlCHI}$ & 1995 & 9522553 & Pad Mount \\
\hline HANSEN & $2818 \mathrm{TS}$ & $300 \mathrm{KVA}$ & $\mathrm{AlCHI}$ & 2005 & 05207530 & Pad Mount \\
\hline HANSEN & 2627 TS & $300 \mathrm{KVA}$ & HITACHI & 2002 & 24257121 & Pad Mount \\
\hline HANSEN-1 & SHOP STOCK & $300 \mathrm{KVA}$ & COOPER & JUL 2010 & 1050006856 & Pad Mount \\
\hline HENOKO & $10 \mathrm{TS}$ & $300 \mathrm{KVA}$ & VANTRAN & & $88 \mathrm{~V} 3350$ & Pad Mount \\
\hline KINSER & $1229 \mathrm{TS}$ & $300 \mathrm{KVA}$ & DAIHEN & & 3ND0859A1 & Pad Mount \\
\hline KINSER & 708 TS & $300 \mathrm{KVA}$ & HITACHI & 2001 & $\mid 24255121$ & Pad Mount \\
\hline KINSER & $18 \mathrm{TS}$ & $300 \mathrm{KVA}$ & G.E. & & L190261T73AA & Pad Mount \\
\hline KINSER & 26-A TS & $300 \mathrm{KVA}$ & COOPER & DEC2004 & 0437020530 & Pad Mount \\
\hline KINSER & 202 TS & $300 \mathrm{KVA}$ & FUJI & 1993 DEC & B30228A02 & Pad Mount \\
\hline KINSER & 5 -E TS & $300 \mathrm{KVA}$ & FUJI & 1983 DEC & B30119A01 & Pad Mount \\
\hline KINSER & $\mathrm{M}-21 \mathrm{TS}$ & $300 \mathrm{KVA}$ & COOPER & DEC2004 & 0437020533 & Pad Mount \\
\hline KINSER & $1307 \mathrm{TS}$ & $300 \mathrm{KVA}$ & FUJI ELECTRIC & 1995 FEB & B40194A01 & Pad Mount \\
\hline KINSER & $1220 \mathrm{TS}$ & $300 \mathrm{KVA}$ & $\mathrm{AlCHI}$ & 1997 & 9720757 & Pad Mount \\
\hline KINSER & 92-A TS & $300 \mathrm{KVA}$ & $\mathrm{AICHI}$ & 1989 & 8822081 & Pad Mount \\
\hline KINSER & 4-A TS & $300 \mathrm{KVA}$ & MITSUBISHI & 1981 & DA3057001 & Pad Mount \\
\hline KINSER & 21-A TS & $300 \mathrm{KVA}$ & SIERRA & & $9004-1$ & Pad Mount \\
\hline MCAS & 13-3 TS & $300 \mathrm{KVA}$ & $A B B$ & SEP 2003 & 03J872095 & Pad Mount \\
\hline
\end{tabular}


Page 14 of 24

\begin{tabular}{|c|c|c|c|c|c|c|}
\hline MCAS & $42 \mathrm{TS}$ & $300 \mathrm{KVA}$ & $A B B$ & OCT 2003 & 03J913182 & Pad Mount \\
\hline MCAS & 539A TS & $300 \mathrm{KVA}$ & COOPER & DEC 2007 & 0750023250 & Pad Mount \\
\hline MCAS & 633 TS & $300 \mathrm{KVA}$ & FUJI & & A53659T1 & Pad Mount \\
\hline MCAS & $2 A-T R$ & $300 \mathrm{KVA}$ & VANTRAN & & 01V5667 & Pad Mount \\
\hline MCAS & 6-C TS & $300 \mathrm{KVA}$ & ABB & JAN 2002 & 02J582146 & Pad Mount \\
\hline MCAS & 684 TS & $300 \mathrm{KVA}$ & VANTRAN & & 04V6815 & Pad Mount \\
\hline MCAS & $11-3 \mathrm{TS}$ & $300 \mathrm{KVA}$ & COOPER & & 0526000267 & Pad Mount \\
\hline MCAS & 676 TS & $300 \mathrm{KVA}$ & MITSUBISHI & & DC6959001 & Pad Mount \\
\hline MCAS & 77-A TS & $300 \mathrm{KVA}$ & COOPER & APR 2005 & 0526000429 & Pad Mount \\
\hline MCAS & $159 \mathrm{TS}$ & $300 \mathrm{KVA}$ & DAIHEN & & 3SD1831B1 & Pad Mount \\
\hline MCAS & 20-C TS & $300 \mathrm{KVA}$ & G.E. & & E-693088 & Pad Mount \\
\hline MCAS & 546 TS & $300 \mathrm{KVA}$ & DAIHEN & & 3SD1330A1 & Pad Mount \\
\hline MCAS & $20-G$ TS & $300 \mathrm{KVA}$ & SUNBELT & & 3279783104 & Pad Mount \\
\hline MCAS & 429 TS & $300 \mathrm{KVA}$ & COOPER & NOV 2009 & 0950010825 & Pad Mount \\
\hline FOSTER & 104 TS & $3000 \mathrm{KVA}$ & NIAGARA & & 60310 & Pad Mount \\
\hline $\begin{array}{l}\text { FOSTER } \\
\end{array}$ & 104 TS & $3000 \mathrm{KVA}$ & NIAGARA & & 50309 & Pad Mount \\
\hline CTNY & 4213 TS & $350 \mathrm{KVA}$ & OSAKA & 1985 & 3ND0539A-1 & Pad Mount \\
\hline HANSEN & 2495 TS & $350 \mathrm{KVA}$ & MITSUBISHI & 1992 & D90879003 & Pad Mount \\
\hline MCAS & $518 \mathrm{TV}$ & $37.5 \mathrm{KW}$ & COOPER & & 4153 & Pad Mount \\
\hline MCAS & $518 \mathrm{TV}$ & $37.5 \mathrm{KW}$ & COOPER & & 4152 & Pad Mount \\
\hline MCAS & $518 \mathrm{TV}$ & $4 \mathrm{KW}$ & COOPER & & 1485 & Pad Mount \\
\hline MCAS & $518 \mathrm{TV}$ & $4 \mathrm{KW}$ & COOPER & & 1480 & Pad Mount \\
\hline MCAS & $518 \mathrm{TV}$ & $4 \mathrm{KW}$ & COOPER & & 1479 & Pad Mount \\
\hline MCAS & $518 \mathrm{TV}$ & $4 \mathrm{KW}$ & COOPER & & 1481 & Pad Mount \\
\hline MCAS & $518 \mathrm{TV}$ & $4 \mathrm{KW}$ & COOPER & & 1484 & Pad Mount \\
\hline MCAS & $518 \mathrm{TV}$ & $4 \mathrm{KW}$ & COOPER & & 1487 & Pad Mount \\
\hline MCAS & $518 \mathrm{TV}$ & $4 \mathrm{KW}$ & COOPER & & 1483 & Pad Mount \\
\hline MCAS & $518 \mathrm{TV}$ & $4 \mathrm{KW}$ & COOPER & & 1482 & Pad Mount \\
\hline
\end{tabular}


Page 15 of 24

\begin{tabular}{|c|c|c|c|c|c|c|}
\hline MCAS & $518 \mathrm{TV}$ & $4 \mathrm{KW}$ & COOPER & & 1486 & Pad Mount \\
\hline $\begin{array}{l}\text { FOSTER } \\
\end{array}$ & 131-B1 TS & $400 \mathrm{KVA}$ & MEIDEN & NOV 1986 & 1M9301T2 & Pad Mount \\
\hline FOSTER & 132-A TS & $400 \mathrm{KVA}$ & MEIDEN & NOV 1986 & 1M9301T1 & Pad Mount \\
\hline FOSTER-1 & SHOP STOCK & $400 \mathrm{KVA}$ & $\mathrm{AICHI}$ & & 8421351 & Pad Mount \\
\hline HANSEN & 2888 TS & $400 \mathrm{KVA}$ & OSAKA & 1981 & 3D0134A01 & Pad Mount \\
\hline HANSEN & 2820 TS & $400 \mathrm{KVA}$ & MEIDEN & OCT 1984 & 1K8888T1 & Pad Mount \\
\hline HANSEN & 2655 TS & $400 \mathrm{KVA}$ & MEIDEN & OCT 1984 & 1K8890T1 & Pad Mount \\
\hline MCAS & 9-D TS & $400 \mathrm{KVA}$ & OSAKA & OCT 1984 & 3ND0499A1 & Pad Mount \\
\hline HANSEN & $2601 \mathrm{TV}$ & $400 \mathrm{~A}$ OS & $\overline{G \& W}$ & & $799-216$ & Pad Mount \\
\hline HANSEN & 2663 TV & $400 \mathrm{~A}$ OS & G\&W & & 799-238 & Pad Mount \\
\hline KINSER & 831 (B) SW & 400A VS & MITSUBISHI & 1998 MAR & ID507102 & Pad Mount \\
\hline KINSER & 831 (A) SW & 400A VS & MITSUBISHI & 1998 MAR & ID507101 & Pad Mount \\
\hline CTNY & 4209 TS & $45 \mathrm{KVA}$ & VANTRAN & & $03 \mathrm{~V} 6460$ & Pad Mount \\
\hline CTNY-TG & $6 \mathrm{E}$ TS & $45 \mathrm{KVA}$ & COOPER & DEC 2007 & 0859000030 & Pad Mount \\
\hline FOSTER & 1013 TS & $45 \mathrm{KVA}$ & SUNBELT & & 3297723104 & Pad Mount \\
\hline FOSTER & $7 \mathrm{TS}$ & $45 \mathrm{KVA}$ & PAUWELS & JUN 2007 & 20110703032 & Pad Mount \\
\hline FOSTER-1 & SHOP STOCK & $45 \mathrm{KVA}$ & PAUWELS & JUN 2009 & 20110916859 & Pad Mount \\
\hline FOSTER-1 & SHOP STOCK & $45 \mathrm{KVA}$ & COOPER & N/A & 949002952 & Pad Mount \\
\hline FOSTER-1 & SHOP STOCK & $45 \mathrm{KVA}$ & COOPER & MAY 2008 & 0850007946 & Pad Mount \\
\hline FOSTER-1 & SHOP STOCK & $45 \mathrm{KVA}$ & PAUWELS & JUN 2007 & 20110703033 & Pad Mount \\
\hline FOSTER-1 & SHOP STOCK & $45 \mathrm{KVA}$ & COOPER & 2005 FEB & 0537003791 & Pad Mount \\
\hline FOSTER-1 & SHOP STOCK & $45 \mathrm{KVA}$ & VANTRAN & N/A & $03 \mathrm{~V} 6541-3$ & Pad Mount \\
\hline KINSER & 54-A TH & $45 \mathrm{KVA}$ & MITSUBISHI & & AZ0970001 & Pad Mount \\
\hline KINSER & $66-\mathrm{F} T S$ & $45 \mathrm{KVA}$ & VANTRAN & & 03V6541-1 & Pad Mount \\
\hline KINSER & $106 \mathrm{TS}$ & $45 \mathrm{KVA}$ & COOPER & AUG 2006 & 0650016474 & Pad Mount \\
\hline MCAS & 64-B TS & $45 \mathrm{KVA}$ & PAUWELS & 2008 FEB & 20110708373 & Pad Mount \\
\hline MCAS & 48-B TS & $45 \mathrm{KVA}$ & ABB & JUN 2003 & 03J806149 & Pad Mount \\
\hline MCAS & 656 TS & $45 \mathrm{KVA}$ & ABB & FEB 1997 & 97J284026 & Pad Mount \\
\hline
\end{tabular}


Page 16 of 24

\begin{tabular}{|c|c|c|c|c|c|c|}
\hline MCAS & 503 TS & $45 \mathrm{KVA}$ & COOPER & 2005 FEB & 0537003495 & Pad Mount \\
\hline MCAS & $37-A$ TS & $45 \mathrm{KVA}$ & PAUWELS & & $93 F 51772$ & Pad Mount \\
\hline MCAS & $151 \mathrm{TS}$ & $45 \mathrm{KVA}$ & PAUWELS & APR 2005 & $05 \mathrm{C} 132899$ & Pad Mount \\
\hline MCAS & 46-A TS & $45 \mathrm{KVA}$ & VANTRAN & & $03 \bigvee 6541-4$ & Pad Mount \\
\hline MCAS & 112 TS & $45 \mathrm{KVA}$ & VANTRAN & & $03 \mathrm{~V} 6546-1$ & Pad Mount \\
\hline MCAS & $120 \mathrm{TS}$ & $45 \mathrm{KVA}$ & VANTRAN & & $03 \mathrm{~V} 6546-2$ & Pad Mount \\
\hline MCAS & $125 \mathrm{TS}$ & $45 \mathrm{KVA}$ & VANTRAN & & $03 \mathrm{~V} 6546-3$ & Pad Mount \\
\hline MCAS & $44 \mathrm{TS}$ & $45 \mathrm{KVA}$ & VANTRAN & & $03 \mathrm{~V} 6541-5$ & Pad Mount \\
\hline MCAS & 9-7C TS & $45 \mathrm{KVA}$ & PAUWELS & & 06J144236 & Pad Mount \\
\hline MCAS & $501 \mathrm{TS}$ & $45 \mathrm{KVA}$ & VANTRAN & & 87 V2308 & Pad Mount \\
\hline CTNY & 4425 TS & $50 \mathrm{KVA}$ & DAIHEN & 1997 & 3SD2138R1 & Pad Mount \\
\hline FOSTER & TAKE TO 5609 & $50 \mathrm{KVA}$ & H. K.PORTER & & W-237186 & Pad Mount \\
\hline FOSTER & 151B TH & $50 \mathrm{KVA}$ & $\mathrm{AlCHI}$ & & 03101481 & Pad Mount \\
\hline FOSTER & 27 POLE & $50 \mathrm{KVA}$ & DAIHEN & & QNB399701 & Pad Mount \\
\hline FOSTER-1 & SHOP STOCK & $50 \mathrm{KVA}$ & MITSUBISHI & & DZ3922001 & Pad Mount \\
\hline FOSTER-1 & SHOP STOCK & $50 \mathrm{KVA}$ & DAIHEN & 2002 & GNB399701 & Pad Mount \\
\hline HANSEN & 264C POLE & $50 \mathrm{KVA}$ & DAIHEN & 1994 & 2TF52570 & Pad Mount \\
\hline HANSEN & 2385 TS & $50 \mathrm{KVA}$ & DAIHEN & 1994 & 3SD1715B1 & Pad Mount \\
\hline HANSEN & $2200 \mathrm{TS}$ & $50 \mathrm{KVA}$ & JIMELCO & MAR 0991 & AA000201412 & Pad Mount \\
\hline HANSEN & $2498 \mathrm{TS}$ & $50 \mathrm{KVA}$ & FUJI & 1993 FEB & A24005T1 & Pad Mount \\
\hline HANSEN & 2453 TS & $50 \mathrm{KVA}$ & $\mathrm{AlCHI}$ & 2008 & 08212761 & Pad Mount \\
\hline HENOKO & $18 \mathrm{TS}$ & $50 \mathrm{KVA}$ & ABB & & 02J643186 & Pad Mount \\
\hline KINSER & 1205 TS & $50 \mathrm{KVA}$ & $\mathrm{AlCHI}$ & 1993 & 9320747 & Pad Mount \\
\hline CTNY & $4433 \mathrm{TS}$ & $500 \mathrm{KVA}$ & MITSUBISHI & & DX3707001 & Pad Mount \\
\hline CTNY & 4228 TS & $500 \mathrm{KVA}$ & HOWARD & & 3770674895 & Pad Mount \\
\hline CTNY & 4219 TS & $500 \mathrm{KVA}$ & COOPER & NOV 2007 & 0759001145 & Pad Mount \\
\hline CTNY-TG & 4102 TS & $500 \mathrm{KVA}$ & COOPER & 2008 JAN & 0750024439 & Pad Mount \\
\hline CTNY-TG & $4131 \mathrm{TS}$ & $500 \mathrm{KVA}$ & MEIDEN & AUG 1987 & 1M9938T1 & Pad Mount \\
\hline
\end{tabular}


Page 17 of 24

\begin{tabular}{|c|c|c|c|c|c|c|}
\hline CTNY-TG & 4148 TS & $500 \mathrm{KVA}$ & HITACHI & 2003 & 24645221 & Pad Mount \\
\hline FOSTER & $445 \mathrm{TS}$ & $500 \mathrm{KVA}$ & MEIDEN & 1998 DEC & 1N6330T1 & Pad Mount \\
\hline FOSTER & $478 \mathrm{TS}$ & $500 \mathrm{KVA}$ & $\mathrm{AlCHI}$ & 1997 AUG & 9721148 & Pad Mount \\
\hline FOSTER & 217 TS & $500 \mathrm{KVA}$ & FUJI & & A30952T1 & Pad Mount \\
\hline FOSTER & 494 TS & $500 \mathrm{KVA}$ & $\mathrm{AlCHI}$ & 2004 & 04206082 & Pad Mount \\
\hline FOSTER & $1 \mathrm{TV}$ & $500 \mathrm{KVA}$ & MOLONEY & & 1934243 & Pad Mount \\
\hline FOSTER & 359 TS & $500 \mathrm{KVA}$ & HITACHI & JULY 2004 & 23013421 & Pad Mount \\
\hline FOSTER & TAKE TO 5609 & $500 \mathrm{KVA}$ & G.E. & & Q112067-TRH & Pad Mount \\
\hline FOSTER & $220 \mathrm{TS}$ & $500 \mathrm{KVA}$ & $\mathrm{AlCHI}$ & & 9122773 & Pad Mount \\
\hline FOSTER & $481 \mathrm{TS}$ & $500 \mathrm{KVA}$ & FUJI & JAN 1996 & B50348AT1 & Pad Mount \\
\hline FOSTER & 5973 TS & $500 \mathrm{KVA}$ & $\mathrm{AlCHI}$ & 2006 & 06209924 & Pad Mount \\
\hline FOSTER & $1000 \mathrm{TS}$ & $500 \mathrm{KVA}$ & COOPER & AUG 2002 & 0237013493 & Pad Mount \\
\hline FOSTER & $222 \mathrm{TS}$ & $500 \mathrm{KVA}$ & MITSUBISHI & & DC1671001 & Pad Mount \\
\hline FOSTER & $490 \mathrm{TS}$ & $500 \mathrm{KVA}$ & MITSUBISHI & 1999 & DD9891001 & Pad Mount \\
\hline FOSTER & $495 \mathrm{TS}$ & $500 \mathrm{KVA}$ & $\mathrm{AlCHI}$ & 2002 & 02704123 & Pad Mount \\
\hline FOSTER & 5903 TS & $500 \mathrm{KVA}$ & MITSUBISHI & 1988 & DM9906001 & Pad Mount \\
\hline FOSTER & 5822 TS & $500 \mathrm{KVA}$ & HITACHI & 2003 APR & 24751321 & Pad Mount \\
\hline FOSTER & 5644 TS & $500 \mathrm{KVA}$ & HITACHI & 2004 & 23062421 & Pad Mount \\
\hline FOSTER & 5696 TS & $500 \mathrm{KVA}$ & DAIHEN & 1991 & 3SD1250A1 & Pad Mount \\
\hline FOSTER & 5670 TS & $500 \mathrm{KVA}$ & WESTING HOUSE & OCT 1989 & 89J495273 & Pad Mount \\
\hline FOSTER & 5704 TS & $500 \mathrm{KVA}$ & $\mathrm{AlCHI}$ & 1989 & 892221701 & Pad Mount \\
\hline FOSTER & 5703 TS & $500 \mathrm{KVA}$ & $\mathrm{AlCHI}$ & 1989 & 892221702 & Pad Mount \\
\hline FOSTER & 5836 TS & $500 \mathrm{KVA}$ & HITACHI & 2003 & 24820321 & Pad Mount \\
\hline FOSTER & $9 \mathrm{TS}$ & $500 \mathrm{KVA}$ & COOPER & & 0526001680 & Pad Mount \\
\hline FOSTER & 131-E TS & $500 \mathrm{KVA}$ & $\mathrm{AlCHI}$ & 1988 & 8721968 & Pad Mount \\
\hline FOSTER & $38 \mathrm{TS}$ & $500 \mathrm{KVA}$ & VANTRAN & & 07V8039 & Pad Mount \\
\hline FOSTER & 370 TS & $500 \mathrm{KVA}$ & DAIHEN & 1991 & 3SD1253A1 & Pad Mount \\
\hline FOSTER & 5906 TS & $500 \mathrm{KVA}$ & COOPER & SEP 2008 & 0850014636 & Pad Mount \\
\hline
\end{tabular}


Page 18 of 24

\begin{tabular}{|c|c|c|c|c|c|c|}
\hline FOSTER-1 & SHOP STOCK & $500 \mathrm{KVA}$ & COOPER & MAR 2009 & 0950003263 & Pad Mount \\
\hline FOSTER-1 & SHOP STOCK & $500 \mathrm{KVA}$ & DAIHEN & 1988 & 3ND0754A1 & Pad Mount \\
\hline FOSTER-1 & SHOP STOCK & $500 \mathrm{KVA}$ & DAIHEN & 1991 & 3SD1254A1 & Pad Mount \\
\hline FOSTER-1 & SHOP STOCK & $500 \mathrm{KVA}$ & FUJI & & A30951T1 & Pad Mount \\
\hline FOSTER-1 & SHOP STOCK & $500 \mathrm{KVA}$ & COOPER & MAY 2009 & 0950005190 & Pad Mount \\
\hline FOSTER-1 & SHOP STOCK & $500 \mathrm{KVA}$ & MITSUBISHI & 2005 & DA3633001 & Pad Mount \\
\hline FOSTER-1 & SHOP STOCK & $500 \mathrm{KVA}$ & COOPER & MAY 2006 & 0650008288 & Pad Mount \\
\hline FOSTER-1 & SHOP STOCK & $500 \mathrm{KVA}$ & COOPER & NOV 2007 & 0750021298 & Pad Mount \\
\hline FOSTER-1 & SHOP STOCK & $500 \mathrm{KVA}$ & MITSUBISHI & 2005 & DA3631001 & Pad Mount \\
\hline FOSTER-1 & SHOP STOCK & $500 \mathrm{KVA}$ & COOPER & JUN 2010 & 1050006859 & Pad Mount \\
\hline FOSTER-1 & SHOP STOCK & $500 \mathrm{KVA}$ & $A B B$ & SEP 2003 & 03J871119 & Pad Mount \\
\hline HANSEN & $2441 \mathrm{TS}$ & $500 \mathrm{KVA}$ & FUJI & $1993 \mathrm{MAR}$ & A24006T1 & Pad Mount \\
\hline HANSEN & 2205 TS & $500 \mathrm{KVA}$ & $\mathrm{AlCHI}$ & 1997 & 9721571 & Pad Mount \\
\hline HANSEN & 2819 TS & $500 \mathrm{KVA}$ & TOSHIBA & 2003 & 02033660 & Pad Mount \\
\hline HANSEN & 2610 TS & $500 \mathrm{KVA}$ & MEIDEN & JUL 1999 & 1N6714T1 & Pad Mount \\
\hline HANSEN & 2519 TS & $500 \mathrm{KVA}$ & $\mathrm{AlCHI}$ & 1999 & 9821621 & Pad Mount \\
\hline HANSEN & 2729 TS & $500 \mathrm{KVA}$ & $\mathrm{AlCHI}$ & 2002 & 02704782 & Pad Mount \\
\hline HANSEN & $2221 \mathrm{TS}$ & $500 \mathrm{KVA}$ & MEIDEN & 1996 JAN & 1T8311T1 & Pad Mount \\
\hline HANSEN & $2518 \mathrm{TS}$ & $500 \mathrm{KVA}$ & MITSUBISHI & 1996 & DN3220001 & Pad Mount \\
\hline HANSEN & 2445 TS & $500 \mathrm{KVA}$ & MITSUBISHI & 1988 & DM1953001 & Pad Mount \\
\hline HANSEN & $2141 \mathrm{TS}$ & $500 \mathrm{KVA}$ & G.E. & 1991 NOV & P817332TYF & Pad Mount \\
\hline HANSEN & $2431 \mathrm{TS}$ & $500 \mathrm{KVA}$ & DAIHEN & 1992 & 3SD153501 & Pad Mount \\
\hline HANSEN & 2530 TS & $500 \mathrm{KVA}$ & DAIHEN & 1992 & 3SD1424A1 & Pad Mount \\
\hline HANSEN & 2442 TS & $500 \mathrm{KVA}$ & MEIDEN & $1990 \mathrm{DEC}$ & 1P9691T1 & Pad Mount \\
\hline HANSEN & 2622 TS & $500 \mathrm{KVA}$ & DAIHEN & 1990 & 3SD1232A1 & Pad Mount \\
\hline HANSEN & 2665 TS & $500 \mathrm{KVA}$ & DAIHEN & 1990 & 3SD1122A1 & Pad Mount \\
\hline HANSEN & $2339 \mathrm{TS}$ & $500 \mathrm{KVA}$ & HITACHI & 2003 & 024775321 & Pad Mount \\
\hline HANSEN & 2245 TS & $500 \mathrm{KVA}$ & TOSHIBA & 1999 & 99000692 & Pad Mount \\
\hline
\end{tabular}


Page 19 of 24

\begin{tabular}{|c|c|c|c|c|c|c|}
\hline HANSEN & 2872 TS & $500 \mathrm{KVA}$ & $\mathrm{AlCHI}$ & 2003 & 03204219 & Pad Mount \\
\hline HANSEN & 2165 TS & $500 \mathrm{KVA}$ & MITSUBISHI & 1994 & DH4649001 & Pad Mount \\
\hline HANSEN & 2860 TS & $500 \mathrm{KVA}$ & $\mathrm{AlCHI}$ & 1994 & 9421620 & Pad Mount \\
\hline HANSEN & 2814-A TS & $500 \mathrm{KVA}$ & VANTRAN & & 00V5179 & Pad Mount \\
\hline HANSEN & $2416 \mathrm{TS}$ & $500 \mathrm{KVA}$ & COOPER & $2007 \mathrm{JAN}$ & 0750002595 & Pad Mount \\
\hline HANSEN & 2340 TS & $500 \mathrm{KVA}$ & FUJI & 1990 JAN & B90330A01 & Pad Mount \\
\hline KINSER & $401 \mathrm{TV}$ & $500 \mathrm{KVA}$ & G.E. & & M154620 & Pad Mount \\
\hline KINSER & $51-\mathrm{H}$ TS & $500 \mathrm{KVA}$ & COOPER & DEC2004 & 0437020881 & Pad Mount \\
\hline KINSER & $99 \mathrm{TS}$ & $500 \mathrm{KVA}$ & MITSUBISHI & 1988 & DM4692001 & Pad Mount \\
\hline KINSER & 616 TS & $500 \mathrm{KVA}$ & FUJI ELECTRIC & NOV 1993 & $\mathrm{~B} 30146 \mathrm{~A} 01$ & Pad Mount \\
\hline KINSER & 1225 TS & $500 \mathrm{KVA}$ & FUJI & DEC 1990 & B00288A01 & Pad Mount \\
\hline KINSER & $618 \mathrm{TS}$ & $500 \mathrm{KVA}$ & TOSHIBA & 2002 & 01057452 & Pad Mount \\
\hline KINSER & 29-A TH & $500 \mathrm{KVA}$ & JIMELCO & & AA00201407 & Pad Mount \\
\hline KINSER & 208 TS & $500 \mathrm{KVA}$ & COOPER & SEP2004 & 0437015743 & Pad Mount \\
\hline KINSER & 24-D TS & $500 \mathrm{KVA}$ & COOPER & APR 2008 & 0850007597 & Pad Mount \\
\hline MCAS & 433-A TS & $500 \mathrm{KVA}$ & COOPER & 2007 OCT & 0759002306 & Pad Mount \\
\hline MCAS & 36-B TS & $500 \mathrm{KVA}$ & ABB & 1997 FEB & 97J269325 & Pad Mount \\
\hline MCAS & 64-A TS & $500 \mathrm{KVA}$ & $\mathrm{AlCHI}$ & 1990 & 8922620 & Pad Mount \\
\hline MCAS & $433 \mathrm{TS}$ & $500 \mathrm{KVA}$ & COOPER & 2007 OCT & 0759002435 & Pad Mount \\
\hline MCAS & 219 TS & $500 \mathrm{KVA}$ & $A B B$ & JUN 2005 & 05J319105 & Pad Mount \\
\hline MCAS & $39 \mathrm{TV}$ & $500 \mathrm{KVA}$ & JIMELCO & & AA00201410 & Pad Mount \\
\hline MCAS & 4-A TV & $500 \mathrm{KVA}$ & JIMELCO & MAR 1991 & AA00201409 & Pad Mount \\
\hline MCAS & 633A TS & $500 \mathrm{KVA}$ & VANTRAN & & $01 \mathrm{~V} 5700$ & Pad Mount \\
\hline MCAS & $417 \mathrm{TS}$ & $500 \mathrm{KVA}$ & ABB & & 03J767219 & Pad Mount \\
\hline MCAS & 509 TS & $500 \mathrm{KVA}$ & COOPER & NOV 2007 & 0750022806 & Pad Mount \\
\hline MCAS & 223 TS & $500 \mathrm{KVA}$ & $\mathrm{AlCHI}$ & & 9222303 & Pad Mount \\
\hline MCAS & $525 \mathrm{TS}$ & $500 \mathrm{KVA}$ & $\mathrm{AlCHI}$ & 1991 & 9023315 & Pad Mount \\
\hline MCAS & $218 \mathrm{TS}$ & $500 \mathrm{KVA}$ & COOPER & & 0537015240 & Pad Mount \\
\hline
\end{tabular}


Page 20 of 24

\begin{tabular}{|c|c|c|c|c|c|c|}
\hline MCAS & 4-B TS & $500 \mathrm{KVA}$ & H. K.PORTER & & W224357 & Pad Mount \\
\hline MCAS & 740 TS & $500 \mathrm{KVA}$ & MEIDEN & SEP 1991 & 1Q8154T1 & Pad Mount \\
\hline MCAS & 505-A TS & $500 \mathrm{KVA}$ & ABB & DEC 2008 & 08J163083 & Pad Mount \\
\hline MCAS & 229 TS & $500 \mathrm{KVA}$ & MEIDEN & 1994 NOV & 1R9945T1 & Pad Mount \\
\hline MCAS & $3 \mathrm{TS}$ & $500 \mathrm{KVA}$ & BALTEAU & & $\begin{array}{l}\text { PYB-0143 } \\
\end{array}$ & Pad Mount \\
\hline MCAS & 6-B TS & $500 \mathrm{KVA}$ & COOPER & MAY 2008 & 0850007611 & Pad Mount \\
\hline N.T.A & 502 TS & $500 \mathrm{KVA}$ & COOPER & JUL 2007 & 0750013417 & Pad Mount \\
\hline FOSTER & $21 \mathrm{GS}$ & $600 \mathrm{~A}$ & G\&W (SF6) & & $625-00-0162$ & Pad Mount \\
\hline FOSTER-1 & SHOP STOCK & $600 \mathrm{~A}$ & G\&W & & $626-02-0016$ & Pad Mount \\
\hline FOSTER-1 & SHOP STOCK & $600 \mathrm{~A}$ & G\&W & OCT 2007 & $627-07-0962$ & Pad Mount \\
\hline FOSTER-1 & SHOP STOCK & $600 \mathrm{~A}$ & G\&W & APR 2000 & $625-00-0184$ & Pad Mount \\
\hline FOSTER-1 & SHOP STOCK & $600 \mathrm{~A}$ & G\&W & APR 2000 & $622-00-0013$ & Pad Mount \\
\hline CTNY & $4229 \mathrm{TS}$ & $75 \mathrm{KVA}$ & $\mathrm{AlCHI}$ & 1986 & 8622045 & Pad Mount \\
\hline CTNY & 4467 TS & $75 \mathrm{KVA}$ & MEIDEN & 1978 & 1T8016T1 & Pad Mount \\
\hline CTNY & 4496 TS & $75 \mathrm{KVA}$ & MEIDEN & OCT 1988 & 1N8922T1 & Pad Mount \\
\hline CTNY & $4331 \mathrm{TS}$ & $75 \mathrm{KVA}$ & COOPER & 2000 FEB & 0037003186 & Pad Mount \\
\hline CTNY & 4423 TS & $75 \mathrm{KVA}$ & COOPER & SEP 1995 & 959004585 & Pad Mount \\
\hline CTNY & 4460 TS & $75 \mathrm{KVA}$ & FUJI & & A01329T1-1 & Pad Mount \\
\hline CTNY & 4227 TS & $75 \mathrm{KVA}$ & PAUWELS & & 05J136842 & Pad Mount \\
\hline CTNY & $4461 \mathrm{TS}$ & $75 \mathrm{KVA}$ & PAUWELS & OCT 2005 & 05G135692 & Pad Mount \\
\hline CTNY & 4204 TS & $75 \mathrm{KVA}$ & COOPER & SEP 04 & 0437014505 & Pad Mount \\
\hline CTNY & $4408 \mathrm{TS}$ & $75 \mathrm{KVA}$ & PAUWELS & NOV 2004 & 04J129806 & Pad Mount \\
\hline CTNY-TG & $4151 \mathrm{TS}$ & $75 \mathrm{KVA}$ & $\mathrm{AlCHI}$ & 1993 & 9320157 & Pad Mount \\
\hline FOSTER & 546 TS & $75 \mathrm{KVA}$ & CUTLER-HAMMER & OCT 2003 & 03J907081 & Pad Mount \\
\hline FOSTER & 267-B2 TS & $75 \mathrm{KVA}$ & RTE & & 896005650 & Pad Mount \\
\hline FOSTER & $262 \mathrm{TS}$ & $75 \mathrm{KVA}$ & $\mathrm{AlCHI}$ & 2001 & 01703756 & Pad Mount \\
\hline FOSTER & $5608 \mathrm{TS}$ & $75 \mathrm{KVA}$ & MITSUBISHI & 1988 & DM0066001 & Pad Mount \\
\hline FOSTER & 5606 TS & $75 \mathrm{KVA}$ & DAIHEN & 1988 & 35SD0926A1 & Pad Mount \\
\hline
\end{tabular}


Page 21 of 24

\begin{tabular}{|c|c|c|c|c|c|c|}
\hline FOSTER & 154-D TS & $75 \mathrm{KVA}$ & COOPER & FEB2005 & 0537000684 & Pad Mount \\
\hline FOSTER & 456 TS & $75 \mathrm{KVA}$ & COOPER & AUG 2004 & 043701514 & Pad Mount \\
\hline FOSTER & 5828 TS & $75 \mathrm{KVA}$ & COOPER & SEP 2004 & 0437014506 & Pad Mount \\
\hline FOSTER & $5638 \mathrm{TS}$ & $75 \mathrm{KVA}$ & MEIDEN & JUL 1994 & 1S8380T1 & Pad Mount \\
\hline FOSTER & 146-B TS & $75 \mathrm{KVA}$ & RTE & & 866001907 & Pad Mount \\
\hline $\begin{array}{l}\text { FOSTER } \\
\end{array}$ & 267-B1 TS & $75 \mathrm{KVA}$ & WESTING HOUSE & & 86JC624058 & Pad Mount \\
\hline FOSTER-1 & SHOP STOCK & $75 \mathrm{KVA}$ & PAUWELS & AUG 2008 & 20110811454 & Pad Mount \\
\hline FOSTER-1 & SHOP STOCK & $75 \mathrm{KVA}$ & COOPER & SEP 2006 & 0650016411 & Pad Mount \\
\hline FOSTER-1 & SHOP STOCK & $75 \mathrm{KVA}$ & COOPER & SEP 2004 & 0437015140 & Pad Mount \\
\hline FOSTER-1 & SHOP STOCK & $75 \mathrm{KVA}$ & PAUWELS & JUL 2005 & 05E134381 & Pad Mount \\
\hline FOSTER-1 & SHOP STOCK & $75 \mathrm{KVA}$ & COOPER & 2005 FEB & 0537003657 & Pad Mount \\
\hline FOSTER-1 & SHOP STOCK & $75 \mathrm{KVA}$ & COOPER & 2005 FEB & 0537003658 & Pad Mount \\
\hline FOSTER-1 & SHOP STOCK & $75 \mathrm{KVA}$ & HOWARD & & 2212572195 & Pad Mount \\
\hline FOSTER-1 & SHOP STOCK & $75 \mathrm{KVA}$ & COOPER & AUG 2006 & 0650016412 & Pad Mount \\
\hline HANSEN & 124 POLE & $75 \mathrm{KVA}$ & FUJI & JUN 1999 & A90889T1 & Pad Mount \\
\hline HANSEN & 2113 TS & $75 \mathrm{KVA}$ & COOPER & 2005 FEB & 0537003793 & Pad Mount \\
\hline HANSEN & 2425 TS & $75 \mathrm{KVA}$ & COOPER & SEP 2007 & 0750017628 & Pad Mount \\
\hline HANSEN & 2386 TS & $75 \mathrm{KVA}$ & HITACHI & 2000 & 23601911 & Pad Mount \\
\hline HANSEN & 2895 TS & $75 \mathrm{KVA}$ & RTE & & 886006888 & Pad Mount \\
\hline HANSEN & 2448 TS & $75 \mathrm{KVA}$ & OSAKA & 1983 & 3D0332A01 & Pad Mount \\
\hline HANSEN & 2663 TS & $75 \mathrm{KVA}$ & PAUWELS & 2008 FEB & 20110708980 & Pad Mount \\
\hline HANSEN & 2203 TS & $75 \mathrm{KVA}$ & SUNBELT & AUG 1982 & ST029756407 & Pad Mount \\
\hline HANSEN & 261-B POLE & $75 \mathrm{KVA}$ & FUJI & JUN 1993 & A30945T1 & Pad Mount \\
\hline HANSEN & 2542 TS & $75 \mathrm{KVA}$ & ABB & AUG 1997 & $97 J 406217$ & Pad Mount \\
\hline HANSEN & 2206 TS & $75 \mathrm{KVA}$ & ABB & 1997 FEB & 97J284160 & Pad Mount \\
\hline HANSEN & 2395-A TS & $75 \mathrm{KVA}$ & COOPER & DEC 2007 & 0859000042 & Pad Mount \\
\hline HANSEN & 2395 TS & $75 \mathrm{KVA}$ & COOPER & MAR 2010 & 1050002252 & Pad Mount \\
\hline HANSEN & 2466 TS & $75 \mathrm{KVA}$ & MEIDEN & 1999 JUN & 1N6648T1 & Pad Mount \\
\hline
\end{tabular}


Page 22 of 24

\begin{tabular}{|c|c|c|c|c|c|c|}
\hline HENOKO & 1020 TS & $75 \mathrm{KVA}$ & DAIHEN & & 3SD2137B1 & Pad Mount \\
\hline HENOKO & $19 \mathrm{TS}$ & $75 \mathrm{KVA}$ & PAUWELS & & 99C79864 & Pad Mount \\
\hline KINSER & 626 TS & $75 \mathrm{KVA}$ & DAIHEN & 1993 & 3SD162401 & Pad Mount \\
\hline KINSER & 407 TS & $75 \mathrm{KVA}$ & $\mathrm{AlCHI}$ & 1993 & 9320156 & Pad Mount \\
\hline KINSER & 79-B TS & $75 \mathrm{KVA}$ & MITSUBISHI & & DZ8626001 & Pad Mount \\
\hline KINSER & 204 TS & $75 \mathrm{KVA}$ & $\mathrm{AlCHI}$ & & 017003828 & Pad Mount \\
\hline MCAS & 12-B TS & $75 \mathrm{KVA}$ & FUJI & & B70346AT1 & Pad Mount \\
\hline MCAS & 53-A TS & $75 \mathrm{KVA}$ & ABB & SEP 2002 & 02J648031 & Pad Mount \\
\hline MCAS & $553 \mathrm{TS}$ & $75 \mathrm{KVA}$ & COOPER & NOV 1995 & 959006333 & Pad Mount \\
\hline MCAS & 202 TS & $75 \mathrm{KVA}$ & COOPER & NOV 2007 & 0750022395 & Pad Mount \\
\hline MCAS & $538 \mathrm{TS}$ & $75 \mathrm{KVA}$ & COOPER & 2007 JAN & 0759000112 & Pad Mount \\
\hline MCAS & $450 \mathrm{TS}$ & $75 \mathrm{KVA}$ & COOPER & APR 2009 & 0950003759 & Pad Mount \\
\hline MCAS & 38-L TS & $75 \mathrm{KVA}$ & PAUWELS & & 20110703031 & Pad Mount \\
\hline MCAS & $13 \mathrm{TS}$ & $75 \mathrm{KVA}$ & DAIHEN & 1999 & KSB0141B1 & Pad Mount \\
\hline MCAS & 57-A TS & $75 \mathrm{KVA}$ & HOWARD & & 3005213694 & Pad Mount \\
\hline MCAS & $553 \mathrm{~A}$ TS & $75 \mathrm{KVA}$ & COOPER & OCT 2008 & 0850016308 & Pad Mount \\
\hline MCAS & 44A TS & $75 \mathrm{KVA}$ & WESTING HOUSE & & 86JH733156 & Pad Mount \\
\hline MCAS & 665 TS & $75 \mathrm{KVA}$ & PAUWELS & JUN 2008 & 20110810763 & Pad Mount \\
\hline N.T.A & $532 \mathrm{TS}$ & $75 \mathrm{KVA}$ & JIMELCO & $1991 \mathrm{MAR}$ & AA00201414 & Pad Mount \\
\hline CTNY & 4417 TS & $750 \mathrm{KVA}$ & FUJI & 2002 NOV & A20021 & Pad Mount \\
\hline CTNY & $4225 \mathrm{TS}$ & $750 \mathrm{KVA}$ & COOPER & AUG 03 & 0326001576 & Pad Mount \\
\hline CTNY & $4211 \mathrm{TS}$ & $750 \mathrm{KVA}$ & HOWARD & & 1530390804 & Pad Mount \\
\hline CTNY-TG & $4132 \mathrm{TS}$ & $750 \mathrm{KVA}$ & PAUWELS & AUG 2006 & 06C140508 & Pad Mount \\
\hline FOSTER & 464 TS & $750 \mathrm{KVA}$ & ABB & 1998 JUN & 98J597224 & Pad Mount \\
\hline FOSTER & $11 \mathrm{TS}$ & $750 \mathrm{KVA}$ & MEIDEN & 2001 AUG & 1P5419T1 & Pad Mount \\
\hline FOSTER & 5907 TS & $750 \mathrm{KVA}$ & $\mathrm{AlCHI}$ & 2001 & 01703349 & Pad Mount \\
\hline FOSTER & 363 TS & $750 \mathrm{KVA}$ & MITSUBISHI & 2001 & DY1357001 & Pad Mount \\
\hline FOSTER & 480 TS & $750 \mathrm{KVA}$ & MITSUBISHI & 1996 & DM2743001 & Pad Mount \\
\hline
\end{tabular}


Page 23 of 24

\begin{tabular}{|c|c|c|c|c|c|c|}
\hline FOSTER & 5966 TS & $750 \mathrm{KVA}$ & HITACHI & 2004 & 23014421 & Pad Mount \\
\hline FOSTER & 5902 TS & $750 \mathrm{KVA}$ & $\mathrm{AlCHI}$ & 2005 & 05206083 & Pad Mount \\
\hline FOSTER & $460 \mathrm{TS}$ & $750 \mathrm{KVA}$ & $A B B$ & DEC 2004 & 04J185376 & Pad Mount \\
\hline FOSTER & 5906 TS & $750 \mathrm{KVA}$ & COOPER & SEP 2008 & 0850014902 & Pad Mount \\
\hline FOSTER-1 & SHOP STOCK & $750 \mathrm{KVA}$ & $\mathrm{AlCHI}$ & 1997 & 9623180 & Pad Mount \\
\hline FOSTER-1 & SHOP STOCK & $750 \mathrm{KVA}$ & COOPER & AUG 2010 & 1050008156 & Pad Mount \\
\hline FOSTER-1 & SHOP STOCK & $750 \mathrm{KVA}$ & COOPER & NOV 2009 & 0950010955 & Pad Mount \\
\hline HANSEN & 2635 TS & $750 \mathrm{KVA}$ & HITACHI & 1999 & 23339821 & Pad Mount \\
\hline HANSEN & 2447 TS & $750 \mathrm{KVA}$ & COOPER & 2005 FEB & 0537004265 & Pad Mount \\
\hline HANSEN & 2667 TS & $750 \mathrm{KVA}$ & $\overline{\mathrm{AlCHI}}$ & 1993 & 9223017 & Pad Mount \\
\hline HANSEN & 2893 TS & $750 \mathrm{KVA}$ & MEIDEN & OCT 1992 & 1R8626T1 & Pad Mount \\
\hline HANSEN & $2327 \mathrm{TS}$ & $750 \mathrm{KVA}$ & DAIHEN & 1993 & 3SD1605A1 & Pad Mount \\
\hline HANSEN & $2537 \mathrm{TS}$ & $750 \mathrm{KVA}$ & DAIHEN & 1992 & 3SD1429A1 & Pad Mount \\
\hline HANSEN & $2450 \mathrm{TS}$ & $750 \mathrm{KVA}$ & COOPER & DEC 2008 & 085002123 & Pad Mount \\
\hline HANSEN & 2825 TS & $750 \mathrm{KVA}$ & $\mathrm{AlCHI}$ & 2002 & 02704784 & Pad Mount \\
\hline HANSEN & 2153 TS & $750 \mathrm{KVA}$ & $\mathrm{AlCHI}$ & 2005 & 05208270 & Pad Mount \\
\hline HANSEN & 2725 TS & $750 \mathrm{KVA}$ & FUJI & SEP 1992 & B20168A01 & Pad Mount \\
\hline HANSEN & $2118 \mathrm{TS}$ & $750 \mathrm{KVA}$ & $\mathrm{AlCHI}$ & 2007 & 07211927 & Pad Mount \\
\hline HANSEN & $2443 \mathrm{TS}$ & $750 \mathrm{KVA}$ & VANTRAN & & $06 \mathrm{~V} 7282$ & Pad Mount \\
\hline KINSER & $100 \mathrm{TS}$ & $750 \mathrm{KVA}$ & MITSUBISHI & 1989 & DP3113001 & Pad Mount \\
\hline KINSER & $66-\mathrm{H}$ TS & $750 \mathrm{KVA}$ & COOPER & OCT 2008 & 0850017701 & Pad Mount \\
\hline KINSER & 300 TS & $750 \mathrm{KVA}$ & COOPER & MAY 2008 & 0850008332 & Pad Mount \\
\hline KINSER & $1226 \mathrm{TS}$ & $750 \mathrm{KVA}$ & DAIHEN & 1991 & 3SD1272A1 & Pad Mount \\
\hline KINSER & 1202 TS & $750 \mathrm{KVA}$ & $\mathrm{AlCHI}$ & 1993 & 9222379 & Pad Mount \\
\hline KINSER & $1210 \mathrm{TS}$ & $750 \mathrm{KVA}$ & FUJI & MAR 1992 & B10310A01 & Pad Mount \\
\hline KINSER & 40-E TS & $750 \mathrm{KVA}$ & PAUWELS & & 00A101228 & Pad Mount \\
\hline KINSER & 104-A TS & $750 \mathrm{KVA}$ & FUJI & $1993 \mathrm{JUN}$ & A30954T1 & Pad Mount \\
\hline KINSER & 502 TS & $750 \mathrm{KVA}$ & COOPER & 2005 FEB & 0537003496 & Pad Mount \\
\hline
\end{tabular}


Page 24 of 24

\begin{tabular}{|c|c|c|c|c|c|c|}
\hline KINSER & $29-F$ TS & $750 \mathrm{KVA}$ & COOPER & & 0537007394 & Pad Mount \\
\hline KINSER & 41-A TH & $750 \mathrm{KVA}$ & COOPER & APR 2010 & 1050003055 & Pad Mount \\
\hline KINSER & $18 \mathrm{TS}$ & $750 \mathrm{KVA}$ & RTE & & 906008395 & Pad Mount \\
\hline MCAS & $400 \mathrm{TS}$ & $750 \mathrm{KVA}$ & COOPER & JAN 2007 & 0759000102 & Pad Mount \\
\hline MCAS & 2B-4A TS & $750 \mathrm{KVA}$ & COOPER & NOV 2007 & 0759001159 & Pad Mount \\
\hline MCAS & 548 TS & $750 \mathrm{KVA}$ & MITSUBISHI & & D28363001 & Pad Mount \\
\hline MCAS & 510 TS & $750 \mathrm{KVA}$ & ABB & SEP 2005 & 05J388001 & Pad Mount \\
\hline MCAS & 562 TS & $750 \mathrm{KVA}$ & MITSUBISHI & & DP1479001 & Pad Mount \\
\hline MCAS & 20-B2 TS & $750 \mathrm{KVA}$ & COOPER & APR 2008 & 0850007313 & Pad Mount \\
\hline MCAS & 443 TS & $750 \mathrm{KVA}$ & COOPER & DEC 2008 & 0850020328 & Pad Mount \\
\hline MCAS & 439 TS & $750 \mathrm{KVA}$ & COOPER & DEC 2008 & 0850020568 & Pad Mount \\
\hline HANSEN & 2493 TS & $850 \mathrm{KVA}$ & $\mathrm{AlCHI}$ & 1985 & 8521374 & Pad Mount \\
\hline
\end{tabular}

\section{END}




\begin{tabular}{|c|c|c|c|c|c|c|c|c|c|c|c|}
\hline CAMP & LOC & $\begin{array}{c}\text { CAPACITY } \\
\text { KVA }\end{array}$ & MFR_DATE & MANUFACT & Serial_ID & Type & \# & $\#$ & $\#$ & Age & Age Sort \\
\hline FOSTER & 5704 TS & 500 & 1989 & $\mathrm{AlCHI}$ & 892221701 & Pad Mount & 1 & & & 23 & 11 \\
\hline FOSTER & 5703 TS & 500 & 1989 & AICHI & 892221702 & PadMount & 2 & & & 23 & 11 \\
\hline FOSTER & 478 TS & 500 & 1997 & AICHI & 9721148 & PadMount & 3 & & & 15 & 11 \\
\hline FOSTER & 5696 TS & 500 & 1991 & DAIREN & 3SD1250A1 & Pad Mount & 4 & & & 21 & 11 \\
\hline FOSTER & $370 \mathrm{TS}$ & 500 & 1991 & DAIREN & 3SD1253A1 & Pad Mount & 5 & & & 21 & 12 \\
\hline FOSTER & 481 TS & 500 & 1996 & FUJI & B50348AT1 & Pad Mount & 6 & & & 16 & 13 \\
\hline FOSTER & $445 \mathrm{TS}$ & 500 & 1998 & MEIDEN & 1N6330T1 & Pad Mount & 7 & & & 14 & 13 \\
\hline FOSTER & $490 \mathrm{TS}$ & 500 & 1999 & MITSUBISHI & DD9891001 & Pad Mount & 8 & & & 13 & 13 \\
\hline FOSTER & 5670 TS & 500 & 1989 & WESTING HOUSE & $89 \mathrm{~J} 495273$ & Pad Mount & 9 & & & 23 & 13 \\
\hline HANSEN & $2860 \mathrm{TS}$ & 500 & 1994 & AICHI & 9421620 & PadMount & 10 & & & 18 & 13 \\
\hline HANSEN & 2519 TS & 500 & 1999 & AICHI & 9821621 & Pad Mount & 11 & & & 13 & 14 \\
\hline HANSEN & $2622 \mathrm{TS}$ & 500 & 1990 & DAIHEN & 3SD1232A1 & Pad Mount & 12 & & & 22 & 14 \\
\hline HANSEN & 2665 TS & 500 & 1990 & DAIHEN & 3SD1122A1 & PadMount & 13 & & & 22 & 15 \\
\hline HANSEN & 2530 TS & 500 & 1992 & DAIHEN & 3SD1424A1 & PadMount & 14 & & & 20 & 16 \\
\hline HANSEN & $2340 \mathrm{TS}$ & 500 & 1990 & FUJ & B90330A01 & Pad Mount & 15 & & & 22 & 16 \\
\hline HANSEN & $2441 \mathrm{TS}$ & 500 & 1993 & FUJT & A24006T1 & Pad Mount & 16 & & & 19 & 16 \\
\hline HANSEN & $2141 \mathrm{TS}$ & 500 & 1991 & G.E. & P817332TYF & Pad Mount & 17 & & & 21 & 16 \\
\hline HANSEN & $2442 \mathrm{TS}$ & 500 & 1990 & MEIDEN & 1P9691T1 & Pad Mount & 18 & & & 22 & 18 \\
\hline HANSEN & $2221 \mathrm{TS}$ & 500 & 1996 & MEIDEN & $1 T 8311 T 1$ & Pad Mount & 19 & & & 16 & 18 \\
\hline HANSEN & 2610 TS & 500 & 1999 & MEIDEN & 1N6714T1 & Pad Mount & 20 & & & 13 & 19 \\
\hline HANSEN & $2165 \mathrm{TS}$ & 500 & 1994 & MITSUBISHI & DH4649001 & Pad Mount & 21 & & & 18 & 19 \\
\hline HANSEN & $2518 \mathrm{TS}$ & 500 & 1996 & MITSUBISHI & DN3220001 & Pad Mount & 22 & & & 16 & 19 \\
\hline HANSEN & $2245 \mathrm{TS}$ & 500 & 1999 & TOSHIBA & 99000692 & Pad Mount & 23 & & & 13 & 19 \\
\hline KINSER & $1225 \mathrm{TS}$ & 500 & 1990 & FUJ & B00288A01 & Pad Mount & 24 & & & 22 & 19 \\
\hline KINSER & 616TS & 500 & 1993 & FUJT & B30146A01 & Pad Mount & 25 & & & 19 & 19 \\
\hline KIINSER & प्र915 & 500 & T98ठ & TVIITSUBISATI & DIVI40YZOUT & Pad mount & 26 & & & 24 & 19 \\
\hline FOSTER & $464 \mathrm{TS}$ & 750 & 1998 & ABB & $98 \mathrm{~J} 597224$ & Pad Mount & 27 & 1 & & 14 & 20 \\
\hline FOSTER & 5907 TS & 750 & 2001 & AICHI & 1703349 & Pad Mount & 28 & 2 & & 11 & 20 \\
\hline FOSTER & $11 \mathrm{TS}$ & 750 & 2001 & MEIDEN & 1P5419T1 & Pad Mount & 29 & 3 & & 11 & 20 \\
\hline FOSTER & $480 \mathrm{TS}$ & 750 & 1996 & MITSUBISHI & DM2743001 & Padmount & 30 & 4 & & 16 & 20 \\
\hline FOSTER & $363 \mathrm{TS}$ & 750 & 2001 & MITSUBISHI & DY1357001 & Pad Mount & 31 & 5 & & 11 & 20 \\
\hline HANSEN & 2667 TS & 750 & 1993 & AlCHI & 9223017 & Pad Mount & 32 & 6 & & 19 & 21 \\
\hline HANSEN & 2537 TS & 750 & 1992 & DAIREN & 3SD1429A1 & Pad Mount & 33 & 7 & & 20 & 21 \\
\hline HANSEN & $2327 \mathrm{TS}$ & 750 & 1993 & DAIREN & 3SD1605A1 & PadMount & 34 & 8 & & 19 & 21 \\
\hline FAIVSEIV & ZIZSTS & 150 & Ty9L & FUJ & BZUTOठAU & Pad Iviount & 35 & 9 & & 20 & 21 \\
\hline HANSEN & $2635 \mathrm{TS}$ & 750 & 1999 & माTACHI & 23339821 & Pad Mount & 36 & 10 & & 13 & 22 \\
\hline HANSEN & 2893 TS & 750 & 1992 & MEIDEN & 1R8626T1 & Padmount & 37 & 11 & & 20 & 22 \\
\hline KINSER & 1202 TS & 750 & 1993 & AICHI & 9222379 & Pad Mount & 38 & 12 & & 19 & 22 \\
\hline KINSER & $1226 \mathrm{TS}$ & 750 & 1991 & DAIREN & 3SD1272A1 & Pad Mount & 39 & 13 & & 21 & 22 \\
\hline KINSER & 1210TS & 750 & 1992 & FUJ & B10310A01 & Pad Mount & 40 & 14 & & 20 & 22 \\
\hline KINSER & 104-A TS & 750 & 1993 & FUJT & A30954T1 & Pad Mount & 41 & 15 & & 19 & 23 \\
\hline KINSER & $100 \mathrm{TS}$ & 750 & 1989 & MITSUBISHI & DP3113001 & Padmount & 42 & 16 & & 23 & 23 \\
\hline FOSTER & 1002 TS & 1000 & 1993 & G.E. & Q108279-TNI & Pad Mount & 43 & & 1 & 19 & 23 \\
\hline FOSTER & $1004 \mathrm{TV}$ & 1000 & 1989 & WESTING HOUSE & PAW4176-0101 & Pad Mount & 44 & & 2 & 23 & 23 \\
\hline HANSEN & 2365 TS & 1000 & 2001 & FUJT & B10215A01 & Padmount & 45 & & 3 & 11 & 23 \\
\hline HANSEN & $2654 \mathrm{TS}$ & 1000 & 2000 & TOSHIBA & 23589 & Pad Mount & 46 & & 4 & 12 & 24 \\
\hline
\end{tabular}




\section{Appendix B}

Military Base Drawings 


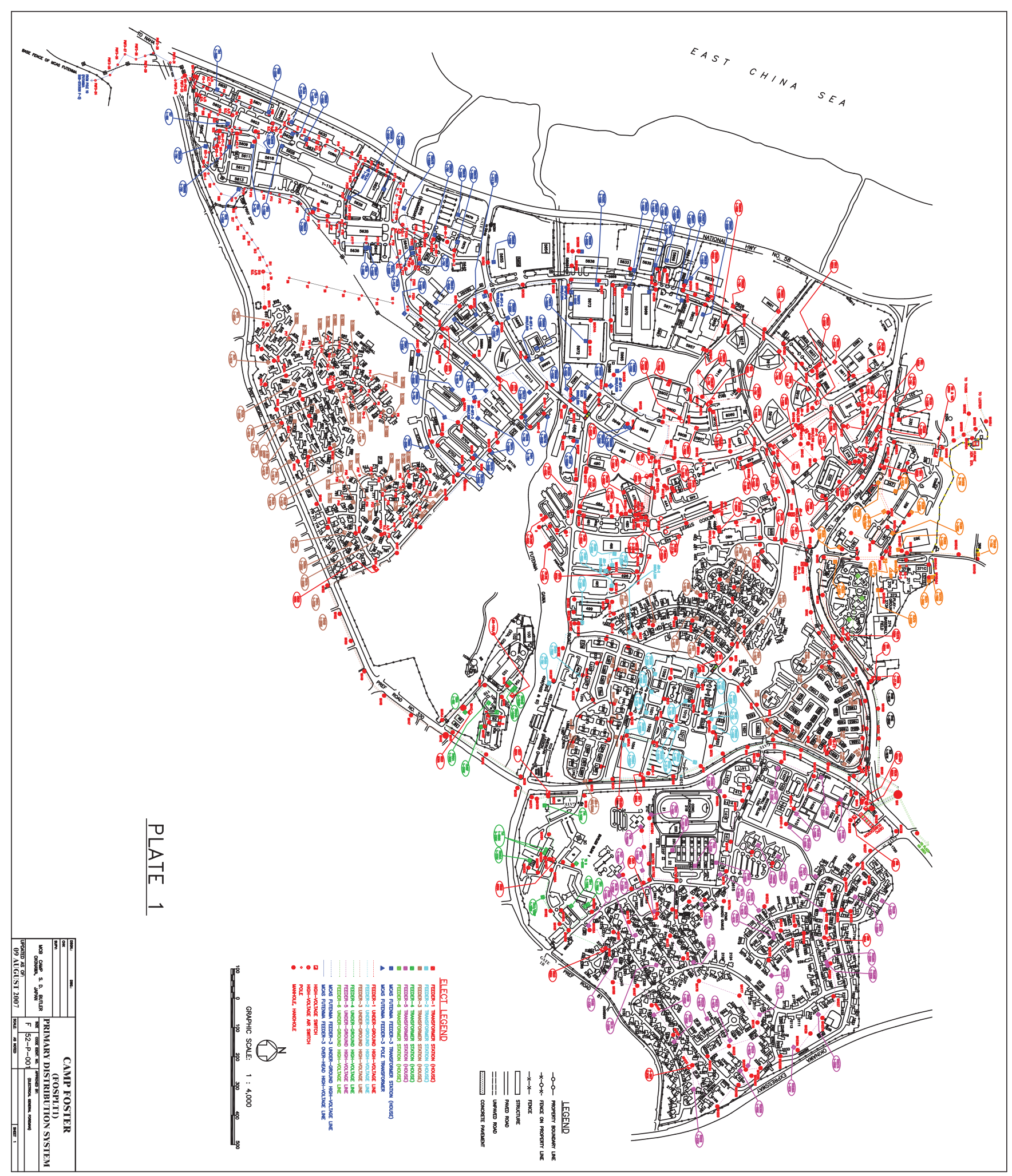




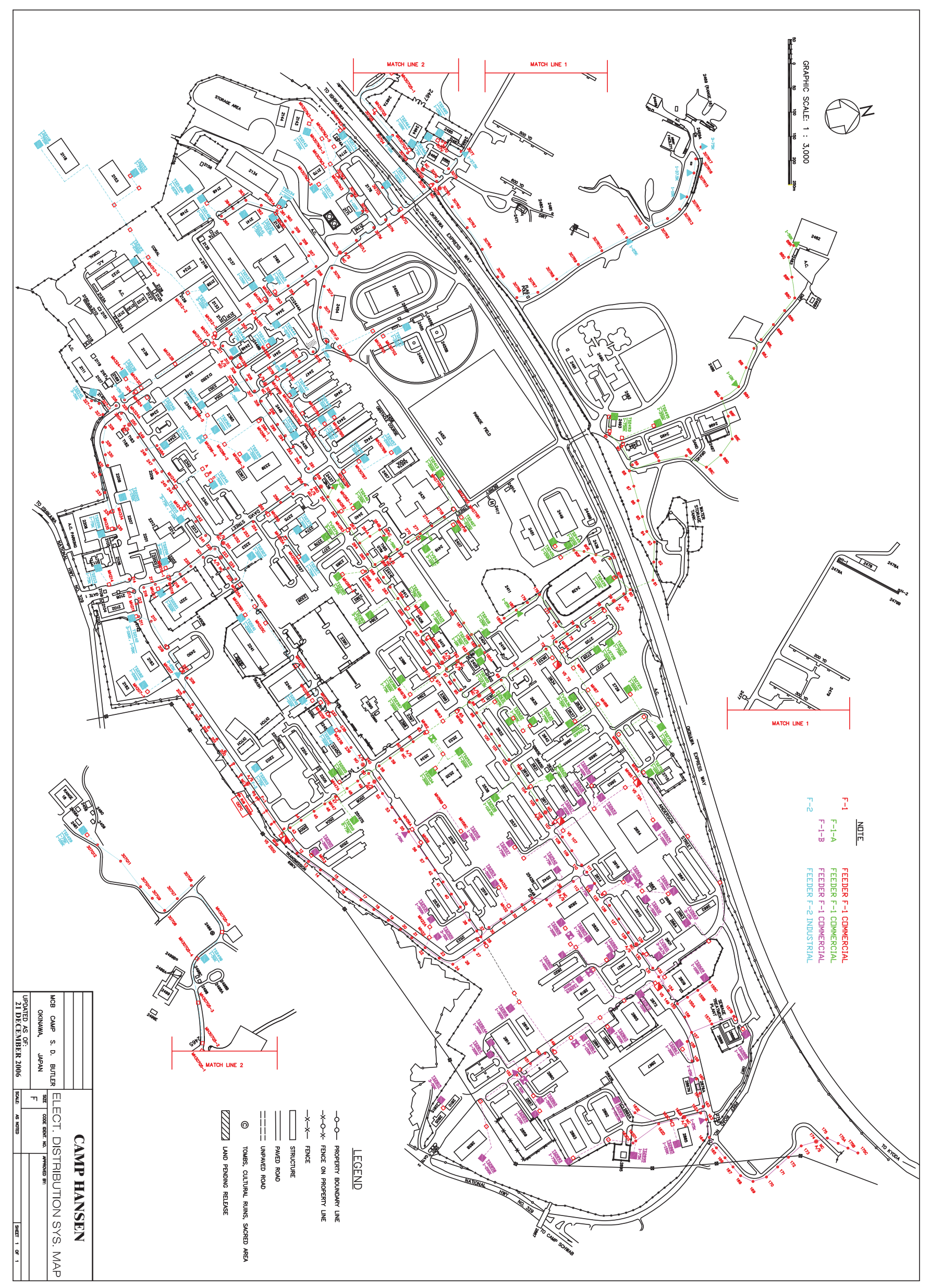




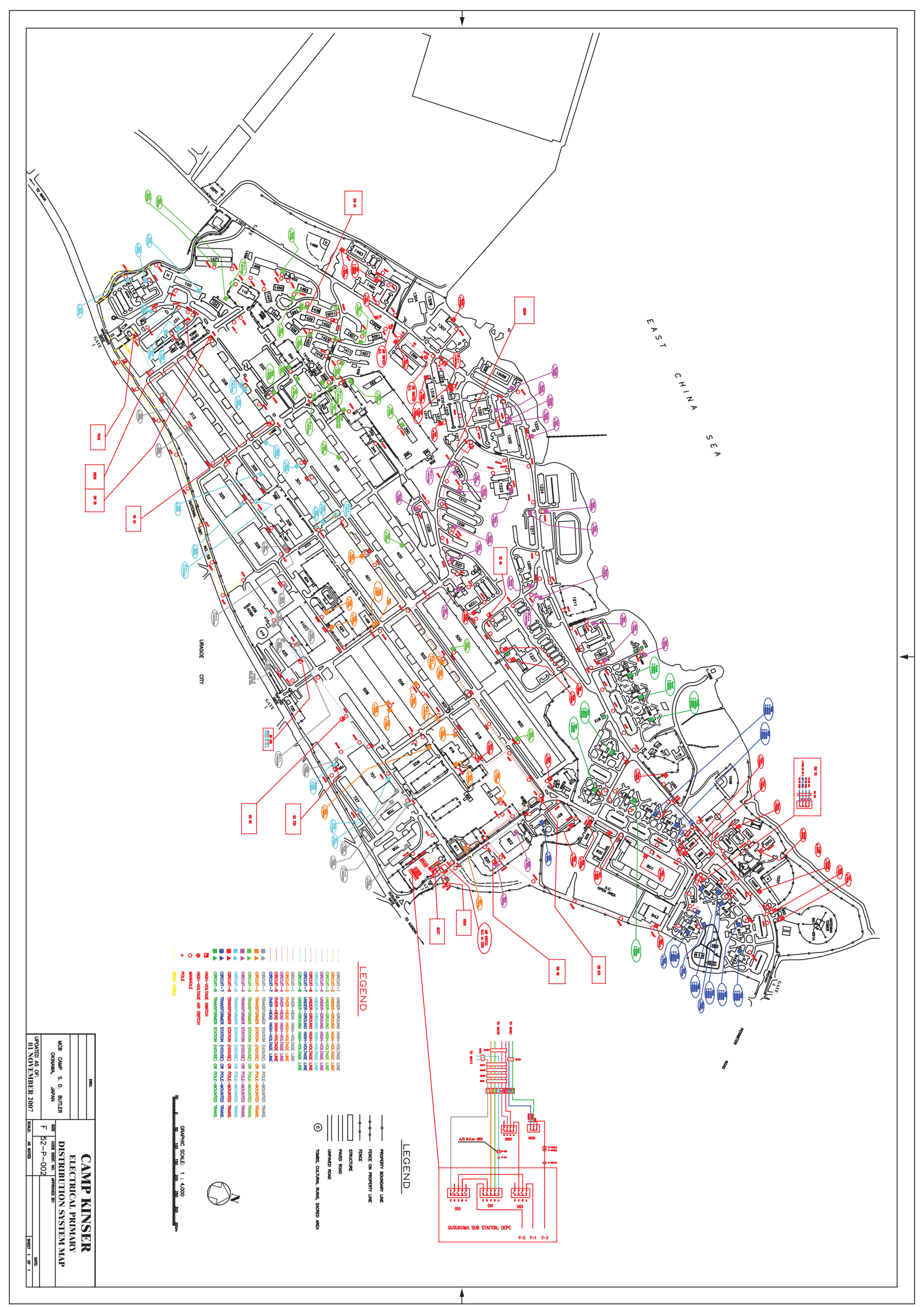



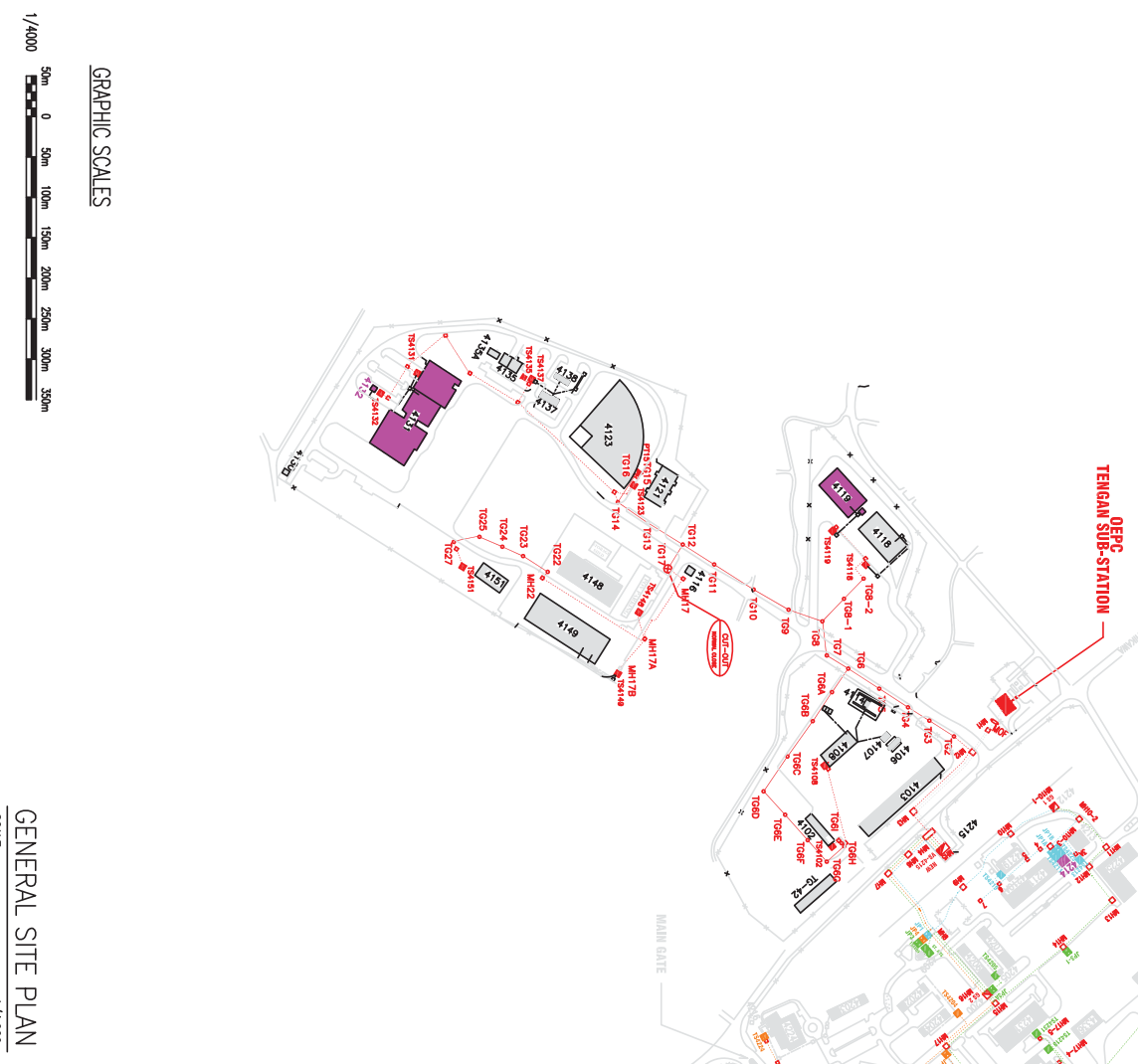

$\theta$

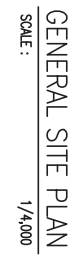

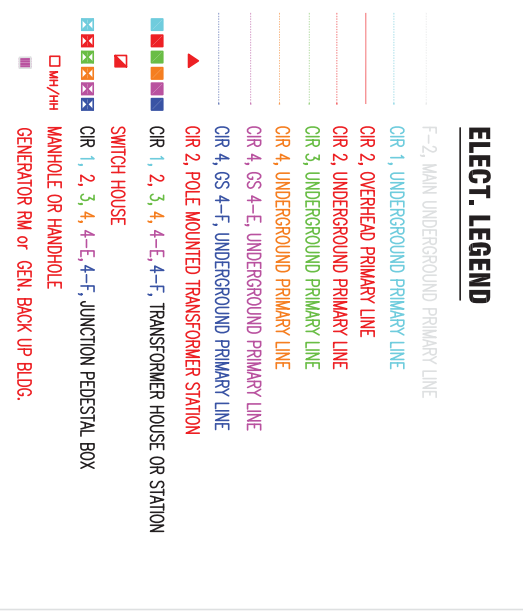




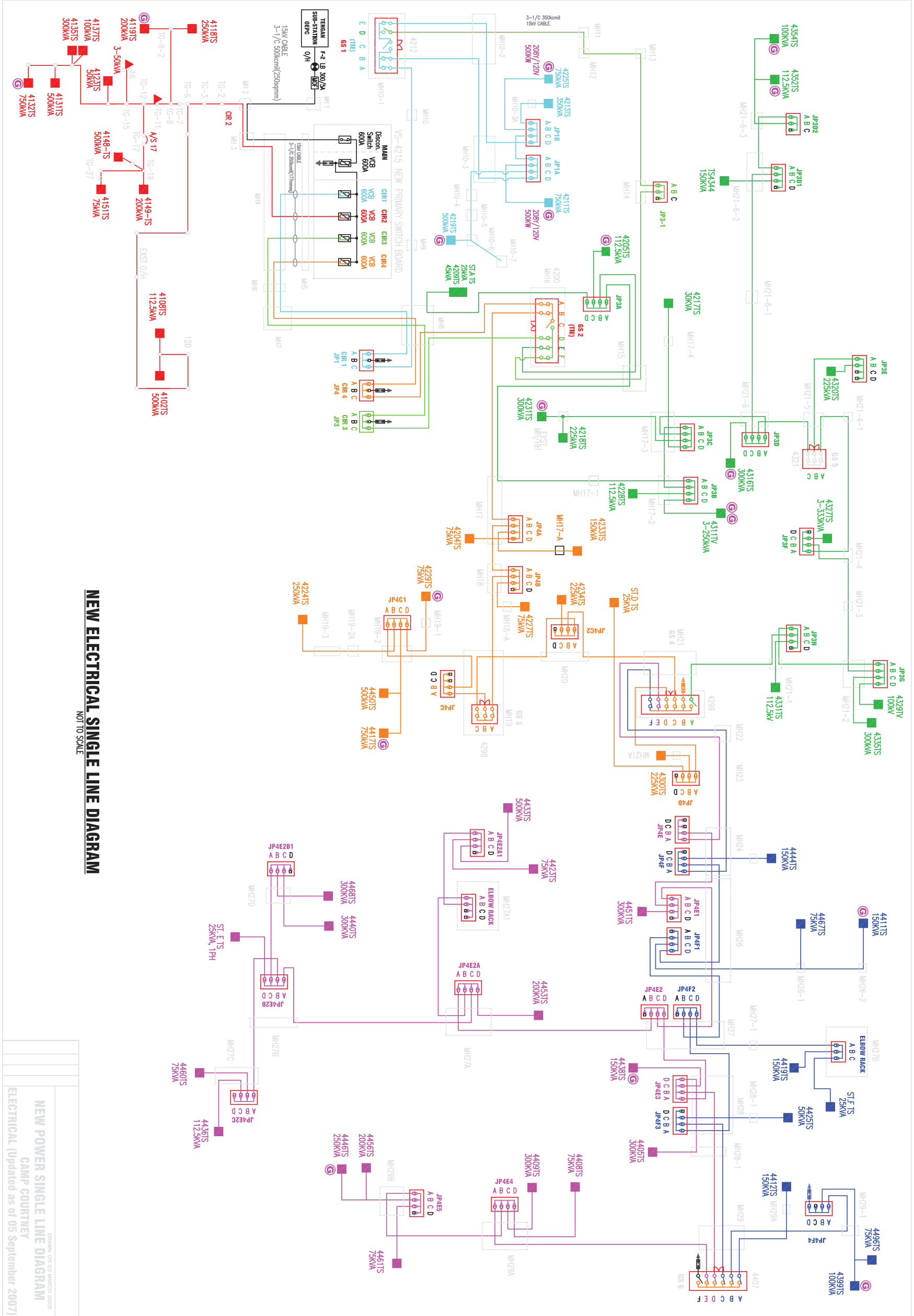




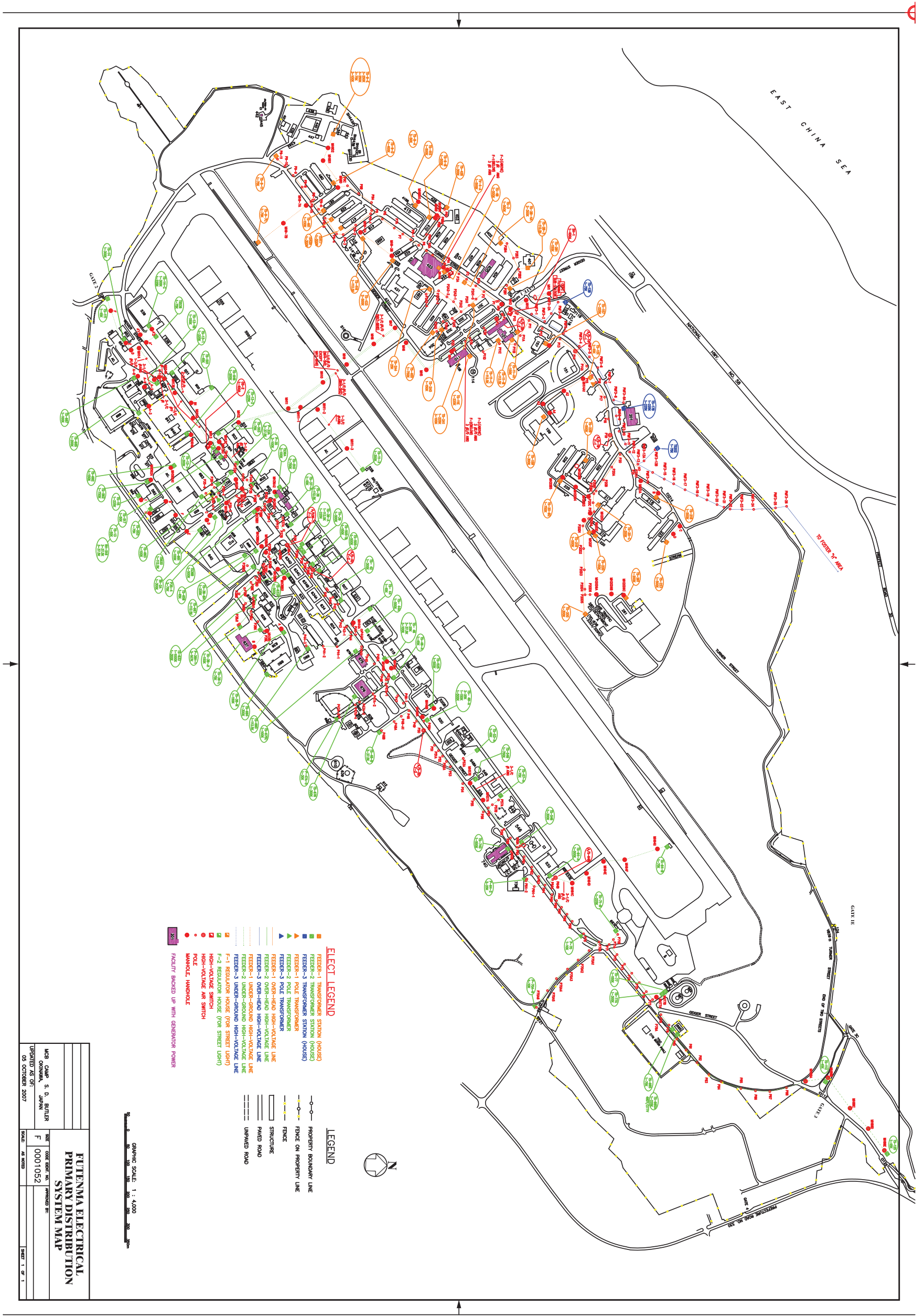




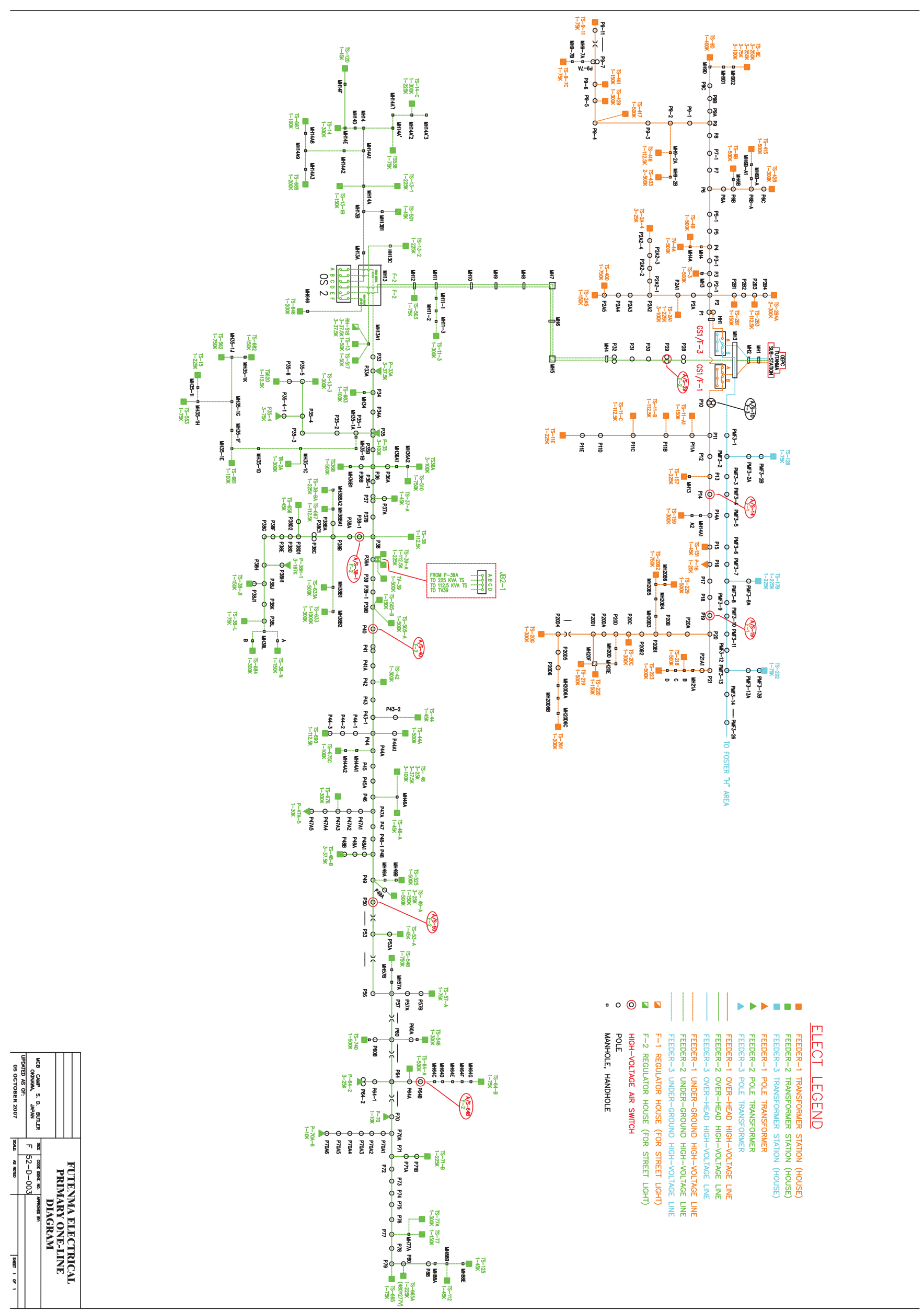




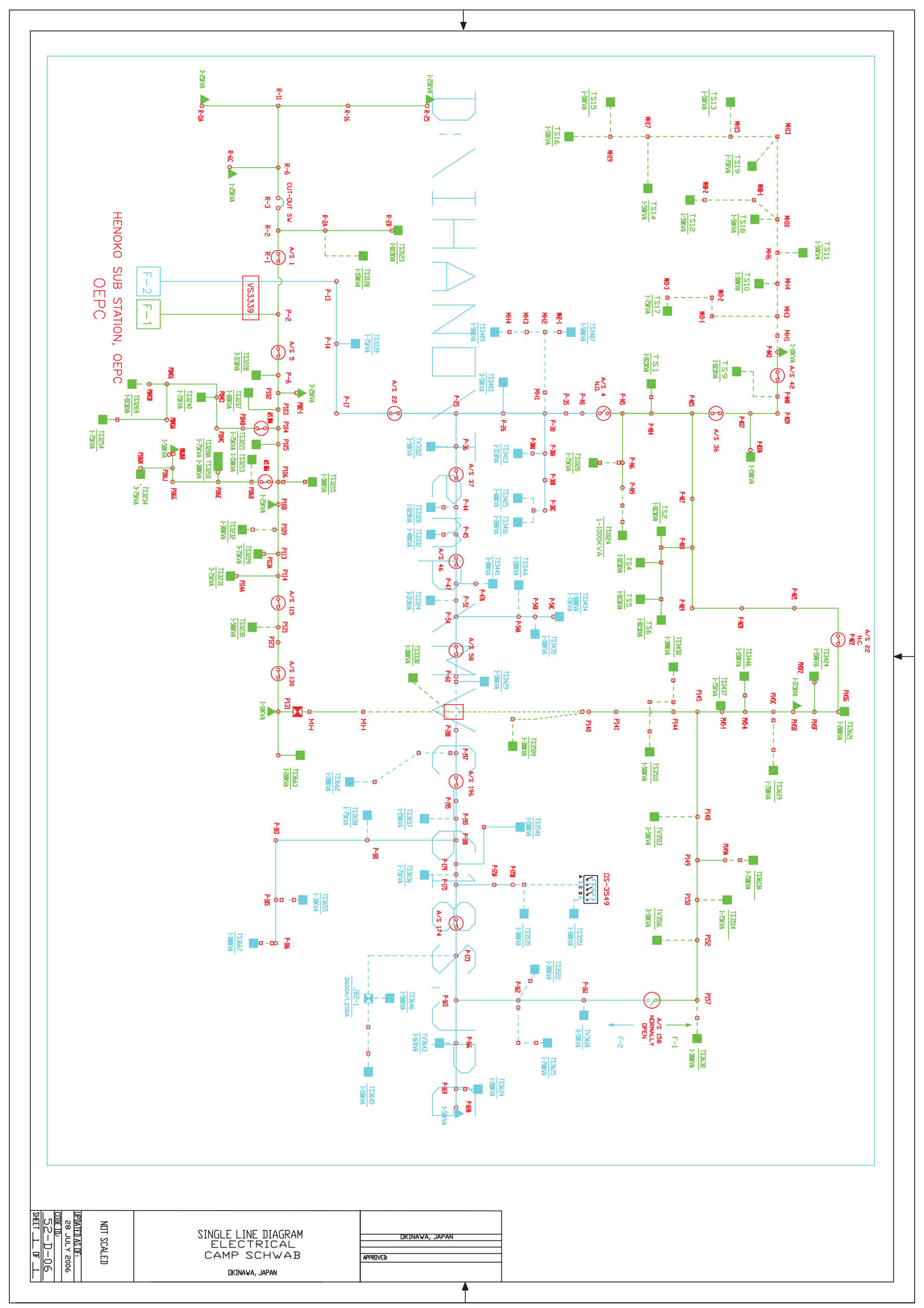




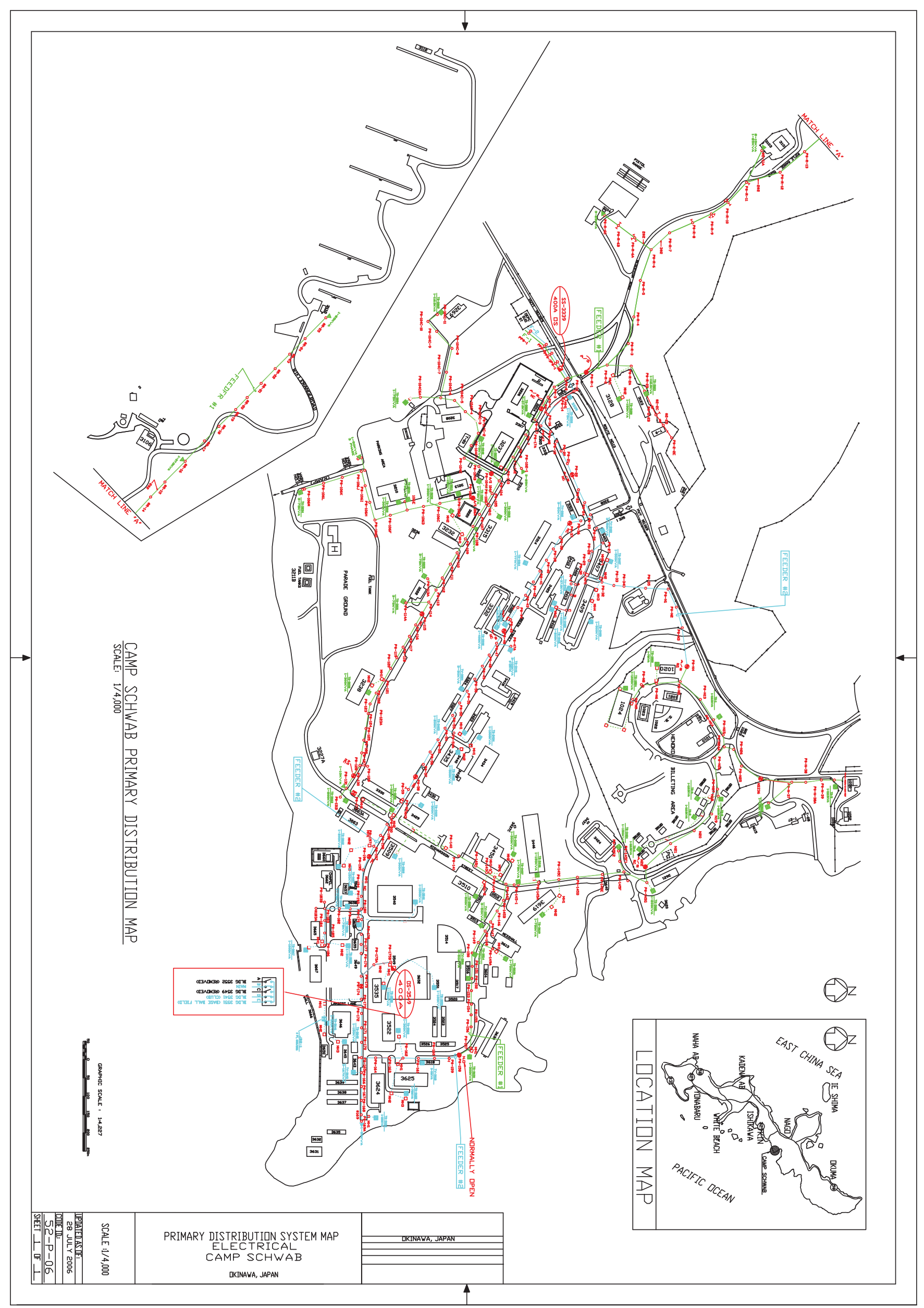




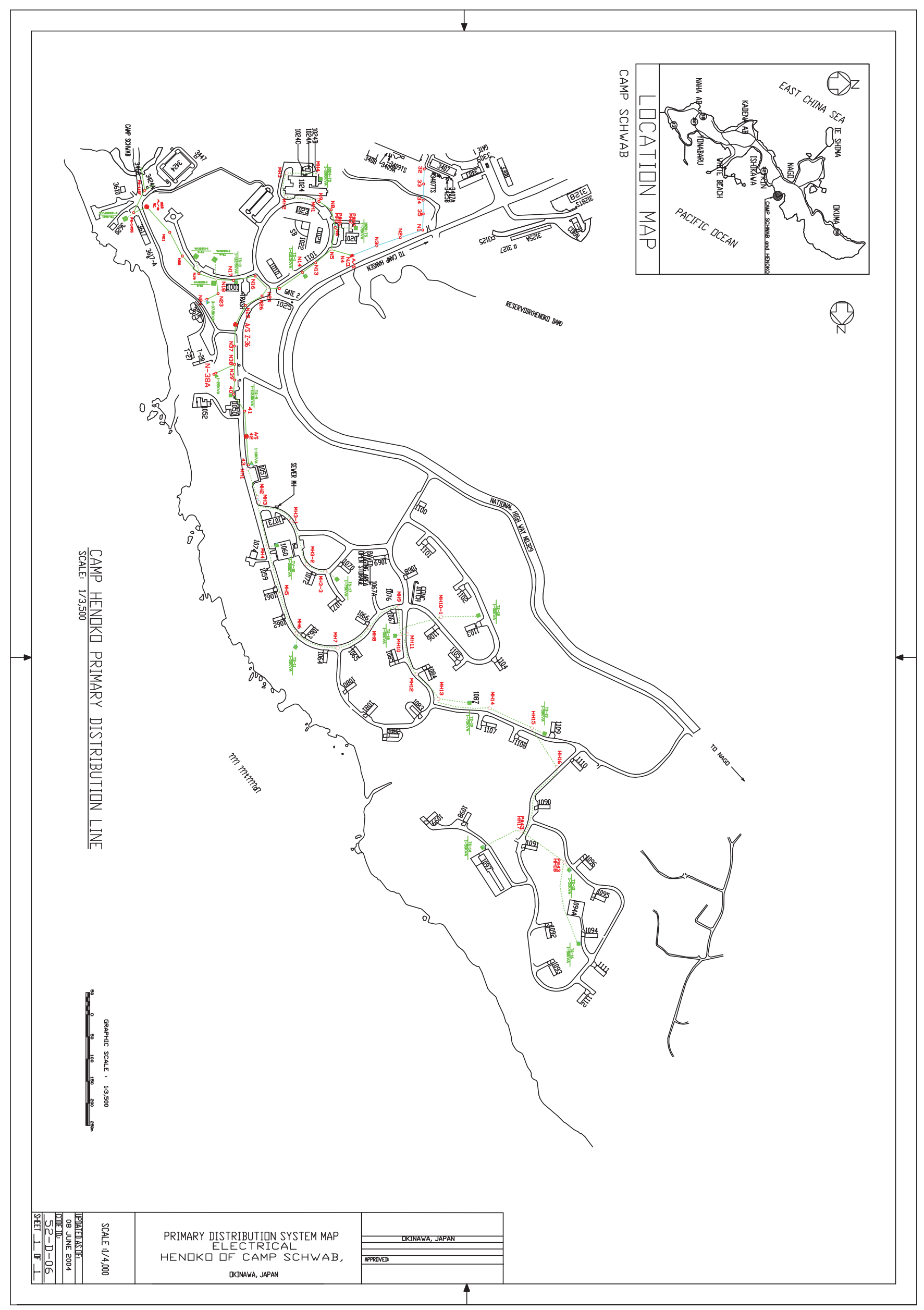




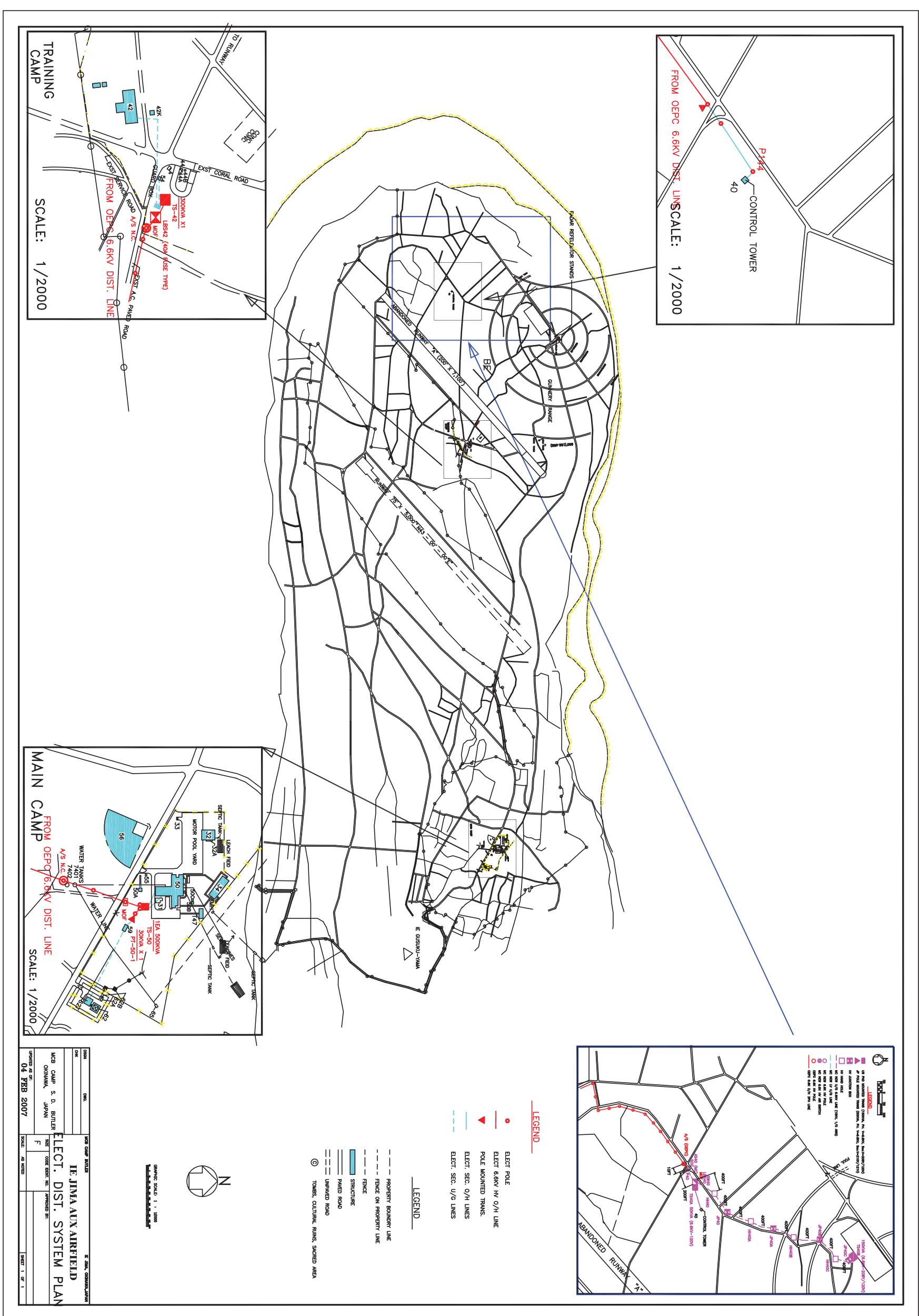




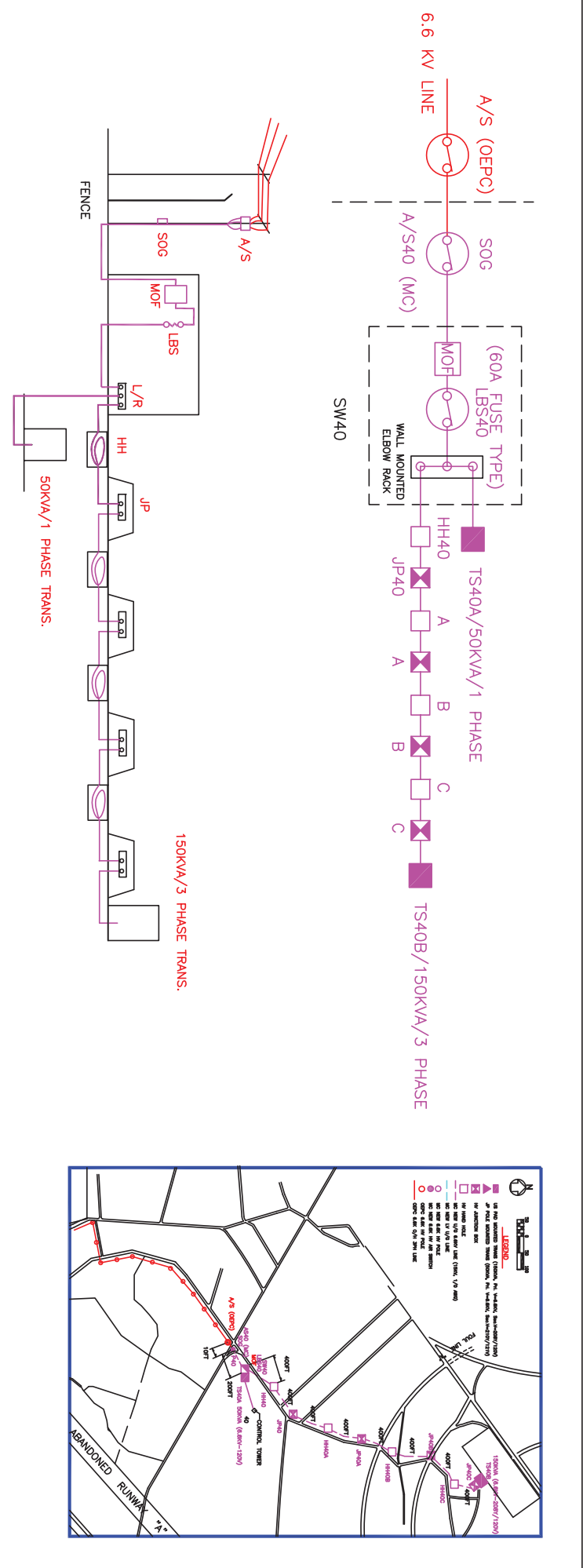




\section{Appendix C \\ U.S. Manufacturer Data}




\section{Distribution Transformer National Efficiency Standards}

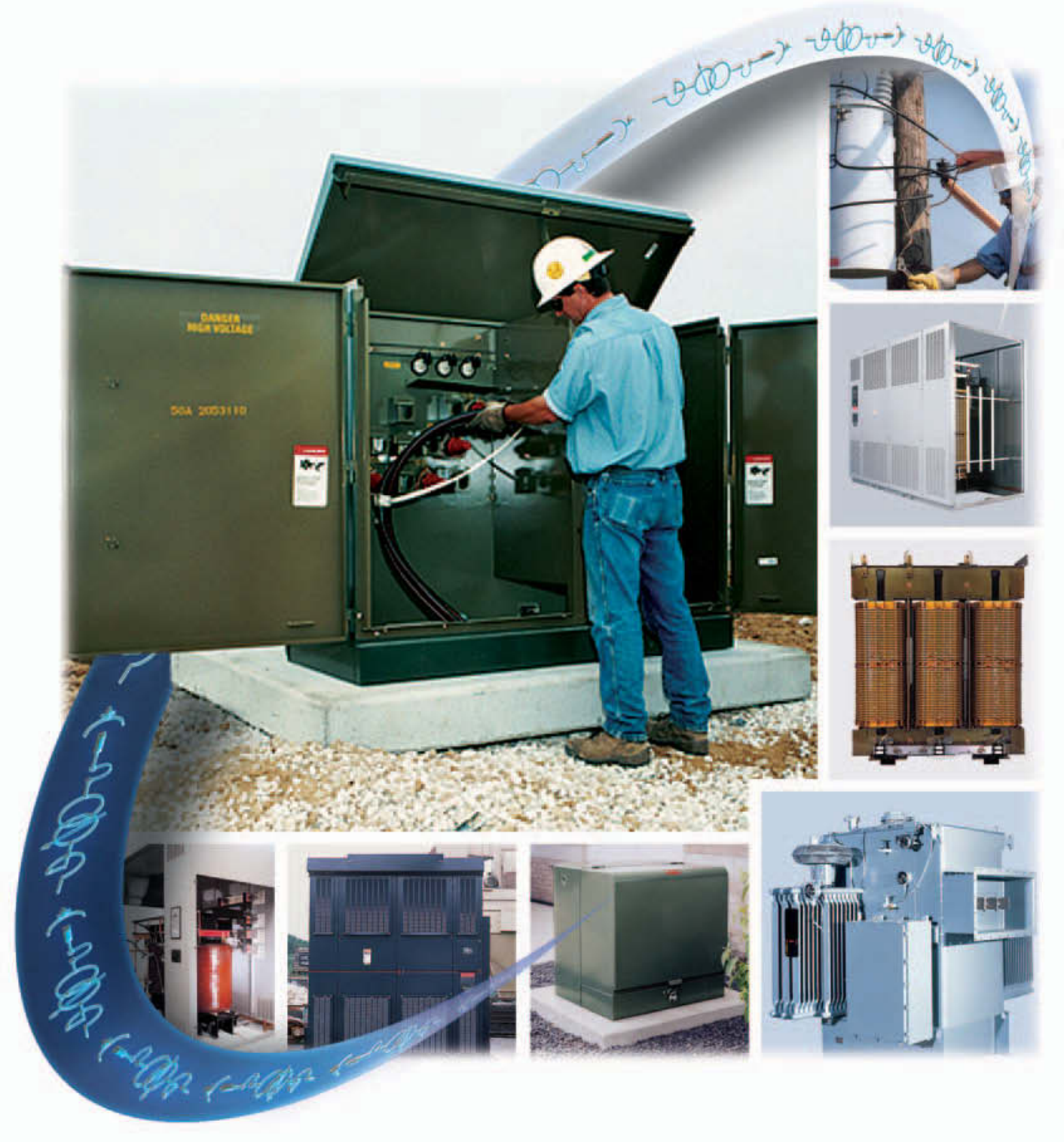




\section{An overview}

On October 12, 2007, the United States Department of Energy (DOE) issued its final ruling on distribution transformer efficiency for liquid and dry distribution transformers. The ruling impacts the following:

- Single phase - 10 to $833 \mathrm{kVA}$

- Three phase - 15 to $2500 \mathrm{kVA}$

Distribution transformers that are manufactured in or imported into the U.S. and its territories on or after January 1, 2010, will be required to comply with these new DOE standards.

\section{Benefits of the National Efficiency Standard}

\section{Saves 2.74 quads (10 $10^{15}$ BTU's) of energy over 29 years}

- Energy of 27 million US households in a single year

- Eliminating need for 6 new 400 MW power plants

\section{Reduce greenhouse gas emission of $\sim 238$ million tons of $\mathrm{CO}_{2}$}

- Equivalent to removing 80\% of all light vehicles for one year

- Others emission reductions not included in final justification

- Greater than 46 thousand tons (kt) of nitrous oxide $\left(\mathrm{NO}_{2}\right)$

- Greater than 4 tons of mercury $(\mathrm{Hg})$

Payback ranges from 1 to 15 years based on design line

- Net present value of $\$ 1.39$ billion using a $7 \%$ discount rate

- Net present value of $\$ 7.8$ billion using a 3\% discount rate

- Cumulative from 2010 to 2073 in 2006

\section{Definition of transformer efficiency}

\%Efficiency $=100 \times$ output watts $/$ input watts

Output being less than input due to losses in form of heat

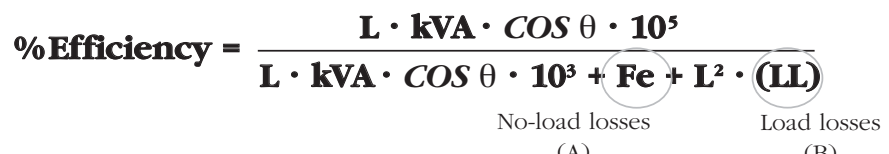

(A)

(B)

$\mathrm{Fe}=$ No-load losses $(\mathrm{A})$

$\mathrm{LL}=\mathrm{Load}$ losses $(\mathrm{B})$
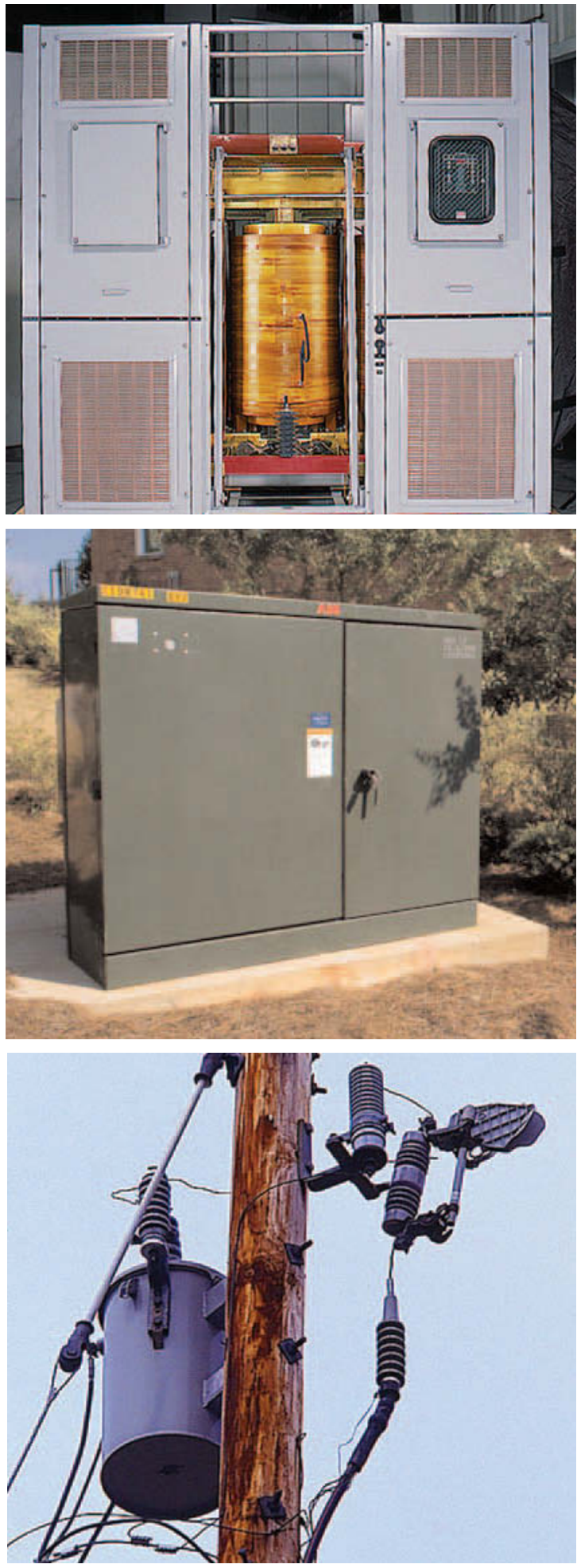


\section{Definition of transformer losses}

Total transformer losses are a combination of no-load losses and load losses.

No-load losses, or core losses, are a function of hysteresis loss from the steel, chemistry, coating and processing, and eddy loss from steel thickness.

Load losses, or conductor losses, are caused by $\mathrm{I}^{2} \mathrm{R}$ loss from the copper or aluminum material plus current density and length, and eddy loss from the design geometry and proximity to steel parts.

\section{Development of new national standards}

When the DOE first began this process, they established six levels of efficiency.

- TSL1 = NEMA TP1

- $\operatorname{TSL} 2=1 / 3$ difference between TSL1 and TSL4

- $\operatorname{TSL} 3=2 / 3$ difference between TSL1 and TSL4

- TSL4 = minimum LCC (Life Cycle Cost)

- TSL5 = maximum efficiency with no change in the LCC

- TSL6 $=$ theoretical maximum possible efficiency $($ TSL $=$ Trial Standard Level $)$

At the conclusion the following standards were adapted.

\section{National standard - liquid-filled}

\begin{tabular}{|c|c|c|c|c|c|c|c|}
\hline $\begin{array}{l}\text { Product class } 1 \\
\text { table EA.3 }\end{array}$ & \multicolumn{7}{|c|}{$\begin{array}{l}\text { Liquid-immersed medium voltage single phase transformer } \\
\text { TSL }\end{array}$} \\
\hline KVA & 1 & 2 & 3 & 4 & 5 & 6 & Standard \\
\hline 5 & & & & & & & \\
\hline 10 & $98.40 \%$ & $98.40 \%$ & $98.44 \%$ & $98.48 \%$ & $98.69 \%$ & $99.32 \%$ & $98.62 \%$ \\
\hline 15 & $98.60 \%$ & $98.56 \%$ & $98.59 \%$ & $98.63 \%$ & $98.82 \%$ & $99.39 \%$ & $98.76 \%$ \\
\hline 25 & $98.70 \%$ & $98.73 \%$ & $98.76 \%$ & $98.79 \%$ & $98.96 \%$ & $99.46 \%$ & $98.91 \%$ \\
\hline 37.5 & $98.80 \%$ & $98.85 \%$ & $98.88 \%$ & $98.91 \%$ & $99.06 \%$ & $99.51 \%$ & $99.01 \%$ \\
\hline 50 & $98.90 \%$ & $98.90 \%$ & $98.90 \%$ & $99.04 \%$ & $99.19 \%$ & $99.59 \%$ & $99.08 \%$ \\
\hline 75 & $99.00 \%$ & $99.04 \%$ & $99.06 \%$ & $99.08 \%$ & $99.21 \%$ & $99.59 \%$ & $99.17 \%$ \\
\hline 100 & $99.00 \%$ & $99.10 \%$ & $99.12 \%$ & $99.14 \%$ & $99.26 \%$ & $99.62 \%$ & $99.23 \%$ \\
\hline 167 & $99.10 \%$ & $99.21 \%$ & $99.23 \%$ & $99.25 \%$ & $99.35 \%$ & $99.66 \%$ & $99.25 \%$ \\
\hline 250 & $99.20 \%$ & $99.26 \%$ & $99.36 \%$ & $99.45 \%$ & $99.69 \%$ & $99.70 \%$ & $99.32 \%$ \\
\hline 333 & $99.20 \%$ & $99.31 \%$ & $99.40 \%$ & $99.49 \%$ & $99.71 \%$ & $99.72 \%$ & $99.36 \%$ \\
\hline 500 & $99.30 \%$ & $99.38 \%$ & $99.46 \%$ & $99.54 \%$ & $99.74 \%$ & $99.75 \%$ & $99.42 \%$ \\
\hline 667 & $99.40 \%$ & $99.42 \%$ & $99.50 \%$ & $99.57 \%$ & $99.76 \%$ & $99.77 \%$ & $99.46 \%$ \\
\hline 833 & $99.40 \%$ & $99.45 \%$ & $99.52 \%$ & $99.60 \%$ & $99.77 \%$ & $99.78 \%$ & $99.49 \%$ \\
\hline
\end{tabular}

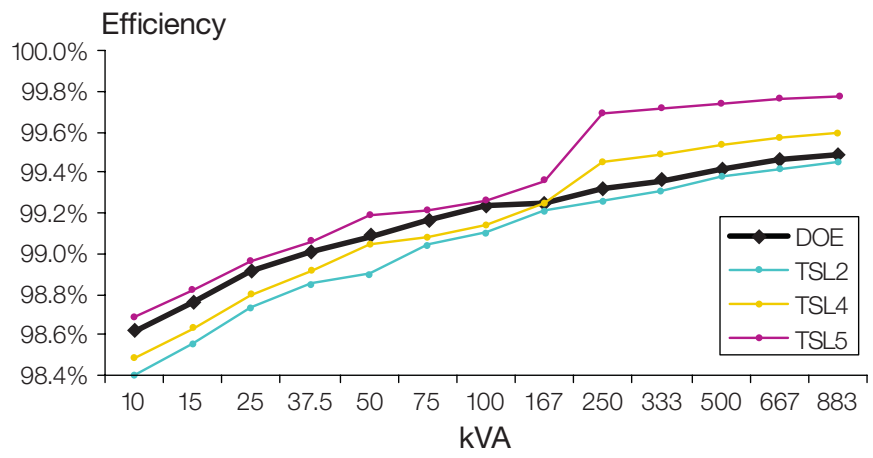

\begin{tabular}{|c|c|c|c|c|c|c|c|}
\hline \multirow{2}{*}{$\begin{array}{r}\begin{array}{l}\text { Product class } 2 \\
\text { table EA.4 } \\
\text { KVA }\end{array} \\
\end{array}$} & \multicolumn{7}{|c|}{$\begin{array}{l}\text { Liquid-immersed medium voltage three phase transformer } \\
\text { TSL }\end{array}$} \\
\hline & 1 & 2 & 3 & 4 & 5 & 6 & Standard \\
\hline 15 & $98.10 \%$ & $98.36 \%$ & $98.68 \%$ & $98.68 \%$ & $99.25 \%$ & $99.31 \%$ & $98.36 \%$ \\
\hline 30 & $98.40 \%$ & $98.62 \%$ & $98.89 \%$ & $98.89 \%$ & $99.37 \%$ & $99.42 \%$ & $98.62 \%$ \\
\hline$\overline{45}$ & $98.60 \%$ & $98.76 \%$ & $99.00 \%$ & $99.00 \%$ & $99.43 \%$ & $99.47 \%$ & $98.76 \%$ \\
\hline 75 & $98.70 \%$ & $98.91 \%$ & $99.12 \%$ & $99.12 \%$ & $99.50 \%$ & $99.54 \%$ & $98.91 \%$ \\
\hline 112.5 & $98.80 \%$ & $99.01 \%$ & $99.20 \%$ & $99.20 \%$ & $99.55 \%$ & $99.58 \%$ & $99.01 \%$ \\
\hline 150 & $98.90 \%$ & $99.08 \%$ & $99.26 \%$ & $99.26 \%$ & $99.58 \%$ & $99.61 \%$ & $99.08 \%$ \\
\hline 225 & $99.00 \%$ & $99.17 \%$ & $99.33 \%$ & $99.33 \%$ & $99.62 \%$ & $99.65 \%$ & $99.17 \%$ \\
\hline 300 & $99.00 \%$ & $99.23 \%$ & $99.38 \%$ & $99.38 \%$ & $99.65 \%$ & $99.67 \%$ & $99.23 \%$ \\
\hline 500 & $99.10 \%$ & $99.32 \%$ & $99.45 \%$ & $99.45 \%$ & $99.69 \%$ & $99.71 \%$ & $99.25 \%$ \\
\hline 750 & $99.20 \%$ & $99.24 \%$ & $99.31 \%$ & $99.37 \%$ & $99.66 \%$ & $99.66 \%$ & $99.32 \%$ \\
\hline 1000 & $99.20 \%$ & $99.29 \%$ & $99.36 \%$ & $99.41 \%$ & $99.68 \%$ & $99.68 \%$ & $99.36 \%$ \\
\hline 1500 & $99.30 \%$ & $99.36 \%$ & $99.42 \%$ & $99.47 \%$ & $99.71 \%$ & $99.71 \%$ & $99.42 \%$ \\
\hline 2000 & $99.40 \%$ & $99.40 \%$ & $99.46 \%$ & $99.51 \%$ & $99.73 \%$ & $99.73 \%$ & $99.46 \%$ \\
\hline 2500 & $99.40 \%$ & $99.44 \%$ & $99.49 \%$ & $99.53 \%$ & $99.74 \%$ & $99.74 \%$ & $99.49 \%$ \\
\hline
\end{tabular}

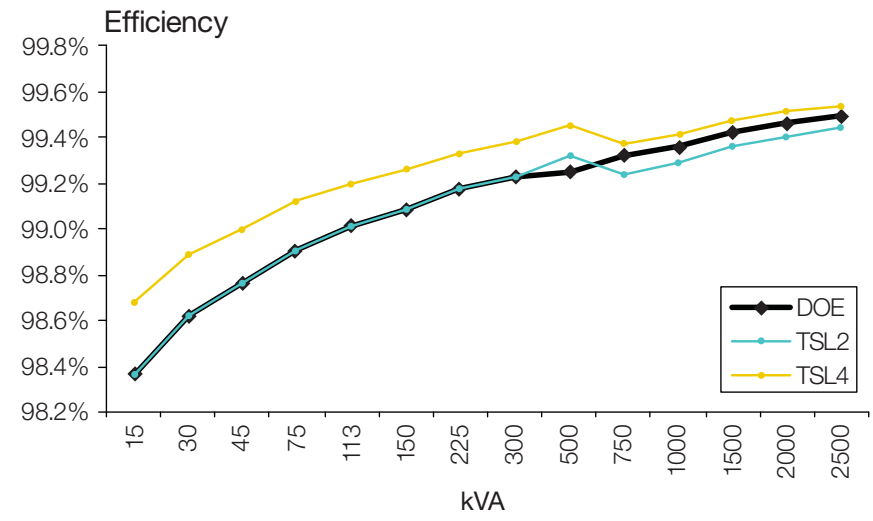

Shaded columns indicate Trial Standard Levels that make up the standard. 


\begin{tabular}{|c|c|c|c|c|c|c|c|c|c|c|}
\hline \multicolumn{2}{|c|}{$\begin{array}{c}\text { Product class } 7 \\
\text { table EA.7 }\end{array}$} & \multicolumn{9}{|c|}{$\begin{array}{l}\text { Dry-type medium voltage single phase transformer (46-96 kV BIL) } \\
\text { TSL }\end{array}$} \\
\hline & KVA & $\overline{0-A v g}$ & 0-Min & 1 & 2 & 3 & 4 & 5 & 6 & Standarc \\
\hline & 15 & $97.46 \%$ & $96.87 \%$ & $97.60 \%$ & $97.86 \%$ & $98.14 \%$ & $98.41 \%$ & $98.54 \%$ & $98.54 \%$ & $97.86 \%$ \\
\hline & 25 & $97.77 \%$ & $97.24 \%$ & $97.90 \%$ & $98.12 \%$ & $98.36 \%$ & $98.60 \%$ & $98.71 \%$ & $98.71 \%$ & $98.12 \%$ \\
\hline & 37.5 & $97.98 \%$ & $97.51 \%$ & $98.10 \%$ & $98.30 \%$ & $98.52 \%$ & $98.73 \%$ & $98.84 \%$ & $98.84 \%$ & $98.30 \%$ \\
\hline & 50 & $98.12 \%$ & $97.68 \%$ & $98.20 \%$ & $98.42 \%$ & $98.62 \%$ & $98.82 \%$ & $98.92 \%$ & $98.92 \%$ & $98.42 \%$ \\
\hline & 75 & $98.30 \%$ & $97.90 \%$ & $98.40 \%$ & $98.57 \%$ & $98.75 \%$ & $98.94 \%$ & $99.02 \%$ & $99.02 \%$ & $98.57 \%$ \\
\hline \multirow[t]{4}{*}{ DL11 } & 100 & $98.42 \%$ & $98.05 \%$ & $98.05 \%$ & $98.67 \%$ & $98.84 \%$ & $99.01 \%$ & $99.09 \%$ & $99.09 \%$ & $98.67 \%$ \\
\hline & 167 & $98.61 \%$ & $98.28 \%$ & $98.80 \%$ & $98.83 \%$ & $98.98 \%$ & $99.13 \%$ & $99.20 \%$ & $99.20 \%$ & $98.83 \%$ \\
\hline & 250 & $99.02 \%$ & $98.58 \%$ & $98.90 \%$ & $98.95 \%$ & $99.08 \%$ & $99.23 \%$ & $99.42 \%$ & $99.42 \%$ & $98.95 \%$ \\
\hline & 333 & $99.09 \%$ & $98.68 \%$ & $99.00 \%$ & $99.03 \%$ & $99.15 \%$ & $99.28 \%$ & $99.46 \%$ & $99.46 \%$ & $99.03 \%$ \\
\hline \multirow[t]{3}{*}{ DL12 } & 500 & $99.18 \%$ & $98.81 \%$ & $99.10 \%$ & $99.12 \%$ & $99.23 \%$ & $99.35 \%$ & $99.51 \%$ & $99.51 \%$ & $99.12 \%$ \\
\hline & 667 & $99.24 \%$ & $98.89 \%$ & $99.20 \%$ & $99.18 \%$ & $99.28 \%$ & $99.40 \%$ & $99.54 \%$ & $99.54 \%$ & $99.18 \%$ \\
\hline & 833 & $99.28 \%$ & $98.95 \%$ & $99.20 \%$ & $99.23 \%$ & $99.43 \%$ & $99.57 \%$ & $99.57 \%$ & $99.57 \%$ & $99.23 \%$ \\
\hline
\end{tabular}

\begin{tabular}{|c|c|c|c|c|c|c|c|c|c|c|}
\hline \multicolumn{2}{|c|}{$\begin{array}{l}\text { Product class } 8 \\
\text { table EA. } 8\end{array}$} & \multicolumn{9}{|c|}{$\begin{array}{l}\text { Dry-type medium voltage three phase transformer (46-96 kV BIL) } \\
\text { TSL }\end{array}$} \\
\hline & & $0-A V g$ & 0-Min & 1 & 2 & 3 & 4 & . & 6 & Standard \\
\hline & 15 & $96.66 \%$ & $95.88 \%$ & $96.80 \%$ & $97.19 \%$ & $97.55 \%$ & $97.91 \%$ & $98.08 \%$ & $98.08 \%$ & $97.18 \%$ \\
\hline & 30 & $97.19 \%$ & $96.53 \%$ & $97.30 \%$ & $97.63 \%$ & $97.94 \%$ & $98.24 \%$ & $98.38 \%$ & $98.38 \%$ & $97.63 \%$ \\
\hline & 45 & $97.46 \%$ & $96.87 \%$ & $97.60 \%$ & $97.86 \%$ & $98.14 \%$ & $98.41 \%$ & $98.54 \%$ & $98.54 \%$ & $97.86 \%$ \\
\hline & 75 & $97.77 \%$ & $97.24 \%$ & $97.90 \%$ & $98.12 \%$ & $98.36 \%$ & $98.60 \%$ & $98.71 \%$ & $98.71 \%$ & $98.12 \%$ \\
\hline & 112.5 & $97.98 \%$ & $97.51 \%$ & $98.10 \%$ & $98.30 \%$ & $98.52 \%$ & $98.73 \%$ & $98.84 \%$ & $98.84 \%$ & $98.30 \%$ \\
\hline & 150 & $98.12 \%$ & $97.68 \%$ & $98.20 \%$ & $98.42 \%$ & $98.62 \%$ & $98.82 \%$ & $98.92 \%$ & $98.92 \%$ & $98.42 \%$ \\
\hline & 225 & $98.30 \%$ & $98.90 \%$ & $98.40 \%$ & $98.57 \%$ & $98.75 \%$ & $98.94 \%$ & $99.02 \%$ & $99.02 \%$ & $98.57 \%$ \\
\hline \multirow[t]{4}{*}{ DL11 } & 300 & $98.42 \%$ & $98.05 \%$ & $98.50 \%$ & $98.67 \%$ & $99.84 \%$ & $99.01 \%$ & $99.09 \%$ & $99.09 \%$ & $98.67 \%$ \\
\hline & 500 & $98.61 \%$ & $98.28 \%$ & $98.80 \%$ & $98.83 \%$ & $98.98 \%$ & $99.13 \%$ & $99.20 \%$ & $99.20 \%$ & $98.83 \%$ \\
\hline & 750 & $99.02 \%$ & $98.58 \%$ & $98.90 \%$ & $98.95 \%$ & $99.08 \%$ & $99.23 \%$ & $99.42 \%$ & $99.42 \%$ & $98.95 \%$ \\
\hline & 1000 & $99.09 \%$ & $98.68 \%$ & $99.00 \%$ & $99.03 \%$ & $99.15 \%$ & $99.28 \%$ & $99.46 \%$ & $99.46 \%$ & $99.03 \%$ \\
\hline \multirow[t]{3}{*}{$\underline{\text { DL12 }}$} & 1500 & $99.18 \%$ & $98.81 \%$ & $99.00 \%$ & $99.12 \%$ & $99.23 \%$ & $99.35 \%$ & $99.51 \%$ & $99.51 \%$ & $99.12 \%$ \\
\hline & 2000 & $99.24 \%$ & $98.89 \%$ & $99.20 \%$ & $99.18 \%$ & $99.28 \%$ & $99.40 \%$ & $99.54 \%$ & $99.54 \%$ & $99.18 \%$ \\
\hline & 2500 & $99.28 \%$ & $98.95 \%$ & $99.20 \%$ & $99.23 \%$ & $99.32 \%$ & $99.43 \%$ & $99.57 \%$ & $99.57 \%$ & $99.23 \%$ \\
\hline
\end{tabular}
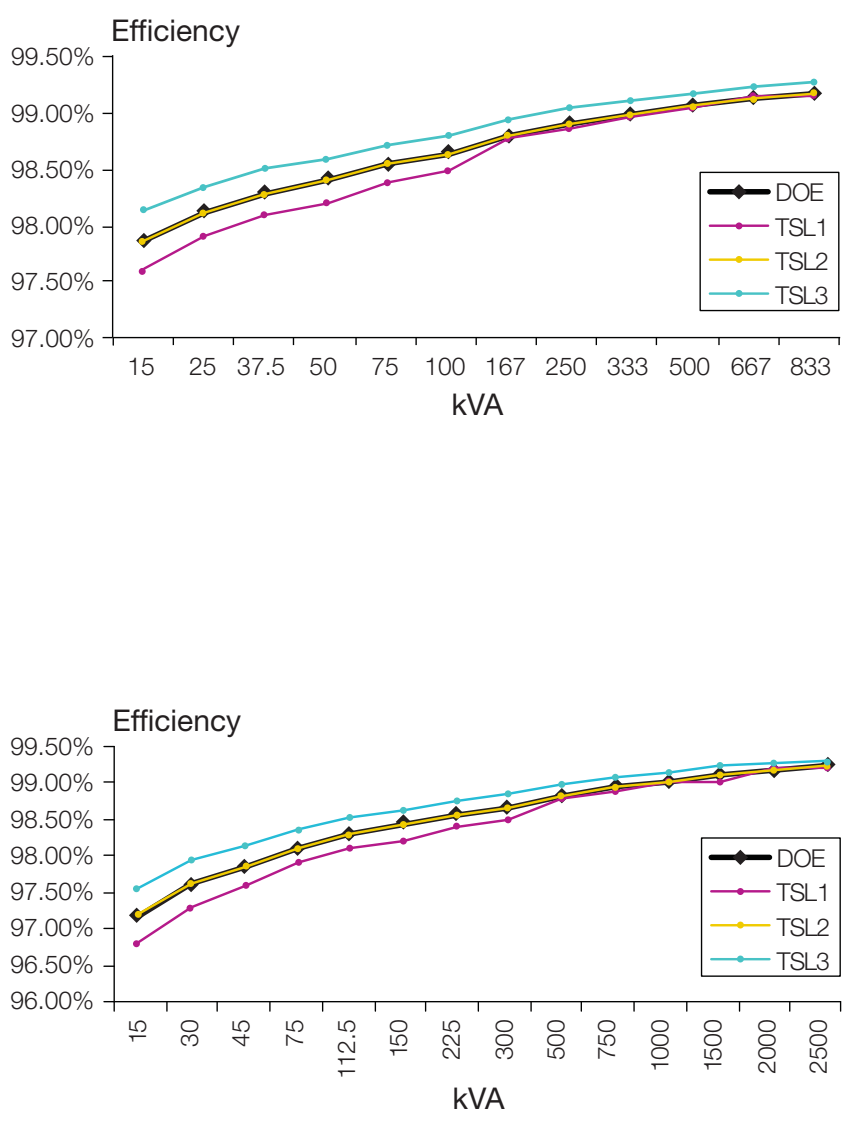

Shaded columns indicate Trial Standard Levels that make up the standard.

\section{Impact of the new efficiency standards}

The new efficiency standards will increase the conductor cross section thus increasing copper and aluminum consumption. In addition, weights and dimensions will increase in most cases.

Due to these increases, transportation costs will rise as there will be fewer units per truckload. And since the wider and deeper tanks will not be offset by a reduction in tank height, the average oil volume per unit will increase as well. In some cases, higher efficiency will lead to lower losses, less heating and a reduction or elimination of radiators.

Overall, transformers will get bigger and heavier due to an increase in the total amount of material used. Also, there will be more need for higher grade and higher cost electrical steel. We project a 16 percent increase in tonnage of electrical steel, 16 percent increase in copper and 19 percent increase in aluminum.

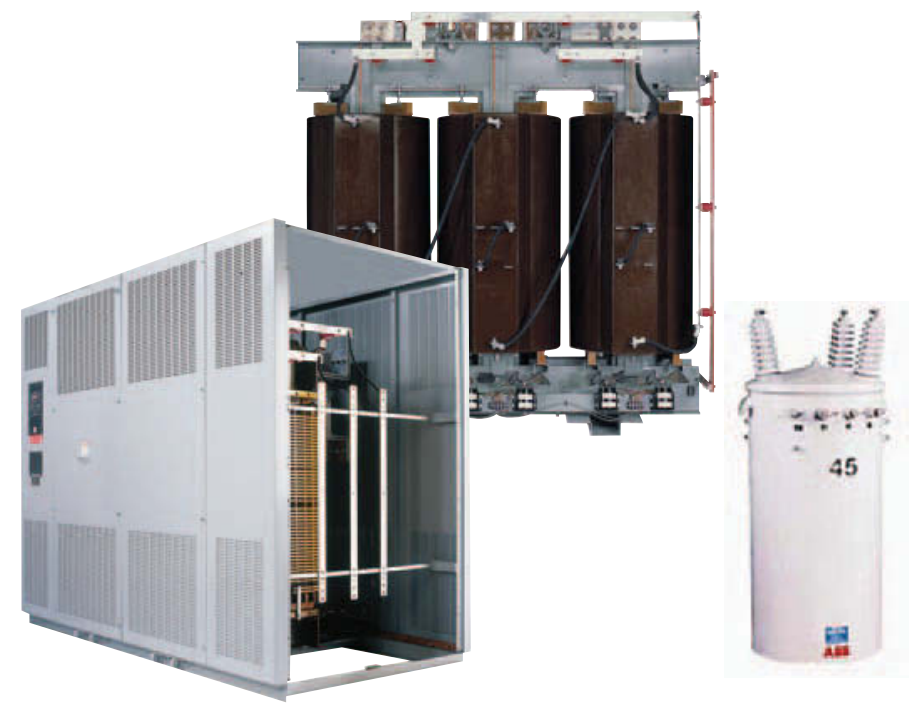




\section{ABB is ideally positioned to help you through this process}

At $\mathrm{ABB}$, we have taken these additional material requirements into consideration with our suppliers to make sure we have the necessary materials to meet the DOE standards.

Currently, in our present design base, the following ABB transformers meet or exceed the standards:

- $50 \%$ of liquid single phase

- $40 \%$ of liquid three phase

- $25 \%$ of dry-type

\section{ABB will assure compliance}

According to the regulations, the manufacturer (ABB) will determine the efficiency of a basic model whether by testing or by an Alternative Efficiency Determination Method (AEDM).

The basic model is defined as the transformer design with the same energy consumption along with electrical features including kVA, BIL, voltage and taps. The load is calculated at 50 percent, the power factor is equal to 1 (one). The no-load loss is calculated at $20^{\circ} \mathrm{C}$ and load losses are calculated at $55^{\circ} \mathrm{C}$ for liquid-filled units and $75^{\circ} \mathrm{C}$ for dry-type transformers. Auxiliary devices, such as circuit breakers, fuses and switches, are excluded from the calculation of efficiency.

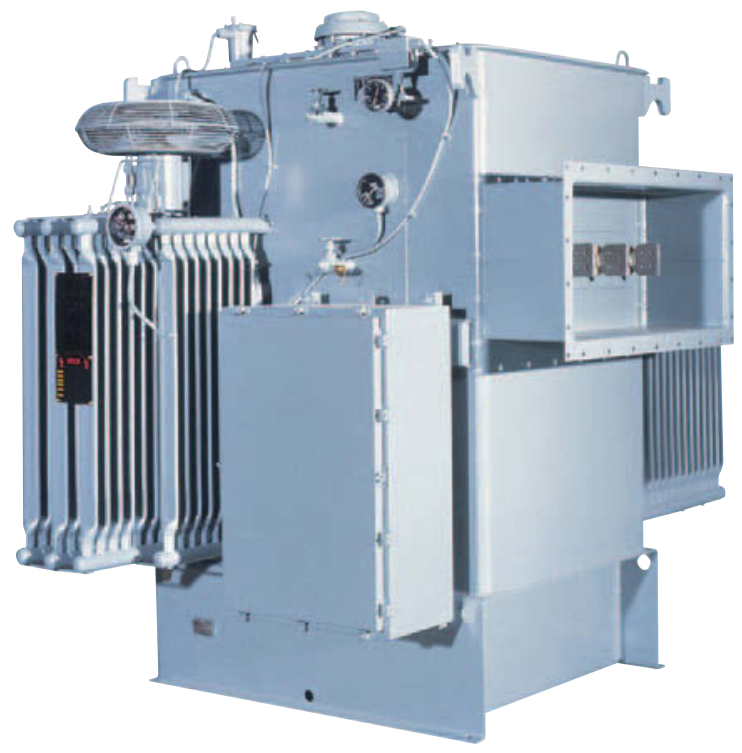

\section{The ABB response to the efficiency standards}

All of us at ABB support this move to higher efficiency standards, since this has been part of our strategy as a sustainable corporation from the beginning. We understand the impact on you, in terms of cost, and we are committed to using our design technology and material supply management expertise to minimize any negative factors for our customers.

We are confident that we will be able to meet the needs of our customers in 2010 and for many years thereafter. In short, $\mathrm{ABB}$ will be able to provide you with standardscompliant distribution transformers when and where you need them.

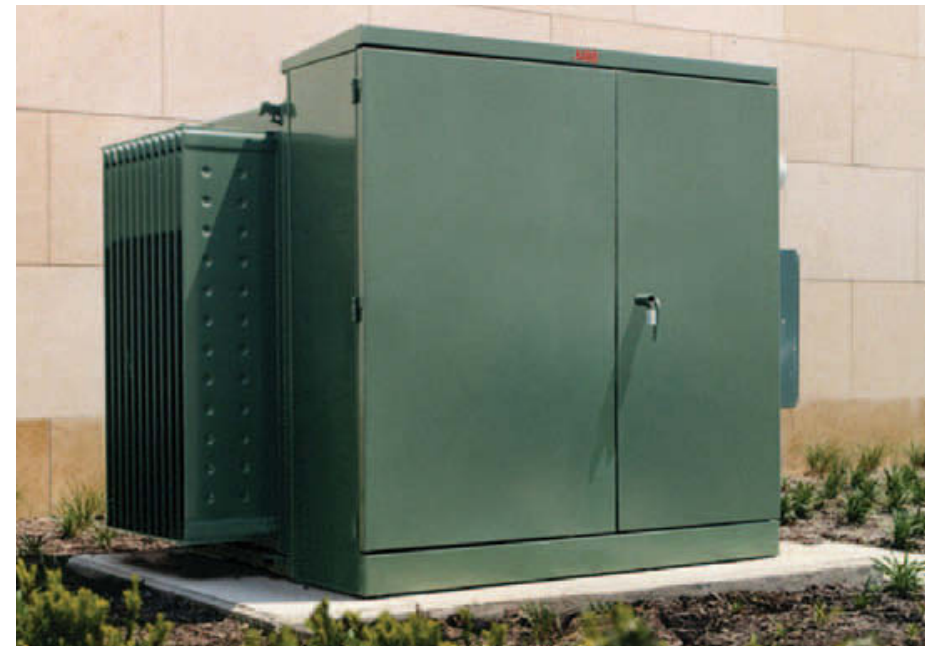




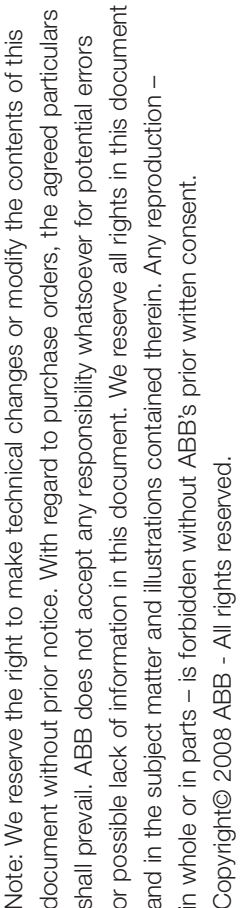

ABB Inc.

Distribution Transformers 500 West Highway 94 Jefferson City, MO 65101 USA

Phone +1 5736342111

Fax +1 5736596275 www.abb.com/transformers
ABB Inc.

Small Power Transformers 2135 Philpott Road South Boston, VA 24592 USA

Phone +1 4345757971

Fax +1 4345752208
ABB Inc.

Dry and Cast Technologies 171 Industry Drive

P. O. Box 38

Bland, VA 24315

USA

Phone +1 2766883325

Fax +12766884588 


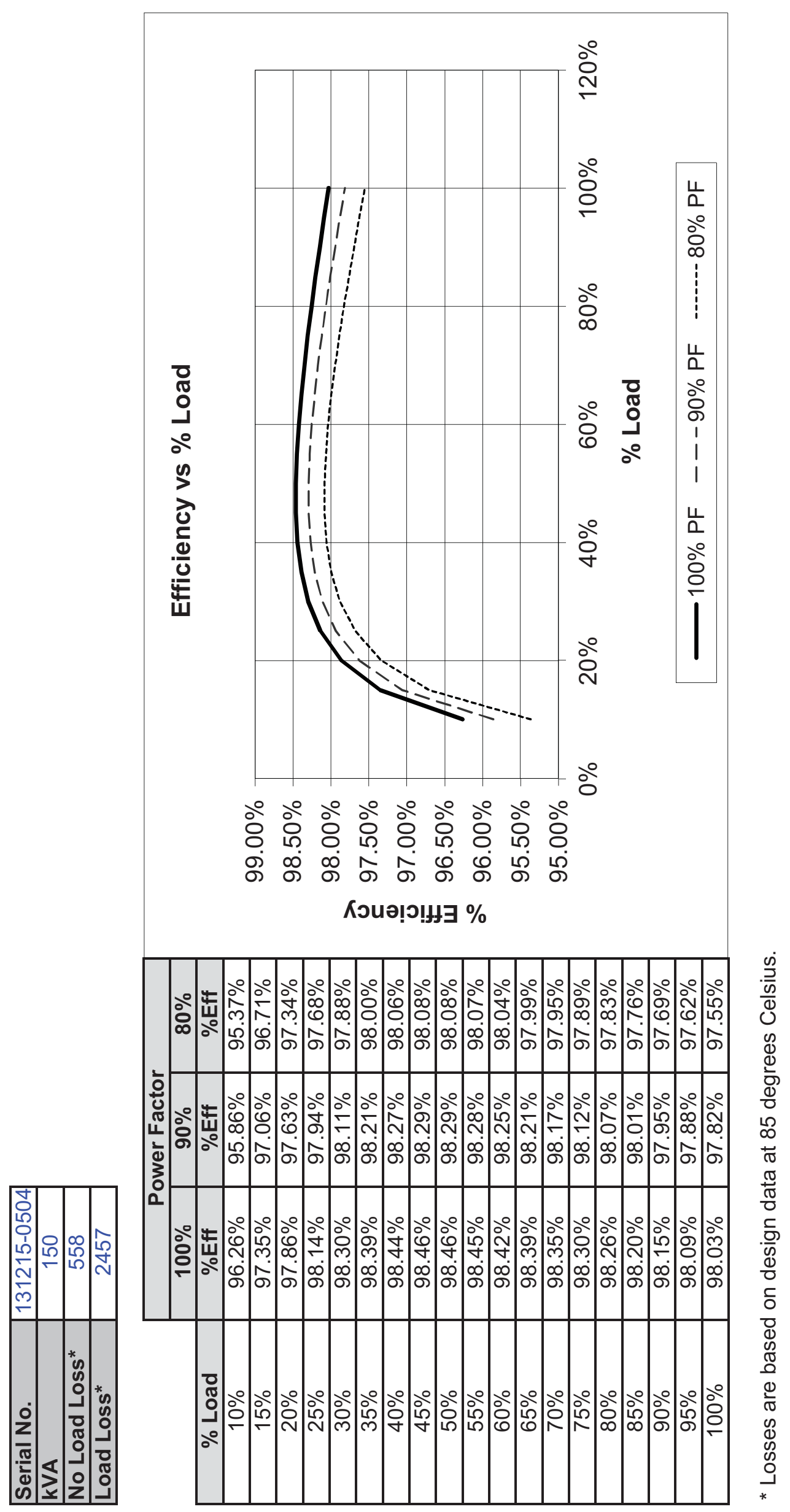

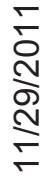

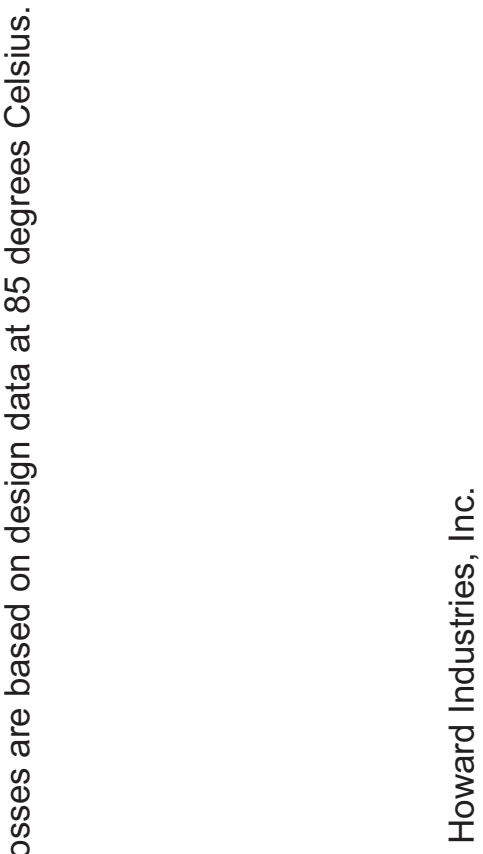




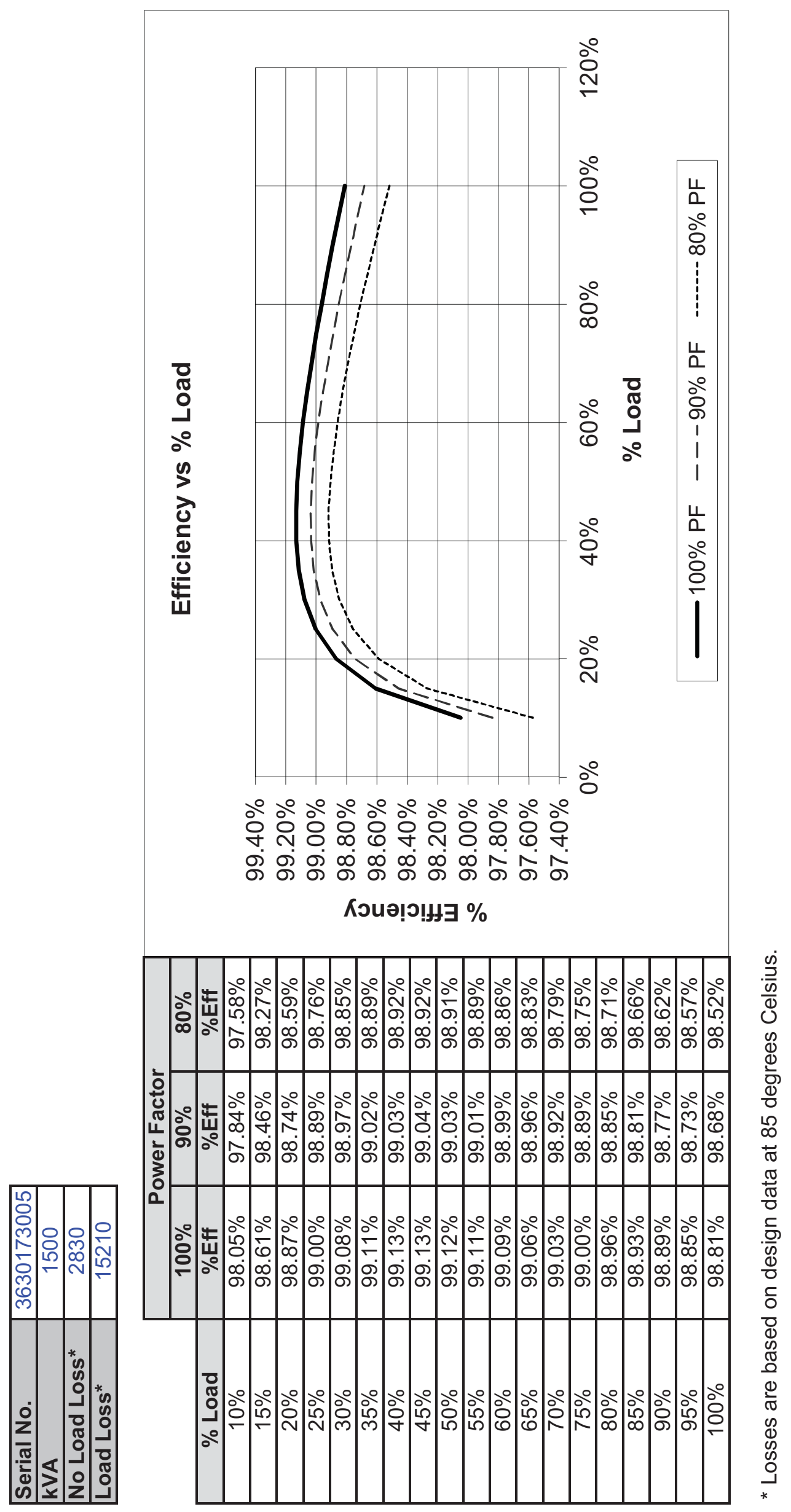

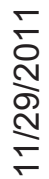

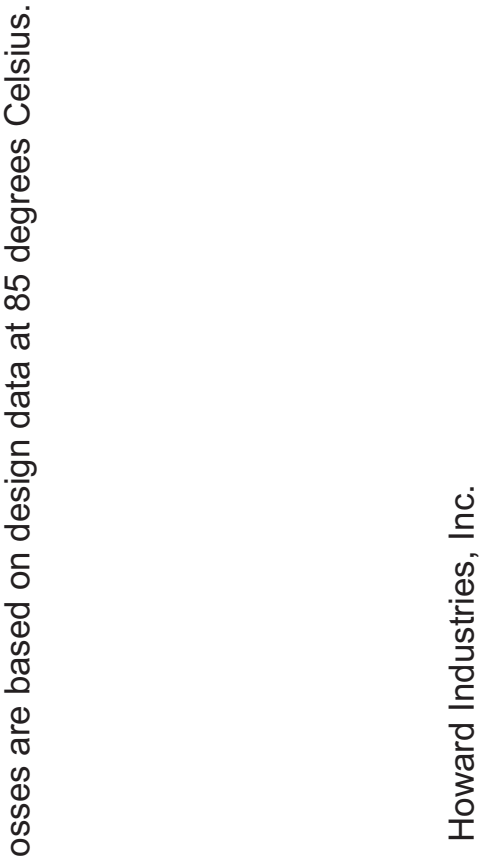




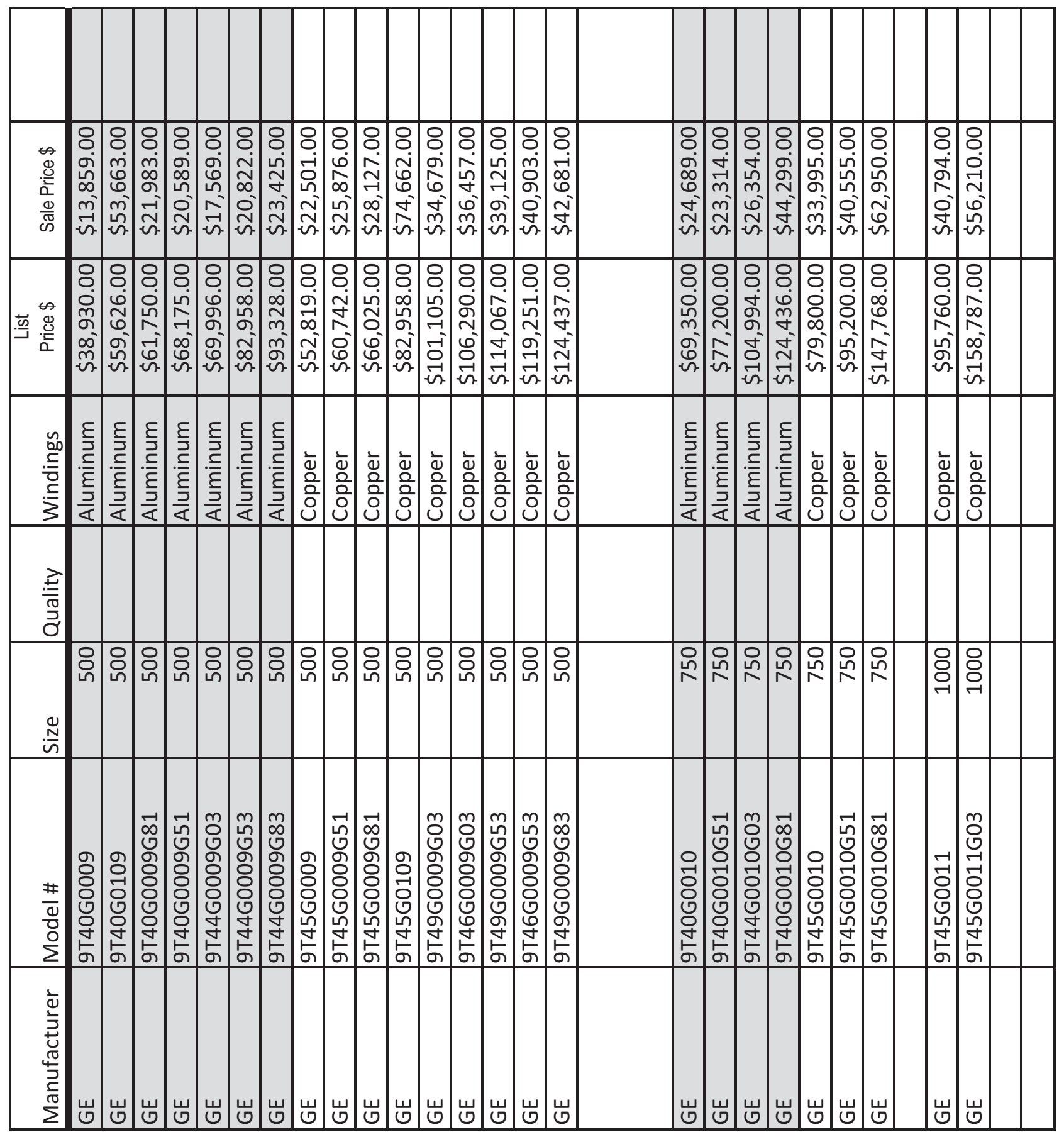




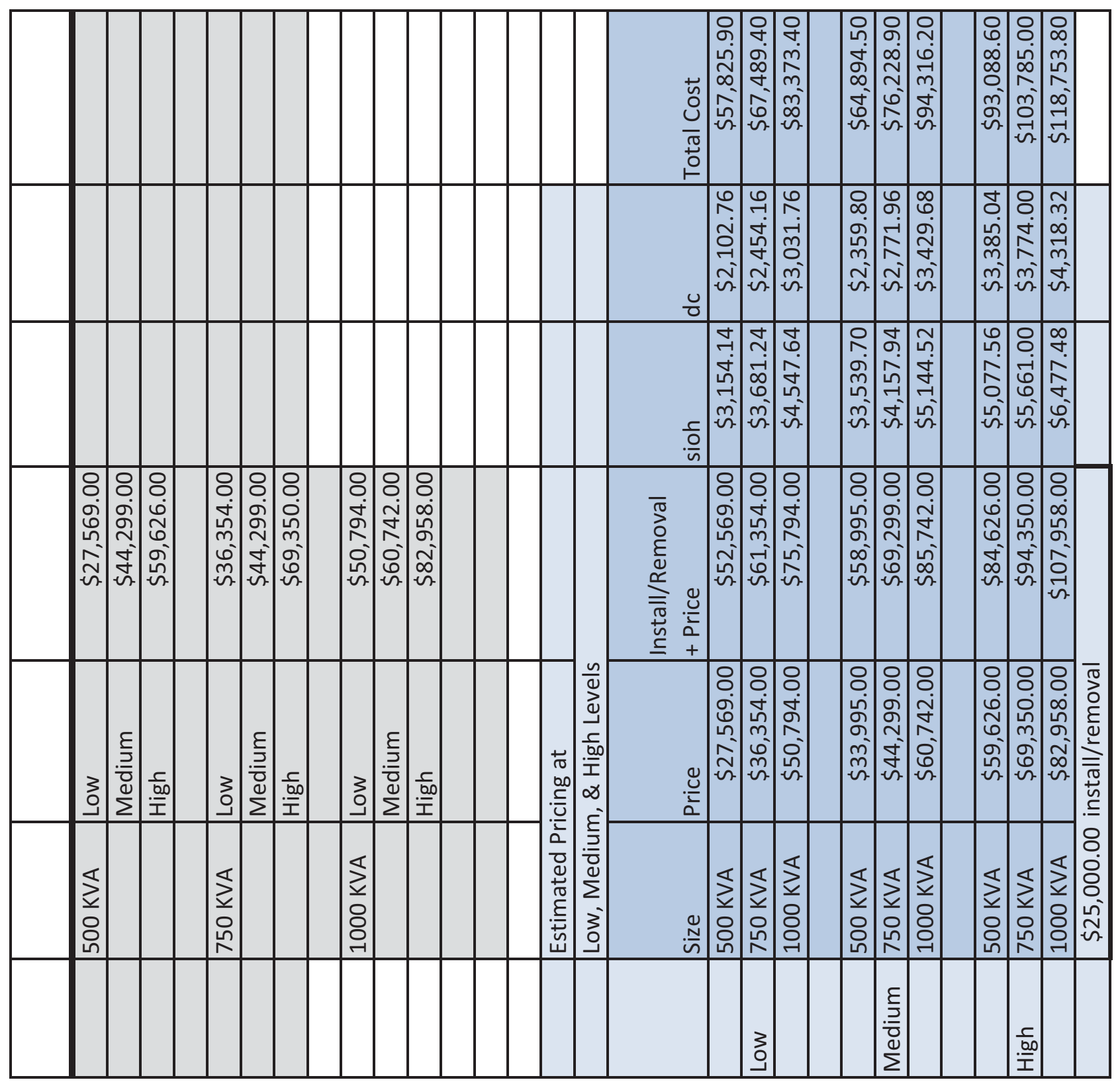




\begin{tabular}{|c|c|c|c|c|c|}
\hline $\begin{array}{l}\text { 150kva } \\
\text { Load \% }\end{array}$ & $\begin{array}{l}\text { PF } 0.80 \\
\text { Eff } \%\end{array}$ & $\begin{array}{l}\text { PF } 0.85 \\
\text { Eff \% }\end{array}$ & $\begin{array}{l}\text { PF } 0.90 \\
\text { Eff \% }\end{array}$ & $\begin{array}{l}\text { PF } 1.0 \\
\text { Eff } \%\end{array}$ & \\
\hline $10 \%$ & 95.37 & 95.615 & 95.86 & 96.26 & \\
\hline $15 \%$ & 96.71 & 96.885 & 97.06 & 97.35 & \\
\hline $20 \%$ & 97.34 & 97.485 & 97.63 & 97.86 & \\
\hline $25 \%$ & 97.68 & 97.81 & 97.94 & 98.14 & \\
\hline $30 \%$ & 97.88 & 97.995 & 98.11 & 98.3 & \\
\hline $35 \%$ & 98 & 98.105 & 98.21 & 98.39 & \\
\hline $40 \%$ & 98.06 & 98.165 & 98.27 & 98.44 & \\
\hline $45 \%$ & 98.08 & 98.185 & 98.29 & 98.46 & \\
\hline $50 \%$ & 98.08 & 98.185 & 98.29 & 98.46 & \\
\hline $55 \%$ & 98.07 & 98.175 & 98.28 & 98.45 & \\
\hline $60 \%$ & 98.04 & 98.145 & 98.25 & 98.42 & \\
\hline $65 \%$ & 97.99 & 98.1 & 98.21 & 98.39 & \\
\hline $70 \%$ & 97.95 & 98.06 & 98.17 & 98.35 & \\
\hline $75 \%$ & 97.89 & 98.005 & 98.12 & 98.3 & \\
\hline $80 \%$ & 97.83 & 97.95 & 98.07 & 98.26 & \\
\hline $85 \%$ & 97.76 & 97.885 & 98.01 & 98.2 & \\
\hline $90 \%$ & 97.69 & 97.82 & 97.95 & 98.15 & \\
\hline $95 \%$ & 97.62 & 97.75 & 97.88 & 98.09 & \\
\hline $100 \%$ & 97.55 & 97.685 & 97.82 & 98.03 & \\
\hline
\end{tabular}

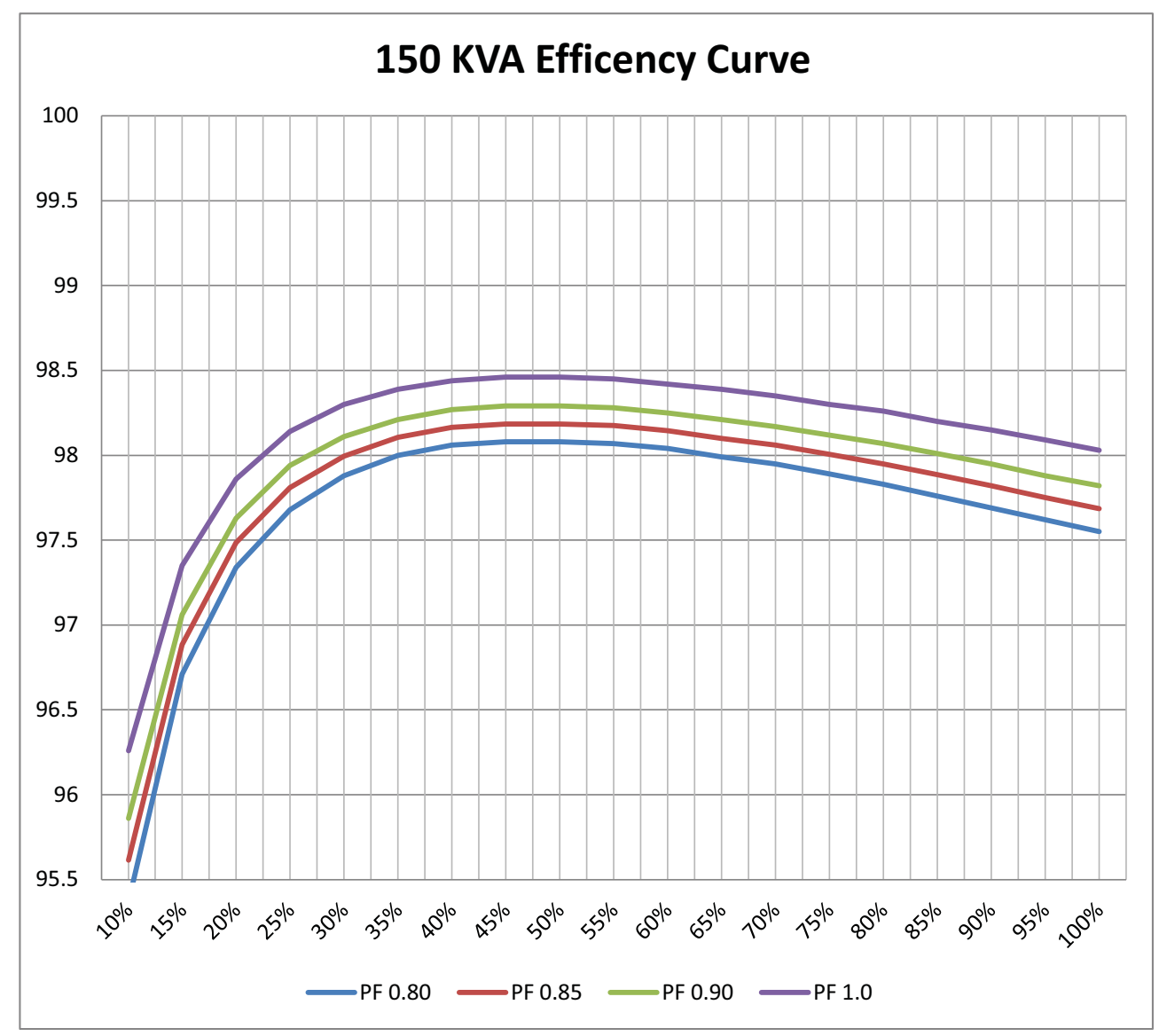




\begin{tabular}{|c|c|c|c|c|c|}
\hline $500 \mathrm{KVA}$ & PF 0.80 & PF 0.85 & PF 0.90 & PF 1.0 & \\
\hline Load \% & Eff \% & Eff \% & Eff \% & Eff \% & \\
\hline $10 \%$ & 95.94296 & 96.15815 & 96.37333 & 96.72407 & \\
\hline $15 \%$ & 97.11444 & 97.2687 & 97.42296 & 97.67667 & \\
\hline $20 \%$ & 97.66407 & 97.79093 & 97.91778 & 98.12185 & \\
\hline $25 \%$ & 97.96 & 98.07315 & 98.1863 & 98.36296 & \\
\hline $30 \%$ & 98.13148 & 98.23222 & 98.33296 & 98.50222 & \\
\hline $35 \%$ & 98.23074 & 98.32537 & 98.42 & 98.57667 & \\
\hline $40 \%$ & 98.28296 & 98.375 & 98.46704 & 98.61889 & \\
\hline $45 \%$ & 98.29778 & 98.39111 & 98.48444 & 98.6337 & \\
\hline $50 \%$ & 98.29519 & 98.38852 & 98.48185 & 98.63111 & \\
\hline $55 \%$ & 98.28259 & 98.37593 & 98.46926 & 98.62111 & \\
\hline $60 \%$ & 98.25259 & 98.34722 & 98.44185 & 98.5937 & \\
\hline $65 \%$ & 98.20778 & 98.30611 & 98.40444 & 98.5637 & \\
\hline $70 \%$ & 98.16778 & 98.26611 & 98.36444 & 98.5263 & \\
\hline $75 \%$ & 98.11296 & 98.2163 & 98.31963 & 98.48148 & \\
\hline $80 \%$ & 98.05815 & 98.16519 & 98.27222 & 98.44148 & \\
\hline $85 \%$ & 97.99333 & 98.10537 & 98.21741 & 98.38926 & \\
\hline $90 \%$ & 97.93111 & 98.04685 & 98.16259 & 98.34185 & \\
\hline $95 \%$ & 97.8663 & 97.98333 & 98.10037 & 98.28704 & \\
\hline $100 \%$ & 97.80148 & 97.92222 & 98.04296 & 98.23222 & \\
\hline
\end{tabular}

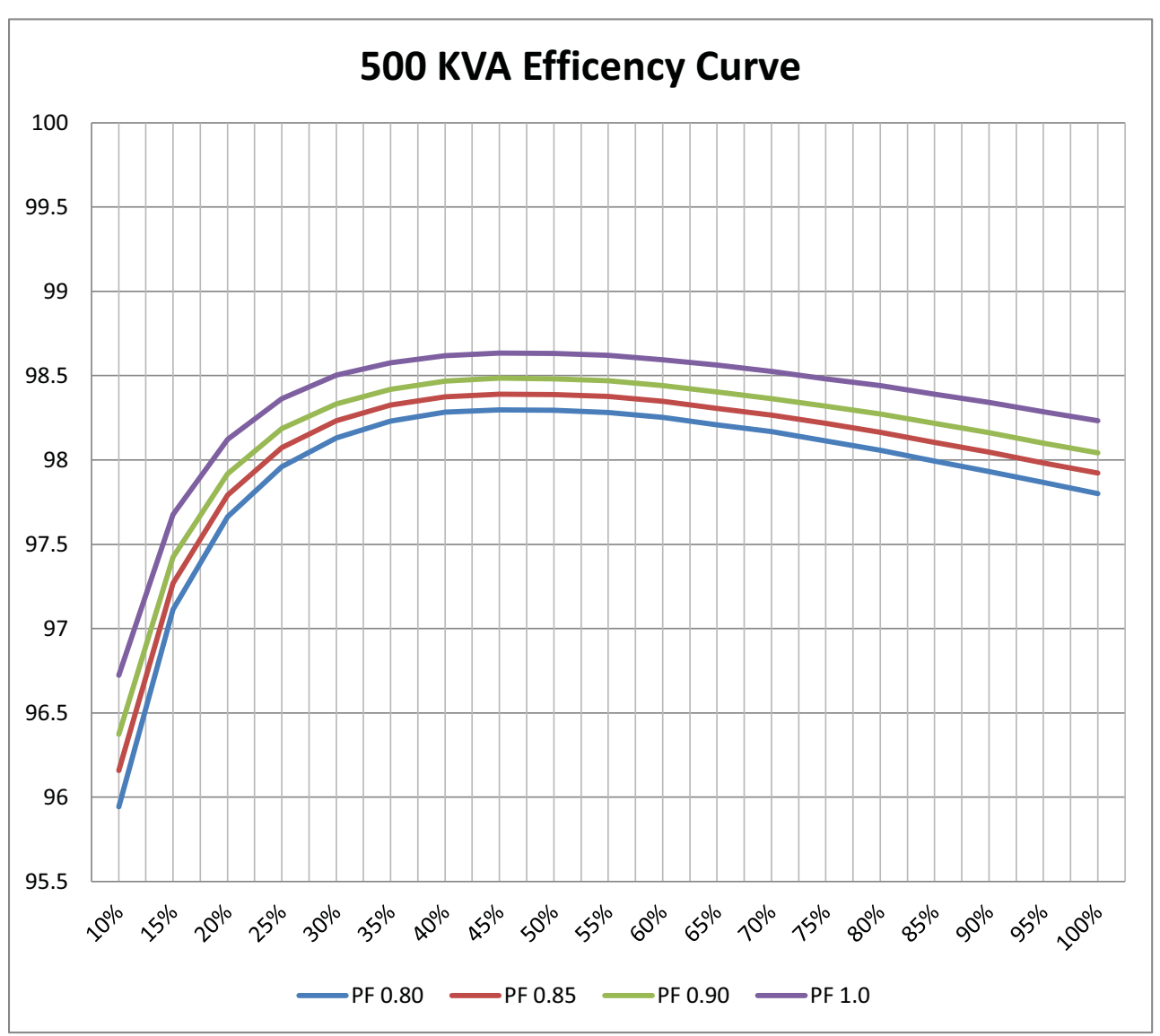




\begin{tabular}{|c|c|c|c|c|c|}
\hline 750 KVA & PF 0.80 & PF 0.85 & PF 0.90 & PF 1.0 & \\
\hline Load \% & Eff \% & Eff \% & Eff \% & Eff \% & \\
\hline $10 \%$ & 96.35222 & 96.54611 & 96.74 & 97.05556 & \\
\hline $15 \%$ & 97.40333 & 97.54278 & 97.68222 & 97.91 & \\
\hline $20 \%$ & 97.89556 & 98.00944 & 98.12333 & 98.30889 & \\
\hline $25 \%$ & 98.16 & 98.26111 & 98.36222 & 98.52222 & \\
\hline $30 \%$ & 98.31111 & 98.40167 & 98.49222 & 98.64667 & \\
\hline $35 \%$ & 98.39556 & 98.48278 & 98.57 & 98.71 & \\
\hline $40 \%$ & 98.44222 & 98.525 & 98.60778 & 98.74667 & \\
\hline $45 \%$ & 98.45333 & 98.53833 & 98.62333 & 98.75778 & \\
\hline $50 \%$ & 98.44889 & 98.53389 & 98.61889 & 98.75333 & \\
\hline $55 \%$ & 98.43444 & 98.51944 & 98.60444 & 98.74333 & \\
\hline $60 \%$ & 98.40444 & 98.49167 & 98.57889 & 98.71778 & \\
\hline $65 \%$ & 98.36333 & 98.45333 & 98.54333 & 98.68778 & \\
\hline $70 \%$ & 98.32333 & 98.41333 & 98.50333 & 98.65222 & \\
\hline $75 \%$ & 98.27222 & 98.36722 & 98.46222 & 98.61111 & \\
\hline $80 \%$ & 98.22111 & 98.31889 & 98.41667 & 98.57111 & \\
\hline $85 \%$ & 98.16 & 98.26278 & 98.36556 & 98.52444 & \\
\hline $90 \%$ & 98.10333 & 98.20889 & 98.31444 & 98.47889 & \\
\hline $95 \%$ & 98.04222 & 98.15 & 98.25778 & 98.42778 & \\
\hline $100 \%$ & 97.98111 & 98.09167 & 98.20222 & 98.37667 & \\
\hline
\end{tabular}

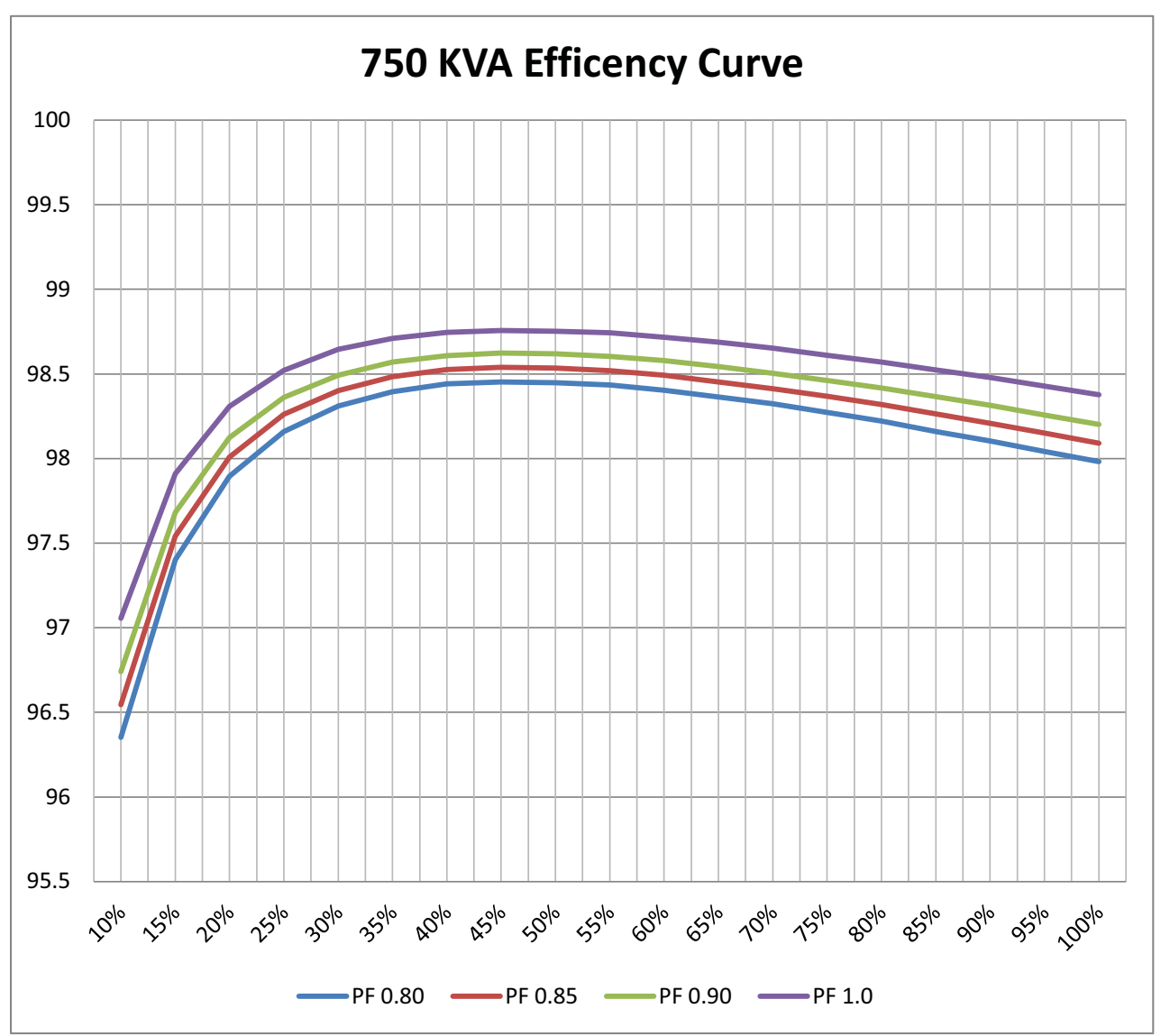




\begin{tabular}{|c|c|c|c|c|c|}
\hline 1000 KVA & PF 0.80 & PF 0.85 & PF 0.90 & PF 1.0 & \\
\hline Load \% & Eff \% & Eff \% & Eff \% & Eff \% & \\
\hline $10 \%$ & 96.76148 & 96.93407 & 97.10667 & 97.38704 & \\
\hline $15 \%$ & 97.69222 & 97.81685 & 97.94148 & 98.14333 & \\
\hline $20 \%$ & 98.12704 & 98.22796 & 98.32889 & 98.49593 & \\
\hline $25 \%$ & 98.36 & 98.44907 & 98.53815 & 98.68148 & \\
\hline $30 \%$ & 98.49074 & 98.57111 & 98.65148 & 98.79111 & \\
\hline $35 \%$ & 98.56037 & 98.64019 & 98.72 & 98.84333 & \\
\hline $40 \%$ & 98.60148 & 98.675 & 98.74852 & 98.87444 & \\
\hline $45 \%$ & 98.60889 & 98.68556 & 98.76222 & 98.88185 & \\
\hline $50 \%$ & 98.60259 & 98.67926 & 98.75593 & 98.87556 & \\
\hline $55 \%$ & 98.5863 & 98.66296 & 98.73963 & 98.86556 & \\
\hline $60 \%$ & 98.5563 & 98.63611 & 98.71593 & 98.84185 & \\
\hline $65 \%$ & 98.51889 & 98.60056 & 98.68222 & 98.81185 & \\
\hline $70 \%$ & 98.47889 & 98.56056 & 98.64222 & 98.77815 & \\
\hline $75 \%$ & 98.43148 & 98.51815 & 98.60481 & 98.74074 & \\
\hline $80 \%$ & 98.38407 & 98.47259 & 98.56111 & 98.70074 & \\
\hline $85 \%$ & 98.32667 & 98.42019 & 98.5137 & 98.65963 & \\
\hline $90 \%$ & 98.27556 & 98.37093 & 98.4663 & 98.61593 & \\
\hline $95 \%$ & 98.21815 & 98.31667 & 98.41519 & 98.56852 & \\
\hline $100 \%$ & 98.16074 & 98.26111 & 98.36148 & 98.52111 & \\
\hline
\end{tabular}

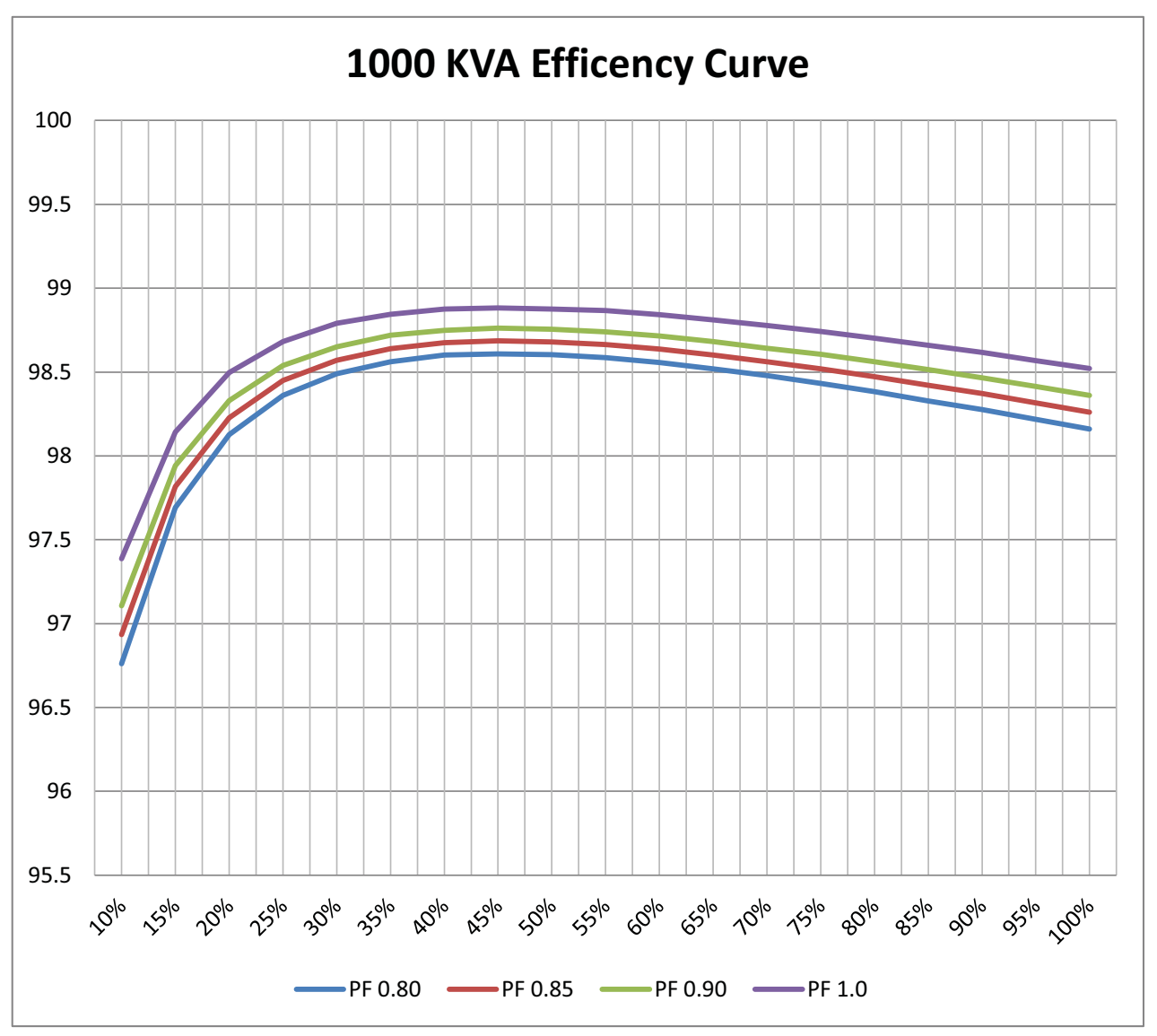




\begin{tabular}{|c|c|c|c|c|}
\hline $\begin{array}{l}\text { 1500kva } \\
\text { Load \% }\end{array}$ & $\begin{array}{l}\text { PF } 0.80 \\
\text { Eff } \%\end{array}$ & $\begin{array}{l}\text { PF } 0.85 \\
\text { Eff } \%\end{array}$ & $\begin{array}{l}\text { PF } 0.90 \\
\text { Eff \% }\end{array}$ & $\begin{array}{l}\text { PF } 1.0 \\
\text { Eff } \%\end{array}$ \\
\hline $10 \%$ & 97.58 & 97.71 & 97.84 & 98.05 \\
\hline $15 \%$ & 98.27 & 98.365 & 98.46 & 98.61 \\
\hline $20 \%$ & 98.59 & 98.665 & 98.74 & 98.87 \\
\hline $25 \%$ & 98.76 & 98.825 & 98.89 & 99 \\
\hline $30 \%$ & 98.85 & 98.91 & 98.97 & 99.08 \\
\hline $35 \%$ & 98.89 & 98.955 & 99.02 & 99.11 \\
\hline $40 \%$ & 98.92 & 98.975 & 99.03 & 99.13 \\
\hline $45 \%$ & 98.92 & 98.98 & 99.04 & 99.13 \\
\hline $50 \%$ & 98.91 & 98.97 & 99.03 & 99.12 \\
\hline $55 \%$ & 98.89 & 98.95 & 99.01 & 99.11 \\
\hline $60 \%$ & 98.86 & 98.925 & 98.99 & 99.09 \\
\hline $65 \%$ & 98.83 & 98.895 & 98.96 & 99.06 \\
\hline $70 \%$ & 98.79 & 98.855 & 98.92 & 99.03 \\
\hline $75 \%$ & 98.75 & 98.82 & 98.89 & 99 \\
\hline $80 \%$ & 98.71 & 98.78 & 98.85 & 98.96 \\
\hline $85 \%$ & 98.66 & 98.735 & 98.81 & 98.93 \\
\hline $90 \%$ & 98.62 & 98.695 & 98.77 & 98.89 \\
\hline $95 \%$ & 98.57 & 98.65 & 98.73 & 98.85 \\
\hline $100 \%$ & 98.52 & 98.6 & 98.68 & 98.81 \\
\hline
\end{tabular}

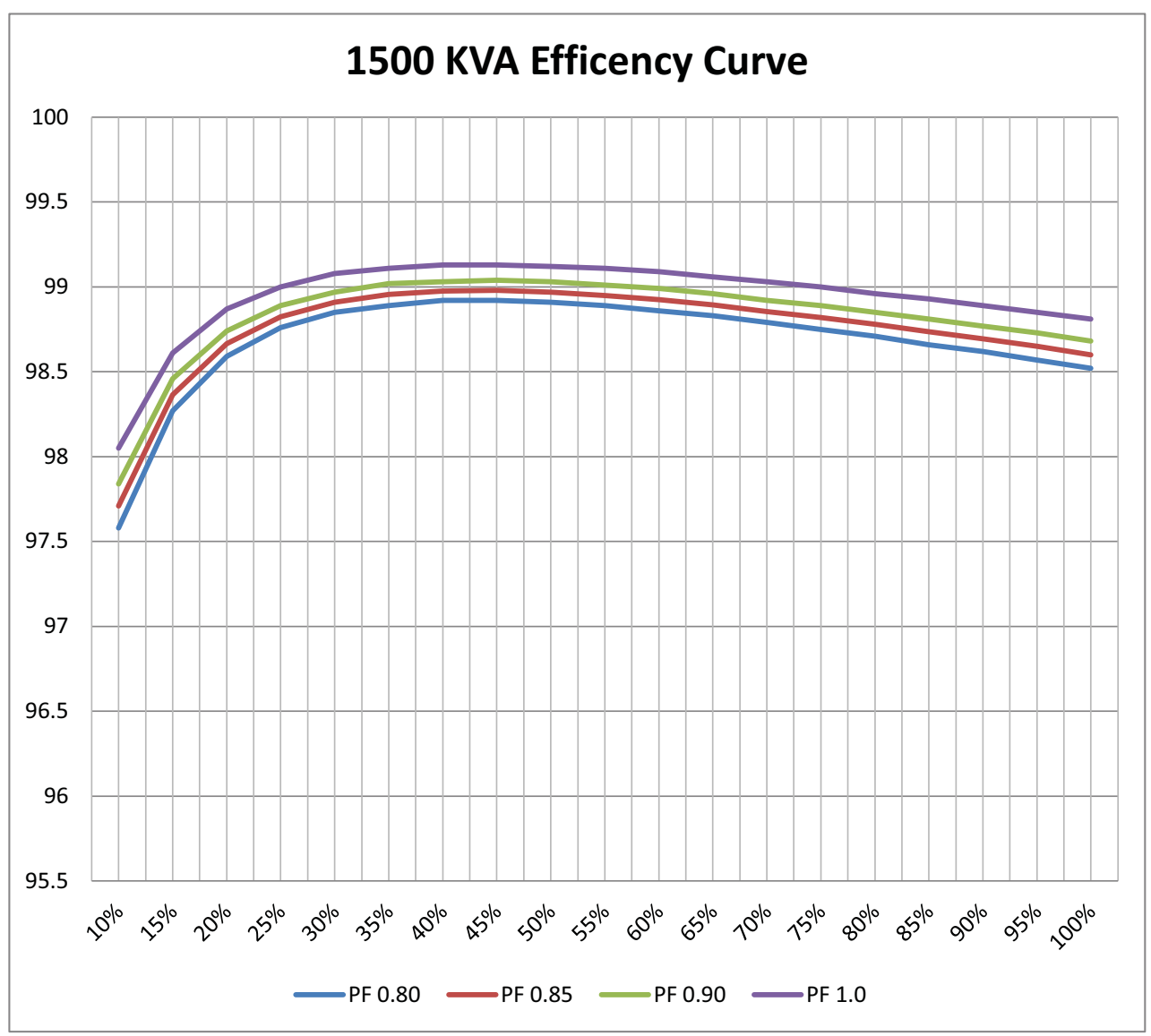




\section{NEMA PREMIUM ${ }^{\circledR}$ EFFICIENCY TRANSFORMER PROGRAM GUIDELINES}

\section{PROGRAM DESCRIPTION AND SPECIFICATION DOCUMENT}

Below is the program description and product specification for the NEMA Premium $®$ Efficiency Transformer program. A product must meet all of the identified criteria if it is to be qualified as a NEMA Premium Compliant Transformer by its manufacturer.

1) Qualifying Products: For the purposes of this Program, Qualifying Products are transformers meeting the following criteria:

Dry-type distribution transformers: for single phase, between 15 kVA and 333 kVA; for three-phase, between $15 \mathrm{kVA}$ and $1000 \mathrm{kVA}$

2) Premium Efficiency Specifications for Qualifying Products: Qualifying Products which meet or exceed the nominal Premium Efficiency levels presented in 10 CFR § 431.196 and reproduced here. In case of discrepancy, the CFR values shall prevail

Table 1: Low-Voltage Dry-Type Distribution Transformers

\begin{tabular}{|c|r|c|r|}
\hline \multicolumn{2}{|c|}{ Single-phase } & \multicolumn{2}{c|}{ Three-phase } \\
\hline kVA & Efficiency (\%) & kVA & Efficiency (\%) \\
\hline 15 & $98.39 \%$ & 15 & $97.90 \%$ \\
\hline 25 & $98.60 \%$ & 30 & $98.25 \%$ \\
\hline 37.5 & $98.74 \%$ & 45 & $98.39 \%$ \\
\hline 50 & $98.81 \%$ & 75 & $98.60 \%$ \\
\hline 75 & $98.95 \%$ & 112.5 & $98.74 \%$ \\
\hline 100 & $99.02 \%$ & 150 & $98.81 \%$ \\
\hline 167 & $99.09 \%$ & 225 & $98.95 \%$ \\
\hline 250 & $99.16 \%$ & 300 & $99.02 \%$ \\
\hline 333 & $99.23 \%$ & 500 & $99.09 \%$ \\
\hline & & 750 & $99.16 \%$ \\
\hline & & 1000 & $99.23 \%$ \\
\hline
\end{tabular}

3) Test Procedure: The manufacturer shall determine the energy efficiency in accordance with 10 C.F.R. Part 431 ("Test Procedures for Distribution Transformers).

4) Buyer Information: NEMA recommends that the manufacturer place the NEMA Premium ${ }^{\circledR}$ Mark on all Qualifying Product models, which meet or exceed the Premium Efficiency Specifications set forth in Tables 1 above, their packaging, and product-related materials such as brochures, manuals, catalogs, advertisements, and Web sites. In addition, the manufacturer is encouraged to disseminate educational materials to educate purchasers about energy efficiency and its benefits. The manufacturer may determine the best manner to disseminate such 
educational material so that it complements the manufacturer's promotional and marketing strategy.

5) The NEMA Premium ${ }^{\circledR}$ Mark is the trademark of NEMA. As such, the name and NEMA Premium Mark may only be used in accordance with the following guidelines and Memorandum of Understanding and License signed by Partners and NEMA. NEMA oversees the proper use of the NEMA Premium ${ }^{\circledR}$ Mark. This includes monitoring the use of the mark in the marketplace and directly contacting those organizations that are using them improperly or without authorization. Consequences to Partners of misusing the NEMA Premium ${ }^{\circledR}$ Mark may include termination of the partner's participation in the NEMA Premium program.

6) Effective Date: The date that manufacturers may begin to qualify products as NEMA Premium Compliant Transformers is defined as the effective date of the specifications. The effective date is May 1, 2010.

7) Specification Revisions: The NEMA Transformer Section reserves the right to change the specifications should technological and/or market developments affect its usefulness to purchasers, customers, industry or the environment. Revisions are to be arrived at through industry discussions and consensus. 


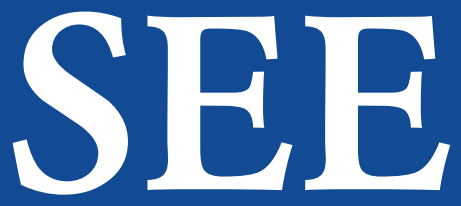

Intelligent Energy

Strategies for development and diffusion of Energy Efficient Distribution Transformers

Selecting Energy Efficient Distribution Transformers A Guide for Achieving Least-Cost Solutions

Prepared for Intelligent Energy Europe Programme

Strategies for Development and Diffusion of

Energy Efficient Distribution Transformers

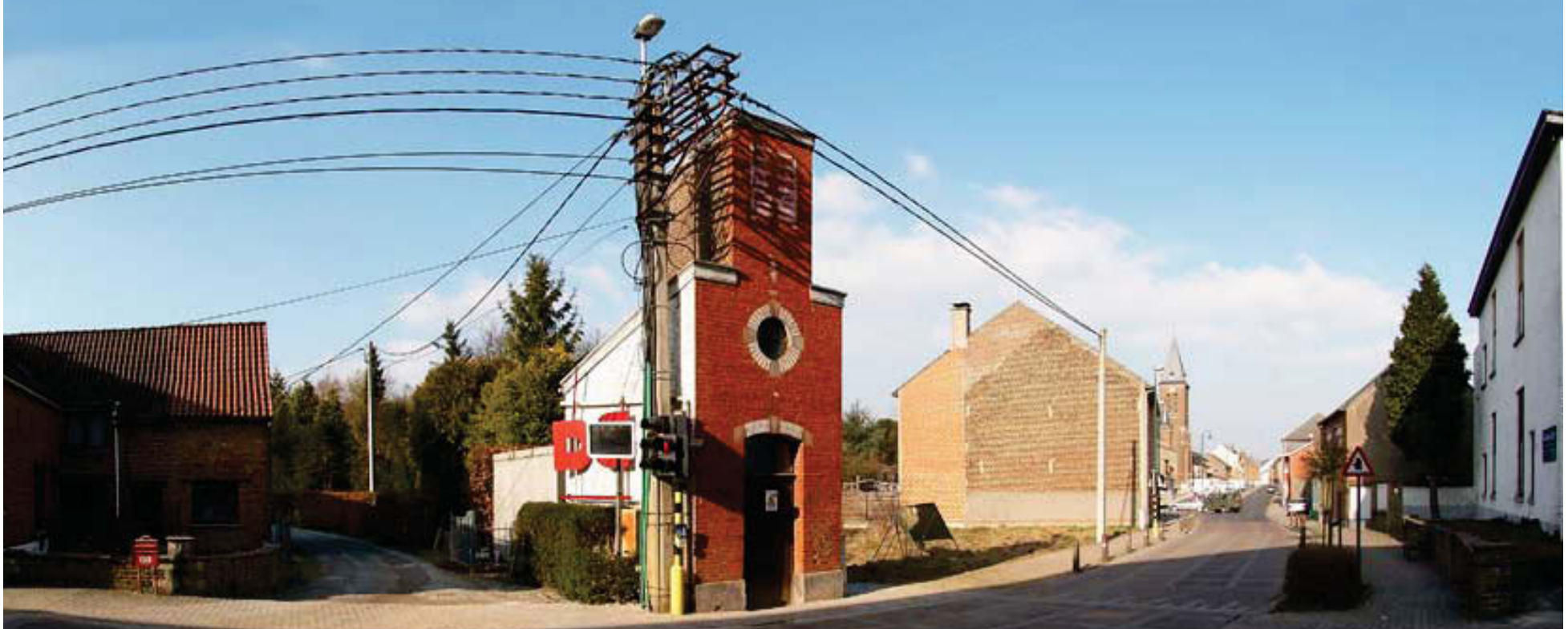

Intelligent Energy 


\section{Intelligent Energy Europe}

\section{About Intelligent Energy Europe Programme}

There are many untapped opportunities to save energy and encourage the use of renewable energy sources in Europe, but market conditions do not always help.

The Intelligent Energy - Europe programme is the EU's tool for funding action to improve these conditions and move us towards a more energy intelligent Europe.

\section{LEGAL NOTICE}

Neither the European Commission, nor any person acting on behalf of the Commission, nor any person from SEEDT Project Partners organizations is responsible for the use which might be made of the information contained in this publication.

The views given in this publication do not necessarily represent the views of the European Commission.

Reproduction is authorised provided the source is acknowledged. Intelligent Energy Europe Programme 


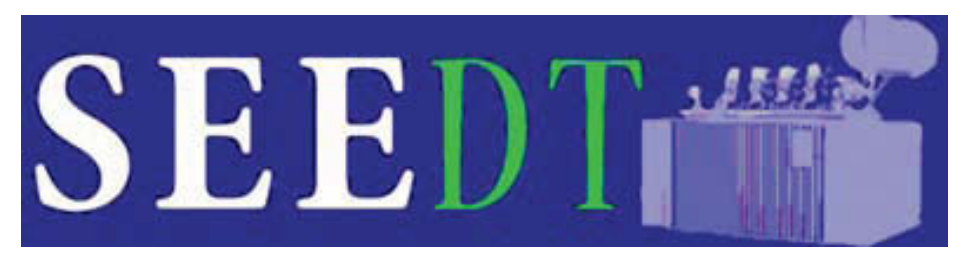

\title{
Selecting Energy Efficient Distribution Transformers A Guide for Achieving Least-Cost Solutions
}

\author{
PROJECT No EIE/05/056/SI2.419632
}

First Published June 2008

\section{Prepared for Intelligent Energy Europe Programme \\ Strategies for Development and Diffusion of \\ Energy Efficient Distribution Transformers}

by

\section{Polish Copper Promotion Centre and European Copper Institute}

in collaboration with

National Technical University of Athens, NTUA -SEEDT Co-ordinator

Greece

Agence De l'Environnement et de la Maitrise de l'Energie, ADEME

France

Alternatives pour l'énergie, les énergies renouvelables et l'environnement, AERE

France

AREVA T\&D Spółka z ograniczoną odpowiedzialnością, AREVA

Poland

ENDESA Distribución Electrica, ENDESA

Spain

Federazione delle Associazioni Scientifiche e Techniche, FAST

Italy

Łódzki Zakład Energetyczny S.A., ŁZE S.A.

Poland

Thelcon Ltd

Greece

Wuppertal Institut für Umwelt, Klima, Energie GmbH

Germany

with the support of the EUROPEAN COMMISSION 


\section{This SEEDT Guide is Something Good for You:}

If ...

...you would like to:

purchase a distribution transformer at the least lifecycle costs

achieve further energy and budget savings in your organisation

contribute to increasing energy security and reducing greenhouse gas emissions...

... and you are:

working in an electricity distribution company, industry, commercial or public organisation wanting to purchase a distribution transformer

working as a facility manager, planner or equipment installer in charge of technical planning or preparing the purchase of a distribution transformer in a client's organisation ...

... then we recommend you to read this brochure.

It presents recommendations and results of the European project SEEDT (Strategies for development and diffusion of energy-efficient distribution transformers), carried out with financial support from the EUROPEAN COMMISSION under the Intelligent Energy - Europe programme and national co-financiers. The aim of SEEDT is to promote the use of energy-efficient distribution transformers, which can be profitable for investors, and by contributing to European Community energy savings, may help to fulfil EU energy policy targets.

\section{Disclaimer}

The sole responsibility for the content of this publication lies with the authors. It does not necessarily reflect the opinion of the European Communities. The European Commission is not responsible for any use that may be made of the information contained therein. While this document has been prepared with care, SEEDT team and any other contributing institutions give no warranty in regards to the contents and shall not be liable for any direct, incidental or consequential damages arising out of its use. 


\section{Contents}

I INTRODUCTION 7

I.I Profitable Solutions Contributing to European Targets 7

I.2 European Distribution Transformer Characteristics 7

2 Losses in transformers 9

2.I Definition of transformer losses 9

2.I.I Extra losses due to harmonics 10

2.2 Losses in European Distribution Transformers I I

2.3 Transformer losses standards $\quad 13$

3 Cost of losses $\quad$ I5

3.I Annual energy losses and cost of these losses 15

3.2 Life cycle cost of transformers 15

$\begin{array}{ll}3.3 \text { Price of a transformer } & 17\end{array}$

$\begin{array}{ll}3.4 \text { Electricity price } & 18\end{array}$

3.5 Time value of money - interest rate 19

$\begin{array}{ll}3.6 \text { Lifetime } & 19\end{array}$

3.7 Loading profile and Load factor 19

3.8 Other aspects; technical (cos phi, harmonics), operational and environmental (climate change mitigation, ecodesign) 2I

3.9 Sensitivity analysis 21

4 Energy-efficient transformers 23

4.I Traditional technologies 23

4.2 Superconducting transformers 23

4.3 Amorphous transformers 23

5 Policy support proposed by SEEDT 25

6 Conclusions 27

7 Bibliography 28

Annex 29 


\section{LIST OF FIGURES}

Figure I. Distribution transformer population / rated power

Figure 2. Fire of a pole-mounted distribution transformer in the streets of Moscow

Figure 3. Breakdown of distribution sector distribution transformer losses, EU-27 and Norway

Figure 4. Operating efficiency of distribution sector distribution transformers, EU-27 and Norway

Figure 5. Simplified chart for calculation of factor $A$

Figure 6. Explanation of relation between time of peak losses and time of peak load

Figure 7. Sensitivity analysis - capitalised cost

Figure 8. Continuously transposed conductors (courtesy DKI) 22

Figure 9. Casting amorphous metal - Courtesy Metglas, Inc 24

Figure 10.Amorphous transformer from the inside $\quad 24$

Figure I I. Energy-efficient transformers can help our environment. 25

\section{LIST OF TABLES}

Table I: EU27 distribution transformer populations and annual market (transformers installed in 2004)

Table 2: EU-27 distribution transformer losses

Table A.I HD428/HD538

Table A.2 EN 50464-I No-load losses P (W) and sound power level (Lw ) for $\mathbf{U} \leq 24 \mathrm{kV}$

Table A.3 EN 50464-I Load losses Pk (W) at $75{ }^{\circ} \mathrm{C}$ for Um $\leq 24$ kV

Table A.4 EN 50464-I Load losses Pk36 (W) at $75^{\circ} \mathrm{C}$ for Um = $36 \mathrm{kV}$

Table A.5 EN 50464-I No-load losses P036 (W) and sound power level (Lw (A) ) for Um = $36 \mathrm{kV}$

\section{LIST OF ILLUSTRATIONS}

Photography used under the terms of the GNU Free Documentation license version 1.2:

- Title page photo: Transformer building in Eizer (Overijse), Belgium - author Wouter Hagens.

- Page 27 top photo: Transformer in Wroclaw, Poland - author Kamil Kozłowski.

- Page 27 bottom photo: Three-Phase, Medium-Voltage Transformer - author Stahlkocher. 


\section{INTRODUCTION}

\subsection{Profitable Solutions Contributing to European Targets}

SEEDT - Strategies for development and diffusion of Energy-efficient Distribution Transformers - is one of the projects in the Intelligent Energy Europe programme.

The aim of this project is to promote the use of energy-efficient distribution transformers, which can be profitable for investors, and, by contributing to European Community energy savings, may help to fulfil EU energy policy targets. Strategies developed in SEEDT include proposals for changes to the regulatory scheme for electricity distribution companies, labelling, mandatory standards, further support mechanisms, and dissemination activities.

Energy efficiency has now become the top priority for European energy policy. The Action Plan on Energy Efficiency, released in 2006 by the European Commission, envisaged launching a debate on how the EU could achieve a reduction in its energy consumption by $20 \%$ (390 Mtoe) compared to the 'business as usual' projections for 2020 on a costeffective basis and, by so doing, limit the growth in energy consumption to a level below 1500 Mtoe/year by 2020 . In order to achieve this target, an annual improvement of $3.3 \%$ is needed, which is $1.5 \%$ more than in the baseline scenario.

Energy-efficient transformers can make a valuable contribution to European energy savings if they are effectively promoted and are given sufficient regulatory support. The calculations by the SEEDT project team show that energy savings of European distribution transformers can reach about 18.5 TWh of electricity per year if all transformers operating today are replaced by the most energy-efficient transformers available today (but excluding the superconductivity option). In other words, an energy-efficient transformer has 55.5\% lower energy losses and thus substantially lower running costs than the average transformer installed today. If, for every future distribution transformer purchase, the most energy-efficient technical option is chosen, then, assuming the anticipated development of the electricity system and replacement rates, up to 11.6 TWh electricity per year can be saved over 15 years.
Additional incentives and further support are needed to realise the energy saving potentials and to reduce greenhouse gas emissions and to increase energy security respectively. This is in spite of the fact that, in many cases, energy-efficient transformers are economical. Energy-efficient transformers usually have lower lifetime costs, despite their higher capital cost, because of the lower cost of losses over time.

In Europe, unlike in many countries around the world, there is no general mandatory standard or even voluntary approach for energy efficiency of distribution transformers. The two main documents which describe losses in transformers are European Standard EN 50464-1 for oil cooled transformers, (which has superseded the harmonised document HD428), and harmonised document HD538 for dry type transformers (or their country equivalents, e.g., DIN, etc.).

\subsection{European Distribution Transformer Characteristics}

The population of distribution transformers in Europe is about 4.5 million units. The annual market for new investments and replacements is estimated at about $3 \%$ of existing stock. Important determinants of market development are the change in electricity consumption, the increase in decentralized generation, the development of electricity and material prices, and the pressure from re-regulation on the unbundled electricity distribution companies in the various countries.

The overall population of distribution transformers in the energy distribution sector in EU-27 is estimated at 3.7 million units. The average transformer power rating of these distribution transformers is between 116 and 369 kVA depending on the country. The population of privately owned (usually referred to as industrial) oil filled distribution transformers installed in the EU-27 is estimated at 800000 units with an average transformer rating of about $400 \mathrm{kVA}$. The number of privately owned dry type transformers is estimated at less than 200000 units but the average rating is just over $800 \mathrm{kVA}$, more than double that of the private oil-filled fleet.

More than two-thirds of the installed transformers have a rated power below $400 \mathrm{kVA}$ (practically up to $250 \mathrm{kVA}$ with a few percent $315 \mathrm{kVA}$ units). The newly purchased units have higher power ratings with less than $50 \%$ of them in the lower power category. 
Table 1: EU27 distribution transformer populations and annual market (transformers installed in 2004)

\begin{tabular}{|c|c|c|c|c|c|}
\hline & & \multicolumn{2}{|c|}{ Fleet EU-27 } & \multicolumn{2}{|c|}{ Market EU-27 } \\
\hline & & pcs & MVA & pcs & MVA \\
\hline \multirow{4}{*}{ Distribution sector oil ${ }^{*}$} & $<400 \mathrm{kVA}$ & 2688000 & 313000 & 56000 & 7000 \\
\hline & $\geq 400 \mathrm{kVA} \& \leq 630 \mathrm{kVA}$ & 861000 & 441000 & 23000 & 12000 \\
\hline & $>630 \mathrm{kVA}$ & 127000 & 157000 & 6000 & 8000 \\
\hline & Subtotal & 3676000 & 911000 & 85000 & 27000 \\
\hline \multirow{4}{*}{ Industry oil } & $<400 \mathrm{kVA}$ & 493000 & 66000 & 24000 & 3000 \\
\hline & $\geq 400 \mathrm{kVA} \& \leq 630 \mathrm{kVA}$ & 181000 & 90000 & 8000 & 4000 \\
\hline & $>630 \mathrm{kVA}$ & 127000 & 173000 & 6000 & 8000 \\
\hline & Total industry oil & 802000 & 330000 & 38000 & 15000 \\
\hline \multirow{4}{*}{ Industry dry } & $<400$ kVA & 39000 & 13000 & 3000 & 1000 \\
\hline & $\geq 400 \mathrm{kVA} \& \leq 630 \mathrm{kVA}$ & 69000 & 41000 & 5000 & 3000 \\
\hline & $>630 \mathrm{kVA}$ & 66000 & 90000 & 8000 & 11000 \\
\hline & Total industry dry & 174000 & 144000 & 16000 & 14000 \\
\hline \multicolumn{2}{|l|}{ Total } & 4652000 & 1384000 & 140000 & 57000 \\
\hline
\end{tabular}

* The population of dry-type transformers in the energy distribution sector is estimated at marginally low level ( 1\% of utility fleet).

Distribution transformers operated and owned by electricity distribution companies are responsible for supplying $66 \%$ of electricity to final users at low voltage level and represent almost $80 \%$ of distribution transformers stock.

It is very clear from the table that smaller units dominate the distribution sector, while, in the industrial sector, especially for dry type transformers, larger units are more common.
From the 'market' columns it is evident that the growth of larger kVA ratings is strong - roughly estimated at $0.4 \%$ per annum.

Figure 1 presents the distribution of transformer power ratings across different transformer segments. Note that these are relative figures so, for absolute rating distribution compare, only bars of the same colour.

Figure 1. Distribution transformer population / rated power

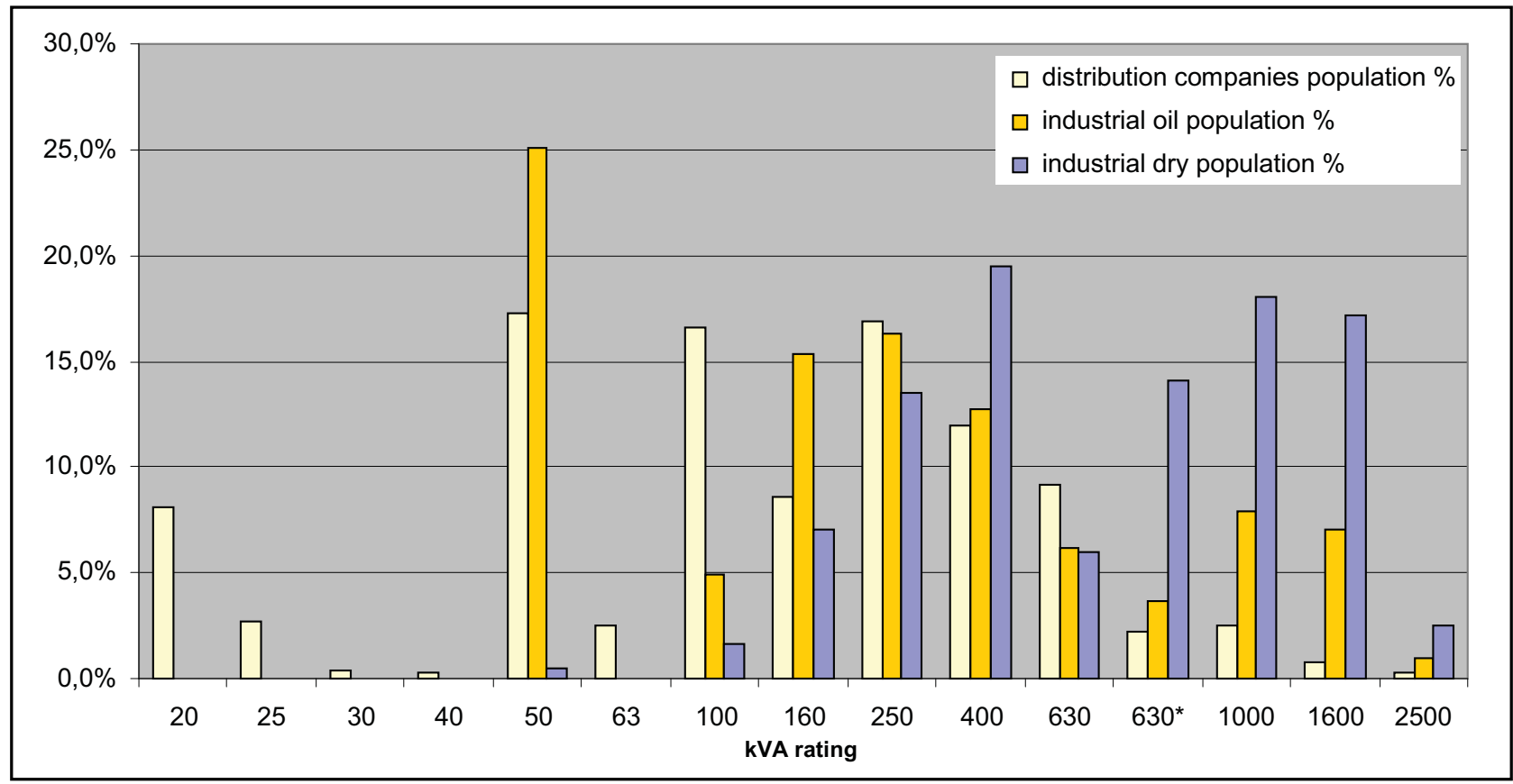

* and upwards indicate $6 \%$ (compared to 4 or $4.5 \%$ for lower ratings) short circuit impedance 


\section{Losses in transformers}

\subsection{Definition of transformer losses}

Transformer losses can be divided into two main components: no-load losses and load losses. These types of losses are common to all types of transformers, regardless of transformer application or power rating. There are, however, two other types of losses; extra losses created by harmonics and losses which may apply particularly to larger transformers - cooling or auxiliary losses, caused by the use of cooling equipment like fans and pumps.

\section{No-Load losses}

These losses occur in the transformer core whenever the transformer is energised (even when the secondary circuit is open). They are also called iron losses or core losses and are constant.

They are composed of:

- Hysteresis losses, caused by the frictional movement of magnetic domains in the core laminations being magnetized and demagnetized by alternation of the magnetic field. These losses depend on the type of material used to build a core. Silicon steel has much lower hysteresis than normal steel but amorphous metal has much better performance than silicon steel. Nowadays hysteresis losses can be reduced by material processing such as cold rolling, laser treatment or grain orientation. Hysteresis losses are usually responsible for more than a half of total no-load losses ( $\sim 50 \%$ to $\sim 70 \%$ ). This ratio was smaller in the past (due to the higher contribution of eddy current losses particularly in relatively thick and not laser treated sheets).

- Eddy current losses, caused by varying magnetic fields inducing eddy currents in the laminations and thus generating heat. These losses can be reduced by building the core from thin laminated sheets insulated from each other by a thin varnish layer to reduce eddy currents. Eddy current losses nowadays usually account for $30 \%$ to $50 \%$ of total no-load losses. When assessing efforts in improving distribution transformer efficiency, the biggest progress has been achieved in reduction of these losses.

- There are also marginal stray and dielectric losses which occur in the transformer core, accounting usually for no more than $1 \%$ of total no-load losses.

\section{Load losses}

These losses are commonly called copper losses or short circuit losses. Load losses vary according to the transformer loading. They are composed of:

- Ohmic heat loss, sometimes referred to as copper loss, since this resistive component of load loss dominates. This loss occurs in transformer windings and is caused by the resistance of the conductor. The magnitude of these losses increases with the square of the load current and is proportional to the resistance of the winding. It can be reduced by increasing the cross sectional area of conductor or by reducing the winding length. Using copper as the conductor maintains the balance between weight, size, cost and resistance; adding an additional amount to increase conductor diameter, consistent with other design constraints, reduces losses.

- Conductor eddy current losses. Eddy currents, due to magnetic fields caused by alternating current, also occur in the windings. Reducing the cross-section of the conductor reduces eddy currents, so stranded conductors are used to achieve the required low resistance while controlling eddy current loss. Effectively, this means that the 'winding' is made up of a number of parallel windings. Since each of these windings would experience a slightly different flux, the voltage developed by each would be slightly different and connecting the ends would result in circulating currents which would contribute to loss. This is avoided by the use of continuously transposed conductor (CTC), in which the strands are frequently transposed to average the flux differences and equalise the voltage.

\section{Auxiliary losses}

These losses are caused by using energy to run cooling fans or pumps which help to cool larger transformers.

\section{Extra losses due to harmonics and reactive power}

This category of losses includes those extra losses which are caused by reactive power and harmonics.

The reactive component of the load current generates a real loss even though it makes no contribution to useful load power. Losses are proportional to 1/(cos phi $)^{2}$. Low power factor loads should be avoided to reduce losses related to reactive power.

Power losses due to eddy currents depend on the square of frequency so the presence of harmonic frequencies which are higher than normal $50 \mathrm{~Hz}$ frequency cause extra losses in the core and winding. Harmonics deserve separate attention and are discussed below. 


\subsubsection{Extra losses due to harmonics}

Non-linear loads, such as power electronic devices, such as variable speed drives on motor systems, computers, UPS systems, TV sets and compact fluorescent lamps, cause harmonic currents on the network. Harmonic voltages are generated in the impedance of the network by the harmonic load currents. Harmonics increase both load and no-load losses due to increased skin effect, eddy current, stray and hysteresis losses.

The most important of these losses is that due to eddy current losses in the winding; it can be very large and consequently most calculation models ignore the other harmonic induced losses.

The precise impact of a harmonic current on load loss depends on the harmonic frequency and the way the transformer is designed.

In general, the eddy current loss increases by the square of the frequency and the square of the load current. So, if the load current contained $20 \%$ fifth harmonic, the eddy current loss due to the harmonic current component would be $5 \times 5 \times$ $0.2 \times 0.2$ multiplied by the eddy current loss at the fundamental frequency - meaning that the eddy current loss would have doubled.
In a transformer that is heavily loaded with harmonic currents, the excess loss can cause high temperature at some locations in the windings. This can seriously reduce the life span of the transformer and even cause immediate damage and sometimes fire.

- Reducing themaximumapparent powertransferred by the transformer, often called de-rating. To estimate the required de-rating of the transformer, the load's de-rating factor may be calculated. This method, used commonly in Europe, is to estimate by how much a standard transformer should be derated so that the total loss on harmonic load does not exceed the fundamental design loss. This derating parameter is known as "factor K".

The transformer de-rating factor is calculated according to the formula in HD 538.3.S1. The factor $\mathrm{K}$ is given by:

$$
K=\left[1+\frac{e}{1+e}\left(\frac{I_{h}}{I}\right)^{2} \sum_{n=2}^{n=N}\left(n^{q}\left(\frac{I_{n}}{I_{1}}\right)^{2}\right)\right]^{0.5}
$$

where:

e - the eddy current loss at the fundamental frequency divided by the loss due to a DC current equal to the RMS value of the sinusoidal current, both at reference temperature.

$\mathrm{n}$ - the harmonic order

\section{Figure 2. Fire of a pole-mounted distribution transformer in the streets of Moscow}

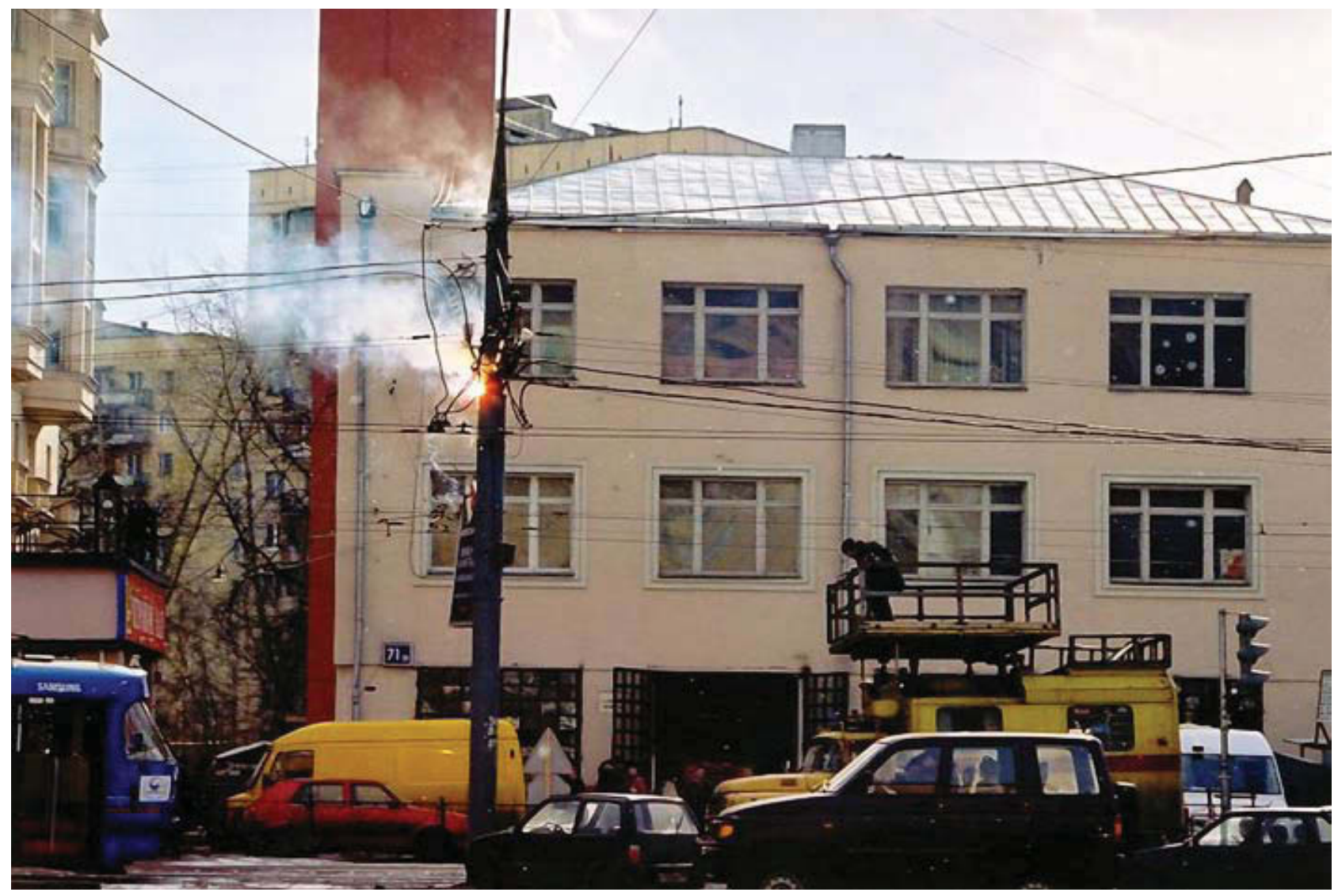


I - the RMS value of the sinusoidal current including all harmonics given by:

$$
I=\left(\sum_{n=1}^{n=N}\left(I_{n}\right)^{2}\right)^{0.5}=I_{1}\left[\sum_{n=1}^{n=N}\left(\frac{I_{n}}{I_{1}}\right)^{2}\right]^{0.5}
$$

$I_{n}$ - the magnitude of the $n$-th harmonic

$\mathrm{I}_{1}$ - the magnitude of the fundamental current

$q$ - exponential constant that is dependent on the type of winding and frequency. Typical values are 1.7 for transformers with round rectangular cross-section conductors in both windings and 1.5 for those with foil low voltage windings.

- Developing special transformer designs rated for non-sinusoidal load currents. This process requires analysis and minimising of the eddy loss in the windings, calculation of the hot spot temperature rise, individual insulation of laminations, and/or increasing the size of the core or windings. Each manufacturer will use any or all of these techniques according to labour rates, production volume and the capability of his plant and equipment. These products are sold as 'K rated' transformers. During the transformer selection process, the designer should estimate the $\mathrm{K}$ factor of the load and select a transformer with the same or higher $\mathrm{K}$ factor.

$\mathrm{K}$ factor is defined as:

$$
K=\sum_{n=1}^{n=n_{\text {max }}} I_{n}^{2} n^{2}
$$

There are some simple tools available which help to calculate the de-rating factor- $\mathrm{K}$ and $\mathrm{K}$-factor. To use them it is necessary to know the harmonic spectrum of the load current. An example of a K-Factor \& Factor $\mathrm{K}$ calculator can be found at:

\section{http://www.leonardo-energy.org/drupal/node/456}

AsanexampleIEC61378-1 dealswith thespecification, design and testing of power transformers and reactors, which are intended for integration within semiconductor converter plants; it is not designed for industrial or public distribution of $A C$ power in general.

The scope of this standard is limited to applications of power converters, of any power rating, for local distribution, at moderate rated converter voltage, generally for industrial applications and typically with a highest voltage for equipment not exceeding $36 \mathrm{kV}$.

The converter transformers covered by this standard may be of the oil immersed or dry-type design. The oil-immersed transformers are required to comply with IEC 60076, and with IEC 60726 for dry-type transformers.

\subsection{Losses in European Distribution Transformers}

The study performed by the SEEDT team has estimated the distribution transformer losses in EU-27 countries and Norway.

The overall losses in EU-27 distribution transformers are estimated at about $33 \mathrm{TWh} /$ year. However, this figure does not include reactive power and harmonic losses which, at a conservative estimate, add a further $5 \mathrm{TWh} /$ year (or $15 \%$ of calculated total of no-load and load losses) for all electricity distribution companies and private distribution transformers. Therefore, total losses of distribution transformers in EU-27 might total about $38 \mathrm{TWh} /$ year.

Table 2 below presents the EU-27 losses in distribution transformers divided into three sectors with the existing population (fleet) and newly installed units (market) listed separately. No-load losses $\left(P_{0}\right)$ account for more than $70 \%$ of total losses ( $1-\Sigma \mathrm{P}_{k} / \Sigma \mathrm{P}$ ratios).

Table 2: EU-27 distribution transformer losses

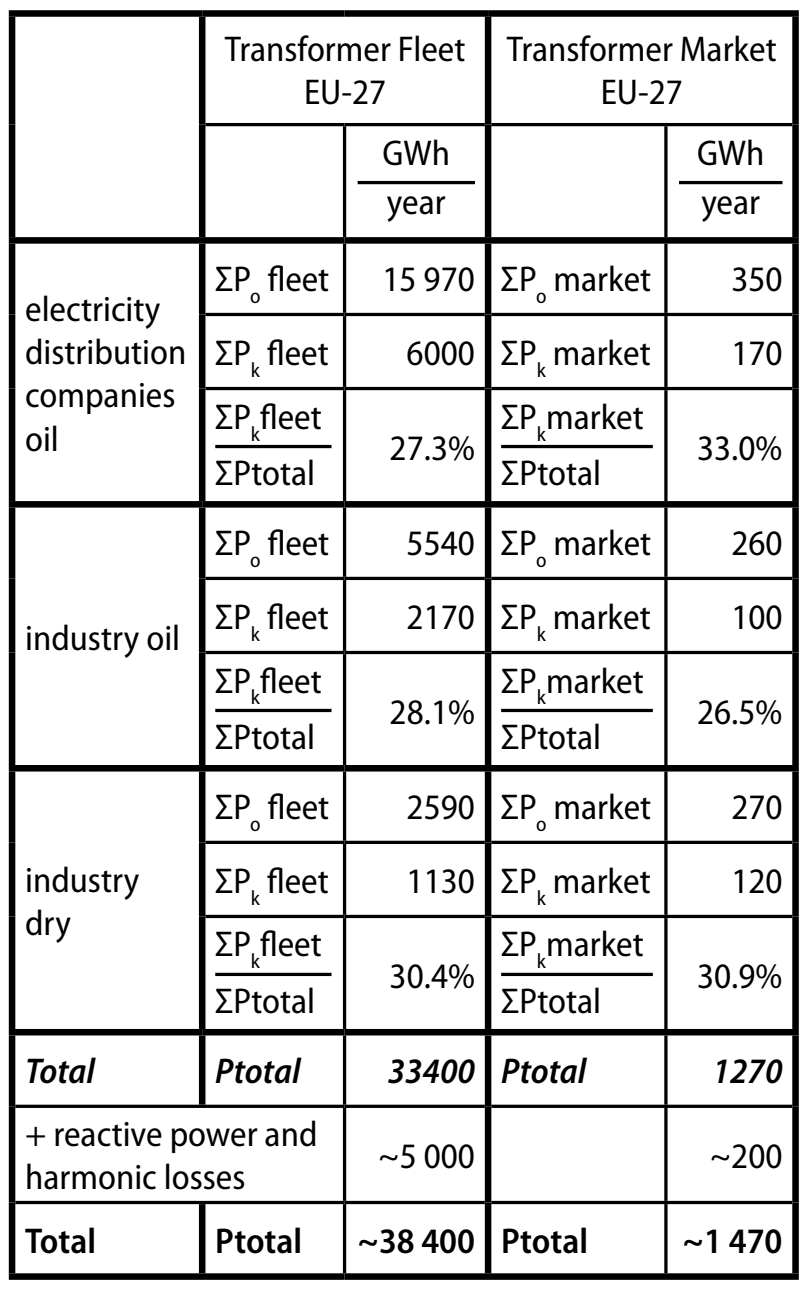


Total losses in newly installed transformers are estimated at $1.24 \mathrm{TWh} /$ year or $1.43 \mathrm{TWh} /$ year including estimated reactive power and harmonic losses. Transformers purchased by electricity distribution companies account for more than 500 $\mathrm{GWh} /$ year of energy losses in the EU-25. Although transformers in electricity distribution companies have rather lower rated losses than those used by industry, their overall running efficiency is similar because the industrial population is made up of higher power transformers which are generally more efficient, run at higher loadings than transformers owned by electricity distribution companies.
Figure 3 presents the total, load and no-load losses of distribution transformers in electricity distribution companies in the EU-27 countries plus Norway (the labels apply to the total and load losses).

The ratio of no-load losses to load losses is close to 3 , while the average operating efficiency for EU-27 countries is $93.38 \%$ (Figure 4).

This leads to the general conclusion that the reduction of no-load losses, especially for small, lightly loaded transformers, should be closely examined.

Figure 3. Breakdown of distribution sector distribution transformer losses, EU-27 and Norway

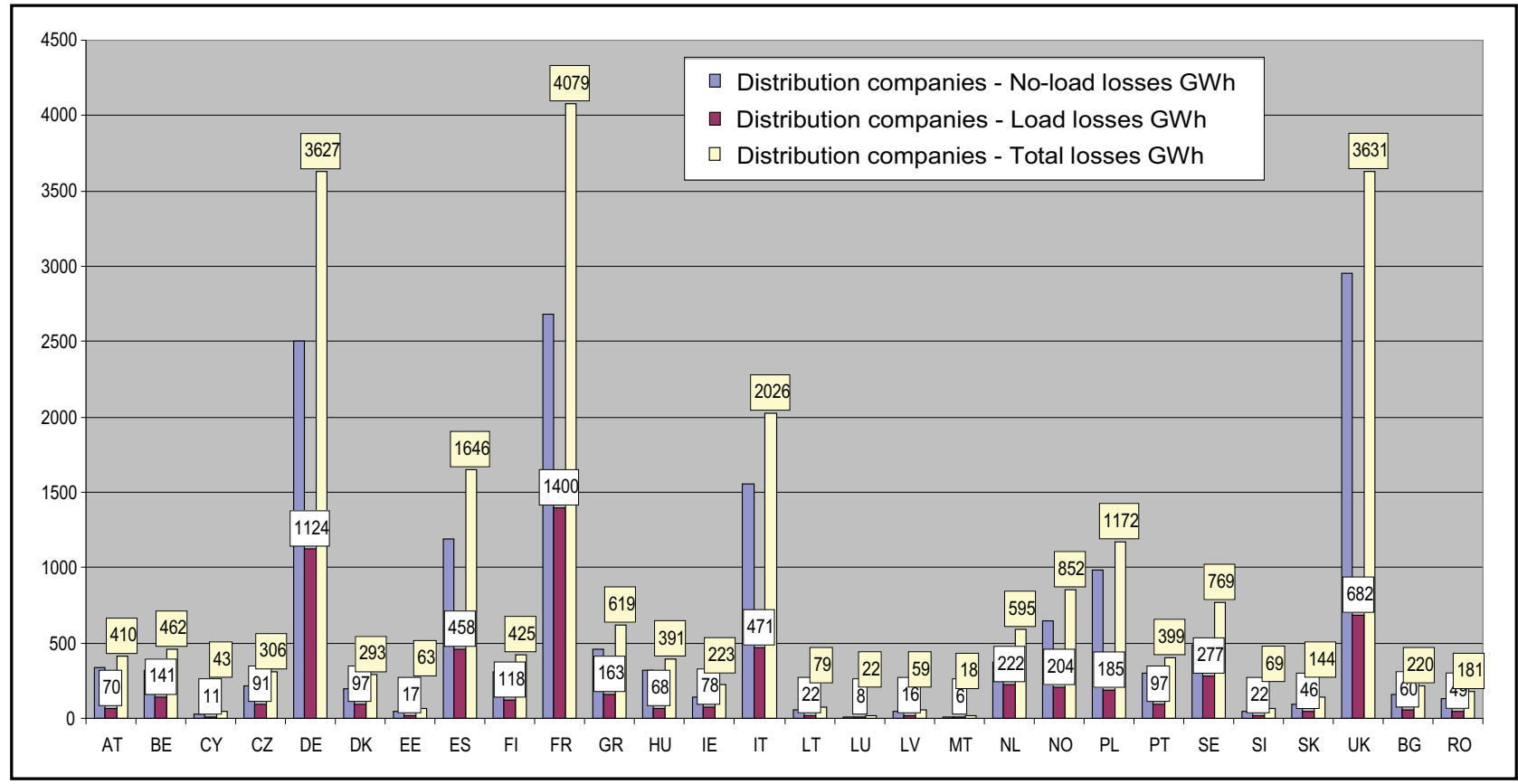

Figure 4. Operating efficiency of distribution sector distribution transformers, EU-27 and Norway

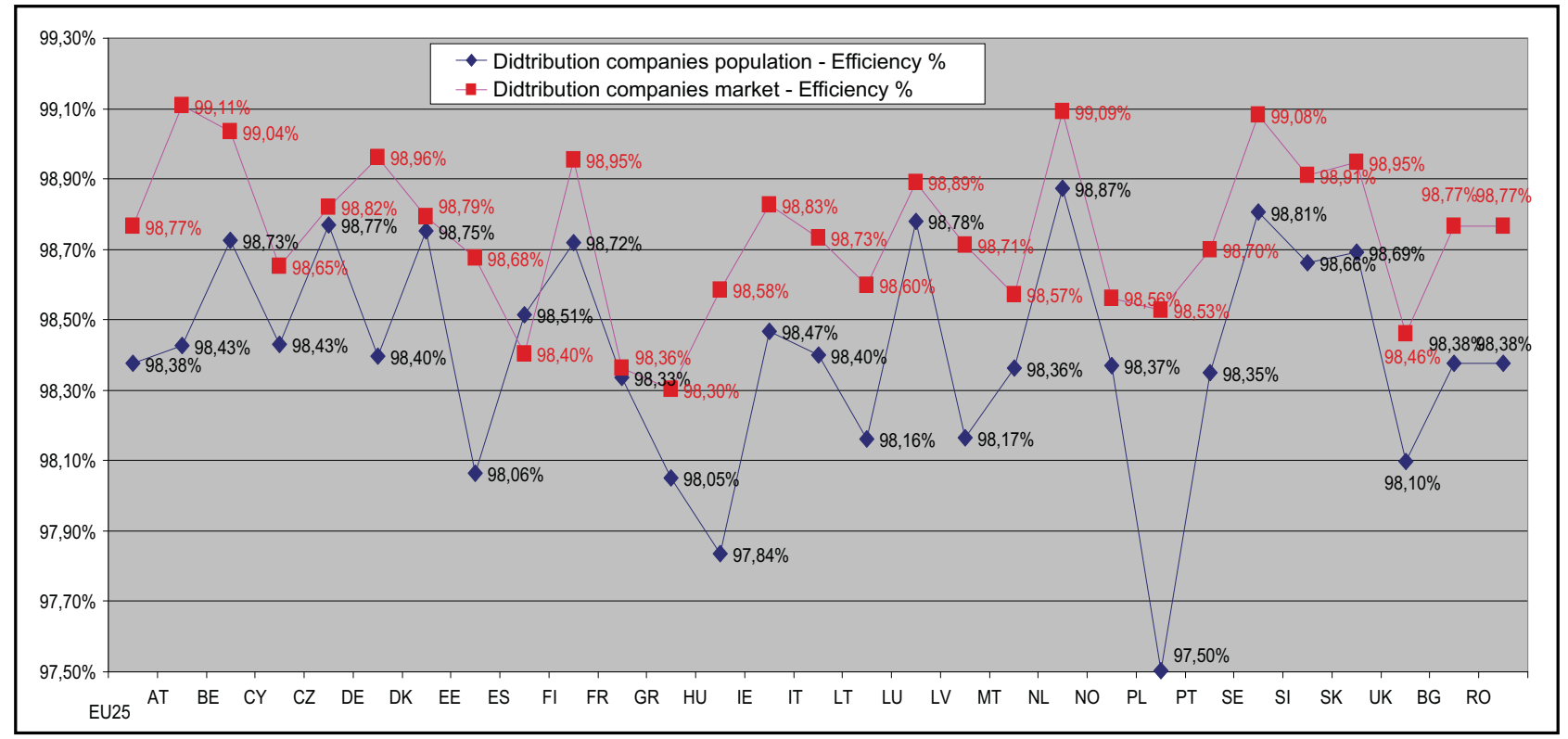


It is worth mentioning that this ratio of no-load to load losses is specific for distribution transformers, mainly because of the loading characteristics. In larger transformers like those used to step up voltage in power plants or in substation transformers no-load losses are smaller or much smaller than load losses. In typical generator step-up transformers the ratio of no-load to load losses can be as small as 0.2 to 0.3 .

Other observations are that electricity distribution companies in different countries take different approaches to taking losses into account when buying a transformer. Some of them apply rational (life-cycle cost) considerations, but others just follow traditional purchasing habits. Transformer manufacturers therefore have to adapt their sales strategies according to these different approaches. The analysis of the existing market situation and purchasing procedures leads to the conclusion that much remains to be done to reduce the existing levels of both no-load and load losses. A comparison of the market situation in electricity distribution companies and industry/commerce does not lead to very clear trends. However two observations are worth mentioning:

- Distribution companies generally pay more attention to reduction of losses in transformers than industry. However, due to higher loading and larger units of industry and commerce population, the operating efficiencies remain at a similar level.

- In general, larger units are specified with lower rated losses. This trend is even more evident in case of load losses.

\subsection{Transformer losses standards}

Unlike many countries around the world, Europe has no mandatory standard on energy efficiency of distribution transformers. The two main documents which describe losses in transformers are: the European Standard EN 50464-1, which has superseded the harmonised document HD428 for oil cooled transformers, and the harmonised document HD538 for dry type transformers, which is still valid (or their various country equivalents, e.g., DIN, etc.). Data from these norms is given in the Annex.

Despitethefact that therearenomandatorystandards in Europe, there are some procurement procedures (internal standards of electricity distribution companies) which are highly demanding in Benelux, Germany, Austria, Switzerland and Scandinavia. Most of the electricity distribution companies in these countries buy transformers at $C^{1}$ [ $C^{\prime}$ minus 30\%] (HD 428) or AoBk (new 50464) standards. ENDESA in Spain purchases HD 428 CC' for 400 kVA units. EdF has introduced a certain purchasing policy which specifies no load losses between Co and Eo and load losses between Dk and Bk. The mix of losses is focused on low no-load losses for small ratings and low load losses for higher ratings. Also tolerance of losses has changed recently. More often utilities reduce the tolerance of losses to, e.g., $0 \%$ instead of $15 \%$.

Efficiency standards outside Europe may be expressed in terms of electrical efficiency, at a certain load level, or in terms of maximum values for no-load and load loss. Some examples follow below.

Australia"recalculated"the American $60 \mathrm{~Hz}$ efficiency NEMA TP-1 standard - which has never become mandatory in USA at federal level - to $50 \mathrm{~Hz}$ and also interpolated linearly the efficiencies for ratings which are different from those used in the USA.

New Zealand follows the Australian regulations for distribution transformers as a matter of policy.

In China, the standards are regularly upgraded since 1999 with $S 7$ and then $\$ 9$ having been replaced by the current standard S11, which defines allowable levels for no-load and load losses slightly below Europe's AC' level. S11 will soon be replaced by S13 which is expected to specify lower loss levels.

The Indian Bureau of Energy Efficiency (BEE), classifies distribution transformers in the range from 25 up to 200 kVA into 5 categories from 1 Star (high loss) to 5 Stars (low loss). 5 Stars represents worldclass performance. 3 Stars is being proposed as a minimum efficiency standard, and is being widely followed by utilities.

Japan has a different type of distribution system, with the last step of voltage transformation much closer to the consumer. The majority of units are pole mounted single phase transformers. The driver for setting up minimum efficiency standards was the Kyoto commitment. Transformers, together with 17 other categories of electrical equipment, should meet minimum efficiencies. In the case of transformers, the efficiency is defined at $40 \%$ load. Target average efficiency has been defined for the year 2006 (oil) or 2007 (dry type), based on the best products on the market in 2003.

1 Designations are explained in Annex - European Distribution Transformer Loss standards 
The standard is designed differently from other standards, with efficiencies for different products being described by equations. This is currently the most demanding of all regulated standards.

Mexico sets the minimum efficiencies at slightly less stringent levels; $0.1 \%$ to $0.2 \%$ below NEMA TP-1 efficiency. As in Australia, the Mexican standard includes voluntary and mandatory elements.

In 1997, Oak Ridge National Laboratory performed extensive studies to determine whether energy conservation standards for distribution transformers would offer significant energy savings, be technically achievable and economically justified. The energy savings potential in the USA from switching to high efficient transformers was estimated to be $141 \mathrm{TWh}$ cumulatively. One of the reasons for this high figure is the high number of distribution transformers in the utility networks in the US.

To reduce these losses, the National Electrical Manufacturers Association (NEMA) created the TP1 standard which defines a minimum efficiency for dry and oil-filled type transformers in the range from 10 to $2500 \mathrm{kVA}$. This became the basis for the rule making process on minimum standards. NEMA TP-1 has been used as a guideline by Canada, Australia, New Zealand and (partially) Mexico and was adopted by Massachusets, Minnesota, Wisconsin, New York, Vermont, California and Oregon. Subsequently, this standard was perceived as insufficiently demanding and, in 2006, the US Department of Energy (DoE) proposed a new standard. This proposal was a compromise between less stringent TP- 1 and the level of losses which, on average, represent the minimum life cycle cost (MLLC), with the proposed loss levels set to represent one third of the improvement between TP-1 and MLCC.

Now, a new standard, closely based on the DoE proposal, has been introduced which will apply to all transformers manufactured for sale in the USA or imported into the USA on or after January 1, 2010. The requirement of the standard is very close to $C^{\prime}$ $-30 \%$ or AoBk.

In addition to this standard, transformers are also a part of the broader EnergyStar labelling programme. EnergyStar is a voluntary programme that encourages the participating utilities to calculate the total cost of ownership of their transformers and buy the type if it is cost-effective to do so. EnergyStar is based on TP1 but may be tightened in the future.
A third programme in the US, set up by the Consortium for Energy Efficiency (CEE), aims to increase the awareness of the potential of efficient transformers in industry. It consists of a campaign to measure the efficiency of industrial transformers and to stimulate companies to upgrade their transformer park to the best available in the market.

Canada follows TP-1 strictly but the mandatory levels apply only for dry type transformers. As far as oil transformers are concerned Canada has conducted an analysis of MEPS implementation potential and found that the great majority of Canadian oil distribution transformers already comply with NEMA TP-1 so the standard would have almost no influence on the market. The yearly MEPS standard impact would only be $0.98 \mathrm{GWh}$ for liquid filled transformers compared to a saving potential at $132 \mathrm{GWh}$ for drytype transformers. Also Energy Star products are very actively promoted in Canada. 


\section{Cost of losses}

\subsection{Annual energy losses and cost of these losses}

The annual energy losses of a transformer can be estimated from the following formula:

$$
W_{\text {Loss }}=\left(P_{0}+P_{K} * L^{2}\right) * 8760 h
$$

in which:

$\mathrm{W}_{\text {Loss }}$ - is the annual energy loss in $\mathrm{kWh}$

$\mathrm{P}_{\mathrm{o}} \quad$ - is the no-load loss in kW. This factor is availablefrom the transformerspecifications or can be measured.

$P_{k} \quad$ - is the short-circuit loss (or load loss) in kW. This factor is available from the transformer specifications or can be measured.

L - is the average per-unit load on the transformer.

8760 - is the number of hours in a year

To calculate the cost of these losses, they need to be converted to the moment of purchase by assigning capital values, to be able to put them into the same perspective as the purchase price. This is called the Total Capitalised Cost of the losses, TCCloss. This can be calculated using the following formula:

where:

$$
T C C_{\text {loss }}=W_{\text {loss }} \times \frac{(1+i)^{n}-1}{i \cdot(1+i)^{n}} \times C \times 8760
$$

C - is the estimated average cost per kWh in each year

i $\quad$ - is the estimated interest rate

n $\quad$ - is the expected life time of the transformer

\subsection{Life cycle cost of transformers}

To perform the economical analysis of transformer, it is necessary to calculate its life cycle cost, sometimes called total cost of ownership, over the life span of transformer or, in other words, the capitalised cost of the transformer. All these terms mean the same - in one formula, costs of purchasing, operating and maintaining the transformer need to be compared taking into account the time value of money.

The concept of the 'time value of money' is that a sum of money received today has a higher value because it is available to be exploited - than a similar sum of money received at some future date.

In practice, some simplification can be made. While each transformer will have its own purchase price and loss factors, other costs, such as installation, maintenance and decommissioning will be similar for similar technologies and can be eliminated from the calculation. Only when different technologies are compared e.g. air cooled dry type transformers with oil cooled transformers will these elements need to be taken into account.

Taking only purchase price and the cost of losses into account the Total Cost of Ownership can be calculated by:

$$
T C O=P P+A^{*} P_{0}+B * P_{K}
$$

where:

PP - is the purchase price of transformer,

A - represents the assigned cost of no-load losses per watt,

Po - is the rated no-load loss,

B - is the assigned cost of load losses per watt,

$\mathrm{Pk} \quad$ - is the rated load loss.

This formula can also be found in HD428 and HD538.

$P_{0}$ and $P_{k}$ are transformer rated losses. $A$ and $B$ values depend on the expected loading of the transformer and energy prices.

The choice of the factors $A$ and $B$ is difficult since they depend on the expected loading of the transformer, which is often unknown, and energy prices, which are volatile, as well as interest rate and the anticipated economic lifetime. If the load grows over time, the growth rate must be known or estimated and the applicable energy price over the lifetime must be forecast. Typically, the value of A ranges from less than 1 to 8 EUR/Watt and $B$ is between 0.2 and 5 EUR/Watt.

Below we propose a relatively simple method for determining the $A$ and $B$ factor for distribution transformers.

$A$ and $B$ factors are calculated as follows: (no-load loss capitalisation)

$$
A=\frac{(1+i)^{n}-1}{i \cdot(1+i)^{n}} \times C_{k W h} \times 8760
$$

and (no-load loss capitalisation)

where:

$$
B=\frac{(1+i)^{n}-1}{i \cdot(1+i)^{n}} \times C_{k W h} \times 8760 \times\left(\frac{I_{l}}{I_{r}}\right)^{2}
$$

i $\quad$ - interest rate [\%/year]

$\mathrm{n}$ - lifetime [years]

$\mathrm{C}_{\mathrm{kWh}}-\mathrm{kWh}$ price [EUR/kWh]

8760 - number of hours in a year [h/year]

$I_{1} \quad$ - loading current $[A]$

$\mathrm{I}_{\mathrm{r}}$ - rated current $[\mathrm{A}]$ 
These formulae assume that energy prices and the loading are constant over the transformer life.

Usually, the loss evaluation figures $A$ and $B$ form part of the request for quotation are submitted to the transformer manufacturers, who can then start the complicated process of designing a transformer to give the required performance. The result of this open process should be the cheapest transformer, i.e. with the lowest total cost of ownership, optimised for a given application. The drawback of this process is, as mentioned, the difficulty in predicting the future load profile and electricity costs and tarrifs with any confidence. On the other hand, these optimisation efforts depend on material prices, particularly active materials, i.e. conductor and core material. Dynamic optimisation makes sense when there is the different price volatility of different materials like aluminium and copper or high and low loss magnetic steel..

For large transformers, above a few MVA, the cost of losses are so high that transformers are custom-built, tailored to the loss evaluation figures specified in the request for quotation for a specific project.

For distribution transformers, often bought in large batches, the process is undertaken once every few years. This yields an optimum transformer design, which is then retained for several years - less so nowadays because of the volatility of metal prices until energy prices and load profiles have changed dramatically. In fact the loss levels established in HD428, HD538 and national standards reflect established practice of preferred designs with respect to loss evaluation values.

To make the capitalisation more attractive, so that the use of TCO is easier, we propose the use of a graph, shown in Figure 5, which allows determination of factor $A$.

Factor A expresses the relation between the cost of no load losses and the following:

- Electricity price

- Discount rate or company interest rate or average cost of capital

- Capitalisation period or expected lifetime of the transformer

This example illustrates that for an electricity price of $100 € / \mathrm{MWh}$, an interest rate of $5 \%$ and a 10 year capitalisation period, the cost of no load loss will be $6,75 € /$ Watt.

Factor $A$ is directly proportional to electricity price so the A factor can simply be scaled to account for electricity price changes as long as the interest rate and capitalisation period remain unchanged.

Figure 5. Simplified chart for calculation of factor $A$

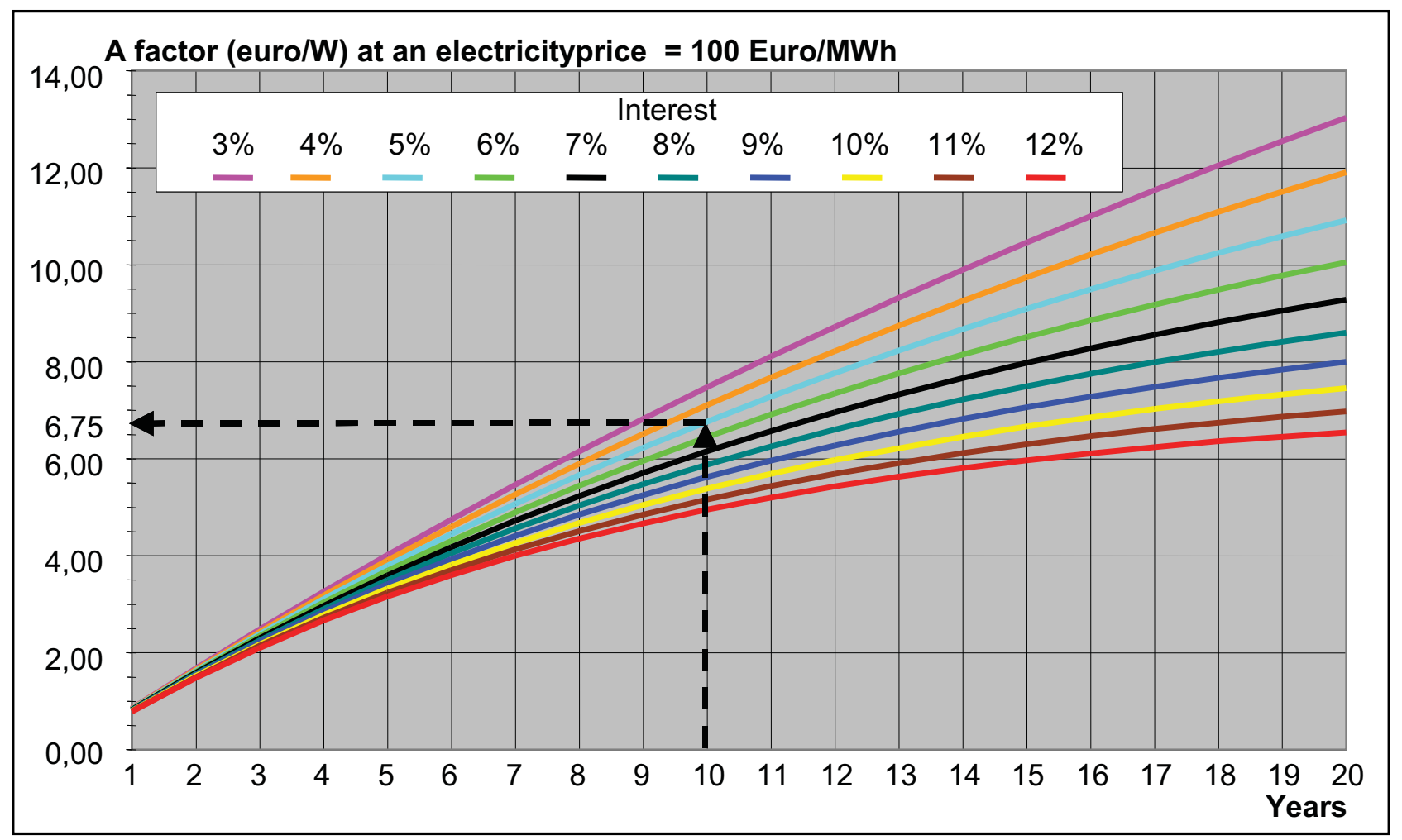


It is important to note that, for small interest rates, a doubling of the capitalisation period will result in almost doubling the cost of losses. On the other hand, applying too high a capital rate, by making, for example, too high a provision for risk, will produce a low value of loss.

Factor B, as explained previously, is simply the product of factor $A$ and the square of the loading factor. ( $B=A *$ (Loading)2) The loading factor used here is the expected average load over the life span of the transformer, possibly taking harmonics into account.

Leonardo ENERGY offers a downloadable spreadsheet to be used as a calculator of TCO. The tool incorporates a more sophisticated modelling of loads and harmonics.

Furthermore, in the course of the SEEDT project, the tool SEEDT TLCalc has been developed. This transformer loss calculator is an interactive tool that allows comparison of two distribution transformers from both an economic and environmental point of view.

The Spreadsheet tools for life-cycle evaluation can be found at:

\section{http://www.leonardo-energy.org/drupal/node/446}

\section{http://seedt.ntua.gr \\ (cf. main menu with the title "TLCalc")}

Having determined the required values it is important to know how sensitive they are to changes in the assumptions on which they were based.

\subsection{Price of a transformer}

Today the production of transformers is characterised by a large variety of designs, manufactured in relatively short batches to meet the demands of a variety of customers with ever-changing expectations. Of course, fluctuations in commodity prices have a significant effect.

The designer has a certain choice of solutions which can be applied in order to build a transformer with expected rated losses. It is possible to use, for instance, different magnetic materials or change the proportions between the level of magnetic induction and current density. It is, however, important from the point of view of the manufacturer that the transformer with expected parameters will be designed in an optimum way.
It is also possible to vary the ratio of copper to steel quantities. According to an old rule of thumb within the transformer industry, the production cost optimum lies somewhere around a ratio of steel to copper usage of 2:1. However, it is a fairly flat optimum and, of course, varies with the price ratio of steel to copper. Independently of this, it should be taken into consideration that the operating properties of the transformer also vary when the shares are varied, especially with respect to losses: Holding the current densities in the windings and the magnetic flux density in the core constant, the loss per kilogram of copper or steel, respectively, will be more or less constant. Thereby a transformer designed according to this philosophy, but with more iron and less copper, tends to have higher iron losses, and one with more copper and less iron will have higher copper losses. But this does not mean that skimping on copper and steel pays off! Rather, enhancing the core cross-section while keeping the number of turns constant will reduce core losses, and enhancing the copper cross section while keeping the core cross section constant will reduce copper losses. In short: the bulkier transformer will always be more efficient, and metal prices will always be an obstacle against its implementation.

The progress in the scope of calculation techniques allows for optimisation to get a precise model of the transformer geometry, taking into account the actual core cross-section and the location of windings. It allows precise calculation of the transformer parameters. Manufacturers have many methods of design optimisation at their disposal which may be used in solving the issues in question.

At today's commodity prices (low loss magnetic steel 2500-3000€/tonne, copper 6000-7000€/tonne) the indicative transformer price for $\mathrm{AC}^{\prime}$ class $100 \mathrm{kVA}$ typical distribution transformer is around $3000 €$, $400 \mathrm{kVA}$ is around $7000 €$ and $1000 \mathrm{kVA}$ around $12000 €$. The price / rating characteristics can be roughly described as:

where:

$$
C_{1}=C_{0} *\left(\frac{S_{1 n}}{S_{0 n}}\right)^{x}
$$

$C_{1} \quad$ - is cost of transformer " 1 "

$\mathrm{C}_{0} \quad$ - is cost of transformer " 0 "

$\mathrm{S}_{1 \mathrm{n}}$ - is rated power of transformer "1"

$\mathrm{S}_{0 \mathrm{n}}$ - is rated power of transformer "0"

$x \quad$ - exponent (cost factor) 
The $X$ factor is about 0.4 to 0.5 . For more efficient units this factor has a tendency to increase up to 0.6 or even higher.

Dry type transformers representing rated losses specified by HD 538 standard are usually a few percent lighter than oil transformers (as they do not contain oil) but, because of the more costly manufacturing process, they are about $10 \%$ to $30 \%$ more expensive than $\mathrm{CC}^{\prime}$ oil-filled machines. Because of some differences in design, particularly of magnetic circuit, no-load losses of dry transformers are substantially higher than in oil transformers.

Thanks to optimisation efforts and technology improvements, there is an area for transformer manufacturing cost reduction. Commodity prices have gone up for the last few years and have outweighed the cost-reducing effect of optimisation and technological improvements. The cost of active materials in typical transformers increased by more than $50 \%$ between the years 2003 and 2007. In these conditions transformer design optimisation can bring around $5 \%$ savings in manufacturing cost. This effect is better in less efficient machines.

Please note that, when the price of both active materials (magnetic steel and winding material) increasesinsimilarproportion, theoptimisationbrings only marginal savings in manufacturing cost, when the same loss levels are specified. So manufacturers rather prefer to agree certain capitalisation based formula when optimisation of transformer may bring some savings in manufacturing cost.

Today, the cost active materials in an average distribution transformer represents about $50 \%$ of the transformer total price; taking all materials into account increases this to $70 \%$ of the total price.

Let's take the example of a typical medium size distribution transformer. Efficiency improvement today by reducing no-load losses adds to the cost by about $0.3 \%$ to $0.7 \%$ for $1 \%$ loss reduction in the area of moderate losses (between $\mathrm{A}^{\prime}$ and $\mathrm{C}^{\prime}$ level) and from $0.7 \%$ (close to $C^{\prime}$ ) to $1.4 \%$ in area of very low losses (more than $20 \%$ below $C^{\prime}$ ). For load losses, this proportion is higher, even at smaller load loss reduction and ranges from $0.6 \%$ to $1 \%$. When we start to reduce load loss in the area of level $\mathrm{C}$ minus 15\% (new Ak level) this cost may increase to $1.6 \%$. Very roughly, if both losses are reduced at the same time, the cost should theoretically be higher (if no- load losses are reduced by adding more steel, the core becomes wider and requires longer conductor path resulting in increase of resistance and load loss, when load losses are reduced by increasing conductor cross-section the core becomes larger and requires more steel) but in fact this effect is almost negligible until a certain level of both losses. Beyond that, reducing both losses at the same time results in increasing suboptimum and going further towards both reduction extremes becomes extremely expensive and absolutely impractical.

It is, however, worthwhile mentioning that load loss reduction by increasing the amount of conductor material is not necessarily the most cost-effective measure. As already mentioned, to reduce load losses by design optimisation it is possible to reduce the amount of copper but change other transformer parameters e.g. adding more or higher grade magnetic steel.

As a very general comparison, ten years ago, amorphous transformers were more expensive than the European average transformers (with $\mathrm{AC}^{\prime}$ losses) by a factor of 2 or more. Today, this proportion has reduced to a factor of 1.5 or less.

\section{Note that values given above are indicative only!}

\subsection{Electricity price}

The process of electricity market liberalisation led to an adjustment of electricity prices in Europe. Prices for end users consist of certain standardised components:

- energy and load price

- transmission price

- distribution price

- metering and billing price

Prices for industry and commerce are usually subject to bilateral contracts between supplier and end user. Large industry partly buys directly in the energy market. Electricity distribution companies usually tender for covering loss energy.

Prices may differ depending on the type and size of end user, the time when the electricity is consumed (e.g. night tariffs) and the voltage level at which electrical energy delivery and metering is made. VAT and other local or national taxes are added. 
Electricity prices for industry in Europe vary between $60 € / M W h$ to almost $120 €$ / MWh depending on country and industry size with the mean value of $90 € /$ MWh for users consuming around 1GWh annually. Price evolution scenarios are not identical as EU energy policy may have different costs and different results but, in general, no dramatic increase is expected and after 2010/2015 prices should become rather stable.

Which price should be used when calculating TCO? For industrial and commercial users the contractual price, if possible with a price evolution scenario when changes are thought likely. For an electricity distribution company tendering for covering electricity losses, the price received under the tender should be used. For other electricity distribution companies, the question is more complex. First of all this should be long-term marginal cost not a price. It should not be an electricity cost having generation and transmission part included only. Marginal costs of distribution network should be included as well. If electricity prices reflect fair electrical energy supply business i.e. are based on cost calculation, the cost in question should be final end user price less profit of distribution company.

The real situation for electricity distribution companies is further complicated by regulation schemes, which do not allow for direct and full return on capital invested in - for example - more efficient transformers.

\subsection{Time value of money - interest rate}

Industrial investors have always in mind the rate of investment which should be applied when purchasing machines. This is either the weighted average cost of capital or the required rate of return on different investments. Investment in transformer is however essential to every business as it cannot be avoided. Applying too high rates with a lot of risk provisions is not advisable.

For electricity distribution companies, energy regulators usually recommend certain interest rates to value investments into electricity distribution assets. An indicative value is around $5 \%$ to $6 \%$ real interest rate.

\subsection{Lifetime}

Lifetime is a crucial component of the TCO calculation. Transformers are durable and have long working lives. For financial purposes, the amortisation period for an investment in a transformer is often set at ten years. After that period, companies are no longer motivated to invest in new, more efficient, transformers since the 10 year-old one bears no cost and cannot be logically compared to the cost of a new investment. The average technical life of a transformer is 30 years or more; the SEEDT study has shown that more than $10 \%$ of the European transformer fleet is 40 years old or more. This $10 \%$ of the transformer fleet contributes more than $20 \%$ of the total no-load losses and more than $15 \%$ of load losses in European distribution companies.

Concluding, the minimum reasonable transformer lifetime in TCO calculations should be 20 years and arguments mentioned above indicate that applying 30 years lifetime in industry and commerce, and 40 years lifetime in electricity distribution companies can be justified as well

\subsection{Loading profile and Load factor}

Transformer loading conditions are probably most influential as far as optimum selection of distribution transformer losses is concerned. Very generally, for heavily loaded transformers, the focus should be on load losses while for those which are lightly loaded, it should be on no-load losses. What does heavily mean? Where is a difference?

Ideally, to calculate the load losses it would be necessary to integrate the squares of all momentary ratios of actual load to the rated load. This is practically impossible so the methodology to analyse load losses based on summation of energy consumed in transformers has been developed.

The formula to calculate load losses is presented below:

where:

$$
\sum P_{k}=\beta_{S}^{2} \times \tau \times P_{k}
$$

$\sum P_{k}$ - is sum of load losses in given period of time, usually one year

$\beta_{s} \quad$ - is the peak load of a transformer in given period of time

$\tau \quad$ - is time duration of peak loss

$P_{k} \quad$ - is rated load loss of a transformer

It should be noted that $\left(I_{1} / I_{\mathrm{r}}\right)^{2}$ value from "B" factor calculation formula can be expressed by $\beta_{S}{ }^{2} \times \tau$ 
The sense of time duration of peak losses $\tau$ and its relation to time duration of peak load Ts is explained in Figure 6 below.

Ts represents the fraction of yearly time in which energy is transformed at peak load conditions equivalent to actual energy transformed. ts represents the fraction of yearly time of peak loss (which occurs at peak load) equivalent to actual load losses.

The equation describing this relation:

$$
\tau_{s}=f\left(T_{s}\right)
$$

is empirical and more than 20 different mathematical formulae exist to describe it.

Some of them propose the following formula:

$$
\frac{\tau_{s}}{8760}=\left(\frac{T_{s}}{8760}\right)^{X}
$$

with $\mathrm{x}$ varying around value of 1.7 to 1.8

Some other

$$
\boldsymbol{\tau}_{s}=A \cdot\left(\frac{T_{s}}{8760}\right)+B \cdot\left(\frac{T_{s}}{8760}\right)^{2}
$$

with $A$ value between 0.15 to 0.5 and $B$ value between 0.5 and 0.85 with additional feature of $A+B=1$ (but there are exclusions from the last condition). The physical interpretation of all these formulae is hard and not always proven. SEEDT has analysed these formulas from the accuracy point of view. The $x$ value which gives best results is 1.73 or $A$ value of 0.3 and $B$ value of 0.7 .
Average loading values from SEEDT study for distribution companies are presented below:

\begin{tabular}{|l|r|}
\hline Average load & $18,86 \%$ \\
\hline$\beta s$ & 0,53 \\
\hline$T s$ & 0,36 \\
\hline$\tau$ & 0,2 \\
\hline
\end{tabular}

The average loading of distribution transformers in electricity distribution companies in the EU-27 is $18,9 \%$ and peak load is 0,53 (53\%). The time of peak load is 0.36 and time of peak loss is 0.2 . The definitions of loading parameters like time of peak load and peak loss are given under point 3.7. For comparison, industry transformers in EU-27 are loaded at 37.7\% on average. Transformers in electricity distribution companies have such low loadings for many reasons, such as the anticipated high variability of load and the need to reserve capacity to provide resilience against failure of other equipment. Another reason could also be that users try to limit the loading on transformers that are in a poor technical condition (e.g. moist insulation and risk of bubbling effect). A further reason might be that distribution transformers are protected against short circuit, but not against overload or excessive temperature.

Figure 6. Explanation of relation between time of peak losses $\tau$ and time of peak load $T$

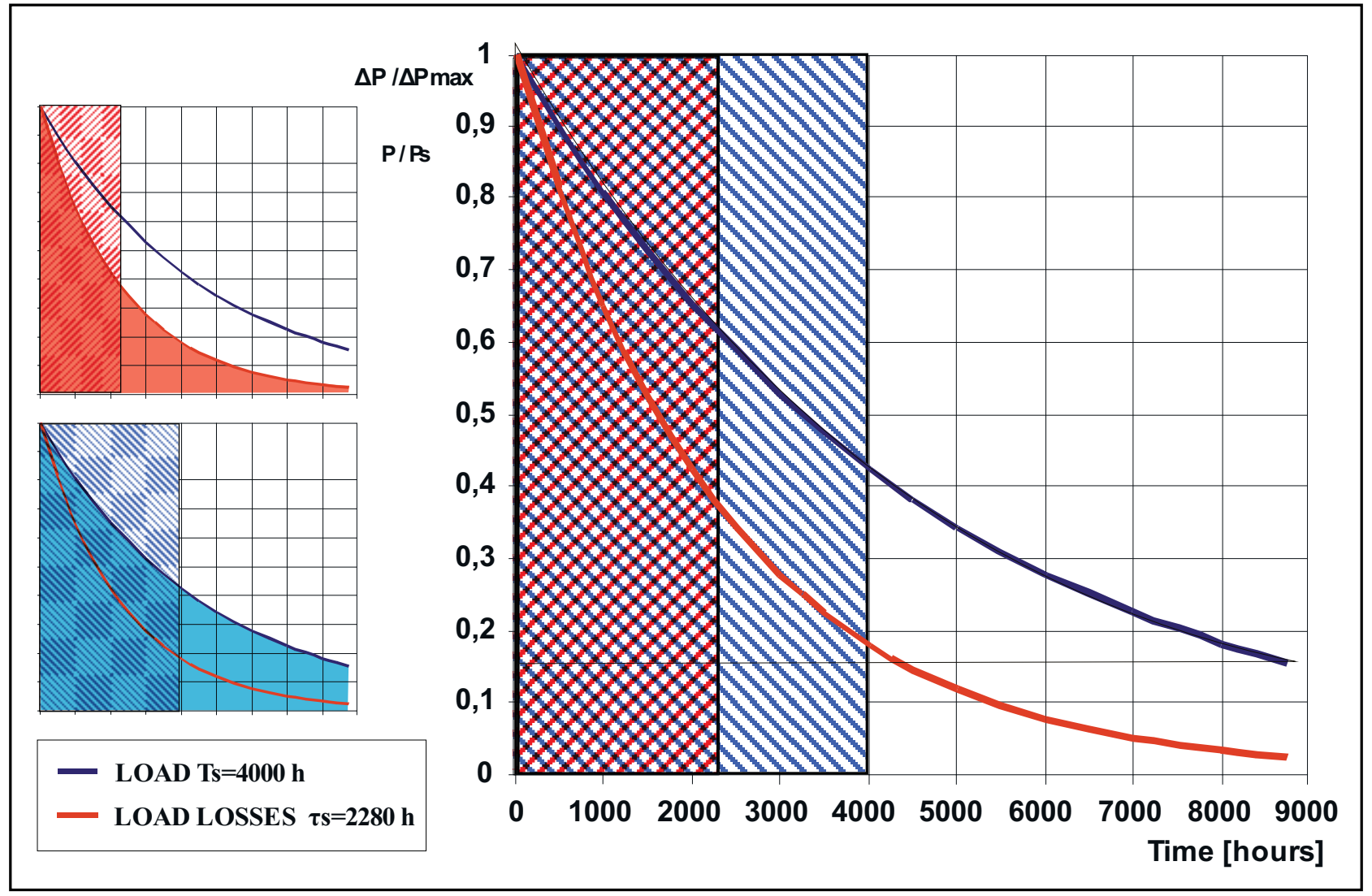


Industrial transformers are loaded higher than transformers owned by distribution companies. Average load is $37.7 \%$, peak load above 0.7 , while time of peak load and peak loss are about 0.3 and 0.15 , which suggests that industry as a whole time capacity and subsequent load are fairly intermittent.

It is quite visible that $\tau$ value is around 50 to $60 \%$ of $\mathrm{T}$ value. Theoretically $\tau$ is $50 \%$ of $\mathrm{T}$ in situations where the $T$ curve (blue) in Figure 6 is a straight line between peak load and zero (load is continuously and uniformly distributed between peak load and zero). On the contrary, when the T curve is a straight horizontal line (equal load all the year), both values $T$ and $\tau$ will be equal.

Understanding the influence of loading conditions is necessary to calculate the " $B$ " factor. In practical situations additional effort should also be made to anticipate loading changes over time.

\subsection{Other aspects;}

technical (cos phi, harmonics), operational and environmental (climate change mitigation, ecodesign)

Apart from the parameters discussed in sections 3.3 to 3.7, which are inputs into the TCO calculation, other parameters, such as power factor (or reactive power), harmonics and even voltage level, have an influence on the energy efficiency of transformers and thus on the TCO results.

The influence of power factor and harmonics has already been explained in section 2.1. Voltage level also influences losses; simply reducing the voltage results in a reduction in flux density by almost the same proportion and core losses will be lower. Approaches to the design of transformers for different voltage ratios, but with the same losses, will be different. Higher ratios will naturally have higher losses.

The mode of operation of a transformer may also have an influence on losses. Load imbalance induces a circulating current in a delta winding, so balancing loads results in lower losses. The balanced components of triple $\mathrm{n}$ harmonics also circulate in the delta winding of a transformer.

The environmental performance of transformers may have an additional bearing on the selection of a more efficient unit. Usually, the cost of $\mathrm{CO}_{2}$ emission certificates are included in the calculation of the electricity price for electricity losses. Furthermore, purchasing an energy-efficient distribution transformer demonstrates the company's willingness to contribute to societal goals of reducing greenhouse gas emissions and increasing energy security in Europe. The ecodesign picture of typical distribution transformer depends in $90 \%$ or more on energy losies which result from transformer operation.

\subsection{Sensitivity analysis}

In 2002, KEMA performed a sensitivity analysis to demonstrate the extent to which changes in input data affect the result given by the capitalisation formula. This analysis was published in 'Energy Saving in Industrial Distribution Transformers.' [1]

Figure 7. Sensitivity analysis - capitalised cost [1]

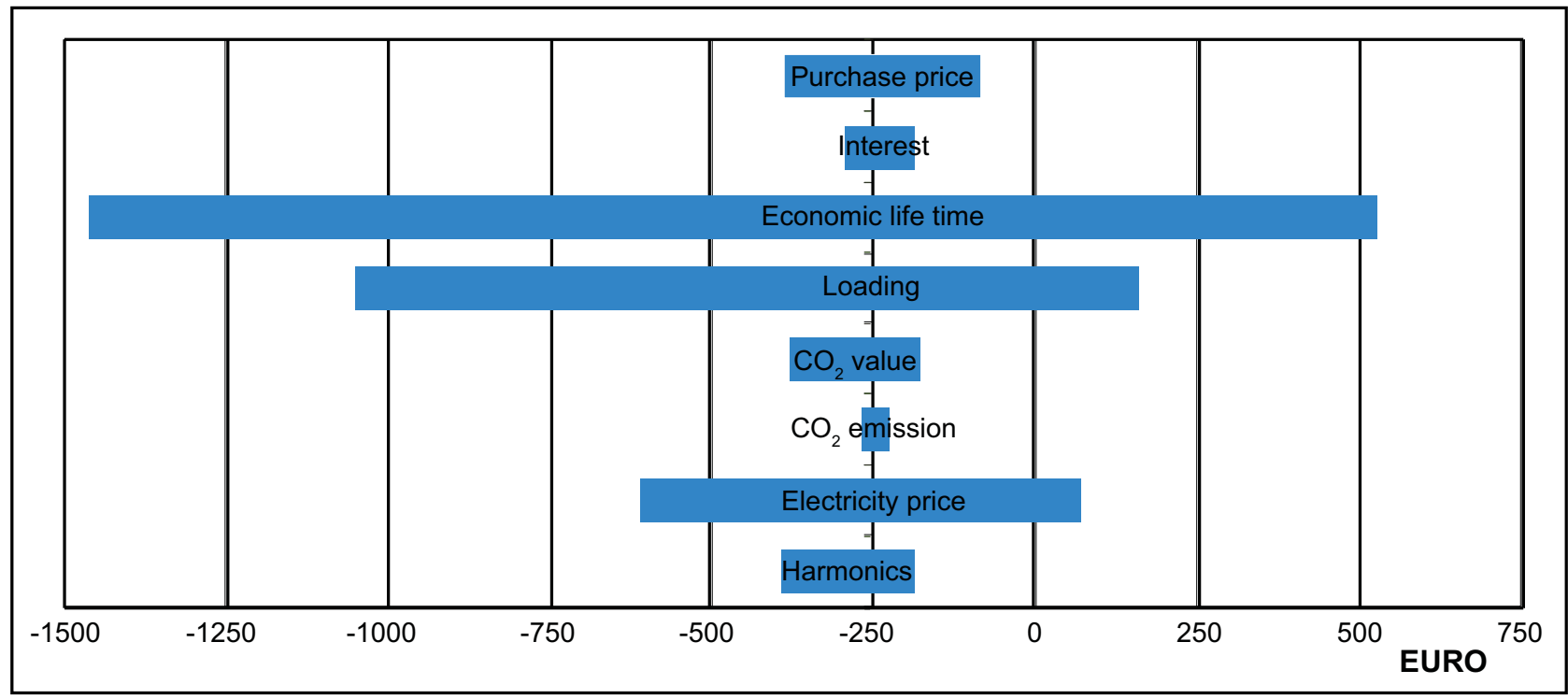


A negative value means it is useful to choose the transformer with the low losses. When the result is positive, the transformer with the losses according to HD 538 (not very efficient) should be used. It is clear that most attention should be given to the economic lifetime, loading pattern and electricity price. Disregarding these values will certainly have a great influence on deciding which transformer should be used and could give a poorly optimised transformer. The influence of the purchase price of the transformer is relatively small and the remaining parameters even smaller.

Figure 8. Continuously transposed conductors (courtesy DKI)

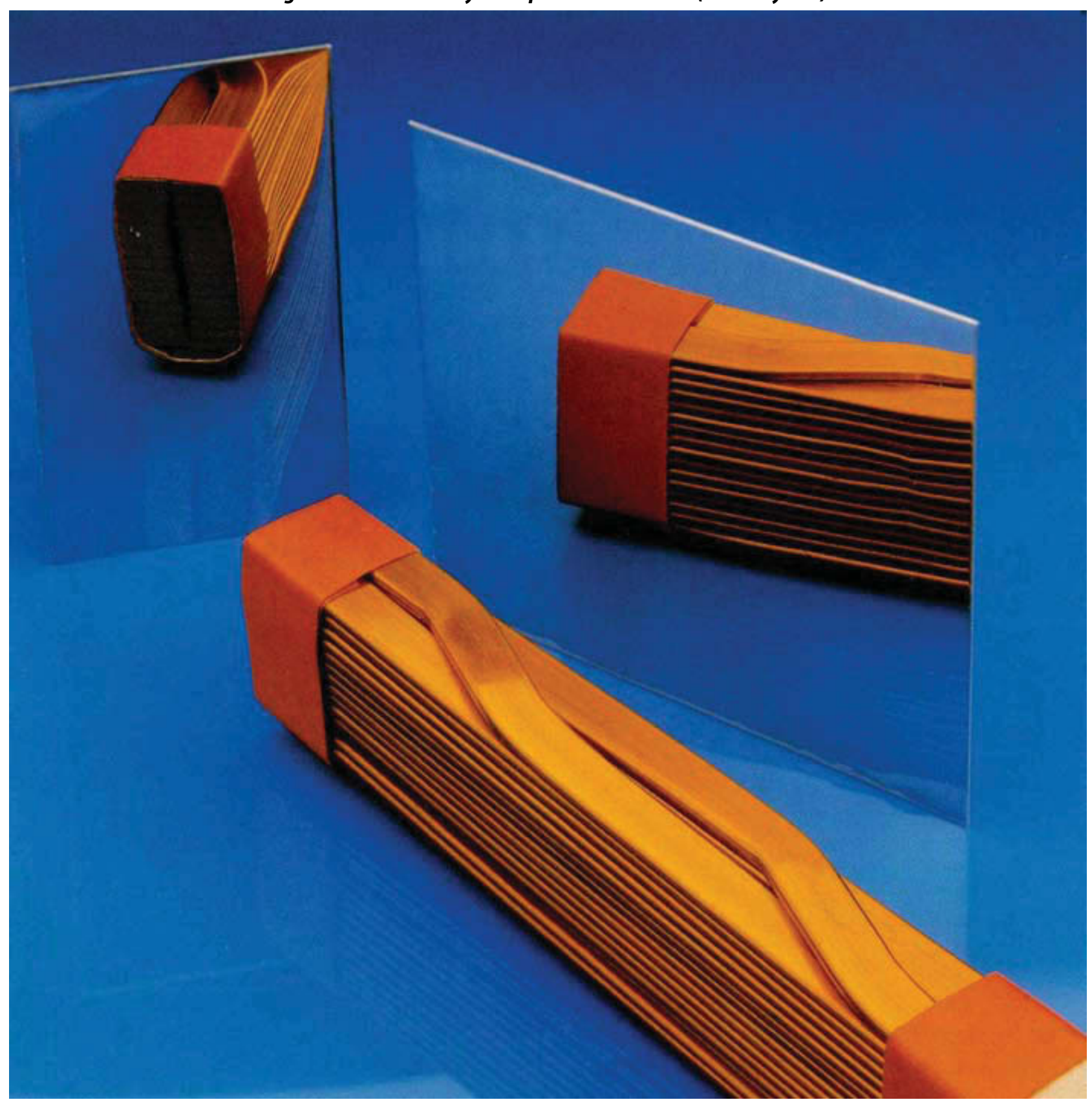




\section{Energy-efficient transformers}

\subsection{Traditional technologies}

According to the SEEDT study the load losses have been reduced by about 30 to $50 \%$ during last 40 years. Copper conductors have replaced aluminium conductors due to their lower resistance and better tensile strength. Conductor design has also been upgraded by the introduction of continuously transposed conductors (a single conductor sub divided into several flat sub-conductors which are regularly transposed), reducing eddy current losses and allowing better packing density of the winding.

Also winding design and insulation improvements, making it thinner, allowing operation at higher temperature, adding some dielectric strength, also helped to reduce load losses by improving heat evacuation and increasing the conductor area.

As far as no-load losses are concerned, their reduction over the last 40 years can be called revolutionary having been reduced by factor 3 to 4. The replacement of hot rolled steel by cold rolled steel, about 40 years ago, has been followed by many material improvements leading to the development of low loss silicon steel.

The introduction of laser cutting for laminations has reduced burrs, improving insulation between laminations and reducing no-load losses. Laminations are also thinner down to $0.1 \mathrm{~mm}$ (usually between 0.2 and $0.3 \mathrm{~mm}$ ) reducing no load-losses further.

\subsection{Superconducting transformers}

In a superconducting transformer the windings, made of a high temperature superconducting material (HTS), are cooled with liquid nitrogen at about $77 \mathrm{~K}$ so that the resistance is almost negligible. Load losses, even after adding losses from nitrogen processing, can be reduced by $50 \%$.

The use of HTS transformers on a larger scale is economically justified and will become more attractive as cooling systems improve and the cost of liquid nitrogen production falls. Another important factor is progress in the processing of long lengths of HTS conductors.
These transformers have smaller weight and volume and are more resistant to overload but cost about $150 \%$ to $200 \%$ of the price of conventional transformers.

So, in applications where weight is crucial (railway vehicles), transformers are much more "squeezed" (by forced cooling) to cut the weight. So efficiencies are much lower, and saving weight saves energy twice.

In our opinion, HTS transformers are suitable only in applications where the load losses make up a high proportion of the total losses, but are not yet ready for general use.

\subsection{Amorphous transformers}

Amorphous alloys differ from conventional crystalline alloys in their magnetic and mechanical properties (such as hardness and strength).

Allied-Signal (US) spent more than 25 years and considerable R\&D effort to achieve the commercial production of METGLAS ${ }^{\circledR}$ amorphous alloys. The Hitachi/Metglas group manufactures amorphous metals and is the biggest promoter of amorphous technology in distribution transformers. The worldwide market share of amorphous core transformers is quite significant with about 3 million single phase units and a few hundred thousand three phase units. It represents about 5\% market share worldwide but this is not reflected in the European market. According to the SEEDT findings, the European market for amorphous transformers is just beginning. For example, ENDESA, the Spanish utility, has started to purchase amorphous transformers from outside Europe and is promoting this technology to local manufacturers.

To achieve an amorphous structure in a solid metal, the molten metal must be solidified very rapidly so that crystallisation cannot take place. The required rate for molten-metal cooling is about one million degrees Celsius per second. Figure 9 illustrates a method of manufacture of amorphous metal.

Amorphous metal core transformers improve electrical power distribution efficiency by reducing transformer core losses. The loss reduction ranges from 65 to $90 \%$ compared with typical silicon steelbased transformers used in Europe under sinusoidal load conditions. Amorphous technology is especially appropriate for transformers operating with low loading because the effect of harmonics on no-load 


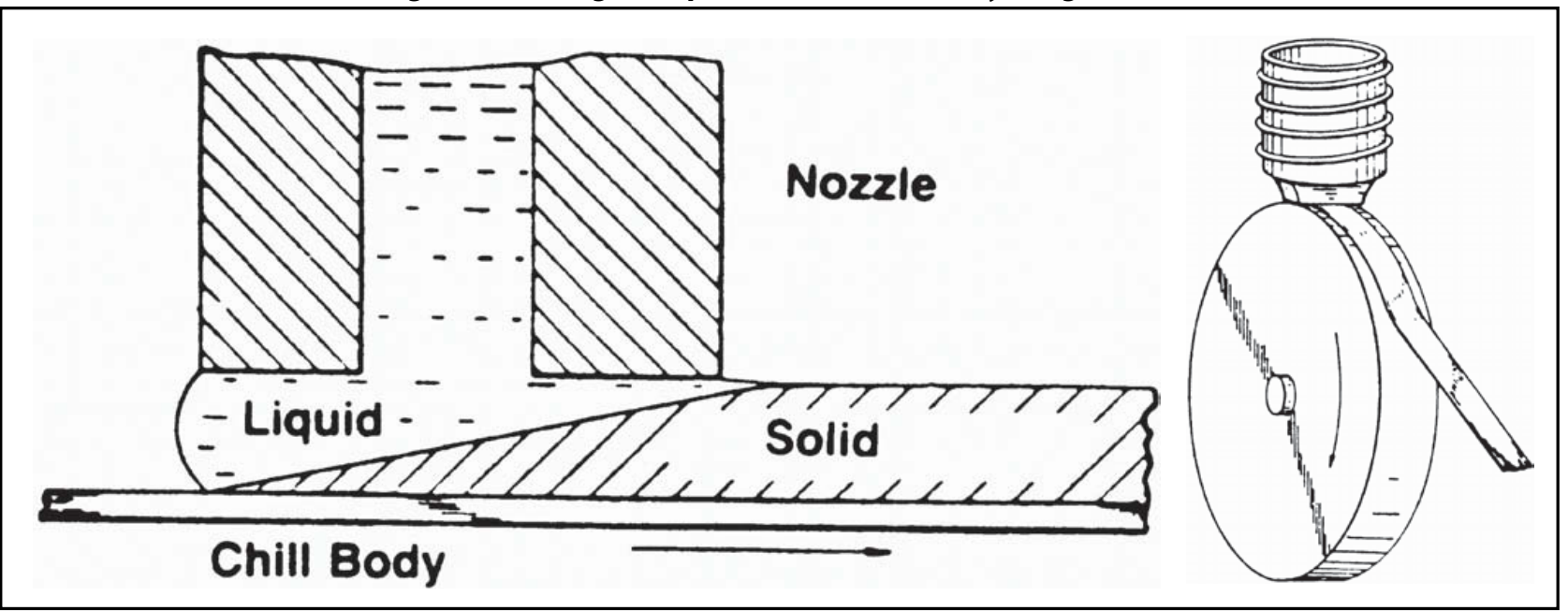

losses is reduced.

to the amorphous nature of the material that gives a higher electrical resistivity and low energy magnetic flux reversal.

From manufacturing cost perspective, amorphous transformers have only slightly higher or almost

Lately, Metglas introduced a new amorphous material, HB1, (improved from the earlier SA1 material) which compensates for the deficiency of reduced saturation induction. The new material reaches saturation at induction levels close to those typical of traditional magnetic steel. This allows more compact cores and smaller and lighter transformers than older amorphous designs. It is also characterised by lower noise level, which was a perceived deficiency of the earlier amorphous metal.

The increase in the use of power electronics has resulted in a considerable amount of higher harmonic distortion in electricity systems. High frequency harmonics lead to increased transformer core losses, especially in distribution transformers that use conventional steel core materials. Amorphous metal core distribution transformers are uniquely suited to providing low loss performance under low loading and at higher frequencies. This is due to improved processing techniques that allow thinner and more consistent strip to be manufactured, as well as the same prices as highly efficient traditional

Figure 10. Amorphous transformer from the inside

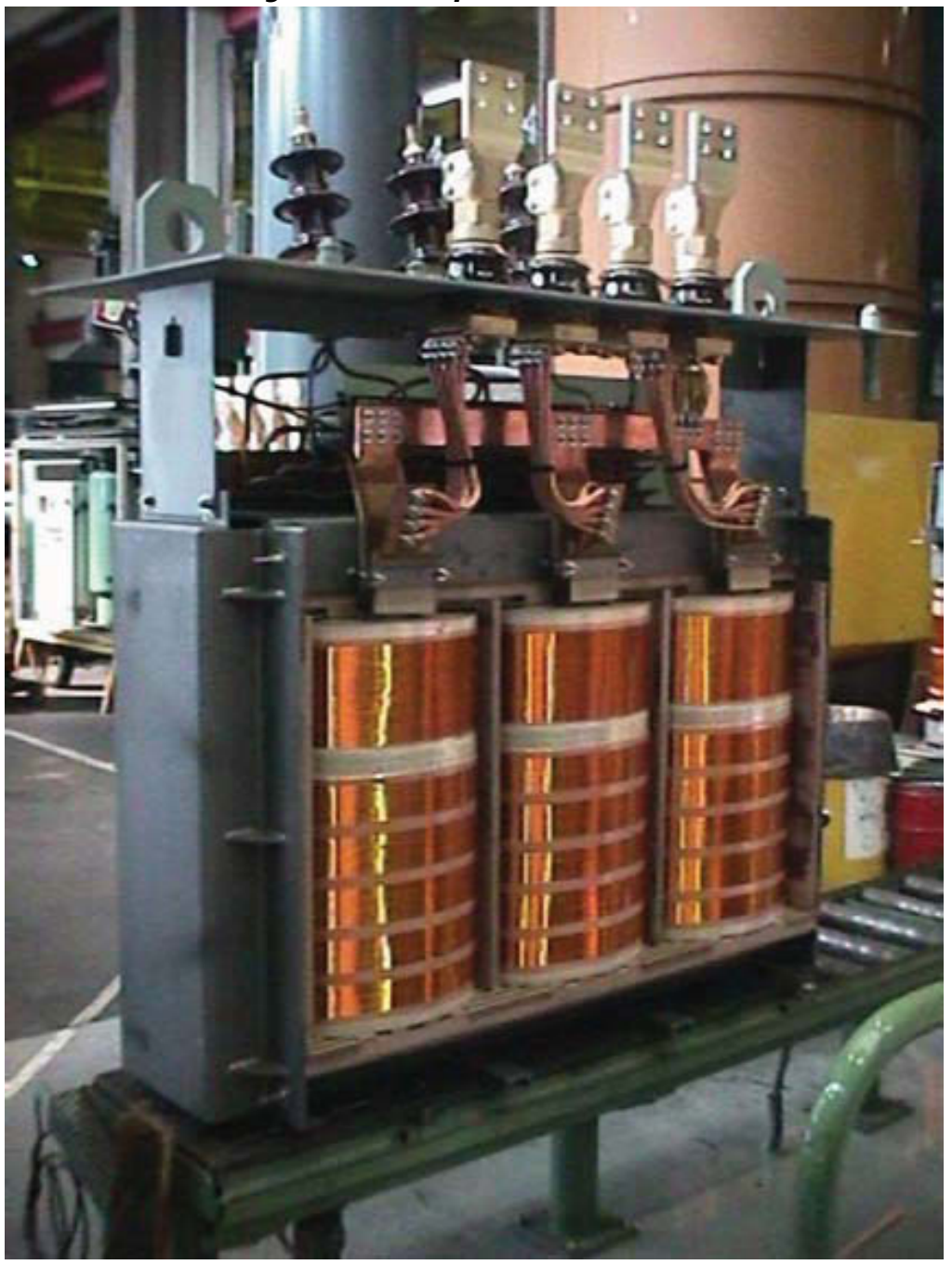


transformers.

Figure 10 shows a 1.6 MVA transformer, with an amorphous iron core, which was produced in 1998 for use in an engine plant in Waterford, Ireland. The load loss for this transformer is $18.2 \mathrm{~kW}$, and the noload loss as low as $384 \mathrm{~W}$ (80\% lower than the typical losses of this size traditional transformer)

\section{Policy support proposed by SEEDT}

The main types of market actors having a direct influence on the decision to purchase a distribution transformer are electricity distribution companies and end users in industry and commerce, as well

Figure 11. Energy-efficient transformers can help our environment.

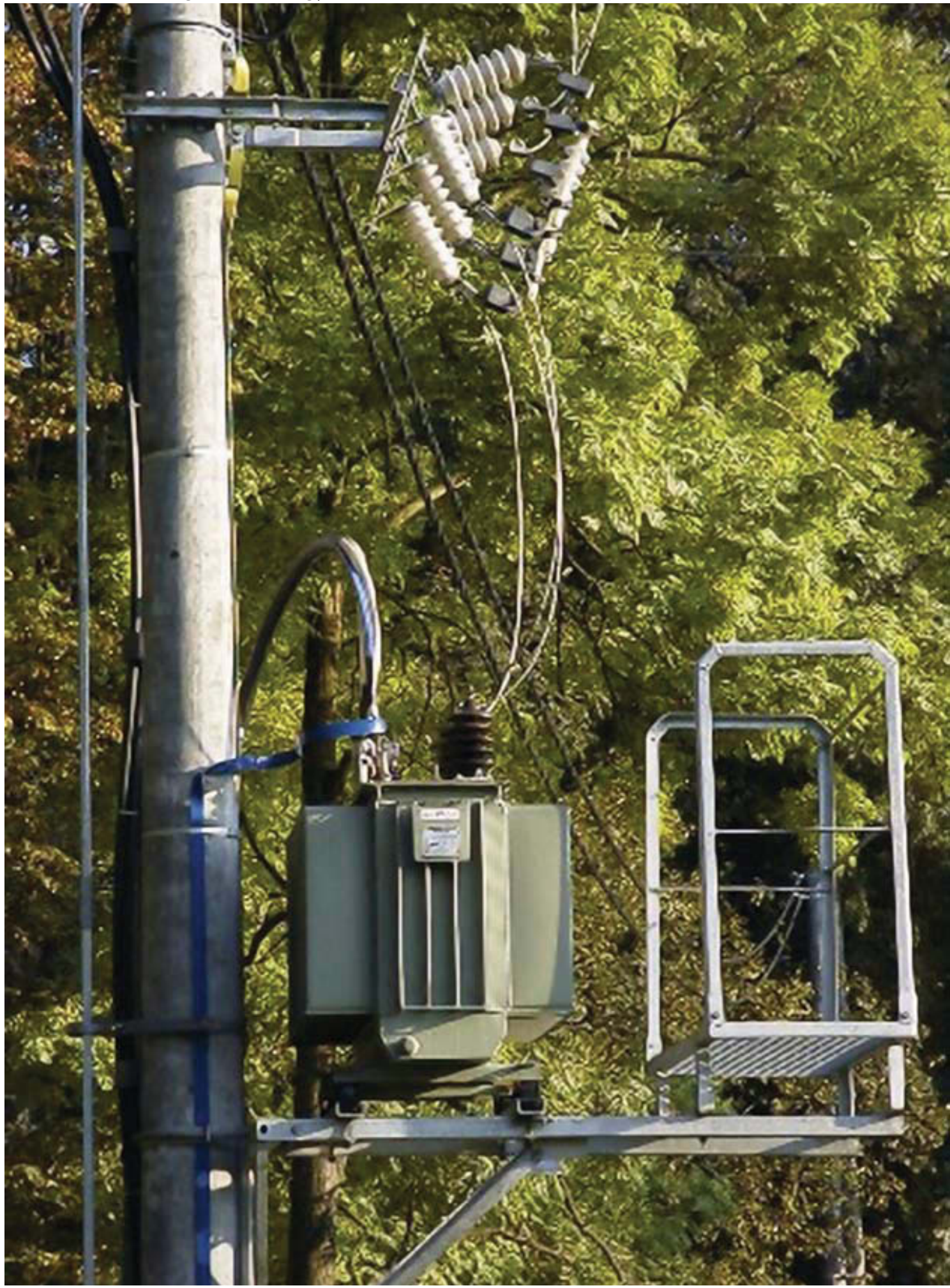


as the engineering firms, energy service companies and consultants who advise to them in the planning and tendering phases of procurement. These market actors face different barriers and obstacles with regard to the development, planning, sales and purchase of energy-efficient distribution transformers. They currently do not receive any support to realise energy-efficient targets.

Therefore, in order to adequately address and overcome these barriers and obstacles and to realise the existing energy efficiency potentials in this field, different policies and measures are needed. These policies and measures should be bundled in an appropriate policy-mix. The SEEDT project proposes:

- Changes in the regulatory schemes are needed to remove disincentives and provide incentives to increase the use of energy-efficiency of distribution transformers by electricity distribution companies. The income of, and investment by, electricity distribution companies is mainly controlled by regulation due to the fact that distribution grids are in most cases natural monopolies. Therefore, the regulation scheme will have a large impact on the investment decisions of electricity distribution companies, including whether or not to buy energy-efficient distribution transformers. In most European Member States, the regulatory mechanisms currently in place do not give any incentives and may even provide a disincentive to the purchase of energy-efficient distribution transformers by electricity distribution companies. Until changes are made to these regulatory regimes, additional fiscal or financial incentives will be needed to offset the current disincentives.

- A bundle of "soft" measures such as:

- the requirement for clearly visible nameplate information,

- a labelling scheme,

- the inclusion in energy advice and audit programmes and

- the provision of a toolkit for buyers.

- Would particularly address those market actors who lack information and knowledge or who tend to follow traditional purchasing routines that do not lead to least-cost solutions. This particularly affects small and medium industry and commerce, but also some smaller electricity distribution companies, engineering firms, energy service companies, energy consultants and planners.
- A European mandatory standard would effectively contribute to realising the saving potentials by addressing the same market actors as the bundle of "soft" measures. A mandatory standard makes it necessary that the regulation of electricity distribution acknowledges the higher investment costs needed for the more efficient distribution transformers and would help Europe to catch up with the developments in the US and in Asia.

- All market actors can implement demonstration or pilot projects together with manufacturers (and their suppliers), but larger companies, particularly, would benefit from increased availability of R\&D support.

In total, very roughly estimated, up to about $10 \mathrm{TWh}$ electricity savings could be realised, per year, by 2025, if the policies and measures proposed by the SEEDT project were broadly implemented, and if general development of the electricity system followed European trend scenarios. These potentials can be realised with technology already available today.

The calculations clearly show that changes in the regulatory schemes are most important to realise the existing saving potentials and to enable investments in transformers with the lowest lifecycle costs. As long as disincentives remain and positive incentives are missing, additional financial or fiscal incentives for electricity distribution companies should be introduced. The largest absolute electricity saving potentials in electricity distribution companies seem to be in France and UK, followed by Spain, Italy and Germany. Therefore, changes in the regulatory schemes are most urgent and should be implemented particularly in these countries.

Compared to saving potentials in other areas, the electricity saving potentials of distribution transformers seem to be small. Nevertheless, every contribution to climate change mitigation and energy security is necessary, particularly if it is economical. Since, in many cases, energy-efficient transformers are economical, it is recommended that the policies and measures proposed by the SEEDT project are implemented. In particular, if avoided external costs were included, or if electricity prices increase compared to the assumptions taken in the SEEDT project, the economic results would be even more favourable for energy-efficient distribution transformers. 


\section{Conclusions}

Distribution transformers today can be more efficient than ever, and at the same time economical. Market reality shows that amorphous technology can be even more efficient and not excessively costly.

Electricity distribution companies and commercial and industrial users should use the methods discussed in Chapter 4 to make transformer purchasing decisions. Total cost of ownership and life cycle cost are important concepts, embodied in the capitalisation formula, that allow losses over the whole of the expected transformer life cycle to be taken into account.

The main barrier to the reduction of transformer losses in electricity distribution companies is regulation. It changes frequently - compared to the lifetime of infrastructure investments - and the changes do not really address transformer losses. The removal of real disincentives in existing regulation is necessary to make a first critical step in transformer efficiency improvement. Additional incentives to encourage investment in energy-efficient transformers should then follow to accelerate market transformation.

Transformer manufacturers offer many choices. Users can usually find sufficient information about transformer operating conditions and economic parameters to make rational decisions. Adequate policies and measures could support end users in this process.

Making appropriate investment in an energyefficient transformer means reducing energy losses and reducing the environmental burden, together with reducing life cycle costs and thus increasing profitability.
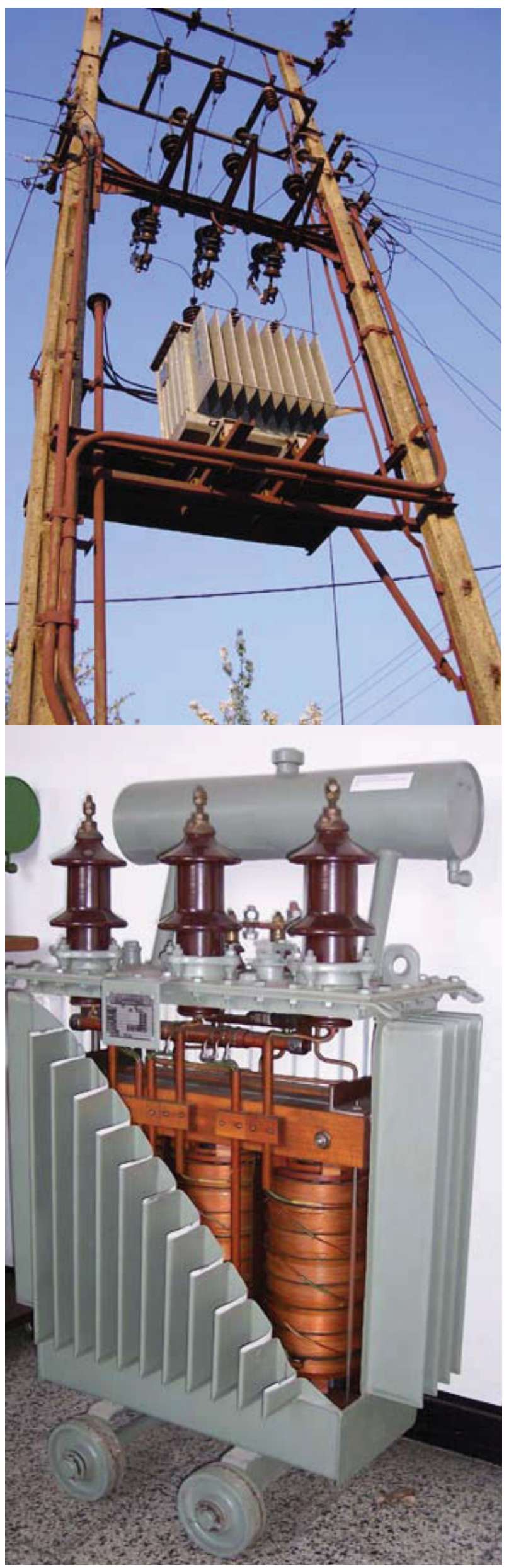


\section{Bibliography}

Energy saving in industrial distribution transformers

W.T.J. Hulshorst, J.F. Groeman

KEMA, 2002

Energy-efficient Transformers

Barry W. Kennedy, Mc Graw

Hill, 1998

The cost of capitalised transformer - compromise between transformer producer and user

MSc. Eng. Jacek DZIURA, E-mail: jacek.dziura@areva-td.com, PhD eng. Dariusz Spałek,

Polish “Transformator 2006" Conference Article

Report on analysis of existing situation of energy-efficient transformers, technical and non technical solutions Roman Targosz

et al 2007 SEEDT D1 Report

Policies and Measures Fostering Energy-Efficient Distribution Transformers

Wolfgang Irrek

et al 2007 SEEDT D6 Report

HD-428 S1, Three phase oil immersed distribution transformers $50 \mathrm{~Hz}$, Part 1 with later changes Cenelec, 1992

HD 538 - Three phase dry type distribution transformers $50 \mathrm{~Hz}$.

Cenelec, 1992

EN 50464-1, Three phase oil immersed distribution transformers $50 \mathrm{~Hz}$

Cenelec, 2005

\section{http://seedt.ntua.gr/}

\section{http://www.leonardo-energy.org/drupal/transformers}


European Distribution Transformer Loss standards:

Table A. 1 HD428/HD538

\begin{tabular}{|c|c|c|c|c|c|c|c|c|}
\hline \multirow{2}{*}{$\begin{array}{c}\text { RATED } \\
\text { POWER }\end{array}$} & \multicolumn{4}{|c|}{ Load Losses for Distribution Transformers } & \multicolumn{3}{c|}{ No-Load Losses for Distribution Transformers } \\
\cline { 2 - 11 } & \multicolumn{3}{|c|}{ OIL-FILLED (HD428) UP TO 24kV } & $\begin{array}{c}\text { DRY TYPE } \\
\text { (HD538) }\end{array}$ & OIL-FILLED (HD428) UP TO 24kV & $\begin{array}{c}\text { DRY TYPE } \\
\text { (HD538) }\end{array}$ \\
\cline { 2 - 11 } & LIST A & LIST B & LISTC & 12 kV PRIMARY & LIST A' & LIST B' & LIST C' & 12kV PRIMARY \\
\hline kVA & W & W & W & W & W & W & W & W \\
\hline 50 & 1100 & 1350 & 875 & N/A & 190 & 145 & 125 & N/A \\
\hline 100 & 1750 & 2150 & 1475 & 2000 & 320 & 260 & 210 & 440 \\
\hline 160 & 2350 & 3100 & 2000 & 2700 & 460 & 375 & 300 & 610 \\
\hline 250 & 3250 & 4200 & 2750 & 3500 & 650 & 530 & 425 & 820 \\
\hline 400 & 4600 & 6000 & 3850 & 4900 & 930 & 750 & 610 & 1150 \\
\hline $630 / 4 \%$ & 6500 & 8400 & 5400 & 7300 & 1300 & 1030 & 860 & 1500 \\
\hline $630 / 6 \%$ & 6750 & 8700 & 5600 & 7600 & 1200 & 940 & 800 & 1370 \\
\hline 1000 & 10500 & 13000 & 9500 & 10000 & 1700 & 1400 & 1100 & 2000 \\
\hline 1600 & 17000 & 20000 & 14000 & 14000 & 2600 & 2200 & 1700 & 2800 \\
\hline 2500 & 26500 & 32000 & 22000 & 21000 & 3800 & 3200 & 2500 & 4300 \\
\hline
\end{tabular}

Table A.2 EN 50464-1

No load losses $P(W)$ and sound power level ( $L w$ ) for $U \leq 24 \mathrm{kV}$

\begin{tabular}{|c|c|c|c|c|c|c|c|c|c|c|c|}
\hline $\begin{array}{l}\text { Rated } \\
\text { power }\end{array}$ & \multicolumn{2}{|c|}{ E0 } & \multicolumn{2}{|c|}{ Do } & \multicolumn{2}{|c|}{$\mathrm{CO}$} & \multicolumn{2}{|c|}{ B0 } & \multicolumn{2}{|c|}{ A0 } & $\begin{array}{l}\text { Short circuit } \\
\text { impedance }\end{array}$ \\
\hline \multirow{2}{*}{ kVA } & P0 & LwA & P0 & LwA & P0 & LwA & P0 & LwA & P0 & LwA & \multirow{2}{*}{$\%$} \\
\hline & W & $\mathrm{dB}(\mathrm{A})$ & W & $d B(A)$ & W & $\mathrm{dB}(\mathrm{A})$ & W & $d B(A)$ & W & $\mathrm{dB}(\mathrm{A})$ & \\
\hline 50 & 190 & 55 & 145 & 50 & 125 & 47 & 110 & 42 & 90 & 39 & \multirow{8}{*}{4} \\
\hline 100 & 320 & 59 & 260 & 54 & 210 & 49 & 180 & 44 & 145 & 41 & \\
\hline 160 & 460 & 62 & 375 & 57 & 300 & 52 & 260 & 47 & 210 & 44 & \\
\hline 250 & 650 & 65 & 530 & 60 & 425 & 55 & 360 & 50 & 300 & 47 & \\
\hline 315 & 770 & 67 & 630 & 61 & 520 & 57 & 440 & 52 & 360 & 49 & \\
\hline 400 & 930 & 68 & 750 & 63 & 610 & 58 & 520 & 53 & 430 & 50 & \\
\hline 500 & 1100 & 69 & 880 & 64 & 720 & 59 & 610 & 54 & 510 & 51 & \\
\hline 630 & 1300 & 70 & 1030 & 65 & 860 & 60 & 730 & 55 & 600 & 52 & \\
\hline 630 & 1200 & 70 & 940 & 65 & 800 & 60 & 680 & 55 & 560 & 52 & \multirow{7}{*}{6} \\
\hline 800 & 1400 & 71 & 1150 & 66 & 930 & 61 & 800 & 56 & 650 & 53 & \\
\hline 1000 & 1700 & 73 & 1400 & 68 & 1100 & 63 & 940 & 58 & 770 & 55 & \\
\hline 1250 & 2100 & 74 & 1750 & 69 & 1350 & 64 & 1150 & 59 & 950 & 56 & \\
\hline 1600 & 2600 & 76 & 2200 & 71 & 1700 & 66 & 1450 & 61 & 1200 & 58 & \\
\hline 2000 & 3100 & 78 & 2700 & 73 & 2100 & 68 & 1800 & 63 & 1450 & 60 & \\
\hline 2500 & 3500 & 81 & 3200 & 76 & 2500 & 71 & 2150 & 66 & 1750 & 63 & \\
\hline
\end{tabular}


Table A.3 EN 50464-1

Load losses Pk (W) at $75^{\circ} \mathrm{C}$ for Um $\leq 24 \mathrm{kV}$

\begin{tabular}{|c|c|c|c|c|c|}
\hline $\begin{array}{l}\text { Rated } \\
\text { power }\end{array}$ & Dk & $\mathrm{Ck}$ & Bk & Ak & $\begin{array}{l}\text { Short circuit } \\
\text { impedance }\end{array}$ \\
\hline KVA & W & W & W & W & $\%$ \\
\hline 50 & 1350 & 1100 & 875 & 750 & \multirow{8}{*}{4} \\
\hline 100 & 2150 & 1750 & 1475 & 1250 & \\
\hline 160 & 3100 & 2350 & 2000 & 1700 & \\
\hline 250 & 4200 & 3250 & 2750 & 2350 & \\
\hline 315 & 5000 & 3900 & 3250 & 2800 & \\
\hline 400 & 6000 & 4600 & 3850 & 3250 & \\
\hline 500 & 7200 & 5500 & 4600 & 3900 & \\
\hline 630 & 8400 & 6500 & 5400 & 4600 & \\
\hline 630 & 8700 & 6750 & 5600 & 4800 & \multirow{7}{*}{6} \\
\hline 800 & 10500 & 8400 & 7000 & 6000 & \\
\hline 1000 & 13000 & 10500 & 9000 & 7600 & \\
\hline 1250 & 16000 & 13500 & 11000 & 9500 & \\
\hline 1600 & 20000 & 17000 & 14000 & 12000 & \\
\hline 2000 & 26000 & 21000 & 18000 & 15000 & \\
\hline 2500 & 32000 & 26500 & 22000 & 18500 & \\
\hline
\end{tabular}

Table A.4 EN 50464-1

Load losses Pk36 (W) at $75^{\circ} \mathrm{C}$ for Um $=36 \mathrm{kV}$

\begin{tabular}{|c|c|c|c|c|}
\hline $\begin{array}{l}\text { Rated } \\
\text { power }\end{array}$ & Ck36 & Bk36 & Ak36 & $\begin{array}{l}\text { Short-circuit } \\
\text { impedance }\end{array}$ \\
\hline KVA & W & W & W & $\%$ \\
\hline 50 & 1450 & 1250 & 1050 & \multirow{6}{*}{4 or 4,5} \\
\hline 100 & 2350 & 1950 & 1650 & \\
\hline 160 & 3350 & 2550 & 2150 & \\
\hline 250 & 4250 & 3500 & 3000 & \\
\hline 400 & 6200 & 4900 & 4150 & \\
\hline 630 & 8800 & 6500 & 5500 & \\
\hline 800 & 10500 & 8400 & 7000 & \multirow{6}{*}{6} \\
\hline 1000 & 13000 & 10500 & 8900 & \\
\hline 1250 & 16000 & 13500 & 11500 & \\
\hline 1600 & 19200 & 17000 & 14500 & \\
\hline 2000 & 24000 & 21000 & 18000 & \\
\hline 2500 & 29400 & 26500 & 22500 & \\
\hline
\end{tabular}

Table A.5 EN 50464-1

No load losses P036 (W) and sound power level ( $(\mathrm{Ww}(A))$ for Um = $36 \mathrm{kV}$

\begin{tabular}{|c|c|c|c|c|c|c|c|}
\hline Rated power & \multicolumn{2}{|c|}{ C036 } & \multicolumn{2}{|c|}{ B036 } & \multicolumn{2}{|c|}{ A036 } & $\begin{array}{l}\text { Short-circuit } \\
\text { impedance }\end{array}$ \\
\hline \multirow{2}{*}{ kVA } & P0 & LwA & P0 & LwA & P0 & LwA & \multirow{2}{*}{$\%$} \\
\hline & W & $d B(A)$ & W & $d B(A)$ & W & $d B(A)$ & \\
\hline 50 & 230 & 52 & 190 & 52 & 160 & 50 & \multirow{6}{*}{4 or 4,5} \\
\hline 100 & 380 & 56 & 320 & 56 & 270 & 54 & \\
\hline 160 & 520 & 59 & 460 & 59 & 390 & 57 & \\
\hline 250 & 780 & 62 & 650 & 62 & 550 & 60 & \\
\hline 400 & 1120 & 65 & 930 & 65 & 790 & 63 & \\
\hline 630 & 1450 & 67 & 1300 & 67 & 1100 & 65 & \\
\hline 800 & 1700 & 68 & 1500 & 68 & 1300 & 66 & \multirow{6}{*}{6} \\
\hline 1000 & 2000 & 68 & 1700 & 68 & 1450 & 67 & \\
\hline 1250 & 2400 & 70 & 2100 & 70 & 1750 & 68 & \\
\hline 1600 & 2800 & 71 & 2600 & 71 & 2200 & 69 & \\
\hline 2000 & 3400 & 73 & 3150 & 73 & 2700 & 71 & \\
\hline 2500 & 4100 & 76 & 3800 & 76 & 3200 & 73 & \\
\hline
\end{tabular}





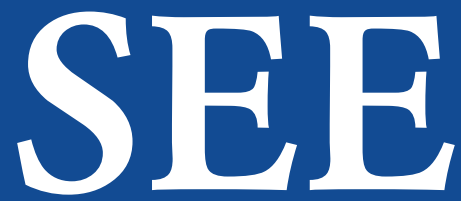

Intelligent Energy Europe

Strategies for development and diffusion of Energy Efficient Distribution Transformers

Technical guide prepared by:

- Roman Targosz, PCPC, targosz@pcpm.pl

- Frangiskos Topalis, NTUA, topalis@ieee.org

-Wolfgang Irrek, Wuppertal Institute, wolfgang.irrek@wupperinst.org

with collaboration of SEEDT project Partners:

- Andrzej Baginski, Andrzej.Baginski@lze.lodz.pl

- Constantinos Bouroussis, costisb@mail.ntua.gr

- Jacques-0livier BUDIN, Jacques-Olivier.Budin@ademe.fr

- Juan Frau, jfrau@gesa.es

- Jordi Gutierrez, jgutierrez@gesa.es

- Anne Rialhe, a.rialhe@aere.fr

- Arkaduisz Klimowicz, arkadiusz.klimowicz@areva-td.com

- Sebastien Kraft, s.kraft@aere.fr

- Theni Oikonomou, theni@thelcon.gr

- Reijalt Marieke, marieke@h2it.org

- Janusz Sobota, janusz.sobota@areva-td.com

- Ilias Sofronis, sofronis@thelcon.gr

- Gerhard Wohlauf, gerhard.wohlauf@wupperinst.org

and SEEDT supporters:

- Zbigniew Szymanski, zbigniew.szymanski@elektryka.com.pl

- Souflaris Thanassis, thanassis.souflaris@gr.schneider-electric.com

Authors give thanks to:

- David Chapman, david.chapman@copperdev.co.uk

- Jacek Dziura, jacek.dziura@areva-td.com

- Stefan Fassbinder, sfassbinder@kupferinstitut.de

- Ivo Pinkiewicz, pinkiewicz@ienot.com.pl

for their review and help in preparation of this guide.

Project status: June 2008

Project co-ordinator: Ass. Professor Frangiskos V. Topalis

National Technical University of Athens (NTUA)

topalis@softlab.ece.ntua.gr

+302107723627

Greece

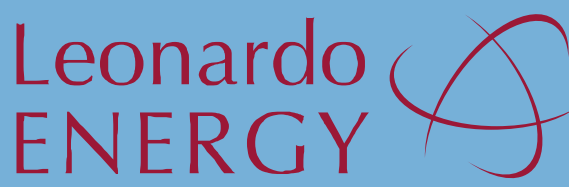

Project website: www.seedt.ntua.gr

www.leonardo-energy.org

Intelligent Energy 


\section{Contents}

\section{Pad-Mounted Transformers}

General Description .............................. 17.0-2

Introduction ............ 17.0

Application. ............. 17.0.2

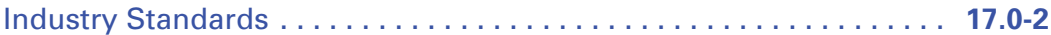

Ratings . . . . . . . . . . . . . . . .

Design Impedances. . . . . . . . . . . . . . . . . . . . . . . .

Application Limitations. . . . . . . . . . . . . . . . . . . . . . . . . 17.0-2

Standard Features. . . . . . . . . . . . . . . . . . . . . . . . 17.0-3

Options................................. 17.0.3

Transformer Cooling Classes . . . . . . . . . . . . . . . . . . .

NEC Requirement Guidelines. . . . . . . . . . . . . . . . 17.0-5

Seismic Qualification ........................ 17.0.5

Primary Protection Options . . . . . . . . . . . . . . . . . . . 17.0-6

Primary Switching Options $\ldots \ldots \ldots \ldots \ldots \ldots \ldots \ldots \ldots \ldots \ldots \ldots$ 17.0-8

Loadbreak Wells and Inserts ........................ 17.0-9

Primary Fuse Details . . . . . . . . . . . . . . . . . . . . . . . . 17.0-10

Layout Data $75-2500$ kVA . . . . . . . . . . . . . . . . . . . . . . . . 17.0 11

Technical Data 75-2500 kVA ......................... 17.0.12

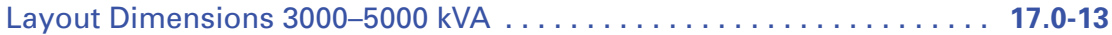

Technical Data 3000-5000 kVA ... . . . . . . . . . . . . . . . . . . . 17.0-14

\section{Specifications}

See Eaton's Product Specification Guide, available on CD or on the Web.

CSI Format. 1995

2010

Section 16321 Section 261219

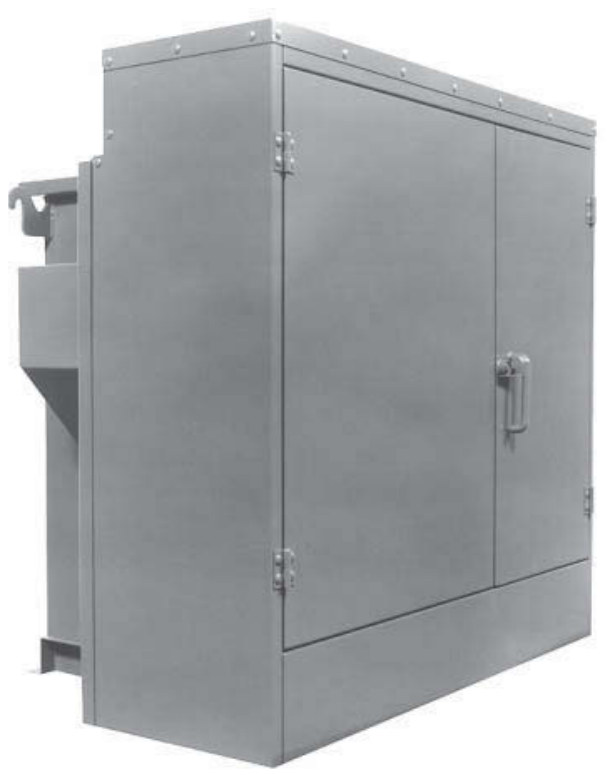




\section{Three-Phase}

\section{Pad-Mounted Transformers}

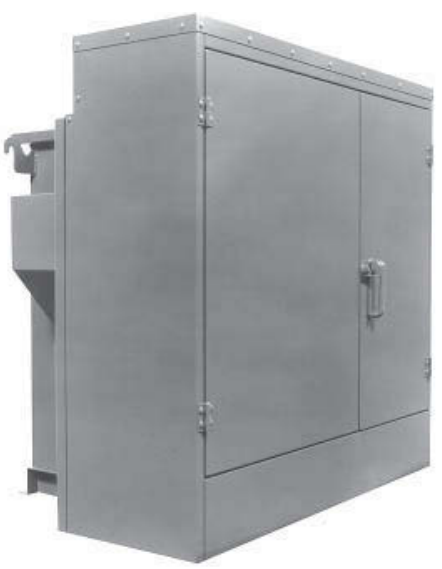

Typical Pad-Mounted Transformer

\section{Introduction}

Eaton's three-phase pad-mounted transformer is offered in a variety of designs and configurations. The following pages describe the standard designs and the common options that are available.

Some special designs and options may require additional engineering, factory coordination, unusual application requirements or special manufacturing needs.

Higher impedances limit secondary fault currents such that coordination with secondary low voltage moldedcase circuit breakers is usually possible. (Low impedances are also available if required for paralleling, and so on.)

Standard color is pad-mounted green [Munsel|® Green (\#7GY3.29/1.5)]. ANSI \#24, 61 and 70 are available as options.

\section{Application}

Liquid-filled, three-phase, commercial pad-mounted distribution transformers are designed for servicing such underground distribution loads as shopping centers, schools, institutions and industrial plants. They are available in both livefront and deadfront construction, for radial or loop-feed applications, with or without taps.

\section{Industry Standards}

Pad-mounted transformers meet industry standards: IEEE ${ }^{\circledR}$ C57.12.00, IEEE C57.12.34, IEEE C57.12.28, IEEE C57.12.29, IEEE C57.12.70, IEEE C57.12.80, IEEE C57.12.90, IEEE C57.91 and NEMA ${ }^{\circledR}$.

\section{Ratings}

- 75-5000 kVA

- High voltages (primary): $\begin{array}{ll}4160 \mathrm{Grd} . \mathrm{Y} / 2400 & 2400 \Delta \\ \text { through } & \text { through } \\ 34,500 \mathrm{Grd} . \mathrm{Y} / 19,920 & 34,500 \Delta\end{array}$

- HV Taps: $2-2-1 / 2 \%$ above and below normal, or $4-2-1 / 2 \%$ below normal

- Standard BIL levels: kV Class BIL (kV) $\begin{array}{rr}1.2 & 30 \\ 2.5 & 45 \\ 5.0 & 60 \\ 8.7 & 75 \\ 15.0 & 95 \\ 25.0 \text { Grd. Y Only } & 125 \\ 25.0 & 150 \\ 34.5 \text { Grd. Y Only } & 150 \\ 34.5 & 150\end{array}$

Low voltages (secondary). All voltages through $5 \mathrm{kV}$ class

- UL labeling available

- Factory Mutual labeling available

\section{Design Impedances}

Impedances are supplied to meet IEEE C57.12.00 standards. Customerspecified impedances are available. (Subject to IEEE/ANSI $\pm 7.5 \%$ impedance tolerance.)

- Typical design impedances:
kVA

Note: Subject to NEMA/IEEE $\pm 7.5 \%$ impedance tolerance.

Note: Non-standard design impedance may be obtained by contacting Eaton.

\section{Application Limitations}

The transformers described herein are designed for the application conditions normally encountered on electric power distribution systems. As such, they are suitable for use under the "usual service conditions" described in IEEE Standard C57.12.00 general requirements for liquid-immersed distribution, power and regulating transformers.

Consult Eaton for unusual service conditions such as:

- Abnormal environmental conditions

- Unusual transient voltages present on the source voltage

- Frequent or planned throughfault duty

- Planned overloading unless in strict accordance with the IEEE loading guide (C57.91)

- Motors whose horsepower rating is greater than half the transformer kVA rating

- Unusual frequency of impact loading may occur when supplying welding apparatus, electric arc furnaces or motors with cyclical loads

- Loads involving abnormal harmonic or DC current that may result where appreciable load currents are controlled by solid-state or similar devices

These lists do not purport to cover all unusual conditions and applicable limitations. Other "unusual service conditions" are described in IEEE Standard C57.12.00.

Table 17.0-1. Temperature Guarantees

\begin{tabular}{|c|c|c|}
\hline Description & Ambient (1) & Rise (2) (3) \\
\hline $\begin{array}{l}\text { Standard } \\
\text { Optional }\end{array}$ & $\begin{array}{l}30^{\circ} \mathrm{C} \\
30^{\circ} \mathrm{C}\end{array}$ & $\begin{array}{l}65^{\circ} \mathrm{C} \\
55^{\circ} \mathrm{C}\end{array}$ \\
\hline \multicolumn{3}{|c|}{$\begin{array}{l}\text { (1) } 30^{\circ} \mathrm{C} \text { average ambient temperature of } \\
\text { cooling air not to exceed } 40^{\circ} \mathrm{C} \text { maximum } \\
\text { over any } 24 \text {-hour period. }\end{array}$} \\
\hline \multicolumn{3}{|c|}{$\begin{array}{l}\text { (2) Degree rise is the average winding } \\
\text { temperature rise by resistance. }\end{array}$} \\
\hline \multicolumn{3}{|c|}{$\begin{array}{l}\text { (3) A dual temperature rating of } 55^{\circ} / 65^{\circ} \mathrm{C} \text { adds } \\
12 \% \text { additional continuous capacity to the } \\
\text { base kVA rating of the transformer. }\end{array}$} \\
\hline \multicolumn{3}{|c|}{ Note: Altitudes not to exceed } \\
\hline
\end{tabular}

Fluids-Liquid Dielectric

The choice of fluid, mineral oil or less flammable natural esther fluid (BIOTEMP ${ }^{\circledR}$, Envirotemp FR3 ${ }^{\circledR}$ ) is made based upon site conditions and proximity to facility walls, windows and flammable structures, and environmentally sensitive areas.

Note: For additional information about transformer applications and types of insulating fluids, see Tab 14. 


\section{General Description}

\section{Standard Features}

(1) Four lifting hooks

(2) Bolted-on terminal compartment with removable front sill

(3) Hinged, lift-off cabinet doors

(4) Interlocked hex-head or penta-head bolt padlock handle operates a cam assembly that is part of the threepoint door latching mechanism

Hex-head or penta-head bolts must be removed from the flange formed on the steel high/low barrier before the HV door can be opened-not shown

Removable neutral ground strapnot shown

(5) Tank ground pads ( 1 in HV, 1 in LV)

(6) Steel high/low voltage compartment barrier

(7) Nameplate

(8) Fill plug and self-actuating pressure relief device

(9) Externally operated no load tap changer

(10) Drain valve and sampling device

\section{Options}

\section{Primary Termination}

(11) For livefront construction, externally clamped high voltage porcelain bushings double eye-bolt or spade for cable (75-225 kVA) or a single eyebolt or spade for cable (300-1500 kVA). Spade bushings are also offered

(12) For deadfront construction, externally clamped high voltage epoxy bushing wells for 200A loadbreak, or 600A non-loadbreak inserts

\section{Secondary Termination}

(13) NEMA spade terminals
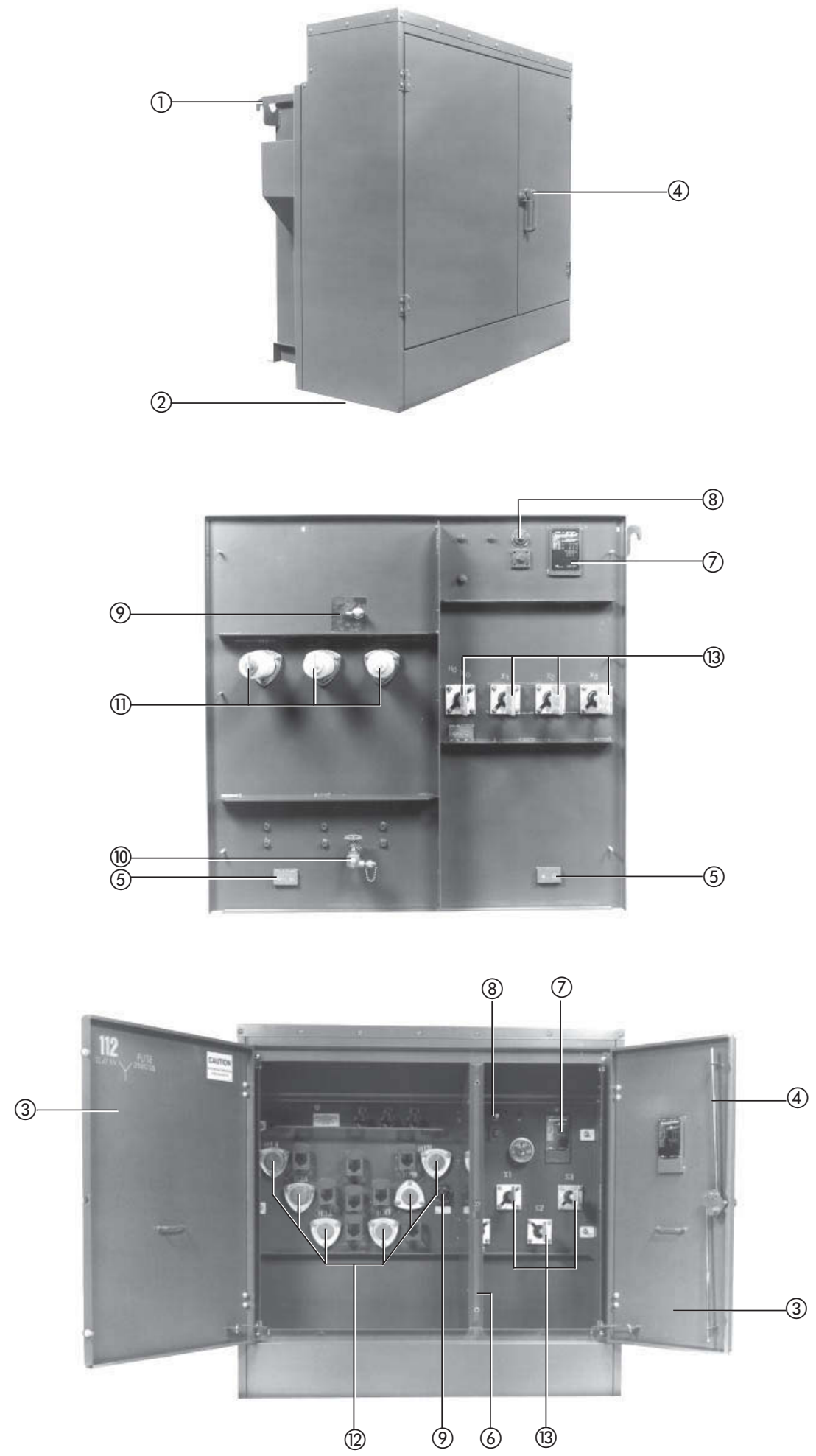

Primary and Secondary Compartment Features 


\section{0-4 Pad-Mounted Transformers}

General Description

Transformer Cooling Classes

Table 17.0-2. Fluids Advantages and Disadvantages

\begin{tabular}{|c|c|}
\hline Advantages & Disadvantages \\
\hline \multicolumn{2}{|l|}{ Mineral Oil } \\
\hline $\begin{array}{l}\text { Low transformer cost } \\
\text { Good dielectric performance } \\
\text { Low maintenance cost } \\
\text { Good heat dissipation } \\
\text { Good cold climate performance } \\
\text { Preventative maintenance-DGA historical data available }\end{array}$ & $\begin{array}{l}\text { - Higher installation cost } \\
\text { - Vaults required for indoor installations per code low fire point }-160^{\circ} \mathrm{C} \\
\text { - }<30 \% \text { biodegradability }\end{array}$ \\
\hline \multicolumn{2}{|l|}{ Silicone Fluid } \\
\hline $\begin{array}{l}\text { Low heat release } \\
\text { Reduced smoke } \\
\text { Low flame } \\
\text { Self extinguishing } \\
\text { Good dielectric performance } \\
\text { Low toxicity } \\
\text { Moderate viscosity } \\
\text { High stability }\end{array}$ & $\begin{array}{l}\text { - Non-biodegradable } \\
\text { - Not suitable for use with internal Bay-O-Net fuses } \\
\text { - Transformer cost } \\
\text { - Disposal cost } \\
\text { - Viton gaskets required } \\
\text { - Retrofil applications } \\
\text { - High transformer cost } \\
\text { - High moisture absorption }\end{array}$ \\
\hline \multicolumn{2}{|l|}{ Environmentally Friendly Fluids } \\
\hline $\begin{array}{l}\text { - High fire point }-360^{\circ} \mathrm{C} \\
\text { - High flash point- } 343^{\circ} \mathrm{C} \\
\text { - Compatible with mineral oil } \\
\text { Excellent retrofil fluid (compatible with oil up to a } 10 \% \text { mixture) } \\
\text { - Excellent dielectric performance } \\
\text { - } 97 \% \text { biodegradable } \\
\text { Renewable resource } \\
\text { - Greater tolerance to moisture }\end{array}$ & $\begin{array}{l}\text { - Transformer cost (lower than silicone fluid) } \\
\text { Pour point }\left(-15^{\circ} \text { to }-25^{\circ} \mathrm{C}\right) \text { transformer energized with full load with top } \\
\text { oil temperature at }-50^{\circ} \mathrm{C} \text { with no problems-no crystals formed at }-68^{\circ} \mathrm{C}\end{array}$ \\
\hline
\end{tabular}

Table 17.0-3. Fluid Properties Comparison

\begin{tabular}{|c|c|c|c|}
\hline Property & $\begin{array}{l}\text { Mineral } \\
\text { Oil }\end{array}$ & $\begin{array}{l}\text { Silicone } \\
\text { Fluid }\end{array}$ & $\begin{array}{l}\text { Environmentally Friendly } \\
\text { Fluids }\end{array}$ \\
\hline $\begin{array}{l}\text { Specific gravity } \\
\text { Flash point }{ }^{\circ} \mathrm{C} \\
\text { Fire point }{ }^{\circ} \mathrm{C}\end{array}$ & $\begin{array}{l}0.91 \\
145 \\
160\end{array}$ & $\begin{array}{l}0.96 \\
300 \\
330\end{array}$ & $\begin{array}{l}0.91 \\
343 \\
360\end{array}$ \\
\hline $\begin{array}{r}\text { Viscosity (cSt.) } 100^{\circ} \mathrm{C} \\
40^{\circ} \mathrm{C} \\
0^{\circ} \mathrm{C}\end{array}$ & $\begin{array}{l}3 \\
12 \\
76\end{array}$ & $\begin{array}{l}16 \\
38 \\
90\end{array}$ & $\begin{array}{l}10 \\
45 \\
300\end{array}$ \\
\hline $\begin{array}{l}\text { Pour point }{ }^{\circ} \mathrm{C} \\
\text { Dielectric strength, } \mathrm{kV} \\
\text { Dissipation factor }(\%) 25^{\circ} \mathrm{C}\end{array}$ & \begin{tabular}{|l|}
-40 \\
30 \\
0.05
\end{tabular} & \begin{tabular}{|l|}
-55 \\
4.3 \\
0.01
\end{tabular} & $\begin{array}{l}-15 \text { to } 25 \\
49 \\
0.025-0.05\end{array}$ \\
\hline \begin{tabular}{|l|} 
Permittivity \\
Resistivity \\
Oxidation inhibitor \\
Biodegradability
\end{tabular} & $\begin{array}{l}2.2 \\
10^{13} \\
\text { Optional } \\
<30 \%\end{array}$ & \begin{tabular}{l|}
2.7 \\
$10^{14}$ \\
No \\
$0 \%$
\end{tabular} & $\begin{array}{l}3.1 \\
10^{13} \\
\text { Required } \\
97 \%\end{array}$ \\
\hline
\end{tabular}




\section{NEC Requirement Guidelines for the Installation of Listed Less-Flammable Liquid-Filled Transformers}

\section{NEC (NFPA) Recognition}

These guidelines focus on the requirements of Article 450.23 of the National Electrical Code ${ }^{\circledR}$ (NEC ${ }^{\circledR}$ ) for the installation of less-flammable liquid-insulated transformers. Lessflammable liquids are used in transformers where an extra margin of fire safety is important. Typical applications include installations indoors, on rooftops, near buildings, bush and forest fire prone areas and in pedestrian traffic areas.

Less-flammable liquids, also known as high fire point liquids, are transformer dielectric coolants that have a minimum fire point of $300^{\circ} \mathrm{C}$. Commonly used fire-resistant fluids include dimethysiloxane and ester-based fluids. Two Nationally Recognized Testing Laboratories (NRTL); Underwriters Laboratories (UL) and FM Approvals (FM) currently list less-flammable liquids. They also list less-flammable liquid-filled transformers.

Less-flammable liquid-filled transformers were formally recognized by the NEC for indoor installation in 1978. In 1990, the NEC integrated specific less-flammable transformer requirements for outdoor installations for Article 450.23, in effect recognizing less-flammable transformers as inherently safer than conventional oil-filled transformers. Less-flammable transformers, long recognized as an additional safeguard for indoor installations, are becoming increasingly recognized for outdoor applications as well.

\section{General NEC Requirements}

The requirements and options for the different types of outdoor installations are outlined in Table 17.0-4. These guidelines also summarize the UL Classification and FM Approvals installation requirements for lessflammable fluids referred to as "listing" requirements in NEC 450.23.

In cases where the transformer installation presents a fire hazard, one or more of the following safeguards will be applied according to the degree of hazard involved:

1. Space requirements.

2. Fire-resistant barriers.

3. Automatic fire suppression systems.

4. Enclosures that confine the oil of a ruptured transformer tank.

NEC Article 450.28, Modification of Transformers, requires that when modifications are made to transformers

Table 17.0-4. NEC Article 450.23 Requirements

\begin{tabular}{|c|c|}
\hline $\begin{array}{l}\text { Installation } \\
\text { Type }\end{array}$ & $\begin{array}{l}\text { NEC } \\
\text { Requirements }\end{array}$ \\
\hline \multicolumn{2}{|l|}{ Outdoor Installations } \\
\hline $\begin{array}{l}\text { Non-combustible building } 1 \text { and no } \\
\text { combustible materials stored in area. }\end{array}$ & $\begin{array}{l}\text { Either of the following listing requirements (2): } \\
\text { Underwriters Laboratories } \\
\text { GM approvals }\end{array}$ \\
\hline $\begin{array}{l}\text { Combustible building (1) or combustible } \\
\text { materials stored in area. }\end{array}$ & $\begin{array}{l}\text { In accordance with NEC Article } 450.27 \text {, oil-insulated } \\
\text { transformers installed outdoors, i.e., space } \\
\text { separation, fire barriers or water spray systems. }\end{array}$ \\
\hline
\end{tabular}

in existing installations that change the transformer type, the transformers must be marked to show the type of insulating liquid installed and the installations must comply with current requirements of the NEC. Examples of changes include replacing a complete transformer (retrofitting) or replacement of the liquid only (retrofilling). Askarel (PCB) and conventional mineral oil-filled transformers are frequently retrofitted or retrofilled using lessflammable liquids. NEC 110.34 sets minimum clear work space dimensions around transformers.

\section{Seismic Qualification}

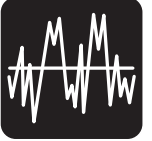

Refer to Tab 1 for information on seismic qualification for this and other Eaton products. Article 450.27." 


\section{Primary Overcurrent Protection Options}

Primary protective devices are applied to distribution transformers in order to:

1. Prevent injury to personnel.

2. Prevent or minimize damage to equipment.

3. Improve the continuity of service by selectively controlling outages.

Factors that affect the protection scheme are:

1. Industry standard.

2. Customer's specification.

3. Customer's system configuration (available fault current, system voltage, system connection, and so on.)

\section{Availability of equipment.}

The first consideration in determining the ampere rating of a fuse is to verify that the fuse in question is capable of withstanding typical inrush currents without element damage. When a transformer is energized, it is exposed to very large currents for very short periods of time. These currents are known as magnetizing inrush (or fuse withstand) and cold load pickup, and are a result of the transformer's magnetic circuit, the electrical system configuration and the connected load.

The second consideration for selecting the fuse ampere rating is the maximum load current the fuse is expected to carry without damage. Transformer fusing tables available from the manufacturer normally list the range of overload provided. If the longtime minimum melt current for a particular fuse size is known, it can be compared to the transformer rated current to determine the exact amount of overload permitted. An ambient of $25^{\circ}-40^{\circ} \mathrm{C}$ is generally assumed for application tables. Care should be taken when fuses are applied in higher ambient conditions, which will reduce the amount of overload permitted. An example of a high ambient condition used frequently in distribution transformers is that of current limiting fuses in dry-well canisters. To accommodate the overload and derating factors referred to, the following ratios are used on general-purpose CL fuses.
Nameplate current rating of fuse/ nameplate current rating of transformer $=1.25$ for enclosures surrounded by air (EFD, clip mount, arc-strangler) or $=1.35$ for enclosures surrounded by oil (canisters).

Derating factors are not applied to expulsion or backup CL fuses because high temperature has minimal effect on their operation.

Finally, it is necessary to verify that the fuse current rating under consideration will, in fact, operate prior to the transformer sustaining any permanent thermal damage (conductor or insulation burning or melting). This is done by comparing the total clearing characteristics of the fuse in question with the IEEE $\left(1^{2} \mathrm{t}\right)$ damage line.

It is important that the total clearing characteristics of the device under consideration lie to the left of the damage line for all expected values of fault current. Note that most fuse characteristics will cross the damage line at some point. It is important to make this occur at the lowest possible value of the current.

The interrupting rating of a device is a measure of the maximum symmetrical fault current at which the device can successfully clear a fault condition without excessive damage to itself, the equipment it is protecting or the surrounding environment.

It is extremely critical that the interrupting rating of a device be greater than the maximum available symmetrical fault current. For devices applied to the transformer primary, the maximum fault current must be supplied by the utility because this value is dependent on the electrical system configuration.

\section{Protective Fuse Link}

- Internal, oil-immersed, expulsion type

- Sized to operate only in the event of a winding failure, isolating the transformer from the primary system

- Interrupting rating is $3500 \mathrm{~A}$ at $8.3 \mathrm{kV}$

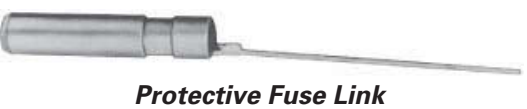

Bay-0-Net-Type Fuse

- Oil immersed, expulsion type

- Drawout for fuse replacement

- Hookstick operable, loadbreak design

- Available with either overloadsensing or fault sensing

- $3500 \mathrm{AIC}$ at $8.3 \mathrm{kV}, 1800 \mathrm{AIC}$ at $15.5 \mathrm{kV}$
Bay-O-Net fuse assemblies are used to protect transformers and distribution systems. They are designed for use in pad-mounted or sub-surface distribution transformers filled with transformer oil or approved equivalent. The assemblies combine the ease of hotstick operation with the safety of deadfront construction.

Removal of the fuse holder from the assembly indicates that the apparatus is electrically disconnected. It also allows convenient fuse element inspection and replacement. When typical safety practices are followed, the assemblies can be load-break operated for working on the transformer secondary; changing distribution voltage with dual voltage switches or tap changers; or disconnecting the apparatus from the line.

The optional Flapper'TM Bay-O-Net Assembly (available as sidewallmounted only) includes a flapper valve inside the housing, which closes when the fuse holder is removed, thus minimizing oil spillage.

Table 17.0-5. Bay-0-Net Fuse Electrical Ratings

\begin{tabular}{|l|l|}
\hline $\begin{array}{l}\text { kV } \\
\text { Rating }\end{array}$ & Specification \\
\hline & Electrical Ratings \\
\hline \begin{tabular}{|l|l|}
150 & BLL and full wave crest \\
50 & $60 \mathrm{~Hz}, \mathrm{AC}, 1-$-minute withstand \\
\hline
\end{tabular}
\end{tabular}

Maximum Single-Phase Interrupting Ratings (1)

\begin{tabular}{|l|l|}
\hline 8.3 & $\begin{array}{l}\text { 3000A rms asymmetrical- } \\
\text { cover mount }\end{array}$ \\
\cline { 2 - 2 } & $\begin{array}{l}\text { 3500A rms symmetrical- } \\
\text { sidewall mount }\end{array}$ \\
\hline 15.5 & $\begin{array}{l}2500 \mathrm{~A} \text { rms asymmetrical- } \\
\text { cover mount }\end{array}$ \\
\cline { 2 - 2 } & $\begin{array}{l}\text { 2500A rms symmetrical- } \\
\text { sidewall mount (2) }\end{array}$ \\
\hline 23.0 & $\begin{array}{l}1000 \mathrm{~A} \text { rms asymmetrical- } \\
\text { cover mount }\end{array}$ \\
\cline { 2 - 2 } & $\begin{array}{l}\text { 1000A rms symmetrical- } \\
\text { sidewall mount }\end{array}$ \\
\hline
\end{tabular}

Load Break Ratings (Phase-to-Phase at $\mathbf{8 0} \%$ PF)

\begin{tabular}{|l|l|}
\hline 10.0 & $160 \mathrm{~A}$ \\
15.5 & $150 \mathrm{~A}$ \\
26.7 & $80 \mathrm{~A}$ \\
34.5 & $50 \mathrm{~A}$ \\
\hline
\end{tabular}

are rated at $2000 \mathrm{~A}$ symmetrical.

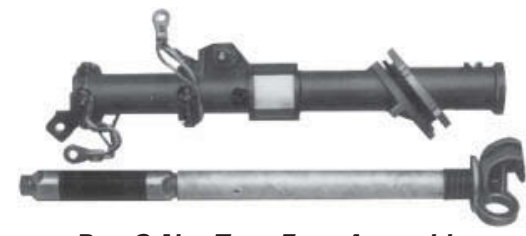

Bay-O-Net-Type Fuse Assembly 
General Description

\section{Current Limiting Fuses}

- Air immersed in drywell canister

- Drawout for fuse replacement

- Hookstick operable

- Limits both the current magnitude and energy associated with low impedance faults

- Effective in minimizing the probability of tank rupture due to internal, high energy, low impedance faults

- Available fuse interrupting ratings of 25,000-50,000A rms (symmetrical)

- Maximum fuse ampere rating at $15 \mathrm{kV}$ (2-50A fuses)

\section{Partial Range Current Limiting Fuses}

- Oil immersed, internally block mounted

- Applied in series with an expulsion type fuse (Bay-O-Net type-see above)

- Protection against tank rupture

The current-limiting backup fuse is used in series with low-current primary protection devices such as a Bay-O-Net fuse.

The fuse's highly efficient current-limiting section minimizes the effects of high fault current stresses on equipment and the distribution system. Its minimum interrupting rating is coordinated with that of a low current interrupter to avoid undesirable low current operation; yet its maximum interrupting rating will clear the highest fault currents likely to occur. Higher continuous current ratings can be achieved by connecting two fuses in parallel.

The current-limiting fuse is used in transformers to protect and isolate faulted equipment. When connected in series with a low current primary protection device, the fuse becomes an element of a two-part protection system that gives a full range of fault protection.

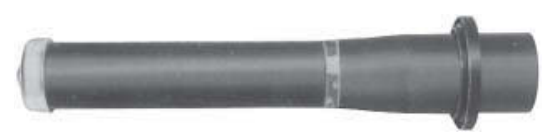

Drawout Current Limiting Fuse Canister

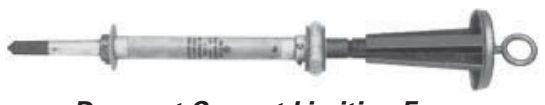

Drawout Current Limiting Fuse Loadbreak Assembly

Table 17.0-6. Current-Limiting Backup Fuse Electrical Ratings and Characteristics

\begin{tabular}{|l|l|}
\hline $\begin{array}{l}\text { Fuse } \\
\text { Type }\end{array}$ & $\begin{array}{l}\text { Maximum } \\
\text { Interrupting Current }\end{array}$ \\
\hline $\begin{array}{l}\text { Backup (partial range) } \\
\text { “C” rated }\end{array}$ & $\begin{array}{l}50,000 \mathrm{~A} \text { rms } \\
\text { symmetrical (1) }\end{array}$ \\
\hline
\end{tabular}

(1) See Table 17.0-8 for fuses with ratings other than 50,000 amperes rms symmetrical.
This two-part system provides low current protection with the replaceable expulsion fuse and it adds the energy-

limiting protection of a current-limiting fuse. Together, they coordinate easily with upstream and downstream devices.

Table 17.0-7. Two- and Four-Position, Load Break, Sectionalizing Switch

\begin{tabular}{|l|c|c|c|}
\hline Ratings & 200 Ampere & 300 Ampere & 600 Ampere \\
\hline Voltage kV phase-phase maximum & 35 & 25 & 15 \\
Voltage kV phase_ground maximum & 21.1 & 15.2 & 8.3 \\
Impulse withstand kV & 150 & 125 & 95 \\
\hline $60 \mathrm{~Hz}$ 1-minute withstand kV & 50 & 40 & 34 \\
Continuous current & 200 & 300 & 600 \\
Loadbreak & 200 & 300 & 600 \\
\hline Momentary, 10 Hz & 10,000 & 10,000 & 15,000 \\
2-second & 10,000 & 10,000 & 10,000 \\
3-shot make and latch ampere & 6000 & 10,000 & 10,000 \\
\hline
\end{tabular}

Table 17.0-8. Current-Limiting Backup Fuse Interrupting Ratings

\begin{tabular}{|l|l|l|l|}
\hline $\begin{array}{l}\text { Continuous Ampere } \\
\text { Current Rating }\end{array}$ & $\begin{array}{l}\text { Minimum Interrupting } \\
\text { (Amperes) }\end{array}$ & $\begin{array}{l}\text { Minimum Melt } \mathbb{R}^{2} t \\
\left(A^{2} \times \text { s }\right)\end{array}$ & $\begin{array}{l}\text { Maximum Clear } \mathbb{I}^{2} t \\
\left(A^{2} \times \text { s }\right)\end{array}$ \\
\hline
\end{tabular}

\begin{tabular}{|l|r|r|r|}
\hline 8.3 kV (2) \\
\hline 30 & 100 & 1200 & 5800 \\
40 & 125 & 1800 & 8200 \\
50 & 165 & 4100 & 16,500 \\
\hline 65 & 300 & 6200 & 26,700 \\
80 & 200 & 9600 & 42,900 \\
100 & 350 & 17,100 & 62,000 \\
\hline 125 & 375 & 30,500 & 97,800 \\
150 & 450 & 43,900 & 148,000 \\
165 & 500 & 68,600 & 245,000 \\
\hline 250 (3) & 800 & 122,000 & 369,000 \\
300 (3) & 1000 & 175,600 & 566,000 \\
$330^{(3)}$ & 1200 & 274,400 & 875,700 \\
\hline
\end{tabular}

\begin{tabular}{|l|l|l|l}
\hline $15.5 \mathrm{kV}(4)$ & \\
\hline
\end{tabular}

\begin{tabular}{|r|l|l|l|}
\hline 30 & 100 & 1200 & 7600 \\
40 & 150 & 1800 & 11,000 \\
50 & 200 & 4100 & 23,000 \\
\hline 65 & 350 & 6200 & 33,000 \\
80 & 250 & 9600 & 52,900 \\
100 & 350 & 17,100 & 93,800 \\
\hline 125 & 400 & 30,500 & 125,700 \\
150 & 450 & 43,900 & 162,300 \\
165 & - & - & - \\
\hline 250 (3) & 122,000 & 408,000 \\
$300^{3}$ & 1000 & 175,600 & 660,700 \\
$330^{3}$ & - & - & - \\
\hline
\end{tabular}

\begin{tabular}{|l|r|r|l|}
\hline $23 \mathbf{~ k V}$ (5) & 125 & 1200 & 10,500 \\
\hline 30 & 200 & 1800 & 15,100 \\
40 & 325 & 4100 & 34,300 \\
\hline 50 & 400 & 6200 & 38,400 \\
\hline 65 & 300 & 9600 & 68,300 \\
80 & 400 & 17,100 & 121,000 \\
100 & 500 & 30,500 & 149,700 \\
\hline 125 & 600 & 43,900 & 196,700 \\
150 & 700 & 68,600 & 307,300 \\
165 & 900 & 122,000 & 391,100 \\
\hline $250(4)$ & 1200 & 175,600 & 563,000 \\
$300(4)$ & 1400 & 274,400 & 882,000 \\
$330(4)$ & &
\end{tabular}

(2) The $8.3 \mathrm{kV}, 30-100 \mathrm{~A}$ ratings have been tested and approved for application at $9.9 \mathrm{kV}$.

The maximum interrupting capacity for the $65-100 \mathrm{~A}$ ratings at $9.9 \mathrm{kV}$ is $18 \mathrm{kA}$.

(3) Parallel fuses.

(4) The $15.5 \mathrm{kV}, 30-125 \mathrm{~A}$ and $250 \mathrm{~A}$ ratings have been tested and approved for application at $17.2 \mathrm{kV}$. The maximum interrupting rating for the $15.5 \mathrm{kV}$ fuse, $30-125 \mathrm{~A}$ at $17.2 \mathrm{kV}$ is $43 \mathrm{kA}$. For the $15 \mathrm{kV}$, $250 \mathrm{~A}$ fuse at $17.2 \mathrm{kV}$, the maximum interrupting rating is $12 \mathrm{kA}$.

(5) The maximum interrupting rating for the $23 \mathrm{kV}$ fuse, $80-165 \mathrm{~A}, 300$ and $330 \mathrm{~A}$, is $30 \mathrm{kA}$. For the $23 \mathrm{kV}, 250 \mathrm{~A}$ fuse, the maximum interrupting rating is $12 \mathrm{kA}$. 


\section{0-8 Pad-Mounted Transformers}

General Description

\section{Primary Switching Options}

Eaton's oil-immersed switches are available for radial or loop-feed system switching in three current ratings. The three-phase gangoperated switch has a spring-loaded mechanism for loadbreak and latch operation. The switch is mounted near the core and coil assembly, for low cable capacitance; and with simultaneous three-phase switching, the possibility of ferroresonance is reduced. Available in ratings through $600 \mathrm{~A}$ at $15 \mathrm{kV}, 300 \mathrm{~A}$ at $25 \mathrm{kV}$, and $200 \mathrm{~A}$ at $35 \mathrm{kV}$.

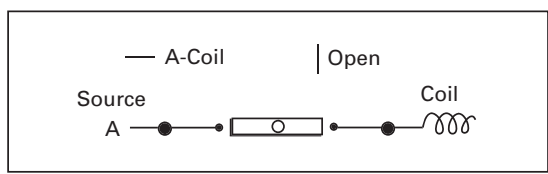

Figure 17.0-1. Two-Position Switch

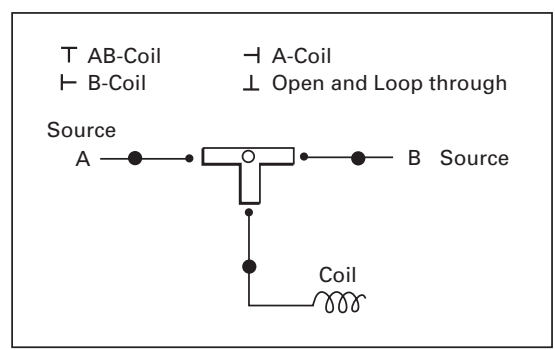

Figure 17.0-2. Four-Position Switch

(Loop Feed) "T Blade"

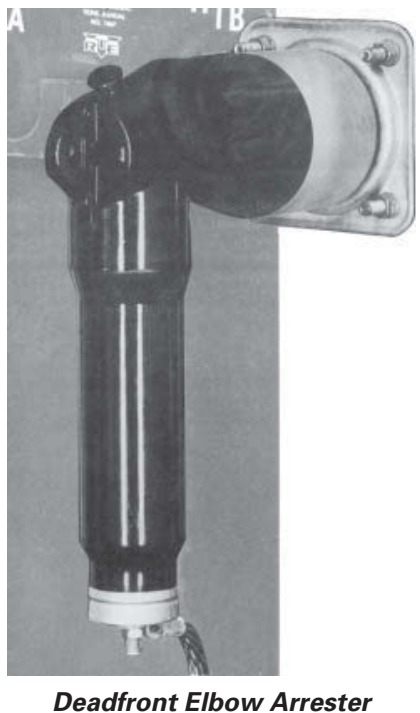

\section{Surge Arresters}

Eaton distribution class surge arresters are supplied on transformers when specified. Transformers with livefront configuration have mounting nuts welded on the tank wall for arrester mounting.

\section{Metal Oxide Varistor (MOV) Deadfront Arrester}

Surge protection is available without losing deadfront construction in the cabinet. The Eaton type MOV arrester is completely deadfront. It is compact, and is usable wherever a loadbreak elbow can be used.

The highly nonlinear characteristics of the varistor elements provide more precise and predictable operating characteristics. The MOV arrester is capable of withstanding temporary overvoltages, so that ratings can be reduced, providing improved margins of protection.

Because it is fully shielded and deadfront, it is mountable at any angle and submersible. Its durable rubber construction means there are no fragile porcelain skirts to chip or crack. The MOV arrester is available in ratings from $3 \mathrm{kV}$ to $27 \mathrm{kV}$.

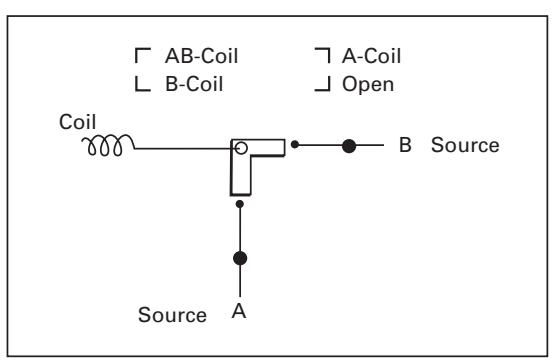

Figure 17.0-3. Four-Position Switch (Loop-Feed) "V Blade" 
SEMI-CONDUCTIVE INSERT

High-quality peroxide-cured EPDM rubber creates a smooth surface around the "current interchange" to evenly distribute electrical stress within the insulation.

\section{NSULATION}

High-quality peroxide-cured EPDM rubber formulated, mixed

and milled in-house for consistent and reliable field performance.

SEMI-CONDUCTIVE SHIELD

High-quality peroxide-cured EPDM rubber provides protective

deadfront shield that meets requirements of IEEE Standard 592.

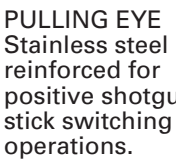

TEST POINT (OPTIONAL)

Corrosion-resistant, conductive electrode provides consistent

capacitive voltage for application of fault indicators and for determining if the circuit is energized (cap not shown).

\section{LOADBREAK BAND}

UV-resistant nylon band identifies

the elbow as three-phase loadbreak

rated and is field replaceable.

CONDUCTIVE INSERT ENDS

Encapsulated with insulating rubber. Reduces cable extrusion and distortion that can be caused by thermocycling. Mitigates the effects of the electrical stresses along the cable to elbow interface, greatly reducing the possibility of interface tracking.

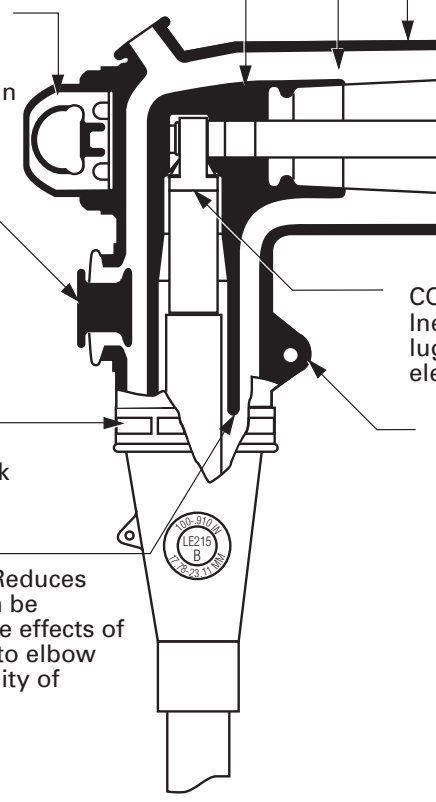

COPPERTOP COMPRESSION CONNECTOR

Inertia-welded aluminum barrel and threaded copper lug makes crimping easy and ensures a tight, reliable electrical connection with loadbreak probe.

GROUNDING TABS

Molded into semi-conductive shield

for the attachment of a ground wire to maintain deadfront safety.

\section{Figure 17.0-4. 200A, 15 kV Class Loadbreak Elbow Connector}

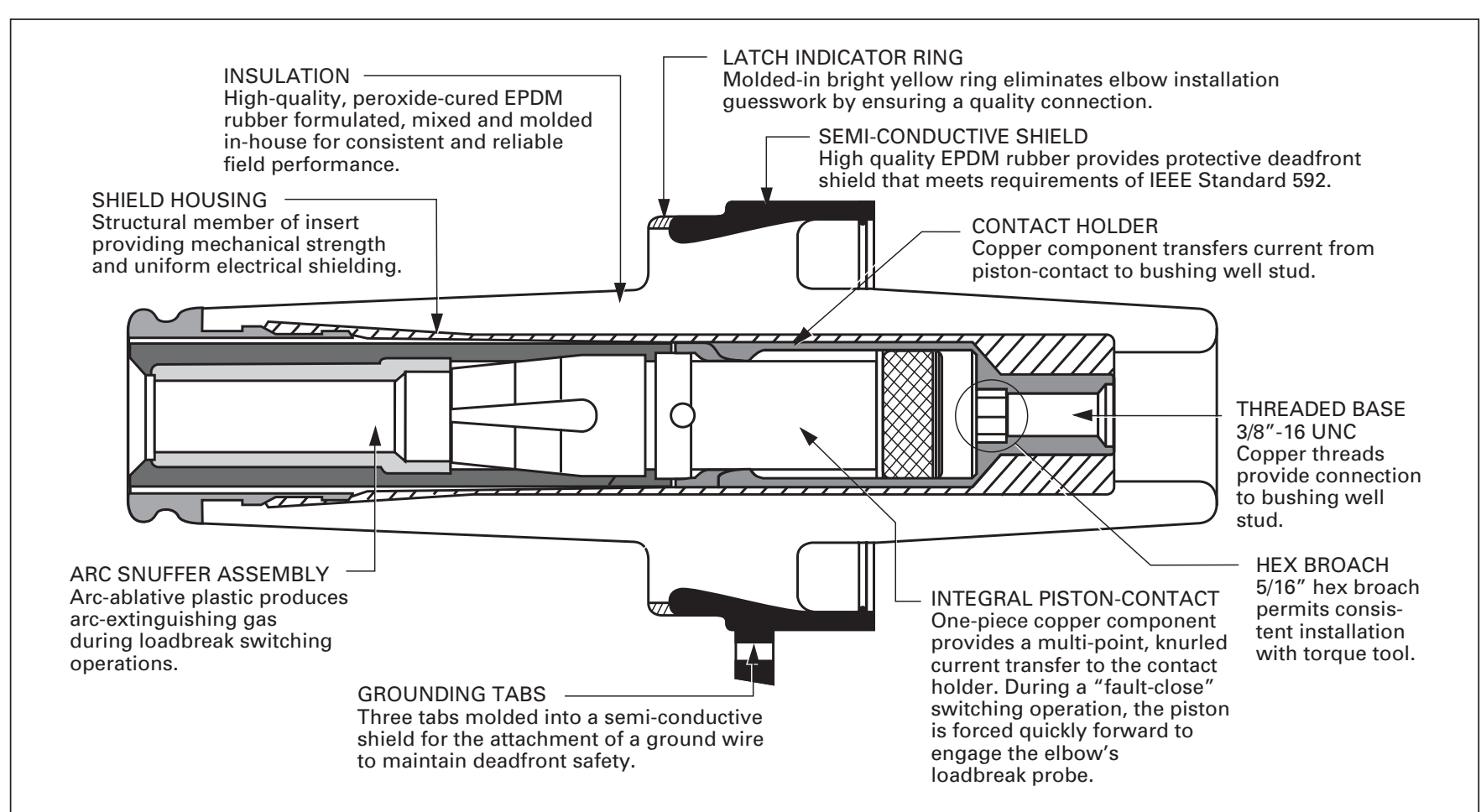

Figure 17.0-5. Bushing Well Insert Cutaway Illustrates Uncomplicated Nature of Current Path 


\section{0-10 Pad-Mounted Transformers}

\section{General Description}

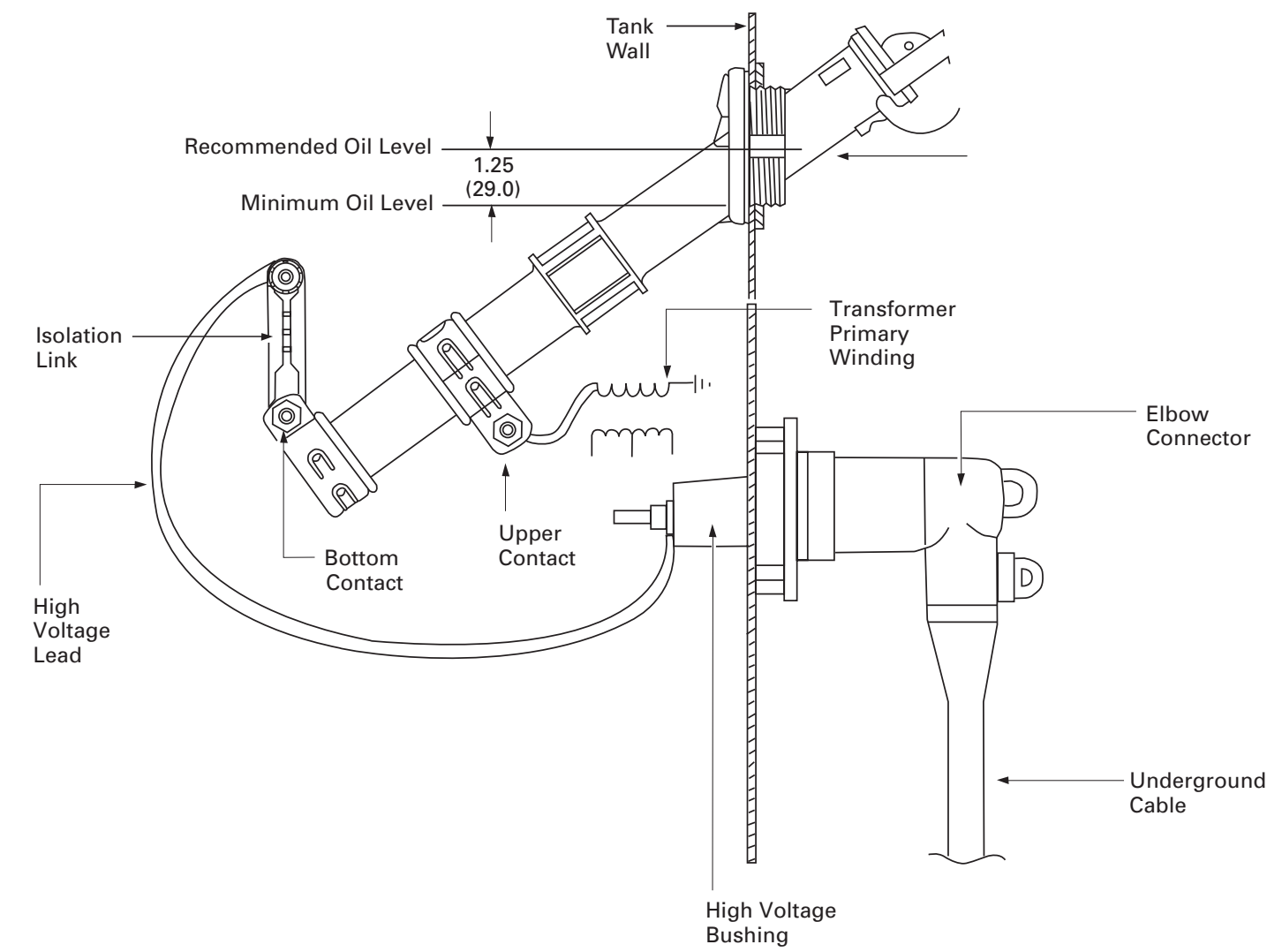

Figure 17.0-6. Bay-0-Net Assembly with Isolation Link

Note: Isolation link is not required if the Bay-O-Net fuse is used in series with a backup energy limiting fuse.

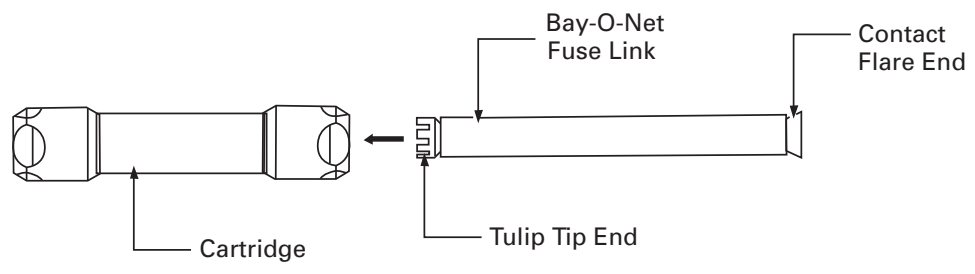

Figure 17.0-7. Insertion of Bay-0-Net Into Cartridge

Tulip End of Bay-O-Net Link

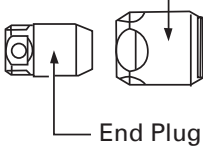

(c)
Flare End of

Bay-O-Net Link

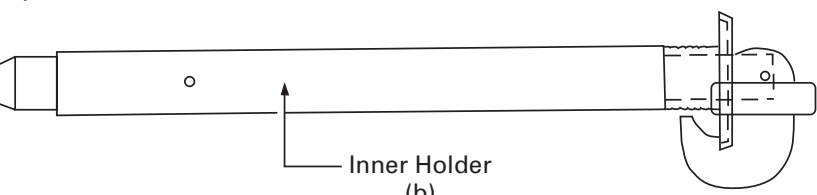

Figure 17.0-8. Assembly of Cartridge with Fuse Onto Inner Holder 

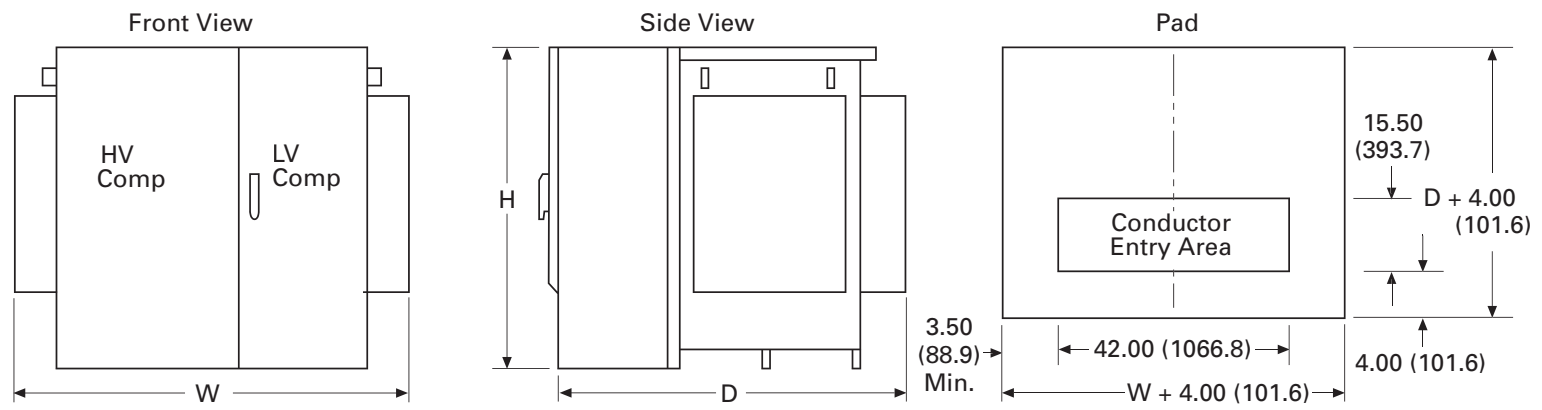

Figure 17.0-9. Pad-Mounted Transformer (75-2500 kVA)—Dimensions in Inches (mm)

Table 17.0-9. Standard Unit, Oil-Immersed $65^{\circ} \mathrm{C}$ Rise, 75-2500 kVA-Dimensions in Inches (mm)

\begin{tabular}{|c|c|c|c|c|c|}
\hline \multirow[t]{2}{*}{ kVA } & \multicolumn{3}{|c|}{ Transformer Dimensions } & \multirow{2}{*}{\begin{tabular}{|l} 
Approximate \\
Weight Lbs (kg)
\end{tabular}} & \multirow{2}{*}{\begin{tabular}{|l} 
Gallons (Liters) of \\
Oil (Approximate)
\end{tabular}} \\
\hline & \begin{tabular}{|l} 
Width (W) \\
\end{tabular} & Depth (D) & Height (H) & & \\
\hline \multicolumn{6}{|c|}{ HV: 5-15 kV Radial Feed, Livefront } \\
\hline $\begin{array}{r}75 \\
112 \\
150\end{array}$ & $\begin{array}{l}56.00(1422.4) \\
56.00(1422.4) \\
56.00(1422.4)\end{array}$ & $\begin{array}{l}50.00(1270.0) \\
50.00(1270.0) \\
50.00(1270.0)\end{array}$ & $\begin{array}{l}56.00(1422.4) \\
56.00(1422.4) \\
56.00(1422.4)\end{array}$ & $\begin{array}{l}2280(1034.2) \\
2400(1088.6) \\
2700(1224.7)\end{array}$ & \begin{tabular}{|l|l|}
115 & $(435.3)$ \\
115 & $(435.3)$ \\
125 & $(473.2)$
\end{tabular} \\
\hline $\begin{array}{l}225 \\
300 \\
500\end{array}$ & $\begin{array}{l}56.00(1422.4) \\
60.00(1524.0) \\
66.00(1676.4)\end{array}$ & \begin{tabular}{|l}
$54.00(1371.6)$ \\
$58.00(1473.2)$ \\
$62.00(1574.8)$
\end{tabular} & \begin{tabular}{|l}
$58.00(1473.2)$ \\
$58.00(1473.2)$ \\
$60.00(1524.0)$
\end{tabular} & $\begin{array}{l}3350 \text { (1519.5) } \\
3650(1655.6) \\
5200(2358.7)\end{array}$ & $\begin{array}{l}150(567.8) \\
165(624.6) \\
200(757.1)\end{array}$ \\
\hline $\begin{array}{r}750 \\
1000 \\
1500\end{array}$ & $\begin{array}{l}81.00(2057.4) \\
84.00(2133.6) \\
86.00(2184.4)\end{array}$ & $\begin{array}{l}64.00(1625.6) \\
66.00(1676.4) \\
72.00(1828.8)\end{array}$ & $\begin{array}{l}68.00 \\
68.00 \\
68.00 \\
6(1727.2) \\
(1727.2)\end{array}$ & \begin{tabular}{|r|}
$7200(3265.9)$ \\
$9000(4082.3)$ \\
$10,250(4649.3)$
\end{tabular} & $\begin{array}{l}360(1362.7) \\
400(1514.2) \\
440(1665.6)\end{array}$ \\
\hline $\begin{array}{l}2000 \\
2500 \\
3000\end{array}$ & \begin{tabular}{|r|}
$92.00(2336.8)$ \\
$98.00(2489.2)$ \\
$102.00(2590.8)$
\end{tabular} & $\begin{array}{l}80.00(2032.0) \\
82.00(2082.8) \\
83.00(2108.2)\end{array}$ & \begin{tabular}{|l|}
72.00 \\
$72.00(1828.8)$ \\
$77.00(1928.8)$ \\
\end{tabular} & $\begin{array}{l}13,400(6078.1) \\
15,000(6803.9) \\
16,500(7484.3)\end{array}$ & $\begin{array}{l}550(2082.0) \\
570(2157.7) \\
625(2365.9)\end{array}$ \\
\hline
\end{tabular}

HV: 5-15 kV Radial Feed, Deadfront

\begin{tabular}{|r|r|l|l|l|l|}
\hline 75 & $62.00(1574.8)$ & $50.00(1270.0)$ & $56.00(1422.4)$ & $2350(1065.9)$ & $115(435.3)$ \\
112 & $62.00(1574.8)$ & $50.00(1270.0)$ & $56.00(142.4)$ & $2450(1111.3)$ & $115(435.3)$ \\
150 & $62.00(1574.8)$ & $50.00(1270.0)$ & $56.00(1422.4)$ & $2700(1224.7)$ & $125(473.2)$ \\
\hline 225 & $62.00(1574.8)$ & $54.00(1371.6)$ & $58.00(1473.2)$ & $3400(1542.2)$ & $150(567.8)$ \\
300 & $62.00(1574.8)$ & $58.00(1473.2)$ & $58.00(1473.2)$ & $3700(1678.3)$ & $165(624.6)$ \\
500 & $66.00(1676.4)$ & $62.00(1574.8)$ & $60.00(1524.0)$ & $5400(2449.4)$ & $200(757.1)$ \\
\hline 750 & $81.00(2057.4)$ & $64.00(1625.6)$ & $68.00(1727.2)$ & $7200(3265.9)$ & $360(1362.7)$ \\
1000 & $84.00(2133.6)$ & $66.00(1676.4)$ & $68.00(1727.2)$ & $9000(4082.3)$ & $400(1514.2)$ \\
1500 & $86.00(2184.4)$ & $72.00(1828.8)$ & $68.00(1727.2)$ & $10,250(4649.3)$ & $440(1665.6)$ \\
\hline 2000 & $92.00(2336.8)$ & $80.00(2032.0)$ & $72.00(1828.8)$ & $13,400(6078.1)$ & $550(2082.0)$ \\
2500 & $98.00(2489.2)$ & $82.00(2082.8)$ & $72.00(1828.8)$ & $15,000(6803.9)$ & $570(2157.7)$ \\
3000 & $102.00(2590.8)$ & $83.00(2108.2)$ & $77.00(1955.8)$ & $16,500(7484.3)$ & $625(2365.9)$ \\
\hline
\end{tabular}

\section{HV: 5-15 kV Loop Feed, Livefront}

\begin{tabular}{|r|r|r|r|r|l|}
\hline 75 & $65.00(1651.0)$ & $50.00(1270.0)$ & $56.00(1422.4)$ & $2400(1088.6)$ & $115(435.3)$ \\
112 & $65.00(1651.0)$ & $50.00(1270.0)$ & $56.00(1422.4)$ & $2500(1134.0)$ & $115(435.3)$ \\
150 & $65.00(1651.0)$ & $50.00(1270.0)$ & $56.00(1422.4)$ & $2800(1270.1)$ & $125(473.2)$ \\
\hline 225 & $65.00(1651.0)$ & $54.00(1371.6)$ & $58.00(1473.2)$ & $3500(1587.6)$ & $150(567.8)$ \\
300 & $66.00(1676.4)$ & $58.00(1473.2)$ & $58.00(1473.2)$ & $3800(1723.7)$ & $165(624.6)$ \\
500 & $68.00(1727.2)$ & $62.00(1574.8)$ & $60.00(1524.0)$ & $5600(2540.1)$ & $200(757.1)$ \\
\hline 750 & $82.00(2082.8)$ & $64.00(1625.6)$ & $68.00(1727.2)$ & $7200(3265.9)$ & $360(1362.7)$ \\
1000 & $86.00(2184.4)$ & $66.00(1676.4)$ & $68.00(1727.2)$ & $9000(4082.3)$ & $400(1514.2)$ \\
1500 & $88.00(2235.2)$ & $72.00(1828.8)$ & $68.00(1727.2)$ & $10,250(4649.3)$ & $440(1665.6)$ \\
\hline 2000 & $92.00(2336.8)$ & $80.00(2032.0)$ & $72.00(1828.8)$ & $13,400(6078.1)$ & $550(2082.0)$ \\
2500 & $98.00(2489.2)$ & $82.00(2082.8)$ & $72.00(1828.8)$ & $15,000(6803.9)$ & $570(2157.7)$ \\
3000 & $102.00(2590.8)$ & $83.00(2108.2)$ & $77.00(1955.8)$ & $16,500(7484.3)$ & $625(2365.9)$ \\
\hline
\end{tabular}

\section{Dimensional Variations}

Height Variations

1. Add 3.00 inches $(76.2 \mathrm{~mm})$ to the height when using bayonet fusing on all kVA ratings.

2. Add 7.00 inches $(177.8 \mathrm{~mm})$ to the height when using dry well canister fusing on 75-500 kVA ratings.

3. Add 8.00 inches $(203.2 \mathrm{~mm})$ to the height when using dry well canister fusing on $750 \mathrm{kVA}$ rating only.

\section{Depth Variations}

4. Canister fuses require deeper tanks on some transformer sizes.

a. Add 4.00 inches $(101.6 \mathrm{~mm})$ to the depth of kVA ratings 75, 150 and 225.

b. Add 2.00 inches $(50.8 \mathrm{~mm})$ to the depth of kVA rating 500

5. Less flammable natural esther fluid requires deeper tanks on some transformer ratings.

a. Add 2.00 inches $(50.8 \mathrm{~mm})$ to the depth of kVA ratings 75-1500. Add 8.00 inches $(203.2 \mathrm{~mm})$ to the depth of kVA ratings 2000 and 2500 . 


\section{0-12 Pad-Mounted Transformers}

Layout Dimensions/Technical Data

Table 17.0-9. Standard Unit, 0il-Immersed 65 C Rise, 75-2500 kVA-Dimensions in Inches (mm) (Continued)

\begin{tabular}{|c|c|c|c|c|c|}
\hline \multirow[t]{2}{*}{ kVA } & \multicolumn{3}{|c|}{ Transformer Dimensions } & \multirow{2}{*}{$\begin{array}{l}\text { Approximate } \\
\text { Weight Lbs (kg) }\end{array}$} & \multirow{2}{*}{$\begin{array}{l}\text { Gallons (Liters) of } \\
\text { Oil (Approximate) }\end{array}$} \\
\hline & Width (W) & Depth (D) & Height (H) & & \\
\hline \multicolumn{6}{|c|}{ HV: 25 kV Radial Feed Deadfront } \\
\hline $\begin{array}{l}75-150 \\
225-300 \\
500\end{array}$ & $\begin{array}{l}68.00(1727.2) \\
72.00(1828.8) \\
72.00(1828.8)\end{array}$ & $\begin{array}{l}52.00(1320.8) \\
54.00(1371.6) \\
56.00(1422.4)\end{array}$ & $\begin{array}{l}55.00(1397.0) \\
55.00(1397.0) \\
55.00(1397.0)\end{array}$ & $\begin{array}{l}3500(1587.6) \\
4500(2041.2) \\
6000(2721.6)\end{array}$ & $\begin{array}{l}135(511.0) \\
185(700.3) \\
190(719.2)\end{array}$ \\
\hline $\begin{array}{r}750 \\
1000 \\
1500 \\
\end{array}$ & $\begin{array}{l}76.00(1930.4) \\
80.00(2032.0) \\
89.00(2260.6)\end{array}$ & $\begin{array}{l}69.00(1752.6) \\
66.00(1676.4) \\
72.00(1828.8)\end{array}$ & $\begin{array}{l}60.00(1524.0) \\
70.00(1778.0) \\
72.00(1828.8)\end{array}$ & $\begin{array}{r}7200(3265.9) \\
9000(4082.3) \\
10,250(4649.3)\end{array}$ & $\begin{array}{l}360(1362.7) \\
400(1514.2) \\
440(1665.6)\end{array}$ \\
\hline \begin{tabular}{|l|}
2000 \\
2500 \\
3000 \\
\end{tabular} & $\begin{array}{r}92.00(2336.8) \\
98.00(2489.2) \\
102.00(2590.8) \\
\end{array}$ & $\begin{array}{l}74.00(1879.6) \\
82.00(2082.8) \\
83.00(2108.2)\end{array}$ & $\begin{array}{l}75.00(1905.0) \\
77.00(1955.8) \\
78.00(1981.2)\end{array}$ & $\begin{array}{l}13,400(6078.1) \\
15,000(6803.9) \\
16,500(7484.3)\end{array}$ & $\begin{array}{l}550(2082.0) \\
570(2157.7) \\
625(2365.9)\end{array}$ \\
\hline \multicolumn{6}{|c|}{ HV: 25 kV Loop Feed Deadfront } \\
\hline $\begin{array}{l}75-150 \\
225-300 \\
500\end{array}$ & $\begin{array}{l}68.00(1727.2) \\
72.00(1828.8) \\
72.00(1828.8)\end{array}$ & $\begin{array}{l}52.00(1320.8) \\
54.00(1371.6) \\
56.00(1422.4)\end{array}$ & $\begin{array}{l}55.00(1397.0) \\
55.00(1397.0) \\
55.00(1397.0)\end{array}$ & $\begin{array}{l}3500(1587.6) \\
4500(2041.2) \\
6000(2721.6)\end{array}$ & $\begin{array}{l}135(511.0) \\
185(700.3) \\
190(719.2)\end{array}$ \\
\hline $\begin{array}{r}750 \\
1000 \\
1500 \\
\end{array}$ & $\begin{array}{l}76.00(1930.4) \\
80.00(2032.0) \\
89.00(2260.6)\end{array}$ & $\begin{array}{l}69.00(1752.6) \\
66.00(1676.4) \\
72.00(1828.8)\end{array}$ & $\begin{array}{l}60.00(1524.0) \\
70.00(1778.0) \\
72.00(1828.8)\end{array}$ & $\begin{array}{r}7200(3265.9) \\
9000(4082.3) \\
10,250(4649.3)\end{array}$ & $\begin{array}{l}360(1362.7) \\
400(1514.2) \\
440(1665.6)\end{array}$ \\
\hline $\begin{array}{l}2000 \\
2500 \\
3000\end{array}$ & $\begin{array}{r}92.00(2336.8) \\
98.00(2489.2) \\
102.00(2590.8)\end{array}$ & $\begin{array}{l}74.00(1879.6) \\
82.00(2082.8) \\
83.00(2108.2)\end{array}$ & $\begin{array}{l}75.00(1905.0) \\
77.00(1955.8) \\
78.00(1981.2)\end{array}$ & $\begin{array}{l}13400(6078.1) \\
15,000(6803.9) \\
16,500(7484.3)\end{array}$ & $\begin{array}{l}550(2082.0) \\
570(2157.7) \\
625(2365.9)\end{array}$ \\
\hline
\end{tabular}

\section{Technical Data}

Table 17.0-10. Liquid Filled $<34.5 \mathrm{kV}$ Primary $55^{\circ} \mathrm{C}$ Temp. Rise

\begin{tabular}{|c|c|c|c|c|}
\hline kVA & $\begin{array}{l}\text { No Load } \\
\text { at } 75^{\circ} \mathrm{C} \\
\text { Ref. Temp. } \\
\text { (Watts) }\end{array}$ & $\begin{array}{l}\text { Load Loss } \\
\text { at } 100 \% \\
\text { Load and } \\
75^{\circ} \mathrm{C} \text { Ref. } \\
\text { Temp. } \\
\text { (Watts) }\end{array}$ & $\begin{array}{l}\text { Total } \\
\text { Losses } \\
\text { at } 100 \% \\
\text { Load and } \\
85^{\circ} \mathrm{C} \\
\text { (Watts) }\end{array}$ & $\begin{array}{l}60-150 \text { kV HV BIL } \\
\text { Total Losses at } \\
50 \% \text { Load and } \\
55^{\circ} \mathrm{C} \text { LL Ref. Temp. } \\
\text { and } 20^{\circ} \mathrm{C} \text { NL Ref. } \\
\text { Temp. per DOE (Watts) }\end{array}$ \\
\hline $\begin{array}{l}75 \\
112.5 \\
150\end{array}$ & $\begin{array}{l}175 \\
250 \\
300\end{array}$ & $\begin{array}{r}960 \\
1250 \\
1630\end{array}$ & $\begin{array}{l}1135 \\
1500 \\
1930\end{array}$ & $\begin{array}{l}413 \\
562 \\
696\end{array}$ \\
\hline $\begin{array}{l}225 \\
300 \\
500\end{array}$ & $\begin{array}{l}330 \\
520 \\
730\end{array}$ & $\begin{array}{l}2500 \\
2600 \\
4900\end{array}$ & $\begin{array}{l}2830 \\
3120 \\
5630\end{array}$ & $\begin{array}{r}942 \\
1164 \\
1889\end{array}$ \\
\hline $\begin{array}{r}750 \\
1000 \\
1500\end{array}$ & $\begin{array}{l}1100 \\
1500 \\
1900\end{array}$ & $\begin{array}{r}6200 \\
6700 \\
10,000\end{array}$ & $\begin{array}{r}7300 \\
8200 \\
11,900\end{array}$ & $\begin{array}{l}2567 \\
3221 \\
4375\end{array}$ \\
\hline $\begin{array}{l}2000 \\
2500 \\
3000\end{array}$ & $\begin{array}{l}2600 \\
2800 \\
3800\end{array}$ & $\begin{array}{l}12,000 \\
15,000 \\
16,000\end{array}$ & $\begin{array}{l}14,600 \\
17,800 \\
19,800\end{array}$ & $\begin{array}{l}5429 \\
6408 \\
-\end{array}$ \\
\hline
\end{tabular}

Note: Losses offered are typical only, not guaranteed.

Table 17.0-11. Liquid Filled $34.5 \mathrm{kV}$ Primary $65^{\circ} \mathrm{C}$ Temp. Rise

\begin{tabular}{|c|c|c|c|c|}
\hline kVA & $\begin{array}{l}\text { No Load } \\
\text { at } 85^{\circ} \mathrm{C} \\
\text { Ref. Temp. } \\
\text { (Watts) }\end{array}$ & $\begin{array}{l}\text { Load Loss } \\
\text { at } 100 \% \\
\text { Load and } \\
85^{\circ} \mathrm{C} \text { Ref. } \\
\text { Temp. } \\
\text { (Watts) }\end{array}$ & $\begin{array}{l}\text { Total } \\
\text { Losses } \\
\text { at } 100 \% \\
\text { Load and } \\
85^{\circ} \mathrm{C} \\
\text { (Watts) } \\
\end{array}$ & $\begin{array}{l}60-150 \text { kV HV BIL } \\
\text { Total Losses at } \\
50 \% \text { Load and } \\
55^{\circ} \mathrm{C} \text { LL Ref. Temp. } \\
\text { and } 20^{\circ} \mathrm{C} \text { NL Ref. } \\
\text { Temp per DOE (Watts) }\end{array}$ \\
\hline $\begin{array}{l}75 \\
112.5 \\
150\end{array}$ & $\begin{array}{l}190 \\
260 \\
320\end{array}$ & $\begin{array}{r}950 \\
1300 \\
1600\end{array}$ & $\begin{array}{l}1140 \\
1560 \\
1920\end{array}$ & $\begin{array}{l}413 \\
562 \\
696\end{array}$ \\
\hline $\begin{array}{l}225 \\
300 \\
500\end{array}$ & $\begin{array}{l}400 \\
500 \\
700\end{array}$ & $\begin{array}{l}2300 \\
3000 \\
5000\end{array}$ & $\begin{array}{l}2700 \\
3500 \\
5700\end{array}$ & $\begin{array}{r}942 \\
1164 \\
1889\end{array}$ \\
\hline $\begin{array}{r}750 \\
1000 \\
1500\end{array}$ & $\begin{array}{l}1000 \\
1300 \\
1900\end{array}$ & $\begin{array}{r}6500 \\
8500 \\
10,500\end{array}$ & $\begin{array}{r}7500 \\
9800 \\
12,400\end{array}$ & $\begin{array}{l}2567 \\
3221 \\
4375\end{array}$ \\
\hline $\begin{array}{l}2000 \\
2500 \\
3000\end{array}$ & $\begin{array}{l}2100 \\
2700 \\
4000\end{array}$ & $\begin{array}{l}14,500 \\
15,500 \\
18,000\end{array}$ & $\begin{array}{l}16,600 \\
18,200 \\
22,000\end{array}$ & $\begin{array}{l}5429 \\
6408 \\
-\end{array}$ \\
\hline
\end{tabular}

Note: Losses offered are typical only, not guaranteed.
Table 17.0-12. Environmentally Friendly Fluid<34.5 kV Primary $55^{\circ} \mathrm{C}$ Temp. Rise

\begin{tabular}{|c|c|c|c|c|}
\hline kVA & $\begin{array}{l}\text { No Load } \\
\text { at } 75^{\circ} \mathrm{C} \\
\text { Ref. Temp. } \\
\text { (Watts) }\end{array}$ & $\begin{array}{l}\text { Load Loss } \\
\text { at } 100 \% \\
\text { Load and } \\
75^{\circ} \mathrm{C} \text { Ref. } \\
\text { Temp. } \\
\text { (Watts) } \\
\end{array}$ & $\begin{array}{l}\text { Total } \\
\text { Losses } \\
\text { at } 100 \% \\
\text { Load and } \\
85^{\circ} \mathrm{C} \\
\text { (Watts) } \\
\end{array}$ & $\begin{array}{l}\text { 60-150 kV HV BIL } \\
\text { Total Losses at } \\
50 \% \text { Load and } \\
55^{\circ} \mathrm{C} \text { LL Ref. Temp. } \\
\text { and } 20^{\circ} \mathrm{C} \text { NL Ref. } \\
\text { Temp. per DOE (Watts) }\end{array}$ \\
\hline $\begin{array}{l}75 \\
112.5 \\
150\end{array}$ & $\begin{array}{l}175 \\
250 \\
300\end{array}$ & $\begin{array}{r}960 \\
1250 \\
1630\end{array}$ & $\begin{array}{l}1135 \\
1500 \\
1930\end{array}$ & $\begin{array}{l}413 \\
562 \\
696\end{array}$ \\
\hline $\begin{array}{l}225 \\
300 \\
500\end{array}$ & $\begin{array}{l}330 \\
520 \\
730\end{array}$ & $\begin{array}{l}2500 \\
2600 \\
4900\end{array}$ & $\begin{array}{l}2830 \\
3120 \\
5630\end{array}$ & $\begin{array}{r}942 \\
1164 \\
1889\end{array}$ \\
\hline $\begin{array}{r}750 \\
1000 \\
1500\end{array}$ & $\begin{array}{l}1100 \\
1500 \\
1900\end{array}$ & $\begin{array}{r}6200 \\
6700 \\
10,000\end{array}$ & $\begin{array}{r}7300 \\
8200 \\
11,900\end{array}$ & $\begin{array}{l}2567 \\
3221 \\
4375\end{array}$ \\
\hline $\begin{array}{l}2000 \\
2500 \\
3000\end{array}$ & $\begin{array}{l}2600 \\
2800 \\
3800\end{array}$ & $\begin{array}{l}12,000 \\
15,000 \\
16,000\end{array}$ & $\begin{array}{l}14,600 \\
17,800 \\
19,800\end{array}$ & $\begin{array}{l}5429 \\
6408 \\
-\end{array}$ \\
\hline
\end{tabular}

Note: Losses offered are typical only, not guaranteed.

Table 17.0-13. Environmentally Friendly Fluid<34.5 kV Primary $65^{\circ} \mathrm{C}$ Temp. Rise

\begin{tabular}{|c|c|c|c|c|}
\hline kVA & $\begin{array}{l}\text { No Load } \\
\text { at } 85^{\circ} \mathrm{C} \\
\text { Ref. Temp. } \\
\text { (Watts) }\end{array}$ & $\begin{array}{l}\text { Load Loss } \\
\text { at } 100 \% \\
\text { Load and } \\
85^{\circ} \mathrm{C} \text { Ref. } \\
\text { Temp. } \\
\text { (Watts) }\end{array}$ & $\begin{array}{l}\text { Total } \\
\text { Losses } \\
\text { at } 100 \% \\
\text { Load and } \\
85^{\circ} \mathrm{C} \\
\text { (Watts) } \\
\end{array}$ & $\begin{array}{l}60-150 \text { kV HV BIL } \\
\text { Total Losses at } \\
50 \% \text { Load and } \\
55^{\circ} \mathrm{C} \text { LL Ref. Temp. } \\
\text { and } 20^{\circ} \mathrm{C} \text { NL Ref. } \\
\text { Temp per DOE (Watts) }\end{array}$ \\
\hline $\begin{array}{l}75 \\
112.5 \\
150\end{array}$ & $\begin{array}{l}190 \\
260 \\
320\end{array}$ & $\begin{array}{r}950 \\
1300 \\
1600\end{array}$ & $\begin{array}{l}1140 \\
1560 \\
1920\end{array}$ & $\begin{array}{l}413 \\
562 \\
696\end{array}$ \\
\hline $\begin{array}{l}225 \\
300 \\
500\end{array}$ & $\begin{array}{l}400 \\
500 \\
700\end{array}$ & $\begin{array}{l}2300 \\
3000 \\
5000\end{array}$ & $\begin{array}{l}2700 \\
3500 \\
5700\end{array}$ & $\begin{array}{r}942 \\
1164 \\
1889\end{array}$ \\
\hline $\begin{array}{r}750 \\
1000 \\
1500 \\
\end{array}$ & $\begin{array}{l}1000 \\
1300 \\
1900\end{array}$ & $\begin{array}{r}6500 \\
8500 \\
10,500\end{array}$ & $\begin{array}{r}7500 \\
9800 \\
12,400\end{array}$ & $\begin{array}{l}2567 \\
3221 \\
4375\end{array}$ \\
\hline $\begin{array}{l}2000 \\
2500 \\
3000\end{array}$ & $\begin{array}{l}2100 \\
2700 \\
4000\end{array}$ & $\begin{array}{l}14,500 \\
15,500 \\
18,000\end{array}$ & $\begin{array}{l}16,600 \\
18,200 \\
22,000\end{array}$ & $\begin{array}{l}5429 \\
6408 \\
-\end{array}$ \\
\hline
\end{tabular}

Note: Losses offered are typical only, not guaranteed. 
Front View

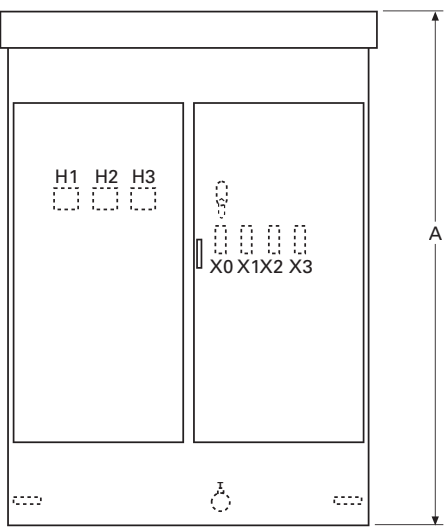

Side View

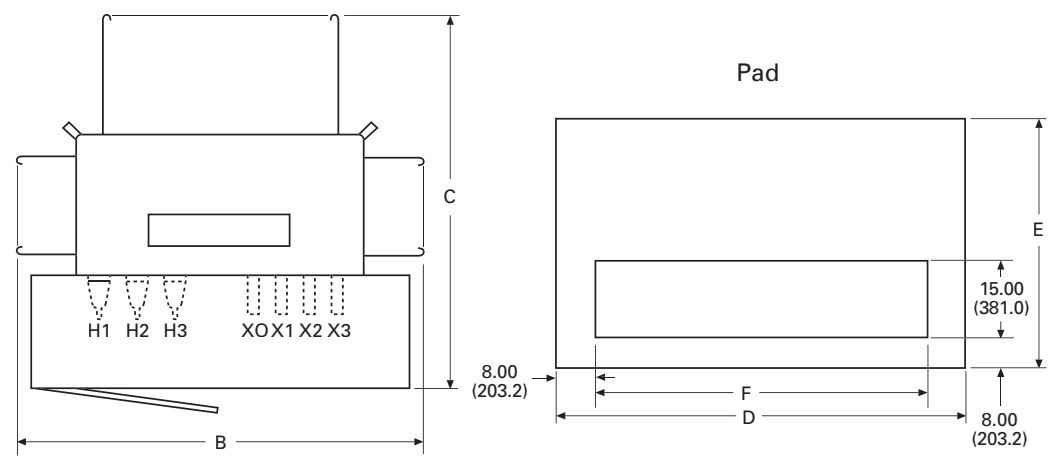

Figure 17.0-10. Pad-Mounted Transformer (3000-5000 kVA)—Dimensions in Inches (mm)

Table 17.0-14. Standard Unit, 0il-Immersed Rated $65^{\circ} \mathrm{C}$ Rise, 3000-5000 kVA—Dimensions in Inches (mm)

\begin{tabular}{|c|c|c|c|c|c|c|c|c|}
\hline \multirow[t]{2}{*}{ kVA } & \multicolumn{3}{|l|}{ Transformer } & \multicolumn{3}{|l|}{ Pad } & \multirow{2}{*}{$\begin{array}{l}\text { Approximate } \\
\text { Weight Lbs (kg) }\end{array}$} & \multirow{2}{*}{$\begin{array}{l}\text { Gallons } \\
\text { (Liters) of Oil }\end{array}$} \\
\hline & A & B & C (1) & D & E (2) & $\mathbf{F}$ & & \\
\hline \multicolumn{9}{|c|}{15 kV Class, Delta Connected HV-HV 95 kV BIL, LV 30 kV BIL (3) } \\
\hline $\begin{array}{l}3000 \\
3750 \\
5000\end{array}$ & $\begin{array}{l}76.00(1930.4) \\
80.00(2032.0) \\
78.00(1981.2)\end{array}$ & $\begin{array}{r}119.00(3022.6) \\
82.00(2082.8) \\
137.00(3479.8) \\
\end{array}$ & \begin{tabular}{|l|}
$100.00(2540.0)$ \\
$111.00(2819.4)$ \\
$108.00(2743.2)$
\end{tabular} & $\begin{array}{l}74.00(1879.6) \\
79.00(2006.6) \\
76.00(1930.4)\end{array}$ & \begin{tabular}{|l|}
$72.00(1828.8)$ \\
$83.00(2108.2)$ \\
$80.00(2032.0)$ \\
\end{tabular} & $\begin{array}{l}58.00(1473.2) \\
63.00(1600.2) \\
60.00(1524.0)\end{array}$ & $\begin{array}{l}12,900(5851) \\
20,000(9072) \\
21,500(9752)\end{array}$ & $\begin{array}{l}385(1457) \\
540(2044) \\
565(2139)\end{array}$ \\
\hline \multicolumn{9}{|c|}{15 kV Class, Wye Connected HV-HV 95 kV BIL, LV 30 kV BIL (3) } \\
\hline $\begin{array}{l}3000 \\
3750 \\
5000\end{array}$ & $\begin{array}{l}74.00(1879.6) \\
97.00(2463.8) \\
91.00(2311.4)\end{array}$ & $\begin{array}{r}117.00(2971.8) \\
81.00(2057.4) \\
119.00(3022.6) \\
\end{array}$ & \begin{tabular}{|l|}
$102.00(2590.8)$ \\
$101.00(2565.4)$ \\
$108.00(2743.2)$
\end{tabular} & $\begin{array}{l}78.00(1981.2) \\
81.00(2057.4) \\
84.00(2133.6)\end{array}$ & \begin{tabular}{|l|}
$74.00(1879.6)$ \\
$77.00(1955.8)$ \\
$80.00(2032.0)$
\end{tabular} & $\begin{array}{l}62.00(1574.8) \\
65.00(1651.0) \\
68.00(1727.2)\end{array}$ & $\begin{array}{l}15,000(6804) \\
21,800(9888) \\
22,000(9979)\end{array}$ & $\begin{array}{l}390(1476) \\
550(2082) \\
585(2214)\end{array}$ \\
\hline \multicolumn{9}{|c|}{25 kV Class, Delta Connected HV-HV 150 kV BIL, LV 30 kV BIL (3) } \\
\hline $\begin{array}{l}3000 \\
3750 \\
5000 \\
\end{array}$ & $\begin{array}{r}83.00(2108.2) \\
96.00(2438.4) \\
101.00(2565.4)\end{array}$ & $\begin{array}{r}84.00(2133.6) \\
84.00(2133.6) \\
101.00(2565.4) \\
\end{array}$ & $\begin{array}{r}101.00(2565.4) \\
98.00(2489.2) \\
107.00(2717.8) \\
\end{array}$ & $\begin{array}{l}86.00(2184.4) \\
86.00(2184.4) \\
84.00(2133.6) \\
\end{array}$ & \begin{tabular}{|l|}
$74.00(1879.6)$ \\
$78.00(1981.2)$ \\
$79.00(2006.6)$ \\
\end{tabular} & $\begin{array}{l}70.00(1778.0) \\
70.00(1778.0) \\
68.00(1727.2) \\
\end{array}$ & $\begin{array}{l}15,400(6985) \\
20,100(9117) \\
22,900(10,387)\end{array}$ & $\begin{array}{l}515(1949) \\
650(2461) \\
670(2536)\end{array}$ \\
\hline \multicolumn{9}{|c|}{25 kV Class, Wye Connected HV-HV 125 kV BIL, LV 30 kV BIL (3) } \\
\hline $\begin{array}{l}3000 \\
3750 \\
5000 \\
\end{array}$ & $\begin{array}{l}84.00(2133.6) \\
93.00(2362.2) \\
90.00(2286.0\end{array}$ & $\begin{array}{r}80.00(2032.0) \\
85.00(2159.0) \\
110.00(2794.0) \\
\end{array}$ & $\begin{array}{r}102.00(2590.8) \\
99.00(2514.6) \\
108.00(2743.2) \\
\end{array}$ & $\begin{array}{l}80.00(2032.0) \\
84.00(2133.6) \\
84.00(2133.6) \\
\end{array}$ & \begin{tabular}{|l|}
$74.00(1879.6)$ \\
$78.00(1981.2)$ \\
$80.00(2032.0)$ \\
\end{tabular} & $\begin{array}{l}64.00(1625.6) \\
68.00(1727.2) \\
68.00(1727.2) \\
\end{array}$ & $\begin{array}{l}16,300(7394) \\
21,200(9616) \\
23,100(10,478)\end{array}$ & $\begin{array}{l}450(1703) \\
575(2177) \\
605(2290)\end{array}$ \\
\hline \multicolumn{9}{|c|}{35 kV Class, Delta Connected HV-HV 200 kV BIL, LV 30 kV BIL } \\
\hline $\begin{array}{l}3000 \\
3750 \\
5000 \\
\end{array}$ & \begin{tabular}{|r|}
$86.00(2184.4)$ \\
$86.00(2184.4)$ \\
$102.00(2590.8)$ \\
\end{tabular} & $\begin{array}{r}86.00(2184.4) \\
82.00(2082.8) \\
122.00(3098.8) \\
\end{array}$ & $\begin{array}{l}101.00(2565.4) \\
102.00(2590.8) \\
106.00(2692.4)\end{array}$ & $\begin{array}{l}78.00(1981.2) \\
82.00(2082.8) \\
83.00(2108.2) \\
\end{array}$ & \begin{tabular}{|l|}
$73.00(1854.2)$ \\
$76.00(1930.4)$ \\
$78.00(1981.2)$ \\
\end{tabular} & $\begin{array}{l}62.00(1574.8) \\
66.00(1676.4) \\
67.00(1701.8) \\
\end{array}$ & $\begin{array}{l}15,700(7121) \\
19,800(8981) \\
22,600(10,251)\end{array}$ & $\begin{array}{l}420(1590) \\
525(1987) \\
580(2196) \\
\end{array}$ \\
\hline \multicolumn{9}{|c|}{35 kV Class, Wye Connected HV-HV 125 kV BIL, LV 30 kV BIL } \\
\hline $\begin{array}{l}3000 \\
3750 \\
5000 \\
\end{array}$ & $\begin{array}{l}82.00(2082.8) \\
91.00(2311.4) \\
92.00(2336.8) \\
\end{array}$ & $\begin{array}{r}86.00(2184.4) \\
82.00(2082.8) \\
122.00(3098.8) \\
\end{array}$ & $\begin{array}{l}101.00(2565.4) \\
102.00(2590.8) \\
106.00(2692.4)\end{array}$ & $\begin{array}{l}78.00(1981.2) \\
82.00(2082.8) \\
83.00(2108.2) \\
\end{array}$ & \begin{tabular}{|l|}
$73.00(1854.2)$ \\
$76.00(1930.4)$ \\
$78.00(1981.2)$ \\
\end{tabular} & $\begin{array}{l}62.00(1574.8) \\
66.00(1676.4) \\
67.00(1701.8) \\
\end{array}$ & $\begin{array}{l}15,700(7121) \\
19,800(8981) \\
22,600(10,251)\end{array}$ & $\begin{array}{l}420(1590) \\
525(1987) \\
580(2196) \\
\end{array}$ \\
\hline \multicolumn{9}{|c|}{35 kV Class, Delta Connected HV-HV 150 kV BIL, LV 30 kV BIL } \\
\hline $\begin{array}{l}3000 \\
3750 \\
5000 \\
\end{array}$ & $\begin{array}{l}84.00(2133.6) \\
84.00(2133.6) \\
92.00(2336.8) \\
\end{array}$ & $\begin{array}{r}84.00(2133.6) \\
84.00(2133.6) \\
122.00(3098.8) \\
\end{array}$ & $\begin{array}{l}100.00(2540.0) \\
101.00(2565.4) \\
106.00(2692.4)\end{array}$ & $\begin{array}{l}86.00(2184.4) \\
86.00(2184.4) \\
81.00(2057.4) \\
\end{array}$ & \begin{tabular}{|l|}
$74.00(1879.6)$ \\
$77.00(1955.8)$ \\
$78.00(1981.2)$ \\
\end{tabular} & $\begin{array}{l}70.00(1778.0) \\
70.00(1778.0) \\
65.00(1651.0) \\
\end{array}$ & $\begin{array}{l}15,400(6985) \\
19,300(8754) \\
20,500(9299) \\
\end{array}$ & $\begin{array}{l}530(2006) \\
630(2385) \\
600(2271) \\
\end{array}$ \\
\hline \multicolumn{9}{|c|}{35 kV Class, Wye Connected HV-HV 150 kV BIL, LV 30 kV BIL } \\
\hline $\begin{array}{l}3000 \\
3750 \\
5000 \\
\end{array}$ & $\begin{array}{l}80.00(2032.0) \\
86.00(2184.4) \\
95.00(2413.0) \\
\end{array}$ & $\begin{array}{r}84.00(2133.6) \\
87.00(2209.8) \\
105.00(2667.0)\end{array}$ & $\begin{array}{l}104.00(2641.6) \\
107.00(2717.8) \\
107.00(2717.8)\end{array}$ & $\begin{array}{l}86.00(2184.4) \\
86.00(2184.4) \\
85.00(2159.0) \\
\end{array}$ & \begin{tabular}{|l|}
$76.00(1930.4)$ \\
$79.00(2006.6)$ \\
$79.00(2006.6)$ \\
\end{tabular} & $\begin{array}{l}70.00(1778.0) \\
70.00(1778.0) \\
69.00(1752.6) \\
\end{array}$ & $\begin{array}{l}17,100(7756) \\
20,600(9344) \\
23,800(10,795)\end{array}$ & $\begin{array}{l}500(1893) \\
560(2120) \\
625(2366)\end{array}$ \\
\hline \multicolumn{9}{|c|}{35 kV Class, Wye Connected HV-HV 200 kV BIL, LV 30 kV BIL } \\
\hline $\begin{array}{l}3000 \\
3750 \\
5000\end{array}$ & $\begin{array}{r}88.00(2235.2) \\
90.00(2286.0) \\
101.00(2565.4)\end{array}$ & $\begin{array}{l}104.00(2641.6) \\
104.00(2641.6) \\
102.00(2590.8)\end{array}$ & $\begin{array}{r}99.00(2514.6) \\
104.00(2641.6) \\
106.00(2692.4)\end{array}$ & $\begin{array}{l}107.00(2717.8) \\
107.00(2717.8) \\
107.00(2717.8)\end{array}$ & \begin{tabular}{|l|}
$83.00(2108.2)$ \\
$90.00(2286.0)$ \\
$90.00(2286.0)$
\end{tabular} & $\begin{array}{l}91.00(2311.4) \\
91.00(2311.4) \\
89.00(2260.6)\end{array}$ & $\begin{array}{l}19,800(8981) \\
24,400(11,068) \\
28,600(12,973)\end{array}$ & $\begin{array}{l}720(2725) \\
840(3180) \\
920(3483)\end{array}$ \\
\hline
\end{tabular}

(1) Standard compartment depth is 22.00 inches $(558.8 \mathrm{~mm}$ ) except $200 \mathrm{kV}$ BIL has a depth of 30.00 inches $(762.0 \mathrm{~mm})$.

Depth may be altered by the addition of switching and fusing.

(2) Extends under base of transformer only. Does not include rear coolers.

(3) Standard low voltages are 480Y and 480 delta (through 3750 kVA only).

Low voltage above 3750 kVA must be $2400 \mathrm{~V}$ or above.

\section{Dimensions are approximate-not for construction.}




\section{0-14 Pad-Mounted Transformers}

Technical Data

\section{Liquid Filled Technical Data}

Table 17.0-15. Liquid Filled 15 kV Primary $55^{\circ} \mathrm{C}$ Temp. Rise

\begin{tabular}{|c|c|c|c|c|}
\hline kVA & $\begin{array}{l}\text { No Load } \\
\text { at } 75^{\circ} \mathrm{C} \\
\text { Ref. Temp. } \\
\text { (Watts) }\end{array}$ & $\begin{array}{l}\text { Load Loss } \\
\text { at } 100 \% \\
\text { Load and } \\
75^{\circ} \mathrm{C} \text { Ref. } \\
\text { Temp. } \\
\text { (Watts) }\end{array}$ & $\begin{array}{l}\text { Total Losses } \\
\text { at } 100 \% \\
\text { Load and } \\
85^{\circ} \mathrm{C} \\
\text { (Watts) }\end{array}$ & $\begin{array}{l}95 \mathrm{kV} \text { HV BIL } \\
\text { Total Losses at } \\
50 \% \text { Load and } \\
55^{\circ} \mathrm{C} \text { LL Ref. Temp. } \\
\text { and } 20^{\circ} \mathrm{C} \text { NL Ref. } \\
\text { Temp. per DOE } \\
\text { (Watts) } \\
\end{array}$ \\
\hline $\begin{array}{l}3000 \\
3750 \\
5000\end{array}$ & $\begin{array}{l}5517 \\
6521 \\
8193\end{array}$ & $\begin{array}{l}22,491 \\
26,340 \\
32,255\end{array}$ & $\begin{array}{l}28,008 \\
32,861 \\
40,448\end{array}$ & $\begin{array}{l}11,140 \\
13,110 \\
16,260\end{array}$ \\
\hline
\end{tabular}

Table 17.0-16. Liquid Filled 5 kV Primary $55^{\circ} \mathrm{C}$ Temp. Rise

\begin{tabular}{|c|c|c|c|c|}
\hline kVA & $\begin{array}{l}\text { No Load } \\
\text { at } 75^{\circ} \mathrm{C} \\
\text { Ref. Temp. } \\
\text { (Watts) }\end{array}$ & $\begin{array}{l}\text { Load Loss } \\
\text { at } 100 \% \\
\text { Load and } \\
75^{\circ} \mathrm{C} \text { Ref. } \\
\text { Temp. } \\
\text { (Watts) }\end{array}$ & $\begin{array}{l}\text { Total Losses } \\
\text { at } 100 \% \\
\text { Load and } \\
85^{\circ} \mathrm{C} \\
\text { (Watts) }\end{array}$ & $\begin{array}{l}60 \mathrm{kV} \text { HV BIL } \\
\text { Total Losses at } \\
50 \% \text { load and } \\
55^{\circ} \mathrm{C} \text { LL Ref. Temp. } \\
\text { and } 20^{\circ} \mathrm{C} \text { NL Ref. } \\
\text { Temp. per DOE } \\
\text { (Watts) }\end{array}$ \\
\hline $\begin{array}{l}3000 \\
3750 \\
5000\end{array}$ & $\begin{array}{l}5461 \\
6455 \\
8111\end{array}$ & $\begin{array}{l}22,269 \\
26,076 \\
31,932\end{array}$ & $\begin{array}{l}27,730 \\
32,531 \\
40,043\end{array}$ & $\begin{array}{l}11,030 \\
12,230 \\
16,090\end{array}$ \\
\hline
\end{tabular}

Table 17.0-17. Liquid Filled $25 \mathrm{kV}$ Primary $55^{\circ} \mathrm{C}$ Temp. Rise

\begin{tabular}{|c|c|c|c|c|}
\hline kVA & $\begin{array}{l}\text { No Load } \\
\text { at } 75^{\circ} \mathrm{C} \\
\text { Ref. Temp. } \\
\text { (Watts) }\end{array}$ & $\begin{array}{l}\text { Load Loss } \\
\text { at } 100 \% \\
\text { Load and } \\
75^{\circ} \mathrm{C} \text { Ref. } \\
\text { Temp. } \\
\text { (Watts) }\end{array}$ & $\begin{array}{l}\text { Total Losses } \\
\text { at } 100 \% \\
\text { Load and } \\
85^{\circ} \mathrm{C} \\
\text { (Watts) }\end{array}$ & $\begin{array}{l}150 \text { kV HV BIL } \\
\text { Total Losses at } \\
50 \% \text { Load and } \\
55^{\circ} \mathrm{C} \text { LL Ref. Temp. } \\
\text { and } 20^{\circ} \mathrm{C} \text { NL Ref. } \\
\text { Temp. per DOE } \\
\text { (Watts) }\end{array}$ \\
\hline $\begin{array}{l}3000 \\
3750 \\
5000\end{array}$ & $\begin{array}{l}5570 \\
6584 \\
8273\end{array}$ & $\begin{array}{l}22,046 \\
25,815 \\
31,612\end{array}$ & $\begin{array}{l}27,616 \\
32,399 \\
39,885\end{array}$ & $\begin{array}{l}11,080 \\
13,040 \\
16,180\end{array}$ \\
\hline
\end{tabular}

Table 17.0-18. Liquid Filled 35 kV Primary $55^{\circ} \mathrm{C}$ Temp. Rise

\begin{tabular}{|c|c|c|c|c|}
\hline kVA & $\begin{array}{l}\text { No Load } \\
\text { at } 75^{\circ} \mathrm{C} \\
\text { Ref. Temp. } \\
\text { (Watts) }\end{array}$ & $\begin{array}{l}\text { Load Loss } \\
\text { at } 100 \% \\
\text { Load and } \\
75^{\circ} \mathrm{C} \text { Ref. } \\
\text { Temp. } \\
\text { (Watts) }\end{array}$ & $\begin{array}{l}\text { Total Losses } \\
\text { at } 100 \% \text { LOAD } \\
\text { and } 85^{\circ} \mathrm{C} \\
\text { (Watts) }\end{array}$ & $\begin{array}{l}200 \mathrm{kV} \text { HV BIL } \\
\text { Total Losses at } \\
50 \% \text { Load and } \\
55^{\circ} \mathrm{C} \text { LL Ref. Temp. } \\
\text { and } 20^{\circ} \mathrm{C} \text { NL Ref. } \\
\text { Temp. per DOE } \\
\text { (Watts) }\end{array}$ \\
\hline $\begin{array}{l}3000 \\
3750 \\
5000\end{array}$ & $\begin{array}{l}5848 \\
6913 \\
8686\end{array}$ & $\begin{array}{l}21,825 \\
25,556 \\
31,295\end{array}$ & $\begin{array}{l}27,673 \\
32,469 \\
39,981\end{array}$ & $\begin{array}{l}11,300 \\
13,300 \\
16,510\end{array}$ \\
\hline
\end{tabular}

Table 17.0-19. Liquid Filled 15 kV Primary $65^{\circ} \mathrm{C}$ Temp. Rise

\begin{tabular}{|c|c|c|c|c|}
\hline kVA & $\begin{array}{l}\text { No Load } \\
\text { at } 85^{\circ} \mathrm{C} \\
\text { Ref. Temp } \\
\text { (Watts) }\end{array}$ & $\begin{array}{l}\text { Load Loss } \\
\text { at } 100 \% \\
\text { Load and } \\
85^{\circ} \mathrm{C} \text { Ref. } \\
\text { Temp. } \\
\text { (Watts) }\end{array}$ & $\begin{array}{l}\text { Total Losses } \\
\text { at } 100 \% \\
\text { Load and } \\
85^{\circ} \mathrm{C} \\
\text { (Watts) }\end{array}$ & $\begin{array}{l}95 \mathrm{kV} \text { HV BIL } \\
\text { Total Losses at } \\
50 \% \text { Load and } \\
55^{\circ} \mathrm{C} \text { LL Ref. Temp. } \\
\text { and } 20^{\circ} \mathrm{C} \text { NL Ref. } \\
\text { Temp. per DOE } \\
\text { (Watts) }\end{array}$ \\
\hline $\begin{array}{l}3000 \\
3750 \\
5000\end{array}$ & $\begin{array}{l}5985 \\
7075 \\
8889\end{array}$ & $\begin{array}{l}24,402 \\
28,578 \\
34,996\end{array}$ & $\begin{array}{l}30,387 \\
35,653 \\
43,885\end{array}$ & $\begin{array}{l}12,090 \\
14,220 \\
17,640\end{array}$ \\
\hline
\end{tabular}

Table 17.0-20. Liquid Filled 5 kV Primary $65^{\circ} \mathrm{C}$ Temp. Rise

\begin{tabular}{|c|c|c|c|c|}
\hline kVA & $\begin{array}{l}\text { No Load } \\
\text { at } 85^{\circ} \mathrm{C} \\
\text { Ref. Temp. } \\
\text { (Watts) }\end{array}$ & $\begin{array}{l}\text { Load Loss } \\
\text { at } 100 \% \\
\text { Load and } \\
85^{\circ} \mathrm{C} \text { Ref. } \\
\text { Temp. } \\
\text { (Watts) }\end{array}$ & $\begin{array}{l}\text { Total Losses } \\
\text { at } 100 \% \\
\text { Load and } \\
85^{\circ} \mathrm{C} \\
\text { (Watts) }\end{array}$ & $\begin{array}{l}95 \mathrm{kV} \text { HV BIL } \\
\text { Total Losses at } \\
50 \% \text { Load and } \\
55^{\circ} \mathrm{C} \text { LL Ref. Temp. } \\
\text { and } 20^{\circ} \mathrm{C} \text { NL Ref. } \\
\text { Temp. per DOE } \\
\text { (Watts) }\end{array}$ \\
\hline $\begin{array}{l}3000 \\
3750 \\
5000\end{array}$ & $\begin{array}{l}5925 \\
7003 \\
8800\end{array}$ & $\begin{array}{l}24,161 \\
28,292 \\
34,646\end{array}$ & $\begin{array}{l}30,086 \\
35,295 \\
43,446\end{array}$ & $\begin{array}{l}11,970 \\
14,080 \\
17,460\end{array}$ \\
\hline
\end{tabular}

Table 17.0-21. Liquid Filled 25 kV Primary $65^{\circ} \mathrm{C}$ Temp. Rise

\begin{tabular}{|l|l|l|l|l|}
\hline kVA & $\begin{array}{l}\text { No Load } \\
\text { at } 85^{\circ} \mathbf{C} \\
\text { Ref. Temp. } \\
\text { (Watts) }\end{array}$ & $\begin{array}{l}\text { Load Loss } \\
\text { at } \mathbf{1 0 0 \%} \\
\text { Load and } \\
\mathbf{8 5}^{\circ} \mathbf{C} \text { Ref. } \\
\text { Temp. } \\
\text { (Watts) }\end{array}$ & $\begin{array}{l}\text { Total Losses } \\
\text { at } \mathbf{1 0 0 \%} \\
\text { Load and } \\
\mathbf{8 5}^{\circ} \mathbf{C} \\
\text { (Watts) }\end{array}$ & $\begin{array}{l}\mathbf{1 5 0} \text { kV HV BIL } \\
\text { Total Losses at } \\
\mathbf{5 0 \%} \text { Load and } \\
\mathbf{5 5} \text { C LL Ref. Temp. } \\
\text { and 20 } \mathbf{C} \text { NL Ref. } \\
\text { Temp. per DOE } \\
\text { (Watts) }\end{array}$ \\
\hline 3000 & 6043 & 23,919 & 29,962 & 12,020 \\
3750 & 7143 & 28,009 & 35,152 & 14,150 \\
5000 & 8976 & 34,299 & 43,275 & 17,550 \\
\hline
\end{tabular}

Table 17.0-22. Liquid Filled $35 \mathrm{kV}$ Primary $65^{\circ} \mathrm{C}$ Temp. Rise

\begin{tabular}{|c|c|c|c|c|}
\hline kVA & \begin{tabular}{|l|} 
No Load \\
at $85^{\circ} \mathrm{C}$ \\
Ref. Temp. \\
(Watts)
\end{tabular} & $\begin{array}{l}\text { Load Loss } \\
\text { at } 100 \% \\
\text { Load and } \\
85^{\circ} \mathrm{C} \text { Ref. } \\
\text { Temp. } \\
\text { (Watts) }\end{array}$ & $\begin{array}{l}\text { Total Losses } \\
\text { at } 100 \% \\
\text { Load and } \\
85^{\circ} \mathrm{C} \\
\text { (Watts) }\end{array}$ & $\begin{array}{l}200 \text { kV HV BIL } \\
\text { Total Losses at } \\
50 \% \text { Load and } \\
55^{\circ} \mathrm{C} \text { LL Ref. Temp. } \\
\text { and } 20^{\circ} \mathrm{C} \text { NL Ref. } \\
\text { Temp. per DOE } \\
\text { (Watts) }\end{array}$ \\
\hline $\begin{array}{l}3000 \\
3750 \\
5000\end{array}$ & \begin{tabular}{|l|}
6345 \\
7500 \\
9424 \\
\end{tabular} & $\begin{array}{l}23,680 \\
27,728 \\
33,955\end{array}$ & $\begin{array}{l}30,025 \\
35,228 \\
43,379\end{array}$ & $\begin{array}{l}12,270 \\
14,430 \\
17,910\end{array}$ \\
\hline
\end{tabular}

Note: Losses offered are typical only, not guaranteed. Losses based on aluminum windings. Losses based on LV rating $0.48 \mathrm{kV}$. 


\section{Appendix D \\ LCC Analysis}


NIST BLCC 5.3-11: ECIP Report

Consistent with Federal Life Cycle Cost Methodology and Procedures, 10 CFR, Part 436, Subpart A

The LCC calculations are based on the FEMP discount rates and energy price escalation rates updated on April 1, 2011.

$\begin{array}{lrr}\text { Location: } & \text { U.S. Average Discount Rate: } & 3 \% \\ \text { Project Title: } & \text { Japan Transformer Analyst: } & \text { INL } \\ \text { Base Date: } & \text { January 1, 2013 Preparation Date: Fri Jun } 2214: 36: 55 \text { MDT } 2012 \\ \text { BOD: } & \text { January 1, 2013 Economic Life: } & 30 \text { years } 0 \text { months }\end{array}$

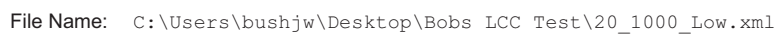

1. Investment

Construction Cost $\$ 50,794$

$\mathrm{SIOH}$

Design Cost $\$ 0$

Total Cost \$50,794

Salvage Value of Existing Equipment $\quad \$ 0$

Public Utility Company $\$ 0$

Total Investment $\$ 50,794$

2. Energy and Water Savings (+) or Cost (-)

Base Date Savings, unit costs, \& discounted savings

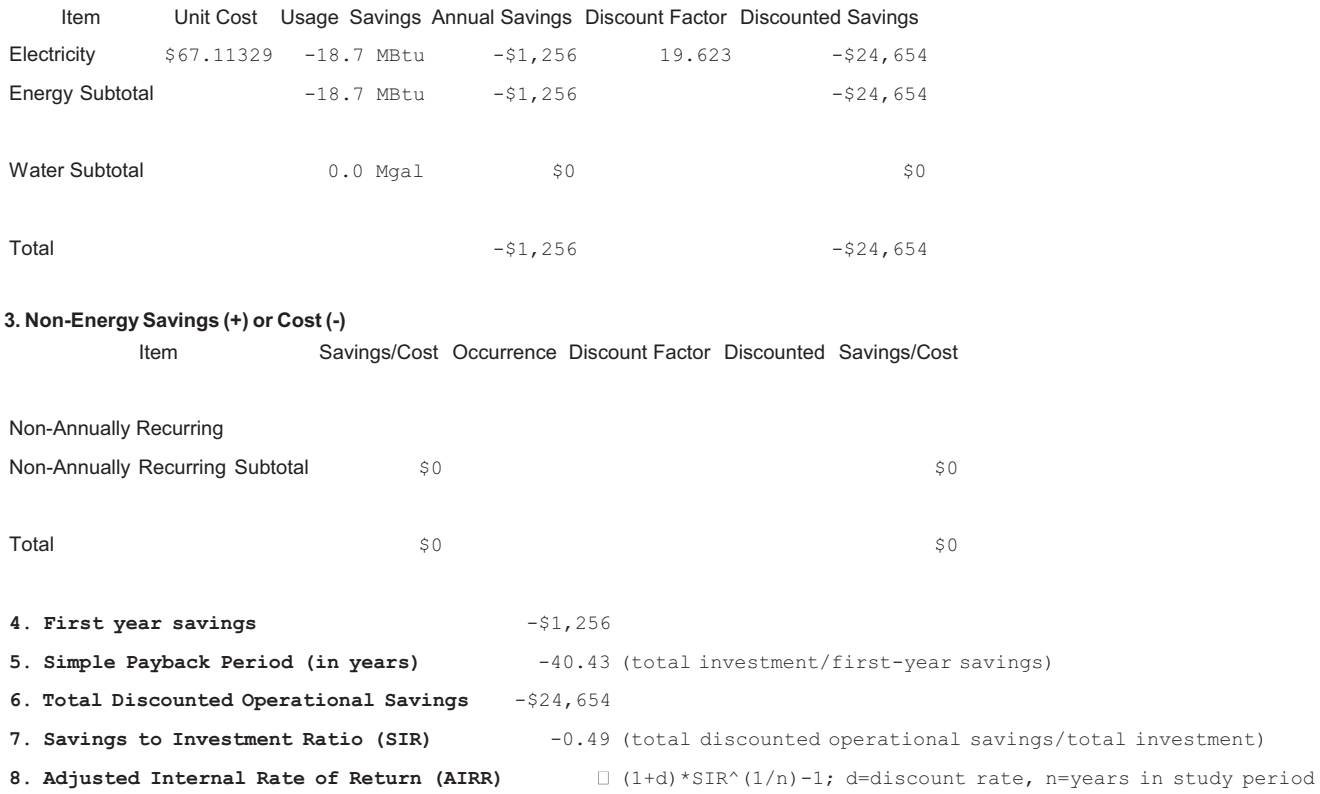

8. Adjusted Internal Rate of Return (AIRR) $\square(1+d) \star S I R^{\wedge}(1 / n)-1$; d=discount rate, n=years in study period 
NIST BLCC 5.3-11: ECIP Report

Consistent with Federal Life Cycle Cost Methodology and Procedures, 10 CFR, Part 436, Subpart A

The LCC calculations are based on the FEMP discount rates and energy price escalation rates updated on April 1, 2011.

$\begin{array}{lrr}\text { Location: } & \text { U.S. Average Discount Rate: } & 3 \% \\ \text { Project Title: } & \text { Japan Transformer Analyst: } & \text { INL } \\ \text { Base Date: } & \text { January 1, 2013 Preparation Date: Fri Jun } 2214: 38: 46 \text { MDT } 2012 \\ \text { BOD: } & \text { January 1, 2013 Economic Life: } & 30 \text { years } 0 \text { months }\end{array}$

File Name: C: $\backslash$ Users $\backslash$ bushjw $\backslash$ Desktop $\backslash$ Bobs LCC Test $\backslash 20 \_1000 \_$Med.xml

1. Investment

Construction Cost $\$ 60,742$

$\mathrm{SIOH}$

Design Cost $\$ 0$

Total Cost $\$ 60,742$

Salvage Value of Existing Equipment $\$ 0$

Public Utility Company $\$ 0$

Total Investment $\$ \$ 60,742$

2. Energy and Water Savings (+) or Cost (-)

Base Date Savings, unit costs, \& discounted savings

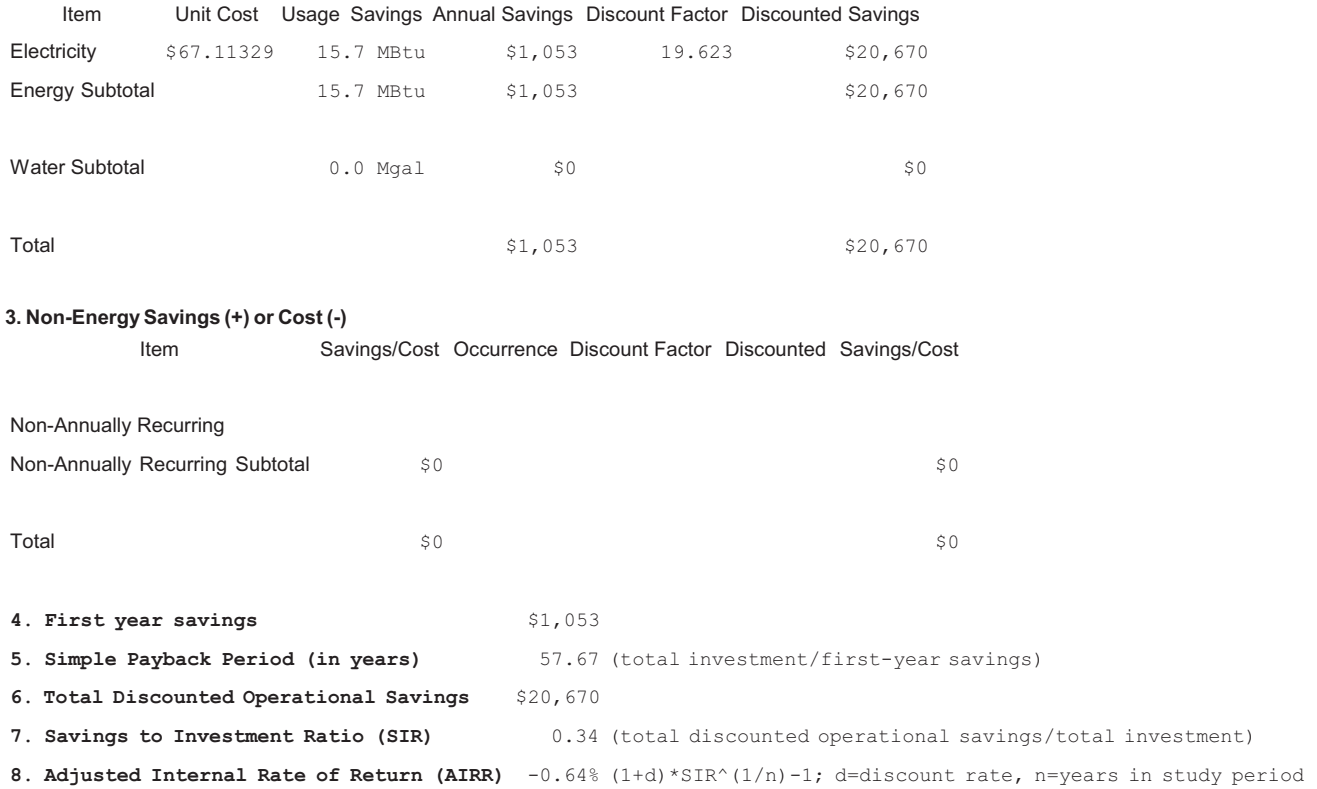

8. Adjusted Internal Rate of Return (AIRR) $-0.64 \%(1+d) * \operatorname{SIR}^{\wedge}(1 / \mathrm{n})-1$; d=discount rate, $\mathrm{n}=$ years in study period 
NIST BLCC 5.3-11: ECIP Report

Consistent with Federal Life Cycle Cost Methodology and Procedures, 10 CFR, Part 436, Subpart A

The LCC calculations are based on the FEMP discount rates and energy price escalation rates updated on April 1, 2011.

$\begin{array}{lrr}\text { Location: } & \text { U.S. Average Discount Rate: } & 3 \% \\ \text { Project Title: } & \text { Japan Transformer Analyst: } & \text { INL } \\ \text { Base Date: } & \text { January 1, 2013 Preparation Date: Fri Jun } 2214: 27: 36 \text { MDT } 2012 \\ \text { BOD: } & \text { January 1, 2013 Economic Life: } & 30 \text { years } 0 \text { months }\end{array}$

File Name: C: \Users \bushjw $\backslash$ Desktop $\backslash$ Bobs LCC Test $\backslash 20 \_500 \_H i g h . x m l$

1. Investment

Construction Cost $\quad \$ 59,626$

$\mathrm{SIOH} \quad \$ 0$

Design Cost $\$ \$$

Total Cost \$59,626

Salvage Value of Existing Equipment $\quad \$ 0$

Public Utility Company $\$ 0$

Total Investment $\$ \$ 59,626$

2. Energy and Water Savings (+) or Cost (-)

Base Date Savings, unit costs, \& discounted savings

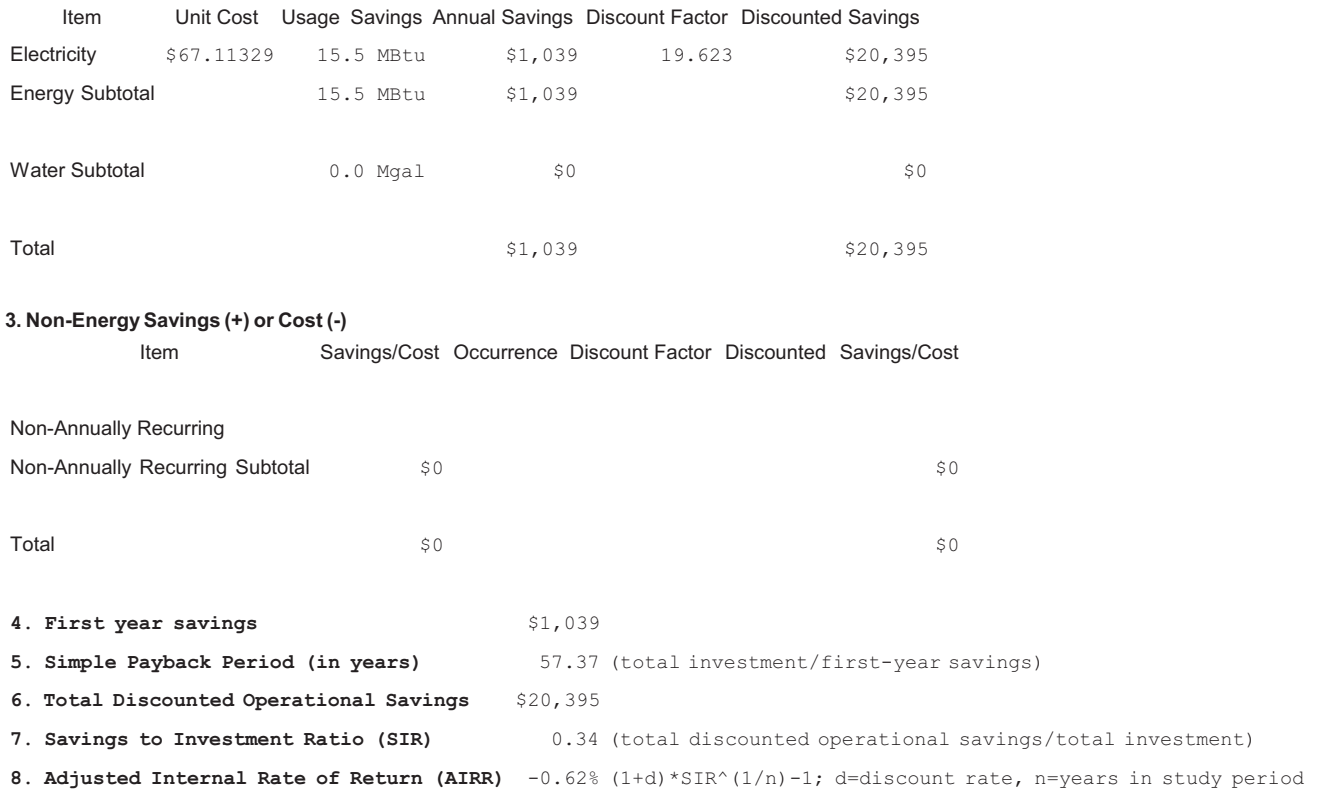


NIST BLCC 5.3-11: ECIP Report

Consistent with Federal Life Cycle Cost Methodology and Procedures, 10 CFR, Part 436, Subpart A

The LCC calculations are based on the FEMP discount rates and energy price escalation rates updated on April 1, 2011.

\begin{tabular}{|c|c|}
\hline \multicolumn{2}{|l|}{ Location: } \\
\hline \multicolumn{2}{|l|}{ Project Title: } \\
\hline \multicolumn{2}{|l|}{ Base Date: } \\
\hline \multicolumn{2}{|l|}{ BOD: } \\
\hline \multicolumn{2}{|c|}{ File Name: $\quad C: \backslash$ Users $\backslash$ bushj $j \backslash \backslash$ Desktop $\backslash$ Bobs } \\
\hline \multicolumn{2}{|l|}{ 1. Investment } \\
\hline Construction Cost & $\$ 27,569$ \\
\hline $\mathrm{SIOH}$ & $\$ 0$ \\
\hline Design Cost & $\$ 0$ \\
\hline Total Cost & $\$ 27,569$ \\
\hline Salvage Value of Existing Equipment & $\$ 0$ \\
\hline Public Utility Company & $\$ 0$ \\
\hline Total Investment & $\$ 27,569$ \\
\hline
\end{tabular}

2. Energy and Water Savings (+) or Cost (-)

Base Date Savings, unit costs, \& discounted savings

Item Unit Cost Usage Savings Annual Savings Discount Factor Discounted Savings

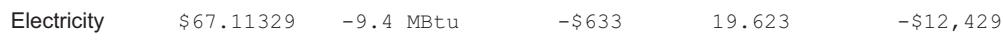

$\begin{array}{llll}\text { Energy Subtotal } & -9.4 \mathrm{MBtu} & -\$ 633 & -\$ 12,429\end{array}$

$\begin{array}{lll}\text { Water Subtotal } & 0.0 \mathrm{Mgal} & \$ 0\end{array}$

$\begin{array}{lll}\text { Total } & -\$ 633 & -\$ 12,429\end{array}$

1. Investme

Wate
30 years 0 months

Japan Transformer Analyst: INL

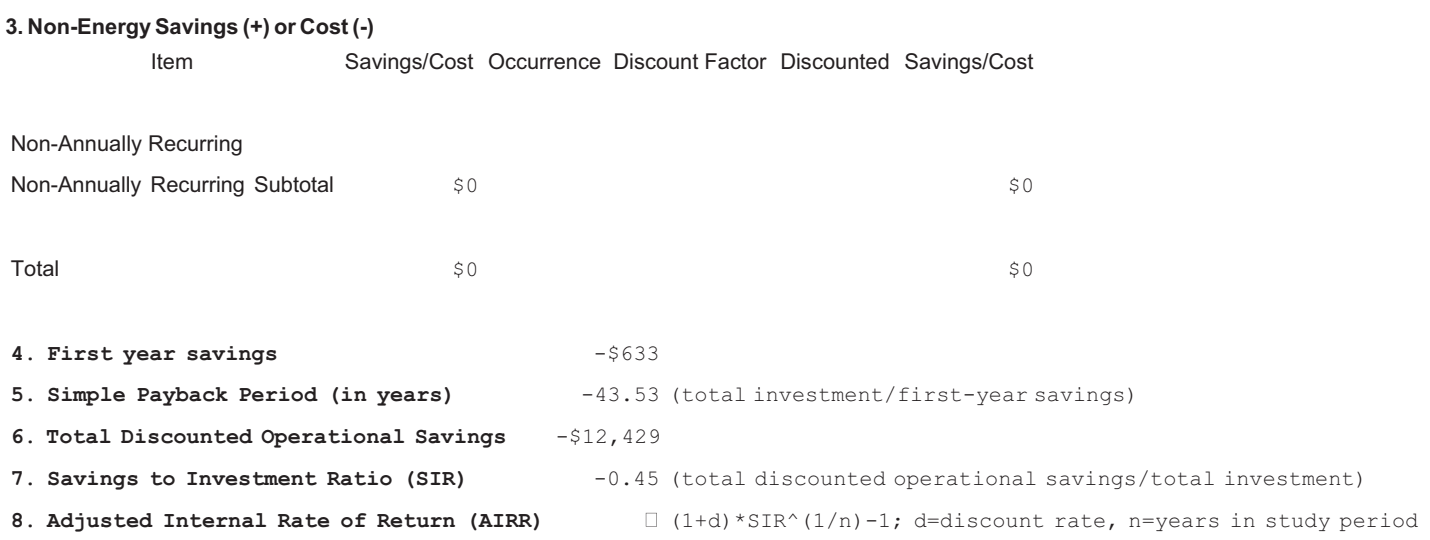

3. Non-Energy Savings (+) or Cost (-)

Item Savings/Cost Occurrence Discount Factor Discounted Savings/Cost

Non-Annually Recurring

$\$ 0$

Total

$\$ 0$

$\$ 0$

$-\$ 633$

-43.53 (total investment/first-year savings)

$-\$ 12,429$

-0.45 (total discounted operational savings/total investment)

$\square(1+d) * \operatorname{SIR}^{\wedge}(1 / \mathrm{n})-1 ; \mathrm{d}=$ discount rate, $\mathrm{n}=$ years in study period 
NIST BLCC 5.3-11: ECIP Report

Consistent with Federal Life Cycle Cost Methodology and Procedures, 10 CFR, Part 436, Subpart A

The LCC calculations are based on the FEMP discount rates and energy price escalation rates updated on April 1, 2011.

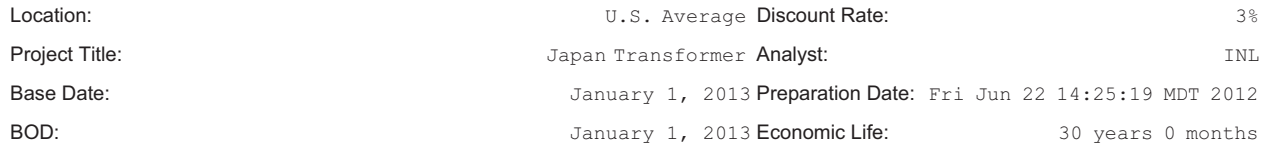

File Name: C: $\backslash$ Users $\backslash$ bushjw $\backslash$ Desktop $\backslash$ Bobs LCC Test $\backslash 20 \_500 \_$Med.xml

1. Investment

Construction Cost \$36,354

$\mathrm{SIOH}$

Design Cost $\$ 0$

Total Cost $\$ 36,354$

Salvage Value of Existing Equipment $\quad \$ 0$

Public Utility Company $\$ 0$

Total Investment $\$ 36,354$

2. Energy and Water Savings (+) or Cost (-)

Base Date Savings, unit costs, \& discounted savings

Item Unit Cost Usage Savings Annual Savings Discount Factor Discounted Savings

$\begin{array}{llllll}\text { Electricity } & \$ 67.11329 & 7.7 \mathrm{MBtu} & \$ 518 & 19.623 & \$ 10,159\end{array}$

$\begin{array}{llll}\text { Energy Subtotal } & 7.7 \mathrm{MBtu} & \$ 518 & \$ 10,159\end{array}$

Water Subtotal $\quad 0.0 \mathrm{Mgal} \quad \$ 0 \quad \$ 0$

$\begin{array}{lr}\text { Total } & \$ 518 \\ & \$ 10,159\end{array}$

3. Non-Energy Savings (+) or Cost (-)

Item Savings/Cost Occurrence Discount Factor Discounted Savings/Cost

Non-Annually Recurring

Non-Annually Recurring Subtotal $\quad \$ 0 \quad \$ 0$

Total

$\$ 0-2-s 0$

4. First year savings

$\$ 518$

5. Simple Payback Period (in years)

70.22 (total investment/first-year savings)

7. Savings to Investment Ratio (SIR)

$\$ 10,159$

0.28 (total discounted operational savings/total investment)

8. Adjusted Internal Rate of Return (AIRR) $-1.29 \%(1+d) * \operatorname{SIR}^{\wedge}(1 / \mathrm{n})-1$; d=discount rate, $\mathrm{n}=y e a r s$ in study period 
NIST BLCC 5.3-11: ECIP Report

Consistent with Federal Life Cycle Cost Methodology and Procedures, 10 CFR, Part 436, Subpart A

The LCC calculations are based on the FEMP discount rates and energy price escalation rates updated on April 1, 2011.

\begin{tabular}{|c|c|}
\hline \multicolumn{2}{|l|}{ Location: } \\
\hline \multicolumn{2}{|l|}{ Project Title: } \\
\hline \multicolumn{2}{|l|}{ Base Date: } \\
\hline \multicolumn{2}{|l|}{ BOD: } \\
\hline \multirow{2}{*}{\multicolumn{2}{|c|}{$\begin{array}{l}\text { File Name: C: } \backslash \text { Users } \backslash \text { bushj } \backslash \text { Wesktop } \backslash \text { Bobs } \\
\text { 1. Investment }\end{array}$}} \\
\hline & \\
\hline Construction Cost & $\$ 82,958$ \\
\hline $\mathrm{SIOH}$ & $\$ 0$ \\
\hline Design Cost & $\$ 0$ \\
\hline Total Cost & $\$ 82,958$ \\
\hline Salvage Value of Existing Equipment & $\$ 0$ \\
\hline Public Utility Company & $\$ 0$ \\
\hline Total Investment & $\$ 82,958$ \\
\hline
\end{tabular}

2. Energy and Water Savings (+) or Cost (-)

Base Date Savings, unit costs, \& discounted savings

Japan Transformer Analyst: INL

January 1, 2013 Preparation Date: Fri Jun 22 15:09:06 MDT 2012

January 1, 2013 Economic Life: 30 years 0 months

t $\backslash 60 \_1000 \_$High.xml

$\$ 0$

58

(1)

(1)

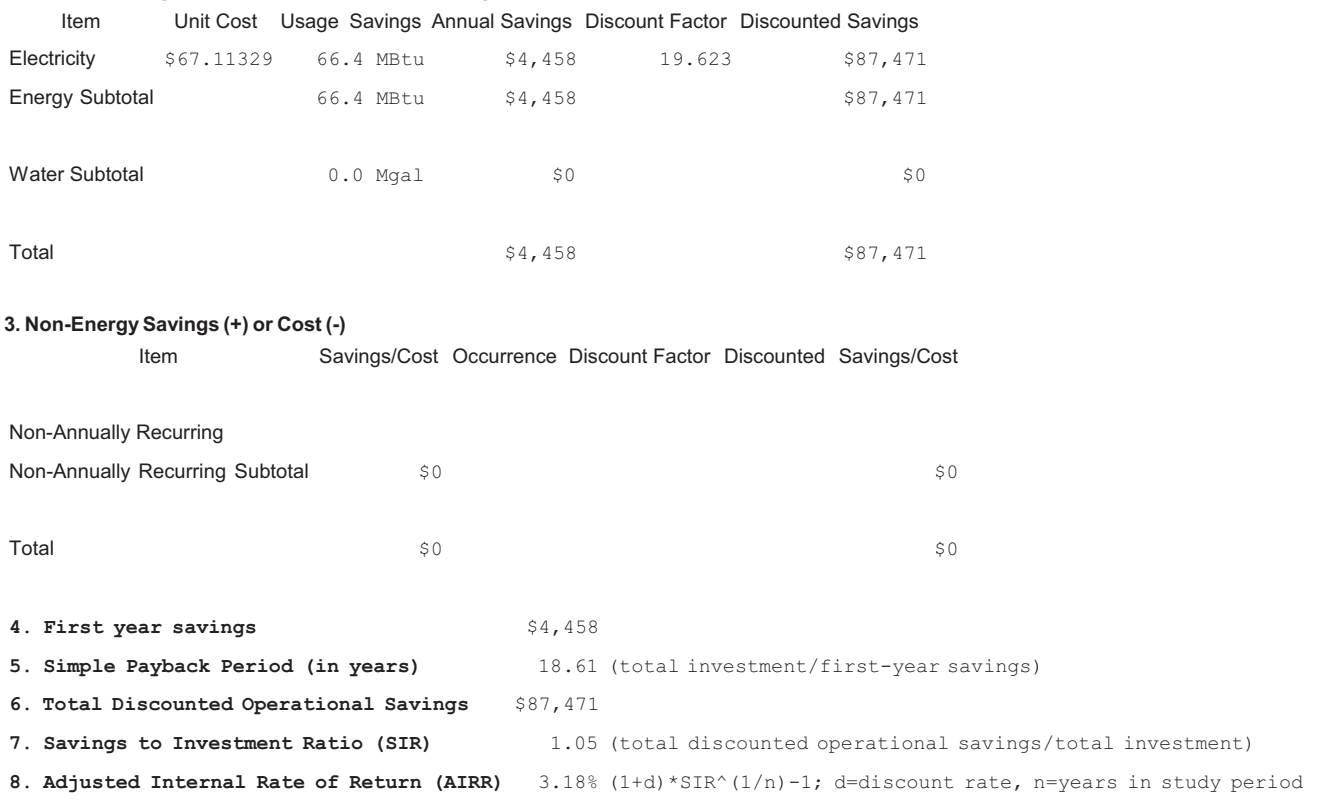

Unit Cost Usage Savings Annual Savings Discount Factor Discounted Savings

$\$ 4,458 \quad 19.623 \quad \$ 87,471$

$\$ 0-\$ 0$

$4,458 \quad \$ 87,471$

Savings (+) or Cost (-)

Savings/Cost Occurrence Discount Factor Discounted Savings/Cost

Non-Annually Recurring

Nor

$\$ 4,458$

1.05 (total discounted operational savings/total investment

8. Adjusted Internal Rate of Return (AIRR) $3.18 \%(1+d) * S^{\wedge}(1 / n)-1$; $d=d i s c o u n t$ rate, n=years in study period
} 
NIST BLCC 5.3-11: ECIP Report

Consistent with Federal Life Cycle Cost Methodology and Procedures, 10 CFR, Part 436, Subpart A

The LCC calculations are based on the FEMP discount rates and energy price escalation rates updated on April 1, 2011.

$\begin{array}{lrr}\text { Location: } & \text { U.S. Average Discount Rate: } & 3 \% \\ \text { Project Title: } & \text { Japan Transformer Analyst: } & \text { INL } \\ \text { Base Date: } & \text { January 1, 2013 Preparation Date: Fri Jun } 2215: 04: 53 \text { MDT } 2012 \\ \text { BOD: } & \text { January 1, 2013 Economic Life: } & 30 \text { years } 0 \text { months }\end{array}$

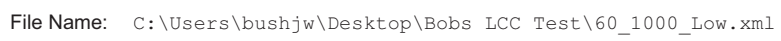

1. Investment

Construction Cost $\$ 50,794$

$\mathrm{SIOH} \quad \$ 0$

Design Cost $\$ 0$

Total Cost \$50,794

Salvage Value of Existing Equipment $\quad \$ 0$

Public Utility Company $\$ 0$

Total Investment $\$ \$ 50,794$

2. Energy and Water Savings (+) or Cost (-)

Base Date Savings, unit costs, \& discounted savings

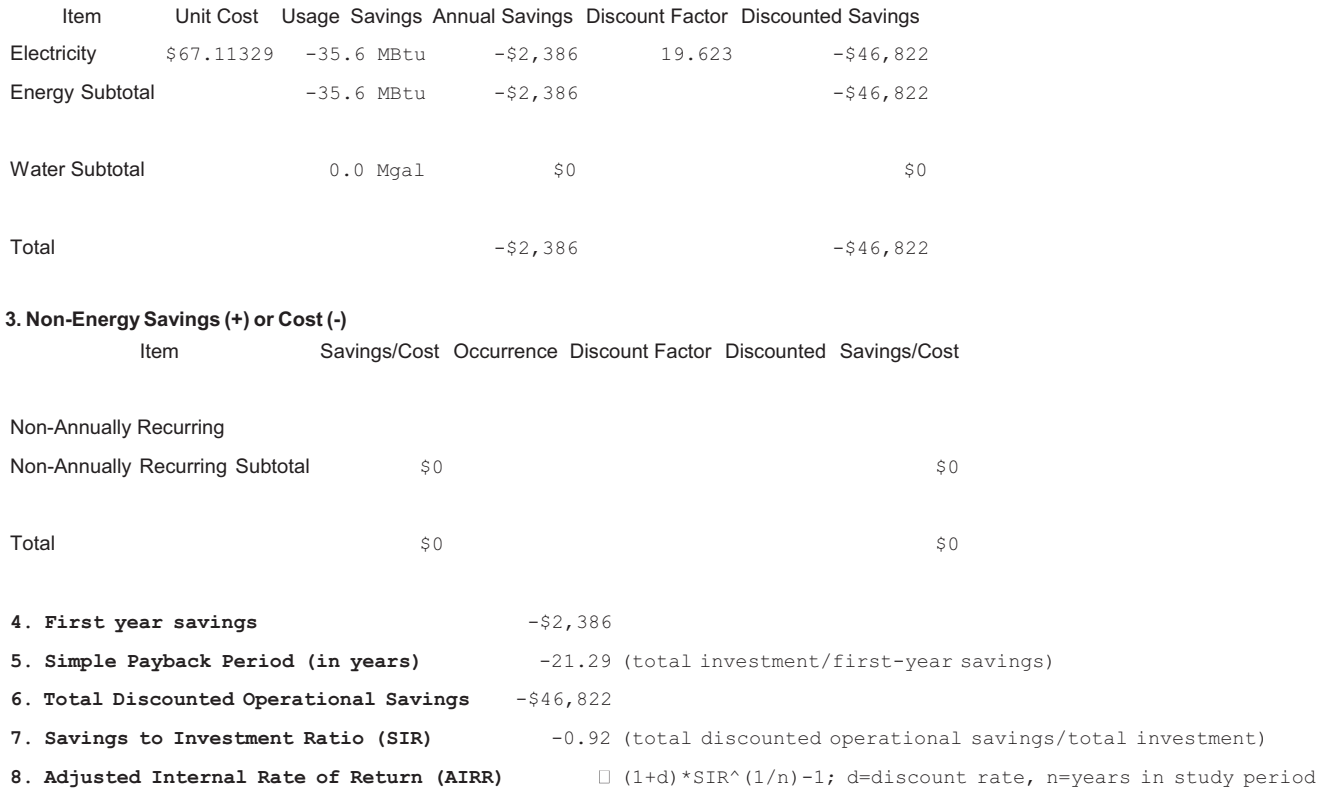

4. First year savings

7. Savings to Investment Ratio (SIR)

8. Adjusted Internal Rate of Return (AIRR) $\square(1+d){ }^{*} \operatorname{SIR}^{\wedge}(1 / n)-1 ; d=d i s c o u n t$ rate, $n=y e a r s$ in study period 
NIST BLCC 5.3-11: ECIP Report

Consistent with Federal Life Cycle Cost Methodology and Procedures, 10 CFR, Part 436, Subpart A

The LCC calculations are based on the FEMP discount rates and energy price escalation rates updated on April 1, 2011.

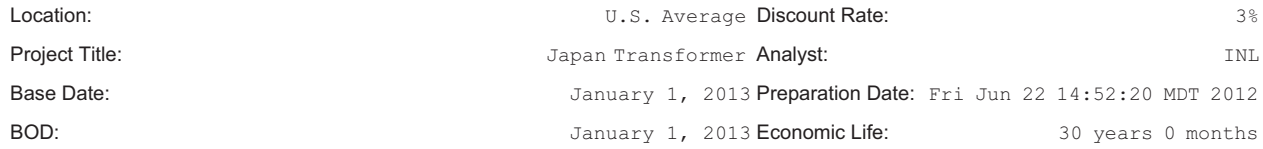

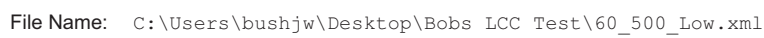

1. Investment

Construction Cost $\$ 27,569$

$\mathrm{SIOH}$

Design Cost $\$ 0$

Total Cost $\$ 27,569$

Salvage Value of Existing Equipment $\quad \$ 0$

Public Utility Company $\$ 0$

Total Investment $\$ 27,569$

2. Energy and Water Savings (+) or Cost (-)

Base Date Savings, unit costs, \& discounted savings

Item Unit Cost Usage Savings Annual Savings Discount Factor Discounted Savings

\begin{tabular}{|c|c|c|c|c|c|}
\hline Electricity & $\$ 67.11329$ & $-18.0 \mathrm{MBtu}$ & $-\$ 1,209$ & 19.623 & $-\$ 23,716$ \\
\hline Energy Su & & $-18.0 \mathrm{MBtu}$ & $-\$ 1,209$ & & $-\$ 23,7$ \\
\hline
\end{tabular}

Energy Subtotal $\quad-18.0 \mathrm{MBtu} \quad-\$ 1,209 \quad-\$ 23,716$

Water Subtotal $\quad 0.0 \mathrm{Mgal} \quad \$ 0 \quad \$ 0$

Total

$-\$ 1,209-\$ 23,716$

3. Non-Energy Savings (+) or Cost (-)

Item

Savings/Cost Occurrence Discount Factor Discounted Savings/Cost

Non-Annually Recurring

Non-Annually Recurring Subtotal $\quad \$ 0 \quad \$ 0$

Total

$\$ 0$

$\$ 0$

4. First year savings

5. Simple Payback Period (in years)

6. Total Discounted Operational Savings

7. Savings to Investment Ratio (SIR)

8. Adjusted Internal Rate of Return (AIRR)

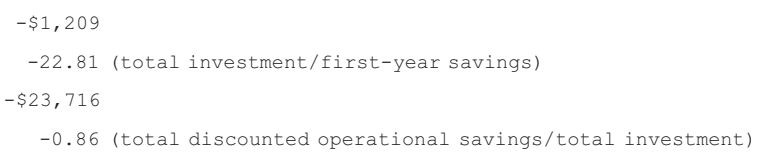

$3 \%$
$\square(1+d) * \operatorname{SIR}^{\wedge}(1 / n)-1 ; d=d i s c o u n t$ rate, $n=y e a r s$ in study period 
NIST BLCC 5.3-11: ECIP Report

Consistent with Federal Life Cycle Cost Methodology and Procedures, 10 CFR, Part 436, Subpart A

The LCC calculations are based on the FEMP discount rates and energy price escalation rates updated on April 1, 2011.

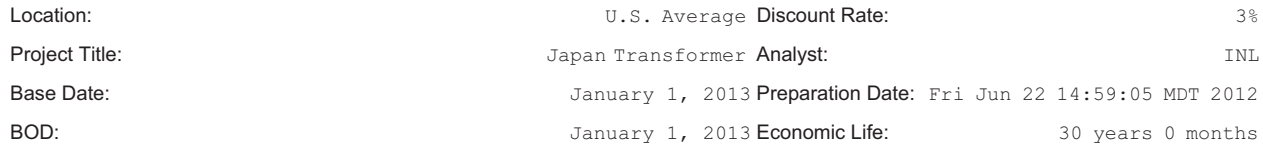

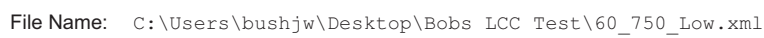

1. Investment

Construction Cost \$36,354

$\mathrm{SIOH}$

Design Cost $\$ 0$

Total Cost $\$ 36,354$

Salvage Value of Existing Equipment $\quad \$ 0$

Public Utility Company $\$ 0$

Total Investment $\$ 36,354$

2. Energy and Water Savings (+) or Cost (-)

Base Date Savings, unit costs, \& discounted savings

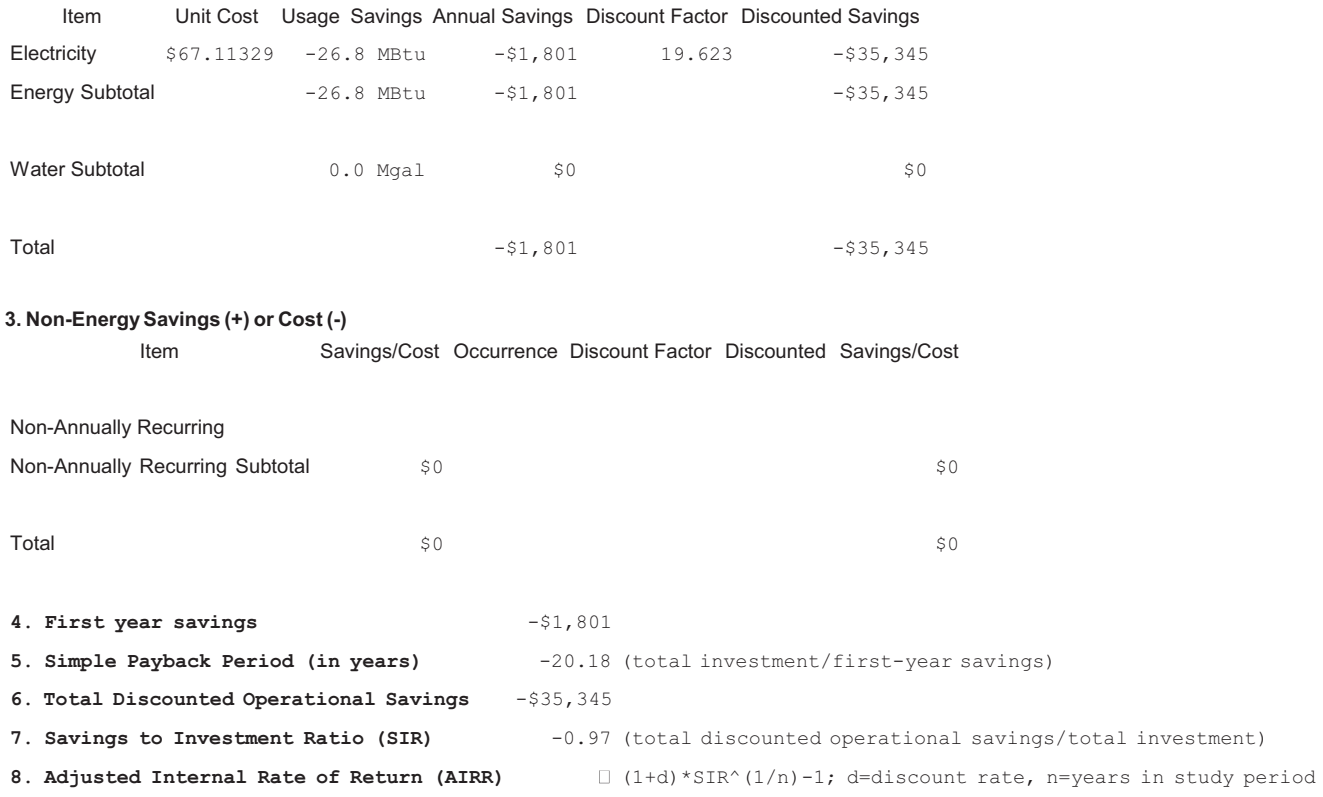

8. Adjusted Internal Rate of Return (AIRR)

$\square(1+d) * \operatorname{SIR}^{\wedge}(1 / n)-1 ; d=d i s c o u n t$ rate, $n=y e a r s$ in study period 
NIST BLCC 5.3-11: ECIP Report

Consistent with Federal Life Cycle Cost Methodology and Procedures, 10 CFR, Part 436, Subpart A

The LCC calculations are based on the FEMP discount rates and energy price escalation rates updated on April 1, 2011.

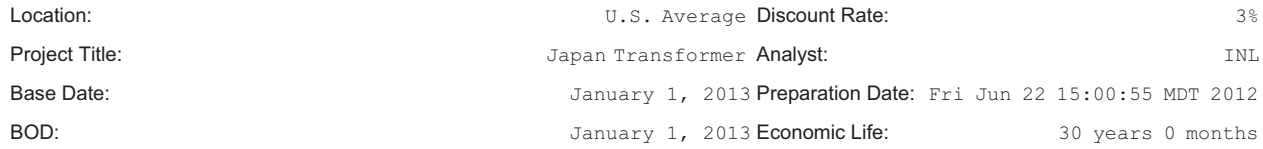

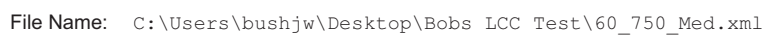

1. Investment

Construction Cost $\$ 44,299$

$\mathrm{SIOH}$

Design Cost $\$ 0$

Total Cost $\$ 44,299$

Salvage Value of Existing Equipment $\quad \$ 0$

Public Utility Company $\$ 0$

Total Investment $\$ 44,299$

2. Energy and Water Savings (+) or Cost (-)

Base Date Savings, unit costs, \& discounted savings

Item Unit Cost Usage Savings Annual Savings Discount Factor Discounted Savings

\begin{tabular}{|c|c|c|c|c|}
\hline Electricity & $\$ 67.11329$ & $30.6 \mathrm{MBtu}$ & $\$ 2,056$ & 19.623 \\
\hline Energy St & & $30.6 \mathrm{MBtu}$ & $\$ 2,056$ & \\
\hline
\end{tabular}

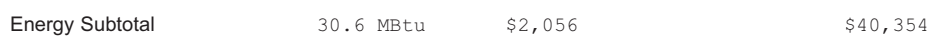

Water Subtotal $\quad 0.0 \mathrm{Mgal} \quad \$ 0 \quad \$ 0$

Water

$\$ 2,056 \quad \$ 40,354$

3. Non-Energy Savings (+) or Cost (-)

Item

Savings/Cost Occurrence Discount Factor Discounted Savings/Cost

Non-Annually Recurring

Non-Annually Recurring Subtotal $\quad \$ 0 \quad \$ 0$

Total

$\$ 0$

4. First year savings

5. Simple Payback Period (in years)

7. Savings to Investment Ratio (SIR)

8. Adjusted Internal Rate of Return (AIRR)

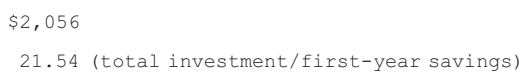
3음

\author{
$2.68 \%(1+d) * \operatorname{SIR}^{\wedge}(1 / n)-1 ; d=d i s c o u n t$ rate, $n=y e a r s$ in study period
}


Consistent with Federal Life Cycle Cost Methodology and Procedures, 10 CFR, Part 436, Subpart A

The LCC calculations are based on the FEMP discount rates and energy price escalation rates updated on April 1, 2011.

Location:

U.S. Average Discount Rate:

Project

Title:

Japan Transformer Analyst:

Base Date:

January 1, 2013 Preparation Date:

Wed May 16 09:18:50 MDT

January 1, 2013 Economic Life:

30 years 0 months

BOD:$$
c: \sqrt{1}
$$

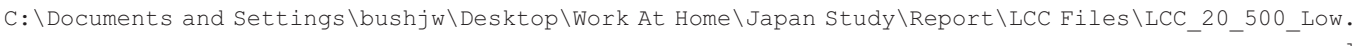

File Name

1. Investment

Construction Cost $\$ 52,569$

$\mathrm{SIOH}$

$\$ 3,154$

Design Cost

$\$ 2,103$

Total Cost

$\$ 57,826$

Salvage Value of Existing Equipment \$0

Public Utility Company $\$ 0$

Total Investment $\quad \$ 57,826$

2. Energy and Water Savings (+) or Cost (-)

Base Date Savings, unit costs, \& discounted savings

Item Unit Cost Usage Savings Annual Savings Discount Factor Discounted Savings

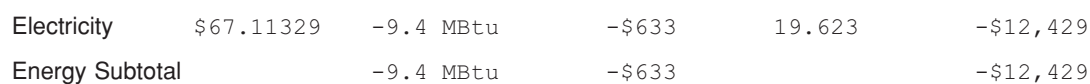

$\begin{array}{llll}\text { Energy Subtotal } & -9.4 \mathrm{MBtu} & -\$ 633 & -\$ 12,429\end{array}$

$\begin{array}{llll}\text { Water Subtotal } & 0.0 \mathrm{Mgal} & \$ 0 & \$ 0\end{array}$

Total

$-\$ 633-\$ 12,429$

3. Non-Energy Savings (+) or Cost (-)

Item Savings/Cost Occurrence Discount Factor Discounted Savings/Cost

Non-Annually Recurring

Non-Annually Recurring Subtotal

4. First year savings

5. Simple Payback Period (in years)

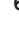

7. Savings to Investment Ratio (SIR)

8. Adjusted Internal Rate of Return (AIRR)

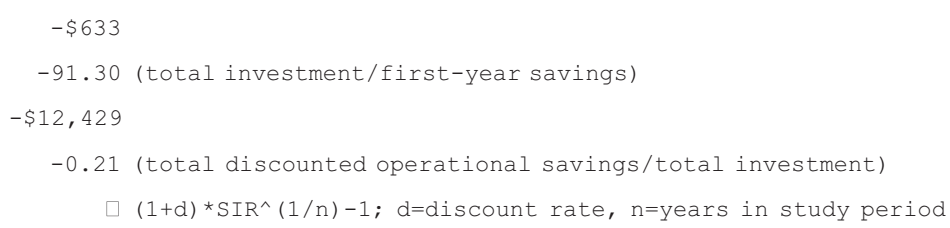



Consistent with Federal Life Cycle Cost Methodology and Procedures, 10 CFR, Part 436, Subpart A

The LCC calculations are based on the FEMP discount rates and energy price escalation rates updated on April 1, 2011.

Location:

U.S. Average Discount Rate:

Project

Title:

Japan Transformer Analyst:

Base Date:

January 1, 2013 Preparation

Wed May $16 \quad 09: 20: 00$ MDT

January 1， 2013 Economic Life:

30 years 0 months

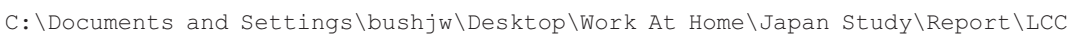

$$
\text { Files } \backslash \text { LCC_20_500_Med.xml }
$$

File Name:

$\$ 58,995$

$\$ 3,540$

$\$ 2,360$

$\$ 64,894$

Total Cost

Salvage Value of Existing Equipment $\$ 0$

Public Utility Company $\$ 0$

Total Investment \$64,894

2. Energy and Water Savings (+) or Cost (-)

Base Date Savings, unit costs, \& discounted savings

Item Unit Cost Usage Savings Annual Savings Discount Factor Discounted Savings

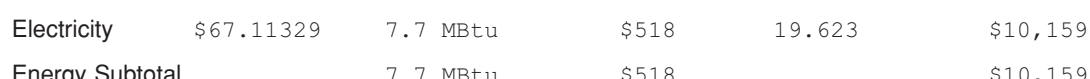

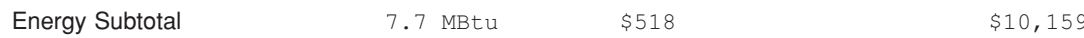

$\begin{array}{llll}\text { Water Subtotal } & 0.0 \mathrm{Mgal} & \$ 0 & \$ 0\end{array}$

Total

$\$ 518 \quad \$ 10,159$

3. Non-Energy Savings (+) or Cost (-)

Item Savings/Cost Occurrence Discount Factor Discounted Savings/Cost

Non-Annually Recurring

Non-Annually Recurring Subtotal

4. First year savings

5. Simple Payback Period (in years)

6.

7. Savings to Investment Ratio (SIR)

8. Adjusted Internal Rate of Return (AIRR)

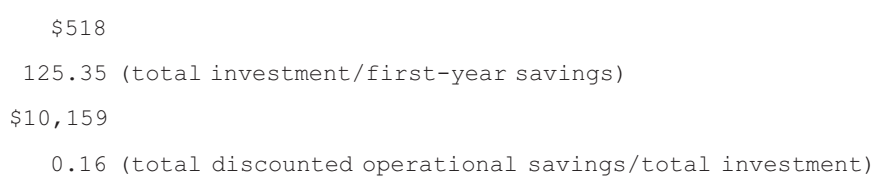

$-3.17 \%(1+d){ }^{*} \operatorname{SIR}^{\wedge}(1 / n)-1 ; d=d i s c o u n t$ rate, $n=y e a r s$ in study period 

Consistent with Federal Life Cycle Cost Methodology and Procedures, 10 CFR, Part 436, Subpart A

The LCC calculations are based on the FEMP discount rates and energy price escalation rates updated on April 1, 2011.

Location:

Title:

Japan Transformer Analyst:

Base Date:

BOD:

January 1, 2013 Preparation
$\$ 84,626$

$\$ 5,078$

$\$ 3,385$

$\$ 93,089$

Total Cost

$\$ 0$

$\begin{array}{lr}\text { Public Utility Company } & \$ 0 \\ \text { Total Investment } & \$ 93,089\end{array}$

$\begin{array}{lr}\text { Public Utility Company } & \$ 0 \\ \text { Total Investment } & \$ 93,089\end{array}$

$\begin{array}{lr}\text { 1. Investment } & \\ \text { Construction Cost } & \$ 84,626 \\ \text { SIOH } & \$ 5,078 \\ \text { Design Cost } & \$ 3,385 \\ \text { Total Cost } & \$ 93,089 \\ \text { Salvage Value of Existing Equipment } & \$ 0 \\ \text { Public Utility Company } & \$ 0 \\ \text { Total Investment } & \$ 93,089\end{array}$

2. Energy and Water Savings (+) or Cost (-)

Base Date Savings, unit costs, \& discounted savings

Item Unit Cost Usage Savings Annual Savings Discount Factor Discounted Savings

\begin{tabular}{|c|c|c|c|c|}
\hline Electricity & $\$ 67.11329$ & 15.5 MBtu & $\$ 1,039$ & 19.623 \\
\hline
\end{tabular}

$\begin{array}{lll}\text { Energy Subtotal } & 15.5 \mathrm{MBtu} & \$ 1,039\end{array}$

$\begin{array}{llll}\text { Water Subtotal } & 0.0 \mathrm{Mgal} & \$ 0 & \$ 0\end{array}$

Total

3. Non-Energy Savings (+) or Cost (-)

Item Savings/Cost Occurrence Discount Factor Discounted Savings/Cost

Non-Annually Recurring

Non-Annually Recurring Subtotal

4. First year savings

5. Simple Payback Period (in years)

6

7. Savings to Investment Ratio (SIR)

8. Adjusted Internal Rate of Return (AIRR)

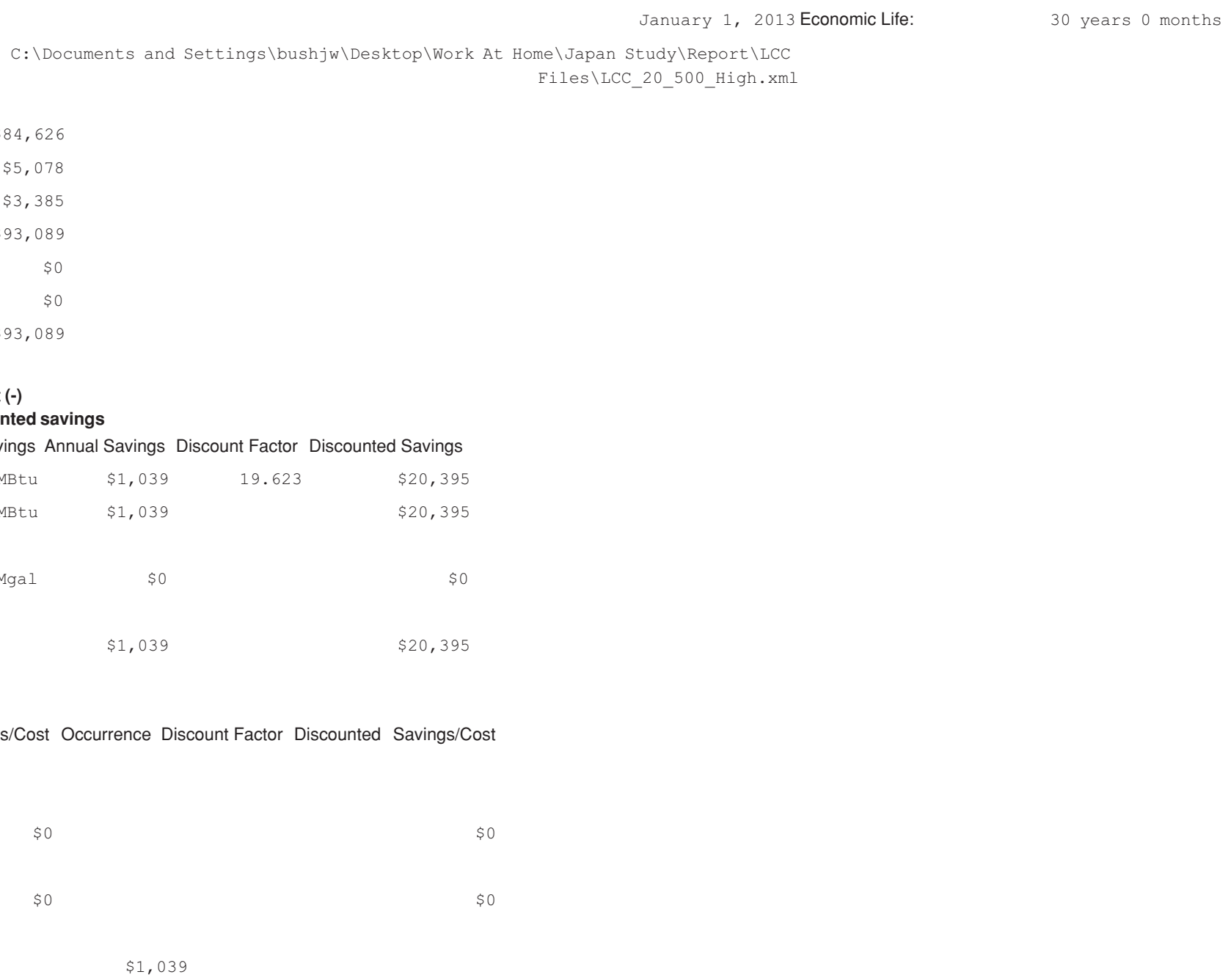

January 1, 2013 Economic Life:

$C: \backslash$ Documents and Settings $\backslash$ bushj $\backslash$ W $\backslash$ Desktop $\backslash$ Work At Home $\backslash$ Japan Study $\backslash$ Report $\backslash$ LCC

$$
\text { Files } \backslash \text { LCC_20_500_High. xmI }
$$

$\$ 1,039 \quad \$ 20,395$

30 years 0 months

Wed May 16 09:07:32 MDT 

Consistent with Federal Life Cycle Cost Methodology and Procedures, 10 CFR, Part 436, Subpart A

The LCC calculations are based on the FEMP discount rates and energy price escalation rates updated on April 1, 2011.

Location:

U.S. Average Discount Rate:

Project

Title:

Japan Transformer Analyst:

Base Date:

January 1, 2013 Preparation Date:

Wed May 16 09:23:51 MDT

January 1, 2013 Economic Life:

30 years 0 months

BOD:

C: $\backslash$ Documents and Settings $\backslash$ bushjw $\backslash$ Desktop $\backslash$ Work At Home $\backslash$ Japan Study $\backslash$ Report $\backslash L C C$ Files $\backslash$ LCC_20_750_Low.

File Name:

1. Investment

Construction Cost $\$ 61,354$

$\mathrm{SIOH}$

$\$ 3,681$

Design Cost

$\$ 2,454$

Total Cost

$\$ 67,489$

Salvage Value of Existing Equipment \$0

Public Utility Company

$\$ 0$

Total Investment

$\$ 67,489$

2. Energy and Water Savings (+) or Cost (-)

Base Date Savings, unit costs, \& discounted savings

Item Unit Cost Usage Savings Annual Savings Discount Factor Discounted Savings

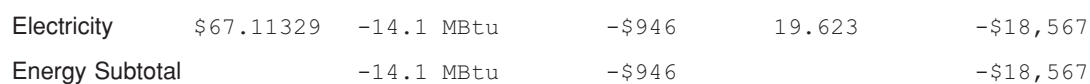

$\begin{array}{llll}\text { Energy Subtotal } & -14.1 \mathrm{MBtu} & -\$ 946 & -\$ 18,567\end{array}$

$\begin{array}{llll}\text { Water Subtotal } & 0.0 \mathrm{Mgal} & \$ 0 & \$ 0\end{array}$

Total

$-\$ 946 \quad-\$ 18,567$

3. Non-Energy Savings (+) or Cost (-)

Item Savings/Cost Occurrence Discount Factor Discounted Savings/Cost

Non-Annually Recurring

Non-Annually Recurring Subtotal

4. First year savings

5. Simple Payback Period (in years)

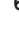

7. Savings to Investment Ratio (SIR)

8. Adjusted Internal Rate of Return (AIRR)

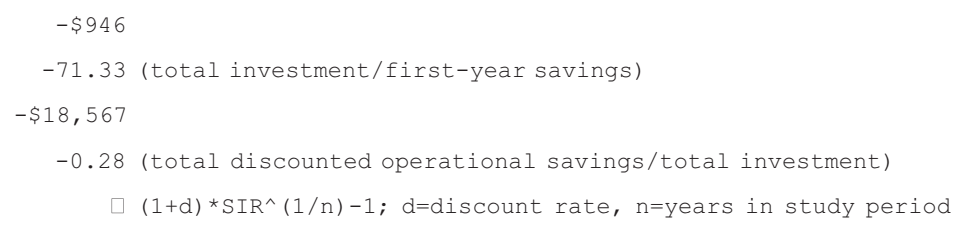



Consistent with Federal Life Cycle Cost Methodology and Procedures, 10 CFR, Part 436, Subpart A

The LCC calculations are based on the FEMP discount rates and energy price escalation rates updated on April 1, 2011.

Location:

U.S. Average Discount Rate:

Project

Title:

Japan Transformer Analyst:

Base Date:

January 1, 2013 Preparation

Wed May $16 \quad 09: 25: 03$ MDT

January 1， 2013 Economic Life:

30 years 0 months

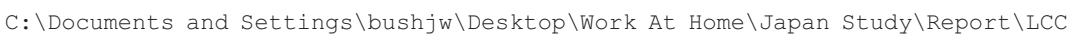

$$
\text { Files } \backslash \text { LCC_20_750_Med.xml }
$$

File Name:

$\$ 69,299$

$\$ 4,158$

$\$ 2,772$

$\$ 76,229$

Total Cost

Salvage Value of Existing Equipment \$0

Public Utility Company $\$ 0$

Total Investment \$76,229

2. Energy and Water Savings (+) or Cost (-)

Base Date Savings, unit costs, \& discounted savings

Item Unit Cost Usage Savings Annual Savings Discount Factor Discounted Savings

\begin{tabular}{|c|c|c|c|c|}
\hline Electricity & $\$ 67.11329$ & $11.6 \mathrm{MBtu}$ & $\$ 779$ & 19.623 \\
\hline
\end{tabular}

$\begin{array}{llll}\text { Energy Subtotal } & 11.6 \mathrm{MBtu} & \$ 779 & \$ 15,285\end{array}$

$\begin{array}{llll}\text { Water Subtotal } & 0.0 \mathrm{Mgal} & \$ 0 & \$ 0\end{array}$

Total

$\$ 779 \quad \$ 15,285$

3. Non-Energy Savings (+) or Cost (-)

Item Savings/Cost Occurrence Discount Factor Discounted Savings/Cost

Non-Annually Recurring

Non-Annually Recurring Subtotal

4. First year savings

5. Simple Payback Period (in years)

6.

7. Savings to Investment Ratio (SIR)

8. Adjusted Internal Rate of Return (AIRR)

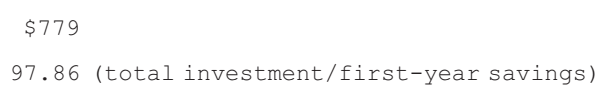

$-2.37 \%(1+d) * \operatorname{SIR}^{\wedge}(1 / n)-1 ; d=d i s c o u n t$ rate, $n=y e a r s$ in study period 

Consistent with Federal Life Cycle Cost Methodology and Procedures, 10 CFR, Part 436, Subpart A

The LCC calculations are based on the FEMP discount rates and energy price escalation rates updated on April 1, 2011.

Location:

Title:

Japan Transformer Analyst:

Base Date:

BOD:

January 1, 2013 Preparation
Date:

January 1, 2013 Economic Life:

$C: \backslash$ Documents and Settings $\backslash$ bushjw $\backslash$ Desktop $\backslash$ Work At Home $\backslash J a p a n$ Study $\backslash$ Report $\backslash$ LCC

$$
\text { Files } \backslash \text { LCC_20_750_High. xmI }
$$

2. Energy and Water Savings (+) or Cost (-)

Base Date Savings, unit costs, \& discounted savings

Item Unit Cost Usage Savings Annual Savings Discount Factor Discounted Savings

\begin{tabular}{|c|c|c|c|c|}
\hline Electricity & $\$ 67.11329$ & $20.9 \mathrm{MBtu}$ & $\$ 1,403$ & 19.623 \\
\hline
\end{tabular}

$\begin{array}{lll}\text { Energy Subtotal } & 20.9 \mathrm{MBtu} & \$ 1,403\end{array}$

$\begin{array}{llll}\text { Water Subtotal } & 0.0 \mathrm{Mgal} & \$ 0 & \$ 0\end{array}$

Total

$\$ 1,403 \quad \$ 27,531$

3. Non-Energy Savings (+) or Cost (-)

Item Savings/Cost Occurrence Discount Factor Discounted Savings/Cost

Non-Annually Recurring

Non-Annually Recurring Subtotal
4. First year savings

5. Simple Payback Period (in years)

6

7.

8.

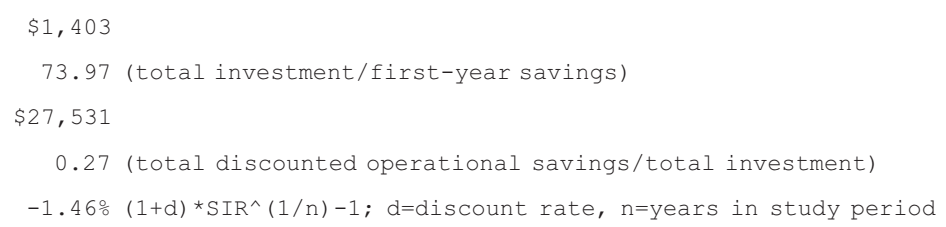

Wed May $16 \quad 09: 21: 01$ MDT

30 years 0 months
$\$ 94,350$

$\$ 5,661$

$\$ 3,774$

$\$ 103,785$

Total Cost

Salvage Value of Existing Equipment $\$ 0$

Public Utility Company $\$ 0$

Total Investment \$103,785

$\begin{array}{lr}\text { 1. Investment } & \\ \text { Construction Cost } & \$ 94,350 \\ \text { SIOH } & \$ 5,661 \\ \text { Design Cost } & \$ 3,774 \\ \text { Total Cost } & \$ 103,785 \\ \text { Salvage Value of Existing Equipment } & \$ 0 \\ \text { Public Utility Company } & \$ 0 \\ \text { Total Investment } & \$ 103,785\end{array}$



Consistent with Federal Life Cycle Cost Methodology and Procedures, 10 CFR, Part 436, Subpart A

The LCC calculations are based on the FEMP discount rates and energy price escalation rates updated on April 1, 2011.

Location:

Title:

Japan Transformer Analyst:

Base Date:

January 1, 2013 Preparation
Date:

January 1, 2013 Economic Life:

BOD:

C: $\backslash$ Documents and Settings $\backslash$ bushjw $\backslash$ Desktop $\backslash$ Work At Home $\backslash$ Japan Study $\backslash$ Report $\backslash$ LCC Files $\backslash$ LCC_20_1000_Low.

File Name:

1. Investment

Construction Cost $\$ 75,794$

$\mathrm{SIOH}$

$\$ 4,548$

Design Cost

$\$ 3,032$

Total Cost

$\$ 83,373$

Salvage Value of Existing Equipment \$0

Public Utility Company

$\$ 0$

Total Investment

$\$ 83,373$

2. Energy and Water Savings (+) or Cost (-)

Base Date Savings, unit costs, \& discounted savings

Item Unit Cost Usage Savings Annual Savings Discount Factor Discounted Savings

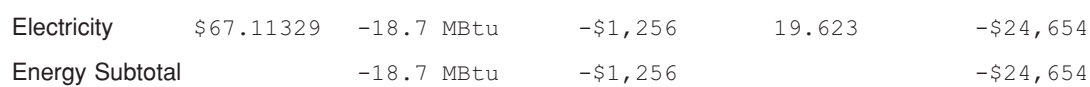

$\begin{array}{llll}\text { Energy Subtotal } & -18.7 \mathrm{MBtu} & -\$ 1,256 & -\$ 24,654\end{array}$

$\begin{array}{llll}\text { Water Subtotal } & 0.0 \mathrm{Mgal} & \$ 0 & \$ 0\end{array}$

Total

$-\$ 1,256 \quad-\$ 24,654$

3. Non-Energy Savings (+) or Cost (-)

Item Savings/Cost Occurrence Discount Factor Discounted Savings/Cost

Non-Annually Recurring

Non-Annually Recurring Subtotal

4. First year savings

5. Simple Payback Period (in years)

6.

7. Savings to Investment Ratio (SIR)

8. Adjusted Internal Rate of Return (AIRR)

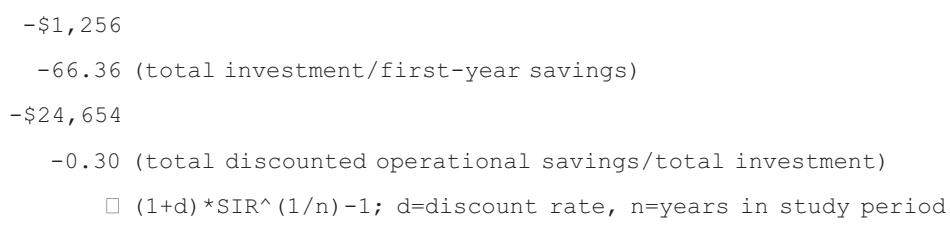

Wed May 16 09:05:33 MDT

30 years 0 months 

Consistent with Federal Life Cycle Cost Methodology and Procedures, 10 CFR, Part 436, Subpart A

The LCC calculations are based on the FEMP discount rates and energy price escalation rates updated on April 1, 2011.

Location:

Title:

Japan Transformer Analyst:

Base Date:

BOD:

January 1, 2013 Preparation

January 1, 2013 Economic Life:

$C: \backslash$ Documents and Settings $\backslash$ bushj $\backslash$ W $\backslash$ Desktop $\backslash$ Work At Home $\backslash J a p a n$ Study $\backslash$ Report $\backslash$ LCC

$$
\text { Files } \backslash \text { LCC_20_1000_Med.xmI }
$$

$\$ 85,742$

$\$ 5,145$

$\$ 3,430$

$\$ 94,316$

Total Cost

Salvage Value of Existing Equipment $\$ 0$

Public Utility Company

Total Investment

$\$ 94,316$
Wed May 16 09:06:27 MDT

30 years 0 months

2. Energy and Water Savings (+) or Cost (-)

Base Date Savings, unit costs, \& discounted savings

Item Unit Cost Usage Savings Annual Savings Discount Factor Discounted Savings

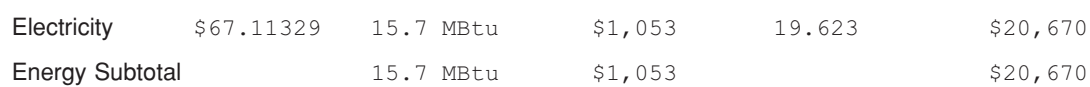

$\begin{array}{lll}\text { Energy Subtotal } & 15.7 \mathrm{MBtu} & \$ 1,053\end{array}$

$\begin{array}{llll}\text { Water Subtotal } & 0.0 \mathrm{Mgal} & \$ 0 & \$ 0\end{array}$

Total

$\$ 1,053 \quad \$ 20,670$

3. Non-Energy Savings (+) or Cost (-)

Item Savings/Cost Occurrence Discount Factor Discounted Savings/Cost

Non-Annually Recurring

Non-Annually Recurring Subtotal

4. First year savings

5. Simple Payback Period (in years)

6.

7. Savings to Investment Ratio (SIR)

8. Adjusted Internal Rate of Return (AIRR)

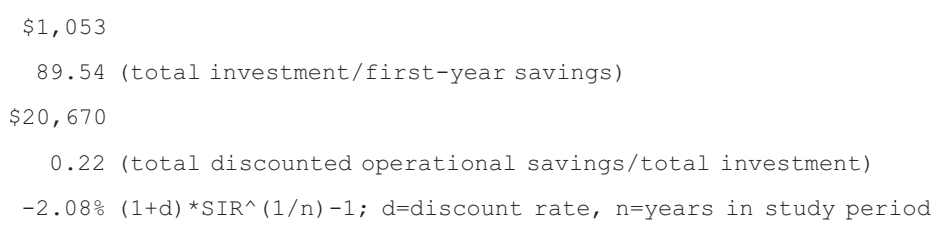




Consistent with Federal Life Cycle Cost Methodology and Procedures, 10 CFR, Part 436, Subpart A

The LCC calculations are based on the FEMP discount rates and energy price escalation rates updated on April 1, 2011.

Location:

U.S. Average Discount Rate:

Project

Title:

Japan Transformer Analyst:

Base Date:

January 1, 2013 Preparation Date:

Wed May 16 09:50:43 MDT

January 1, 2013 Economic Life:

30 years 0 months

BOD:

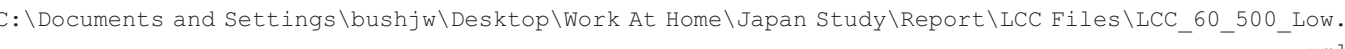

File Name:

1. Investment

Construction Cost $\$ 52,569$

$\mathrm{SIOH}$

$\$ 3,154$

Design Cost

$\$ 2,103$

Total Cost

$\$ 57,826$

Salvage Value of Existing Equipment \$0

Public Utility Company $\$ 0$

Total Investment $\quad \$ 57,826$

2. Energy and Water Savings (+) or Cost (-)

Base Date Savings, unit costs, \& discounted savings

Item Unit Cost Usage Savings Annual Savings Discount Factor Discounted Savings

\begin{tabular}{lllll} 
Electricity $\$ 67.11329$ & $-18.0 \mathrm{MBtu}$ & $-\$ 1,209$ & 19.623 & $-\$ 23,716$ \\
\hline Energy Subtotal & $-18.0 \mathrm{MBtu}$ & $-\$ 1,209$ & & $-\$ 23,716$
\end{tabular}

$\begin{array}{llll}\text { Energy Subtotal } & -18.0 \mathrm{MBtu} & -\$ 1,209 & -\$ 23,716\end{array}$

$\begin{array}{llll}\text { Water Subtotal } & 0.0 \mathrm{Mgal} & \$ 0 & \$ 0\end{array}$

Total

$-\$ 1,209-\$ 23,716$

3. Non-Energy Savings (+) or Cost (-)

Item Savings/Cost Occurrence Discount Factor Discounted Savings/Cost

Non-Annually Recurring

Non-Annually Recurring Subtotal

4. First year savings

5. Simple Payback Period (in years)

6

7. Savings to Investment Ratio (SIR)

8. Adjusted Internal Rate of Return (AIRR)

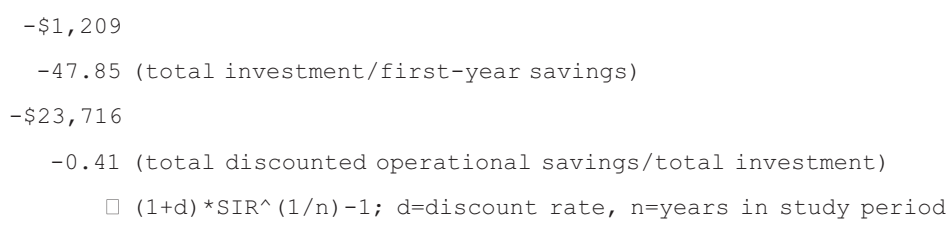



Consistent with Federal Life Cycle Cost Methodology and Procedures, 10 CFR, Part 436, Subpart A

The LCC calculations are based on the FEMP discount rates and energy price escalation rates updated on April 1, 2011.

Location:

U.S. Average Discount Rate:

Project

Title:

Japan Transformer Analyst:

Base Date:

January 1, 2013 Preparation

Wed May 16 09:51:45 MDT

January 1， 2013 Economic Life:

30 years 0 months

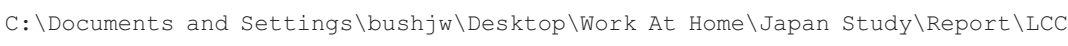

$$
\text { Files } \backslash \text { LCC_60_500_Med.xml }
$$

File Name:

$\$ 58,995$

$\$ 3,540$

$\$ 2,360$

$\$ 64,894$

Total Cost

$\$ 0$

Public Utility Company $\$ 0$

Total Investment $\$ 64,894$

2. Energy and Water Savings (+) or Cost (-)

Base Date Savings, unit costs, \& discounted savings

Item Unit Cost Usage Savings Annual Savings Discount Factor Discounted Savings

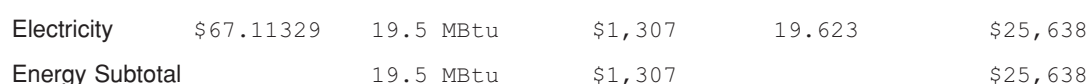

$\begin{array}{llll}\text { Energy Subtotal } & 19.5 \mathrm{MBtu} & \$ 1,307 & \$ 25,638\end{array}$

$\begin{array}{llll}\text { Water Subtotal } & 0.0 \mathrm{Mgal} & \$ 0 & \$ 0\end{array}$

Total

$\$ 1,307 \quad \$ 25,638$

3. Non-Energy Savings (+) or Cost (-)

Item Savings/Cost Occurrence Discount Factor Discounted Savings/Cost

Non-Annually Recurring

Non-Annually Recurring Subtotal

4. First year savings

5. Simple Payback Period (in years)

6.

7. Savings to Investment Ratio (SIR)

8. Adjusted Internal Rate of Return (AIRR)

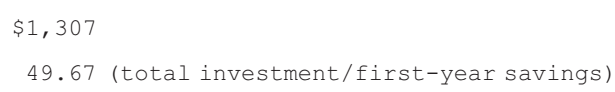

$-0.14 \%(1+d) * \operatorname{SIR}^{\wedge}(1 / n)-1 ; d=d i s c o u n t$ rate, $n=y e a r s$ in study period 

Consistent with Federal Life Cycle Cost Methodology and Procedures, 10 CFR, Part 436, Subpart A

The LCC calculations are based on the FEMP discount rates and energy price escalation rates updated on April 1, 2011.

Location:

Title:

Japan Transformer Analyst:

Base Date:

BOD:

January 1, 2013 Preparation
Date:

January 1, 2013 Economic Life:

$C: \backslash$ Documents and Settings $\backslash$ bushj $\backslash$ Wesktop $\backslash$ Work At Home $\backslash$ Japan Study $\backslash$ Report $\backslash$ LCC

$$
\text { Files } \backslash \text { LCC_60_500_High. xmI }
$$

$\$ 84,626$

$\$ 5,078$

$\$ 3,385$

$\$ 93,089$

Total Cost

Salvage Value of Existing Equipment \$0

Public Utility Company

$\$ 0$

$\$ 93,089$
Wed May 16 09:49:48 MDT

30 years 0 months

2. Energy and Water Savings (+) or Cost (-)

Base Date Savings, unit costs, \& discounted savings

Item Unit Cost Usage Savings Annual Savings Discount Factor Discounted Savings

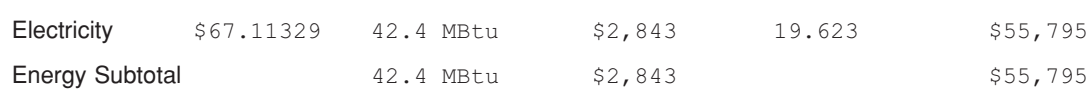

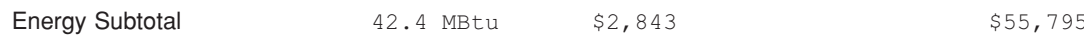

$\begin{array}{llll}\text { Water Subtotal } & 0.0 \mathrm{Mgal} & \$ 0 & \$ 0\end{array}$

Total

$\$ 2,843 \quad \$ 55,795$

3. Non-Energy Savings (+) or Cost (-)

Item Savings/Cost Occurrence Discount Factor Discounted Savings/Cost

Non-Annually Recurring

Non-Annually Recurring Subtotal

4. First year savings

5. Simple Payback Period (in years)

6

7.

8 .

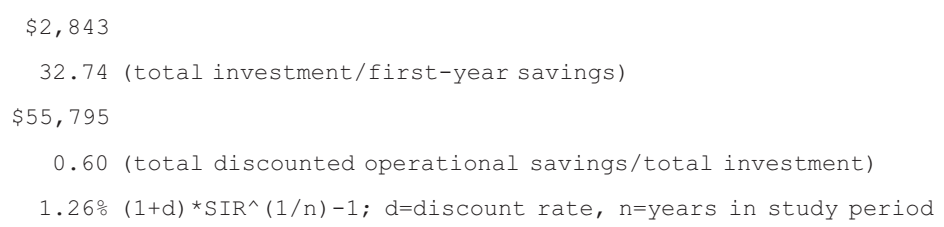



Consistent with Federal Life Cycle Cost Methodology and Procedures, 10 CFR, Part 436, Subpart A

The LCC calculations are based on the FEMP discount rates and energy price escalation rates updated on April 1, 2011.

Location:

U.S. Average Discount Rate:

Project

Title:

Japan Transformer Analyst:

Base Date:

January 1, 2013 Preparation Date:

Wed May 16 09:57:15 MDT

January 1, 2013 Economic Life:

30 years 0 months

BOD:

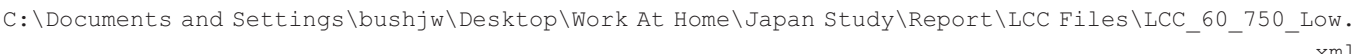

File Name:

1. Investment

Construction Cost $\$ 61,354$

$\mathrm{SIOH}$

$\$ 3,681$

Design Cost

$\$ 2,454$

Total Cost

$\$ 67,489$

Salvage Value of Existing Equipment \$0

Public Utility Company

$\$ 0$

Total Investment

$\$ 67,489$

2. Energy and Water Savings (+) or Cost (-)

Base Date Savings, unit costs, \& discounted savings

Item Unit Cost Usage Savings Annual Savings Discount Factor Discounted Savings

$\begin{array}{llllll}\text { Electricity } & \$ 67.11329 & -26.8 \mathrm{MBtu} & -\$ 1,801 & 19.623 & -\$ 35,345 \\ \text { Energy Subtotal } & -26.8 \mathrm{MBtu} & -\$ 1,801 & & -\$ 35,345\end{array}$

$\begin{array}{llll}\text { Energy Subtotal } & -26.8 \mathrm{MBtu} & -\$ 1,801 & -\$ 35,345\end{array}$

$\begin{array}{llll}\text { Water Subtotal } & 0.0 \mathrm{Mgal} & \$ 0 & \$ 0\end{array}$

Total

$-\$ 1,801 \quad-\$ 35,345$

3. Non-Energy Savings (+) or Cost (-)

Item Savings/Cost Occurrence Discount Factor Discounted Savings/Cost

Non-Annually Recurring

Non-Annually Recurring Subtotal

4. First year savings

5. Simple Payback Period (in years)

6

7. Savings to Investment Ratio (SIR)

8. Adjusted Internal Rate of Return (AIRR)

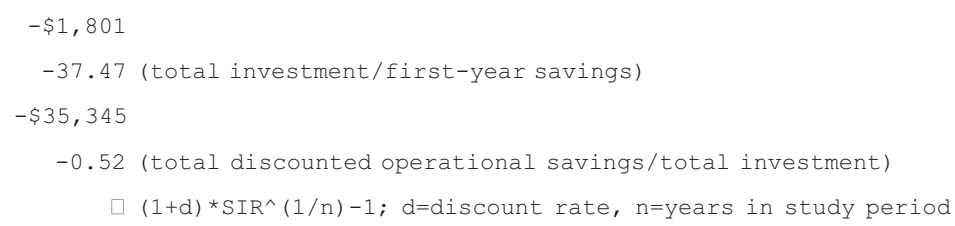



Consistent with Federal Life Cycle Cost Methodology and Procedures, 10 CFR, Part 436, Subpart A

The LCC calculations are based on the FEMP discount rates and energy price escalation rates updated on April 1, 2011.

Location:

U.S. Average Discount Rate:

Project

Title:

Japan Transformer Analyst:

Base Date:

January 1, 2013 Preparation

Wed May $16 \quad 09: 58: 08$ MDT

January 1， 2013 Economic Life:

30 years 0 months

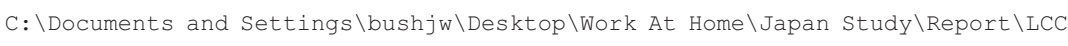

$$
\text { Files } \backslash \text { LCC_60_750_Med.xml }
$$

File Name:

$\$ 69,299$

$\$ 4,158$

$\$ 2,772$

$\$ 76,229$

Total Cost

$\$ 0$

Public Utility Company $\$ 0$

Total Investment $\$ 76,229$

2. Energy and Water Savings (+) or Cost (-)

Base Date Savings, unit costs, \& discounted savings

Item Unit Cost Usage Savings Annual Savings Discount Factor Discounted Savings

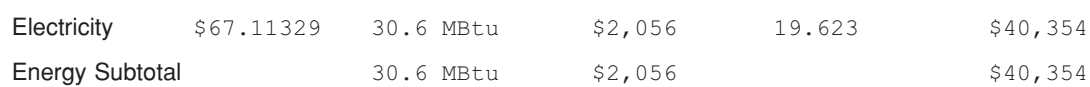

$\begin{array}{lll}\text { Energy Subtotal } & 30.6 \mathrm{MBtu} & \$ 2,056\end{array} \$ 40,354$

$\begin{array}{llll}\text { Water Subtotal } & 0.0 \mathrm{Mgal} & \$ 0 & \$ 0\end{array}$

Total

$\$ 2,056 \quad \$ 40,354$

3. Non-Energy Savings (+) or Cost (-)

Item Savings/Cost Occurrence Discount Factor Discounted Savings/Cost

Non-Annually Recurring

Non-Annually Recurring Subtotal

4. First year savings

5. Simple Payback Period (in years)

6

7. Savings to Investment Ratio (SIR)

8. Adjusted Internal Rate of Return (AIRR)

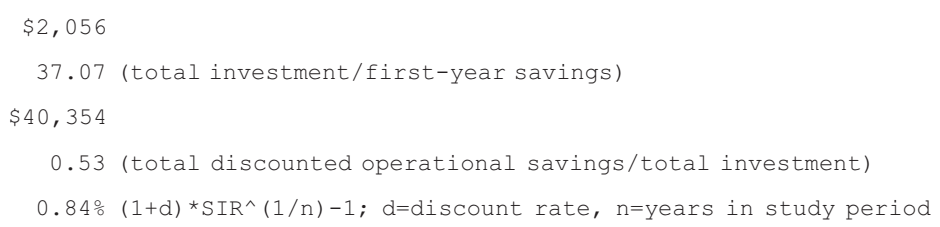



Consistent with Federal Life Cycle Cost Methodology and Procedures, 10 CFR, Part 436, Subpart A

The LCC calculations are based on the FEMP discount rates and energy price escalation rates updated on April 1, 2011.

Location:

Title:

Japan Transformer Analyst:

Base Date:

BOD:

January 1, 2013 Preparation
Date:

January 1, 2013 Economic Life:

$C: \backslash$ Documents and Settings $\backslash$ bushjw $\backslash$ Desktop $\backslash$ Work At Home $\backslash J a p a n$ Study $\backslash$ Report $\backslash$ LCC

$$
\text { Files } \backslash \text { LCC_60_750_High.xml }
$$

$\$ 94,350$

$\$ 5,661$

$\$ 3,774$

$\$ 103,785$

Salvage Value of Existing Equipment $\$ 0$

Public Utility Company $\$ 0$

Total Investment $\$ 103,785$
Wed May 16 09:56:36 MDT

30 years 0 months

2. Energy and Water Savings (+) or Cost (-)

Base Date Savings, unit costs, \& discounted savings

Item Unit Cost Usage Savings Annual Savings Discount Factor Discounted Savings

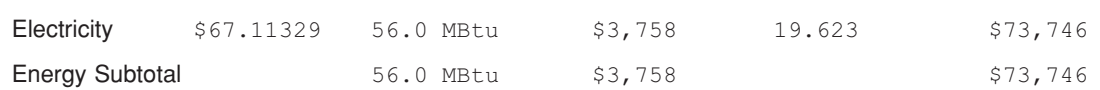

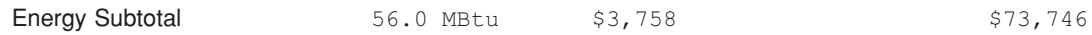

$\begin{array}{llll}\text { Water Subtotal } & 0.0 \mathrm{Mgal} & \$ 0 & \$ 0\end{array}$

Total

$\$ 3,758 \quad \$ 73,746$

3. Non-Energy Savings (+) or Cost (-)

Item Savings/Cost Occurrence Discount Factor Discounted Savings/Cost

Non-Annually Recurring

Non-Annually Recurring Subtotal

4. First year savings

5. Simple Payback Period (in years)

6.

7. Savings to Investment Ratio (SIR)

8. Adjusted Internal Rate of Return (AIRR)

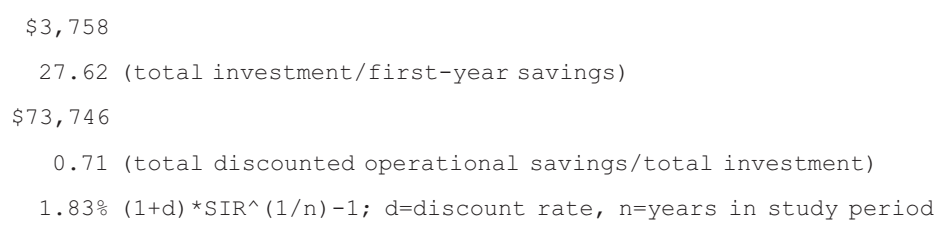



Consistent with Federal Life Cycle Cost Methodology and Procedures, 10 CFR, Part 436, Subpart A

The LCC calculations are based on the FEMP discount rates and energy price escalation rates updated on April 1, 2011.

Location:

Title:

Japan Transformer Analyst:

Base Date:

January 1, 2013 Preparation
Date:

January 1, 2013 Economic Life:

BOD:

C: $\backslash$ Documents and Settings $\backslash$ bushjw $\backslash$ Desktop $\backslash$ Work At Home $\backslash$ Japan Study $\backslash$ Report $\backslash$ LCC Files $\backslash$ LCC_60_1000_Low.

File Name:

1. Investment

Construction Cost $\$ 75,794$

$\mathrm{SIOH}$

$\$ 4,548$

$\$ 3,032$

Design Cost

$\$ 83,373$

Salvage Value of Existing Equipment $\$ 0$

Public Utility Company

$\$ 0$

Total Investment

$$
\$ 83,373
$$

2. Energy and Water Savings (+) or Cost (-)

Base Date Savings, unit costs, \& discounted savings

Item Unit Cost Usage Savings Annual Savings Discount Factor Discounted Savings

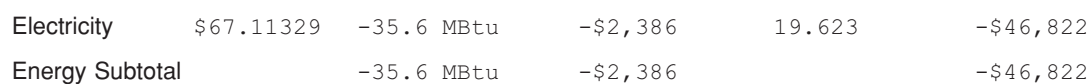

$\begin{array}{llll}\text { Energy Subtotal } & -35.6 \mathrm{MBtu} & -\$ 2,386 & -\$ 46,822\end{array}$

$\begin{array}{llll}\text { Water Subtotal } & 0.0 \mathrm{Mgal} & \$ 0 & \$ 0\end{array}$

Total

$-\$ 2,386 \quad-\$ 46,822$

3. Non-Energy Savings (+) or Cost (-)

Item Savings/Cost Occurrence Discount Factor Discounted Savings/Cost

Non-Annually Recurring

Non-Annually Recurring Subtotal

4. First year savings

5. Simple Payback Period (in years)

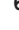

7. Savings to Investment Ratio (SIR)

8. Adjusted Internal Rate of Return (AIRR)

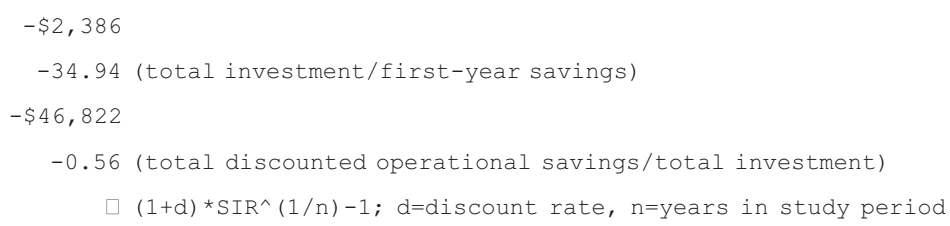

Wed May 16 09:26:49 MDT

30 years 0 months 

Consistent with Federal Life Cycle Cost Methodology and Procedures, 10 CFR, Part 436, Subpart A

The LCC calculations are based on the FEMP discount rates and energy price escalation rates updated on April 1, 2011.

Location:

Title:

Japan Transformer Analyst:

Base Date:

BOD:

January 1, 2013 Preparation
Date:

January 1, 2013 Economic Life:

$C: \backslash$ Documents and Settings $\backslash$ bushj $\backslash$ W $\backslash$ Desktop $\backslash$ Work At Home $\backslash$ Japan Study $\backslash$ Report $\backslash$ LCC

$$
\text { Files } \backslash \text { LCC_60_1000_Med.xmI }
$$

$\$ 85,742$

$\$ 5,145$

$\$ 3,430$

$\$ 94,316$

Total Cost

Salvage Value of Existing Equipment $\$ 0$

Public Utility Company

Total Investment

$\$ 94,316$
Wed May $16 \quad 09: 27: 30$ MDT

30 years 0 months

2. Energy and Water Savings (+) or Cost (-)

Base Date Savings, unit costs, \& discounted savings

Item Unit Cost Usage Savings Annual Savings Discount Factor Discounted Savings

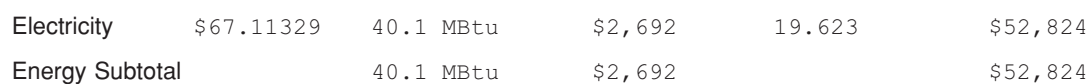

$\begin{array}{lll}\text { Energy Subtotal } & 40.1 \mathrm{MBtu} & \$ 2,692\end{array}$

$\begin{array}{llll}\text { Water Subtotal } & 0.0 \mathrm{Mgal} & \$ 0 & \$ 0\end{array}$

Total

$\$ 2,692$

3. Non-Energy Savings (+) or Cost (-)

Item Savings/Cost Occurrence Discount Factor Discounted Savings/Cost

Non-Annually Recurring

Non-Annually Recurring Subtotal

4. First year savings

5. Simple Payback Period (in years)

6.

7. Savings to Investment Ratio (SIR)

8. Adjusted Internal Rate of Return (AIRR)

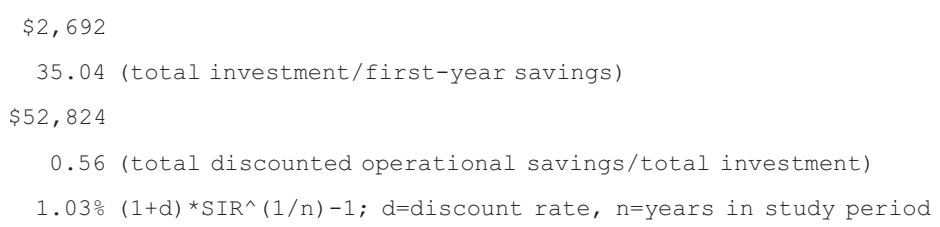



Consistent with Federal Life Cycle Cost Methodology and Procedures, 10 CFR, Part 436, Subpart A

The LCC calculations are based on the FEMP discount rates and energy price escalation rates updated on April 1, 2011.

Location:

U.S. Average Discount Rate:

Project

Title:

Japan Transformer Analyst:

Base Date:

January 1, 2013 Preparation
Date:

Wed May $16 \quad 09: 25: 55$ MDT

January 1, 2013 Economic Life:

30 years 0 months

BOD:

$C: \backslash$ Documents and Settings $\backslash$ bushj $\backslash$ Wesktop $\backslash$ Work At Home $\backslash$ Japan Study $\backslash$ Report $\backslash$ LCC

File Name:

$$
\text { Files } \backslash \text { LCC_60_1000_High.xml }
$$

1. Investment

Construction Cost \$107,958

$\mathrm{SIOH} \$ 6,477$

Design Cost $\$ 4,318$

Total Cost $\$ 118,754$

Salvage Value of Existing Equipment $\$ 0$

Public Utility Company $\$ 0$

Total Investment $\$ 118,754$

2. Energy and Water Savings (+) or Cost (-)

Base Date Savings, unit costs, \& discounted savings

Item Unit Cost Usage Savings Annual Savings Discount Factor Discounted Savings

$\begin{array}{llllll}\text { Electricity } & \$ 67.11329 & 66.4 \mathrm{MBtu} & \$ 4,458 & 19.623 & \$ 87,471 \\ & & 66.4 \mathrm{MBtu} & \$ 4,458 & & \$ 87,471\end{array}$

$\begin{array}{llll}\text { Energy Subtotal } & 66.4 \mathrm{MBtu} & \$ 4,458 & \$ 87,471\end{array}$

$\begin{array}{llll}\text { Water Subtotal } & 0.0 \mathrm{Mgal} & \$ 0 & \$ 0\end{array}$

Total

$\$ 4,458 \quad \$ 87,471$

3. Non-Energy Savings (+) or Cost (-)

Item Savings/Cost Occurrence Discount Factor Discounted Savings/Cost

Non-Annually Recurring

Non-Annually Recurring Subtotal

4. First year savings

5. Simple Payback Period (in years)

6

7. Savings to Investment Ratio (SIR)

8. Adjusted Internal Rate of Return (AIRR)

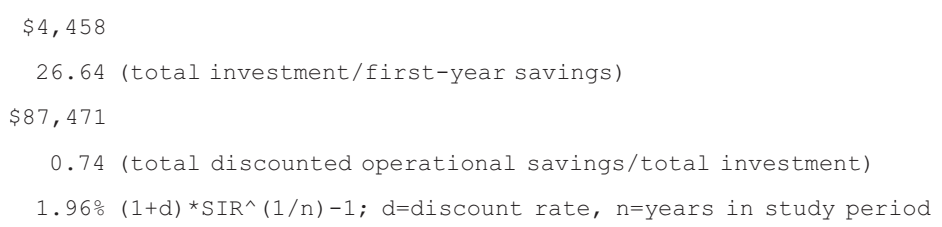





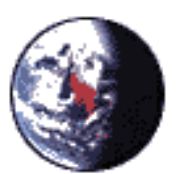

"Robertson CIV Lubka" <lubka.robertson@usmc.mil> 03/19/2012 07:17 PM

\author{
To "Jason W Bush" <Jason.Bush@inl.gov> \\ cc "Karen R HNC Moore" <Karen.R.Moore@usace.army.mil>, \\ "Kurt S Myers" <Kurt.Myers@inl.gov>, "Robert J Turk" \\ bcc \\ <Robert.Turk@inl.gov>, "Robertson CIV Lubka"
}

Subject RE: Transformer Efficiency Assessment PWS -- Onelines

Hi Jason,

We are currently using an average rate of $\$ 0.229$ / kWh for the installation costs. The rate fluctuates from $\sim$ Y16 to $\sim 18$ / $\mathrm{kWh}$, it can go higher or lower, and the dollar costs depends completely on the $\mathrm{Y}$ to $\$$ conversion.

$\$ 0.229$ is acceptable for the purposed of this study.

Thank you, Lubka

-----Original Message-----

From: Jason W Bush [mailto:Jason.Bush@inl.gov]

Sent: Friday, March 16, 2012 8:59

To: Robertson CIV Lubka

CC: Karen R HNC Moore; Kurt S Myers; Robert J Turk

Subject: RE: Transformer Efficiency Assessment PWS -- Onelines

Hi Lubka,

I have found off base energy cost in Okinawa (From Okinawa Electric Power Company) to be pretty high:

26.77 yen/ kWh or $\sim \$ 0.3287 / \mathrm{kWh}$

I believe this is most likely a higher rate than the base pays... is it possible to get a closer figure for what the base's cost per kwh is?

Thanks for your help,

"Robertson CIV Lubka" <lubka.robertson@usmc.mil>

01/22/2012 07:55 PM TO

"Jason W Bush" <Jason.Busheinl.gov>

$\mathrm{CC}$

"Karen R HNC Moore" <Karen.R.Mooredusace.army.mil>, "Kurt S Myers" <Kurt.Myers@inl.gov>, "Robert J Turk" <Robert.Turk@inl.gov> Subject

RE: Transformer Efficiency Assessment PWS -- Onelines 


\section{Appendix E \\ Economic Analysis Spreadsheets}


$* * *$ using today's $\$ / \mathrm{kwh}$ cost and not accounting for past or future inflation...

$20 \%$ load-Current $30 \%$ load-Current $40 \%$ load-Current $50 \%$ load-Current $60 \%$ load-Current $70 \%$ load-Current

\begin{tabular}{|c|c|c|c|c|c|c|c|c|c|c|c|}
\hline CAMP & LOC & $\begin{array}{c}\text { CAPACITY } \\
\text { KVA }\end{array}$ & $\underset{E}{\text { MFR_DAT }}$ & $\begin{array}{l}\text { EOL } \\
\text { Date }\end{array}$ & $\begin{array}{c}\text { Years until } \\
\text { EOL }\end{array}$ & $\begin{array}{l}\text { Cost of loss from } \\
\text { present to EOL }\end{array}$ & $\begin{array}{l}\text { Cost of loss from } \\
\text { present to EOL }\end{array}$ & $\begin{array}{l}\text { Cost of loss from } \\
\text { present to EOL }\end{array}$ & $\begin{array}{l}\text { Cost of loss from } \\
\text { present to EOL }\end{array}$ & $\begin{array}{l}\text { Cost of loss from } \\
\text { present to EOL }\end{array}$ & $\begin{array}{l}\text { Cost of loss from } \\
\text { present to EOL }\end{array}$ \\
\hline KINSER & $99 \mathrm{TS}$ & 500 & 1988 & 2018 & 6 & $\$ 22,600.60$ & $\$ 27,128.68$ & $\$ 33,250.12$ & $\$ 41,216.88$ & $\$ 50,727.74$ & $\$ 62,086.80$ \\
\hline FOSTER & 5704 TS & 500 & 1989 & 2019 & 7 & $\$ 26,367.37$ & $\$ 31,650.13$ & $\$ 38,791.80$ & $\$ 48,086.36$ & $\$ 59,182.36$ & $\$ 72,434.60$ \\
\hline FOSTER & 5703 TS & 500 & 1989 & 2019 & 7 & $\$ 26,367.37$ & $\$ 31,650.13$ & $\$ 38,791.80$ & $\$ 48,086.36$ & $\$ 59,182.36$ & $\$ 72,434.60$ \\
\hline FOSTER & $5670 \mathrm{TS}$ & 500 & 1989 & 2019 & 7 & $\$ 26,367.37$ & $\$ 31,650.13$ & $\$ 38,791.80$ & $\$ 48,086.36$ & $\$ 59,182.36$ & $\$ 72,434.60$ \\
\hline HANSEN & 2622 TS & 500 & 1990 & 2020 & 8 & $\$ 30,134.14$ & $\$ 36,171.58$ & $\$ 44,333.49$ & $\$ 54,955.84$ & $\$ 67,636.98$ & $\$ 82,782.40$ \\
\hline HANSEN & 2665 TS & 500 & 1990 & 2020 & 8 & $\$ 30,134.14$ & $\$ 36,171.58$ & $\$ 44,333.49$ & $\$ 54,955.84$ & $\$ 67,636.98$ & $\$ 82,782.40$ \\
\hline HANSEN & $2340 \mathrm{TS}$ & 500 & 1990 & 2020 & 8 & $\$ 30,134.14$ & $\$ 36,171.58$ & $\$ 44,333.49$ & $\$ 54,955.84$ & $\$ 67,636.98$ & $\$ 82,782.40$ \\
\hline HANSEN & 2442 TS & 500 & 1990 & 2020 & 8 & $\$ 30,134.14$ & $\$ 36,171.58$ & $\$ 44,333.49$ & $\$ 54,955.84$ & $\$ 67,636.98$ & $\$ 82,782.40$ \\
\hline KINSER & $1225 \mathrm{TS}$ & 500 & 1990 & 2020 & 8 & $\$ 30,134.14$ & $\$ 36,171.58$ & $\$ 44,333.49$ & $\$ 54,955.84$ & $\$ 67,636.98$ & $\$ 82,782.40$ \\
\hline FOSTER & 5696 TS & 500 & 1991 & 2021 & 9 & $\$ 33,900.90$ & $\$ 40,693.02$ & $\$ 49,875.17$ & $\$ 61,825.32$ & $\$ 76,091.61$ & $\$ 93,130.20$ \\
\hline FOSTER & 370 TS & 500 & 1991 & 2021 & 9 & $\$ 33,900.90$ & $\$ 40,693.02$ & $\$ 49,875.17$ & $\$ 61,825.32$ & $\$ 76,091.61$ & $\$ 93,130.20$ \\
\hline HANSEN & $2141 \mathrm{TS}$ & 500 & 1991 & 2021 & 9 & $\$ 33,900.90$ & $\$ 40,693.02$ & $\$ 49,875.17$ & $\$ 61,825.32$ & $\$ 76,091.61$ & $\$ 93,130.20$ \\
\hline HANSEN & $2530 \mathrm{TS}$ & 500 & 1992 & 2022 & 10 & $\$ 37,667.67$ & $\$ 45,214.47$ & $\$ 55,416.86$ & $\$ 68,694.80$ & $\$ 84,546.23$ & $\$ 103,478.00$ \\
\hline HANSEN & 2441 TS & 500 & 1993 & 2023 & 11 & $\$ 41,434.44$ & $\$ 49,735.92$ & $\$ 60,958.55$ & $\$ 75,564.28$ & $\$ 93,000.85$ & $\$ 113,825.80$ \\
\hline KINSER & 616 TS & 500 & 1993 & 2023 & 11 & $\$ 41,434.44$ & $\$ 49,735.92$ & $\$ 60,958.55$ & $\$ 75,564.28$ & $\$ 93,000.85$ & $\$ 113,825.80$ \\
\hline HANSEN & 2860 TS & 500 & 1994 & 2024 & 12 & $\$ 45,201.20$ & $\$ 54,257.36$ & $\$ 66,500.23$ & $\$ 82,433.76$ & $\$ 101,455.48$ & $\$ 124,173.60$ \\
\hline HANSEN & 2165 TS & 500 & 1994 & 2024 & 12 & $\$ 45,201.20$ & $\$ 54,257.36$ & $\$ 66,500.23$ & $\$ 82,433.76$ & $\$ 101,455.48$ & $\$ 124,173.60$ \\
\hline FOSTER & 481 TS & 500 & 1996 & 2026 & 14 & $\$ 52,734.74$ & $\$ 63,300.26$ & $\$ 77,583.60$ & $\$ 96,172.72$ & $\$ 118,364.72$ & $\$ 144,869.20$ \\
\hline HANSEN & $2221 \mathrm{TS}$ & 500 & 1996 & 2026 & 14 & $\$ 52,734.74$ & $\$ 63,300.26$ & $\$ 77,583.60$ & $\$ 96,172.72$ & $\$ 118,364.72$ & $\$ 144,869.20$ \\
\hline HANSEN & 2518 TS & 500 & 1996 & 2026 & 14 & $\$ 52,734.74$ & $\$ 63,300.26$ & $\$ 77,583.60$ & $\$ 96,172.72$ & $\$ 118,364.72$ & $\$ 144,869.20$ \\
\hline FOSTER & 478 TS & 500 & 1997 & 2027 & 15 & $\$ 56,501.51$ & $\$ 67,821.71$ & $\$ 83,125.29$ & $\$ 103,042.20$ & $\$ 126,819.35$ & $\$ 155,217.00$ \\
\hline FOSTER & 445 TS & 500 & 1998 & 2028 & 16 & $\$ 60,268.27$ & $\$ 72,343.15$ & $\$ 88,666.98$ & $\$ 109,911.68$ & $\$ 135,273.97$ & $\$ 165,564.80$ \\
\hline FOSTER & $490 \mathrm{TS}$ & 500 & 1999 & 2029 & 17 & $\$ 64,035.04$ & $\$ 76,864.60$ & $\$ 94,208.66$ & $\$ 116,781.16$ & $\$ 143,728.59$ & $\$ 175,912.60$ \\
\hline HANSEN & 2519 TS & 500 & 1999 & 2029 & 17 & $\$ 64,035.04$ & $\$ 76,864.60$ & $\$ 94,208.66$ & $\$ 116,781.16$ & $\$ 143,728.59$ & $\$ 175,912.60$ \\
\hline HANSEN & $2610 \mathrm{TS}$ & 500 & 1999 & 2029 & 17 & $\$ 64,035.04$ & $\$ 76,864.60$ & $\$ 94,208.66$ & $\$ 116,781.16$ & $\$ 143,728.59$ & $\$ 175,912.60$ \\
\hline HANSEN & $2245 \mathrm{TS}$ & 500 & 1999 & 2029 & 17 & $\$ 64,035.04$ & $\$ 76,864.60$ & $\$ 94,208.66$ & $\$ 116,781.16$ & $\$ 143,728.59$ & $\$ 175,912.60$ \\
\hline KINSER & $100 \mathrm{TS}$ & 750 & 1989 & 2019 & 7 & $\$ 35,638.72$ & $\$ 42,924.62$ & $\$ 52,816.53$ & $\$ 65,622.80$ & $\$ 81,015.20$ & $\$ 99,426.39$ \\
\hline KINSER & $1226 \mathrm{TS}$ & 750 & 1991 & 2021 & 9 & $\$ 45,821.21$ & $\$ 55,188.79$ & $\$ 67,906.96$ & $\$ 84,372.17$ & $\$ 104,162.40$ & $\$ 127,833.93$ \\
\hline HANSEN & 2537 TS & 750 & 1992 & 2022 & 10 & $\$ 50,912.46$ & $\$ 61,320.88$ & $\$ 75,452.18$ & $\$ 93,746.85$ & $\$ 115,736.00$ & $\$ 142,037.70$ \\
\hline HANSEN & 2725 TS & 750 & 1992 & 2022 & 10 & $\$ 50,912.46$ & $\$ 61,320.88$ & $\$ 75,452.18$ & $\$ 93,746.85$ & $\$ 115,736.00$ & $\$ 142,037.70$ \\
\hline HANSEN & 2893 TS & 750 & 1992 & 2022 & 10 & $\$ 50,912.46$ & $\$ 61,320.88$ & $\$ 75,452.18$ & $\$ 93,746.85$ & $\$ 115,736.00$ & $\$ 142,037.70$ \\
\hline KINSER & $1210 \mathrm{TS}$ & 750 & 1992 & 2022 & 10 & $\$ 50,912.46$ & $\$ 61,320.88$ & $\$ 75,452.18$ & $\$ 93,746.85$ & $\$ 115,736.00$ & $\$ 142,037.70$ \\
\hline HANSEN & 2667 TS & 750 & 1993 & 2023 & 11 & $\$ 56,003.71$ & $\$ 67,452.97$ & $\$ 82,997.40$ & $\$ 103,121.54$ & $\$ 127,309.60$ & $\$ 156,241.47$ \\
\hline HANSEN & 2327 TS & 750 & 1993 & 2023 & 11 & $\$ 56,003.71$ & $\$ 67,452.97$ & $\$ 82,997.40$ & $\$ 103,121.54$ & $\$ 127,309.60$ & $\$ 156,241.47$ \\
\hline KINSER & 1202 TS & 750 & 1993 & 2023 & 11 & $\$ 56,003.71$ & $\$ 67,452.97$ & $\$ 82,997.40$ & $\$ 103,121.54$ & $\$ 127,309.60$ & $\$ 156,241.47$ \\
\hline KINSER & 104-A TS & 750 & 1993 & 2023 & 11 & $\$ 56,003.71$ & $\$ 67,452.97$ & $\$ 82,997.40$ & $\$ 103,121.54$ & $\$ 127,309.60$ & $\$ 156,241.47$ \\
\hline FOSTER & $480 \mathrm{TS}$ & 750 & 1996 & 2026 & 14 & $\$ 71,277.44$ & $\$ 85,849.23$ & $\$ 105,633.05$ & $\$ 131,245.59$ & $\$ 162,030.40$ & $\$ 198,852.78$ \\
\hline FOSTER & 464 TS & 750 & 1998 & 2028 & 16 & $\$ 81,459.94$ & $\$ 98,113.41$ & $\$ 120,723.49$ & $\$ 149,994.96$ & $\$ 185,177.60$ & $\$ 227,260.32$ \\
\hline HANSEN & $2635 \mathrm{TS}$ & 750 & 1999 & 2029 & 17 & $\$ 86,551.18$ & $\$ 104,245.50$ & $\$ 128,268.71$ & $\$ 159,369.65$ & $\$ 196,751.20$ & $\$ 241,464.09$ \\
\hline FOSTER & 5907 TS & 750 & 2001 & 2031 & 19 & $\$ 96,733.67$ & $\$ 116,509.67$ & $\$ 143,359.14$ & $\$ 178,119.02$ & $\$ 219,898.40$ & $\$ 269,871.63$ \\
\hline FOSTER & $11 \mathrm{TS}$ & 750 & 2001 & 2031 & 19 & $\$ 96,733.67$ & $\$ 116,509.67$ & $\$ 143,359.14$ & $\$ 178,119.02$ & $\$ 219,898.40$ & $\$ 269,871.63$ \\
\hline FOSTER & 363 TS & 750 & 2001 & 2031 & 19 & $\$ 96,733.67$ & $\$ 116,509.67$ & $\$ 143,359.14$ & $\$ 178,119.02$ & $\$ 219,898.40$ & $\$ 269,871.63$ \\
\hline FOSTER & 1004 TV & 1000 & 1989 & 2019 & 7 & $\$ 42,301.85$ & $\$ 51,165.39$ & $\$ 63,260.47$ & $\$ 78,821.40$ & $\$ 97,675.76$ & $\$ 120,267.84$ \\
\hline FOSTER & 1002 TS & 1000 & 1993 & 2023 & 11 & $\$ 66,474.33$ & $\$ 80,402.75$ & $\$ 99,409.31$ & $\$ 123,862.20$ & $\$ 153,490.48$ & $\$ 188,992.32$ \\
\hline HANSEN & 2654 TS & 1000 & 2000 & 2030 & 18 & $\$ 108,776.18$ & $\$ 131,568.14$ & $\$ 162,669.78$ & $\$ 202,683.60$ & $\$ 251,166.24$ & $\$ 309,260.16$ \\
\hline HANSEN & 2365 TS & 1000 & 2001 & 2031 & 19 & $\$ 114,819.30$ & $\$ 138,877.48$ & $\$ 171,706.99$ & $\$ 213,943.80$ & $\$ 265,119.92$ & $\$ 326,441.28$ \\
\hline
\end{tabular}




\begin{tabular}{|c|c|c|c|c|c|}
\hline $\begin{array}{l}20 \% \text { load - High } \\
\text { xfmrs }\end{array}$ & $\begin{array}{l}30 \% \text { load - High } \\
\text { xfmrs }\end{array}$ & $\begin{array}{l}40 \% \text { load - High } \\
\text { xfmrs }\end{array}$ & $\begin{array}{l}50 \% \text { load - High } \\
\text { xfmrs }\end{array}$ & $\begin{array}{l}60 \% \text { load - High } \\
\text { xfmrs }\end{array}$ & $\begin{array}{l}70 \% \text { load - High } \\
\text { xfmrs }\end{array}$ \\
\hline \begin{tabular}{|l|} 
Cost of loss from \\
present to EOL- \\
OLD XFMR
\end{tabular} & $\begin{array}{l}\text { Cost of loss from } \\
\text { present to EOL- } \\
\text { OLD XFMR }\end{array}$ & $\begin{array}{l}\text { Cost of loss from } \\
\text { present to EOL- } \\
\text { OLD XFMR }\end{array}$ & \begin{tabular}{|l|} 
Cost of loss from \\
present to EOL- \\
OLD XFMR
\end{tabular} & $\begin{array}{l}\text { Cost of loss from } \\
\text { present to EOL- } \\
\text { OLD XFMR }\end{array}$ & $\begin{array}{l}\text { Cost of loss from } \\
\text { present to EOL- } \\
\text { OLD XFMR }\end{array}$ \\
\hline$\$ 16,364.47$ & $\$ 18,329.99$ & $\$ 21,695.03$ & $\$ 27,268.16$ & $\$ 33,667.77$ & $\$ 42,059.44$ \\
\hline$\$ 19,091.88$ & $\$ 21,384.99$ & $\$ 25,310.87$ & $\$ 31,812.86$ & $\$ 39,279.07$ & $\$ 49,069.34$ \\
\hline$\$ 19,091.88$ & $\$ 21,384.99$ & $\$ 25,310.87$ & $\$ 31,812.86$ & $\$ 39,279.07$ & $\$ 49,069.34$ \\
\hline$\$ 19,091.88$ & $\$ 21,384.99$ & $\$ 25,310.87$ & $\$ 31,812.86$ & $\$ 39,279.07$ & $\$ 49,069.34$ \\
\hline$\$ 21,819.30$ & $\$ 24,439.99$ & $\$ 28,926.71$ & $\$ 36,357.55$ & $\$ 44,890.36$ & $\$ 56,079.25$ \\
\hline$\$ 21,819.30$ & $\$ 24,439.99$ & $\$ 28,926.71$ & $\$ 36,357.55$ & $\$ 44,890.36$ & $\$ 56,079.25$ \\
\hline$\$ 21,819.30$ & $\$ 24,439.99$ & $\$ 28,926.71$ & $\$ 36,357.55$ & $\$ 44,890.36$ & $\$ 56,079.25$ \\
\hline$\$ 21,819.30$ & $\$ 24,439.99$ & $\$ 28,926.71$ & $\$ 36,357.55$ & $\$ 44,890.36$ & $\$ 56,079.25$ \\
\hline$\$ 21,819.30$ & $\$ 24,439.99$ & $\$ 28,926.71$ & $\$ 36,357.55$ & $\$ 44,890.36$ & $\$ 56,079.25$ \\
\hline$\$ 24,546.71$ & $\$ 27,494.98$ & $\$ 32,542.55$ & $\$ 40,902.24$ & $\$ 50,501.66$ & $\$ 63,089.16$ \\
\hline$\$ 24,546.71$ & $\$ 27,494.98$ & & $\$ 40,902.24$ & $\$ 50,501.66$ & $\$ 63,089.16$ \\
\hline$\$ 24,546.71$ & $\$ 27,494.98$ & $\$ 32$, & $\$ 40,902.24$ & $\$ 50,501.66$ & 089.16 \\
\hline$\$ 27,274.12$ & $\$ 30,549.98$ & $\$ 36,158.39$ & $\$ 45,446.94$ & $\$ 56,112.95$ & $\$ 70,099.06$ \\
\hline$\$ 30,001.53$ & $\$ 33,604.98$ & $\$ 39,774.23$ & $\$ 49,991.63$ & $\$ 61,724.25$ & $\$ 77,108.97$ \\
\hline$\$ 30,001.53$ & $\$ 33,604.98$ & $\$ 39,774.23$ & $\$ 49,991.63$ & $\$ 61,724.25$ & $\$ 77,108.97$ \\
\hline$\$ 32,728.94$ & $\$ 36,659.98$ & $\$ 43,390.07$ & $\$ 54,536.32$ & $\$ 67,335.54$ & $\$ 84,118.87$ \\
\hline$\$ 32,728.94$ & $\$ 36,659.98$ & $\$ 43,390.07$ & $\$ 54,536.32$ & $\$ 67,335.54$ & $\$ 84,118.87$ \\
\hline$\$ 38,183.77$ & $\$ 42,769.98$ & $\$ 50,621.75$ & $\$ 63,625.71$ & $\$ 78,558.13$ & $\$ 98,138.69$ \\
\hline$\$ 38,183.77$ & $\$ 42,769.98$ & $\$ 50,621.75$ & $\$ 63,625.71$ & $\$ 78,558.13$ & $\$ 98,138.69$ \\
\hline$\$ 38,183.77$ & $\$ 42,769.98$ & $\$ 50,621.75$ & $\$ 63,625.71$ & $\$ 78,558.13$ & $\$ 98,138.69$ \\
\hline$\$ 40,911.18$ & $\$ 45,824.97$ & $\$ 54,237.58$ & $\$ 68,170.40$ & $\$ 84,169.43$ & $\$ 105,148.59$ \\
\hline$\$ 43,638.59$ & $\$ 48,879.97$ & $\$ 57,853.42$ & $\$ 72,715.10$ & $\$ 89,780.72$ & $\$ 112,158.50$ \\
\hline$\$ 46,366.00$ & $\$ 51,934.97$ & $\$ 61,469.26$ & $\$ 77,259.79$ & $\$ 95,392.02$ & $\$ 119,168.40$ \\
\hline$\$ 46,366.00$ & $\$ 51,934.97$ & $\$ 61,469.26$ & $\$ 77,259.79$ & $\$ 95,392.02$ & $\$ 119,168.40$ \\
\hline$\$ 46,366.00$ & $\$ 51,934.97$ & $\$ 61,469.26$ & $\$ 77,259.79$ & $\$ 95,392.02$ & $\$ 119,168.40$ \\
\hline$\$ 46,366.00$ & $\$ 51,934.97$ & $\$ 61,469.26$ & $\$ 77,259.79$ & $\$ 95,392.02$ & $\$ 119,168.40$ \\
\hline$\$ 25,817.67$ & $\$ 29,350.51$ & $\$ 35,079.48$ & $\$ 44,083.45$ & $\$ 54,708.37$ & $\$ 68,198.09$ \\
\hline$\$ 33,194.15$ & $\$ 37,736.37$ & $\$ 45,102.19$ & $\$ 56,678.72$ & $\$ 70,339.34$ & $\$ 87,683.26$ \\
\hline$\$ 36,882.39$ & $\$ 41,929.31$ & $\$ 50,113.55$ & $\$ 62,976.35$ & $\$ 78,154.82$ & $\$ 97,425.84$ \\
\hline$\$ 36,882.39$ & $\$ 41,929.31$ & $\$ 50,113.55$ & $\$ 62,976.35$ & $\$ 78,154.82$ & $\$ 97,425.84$ \\
\hline$\$ 36,882.39$ & $\$ 41,929.31$ & $\$ 50,113.55$ & $\$ 62,976.35$ & $\$ 78,154.82$ & $\$ 97,425.84$ \\
\hline$\$ 36,882.39$ & $\$ 41,929.31$ & $\$ 50,1$ & $\$ 62,976.35$ & $\$ 78,154.82$ & $\$ 97,425.84$ \\
\hline$\$ 40,570.63$ & $\$ 46,122.24$ & $\$ 55,124.90$ & $\$ 69,273.99$ & $\$ 85,970.30$ & $\$ 107,168.42$ \\
\hline$\$ 40,570.63$ & $\$ 46,122.24$ & $\$ 55,124.90$ & $\$ 69,273.99$ & $\$ 85,970.30$ & $\$ 107,168.42$ \\
\hline$\$ 40,570.63$ & $\$ 46,122.24$ & $\$ 55,124.90$ & $\$ 69,273.99$ & $\$ 85,970.30$ & $\$ 107,168.42$ \\
\hline$\$ 40,570.63$ & $\$ 46,122.24$ & $\$ 55,124.90$ & $\$ 69,273.99$ & $\$ 85,970.30$ & $\$ 107,168.42$ \\
\hline$\$ 51,635.35$ & $\$ 58,701.03$ & $\$ 70,1$ & $\$ 88,166.89$ & $\$ 109,416.74$ & $\$ 136,396.18$ \\
\hline$\$ 59,011.82$ & $\$ 67,086.89$ & $\$ 80,181.68$ & $\$ 100,762.17$ & $\$ 125,047.71$ & $\$ 155,881.34$ \\
\hline$\$ 62,700.06$ & $\$ 71,279.82$ & $\$ 85,193.03$ & $\$ 107,059.80$ & $\$ 132,863.19$ & $\$ 165,623.93$ \\
\hline$\$ 70,076.54$ & $\$ 79,665.68$ & $\$ 95,2$ & $\$ 119,655.07$ & $\$ 148,494.15$ & $\$ 185,109.10$ \\
\hline$\$ 70,076.54$ & $\$ 79,665.68$ & $\$ 95,215.74$ & $\$ 119,655.07$ & $\$ 148,494.15$ & $\$ 185,109.10$ \\
\hline$\$ 70,076.54$ & $\$ 79,665.68$ & $\$ 95,215.74$ & $\$ 119,655.07$ & $\$ 148,494.15$ & $\$ 185,109.10$ \\
\hline$\$ 30,586.05$ & $\$ 35,001.79$ & $\$ 42,742.59$ & $\$ 53,722.46$ & $\$ 66,472.78$ & $\$ 83,545.25$ \\
\hline$\$ 48,063.80$ & $\$ 55,002.81$ & $\$ 67,166.93$ & $\$ 84,421.01$ & $\$ 104,457.23$ & $\$ 131,285.39$ \\
\hline$\$ 78,64$ & $\$ 90,004$ & $\$ 109,909.53$ & $\$ 138,143.48$ & $\$ 170,930.01$ & $\$ 214,830.64$ \\
\hline$\$ 83,019.28$ & $\$ 95,004.85$ & $\$ 116,015.61$ & $\$ 145,818.11$ & $\$ 180,426.13$ & $\$ 226,765.67$ \\
\hline
\end{tabular}

\begin{tabular}{|c|c|c|c|c|c|}
\hline $\begin{array}{l}20 \% \text { load - Med } \\
\text { xfmrs }\end{array}$ & $\begin{array}{l}30 \% \text { load - Med } \\
\text { xfmrs }\end{array}$ & $\begin{array}{l}40 \% \text { load - Med } \\
\text { xfmrs }\end{array}$ & $\begin{array}{l}50 \% \text { load - Med } \\
\text { xfmrs }\end{array}$ & $\begin{array}{l}60 \% \text { load - Med } \\
\text { xfmrs }\end{array}$ & $\begin{array}{l}70 \% \text { load - Med } \\
\text { xfmrs }\end{array}$ \\
\hline $\begin{array}{l}\text { Cost of loss from } \\
\text { present to EOL- } \\
\text { OLD XFMR }\end{array}$ & $\begin{array}{l}\text { Cost of loss from } \\
\text { present to EOL- } \\
\text { OLD XFMR }\end{array}$ & $\begin{array}{l}\text { Cost of loss from } \\
\text { present to EOL- } \\
\text { OLD XFMR }\end{array}$ & $\begin{array}{l}\text { Cost of loss from } \\
\text { present to EOL- } \\
\text { OLD XFMR }\end{array}$ & $\begin{array}{l}\text { Cost of loss from } \\
\text { present to EOL- } \\
\text { OLD XFMR }\end{array}$ & $\begin{array}{l}\text { Cost of loss from } \\
\text { present to EOL- } \\
\text { OLD XFMR }\end{array}$ \\
\hline$\$ 19,494.47$ & $\$ 22,633.81$ & $\$ 27,566.49$ & $\$ 34,593.67$ & $\$ 42,888.69$ & $\$ 52,507.76$ \\
\hline$\$ 22,743.55$ & $\$ 26,406.11$ & $\$ 32,160.91$ & $\$ 40,359.29$ & $\$ 50,036.80$ & $\$ 61,259.05$ \\
\hline$\$ 22,743.55$ & $\$ 26,406.11$ & $\$ 32,160.91$ & $\$ 40,359.29$ & $\$ 50,036.80$ & $\$ 61,259.05$ \\
\hline$\$ 22,743.55$ & $\$ 26,406.11$ & $\$ 32,160.91$ & $\$ 40,359.29$ & $\$ 50,036.80$ & $\$ 61,259.05$ \\
\hline$\$ 25,992.63$ & $\$ 30,178.42$ & $\$ 36,755.32$ & $\$ 46,124.90$ & $\$ 57,184.91$ & $\$ 70,010.35$ \\
\hline$\$ 25,992.63$ & $\$ 30,178.42$ & $\$ 36,755.32$ & $\$ 46,124.90$ & $\$ 57,184.91$ & $\$ 70,010.35$ \\
\hline$\$ 25,992.63$ & $\$ 30,178.42$ & $\$ 36,755.32$ & $\$ 46,124.90$ & $\$ 57,184.91$ & $\$ 70,010.35$ \\
\hline$\$ 25,992.63$ & $\$ 30,178.42$ & $\$ 36,755.32$ & $\$ 46,124.90$ & $\$ 57,184.91$ & $\$ 70,010.35$ \\
\hline$\$ 25,992.63$ & $\$ 30,178.42$ & $\$ 36,755.32$ & $\$ 46,124.90$ & \begin{tabular}{|l|l|}
$57,184.91$ \\
\end{tabular} & $\$ 70,010.35$ \\
\hline$\$ 29,241.71$ & $\$ 33,950.72$ & $\$ 41,349.74$ & $\$ 51,890.51$ & $\$ 64,333.03$ & $\$ 78,761.64$ \\
\hline$\$ 29,241.71$ & $\$ 33,950.72$ & & & & $\$ 78,761.64$ \\
\hline$\$ 29,241.71$ & $\$ 33,950.72$ & $\$ 41,349.74$ & $\$ 51$, & $54,333.03$ & $\$ 78$ \\
\hline$\$ 32,490.79$ & $\$ 37,723.02$ & $\$ 45,944.15$ & $\$ 57,656.12$ & $\$ 71,481.14$ & $\$ 87,512.93$ \\
\hline$\$ 35,739.87$ & $\$ 41,495.32$ & $\$ 50,538.57$ & $\$ 63,421.73$ & $\$ 78,629.26$ & $\$ 96,264.23$ \\
\hline$\$ 35,739.87$ & $\$ 41,495.32$ & $\$ 50,538.57$ & $\$ 63,421.73$ & $\$ 78,629.26$ & $\$ 96,264.23$ \\
\hline$\$ 38,988.94$ & $\$ 45,267.62$ & $\$ 55,132.98$ & $\$ 69,187.35$ & $\$ 85,777.37$ & $\$ 105,015.52$ \\
\hline$\$ 38,988.94$ & $\$ 45,267.62$ & $\$ 55,132.98$ & $\$ 69,187.35$ & $\$ 85,777.37$ & $\$ 105,015.52$ \\
\hline$\$ 45,487.10$ & $\$ 52,812.23$ & $\$ 64,321.82$ & $\$ 80,718.57$ & $\$ 100,073.60$ & $\$ 122,518.11$ \\
\hline$\$ 45,487.10$ & $\$ 52,812.23$ & $\$ 64,321.82$ & $\$ 80,718.57$ & $\$ 100,073.60$ & $\$ 122,518.11$ \\
\hline$\$ 45,487.10$ & $\$ 52,812.23$ & $\$ 64,321.82$ & $\$ 80,718.57$ & $\$ 100,073.60$ & $\$ 122,518.11$ \\
\hline$\$ 48,736.18$ & $\$ 56,584.53$ & $\$ 68,916.23$ & $\$ 86,484.18$ & $\$ 107,221.71$ & $\$ 131,269.40$ \\
\hline$\$ 51,985.26$ & $\$ 60,356$ & $\$ 73$, & $\$ 92$, & $\$ 114,369.83$ & $\$ 140,020.69$ \\
\hline$\$ 55,234.34$ & $\$ 64,129.13$ & $\$ 78,105.06$ & $\$ 98,015.41$ & $\$ 121,517.94$ & $\$ 148,771.99$ \\
\hline$\$ 55,234.34$ & $\$ 64,129.13$ & $\$ 78,105.06$ & $\$ 98,015.41$ & $\$ 121,517.94$ & $\$ 148,771.99$ \\
\hline$\$ 55,234.34$ & $\$ 64,129.13$ & $\$ 78,105.06$ & $\$ 98,015.41$ & $\$ 121,517.94$ & $\$ 148,771.99$ \\
\hline$\$ 55,234.34$ & $\$ 64,129.13$ & $\$ 78,105.06$ & $\$ 98,015.41$ & $\$ 121,517.94$ & $\$ 148,771.99$ \\
\hline$\$ 30,186.27$ & $\$ 34,759.00$ & $\$ 43,277.68$ & $\$ 53,902.40$ & $\$ 66,619.81$ & $\$ 82,162.36$ \\
\hline$\$ 38,810.92$ & .14 & $\$ 55,6$ & $\$ 69,303.08$ & $35,654.04$ & $\$ 105,637.32$ \\
\hline$\$ 43,123.24$ & $\$ 49,655.71$ & $\$ 61,825.26$ & $\$ 77,003.43$ & $25,171.15$ & $\$ 117,374.80$ \\
\hline$\$ 43,123.24$ & $\$ 49,655.71$ & $\$ 61,825.26$ & $\$ 77,003.43$ & $\$ 95,171.15$ & $\$ 117,374.80$ \\
\hline$\$ 43,123.24$ & $\$ 49,655.71$ & $\$ 61,825.26$ & $\$ 77,003.43$ & $\$ 95,171.15$ & $\$ 117,374.80$ \\
\hline$\$ 43,123.24$ & $\$ 49,655.71$ & $\$ 61,825.26$ & $\$ 77,003.43$ & $\$ 95,171.15$ & $\$ 117,374.80$ \\
\hline$\$ 47,435.56$ & $\$ 54,621.28$ & $\$ 68,007.79$ & $\$ 84,703.77$ & $\$ 104,688.27$ & $\$ 129,112.29$ \\
\hline$\$ 47,435.56$ & $\$ 54,621.28$ & $\$ 68,007.79$ & $\$ 84,703.77$ & $\$ 104,688.27$ & $\$ 129,112.29$ \\
\hline$\$ 47,435.56$ & $\$ 54,621.28$ & $\$ 68,007.79$ & $\$ 84,703.77$ & $\$ 104,688.27$ & $\$ 129,112.29$ \\
\hline$\$ 47,435.56$ & $\$ 54,621.28$ & $\$ 68,007.79$ & $\$ 84,703.77$ & $\$ 104,688.27$ & $\$ 129,112.29$ \\
\hline$\$ 60,372.54$ & $\$ 69,5$ & $\$ 86$, & $\$ 107,804.80$ & $\$ 133,239.61$ & $\$ 164,32$ \\
\hline$\$ 68,997.18$ & $\$ 79,449.13$ & $\$ 98,920.42$ & $\$ 123,205.48$ & $\$ 152,273.84$ & $\$ 187,799.69$ \\
\hline$\$ 73,309.51$ & $\$ 84,414.70$ & $\$ 105,102.94$ & $\$ 130,905.82$ & $\$ 161,790.96$ & $\$ 199,537.17$ \\
\hline$\$ 81,934.16$ & $\$ 94,3$ & $\$ 117,467.99$ & $\$ 146,306.51$ & $\$ 180,825.19$ & $\$ 223,012.13$ \\
\hline$\$ 81,934.16$ & $\$ 94,345.85$ & $\$ 117,467.99$ & $\$ 146,306.51$ & $\$ 180,825.19$ & $\$ 223,012.13$ \\
\hline$\$ 81,934.16$ & $\$ 94,345.8$ & 67.99 & $\$ 146,306.51$ & $\$ 180,825.19$ & $\$ 223,01$ \\
\hline$\$ 34,928.49$ & $\$ 40,559.58$ & $\$ 50,152.90$ & $\$ 63,553.50$ & $\$ 78,832.24$ & $\$ 97,298.68$ \\
\hline$\$ 54,887.62$ & $\$ 63,736.48$ & $\$ 78,811.70$ & $\begin{array}{l}59,869.78 \\
\end{array}$ & $\$ 123,879.23$ & $\$ 152,897.92$ \\
\hline$\$ 89,8$ & $\$ 104,2$ & $\$ 128,964.60$ & $\$ 163,423.28$ & $\$ 202,711.47$ & $\$ 250,19$ \\
\hline$\$ 94,805.89$ & $\$ 110,090.29$ & $\$ 136,129.30$ & $\$ 172,502.35$ & $\$ 213,973.21$ & $\$ 264,096.41$ \\
\hline
\end{tabular}




\begin{tabular}{|c|c|c|c|c|c|}
\hline $20 \%$ load & $30 \%$ load & $40 \%$ load & $50 \%$ load & $60 \%$ load & $70 \%$ load \\
\hline $\begin{array}{l}\text { CurrentXFMR } \\
\text { vs } \\
\text { High XFMR }\end{array}$ & $\begin{array}{l}\text { CurrentXFMR } \\
\text { vs } \\
\text { High XFMR }\end{array}$ & $\begin{array}{l}\text { CurrentXFMR } \\
\text { vs } \\
\text { High XFMR }\end{array}$ & $\begin{array}{l}\text { CurrentXFMR } \\
\text { vs } \\
\text { High XFMR }\end{array}$ & $\begin{array}{l}\text { CurrentXFMR } \\
\text { vs } \\
\text { High XFMR }\end{array}$ & $\begin{array}{l}\text { CurrentXFMR } \\
\text { vs } \\
\text { High XFMR }\end{array}$ \\
\hline$\$ 6,236.13$ & $\$ 8,798.69$ & $\$ 11,555.08$ & $\$ 13,948.72$ & $\$ 17,059.97$ & $\$ 20,027.36$ \\
\hline$\$ 7,275.49$ & $\$ 10,265.14$ & $\$ 13,480.93$ & $\$ 16,273.50$ & $\$ 19,903.30$ & $\$ 23,365.26$ \\
\hline$\$ 7,275.49$ & $\$ 10,265.14$ & $\$ 13,480.93$ & $\$ 16,273.50$ & $\$ 19,903.30$ & $\$ 23,365.26$ \\
\hline$\$ 7,275.49$ & $\$ 10,265.14$ & $\$ 13,480.93$ & $\$ 16,273.50$ & $\$ 19,903.30$ & $\$ 23,365.26$ \\
\hline$\$ 8,314.84$ & $\$ 11,731.59$ & $\$ 15,406.78$ & $\$ 18,598.29$ & $\$ 22,746.62$ & $\$ 26,703.15$ \\
\hline$\$ 8,314.84$ & $\$ 11,731.59$ & $\$ 15,406.78$ & $\$ 18,598.29$ & $\$ 22,746.62$ & $\$ 26,703.15$ \\
\hline$\$ 8,314.84$ & $\$ 11,731.59$ & $\$ 15,406.78$ & $\$ 18,598.29$ & $\$ 22,746.62$ & $\$ 26,703.15$ \\
\hline$\$ 8,314.84$ & $\$ 11,731.59$ & $\$ 15,406.78$ & $\$ 18,598.29$ & $\$ 22,746.62$ & $\$ 26,703.15$ \\
\hline$\$ 8,314.84$ & $\$ 11,731.59$ & $\$ 15,406.78$ & $\$ 18,598.29$ & $\$ 22,746.62$ & $\$ 26,703.15$ \\
\hline$\$ 9,354.20$ & $\$ 13,198.04$ & $\$ 17,332.62$ & $\$ 20,923.08$ & $\$ 25,589.95$ & $\$ 30,041.04$ \\
\hline$\$ 9,354.20$ & $\$ 13,198.04$ & $\$ 17,332.62$ & $\$ 20,923.08$ & $\$ 25,589.95$ & $\$ 30,041.04$ \\
\hline$\$ 9,354.20$ & $\$ 13,198.04$ & $\$ 17,332.62$ & $\$ 20,923.08$ & $\$ 25,589.95$ & $\$ 30,041.04$ \\
\hline$\$ 10,393.55$ & $\$ 14,664.49$ & $\$ 19,258.47$ & $\$ 23,247.86$ & $\$ 28,433.28$ & $\$ 33,378.94$ \\
\hline$\$ 11,432.91$ & $\$ 16,130.94$ & $\$ 21,184.32$ & $\$ 25,572.65$ & $\$ 31,276.61$ & $\$ 36,716.83$ \\
\hline$\$ 11,432.91$ & $\$ 16,130.94$ & $\$ 21,184.32$ & $\$ 25,572.65$ & $\$ 31,276.61$ & $\$ 36,716.83$ \\
\hline$\$ 12,472.26$ & $\$ 17,597.38$ & $\$ 23,110.16$ & $\$ 27,897.44$ & $\$ 34,119.93$ & $\$ 40,054.73$ \\
\hline$\$ 12,472.26$ & $\$ 17,597.38$ & $\$ 23,110.16$ & $\$ 27,897.44$ & $\$ 34,119.93$ & $\$ 40,054.73$ \\
\hline$\$ 14,550.97$ & $\$ 20,530.28$ & $\$ 26,961.86$ & $\$ 32,547.01$ & $\$ 39,806.59$ & $\$ 46,730.51$ \\
\hline$\$ 14,550.97$ & $\$ 20,530.28$ & $\$ 26,961.86$ & $\$ 32,547.01$ & $\$ 39,806.59$ & $\$ 46,730.51$ \\
\hline$\$ 14,550.97$ & $\$ 20,530.28$ & $\$ 26,961.86$ & $\$ 32,547.01$ & $\$ 39,806.59$ & $\$ 46,730.51$ \\
\hline$\$ 15,590.33$ & $\$ 21,996.73$ & $\$ 28,887.71$ & $\$ 34,871.80$ & $\$ 42,649.92$ & $\$ 50,068.41$ \\
\hline$\$ 16,629.68$ & $\$ 23,463.18$ & $\$ 30,813.55$ & $\$ 37,196.58$ & $\$ 45,493.25$ & $\$ 53,406.30$ \\
\hline$\$ 17,669.04$ & $\$ 24,929.63$ & $\$ 32,739.40$ & $\$ 39,521.37$ & $\$ 48,336.57$ & $\$ 56,744.20$ \\
\hline$\$ 17,669.04$ & $\$ 24,929.63$ & $\$ 32,739.40$ & $\$ 39,521.37$ & $\$ 48,336.57$ & $\$ 56,744.20$ \\
\hline$\$ 17,669.04$ & $\$ 24,929.63$ & $\$ 32,739.40$ & $\$ 39,521.37$ & $\$ 48,336.57$ & $\$ 56,744.20$ \\
\hline$\$ 17,669.04$ & $\$ 24,929.63$ & $\$ 32,739.40$ & $\$ 39,521.37$ & $\$ 48,336.57$ & $\$ 56,744.20$ \\
\hline$\$ 9,821.05$ & $\$ 13,574.10$ & $\$ 17,737.04$ & $\$ 21,539.35$ & $\$ 26,306.83$ & $\$ 31,228.30$ \\
\hline$\$ 12,627.06$ & $\$ 17,452.42$ & $\$ 22,804.77$ & $\$ 27,693.45$ & $\$ 33,823.06$ & $\$ 40,150.67$ \\
\hline$\$ 14,030.07$ & $\$ 19,391.57$ & $\$ 25,338.63$ & $\$ 30,770.50$ & $\$ 37,581.18$ & $\$ 44,611.86$ \\
\hline$\$ 14,030.07$ & $\$ 19,391.57$ & $\$ 25,338.63$ & $\$ 30,770.50$ & $\$ 37,581.18$ & $\$ 44,611.86$ \\
\hline$\$ 14,030.07$ & $\$ 19,391.57$ & $\$ 25,338.63$ & $\$ 30,770.50$ & $\$ 37,581.18$ & $\$ 44,611.86$ \\
\hline$\$ 14,030.07$ & $\$ 19,391.57$ & $\$ 25,338.63$ & $\$ 30,770.50$ & $\$ 37,581.18$ & $\$ 44,611.86$ \\
\hline$\$ 15,433.08$ & $\$ 21,330.73$ & $\$ 27,872.50$ & $\$ 33,847.55$ & $\$ 41,339.30$ & $\$ 49,073.05$ \\
\hline$\$ 15,433.08$ & $\$ 21,330.73$ & $\$ 27,872.50$ & $\$ 33,847.55$ & $\$ 41,339.30$ & $\$ 49,073.05$ \\
\hline$\$ 15,433.08$ & $\$ 21,330.73$ & $\$ 27,872.50$ & $\$ 33,847.55$ & $\$ 41,339.30$ & $\$ 49,073.05$ \\
\hline$\$ 15,433.08$ & $\$ 21,330.73$ & $\$ 27,872.50$ & $\$ 33,847.55$ & $\$ 41,339.30$ & $\$ 49,073.05$ \\
\hline$\$ 19,642.10$ & $\$ 27,148.20$ & $\$ 35,474.09$ & $\$ 43,078.70$ & $\$ 52,613.66$ & $\$ 62,456.60$ \\
\hline$\$ 22,448.11$ & $\$ 31,026.52$ & $\$ 40,541.81$ & $\$ 49,232.79$ & $\$ 60,129.89$ & $\$ 71,378.98$ \\
\hline$\$ 23,851.12$ & $\$ 32,965.68$ & $\$ 43,075.68$ & $\$ 52,309.84$ & $\$ 63,888.01$ & $\$ 75,840.16$ \\
\hline$\$ 26,657.13$ & $\$ 36,843.99$ & $\$ 48,143.40$ & $\$ 58,463.94$ & $\$ 71,404.25$ & $\$ 84,762.53$ \\
\hline$\$ 26,657.13$ & $\$ 36,843.99$ & $\$ 48,143.40$ & $\$ 58,463.94$ & $\$ 71,404.25$ & $\$ 84,762.53$ \\
\hline$\$ 26,657.13$ & $\$ 36,843.99$ & $\$ 48,143.40$ & $\$ 58,463.94$ & $\$ 71,404.25$ & $\$ 84,762.53$ \\
\hline$\$ 11,715.80$ & $\$ 16,163.60$ & $\$ 20,517.88$ & $\$ 25,098.94$ & $\$ 31,202.98$ & $\$ 36,722.59$ \\
\hline$\$ 18,410.54$ & $\$ 25,399.94$ & $\$ 32,242.38$ & $\$ 39,441.19$ & $\$ 49,033.25$ & $\$ 57,706.93$ \\
\hline$\$ 30,126.33$ & $\$ 41,563.54$ & $\$ 52,760.25$ & $\$ 64,540.12$ & $\$ 80,236.23$ & $\$ 94,429.52$ \\
\hline$\$ 31,800.02$ & $\$ 43,872.63$ & $\$ 55,691.38$ & $\$ 68,125.69$ & $\$ 84,693.79$ & $\$ 99,675.61$ \\
\hline
\end{tabular}

\begin{tabular}{|c|c|c|c|c|c|}
\hline $0 \%$ load & $30 \%$ load & $40 \%$ load & $50 \%$ load & $60 \%$ load & $70 \%$ load \\
\hline $\begin{array}{l}\text { CurrentXFMR } \\
\text { vs } \\
\text { Med XFMR }\end{array}$ & $\begin{array}{l}\text { CurrentXFMR } \\
\text { vs } \\
\text { Med XFMR }\end{array}$ & $\begin{array}{l}\text { CurrentXFMR } \\
\text { vs } \\
\text { Med XFMR }\end{array}$ & $\begin{array}{l}\text { CurrentXFMR } \\
\text { vs } \\
\text { Med XFMR }\end{array}$ & $\begin{array}{l}\text { CurrentXFMR } \\
\text { vs } \\
\text { Med XFMR }\end{array}$ & $\begin{array}{l}\text { CurrentXFMR } \\
\text { vs } \\
\text { Med XFMR }\end{array}$ \\
\hline$\$ 3,106.13$ & $\$ 4,494.87$ & $\$ 5,683.62$ & $\$ 6,623.21$ & $\$ 7,839.05$ & $\$ 9,579.04$ \\
\hline$\$ 3,623.82$ & $\$ 5,244.01$ & $\$ 6,630.89$ & $\$ 7,727.07$ & $\$ 9,145.56$ & $\$ 11,175.55$ \\
\hline$\$ 3,623.82$ & $\$ 5,244.01$ & $\$ 6,630.89$ & $\$ 7,727.07$ & $\$ 9,145.56$ & $\$ 11,175.55$ \\
\hline$\$ 3,623.82$ & $\$ 5,244.01$ & $\$ 6,630.89$ & $\$ 7,727.07$ & $\$ 9,145.56$ & $\$ 11,175.55$ \\
\hline$\$ 4,141.51$ & $\$ 5,993.16$ & $\$ 7,578.17$ & $\$ 8,830.94$ & $\$ 10,452.07$ & $\$ 12,772.05$ \\
\hline$\$ 4,141.51$ & $\$ 5,993.16$ & $\$ 7,578.17$ & $\$ 8,830.94$ & $\$ 10,452.07$ & $\$ 12,772.05$ \\
\hline$\$ 4,141.51$ & $\$ 5,993.16$ & $\$ 7,578.17$ & $\$ 8,830.94$ & $\$ 10,452.07$ & $\$ 12,772.05$ \\
\hline$\$ 4,141.51$ & $\$ 5,993.16$ & $\$ 7,578.17$ & $\$ 8,830.94$ & $\$ 10,452.07$ & $\$ 12,772.05$ \\
\hline$\$ 4,141.51$ & $\$ 5,993.16$ & $\$ 7,578.17$ & $\$ 8,830.94$ & $\$ 10,452.07$ & $\$ 12,772.05$ \\
\hline$\$ 4,659.19$ & $\$ 6,742.30$ & $\$ 8,525.44$ & $\$ 9,934.81$ & $\$ 11,758.58$ & $\$ 14,368.56$ \\
\hline$\$ 4,659.19$ & $\$ 6,742.30$ & $\$ 8,525.44$ & $\$ 9,934.81$ & $\$ 11,758.58$ & $4,368.56$ \\
\hline$\$ 4,659.19$ & $\$ 6,742$ & $\$ 8,525.44$ & $\$ 9,934.81$ & $\$ 11,758.58$ & 668.56 \\
\hline$\$ 5,176.88$ & $\$ 7,491.45$ & $\$ 9,472.71$ & $\$ 11,038.68$ & $\$ 13,065.09$ & $\$ 15,965.07$ \\
\hline$\$ 5,694.57$ & $\$ 8,24 \mathrm{C}$ & $\$ 10,4$ & $\$ 12,142.55$ & $\$ 14,371.60$ & 661.57 \\
\hline$\$ 5,694.57$ & $\$ 8,240.59$ & $\$ 10,419.98$ & $\$ 12,142.55$ & $\$ 14,371.60$ & $\$ 17,561.57$ \\
\hline$\$ 6,212.26$ & $\$ 8,989.74$ & $\$ 11,367.25$ & $\$ 13,246.41$ & $\$ 15,678.10$ & $\$ 19,158.08$ \\
\hline$\$ 6,212.26$ & $\$ 8,989.74$ & $\$ 11,367.25$ & $\$ 13,246.41$ & $\$ 15,678.10$ & $\$ 19,158.08$ \\
\hline$\$ 7,247.64$ & $\$ 10,488.03$ & $\$ 13,261.79$ & $\$ 15,454.15$ & $\$ 18,291.12$ & $\$ 22,351.09$ \\
\hline$\$ 7,247.64$ & $\$ 10,488.03$ & $\$ 13,261.79$ & $\$ 15,454.15$ & $\$ 18,291.12$ & $\$ 22,351.09$ \\
\hline$\$ 7,247.64$ & $\$ 10,488.03$ & $\$ 13,261.79$ & $\$ 15,454.15$ & $\$ 18,291.12$ & $\$ 22,351.09$ \\
\hline$\$ 7,765.32$ & $1,237.17$ & $\$ 14,209.06$ & $\$ 16,558.02$ & $\$ 19,597.63$ & $\$ 23,947.60$ \\
\hline$\$ 8,283.01$ & $\$ 11,986.32$ & $\$ 15,156.33$ & $\$ 17,661.88$ & $\$ 20,904.14$ & $\$ 25,544.11$ \\
\hline$\$ 8,800.70$ & $\$ 12,735.46$ & $\$ 16,103.60$ & $\$ 18,765.75$ & $\$ 22,210.65$ & $\$ 27,140.61$ \\
\hline$\$ 8,800.70$ & $\$ 12,735.46$ & $\$ 16,103.60$ & $\$ 18,765.75$ & $\$ 22,210.65$ & $\$ 27,140.61$ \\
\hline$\$ 8,800.70$ & & $\$ 16,10$ & $\$ 18,765.75$ & $\$ 22,210.65$ & $\$ 27,140.61$ \\
\hline$\$ 8,800.70$ & $\$ 12,735.46$ & $\$ 16,103.60$ & $\$ 18,765.75$ & $\$ 22,210.65$ & $\$ 27,140.61$ \\
\hline$\$ 5,452.45$ & $\$ 8,165.62$ & & & & $\$ 17,264.03$ \\
\hline$\$ 7,010.30$ & & & 99.08 & 8.36 & 96.61 \\
\hline$\$ 7,789.22$ & $1,665.17$ & $\$ 13,626.92$ & $\$ 16,743.42$ & $\$ 20,564.85$ & $\$ 24,662.90$ \\
\hline$\$ 7,789.22$ & $\$ 11,665$ & $\$ 13,626.92$ & $\$ 16,743.42$ & $\$ 20,564.85$ & $\$ 24,662.90$ \\
\hline$\$ 7,789.22$ & 7 & & .42 & $\$ 20,564.85$ & $\$ 24,662.90$ \\
\hline$\$ 7,789.22$ & 7 & $\$ 1$ & .42 & $\$ 20,564.85$ & $\$ 24,662.90$ \\
\hline$\$ 8,568.14$ & $\$ 12,831.69$ & $\$ 14,989.61$ & $\$ 18,417.77$ & $\$ 22,621.33$ & $\$ 27,129.18$ \\
\hline$\$ 8,568.14$ & $\$ 12,831.69$ & $\$ 14,989.61$ & $\$ 18,417.77$ & $\$ 22,621.33$ & $\$ 27,129.18$ \\
\hline$\$ 8,568.14$ & $\$ 12,831.69$ & $\$ 14,989.61$ & $\$ 18,417.77$ & $\$ 22,621.33$ & $\$ 27,129.18$ \\
\hline$\$ 8,568.14$ & $\$ 12,831.69$ & $\$ 14,989.61$ & $\$ 18,417.77$ & $\$ 22,621.33$ & $\$ 27,129.18$ \\
\hline$\$ 10,904.91$ & & & $\$ 23,440.79$ & $\$ 28,790.79$ & $\$ 34,528.0$ \\
\hline$\$ 12,462.75$ & $\$ 18,664.27$ & $\$ 21,803.07$ & $\$ 26,789.48$ & $\$ 32,903.76$ & $\$ 39,460.63$ \\
\hline$\$ 13,241.67$ & $\$ 19,830.79$ & $\$ 23,165.76$ & $\$ 28,463.82$ & $\$ 34,960.24$ & $\$ 41,926.92$ \\
\hline$\$ 14,799.52$ & $\$ 22,163.83$ & $\$ 25,891.15$ & $\$ 31,812.51$ & $\$ 39,073.21$ & $\$ 46,859.50$ \\
\hline$\$ 14,799.52$ & $\$ 22,163.83$ & $\$ 25,891.15$ & $\$ 31,812.51$ & $\$ 39,073.21$ & $\$ 46,859.50$ \\
\hline$\$ 14,799.52$ & $\$ 22,163.83$ & $\$ 25,891.15$ & $\$ 31,812.51$ & $\$ 39,073.21$ & $\$ 4685950$ \\
\hline$\$ 7,373.36$ & $\$ 10,605.81$ & $\$ 13,107.57$ & $\$ 15,267.90$ & $\$ 18,843.52$ & $\$ 22,969.16$ \\
\hline$\$ 11,586.71$ & $\$ 16,666.27$ & $\$ 20,597.61$ & $\$ 23,992.42$ & $\$ 29,611.25$ & $\$ 36,094.40$ \\
\hline$\$ 18,960.07$ & | & $\$ 33,705.1$ & 53926032 & $\$ 48,454.77$ & $\$ 5006$ \\
\hline$\$ 20,013.41$ & $\$ 28,787.19$ & $\$ 35,577.69$ & $\$ 41,441.45$ & $\$ 51,146.71$ & $\$ 62,344.87$ \\
\hline
\end{tabular}




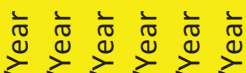

ำ

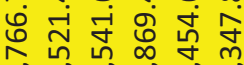

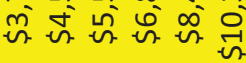

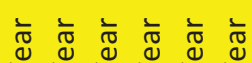

$\geq \geq \geq \geq \geq \geq$

ำ 웅

नें

in

की को को ज्ञ

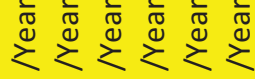

ㄱํ

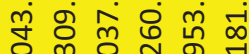

के को जो

虫

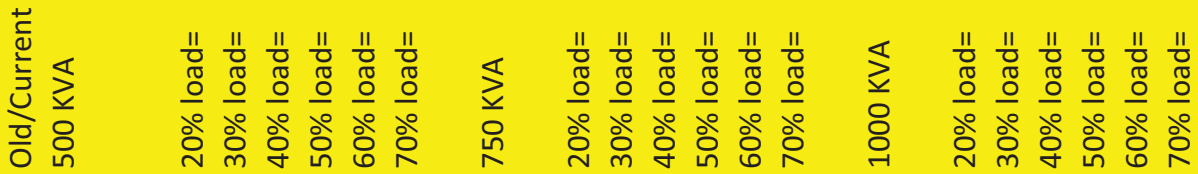

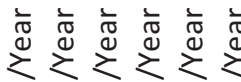

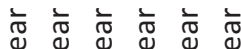

$\geq \geq \geq \geq \geq Z$

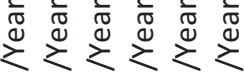

ㄱㅇㅇ

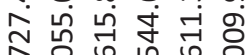

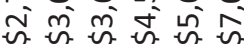

ఫั๊

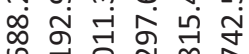

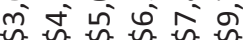

$\forall \mathscr{~}$

के ठి

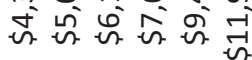

峁

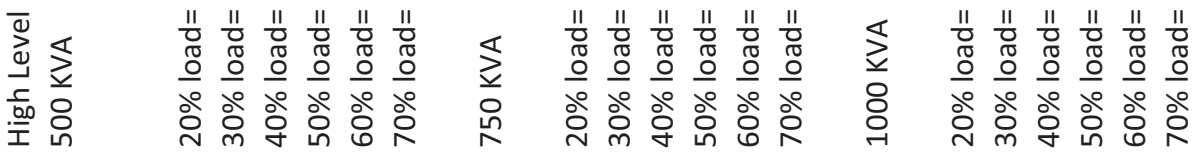

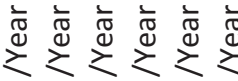

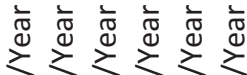

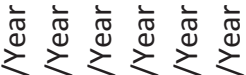

ஃ 유 ₹

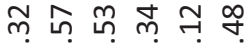

ᄀี

$\infty 刃$ 오상

尌尽命包品

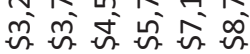

जि की की को

ळ

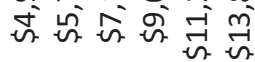

峞

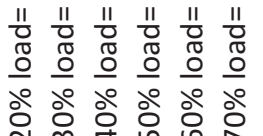

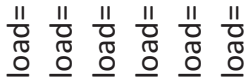

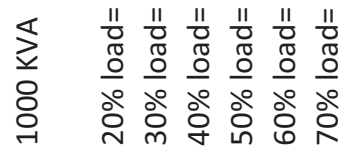

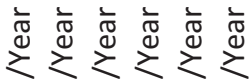

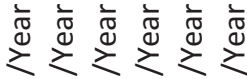

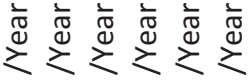

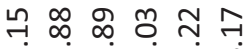

ๆ

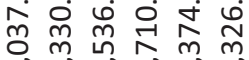

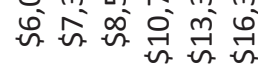

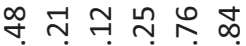

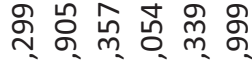

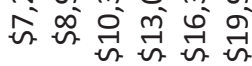

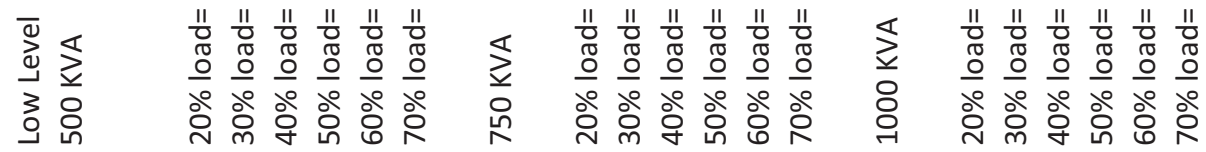




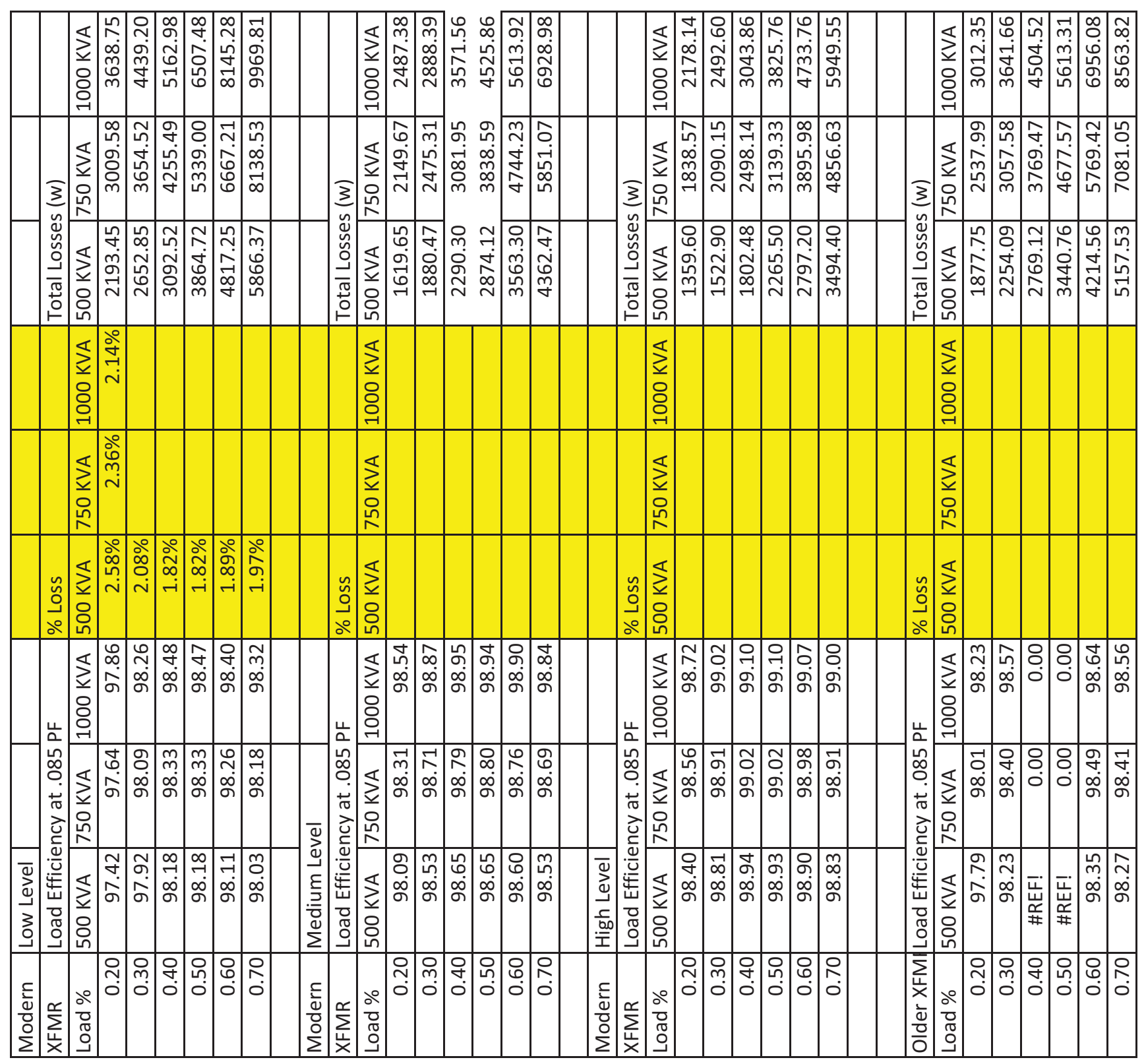

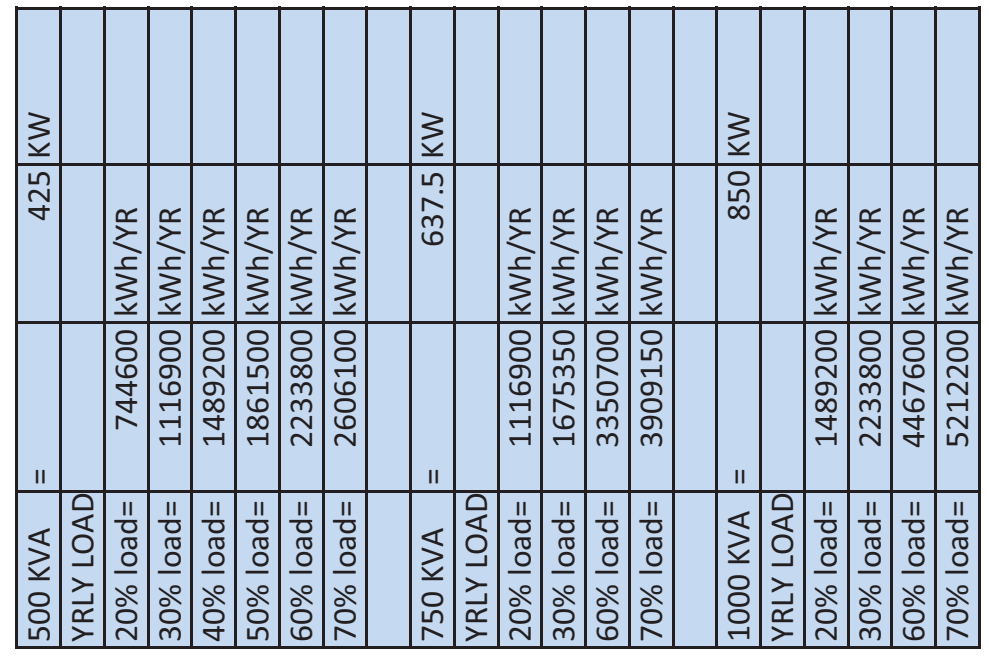




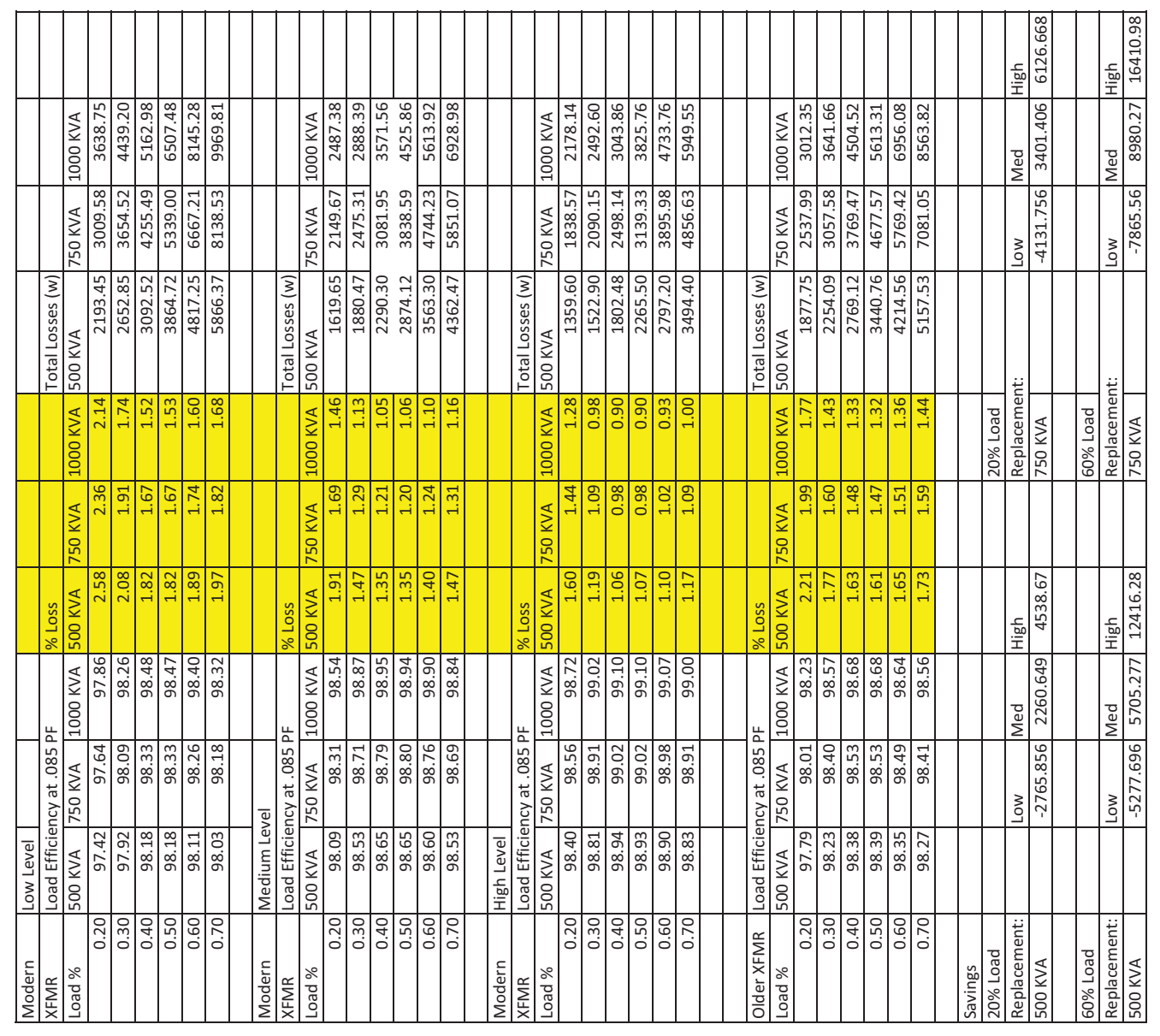




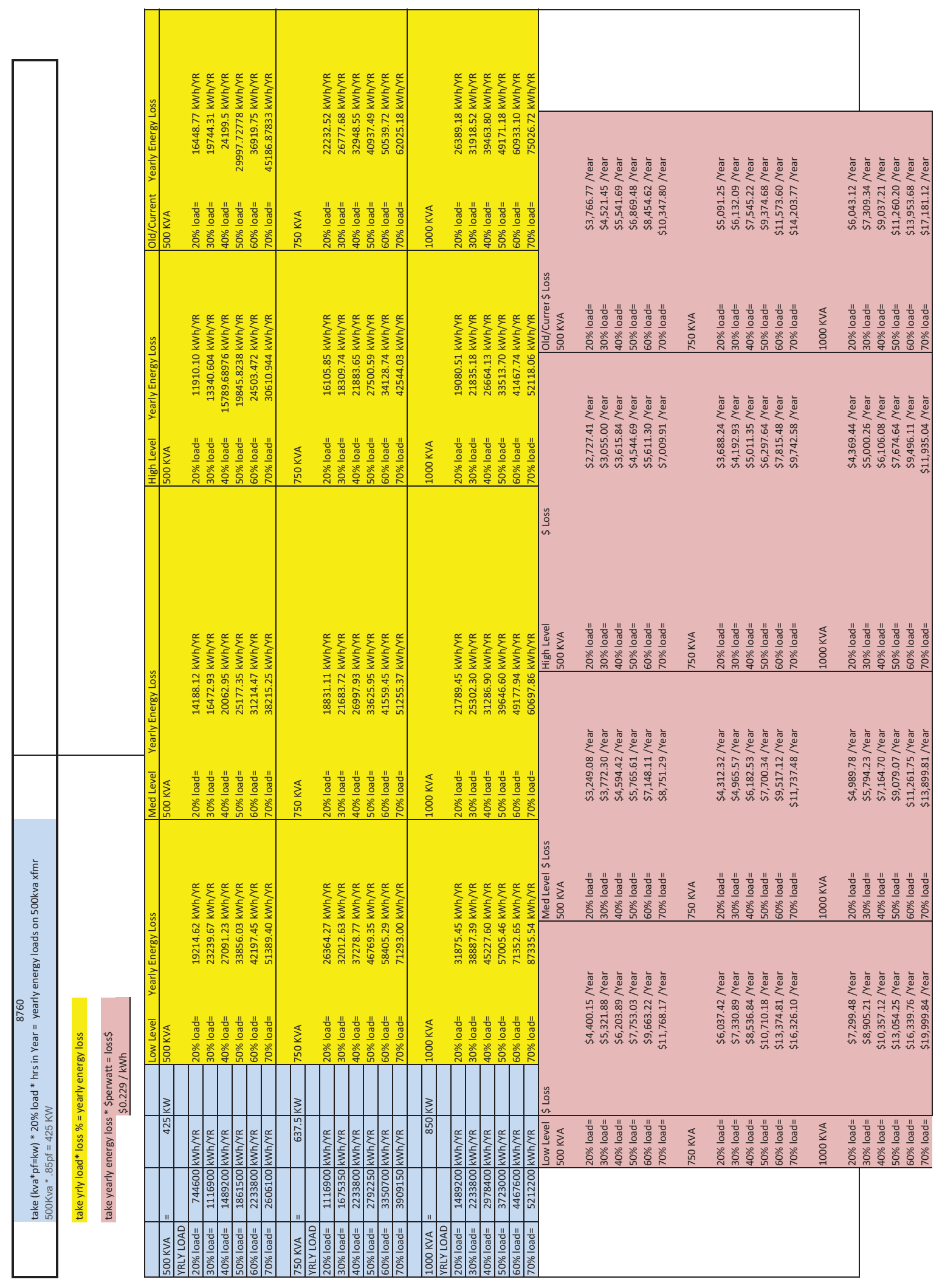




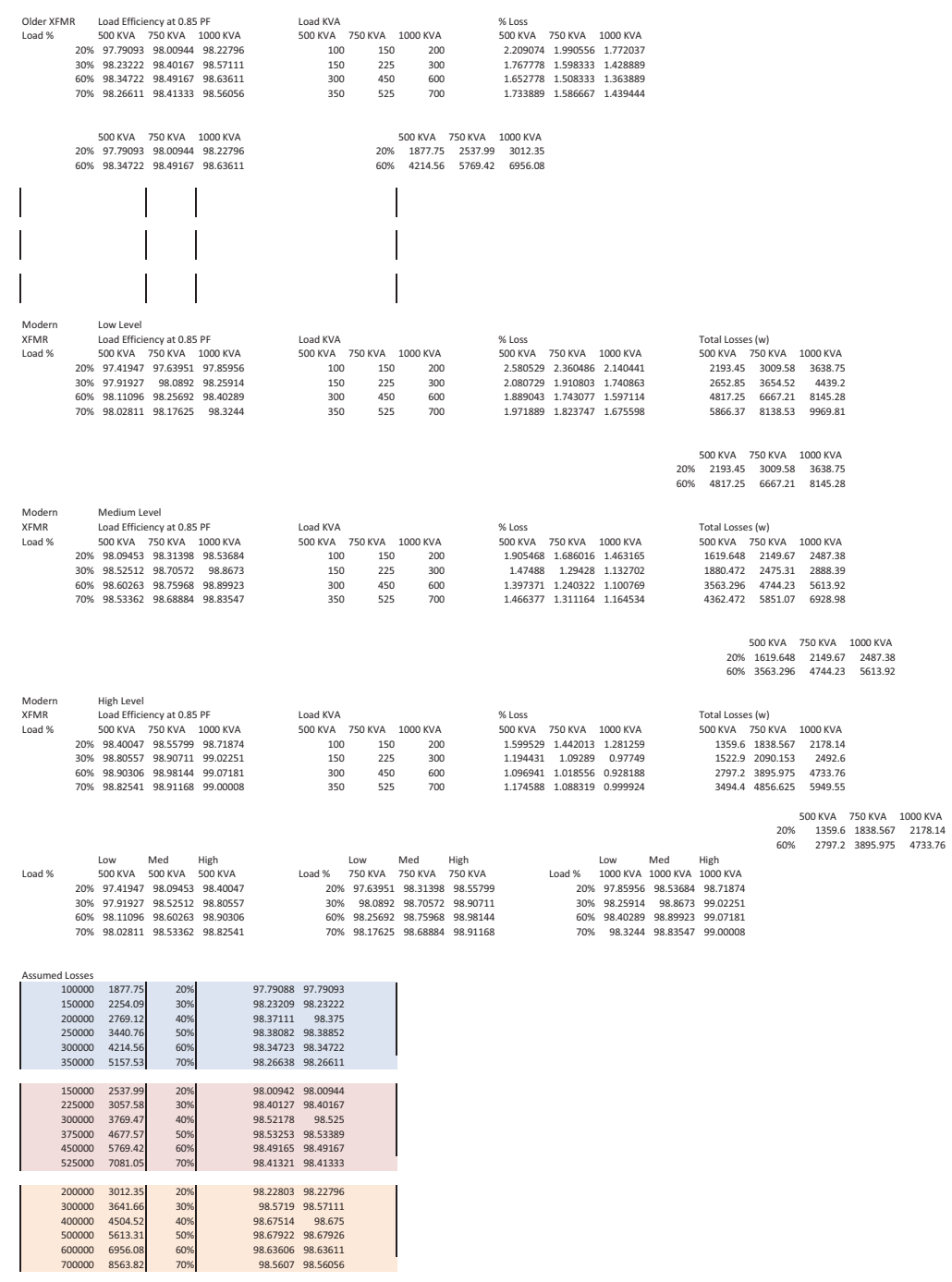



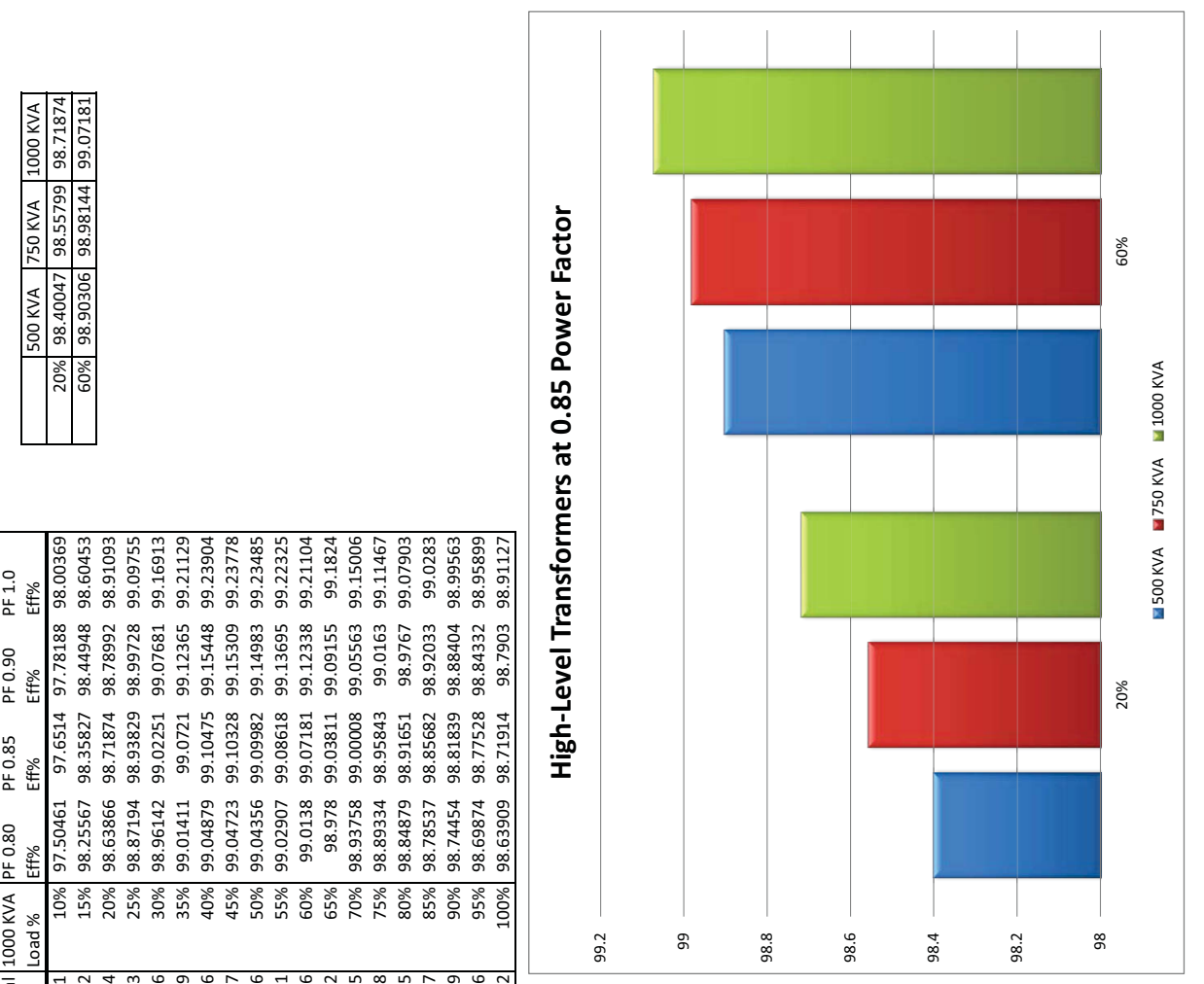

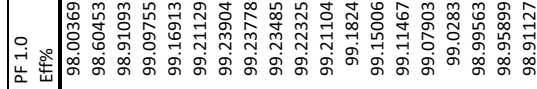

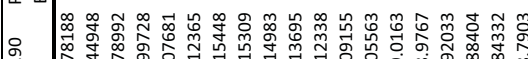

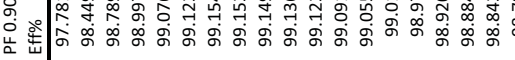

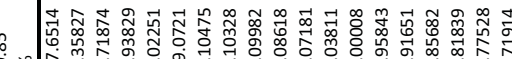

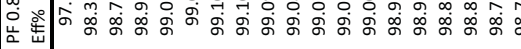

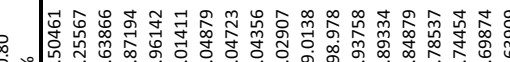

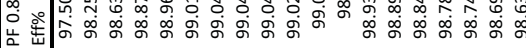

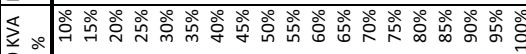
每

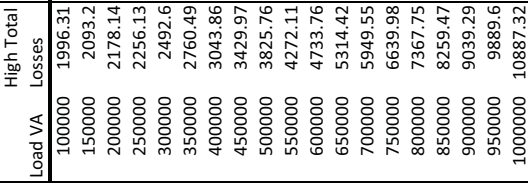

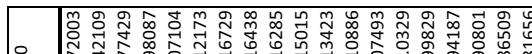

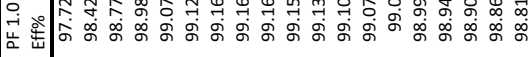

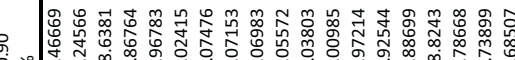

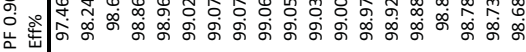
等

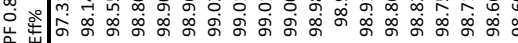

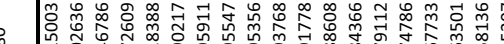

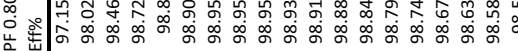

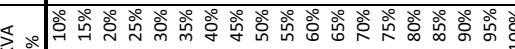

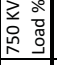

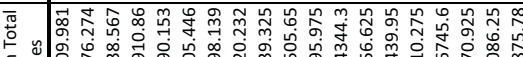

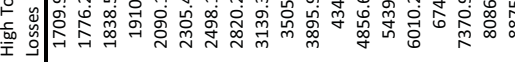

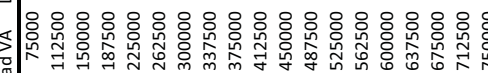

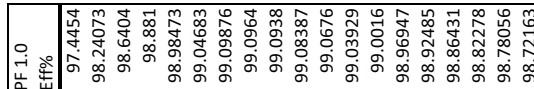
눅 유 然

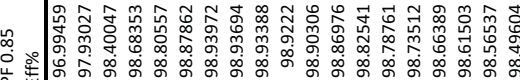

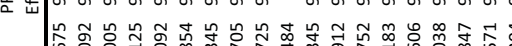

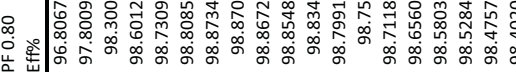

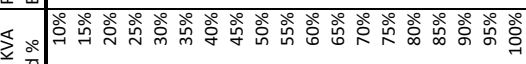

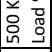

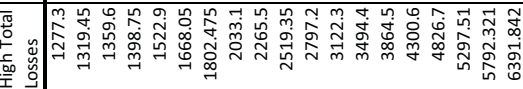

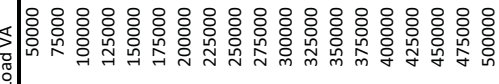

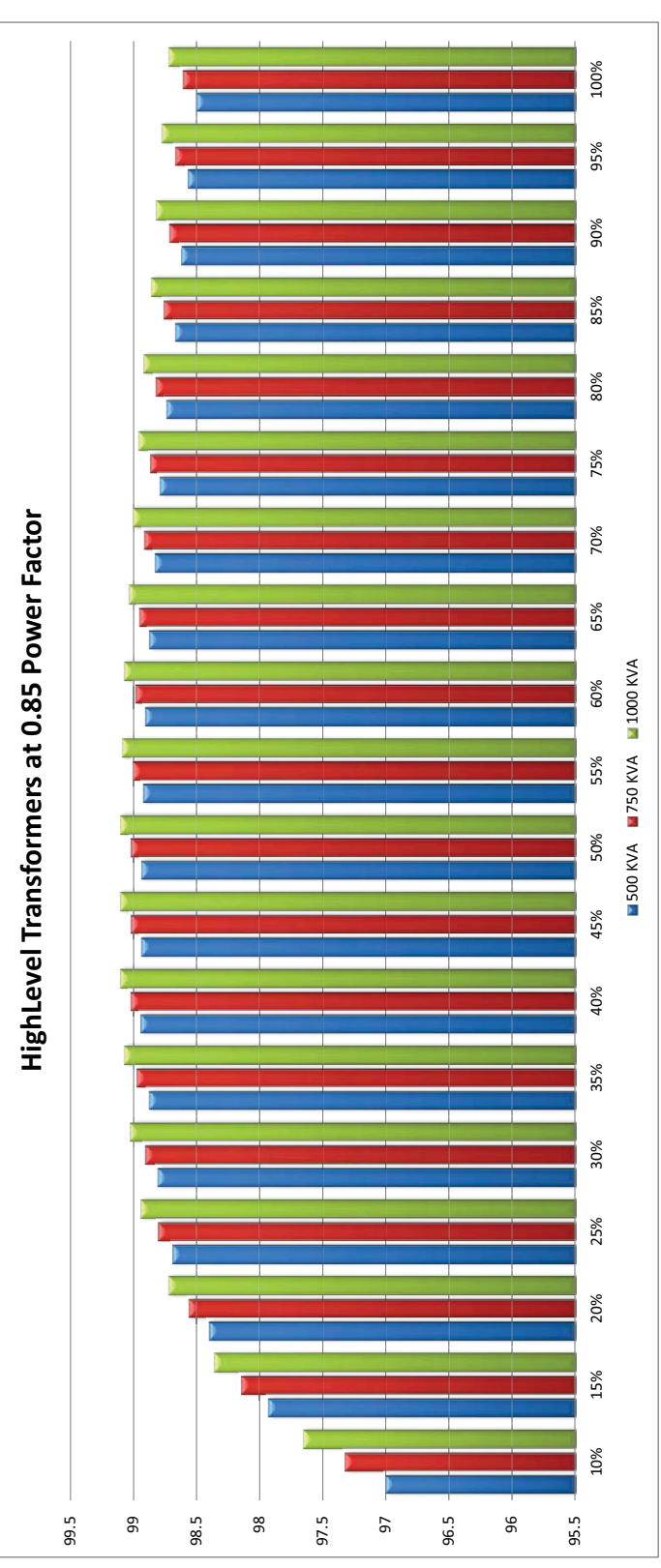




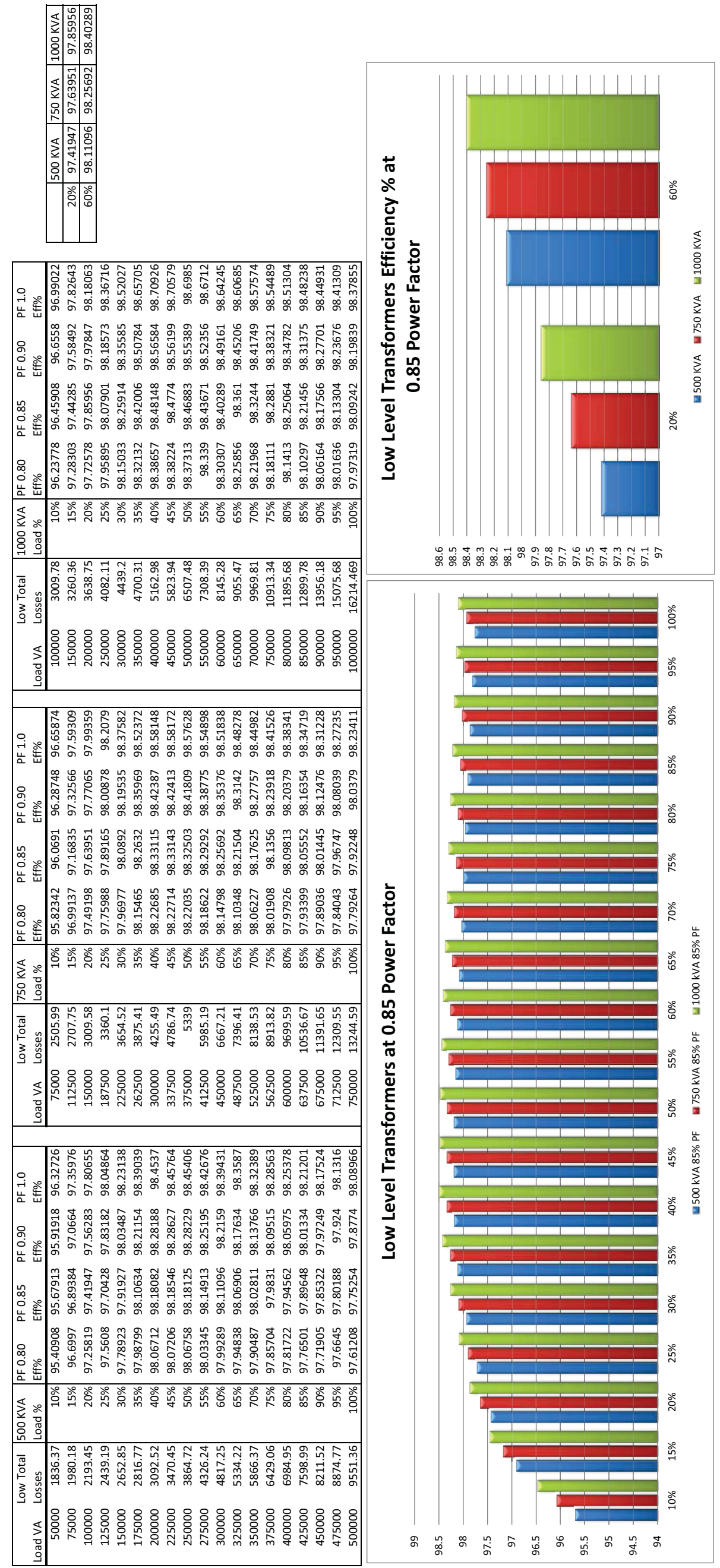




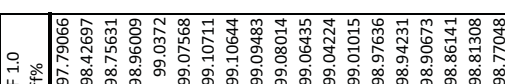

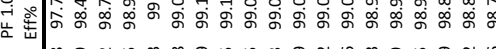

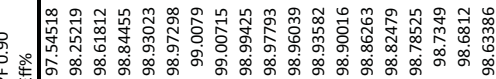

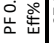

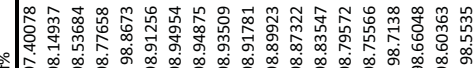
เง

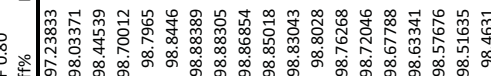

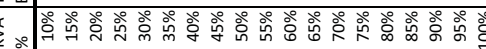

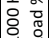

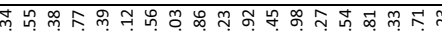
त्ञ (n)

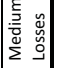

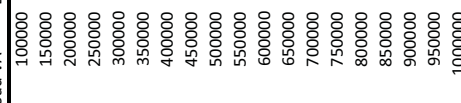

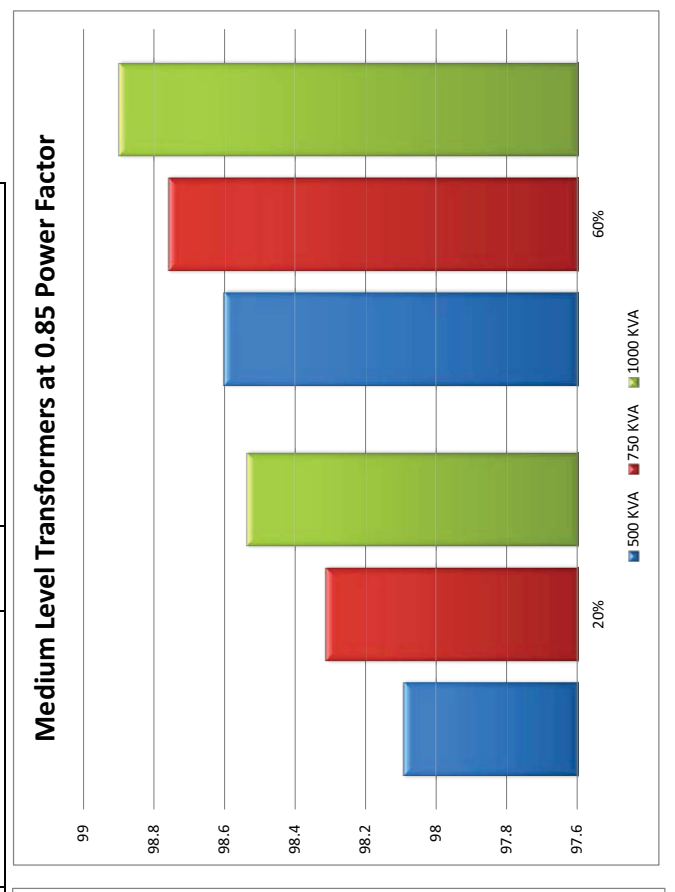

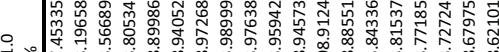
จำง

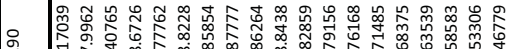

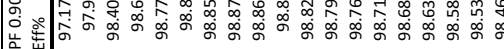

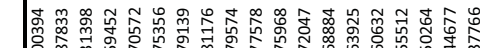

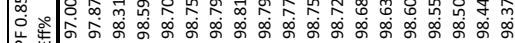
|

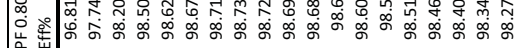

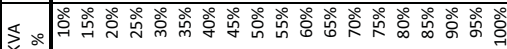
总:

迨

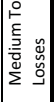

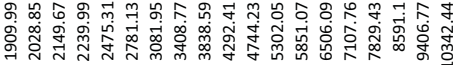

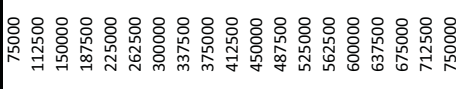

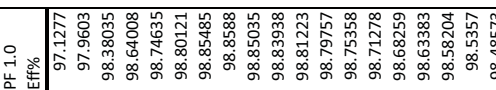
4.

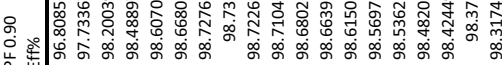

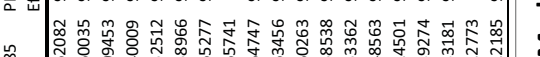

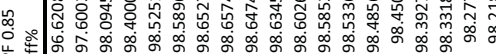

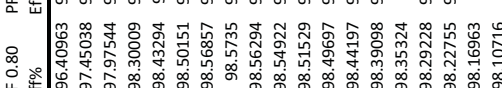

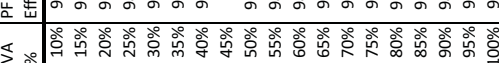
䇏密 is

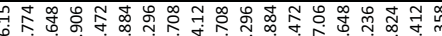
mintwitum

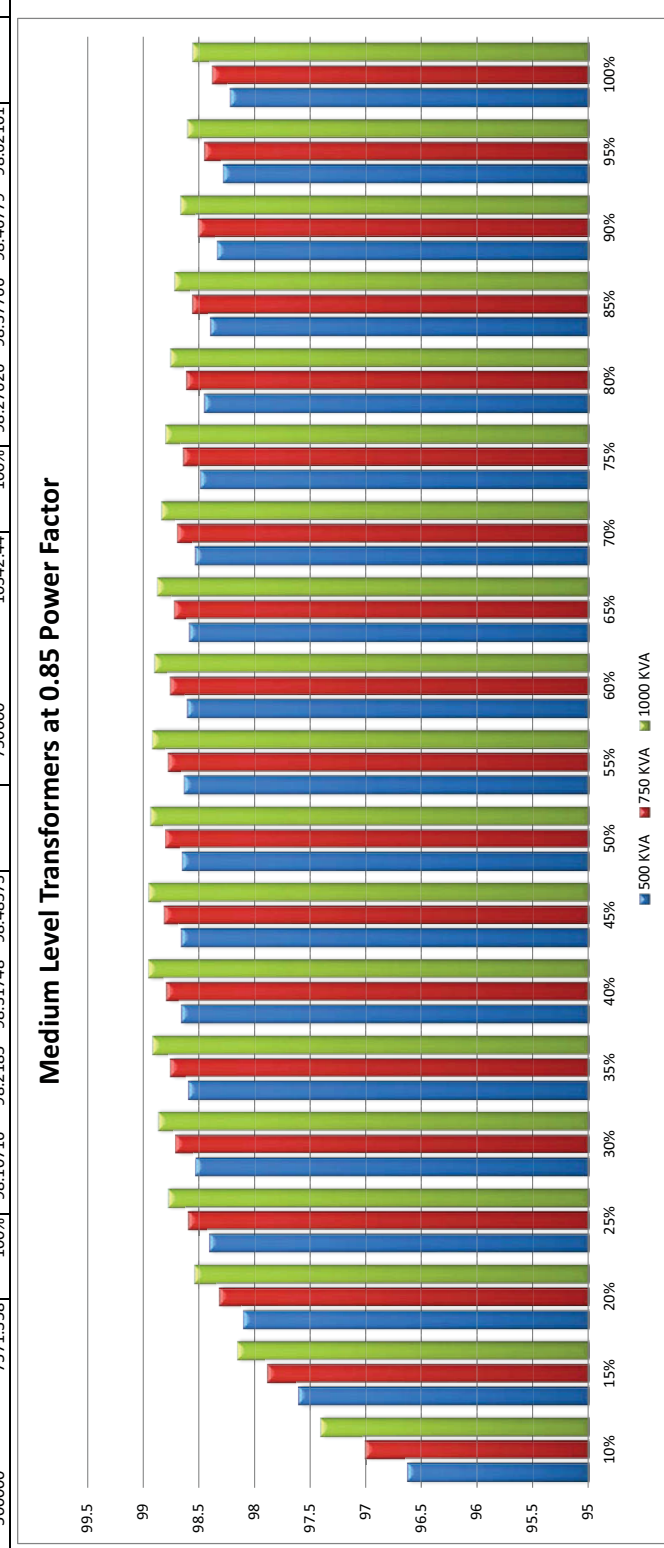




\begin{tabular}{|c|c|c|c|c|c|c|}
\hline Load VA & $\begin{array}{l}\text { Low Total } \\
\text { Losses }\end{array}$ & $\begin{array}{l}1000 \text { KVA } \\
\text { Load } \%\end{array}$ & $\begin{array}{l}\text { PF } 0.80 \\
\text { Eff\% }\end{array}$ & $\begin{array}{l}\text { PF } 0.85 \\
\text { Eff\% }\end{array}$ & $\begin{array}{l}\text { PF } 0.90 \\
\text { Eff\% }\end{array}$ & $\begin{array}{l}\text { PF } 1.0 \\
\text { Eff\% }\end{array}$ \\
\hline 100000 & 3009.78 & $10 \%$ & 96.23778 & 96.45908 & 96.6558 & 96.99022 \\
\hline 150000 & 3260.36 & $15 \%$ & 97.28303 & 97.44285 & 97.58492 & 97.82643 \\
\hline 200000 & 3638.75 & $20 \%$ & 97.72578 & 97.85956 & 97.97847 & 98.18063 \\
\hline 250000 & 4082.11 & $25 \%$ & 97.95895 & 98.07901 & 98.18573 & 98.36716 \\
\hline 300000 & 4439.2 & $30 \%$ & 98.15033 & 98.25914 & 98.35585 & 98.52027 \\
\hline 350000 & 4700.31 & $35 \%$ & 98.32132 & 98.42006 & 98.50784 & 98.65705 \\
\hline 400000 & 5162.98 & $40 \%$ & 98.38657 & 98.48148 & 98.56584 & 98.70926 \\
\hline 450000 & 5823.94 & $45 \%$ & 98.38224 & 98.4774 & 98.56199 & 98.70579 \\
\hline 500000 & 6507.48 & $50 \%$ & 98.37313 & 98.46883 & 98.55389 & 98.6985 \\
\hline 550000 & 7308.39 & $55 \%$ & 98.339 & 98.43671 & 98.52356 & 98.6712 \\
\hline 600000 & 8145.28 & $60 \%$ & 98.30307 & 98.40289 & 98.49161 & 98.64245 \\
\hline 650000 & 9055.47 & $65 \%$ & 98.25856 & 98.361 & 98.45206 & 98.60685 \\
\hline 700000 & 9969.81 & $70 \%$ & 98.21968 & 98.3244 & 98.41749 & 98.57574 \\
\hline 750000 & 10913.34 & $75 \%$ & 98.18111 & 98.2881 & 98.38321 & 98.54489 \\
\hline 800000 & 11895.68 & $80 \%$ & 98.1413 & 98.25064 & 98.34782 & 98.51304 \\
\hline 850000 & 12899.78 & $85 \%$ & 98.10297 & 98.21456 & 98.31375 & 98.48238 \\
\hline 900000 & 13956.18 & $90 \%$ & 98.06164 & 98.17566 & 98.27701 & 98.44931 \\
\hline 950000 & 15075.68 & $95 \%$ & 98.01636 & 98.13304 & 98.23676 & 98.41309 \\
\hline 1000000 & 16214.47 & $100 \%$ & 97.97319 & 98.09242 & 98.19839 & 98.37855 \\
\hline
\end{tabular}

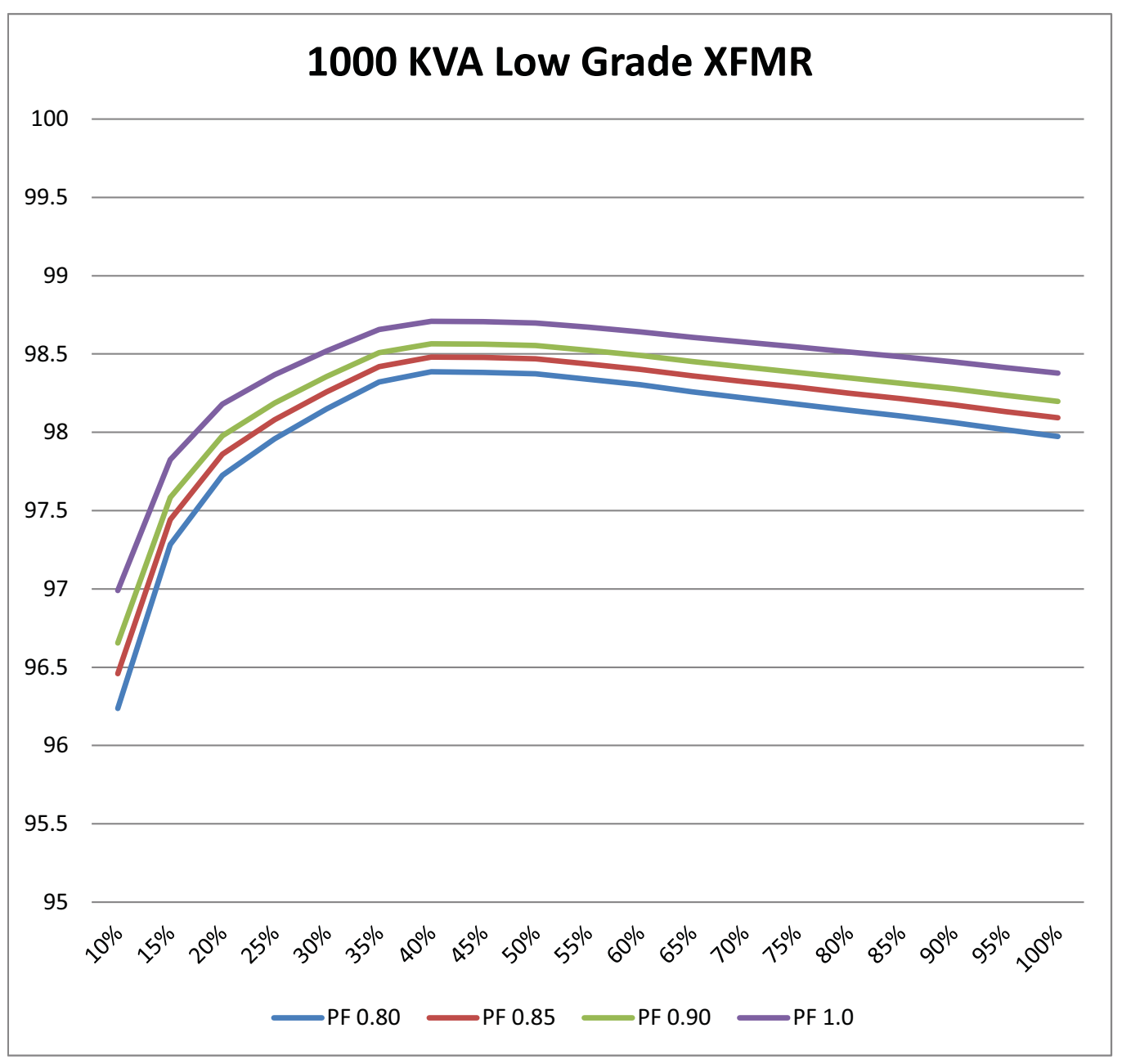




\begin{tabular}{|c|c|c|c|c|c|c|c|}
\hline Load VA & $\begin{array}{l}\text { Medium-Low Total } \\
\text { Losses }\end{array}$ & $\begin{array}{l}1000 \text { KVA } \\
\text { Load } \% \\
\end{array}$ & $\begin{array}{l}\text { PF } 0.80 \\
\text { Eff\% }\end{array}$ & $\begin{array}{l}\text { PF } 0.85 \\
\text { Eff\% }\end{array}$ & $\begin{array}{l}\text { PF } 0.90 \\
\text { Eff\% }\end{array}$ & $\begin{array}{l}\text { PF } 1.0 \\
\text { Eff\% }\end{array}$ & \\
\hline 100000 & 2510.65 & $10 \%$ & 96.86169 & 97.04629 & 97.21039 & 97.48935 & \\
\hline 150000 & 2734.05 & $15 \%$ & 97.72163 & 97.85565 & 97.97478 & 98.1773 & \\
\hline 200000 & 2987.22 & $20 \%$ & 98.13299 & 98.24281 & 98.34043 & 98.50639 & \\
\hline 250000 & 3212.87 & $25 \%$ & 98.39357 & 98.48806 & 98.57206 & 98.71485 & \\
\hline 300000 & 3528.85 & $30 \%$ & 98.52965 & 98.61614 & 98.69302 & 98.82372 & \\
\hline 350000 & 3799.35 & $35 \%$ & 98.64309 & 98.72291 & 98.79386 & 98.91447 & \\
\hline 400000 & 4169.32 & $40 \%$ & 98.69709 & 98.77373 & 98.84186 & 98.95767 & \\
\hline 450000 & 4703.99 & $45 \%$ & 98.69334 & 98.7702 & 98.83852 & 98.95467 & \\
\hline 500000 & 5271.56 & $50 \%$ & 98.68211 & 98.75963 & 98.82854 & 98.94569 & \\
\hline 550000 & 5897.46 & $55 \%$ & 98.65967 & 98.73851 & 98.80859 & 98.92773 & \\
\hline 600000 & 6602.57 & $60 \%$ & 98.62446 & 98.70538 & 98.7773 & 98.89957 & \\
\hline 650000 & 7299.54 & $65 \%$ & 98.59624 & 98.67882 & 98.75222 & 98.87699 & \\
\hline 700000 & 8062.27 & $70 \%$ & 98.56031 & 98.645 & 98.72027 & 98.84825 & \\
\hline 750000 & 8807.59 & $75 \%$ & 98.53207 & 98.61842 & 98.69517 & 98.82565 & \\
\hline 800000 & 9695.76 & $80 \%$ & 98.48504 & 98.57415 & 98.65337 & 98.78803 & \\
\hline 850000 & 10619.92 & $85 \%$ & 98.43825 & 98.53011 & 98.61178 & 98.7506 & \\
\hline 900000 & 11634.83 & $90 \%$ & 98.38405 & 98.47911 & 98.5636 & 98.70724 & \\
\hline 950000 & 12702.21 & $95 \%$ & 98.32866 & 98.42697 & 98.51436 & 98.66293 & \\
\hline 1000000 & 13781.87 & $100 \%$ & 98.27727 & 98.3786 & 98.46868 & 98.62181 & \\
\hline
\end{tabular}

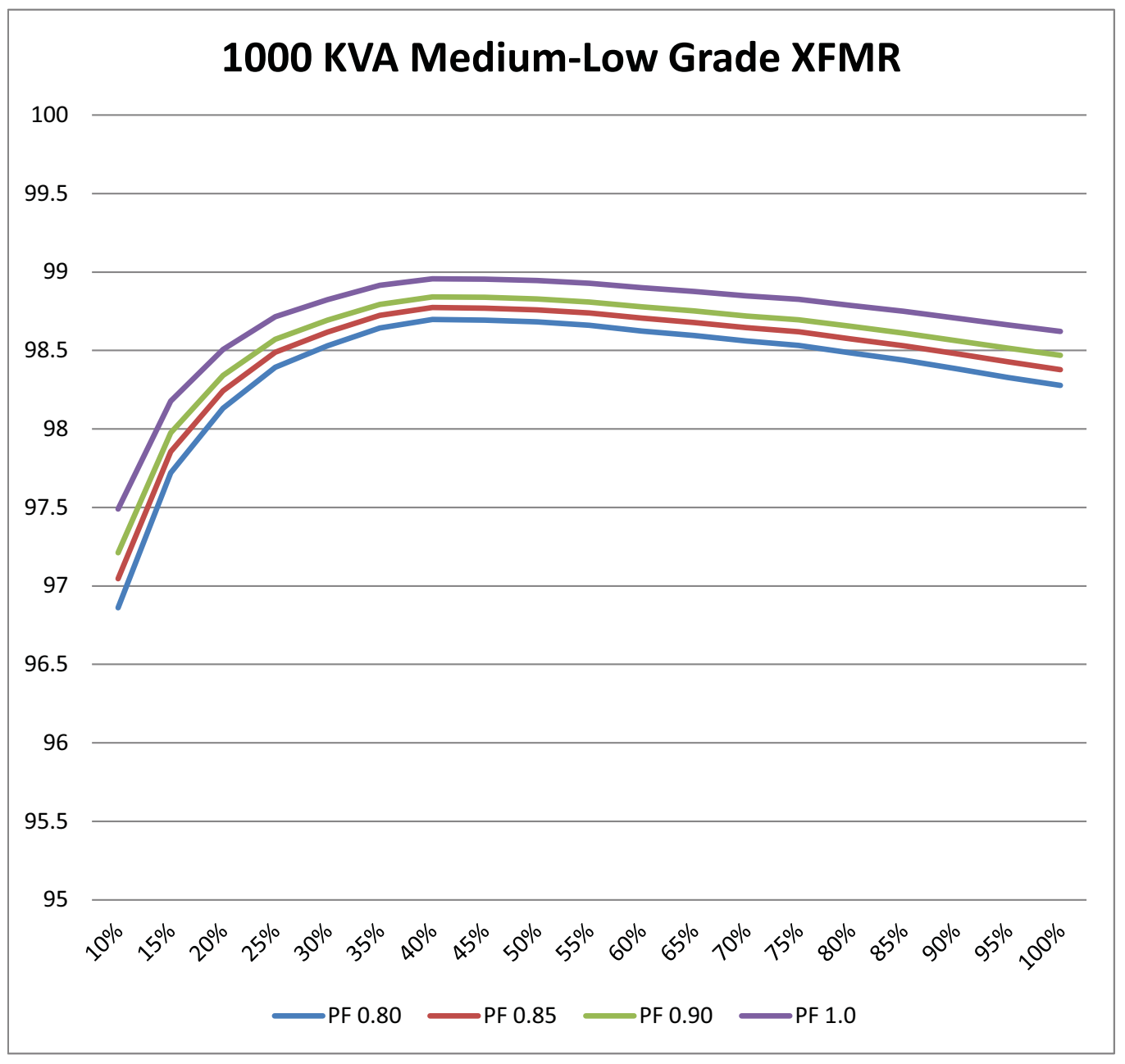




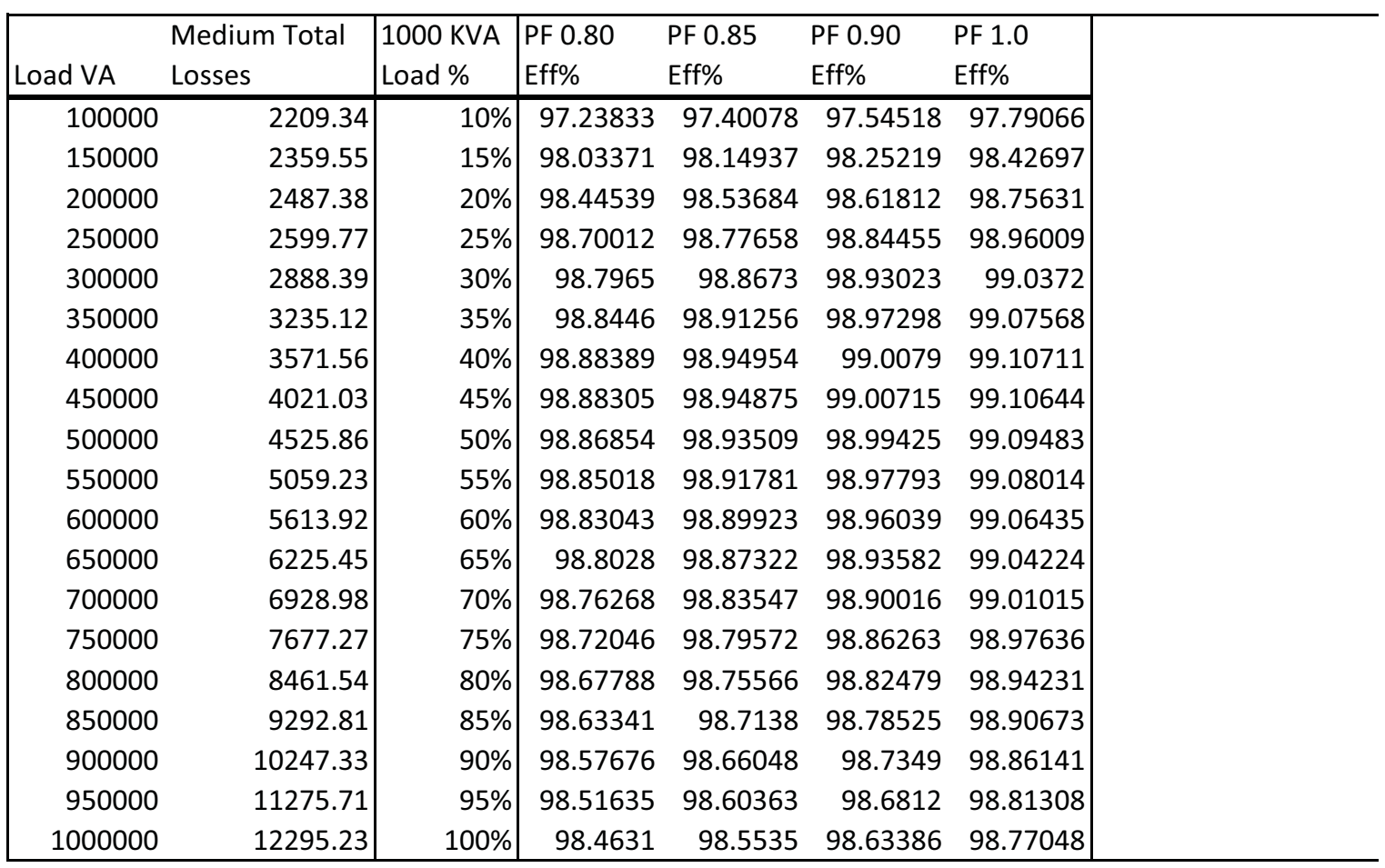

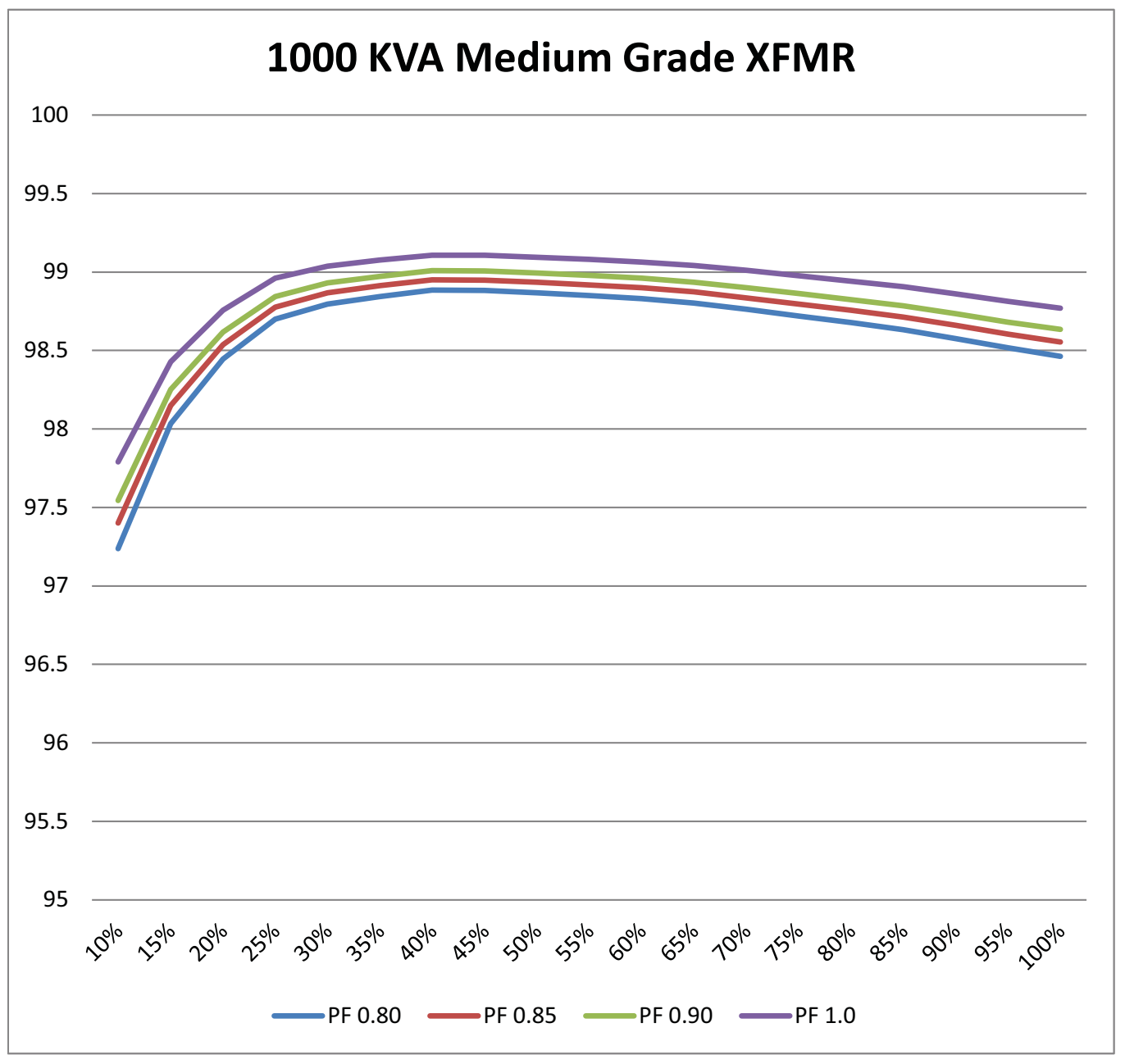




\begin{tabular}{|c|c|c|c|c|c|c|c|}
\hline Load VA & $\begin{array}{l}\text { Medium-High Total } \\
\text { Losses }\end{array}$ & $\begin{array}{l}1000 \text { KVA } \\
\text { Load \% }\end{array}$ & $\begin{array}{l}\text { PF } 0.80 \\
\text { Eff\% }\end{array}$ & $\begin{array}{l}\text { PF } 0.85 \\
\text { Eff\% }\end{array}$ & $\begin{array}{l}\text { PF } 0.90 \\
\text { Eff\% }\end{array}$ & $\begin{array}{l}\text { PF } 1.0 \\
\text { Eff\% }\end{array}$ & \\
\hline 100000 & 2133.08 & $10 \%$ & 97.33365 & 97.49049 & 97.62991 & 97.86692 & \\
\hline 150000 & 2329.78 & $15 \%$ & 98.05852 & 98.17272 & 98.27424 & 98.44681 & \\
\hline 200000 & 2433.23 & $20 \%$ & 98.47923 & 98.56869 & 98.64821 & 98.78339 & \\
\hline 250000 & 2503.59 & $25 \%$ & 98.74821 & 98.82184 & 98.88729 & 98.99856 & \\
\hline 300000 & 2818.82 & $30 \%$ & 98.82549 & 98.89458 & 98.95599 & 99.06039 & \\
\hline 350000 & 3132.44 & $35 \%$ & 98.88127 & 98.94708 & 99.00557 & 99.10502 & \\
\hline 400000 & 3491.25 & $40 \%$ & 98.90898 & 98.97316 & 99.03021 & 99.12719 & \\
\hline 450000 & 3968.03 & $45 \%$ & 98.89777 & 98.96261 & 99.02024 & 99.11822 & \\
\hline 500000 & 4463.67 & $50 \%$ & 98.88408 & 98.94972 & 99.00807 & 99.10727 & \\
\hline 550000 & 4958.73 & $55 \%$ & 98.87302 & 98.93931 & 98.99824 & 99.09841 & \\
\hline 600000 & 5502.12 & $60 \%$ & 98.85373 & 98.92115 & 98.98109 & 99.08298 & \\
\hline 650000 & 6102.86 & $65 \%$ & 98.82637 & 98.89541 & 98.95678 & 99.0611 & \\
\hline 700000 & 6798.99 & $70 \%$ & 98.78589 & 98.85731 & 98.9208 & 99.02872 & \\
\hline 750000 & 7505.45 & $75 \%$ & 98.74909 & 98.82267 & 98.88808 & 98.99927 & \\
\hline 800000 & 8275.72 & $80 \%$ & 98.70692 & 98.78298 & 98.85059 & 98.96554 & \\
\hline 850000 & 9085.11 & $85 \%$ & 98.66395 & 98.74255 & 98.8124 & 98.93116 & \\
\hline 900000 & 10058.52 & $90 \%$ & 98.60298 & 98.68516 & 98.75821 & 98.88239 & \\
\hline 950000 & 11100.73 & $95 \%$ & 98.53938 & 98.6253 & 98.70167 & 98.8315 & \\
\hline 1000000 & 12085.76 & $100 \%$ & 98.48928 & 98.57815 & 98.65714 & 98.79142 & \\
\hline
\end{tabular}

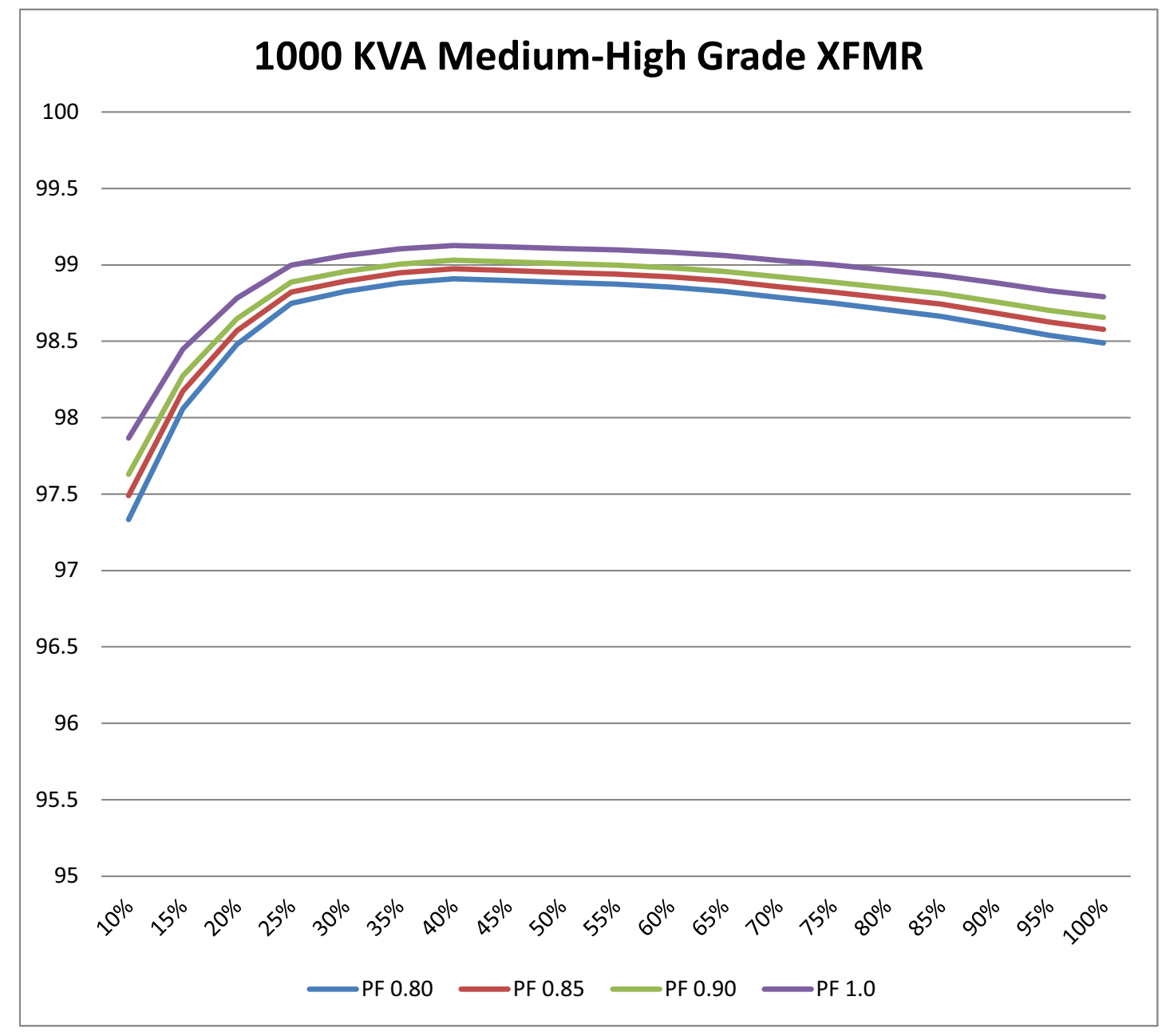




\begin{tabular}{|c|c|c|c|c|c|c|}
\hline Load VA & $\begin{array}{l}\text { High Total } \\
\text { Losses }\end{array}$ & \begin{tabular}{|l|}
1000 KVA \\
Load $\%$
\end{tabular} & \begin{tabular}{|l|} 
PF 0.80 \\
Eff\%
\end{tabular} & $\begin{array}{l}\text { PF } 0.85 \\
\text { Eff\% }\end{array}$ & $\begin{array}{l}\text { PF 0.90 } \\
\text { Eff\% }\end{array}$ & $\begin{array}{l}\text { PF 1.0 } \\
\text { Eff\% }\end{array}$ \\
\hline 100000 & 1996.31 & $10 \%$ & 97.50461 & 97.6514 & 97.78188 & 98.00369 \\
\hline 150000 & 2093.2 & $15 \%$ & 98.25567 & 98.35827 & 98.44948 & 98.60453 \\
\hline 200000 & 2178.14 & $20 \%$ & 98.63866 & 98.71874 & 98.78992 & 98.91093 \\
\hline 250000 & 2256.13 & $25 \%$ & 98.87194 & 98.93829 & 98.99728 & 99.09755 \\
\hline 300000 & 2492.6 & $30 \%$ & 98.96142 & 99.02251 & 99.07681 & 99.16913 \\
\hline 350000 & 2760.49 & $35 \%$ & 99.01411 & 99.0721 & 99.12365 & 99.21129 \\
\hline 400000 & 3043.86 & $40 \%$ & 99.04879 & 99.10475 & 99.15448 & 99.23904 \\
\hline 450000 & 3429.97 & $45 \%$ & 99.04723 & 99.10328 & 99.15309 & 99.23778 \\
\hline 500000 & 3825.76 & $50 \%$ & 99.04356 & 99.09982 & 99.14983 & 99.23485 \\
\hline 550000 & 4272.11 & $55 \%$ & 99.02907 & 99.08618 & 99.13695 & 99.22325 \\
\hline 600000 & 4733.76 & $60 \%$ & 99.0138 & 99.07181 & 99.12338 & 99.21104 \\
\hline 650000 & 5314.42 & $65 \%$ & 98.978 & 99.03811 & 99.09155 & 99.1824 \\
\hline 700000 & 5949.55 & $70 \%$ & 98.93758 & 99.00008 & 99.05563 & 99.15006 \\
\hline 750000 & 6639.98 & $75 \%$ & 98.89334 & 98.95843 & 99.0163 & 99.11467 \\
\hline 800000 & 7367.75 & $80 \%$ & 98.84879 & 98.91651 & 98.9767 & 99.07903 \\
\hline 850000 & 8259.47 & $85 \%$ & 98.78537 & 98.85682 & 98.92033 & 99.0283 \\
\hline 900000 & 9039.29 & $90 \%$ & 98.74454 & 98.81839 & 98.88404 & 98.99563 \\
\hline 950000 & 9889.6 & $95 \%$ & 98.69874 & 98.77528 & 98.84332 & 98.95899 \\
\hline 1000000 & 10887.32 & $100 \%$ & 98.63909 & 98.71914 & 98.7903 & 98.91127 \\
\hline
\end{tabular}

\section{KVA High Grade XFMR}

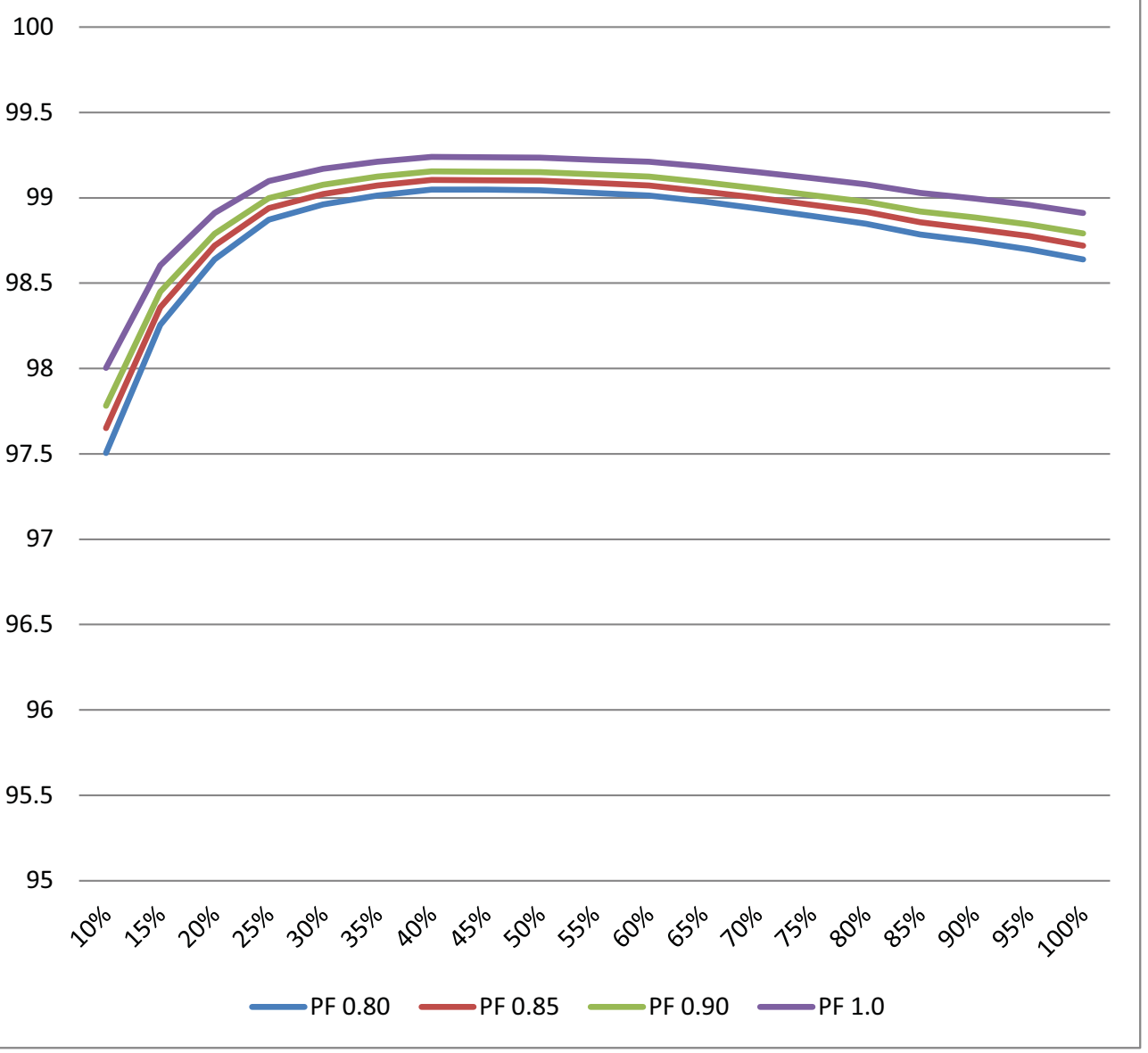




\begin{tabular}{|c|c|c|c|c|c|c|}
\hline Load VA & $\begin{array}{l}\text { Premium Total } \\
\text { Losses }\end{array}$ & $\begin{array}{l}1000 \text { KVA } \\
\text { Load \% }\end{array}$ & $\begin{array}{l}\text { PF } 0.80 \\
\text { Eff\% }\end{array}$ & $\begin{array}{l}\text { PF } 0.85 \\
\text { Eff\% }\end{array}$ & $\begin{array}{l}\text { PF } 0.90 \\
\text { Eff\% }\end{array}$ & $\begin{array}{l}\text { PF } 1.0 \\
\text { Eff\% }\end{array}$ \\
\hline 100000 & 1800.775 & $10 \%$ & 97.74903 & 97.88144 & 97.99914 & 98.19923 \\
\hline 150000 & 1865.332 & $15 \%$ & 98.44556 & 98.53699 & 98.61827 & 98.75645 \\
\hline 200000 & 1935.876 & $20 \%$ & 98.79008 & 98.86125 & 98.92451 & 99.03206 \\
\hline 250000 & 2026.76 & $25 \%$ & 98.98662 & 99.04623 & 99.09922 & 99.1893 \\
\hline 300000 & 2192.92 & $30 \%$ & 99.08628 & 99.14003 & 99.18781 & 99.26903 \\
\hline 350000 & 2398.745 & $35 \%$ & 99.14331 & 99.1937 & 99.23849 & 99.31464 \\
\hline 400000 & 2558.113 & $40 \%$ & 99.20059 & 99.24761 & 99.28941 & 99.36047 \\
\hline 450000 & 2865.309 & $45 \%$ & 99.20408 & 99.2509 & 99.29252 & 99.36326 \\
\hline 500000 & 3221.786 & $50 \%$ & 99.19455 & 99.24193 & 99.28405 & 99.3556 \\
\hline 550000 & 3579.656 & $55 \%$ & 99.18644 & 99.2343 & 99.27684 & 99.34915 \\
\hline 600000 & 4009.5 & $60 \%$ & 99.16469 & 99.21382 & 99.2575 & 99.33175 \\
\hline 650000 & 4544.992 & $65 \%$ & 99.12596 & 99.17738 & 99.22308 & 99.30077 \\
\hline 700000 & 5099.695 & $70 \%$ & 99.08934 & 99.14291 & 99.19052 & 99.27147 \\
\hline 750000 & 5840.88 & $75 \%$ & 99.02652 & 99.08378 & 99.13468 & 99.22122 \\
\hline 800000 & 6517.557 & $80 \%$ & 98.98163 & 99.04154 & 99.09478 & 99.1853. \\
\hline 850000 & 7311.227 & $85 \%$ & 98.92482 & 98.98807 & 99.04428 & 99.13986 \\
\hline 900000 & 8150.349 & $90 \%$ & 98.86801 & 98.93459 & 98.99378 & 99.09441 \\
\hline 950000 & 9009.818 & $95 \%$ & 98.8145 & 98.88423 & 98.94622 & 99.0516 \\
\hline 1000000 & 9850.02 & $100 \%$ & 98.76875 & 98.84117 & 98.90555 & 99.015 \\
\hline
\end{tabular}

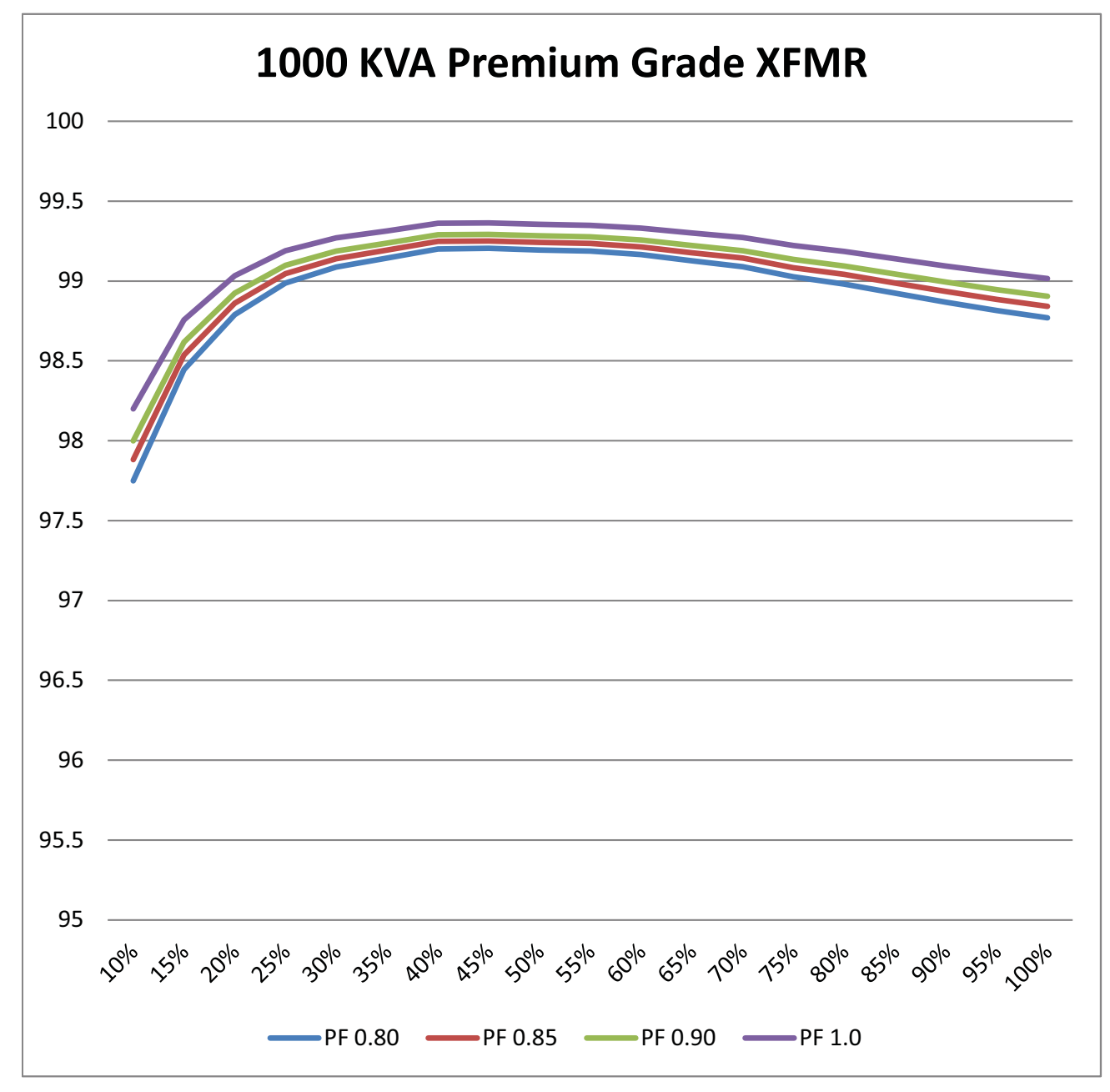




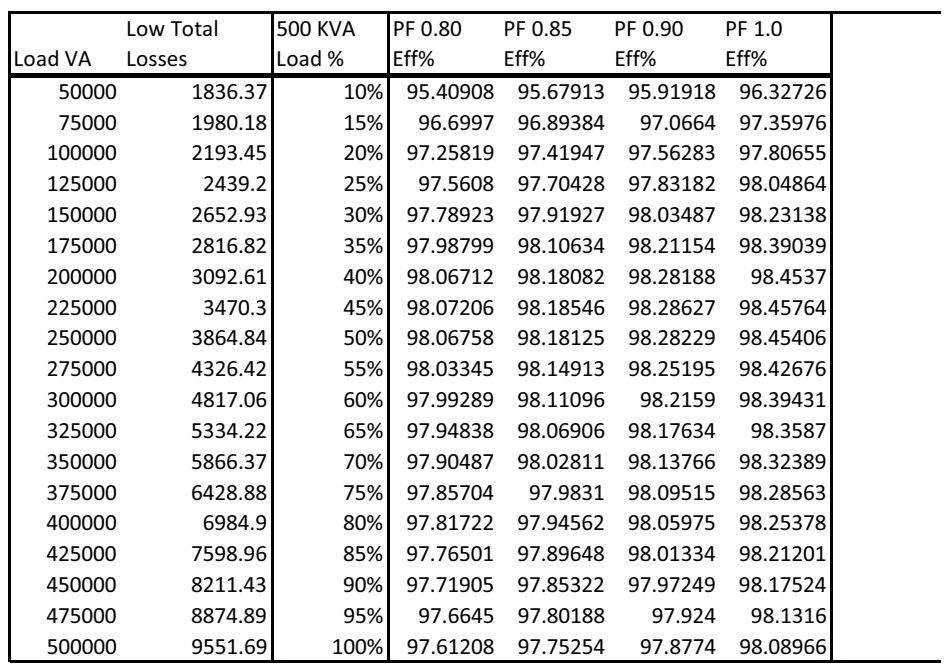

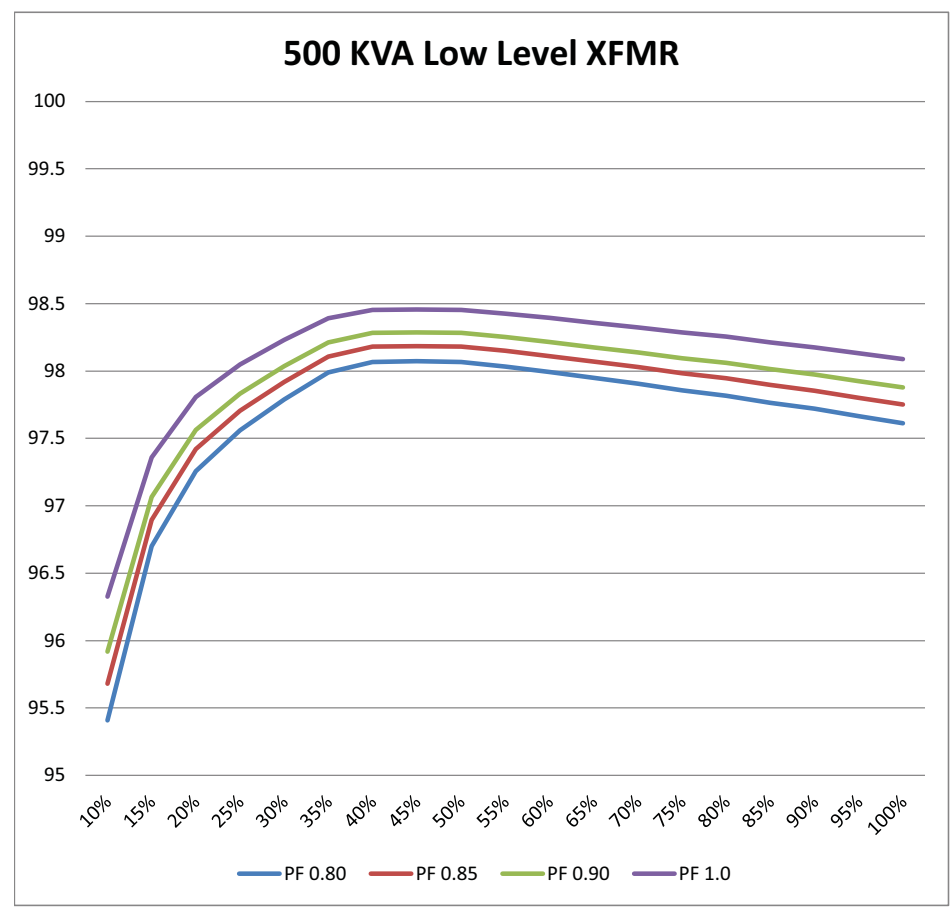




\begin{tabular}{|c|c|c|c|c|c|c|}
\hline Load VA & $\begin{array}{l}\text { Medium-Low Total } \\
\text { Losses }\end{array}$ & $\begin{array}{l}500 \text { KVA } \\
\text { Load \% }\end{array}$ & \begin{tabular}{|l|} 
PF 0.80 \\
Eff\%
\end{tabular} & $\begin{array}{l}\text { PF } 0.85 \\
\text { Eff\% }\end{array}$ & $\begin{array}{l}\text { PF } 0.90 \\
\text { Eff\% }\end{array}$ & $\begin{array}{l}\text { PF 1.0 } \\
\text { Eff\% }\end{array}$ \\
\hline 50000 & 1586.805 & $10 \%$ & 96.03299 & 96.26634 & 96.47377 & 96.82639 \\
\hline 75000 & 1717.03 & $15 \%$ & 97.13828 & 97.30662 & 97.45625 & 97.71063 \\
\hline 100000 & 1867.68 & $20 \%$ & 97.6654 & 97.80273 & 97.9248 & 98.13232 \\
\hline 125000 & 2004.59 & $25 \%$ & 97.99541 & 98.11333 & 98.21814 & 98.39633 \\
\hline 150000 & 2197.75 & $30 \%$ & 98.16854 & 98.27627 & 98.37204 & 98.53483 \\
\hline 175000 & 2366.35 & $35 \%$ & 98.30975 & 98.40918 & 98.49756 & 98.6478 \\
\hline 200000 & 2595.79 & $40 \%$ & 98.37763 & 98.47306 & 98.55789 & 98.70211 \\
\hline 225000 & 2910.33 & $45 \%$ & 98.38315 & 98.47826 & 98.5628 & 98.70652 \\
\hline 250000 & 3246.91 & $50 \%$ & 98.37655 & 98.47204 & 98.55693 & 98.70124 \\
\hline 275000 & 3620.94 & $55 \%$ & 98.35412 & 98.45093 & 98.53699 & 98.68329 \\
\hline 300000 & 4045.74 & $60 \%$ & 98.31428 & 98.41344 & 98.50158 & 98.65142 \\
\hline 325000 & 4456.23 & $65 \%$ & 98.28607 & 98.38689 & 98.4765 & 98.62885 \\
\hline 350000 & 4912.6 & $70 \%$ & 98.2455 & 98.34871 & 98.44044 & 98.5964 \\
\hline 375000 & 5375.99 & $75 \%$ & 98.208 & 98.31341 & 98.40711 & 98.5664 \\
\hline 400000 & 5884.94 & $80 \%$ & 98.16096 & 98.26914 & 98.36529 & 98.52877 \\
\hline 425000 & 6459.03 & $85 \%$ & 98.10029 & 98.21203 & 98.31136 & 98.48023 \\
\hline 450000 & 7050.73 & $90 \%$ & 98.04146 & 98.15667 & 98.25908 & 98.43317 \\
\hline 475000 & 7688.17 & $95 \%$ & 97.9768 & 98.09581 & 98.2016 & 98.38144 \\
\hline 500000 & 8335.38 & $100 \%$ & 97.91616 & 98.03873 & 98.14769 & 98.33292 \\
\hline
\end{tabular}

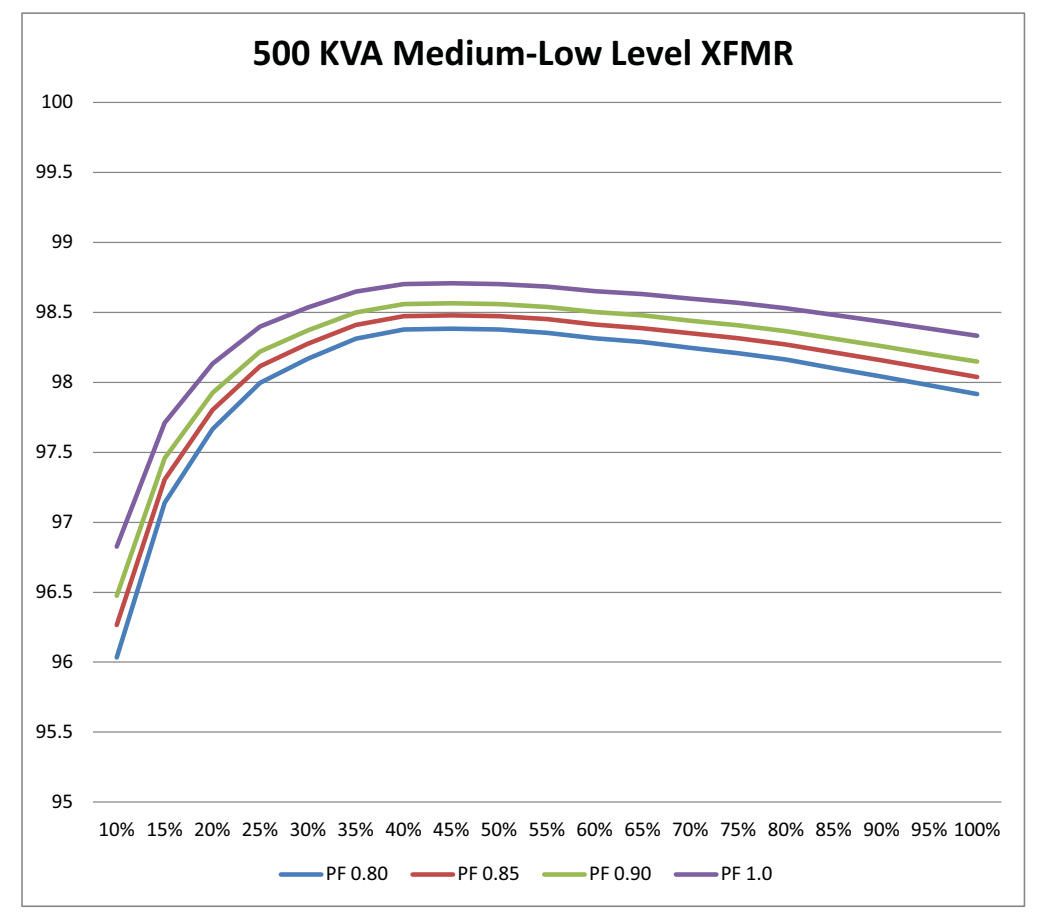




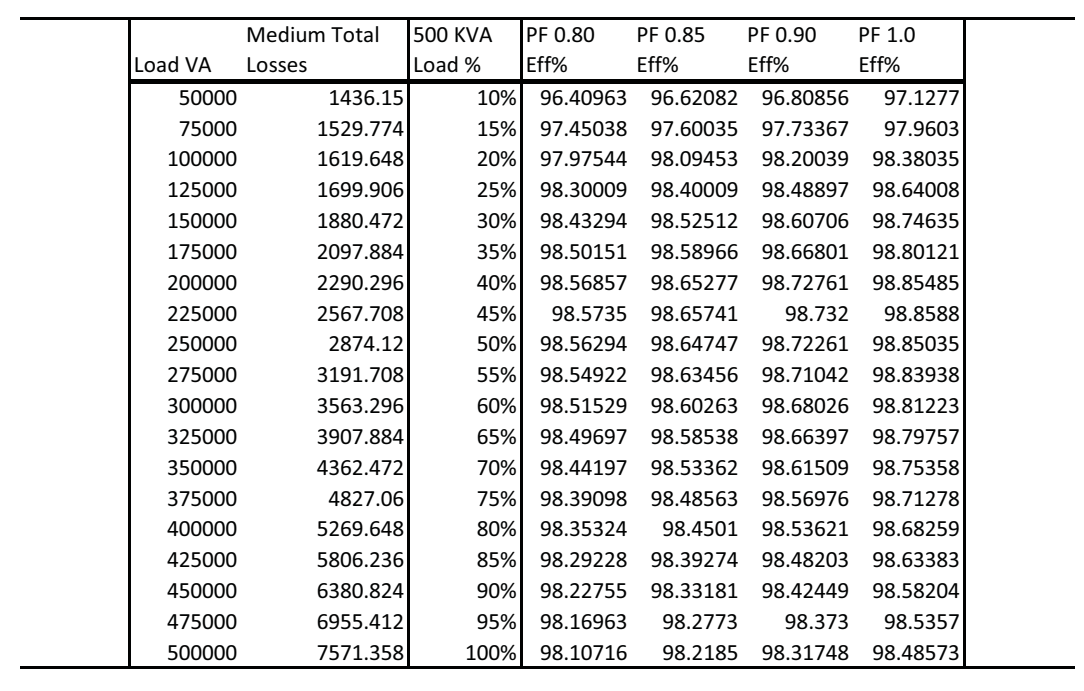

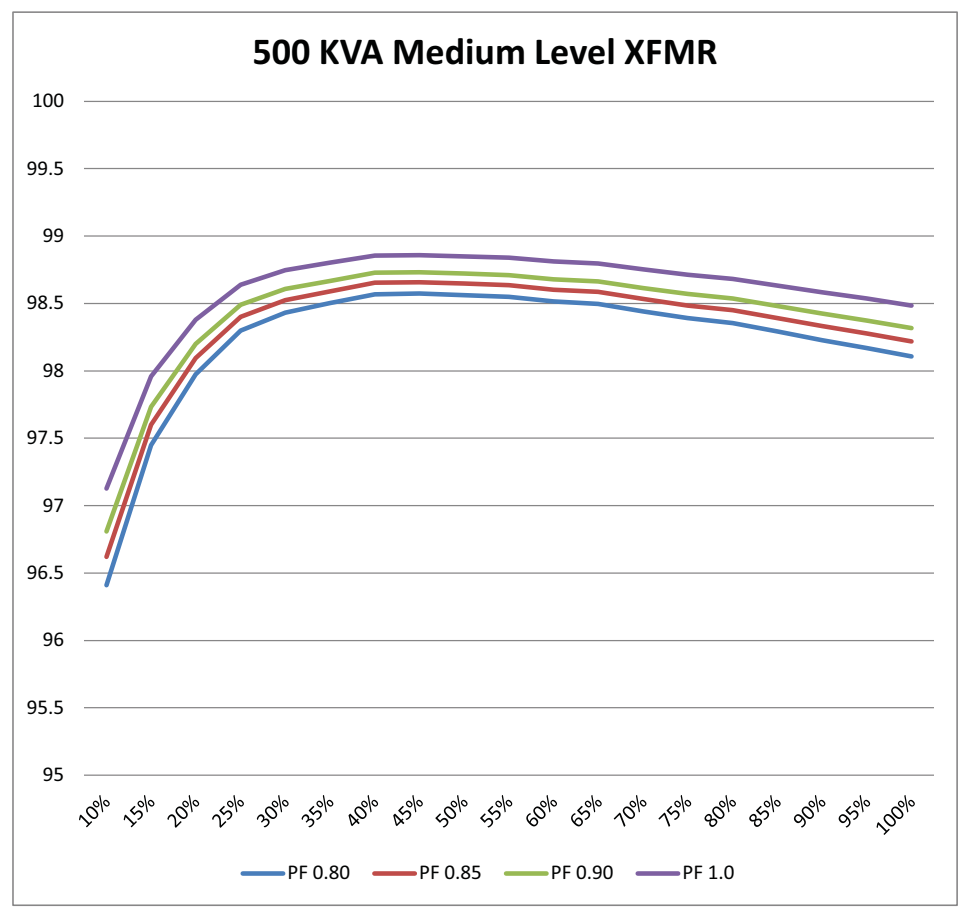




\begin{tabular}{|c|c|c|c|c|c|c|}
\hline Load VA & $\begin{array}{l}\text { Medium-High Total } \\
\text { Losses }\end{array}$ & $\begin{array}{l}500 \text { KVA } \\
\text { Load \% }\end{array}$ & $\begin{array}{l}\text { PF } 0.80 \\
\text { Eff\% }\end{array}$ & $\begin{array}{l}\text { PF } 0.85 \\
\text { Eff\% }\end{array}$ & $\begin{array}{l}\text { PF } 0.90 \\
\text { Eff\% }\end{array}$ & $\begin{array}{l}\text { PF } 1.0 \\
\text { Eff\% }\end{array}$ \\
\hline 50000 & 1490.824 & $10 \%$ & 96.27294 & 96.49218 & 96.68706 & 97.01835 \\
\hline 75000 & 1520.236 & $15 \%$ & 97.46627 & 97.61532 & 97.7478 & 97.97302 \\
\hline 100000 & 1621.648 & $20 \%$ & 97.97294 & 98.09218 & 98.19817 & 98.37835 \\
\hline 125000 & 1677.06 & $25 \%$ & 98.32294 & 98.42159 & 98.50928 & 98.65835 \\
\hline 150000 & 1750.472 & $30 \%$ & 98.54127 & 98.62708 & 98.70335 & 98.83302 \\
\hline 175000 & 1897.884 & $35 \%$ & 98.64437 & 98.72411 & 98.79499 & 98.91549 \\
\hline 200000 & 2110.296 & $40 \%$ & 98.68107 & 98.75865 & 98.82761 & 98.94485 \\
\hline 225000 & 2307.708 & $45 \%$ & 98.71794 & 98.79336 & 98.86039 & 98.97435 \\
\hline 250000 & 2554.12 & $50 \%$ & 98.72294 & 98.79806 & 98.86484 & 98.97835 \\
\hline 275000 & 2798.708 & $55 \%$ & 98.72786 & 98.80269 & 98.86921 & 98.98229 \\
\hline 300000 & 3123.296 & $60 \%$ & 98.69863 & 98.77518 & 98.84322 & 98.9589 \\
\hline 325000 & 3457.884 & $65 \%$ & 98.67004 & 98.74828 & 98.81782 & 98.93604 \\
\hline 350000 & 3892.472 & $70 \%$ & 98.60983 & 98.69161 & 98.76429 & 98.88787 \\
\hline 375000 & 4347.06 & $75 \%$ & 98.55098 & 98.63622 & 98.71198 & 98.84078 \\
\hline 400000 & 4921.648 & $80 \%$ & 98.46199 & 98.55246 & 98.63288 & 98.76959 \\
\hline 425000 & 5496.236 & $85 \%$ & 98.38346 & 98.47855 & 98.56308 & 98.70677 \\
\hline 450000 & 6130.824 & $90 \%$ & 98.29699 & 98.39717 & 98.48622 & 98.63759 \\
\hline 475000 & 6765.412 & $95 \%$ & 98.21963 & 98.32436 & 98.41745 & 98.5757 \\
\hline 500000 & 7401.358 & $100 \%$ & 98.14966 & 98.2585 & 98.35525 & 98.51973 \\
\hline
\end{tabular}

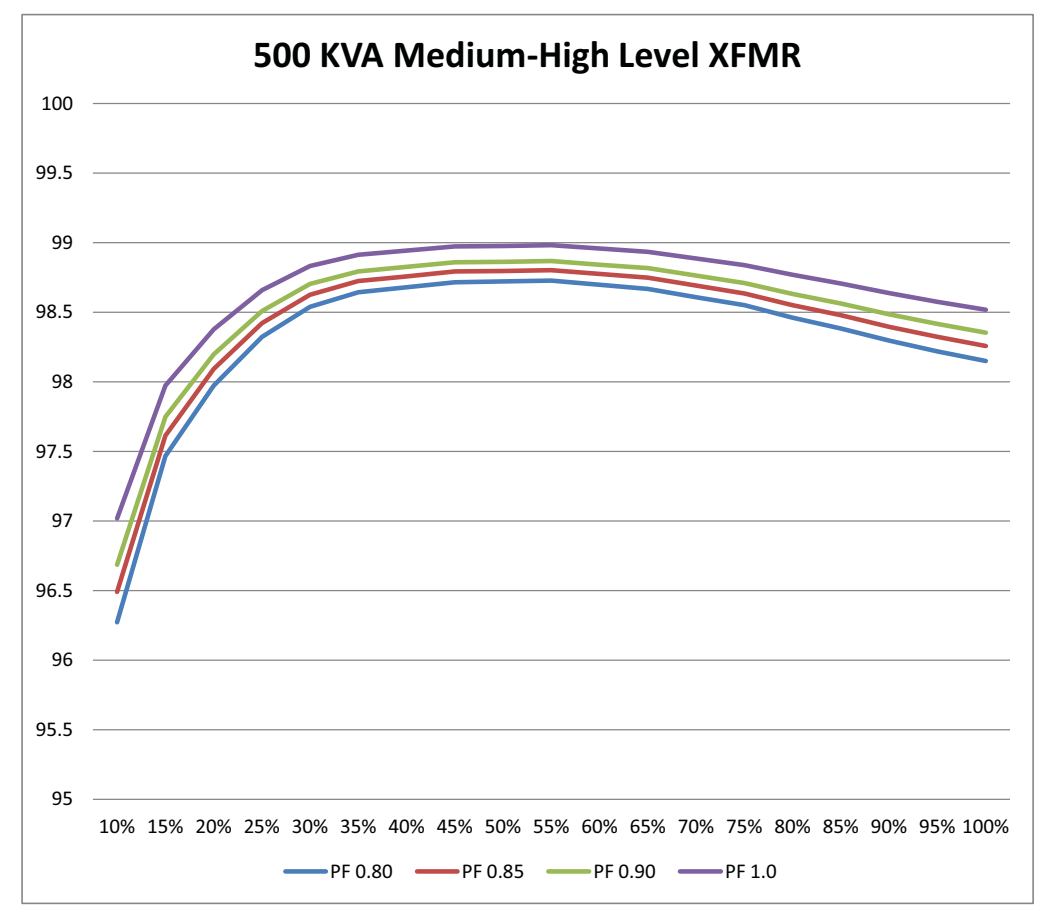




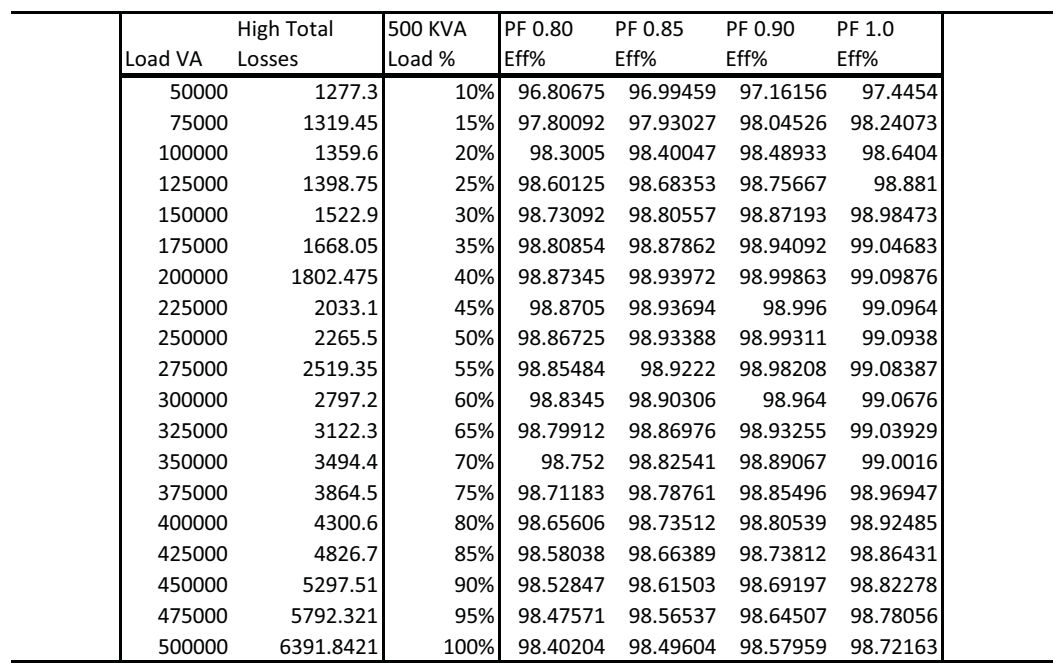

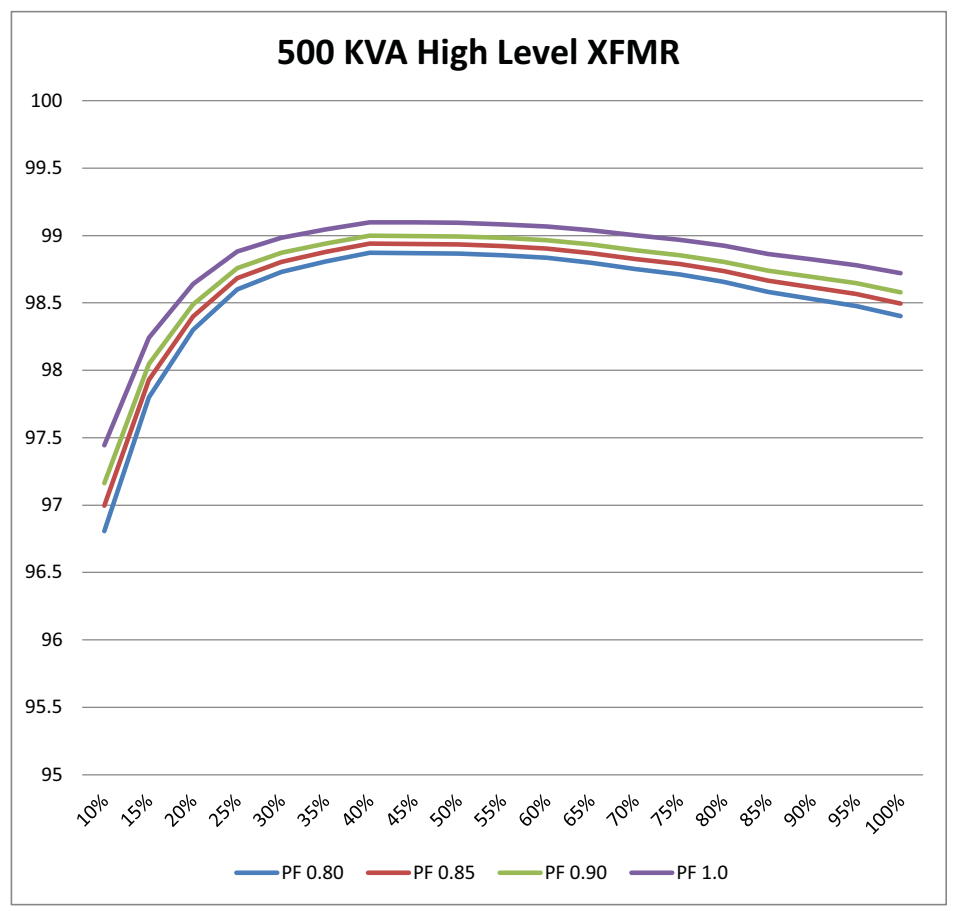




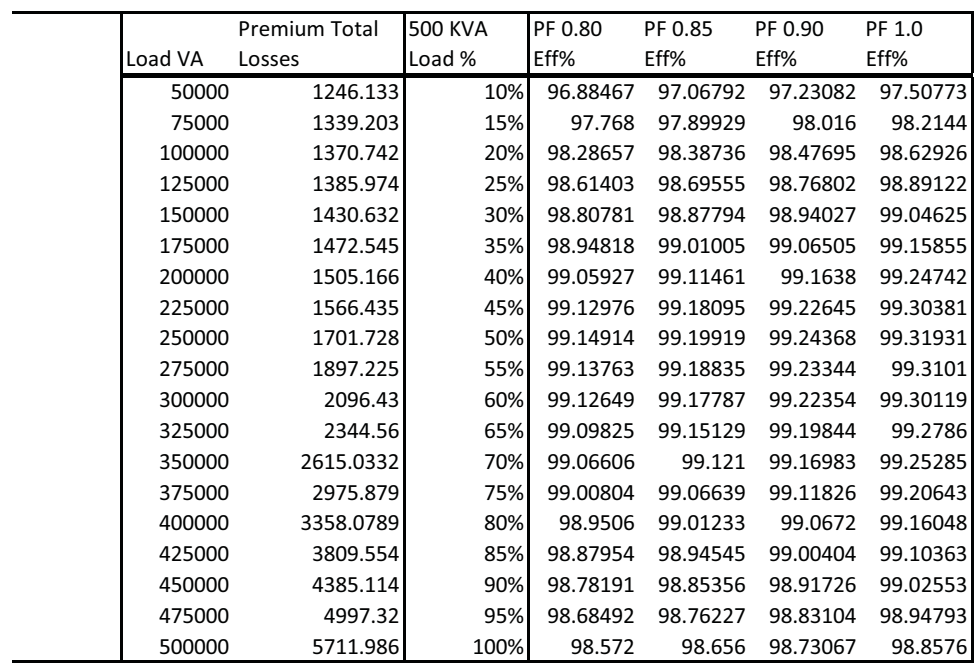

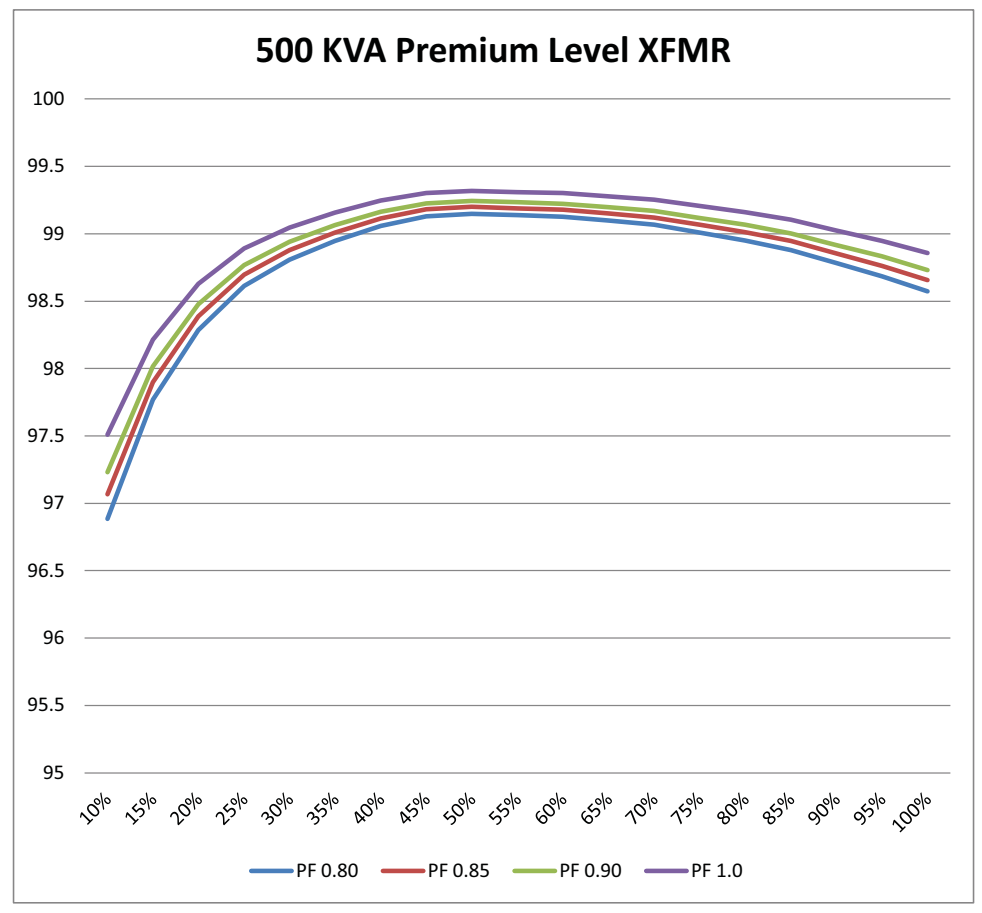




\begin{tabular}{|c|c|c|c|c|c|c|}
\hline Load VA & $\begin{array}{l}\text { Low Total } \\
\text { Losses }\end{array}$ & $\begin{array}{l}750 \text { KVA } \\
\text { Load \% }\end{array}$ & $\begin{array}{l}\text { PF } 0.80 \\
\text { Eff\% }\end{array}$ & $\begin{array}{l}\text { PF } 0.85 \\
\text { Eff\% }\end{array}$ & $\begin{array}{l}\text { PF } 0.90 \\
\text { Eff\% }\end{array}$ & $\begin{array}{l}\text { PF } 1.0 \\
\text { Eff\% }\end{array}$ \\
\hline 75000 & 2505.948 & $10 \%$ & 95.82342 & 96.0691 & 96.28748 & 96.65874 \\
\hline 112500 & 2707.77 & $15 \%$ & 96.99137 & 97.16835 & 97.32566 & 97.59309 \\
\hline 150000 & 3009.62 & $20 \%$ & 97.49198 & 97.63951 & 97.77065 & 97.99359 \\
\hline 187500 & 3360.18 & $25 \%$ & 97.75988 & 97.89165 & 98.00878 & 98.2079 \\
\hline 225000 & 3654.41 & $30 \%$ & 97.96977 & 98.0892 & 98.19535 & 98.37582 \\
\hline 262500 & 3875.24 & $35 \%$ & 98.15465 & 98.2632 & 98.35969 & 98.52372 \\
\hline 300000 & 4255.56 & $40 \%$ & 98.22685 & 98.33115 & 98.42387 & 98.58148 \\
\hline 337500 & 4786.71 & $45 \%$ & 98.22714 & 98.33143 & 98.42413 & 98.58172 \\
\hline 375000 & 5338.96 & $50 \%$ & 98.22035 & 98.32503 & 98.41809 & 98.57628 \\
\hline 412500 & 5985.46 & $55 \%$ & 98.18622 & 98.29292 & 98.38775 & 98.54898 \\
\hline 450000 & 6667.27 & $60 \%$ & 98.14798 & 98.25692 & 98.35376 & 98.51838 \\
\hline 487500 & 7396.43 & $65 \%$ & 98.10348 & 98.21504 & 98.3142 & 98.48278 \\
\hline 525000 & 8138.47 & $70 \%$ & 98.06227 & 98.17625 & 98.27757 & 98.44982 \\
\hline 562500 & 8914.15 & $75 \%$ & 98.01908 & 98.1356 & 98.23918 & 98.41526 \\
\hline 600000 & 9699.56 & $80 \%$ & 97.97926 & 98.09813 & 98.20379 & 98.38341 \\
\hline 637500 & 10536.67 & $85 \%$ & 97.93399 & 98.05552 & 98.16354 & 98.34719 \\
\hline 675000 & 11392.08 & $90 \%$ & 97.89036 & 98.01445 & 98.12476 & 98.31228 \\
\hline 712500 & 12309.53 & $95 \%$ & 97.84043 & 97.96747 & 98.08039 & 98.27235 \\
\hline 750000 & 13244.19 & $100 \%$ & 97.79264 & 97.92248 & 98.0379 & 98.23411 \\
\hline
\end{tabular}

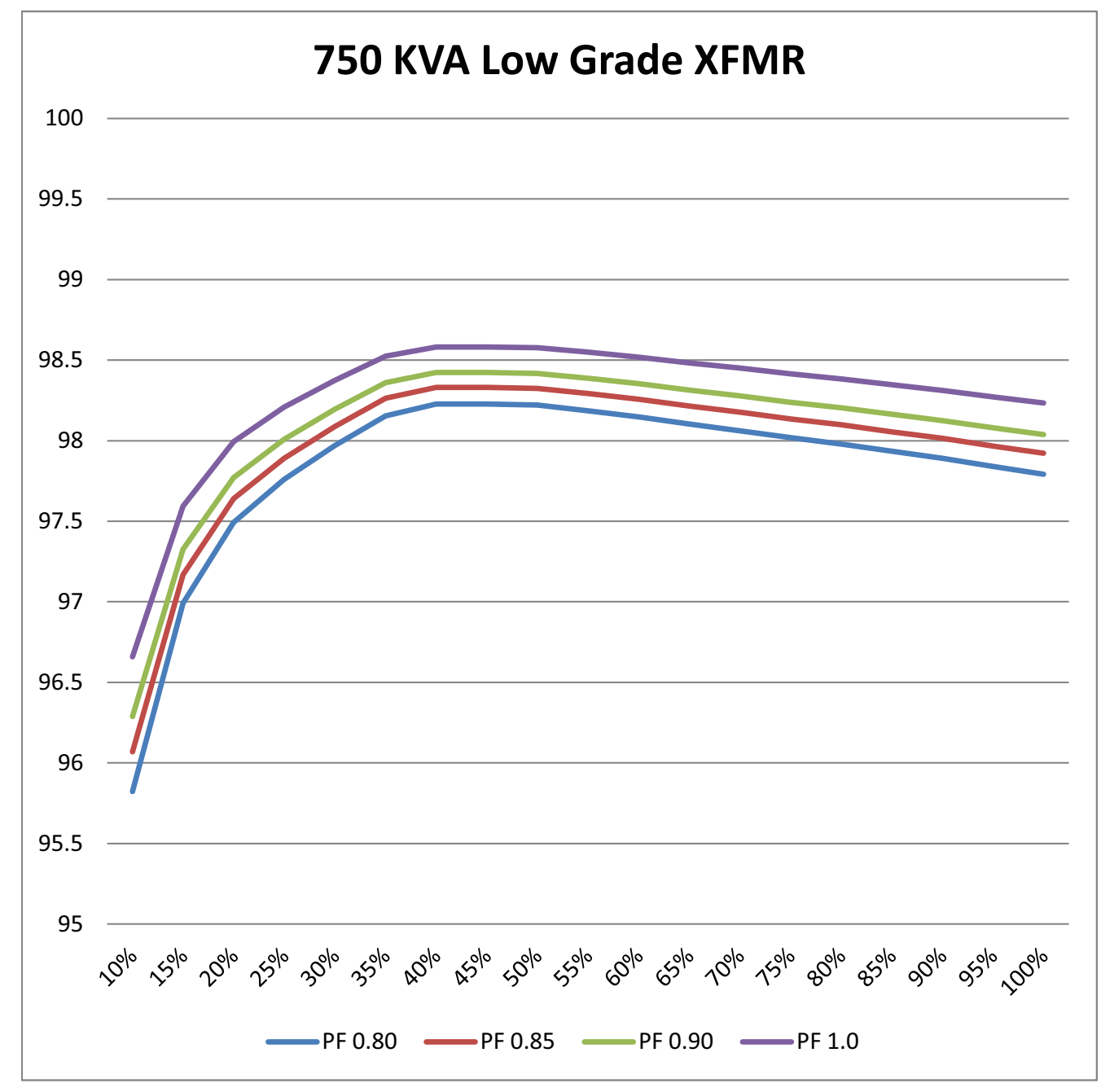




\begin{tabular}{|c|c|c|c|c|c|c|}
\hline Load VA & $\begin{array}{l}\text { Medium-Low Total } \\
\text { Losses }\end{array}$ & $\begin{array}{l}750 \text { KVA } \\
\text { Load \% }\end{array}$ & $\begin{array}{l}\text { PF } 0.80 \\
\text { Eff\% }\end{array}$ & $\begin{array}{l}\text { PF } 0.85 \\
\text { Eff\% }\end{array}$ & $\begin{array}{l}\text { PF } 0.90 \\
\text { Eff\% }\end{array}$ & $\begin{array}{l}\text { PF 1.0 } \\
\text { Eff\% }\end{array}$ \\
\hline 75000 & 2131.6 & $10 \%$ & 96.44733 & 96.65631 & 96.84207 & 97.15787 \\
\hline 112500 & 2313.03 & $15 \%$ & 97.42997 & 97.58115 & 97.71553 & 97.94397 \\
\hline 150000 & 2520.97 & $20 \%$ & 97.89919 & 98.02277 & 98.13261 & 98.31935 \\
\hline 187500 & 2708.27 & $25 \%$ & 98.19449 & 98.30069 & 98.3951 & 98.55559 \\
\hline 225000 & 2971.64 & $30 \%$ & 98.34909 & 98.4462 & 98.53252 & 98.67927 \\
\hline 262500 & 3199.52 & $35 \%$ & 98.47642 & 98.56604 & 98.64571 & 98.78114 \\
\hline 300000 & 3510.34 & $40 \%$ & 98.53736 & 98.6234 & 98.69987 & 98.82989 \\
\hline 337500 & 3946.75 & $45 \%$ & 98.53824 & 98.62423 & 98.70066 & 98.83059 \\
\hline 375000 & 4412 & $50 \%$ & 98.52933 & 98.61584 & 98.69274 & 98.82347 \\
\hline 412500 & 4927.27 & $55 \%$ & 98.50689 & 98.59472 & 98.67279 & 98.80551 \\
\hline 450000 & 5510.27 & $60 \%$ & 98.46937 & 98.55941 & 98.63944 & 98.7755 \\
\hline 487500 & 6079.5 & $65 \%$ & 98.44115 & 98.53285 & 98.61436 & 98.75292 \\
\hline 525000 & 6707.81 & $70 \%$ & 98.4029 & 98.49685 & 98.58036 & 98.72232 \\
\hline 562500 & 7334.84 & $75 \%$ & 98.37004 & 98.46592 & 98.55114 & 98.69603 \\
\hline 600000 & 8049.59 & $80 \%$ & 98.323 & 98.42165 & 98.50934 & 98.6584 \\
\hline 637500 & 8826.78 & $85 \%$ & 98.26926 & 98.37107 & 98.46156 & 98.61541 \\
\hline 675000 & 9651.18 & $90 \%$ & 98.21274 & 98.31788 & 98.41133 & 98.5702 \\
\hline 712500 & 10529.46 & $95 \%$ & 98.15273 & 98.26139 & 98.35798 & 98.52218 \\
\hline 750000 & 11419.69 & $100 \%$ & 98.09672 & 98.20868 & 98.30819 & 98.47737 \\
\hline
\end{tabular}

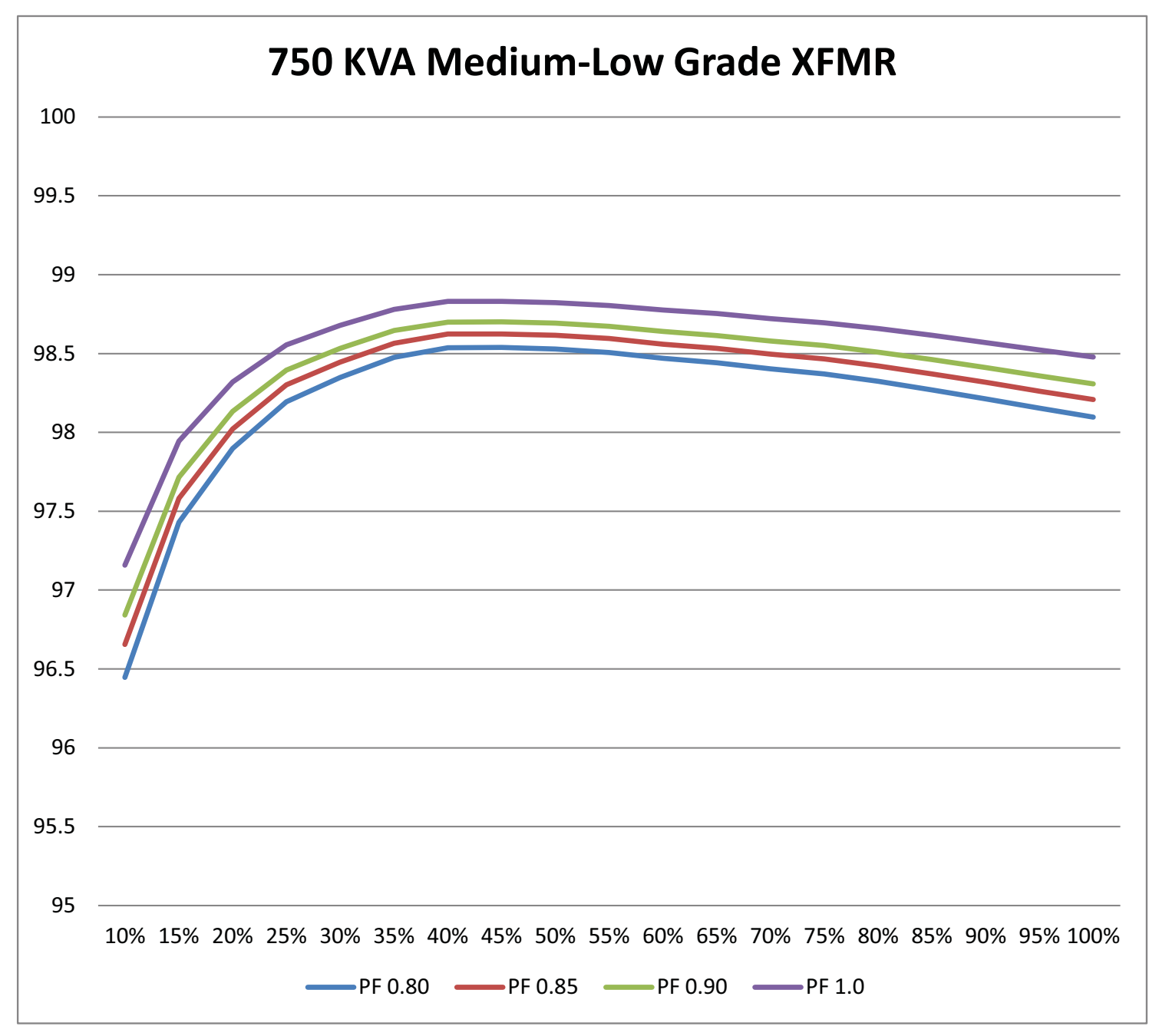




\begin{tabular}{|c|c|c|c|c|c|c|}
\hline Load VA & $\begin{array}{l}\text { Medium Total } \\
\text { Losses }\end{array}$ & $\begin{array}{l}750 \text { KVA } \\
\text { Load \% }\end{array}$ & $\begin{array}{l}\text { PF } 0.80 \\
\text { Eff\% }\end{array}$ & $\begin{array}{l}\text { PF } 0.85 \\
\text { Eff\% }\end{array}$ & $\begin{array}{l}\text { PF } 0.90 \\
\text { Eff\% }\end{array}$ & $\begin{array}{l}\text { PF } 1.0 \\
\text { Eff\% }\end{array}$ \\
\hline 75000 & 1909.99 & $10 \%$ & 96.81668 & 97.00394 & 97.17039 & 97.45335 \\
\hline 112500 & 2028.85 & $15 \%$ & 97.74572 & 97.87833 & 97.9962 & 98.19658 \\
\hline 150000 & 2149.67 & $20 \%$ & 98.20861 & 98.31398 & 98.40765 & 98.56689 \\
\hline 187500 & 2239.99 & $25 \%$ & 98.50667 & 98.59452 & 98.6726 & 98.80534 \\
\hline 225000 & 2475.31 & $30 \%$ & 98.62483 & 98.70572 & 98.77762 & 98.89986 \\
\hline 262500 & 2781.13 & $35 \%$ & 98.67565 & 98.75356 & 98.8228 & 98.94052 \\
\hline 300000 & 3081.95 & $40 \%$ & 98.71585 & 98.79139 & 98.85854 & 98.97268 \\
\hline 337500 & 3408.77 & $45 \%$ & 98.73749 & 98.81176 & 98.87777 & 98.98999 \\
\hline 375000 & 3838.59 & $50 \%$ & 98.72047 & 98.79574 & 98.86264 & 98.97638 \\
\hline 412500 & 4292.41 & $55 \%$ & 98.69927 & 98.77578 & 98.8438 & 98.95942 \\
\hline 450000 & 4744.23 & $60 \%$ & 98.68216 & 98.75968 & 98.82859 & 98.94573 \\
\hline 487500 & 5302.05 & $65 \%$ & 98.6405 & 98.72047 & 98.79156 & 98.9124 \\
\hline 525000 & 5851.07 & $70 \%$ & 98.60689 & 98.68884 & 98.76168 & 98.88551 \\
\hline 562500 & 6506.09 & $75 \%$ & 98.5542 & 98.63925 & 98.71485 & 98.84336 \\
\hline 600000 & 7107.76 & $80 \%$ & 98.51922 & 98.60632 & 98.68375 & 98.81537 \\
\hline 637500 & 7829.43 & $85 \%$ & 98.46482 & 98.55512 & 98.63539 & 98.77185 \\
\hline 675000 & 8591.1 & $90 \%$ & 98.40906 & 98.50264 & 98.58583 & 98.72724 \\
\hline 712500 & 9406.77 & $95 \%$ & 98.34969 & 98.44677 & 98.53306 & 98.67975 \\
\hline 750000 & 10342.44 & $100 \%$ & 98.27626 & 98.37766 & 98.46779 & 98.62101 \\
\hline
\end{tabular}

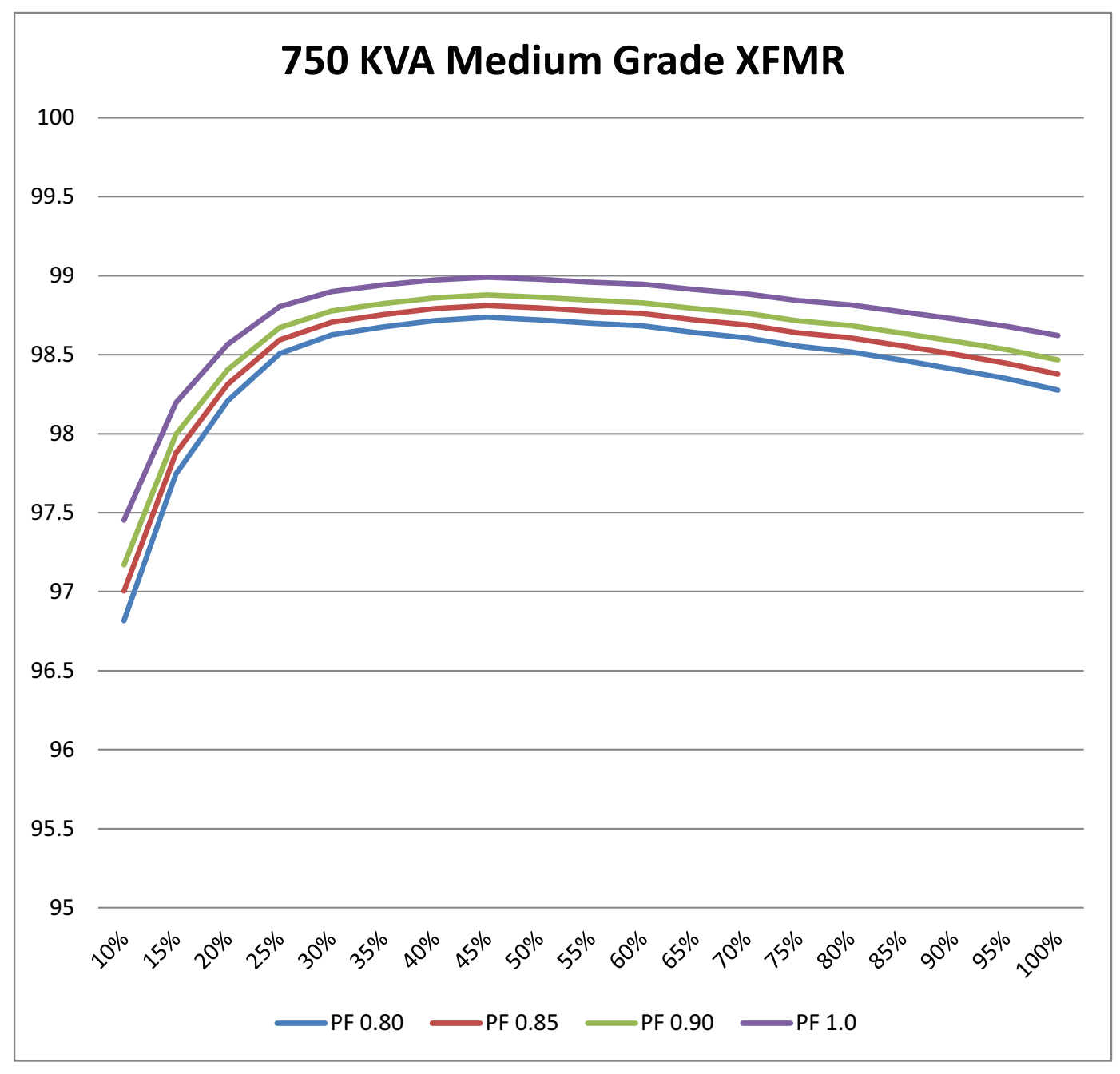




\begin{tabular}{|c|c|c|c|c|c|c|}
\hline Load VA & $\begin{array}{l}\text { Medium-High Total } \\
\text { Losses }\end{array}$ & $\begin{array}{l}750 \text { KVA } \\
\text { Load \% }\end{array}$ & $\begin{array}{l}\text { PF } 0.80 \\
\text { Eff\% }\end{array}$ & $\begin{array}{l}\text { PF } 0.85 \\
\text { Eff\% }\end{array}$ & $\begin{array}{l}\text { PF } 0.90 \\
\text { Eff\% }\end{array}$ & $\begin{array}{l}\text { PF } 1.0 \\
\text { Eff\% }\end{array}$ \\
\hline 75000 & 1995.934 & $10 \%$ & 96.67344 & 96.86912 & 97.04306 & 97.33875 \\
\hline 112500 & 2004.271 & $15 \%$ & 97.77303 & 97.90403 & 98.02047 & 98.21843 \\
\hline 150000 & 2115.608 & $20 \%$ & 98.23699 & 98.3407 & 98.43288 & 98.58959 \\
\hline 187500 & 2238.945 & $25 \%$ & 98.50737 & 98.59517 & 98.67322 & 98.8059 \\
\hline 225000 & 2314.282 & $30 \%$ & 98.71429 & 98.78992 & 98.85714 & 98.97143 \\
\hline 262500 & 2429.619 & $35 \%$ & 98.84304 & 98.9111 & 98.97159 & 99.07443 \\
\hline 300000 & 2556.956 & $40 \%$ & 98.9346 & 98.99727 & 99.05298 & 99.14768 \\
\hline 337500 & 2883.293 & $45 \%$ & 98.93211 & 98.99493 & 99.05077 & 99.14569 \\
\hline 375000 & 3213.63 & $50 \%$ & 98.92879 & 98.9918 & 99.04781 & 99.14303 \\
\hline 412500 & 3600.967 & $55 \%$ & 98.9088 & 98.97299 & 99.03004 & 99.12704 \\
\hline 450000 & 4022.304 & $60 \%$ & 98.88269 & 98.94842 & 99.00684 & 99.10615 \\
\hline 487500 & 4540.941 & $65 \%$ & 98.83566 & 98.90415 & 98.96503 & 99.06852 \\
\hline 525000 & 5050.578 & $70 \%$ & 98.79748 & 98.86822 & 98.93109 & 99.03799 \\
\hline 562500 & 5653.215 & $75 \%$ & 98.74373 & 98.81763 & 98.88332 & 98.99498 \\
\hline 600000 & 6190.852 & $80 \%$ & 98.71024 & 98.78611 & 98.85355 & 98.96819 \\
\hline 637500 & 6849.489 & $85 \%$ & 98.65696 & 98.73597 & 98.80619 & 98.92557 \\
\hline 675000 & 7662.126 & $90 \%$ & 98.58109 & 98.66455 & 98.73874 & 98.86487 \\
\hline 712500 & 8546.763 & $95 \%$ & 98.50057 & 98.58877 & 98.66717 & 98.80045 \\
\hline 750000 & 9506 & $100 \%$ & 98.41567 & 98.50886 & 98.5917 & 98.73253 \\
\hline
\end{tabular}

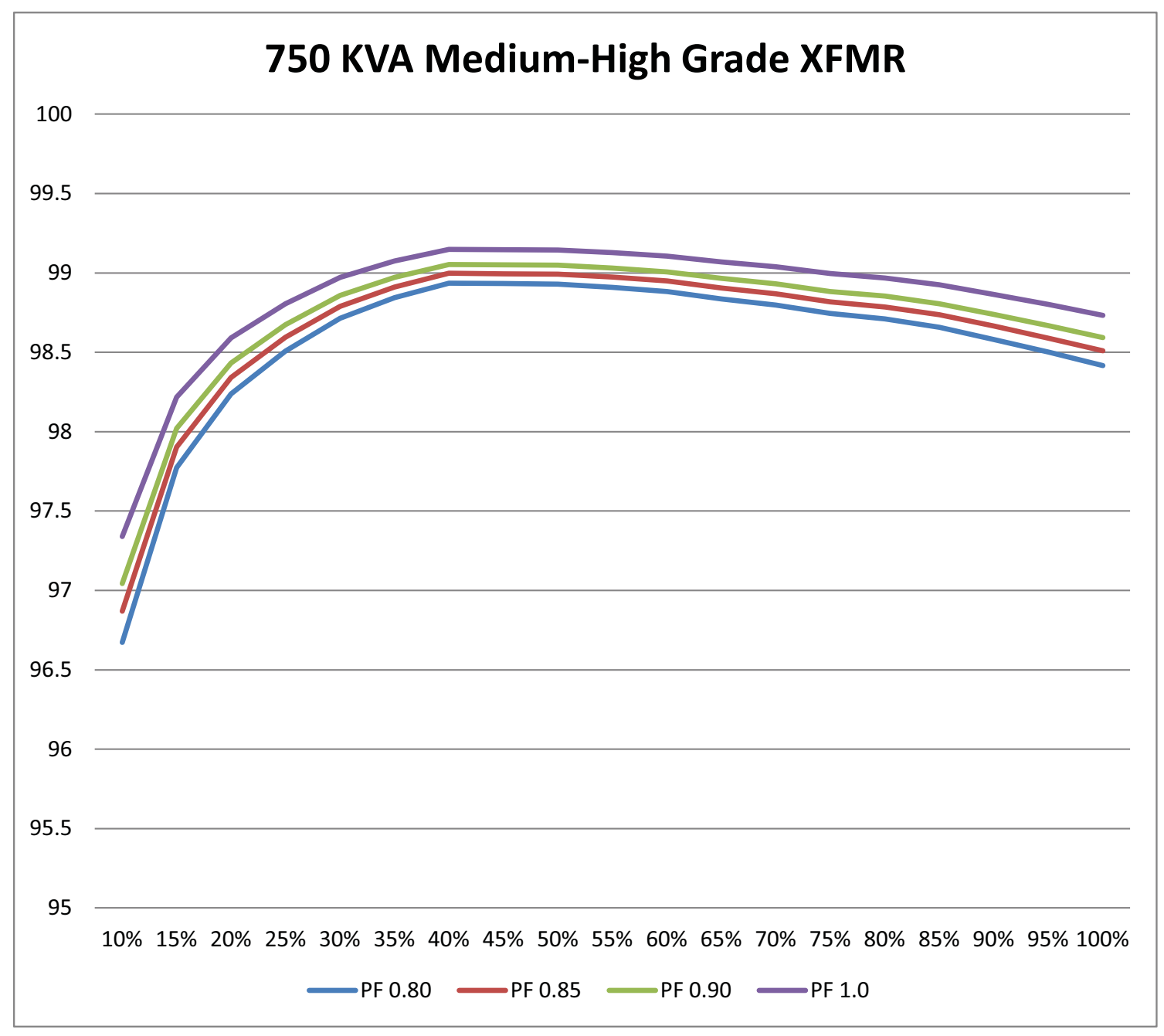




\begin{tabular}{|c|c|c|c|c|c|c|}
\hline Load VA & $\begin{array}{l}\text { High Total } \\
\text { Losses }\end{array}$ & $\begin{array}{l}750 \text { KVA } \\
\text { Load \% }\end{array}$ & $\begin{array}{l}\text { PF } 0.80 \\
\text { Eff\% }\end{array}$ & $\begin{array}{l}\text { PF } 0.85 \\
\text { Eff\% }\end{array}$ & $\begin{array}{l}\text { PF } 0.90 \\
\text { Eff\% }\end{array}$ & $\begin{array}{l}\text { PF } 1.0 \\
\text { Eff\% }\end{array}$ \\
\hline 75000 & 1709.981 & $10 \%$ & 97.15003 & 97.31768 & 97.46669 & 97.72003 \\
\hline 112500 & 1776.274 & $15 \%$ & 98.02636 & 98.14246 & 98.24566 & 98.42109 \\
\hline 150000 & 1838.567 & $20 \%$ & 98.46786 & 98.55799 & 98.6381 & 98.77429 \\
\hline 187500 & 1910.86 & $25 \%$ & 98.72609 & 98.80103 & 98.86764 & 98.98087 \\
\hline 225000 & 2090.153 & $30 \%$ & 98.8388 & 98.90711 & 98.96783 & 99.07104 \\
\hline 262500 & 2305.446 & $35 \%$ & 98.90217 & 98.96675 & 99.02415 & 99.12173 \\
\hline 300000 & 2498.139 & $40 \%$ & 98.95911 & 99.02034 & 99.07476 & 99.16729 \\
\hline 337500 & 2820.232 & $45 \%$ & 98.95547 & 99.01691 & 99.07153 & 99.16438 \\
\hline 375000 & 3139.325 & $50 \%$ & 98.95356 & 99.01511 & 99.06983 & 99.16285 \\
\hline 412500 & 3505.65 & $55 \%$ & 98.93768 & 99.00017 & 99.05572 & 99.15015 \\
\hline 450000 & 3895.975 & $60 \%$ & 98.91778 & 98.98144 & 99.03803 & 99.13423 \\
\hline 487500 & 4344.3 & $65 \%$ & 98.88608 & 98.9516 & 99.00985 & 99.10886 \\
\hline 525000 & 4856.625 & $70 \%$ & 98.84366 & 98.91168 & 98.97214 & 99.07493 \\
\hline 562500 & 5439.95 & $75 \%$ & 98.79112 & 98.86223 & 98.92544 & 99.0329 \\
\hline 600000 & 6010.275 & $80 \%$ & 98.74786 & 98.82151 & 98.88699 & 98.99829 \\
\hline 637500 & 6745.6 & $85 \%$ & 98.67733 & 98.75514 & 98.8243 & 98.94187 \\
\hline 675000 & 7370.925 & $90 \%$ & 98.63501 & 98.71531 & 98.78668 & 98.90801 \\
\hline 712500 & 8086.25 & $95 \%$ & 98.58136 & 98.66481 & 98.73899 & 98.86509 \\
\hline 750000 & 8875.78 & $100 \%$ & 98.5207 & 98.60772 & 98.68507 & 98.81656 \\
\hline
\end{tabular}

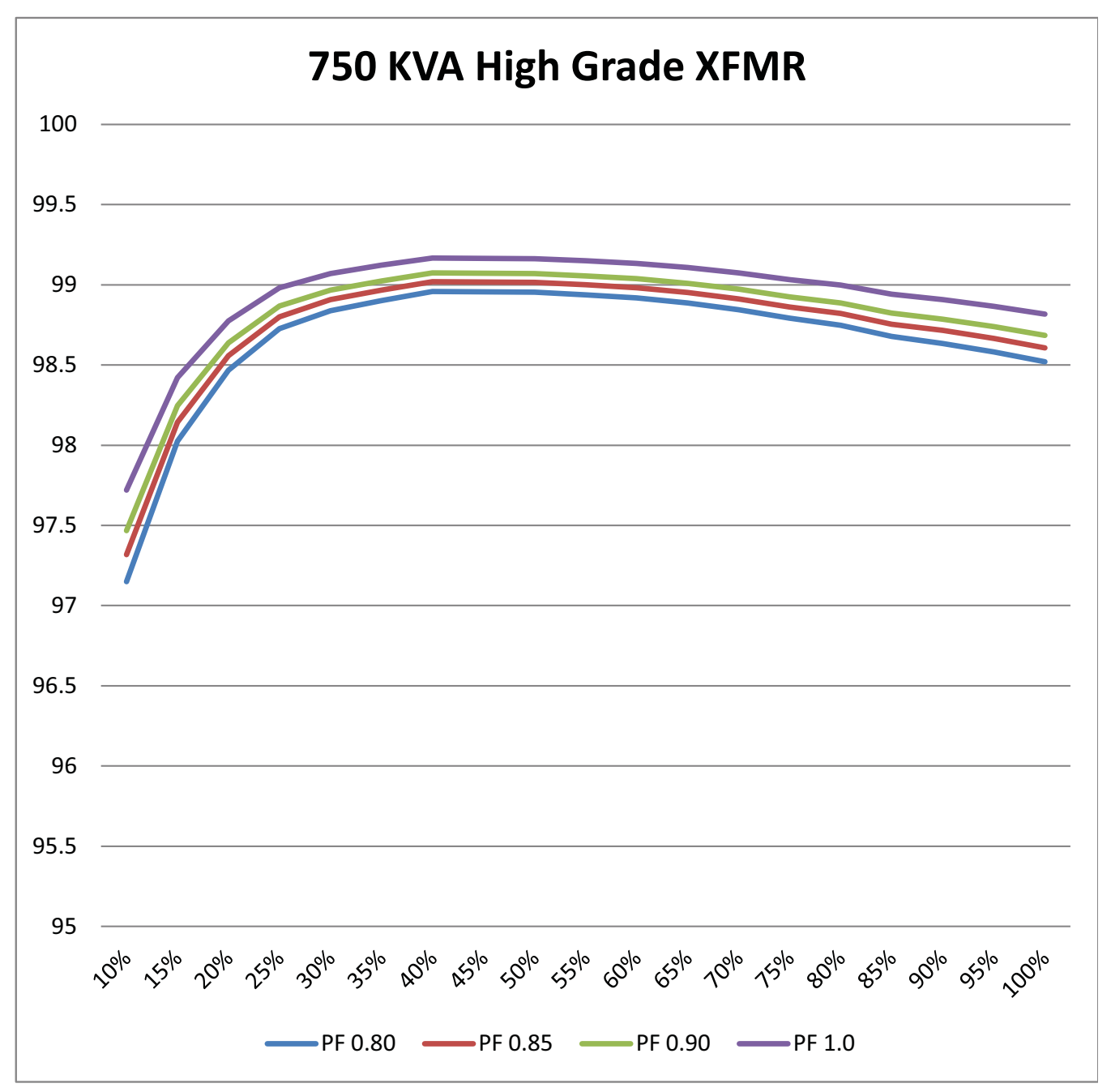




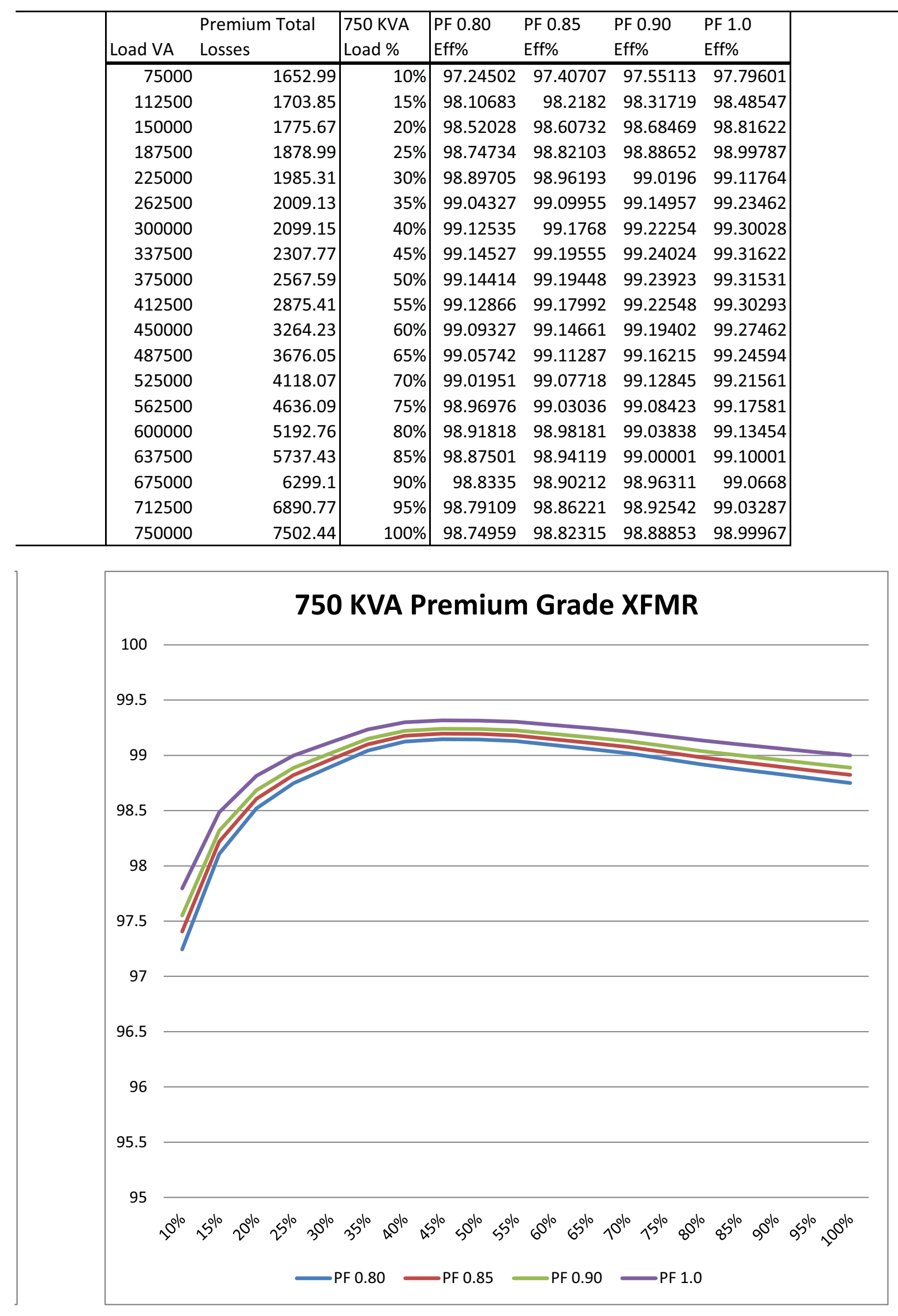




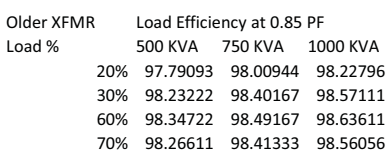

Load KVA
$\begin{array}{ccr}500 \text { KVA } & \text { 750 KVA } & 1000 \text { KVA } \\ 100 & 150 & 200 \\ 150 & 225 & 300 \\ 300 & 450 & 600 \\ 350 & 525 & 700\end{array}$

$\%$ Loss

$500 \mathrm{KVA} \quad 750 \mathrm{KVA} \quad 1000 \mathrm{KVA}$

$2.209074 \quad 1.990556 \quad 1.772037$

$\begin{array}{lll}1.767778 & 1.598333 & 1.428889\end{array}$

$\begin{array}{lll}1.652778 & 1.508333 & 1.363889\end{array}$

$\begin{array}{lll}1.733889 & 1.586667 & 1.439444\end{array}$

$500 \mathrm{KVA} \quad 750 \mathrm{KVA} \quad 1000 \mathrm{KVA}$

$\begin{array}{llll}20 \% & 1877.75 & 2537.99 & 3012.35 \\ & 4214.56 & 5769.42 & 6956.08\end{array}$

$\begin{array}{llll}60 \% & 4214.56 & 5769.42 & 6956.08\end{array}$

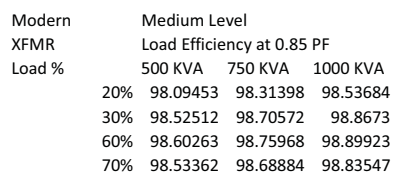
$98.34723 \quad 98.34722$

$98.00942 \quad 98.00944$ 98.4916598 .49167

$98.22803 \quad 98.22796$ 98.6360698 .63611

\begin{tabular}{|c|c|c|}
\hline Load KVA & & \\
\hline $500 \mathrm{KVA}$ & $750 \mathrm{KVA}$ & $1000 \mathrm{KVA}$ \\
\hline 100 & 150 & 200 \\
\hline 150 & 225 & 300 \\
\hline 300 & 450 & 600 \\
\hline 350 & 525 & 700 \\
\hline
\end{tabular}

$\%$ Loss

500 KVA 750 KVA 1000 KVA $\begin{array}{llll}2.580529 & 2.360486 & 2.14044\end{array}$ $\begin{array}{lll}2.080729 & 1.910803 & 1.740863\end{array}$ $\begin{array}{lll}1.889043 & 1.743077 & 1.597114\end{array}$ $\begin{array}{lll}1.971889 & 1.823747 & 1.675598\end{array}$

$\begin{array}{crr}500 \text { KVA } & 750 \text { KVA } & 1000 \text { KVA } \\ 100 & 150 & 200 \\ 150 & 225 & 300 \\ 300 & 450 & 600 \\ 350 & 525 & 700\end{array}$

$\%$ Loss

500 KVA 750 KVA 1000 KVA $\begin{array}{llll}1.599529 & 1.442013 & 1.28125\end{array}$ $\begin{array}{lll}1.194431 & 1.09289 & 0.97749\end{array}$ $\begin{array}{llll}1.096941 & 1.018556 & 0.928188\end{array}$ $\begin{array}{lll}1.174588 & 1.088319 & 0.999924\end{array}$

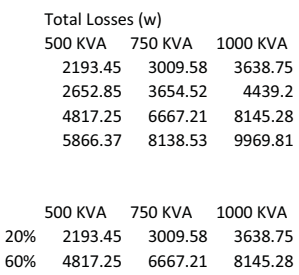
Med High 750 KVA 750 KVA 750 KVA $\begin{array}{llll}20 \% & 97.63951 & 98.31398 & 98.55799\end{array}$ $\begin{array}{lllll}30 \% & 98.0892 & 98.70572 & 98.90711\end{array}$ $\begin{array}{lllll}60 \% & 98.25692 & 98.75968 & 98.98144\end{array}$ $70 \% \quad 98.17625 \quad 98.68884 \quad 98.91168$
Low Med High Load \% 1000 KVA 1000 KVA 1000 KVA $20 \% \quad 97.85956 \quad 98.53684 \quad 98.71874$ $\begin{array}{lllll}30 \% & 98.25914 & 98.8673 & 99.02251\end{array}$ $\begin{array}{lllll}60 \% & 98.40289 & 98.89923 & 99.07181\end{array}$ $\begin{array}{lllll}70 \% & 98.3244 & 98.83547 & 99.00008\end{array}$ 

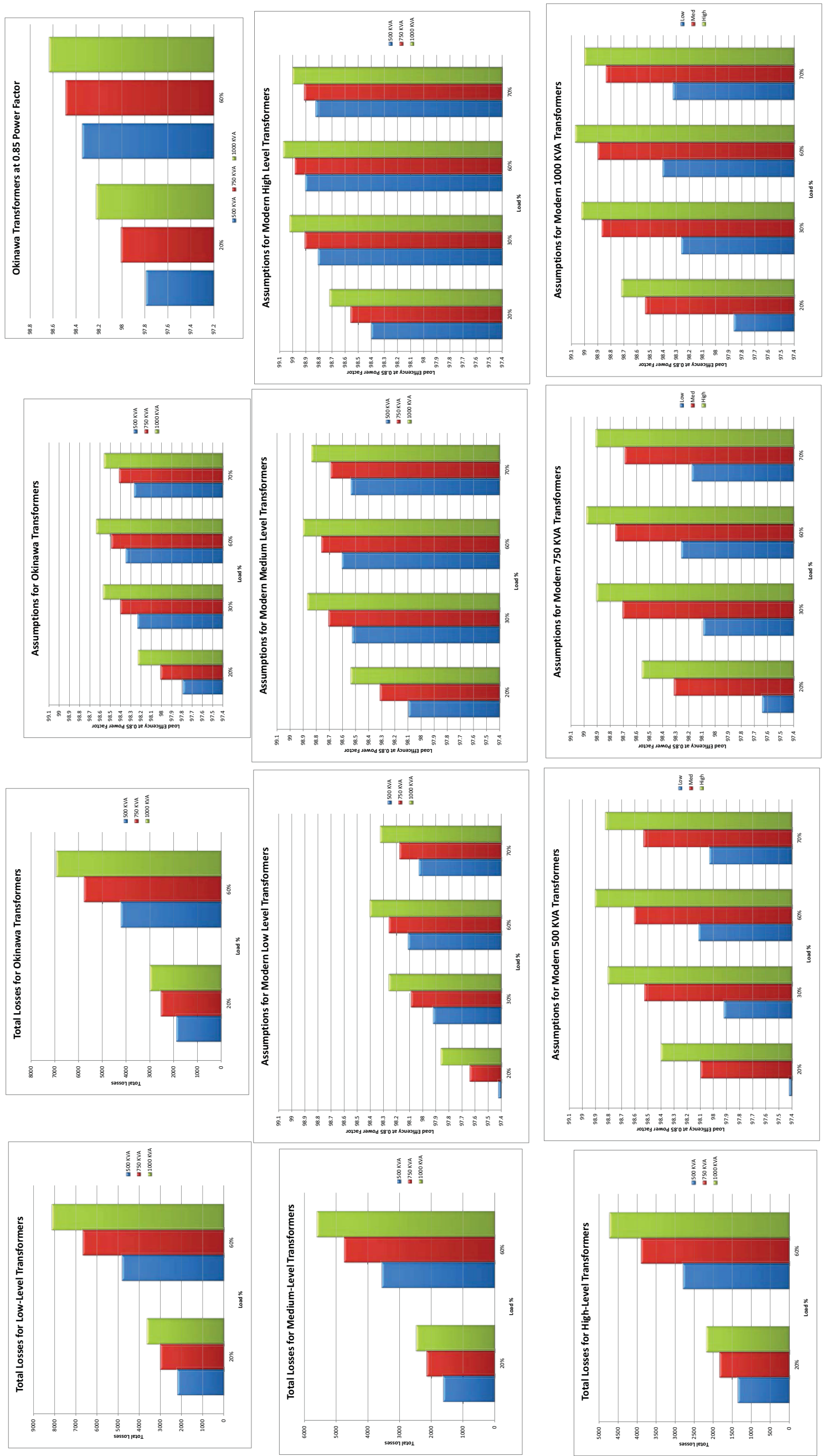


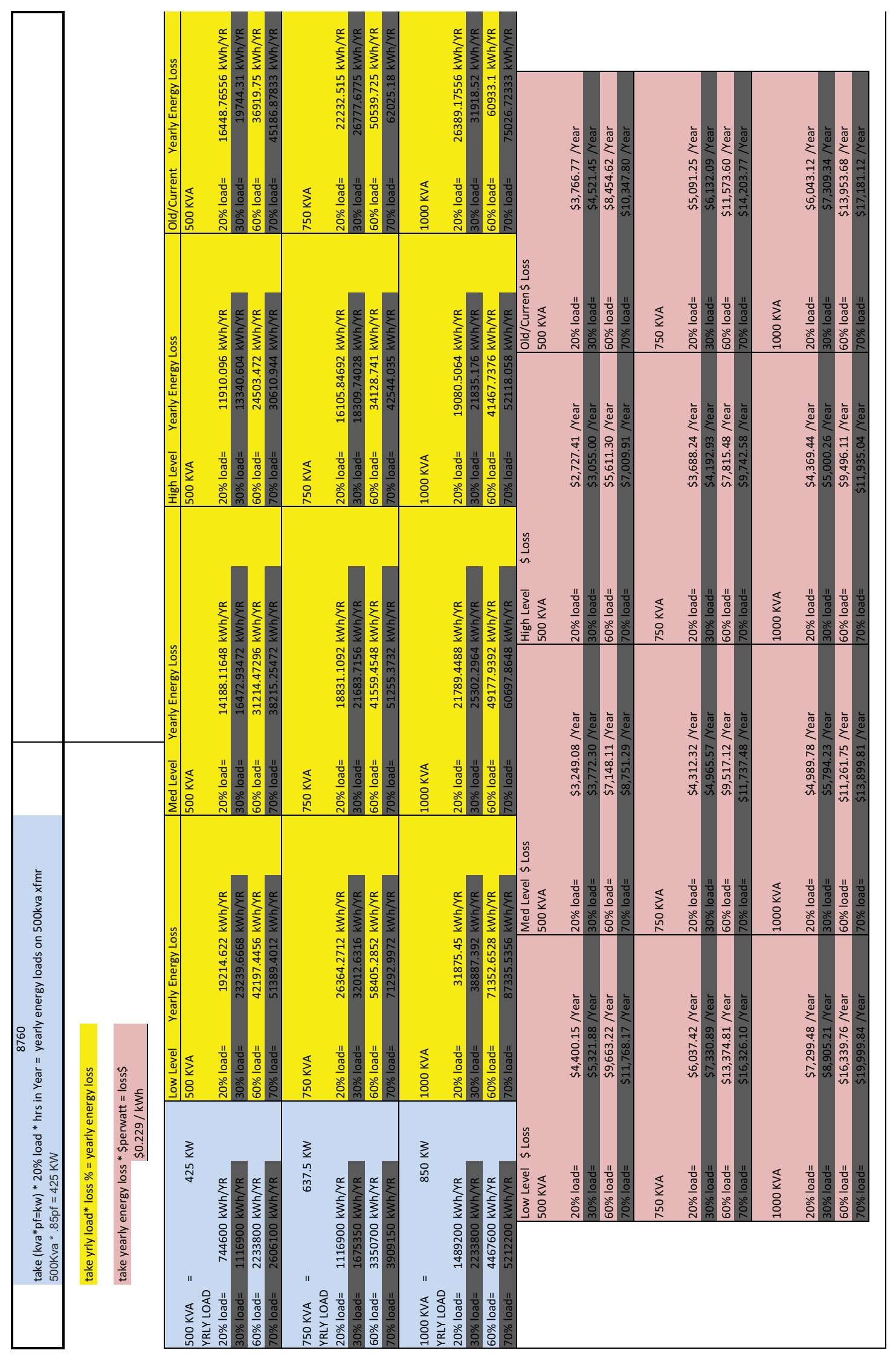




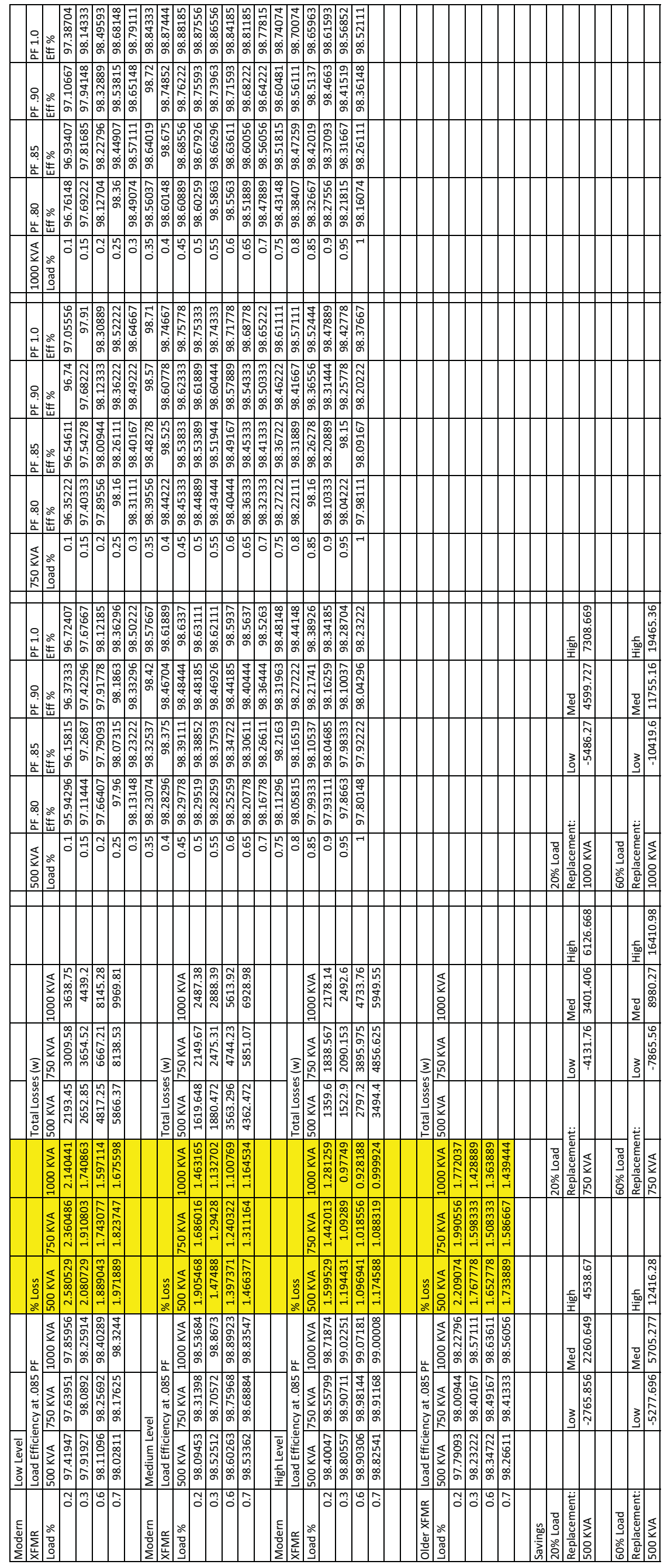

